\title{
Biochemical Characterization and Engineering of L-asparaginases for Amino Acid Depletion Therapy of Acute Lymphoblastic Leukemia
}

\author{
Dissertation \\ for the award of the degree \\ “Doctor of Philosophy" (Ph.D.) \\ Division of Mathematics and Natural Sciences \\ of the Georg-August-Universität Göttingen
}

submitted by

Christos S. Karamitros

from

Athens, Hellas

Göttingen 2014 
Member of the Thesis Committee: Dr. Manfred Konrad (Reviewer)

Enzyme Biochemistry Research Group, Max Planck Institute for Biophysical Chemistry

Member of the Thesis Committee: Dr. Heinz Neumann (Reviewer)

Department of Applied Synthetic Biology, Georg-August-Universität Göttingen

Member of the Thesis Committee: Dr. Marina V. Rodnina

Department of Physical Biochemistry, Max Planck Institute for Biophysical Chemistry

Member of the Examination Committee: Dr. Jean-Christophe Baret

Droplets, Membranes and Interfaces Research Group, Max Planck Institute for Dynamics and Self-Organization

Member of the Examination Committee: Dr. Dirk Görlich

Department of Cellular Logistics, Max Planck Institute for Biophysical Chemistry

Member of the Examination Committee: Dr. Kai Tittmann

Department of Bioanalytics, Georg-August-Universität Göttingen

Day of the oral examination: Wednesday, $\mathbf{1 8}^{\text {th }}$ of June, 2014 


\begin{abstract}
Affidavit
I hereby declare that the Thesis "Biochemical Characterization and Engineering of Lasparaginases for Amino Acid Depletion Therapy of Acute Lymphoblastic Leukemia" has been written independently and with no other sources and aids than quoted.
\end{abstract}

Christos S. Karamitros

Göttingen, the $15^{\text {th }}$ of May 2014 
As you set out for Ithaka

hope the voyage is a long one,

full of adventure, full of discovery.

Laistrygonians and Cyclops,

angry Poseidon - don't be afraid of them:

you'll never find things like that on your way

as long as you keep your thoughts raised high,

as long as a rare excitement

stirs your spirit and your body. (...)

Hope the voyage is a long one. (...)

Keep Ithaka always in your mind.

Arriving there is what you are destined for.

But do not hurry the journey at all.

Better if it lasts for years,

so you are old by the time you reach the island,

wealthy with all you have gained on the way,

not expecting Ithaka to make you rich. (...)

Ithaka gave you the marvelous journey.

Without her you would not have set out.

She has nothing left to give you now.

And if you find her poor, Ithaka won't have fooled you.

Wise as you will have become, so full of experience,

you will have understood by then what these Ithakas mean.

(Constantine P. Cavafy, 1911)

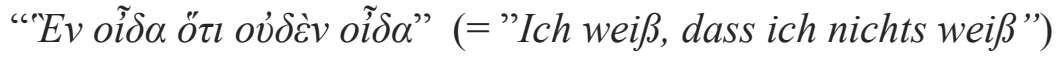

(Sokrates, 5th century B.C.) 


\section{Dedication}

I dedicate this work to all my Teachers who enabled me and made me to appreciate the ultimate meaning of Science. 


\section{Acknowledgements}

As we all realize very often, time is flying extremely fast, making us wonder how this can happen. This was indeed one of the things, that I had been thinking during the last 2 months while writing my Ph.D. Thesis. The, more than, four years period between the time point I addressed to Manfred presenting myself as a potential Ph.D. student candidate on $8^{\text {th }}$ of January 2010 and the day I am writing those acknowledgements passed unbelievably fast, yet is filled with unique experiences. Ph.D. as a life journey (or Ithaka as presented in Cavafy's poem above) has come to an end. I tried to make this voyage long and gather as many experiences as possible. However, the journey on the way to Ithaka is still continuing and this is the journey of Science, which fortunately never ends. Those four years, made me really to understand and feel that I have learned more but I know less, as compared to my starting point; and now I can also understand better the quotation " $E v$ oî $\delta \alpha$ ö $\iota$ ov̉ $\delta \dot{\varepsilon} v$ oí $\delta \alpha$ ".

At this point, I would like to express my deepest and sincere appreciation to certain people, who largely contributed to the success of this work and made me understand what I wrote in the first paragraph. First of all, I own a lot to my supervisor Dr. Manfred Konrad, for his full support, patience, trust and scientific guidance throughout this work. I really appreciate the fact that he gave me the chance to work totally independently, allowing me to unfold experimentally my thoughts. In addition, it must with no doubts granted to him the trust to the "L-asparaginase" project; a totally new project for both of us. I personally consider this as a demonstration of lack of "narrow mind" thinking. Furthermore, his global scientific thinking was a source of motivation for me to conduct research. Manfred.....your contribution was priceless. Thank you for everything.

I am indebted to the other two members of my Ph.D. Thesis committee, that helped me enormously throughout this work. I refer to Dr. Marina V. Rodnina and Dr. Heinz Neumann, who were always there for me, providing their insightful scientific guidance. I am also really thankful to Dr. Jean-Christophe Baret, Dr. Kai Tittmann and Dr. Dirk Görlich for showing interest to my work, thereby participating to the examination committee. Special thanks also to all GGNB members for their constant support and guidance for the whole paper work; they were really helpful and supportive.

I also feel the obligation to thank all the members (former and current) of our lab, who made the work at the lab really exciting: Nazim Nazimuddin, to whom I wish all the best with his 
Ph.D. Thesis, Dr. Theresa McSorley for all our useful discussions about cell culture and Ursula Welscher-Altschäffel for her outstanding technical assistance.

Additionally, I would like to give my special thanks to the Head of Nucleic Acid Chemistry group, Dr. Claudia Höbartner and her lab's members for our two years weekly joint seminars every Tuesday; as well as the Head of the Emeritus Group of Cellular Dynamics, Dr. Thomas Jovin and all the members of his lab for our weekly joint seminars every Wednesday. I had really fruitful scientific discussions with all the members of those two groups, which helped me to expand my horizons towards multidisciplinary fields. Dr. Nickels Jensen from the group of Structure and Dynamics of Mitochondria (Head: Dr. Stefan Jakobs) deserves special thanks and my personal gratitude for his assistance with the FACS data analysis and the operation of their customizable FACS instrument.

I had a really great communication with all our main collaborators and they all deserve my sincere thanks: Dr. Jean-Christophe Baret, Dr. Jiseok Lim and Philipp Gruner from the Droplets, Membranes and Interfaces Research Group (MPI-DS), Dr. Alexey M. Yashchenok from the Department of Interfaces (Max Planck Institute of Colloids and Interfaces, Potsdam) and Dr. Arnon Lavie (University of Illinois, at Chicago) for the crystallographic work.

I am immensely beholden to Dr. George Georgiou (Department of Chemical Engineering, Biomedical Engineering and Institute for Cell and Molecular Biology, University of Texas at Austin) who gave me the great opportunity to work in his lab for 3 months, getting introduced to FACS instrumentation and analysis, as well as for all our fertile discussions about protein engineering. I would like also to acknowledge Dr. Thomas Bornemann for all our useful discussions about ITC, which I really enjoyed. The whole GGNB program and particularly the program "Biomolecules: Structure-Function-Dynamics" deserves enormous grants for the organization of all this massive list of courses offering the possibility to the candidates to step into different labs and get an idea of what other people are doing. My sincere thanks to Max Planck Society (monthly stipend), GGNB program (travel grants) and Dr. George Georgiou (supplementary monthly stipend during my 3-months stay at his lab) for my financial support throughout all those years.

I feel the need to express my gratefulness to my former supervisor Dr. Nicholas Labrou, for having given me the opportunity to work at his lab of Enzyme Technology, which was my first contact with the fascinating world of enzymes. 
Last but not least, I would like to express my deepest appreciation, love and respect to my parents Stergios Karamitros and Maria Karamitrou, who have always been on my side supporting me by any means. They have been an overwhelming source of inspiration for me and I owe them, literally speaking, everything I have achieved up until now. 


\begin{abstract}
The goal of the present work was to form a basis for the development of improved protein therapeutics against acute lymphoblastic leukemia (ALL). Bacterial L-asparaginases, in combination with other chemotherapeutics, are currently used for the treatment of ALL. This chemotherapy approach is limited by the elicitation of many immune responses to patients, mainly attributed to the bacterial origins of the used enzymes. A potential strategy to circumvent such imitations involves the replacement of the bacterial enzymes by human molecules which could drastically eliminate severe side effects arising from the immunogenicity. However, human enzymes which display L-asparaginase activity cannot be used for such treatment, because of their poor catalytic properties and therefore, protein engineering approaches for their catalytic improvement are inevitable. Overall, two novel human L-asparaginases were studied, namely human ASNase1 (hASNase1) and human ASNase3 (hASNase3), with the latter one being also structurally characterized.

Wildtype hASNase3 was used as template for mutagenesis and subsequent screening steps aiming at the identification of catalytically improved variants. A FACS-based highthroughput screening system was employed, which correlates semi-quantitatively the intracellular eGFP fluorescence intensity with the L-asparaginase activity. The system is based on a five-gene-deletion Escherichia coli (E.coli) strain (all genes which contribute to the biosynthesis of L-aspartate have been deleted) whose growth is exclusively dependent on the availability of exogenous L-aspartate, product of the L-asparaginase catalytic activity. The intracellular expression of hASNase 3 variants can rescue the bacterial cells from the lack of L-aspartate since they can produce this amino acid through activity of these variants. The availability of L-aspartate reflects the expression levels of eGFP, and this, in turn, correlates the intracellular fluorescence with the L-asparaginase activity. Applying this screening strategy, overall five mutant libraries were analyzed (one generated via epPCR, and four via site-saturation mutagenesis), and eventually three human ASNase3 variants with improved catalytic properties against the hydrolysis of L-asparagine were identified and isolated, with the best one being 6-fold better than the wild type.

In addition, a novel high-throughput screening platform was developed by capitalizing on the rising field of droplet-based microfluidics. This approach allows the compartmentalization in
\end{abstract}


very small water-in-oil emulsions of different types of chemical and/or enzymatic reactions, thereby monitoring the course of the reactions continuously. To this end, a novel fluorescent, three-step coupled assay for L-asparaginase was developed in order to be able to measure quantitatively enzymatic reactions in volumes of the range 500-600 pL. For standardizing the system at the single-cell level, the current antileukemic drug Escherichia coli L-asparaginase 2 was used, which was displayed on the inner membrane of E.coli cells. Individual cells displaying the enzyme were compartmentalized, and the assay was validated by measuring the activity of the displayed L-asparaginase. Our experimental results demonstrate that this setup allows the quantitative determination of single-cell enzymatic activities, thus being suitable for the screening of directed evolution mutant libraries not only for human Lasparaginases but also for other enzymes in general.

Besides hASNase3, we additionally characterized another human L-asparaginase, namely hASNase1. It was shown that this enzyme which comprises the N-terminal domain of an overall $60-\mathrm{kDa}$ lysophospholipase and resembles the cytoplasmic bacterial E.coli Lasparaginase 1, can form an independent folding and catalytic unit. Strikingly, despite its monomeric state, hASNasel displayed a very pronounced sigmoidal steady-state kinetic profile, hallmark of allosteric enzymes. Its catalytic properties are poorer than those of hASNase3, thus making its engineering task more challenging.

As a complementary strategy to the engineering of human enzymes for improvement of ALL therapy, we focused on the utilization of drug delivery approaches as means for the prolongation of the half-life of L-asparaginases under physiologically relevant conditions. By encapsulating Saccharomyces cerevisiae L-asparaginase 1 (ScASNase1) in multilayer polyelectrolyte microcapsules consisting of biocompatible and biodegradable materials, it was shown that the enzyme's thermal stability and its resistance against proteolysis can be dramatically improved. In addition, the isothermal inactivation rate at $37{ }^{\circ} \mathrm{C}$ of the encapsulated enzyme was considerably lower as compared to the free enzyme, thus suggesting that the encapsulated enzyme can retain its activity at physiologically relevant temperatures longer than its free state. Ultimately, it was demonstrated that unlike preparations of free enzyme, microcapsules filled with active ScASNase1 can kill leukemic cells in-vitro even in the presence of a mixture of proteases which degrade the free enzyme. These results further suggest that encapsulation of the enzymes can prevent their degradation from proteases, thereby prolonging their half-life and consequently allowing them to kill 
leukemic cells. Similar results were obtained when the experiments were done using E.coli Lasparaginase 2 , the current antileukemic drug. 


\section{Table of Contents}

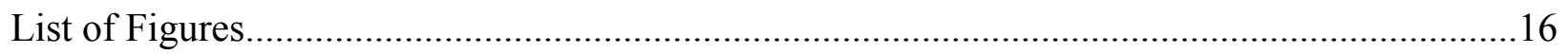

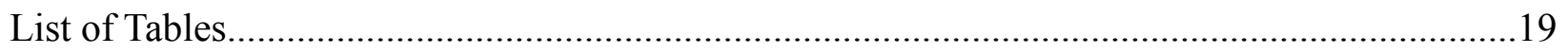

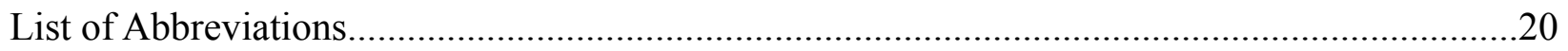

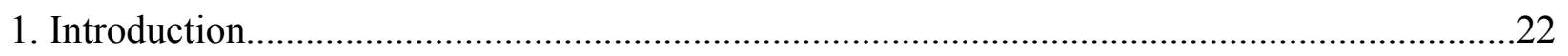

1.1 Physiological role and general classification of L-asparaginases.......................................22

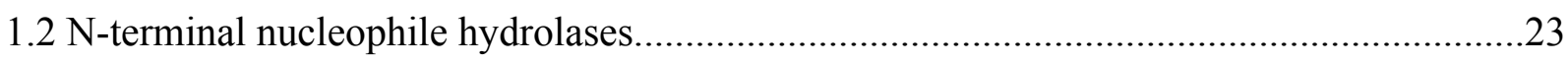

1.2.1 Intramolecular activation mechanism of Ntn-hydrolases.............................................24

1.2.2 Structural and sequence analysis of Ntn-hydrolases.................................................26

1.3 Bacterial-type L-ASNases..........................................................................................

1.3.1 Catalytic mechanism of bacterial-type L-ASNases.......................................................31

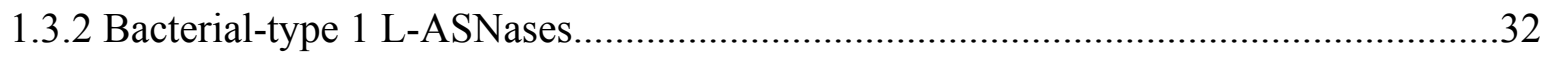

1.3.3 Bacterial-type 2 L-ASNases...................................................................................36

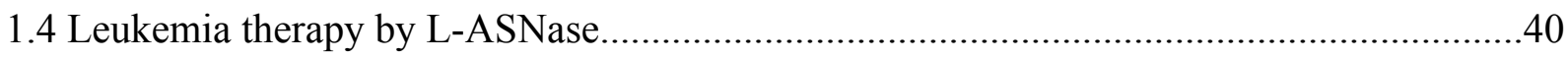

1.4.1 Types of Leukemia................................................................................................41

1.4.1.1 Myeloid Leukemia..........................................................................................

1.4.1.2 Lymphoblastic Leukemia.........................................................................42

1.4.2 Acute Lymphoblastic Leukemia treatment with L-ASNase.........................................43

1.4.3 Side effects as a result of L-ASNase treatment..........................................................45

1.5 Avenues to improve L-ASNase-based ALL therapy............................................................... 46

1.5.1 Polyethylene-glycol (PEG) modification of EcASNase2 ............................................47

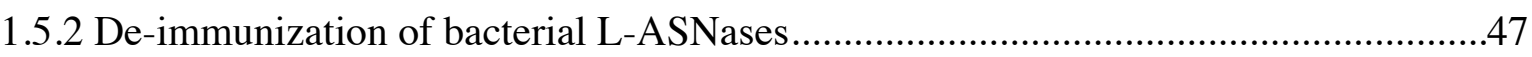

1.5.3 Replacement of the bacterial enzymes by engineered human homologs......................48

1.5.4 Encapsulation of L-ASNases applying drug delivery technologies...............................49

1.6 Development of high-throughput screening platforms for protein directed evolution

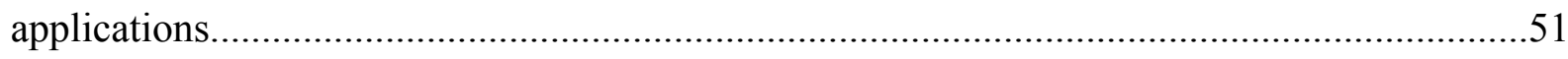

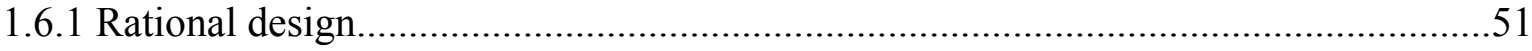

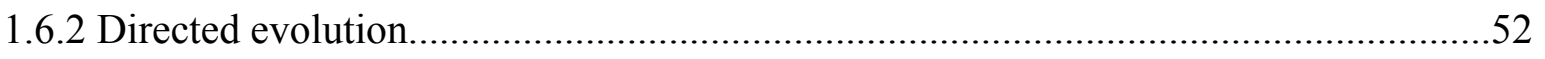

1.6.3 Fluorescence Activated Cell Sorting (FACS)-based 


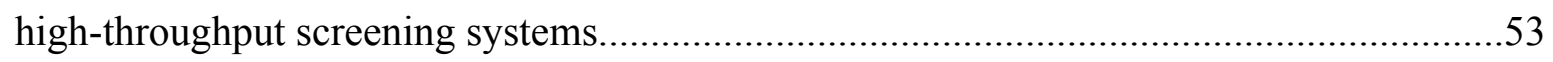

1.6.3.1 Introduction to flow cytometry instrumentation...............................................53

1.6.3.2 Applications of flow cytometry and cell sorting in screening mutant

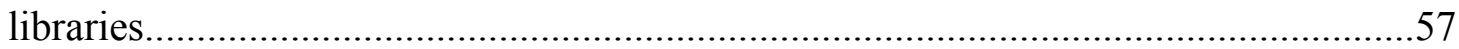

1.6.3.3 Fluorescent screens for enzymatic activity determination by FACS...................58

1.6.4 Droplet-based microfluidic setups as high-throughput screening systems...................63

1.6.4.1 Introduction to droplet-based microfluidics.....................................................63

1.6.4.2 Key characteristics of droplet-based microfluidic platforms.............................65

1.6.4.3 Applications of droplet-based microfluidic platforms.........................................67

1.7 Polyelectrolyte capsules for drug delivery applications..................................................... 72

1.7.1 Formation of polyelectrolyte microcapsules............................................................72

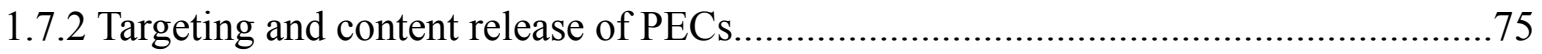

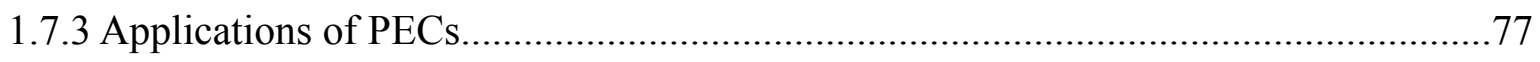

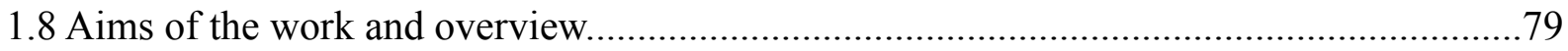

2. Free glycine accelerates the autoproteolytic activation of human asparaginase........................81

3. Bacterial co-expression of the $\alpha$ and $\beta$ protomers of human L-asparaginase-3:

Achieving essential $\mathrm{N}$-terminal exposure of a catalytically critical threonine located

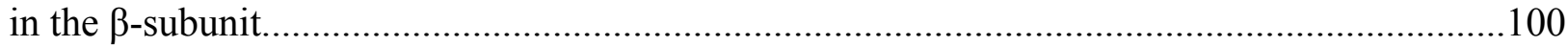

4. An Amplex Red-based fluorometric and spectrophotometric assay for

L-asparaginase using its natural substrate............................................................................116

5. Development and application of high-throughput screening methodologies for

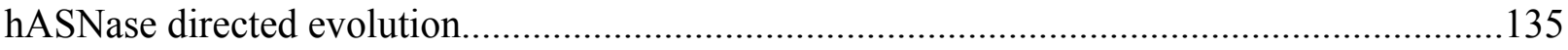

5.1 FACS-based screening of hASNase3 mutant libraries.....................................................136

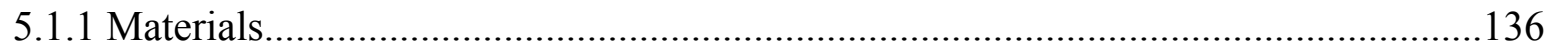

5.1.1.1 Plasmids, E.coli strains, cDNA, and chemicals............................................136

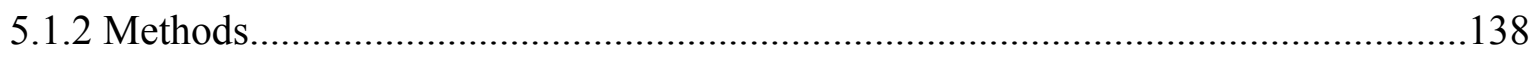

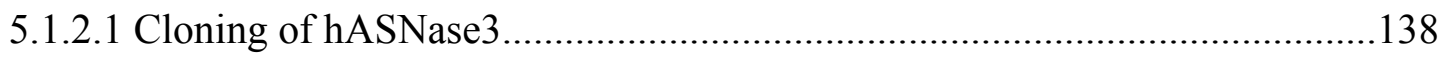

5.1.2.2 Expression and purification of recombinant enzymes....................................138

5.1.2.3 Assays for L-asparaginase activity determination and kinetic 
characterization of the enzyme.

5.1.2.4 Generation of hASNase3 mutant libraries.

5.1.2.5 Screening steps of hASNase3 mutant libraries

5.1.3. Results

5.1.3.1 Genetic complementation of L-ASNase deficiency in E.coli. 158

5.1.3.2 FACS analyses of hASNase3 mutant libraries. 160

5.1.3.3 Identification of catalytically improved mutants from SSM-LIB3 and SSM-LIB4

5.1.3.4 Expression, purification, and kinetic characterization of five hASNase 3 variants selected from the libraries. .168

5.2 Development of a droplet-based microfluidic screening system................................175

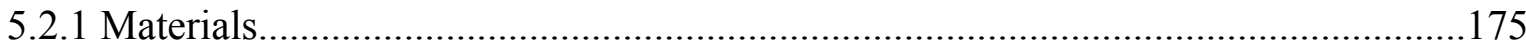

5.2.1.1 Plasmids, E.coli strains and chemicals.................................................... 175

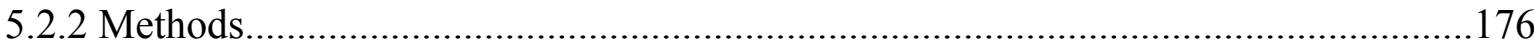

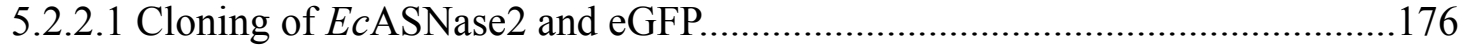

5.2.2.2 Droplet-based microfluidic system: Preparation and quantitative

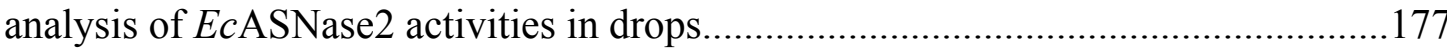

5.2.2.3 Preparation and encapsulation of eGFP-expressing cells............................180

5.2.2.4 Cytoplasmic expression of EcASNase2 and trials for lysing

the cells in droplets for subsequent enzymatic activity determination

5.2.2.5 Anchoring, detection, and quantification of EcASNase2 displayed in the inner membrane of E.coli cells

5.2.2.6 Monitoring L-asparaginase activity of EcASNase2 displayed

in E.coli cells 183

5.2.3 Results 183

5.2.3.1 Validation of the Amplex Red-dependent assay in droplets 183

5.2.3.2 Encapsulation of single E.coli cells displaying EcASNase2

in droplets and determination of the enzymatic activity 186

6. Human 60-kDa Lysophospholipase contains an N-terminal L-asparaginase domain 
which is allosterically regulated by L-asparagine.....

7. Preserving catalytic activity and enhancing biochemical stability of the

therapeutic enzyme asparaginase by biocompatible multilayered polyelectrolyte

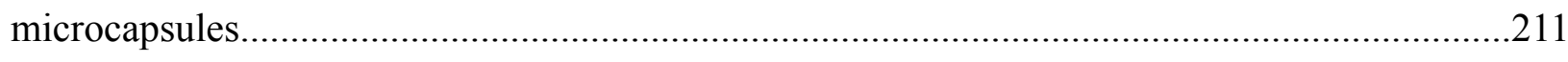

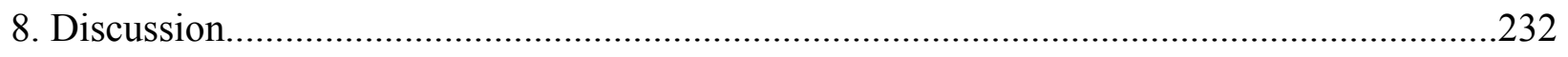

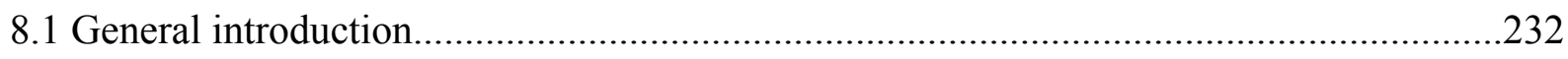

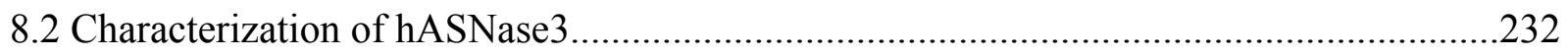

8.2.1 Structural characterization and glycine-induced intramolecular activation of

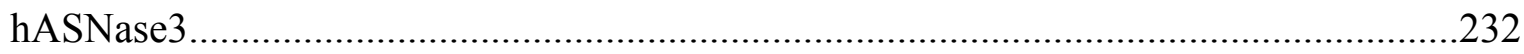

8.2.2 Circular permutant-like version of hASNase 3 ................................................235

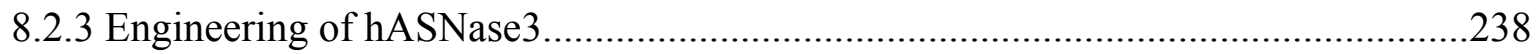

8.2.3.1 FACS-based high-throughput screening of hASNase3 mutant libraries........238

8.2.3.2 Development of a droplet-based microfluidic high-throughput screening

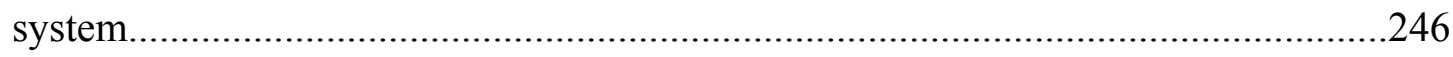

8.3 Biochemical characterization of hASNase1 ..........................................................251

8.4 Characterization of polyelectrolyte microcapsules filled with catalytically active

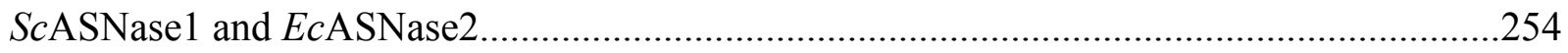

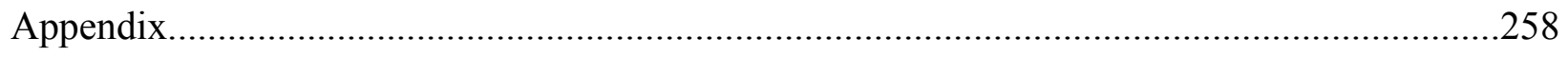

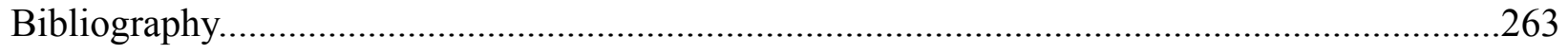

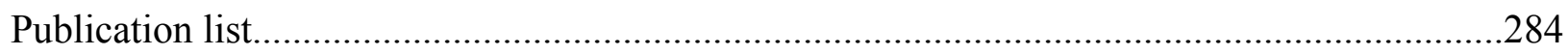

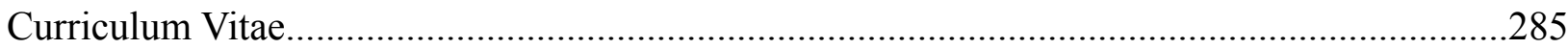




\section{List of Figures}

\section{Introduction}

Figure 1: Schematic representation of the different families of L-ASNases.......................23

Figure 2: Proposed model for the autoproteolytic activation of $\mathbf{N t n - h y d r o l a s e s . . . . . . . . . . . . . . . . . 2 5 ~}$

Figure 3: Amino acid sequence alignment of Ntn-hydrolases........................................27

Figure 4: Structure representation of Ntn-hydrolases..................................................28

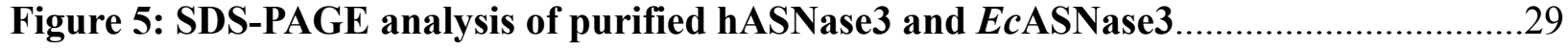

Figure 6: Proposed catalytic mechanism of L-ASNases..................................................32

Figure 7: Sequence alignment of bacterial type-1 L-ASNases.........................................34

Figure 8: Structural representation of bacterial type-1 L-ASNases................................35

Figure 9: Sequence alignment of bacterial type-2 L-ASNases..........................................37

Figure 10: Structural representation of bacterial type-2 L-ASNases...............................38

Figure 11: Human blood cells categorization and development.....................................40

Figure 12: Bone marrow with healthy cells and cancerous cells..................................43

Figure 13: Hydrodynamic focusing process of a flow cytometer......................................54

Figure 14: Application of light generated usually by lasers onto the flow stream...............55

Figure 15: Schematic representation of the sorting process.......................................56

Figure 16: Different FACS-screening approaches which involve

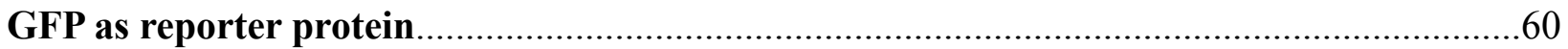

Figure 17: Schematic representation of the Cre recombinase

FACS-based screening system.

Figure 18: Principle of FACS-based high-throughput screening system for

EcASNase2 using GFP as reporter protein.

Figure 19: Snapshot of droplet formation process.....................................................64

Figure 20: Home-made chip used in droplet-based microfluidic setups...........................65

Figure 21: Overview of the most common droplet manipulation procedures.....................66

Figure 22: Schematic representation of the major processes, strategies 
and techniques of directed evolution

Figure 23: Layer-by-Layer (LbL) assembly for polyelectrolyte (PEC) microcapsule

formation. .73

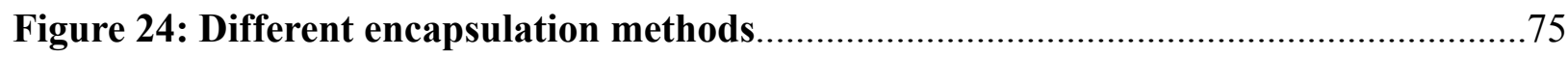

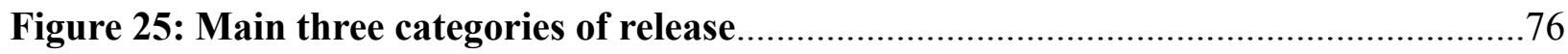

\section{Chapter 5.1: FACS-based screening of hASNase3 mutant libraries}

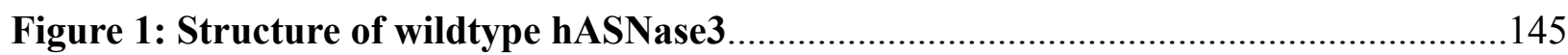

Figure 2: Randomized residues of the SSM-LIB1 ................................................... 146

Figure 3: Randomized residues of the SSM-LIB2 ............................................... 147

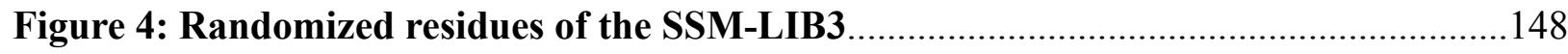

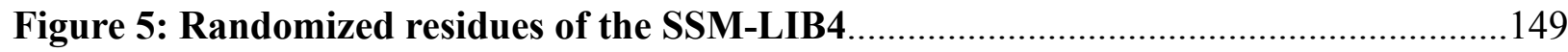

Figure 6: Overlap extension PCR method.................................................................. 150

Figure 7: Principle of the genetic complementation screening system............................152

Figure 8: Principle of the FACS-based screening system

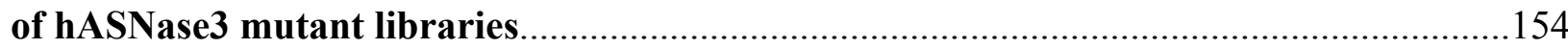

Figure 9: Overall scheme of the whole screening process...........................................157

Figure 10: FACS fluorescence profiles obtained for hASNase3- and

EcASNase2-dependent complementation in the E.coli strain JC1(DE3) which is

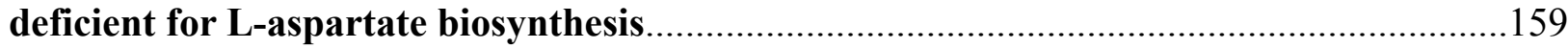

Figure 11: Fluorescence profiles of the four sorting rounds

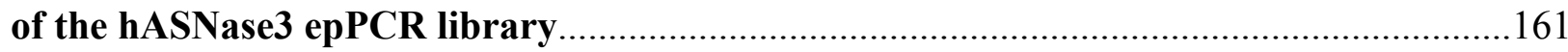

Figure 12: Fluorescence profiles of the three sorting rounds

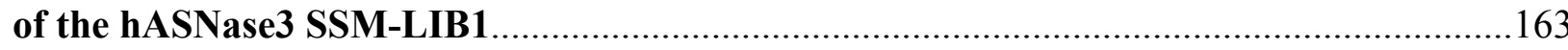

Figure 13: Fluorescence profiles of the three sorting rounds

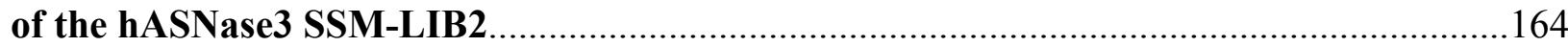

Figure 14: Fluorescence profiles of the two sorting rounds

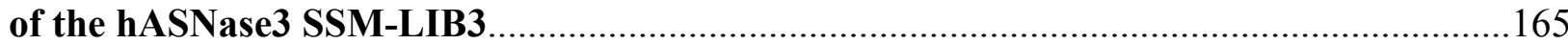


Figure 15: Fluorescence profiles of the two sorting rounds

of the hASNase3 SSM-LIB4. .166

Figure 16: 15\% SDS-PAGE analysis of hASNase 3 wildtype and

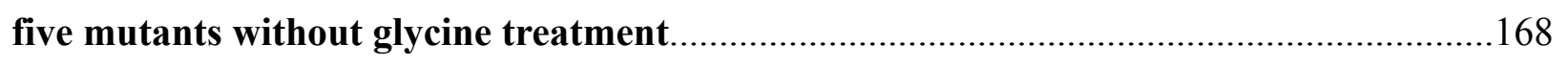

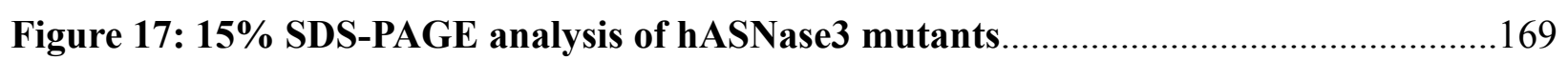

Figure 18: Steady-state kinetic plots for wildtype and mutant hASNase3 enzymes...........171

Figure 19: Steady-state kinetic plots for QDM1 and QDM2 hASNase3 enzymes..............171

Figure 20: Differential scanning fluorimetry melting curves

for wildtype and mutant hASNase 3 enzymes.

\section{Chapter 5.2: Development of a droplet-based microfluidic screening system}

Figure 1: Schemes of plasmids used in this study...

Figure 2: Microfluidic chip $(\sim 1 \times 4 \mathrm{~cm})$ used for the recombinant EcASNase2 activity

measurements.

Figure 3: Schematic experimental setup for monitoring the activity of EcASNase2 in

droplets.

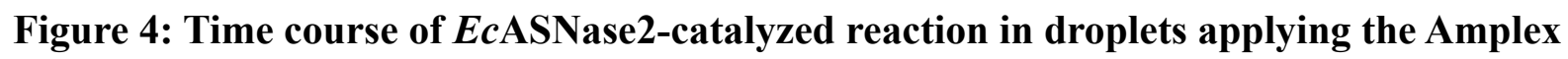

Red assay.

Figure 5: Linearity test and Michaelis-Menten plot for EcASNase2 applying the Amplex

Red assay in microscopic and macroscopic volumes......

Figure 6: Trapping of eGFP-expressing E.coli cells in microchannels...

Figure 7: Comparison of expression levels between cytoplasmically expressed and inner membrane anchored $E c$ ASNase2, immunoblotting analysis of anchored $E c A S N a s e 2-H_{1}$-C and purified $E c A S N a s e 2-H i s_{6}-C$, and standard curve generated based on the band intensities of known amounts of immunoblotted EcASNase2-His6-C.

Figure 8: $E c A S N a s e 2$ activity determination in case of cytoplasmic expression, and display in the inner membrane. 


\section{List of Tables}

\section{Introduction}

Table 1: Biochemical and structural characteristics of different L-ASNases

Table 2: Comparison of cost and time required for screening, using traditional robotic microtiter plates and droplet-based microfluidic setups.

\section{Chapter 5.1: FACS-based screening of hASNase3 mutant libraries}

Table 1: Oligonucleotides used for the generation of $\mathrm{hASNase} 3$ libraries

Table 2. Different epPCR conditions tested for determining the error rates using as

template the pET14b-SUMO plasmid

Table 3: Steady-state kinetic constants for wildtype hASNase3

and all mutants selected by FACS analyses.

Chapter 5.2: Development of a droplet-based microfluidic screening system

Table 1. Oligonucleotides used in this study. 


\section{Abbreviations}

ABS

AFU

ALL

AML

APEx1

CLL

CML

DM1

DM2

DM3

DFS

EcASNase1

EcASNase2

EcASNase3

eGFP

epPCR

FACS

FAD

hASNase1

hASNase3

hAGA

HRP

IPTG

L-AspOx

LbL
Absorbance

Arbitrary Fluorescence Units

Acute Lymphoblastic Leukemia

Acute Myeloid Leukemia

Anchored Periplasmic Expression

Chronic Lymphoblastic Leukemia

Chronic Myeloid Leukemia

Double Mutant 1

Double Mutant 2

Double Mutant 3

Differential Scanning Fluorimetry

Escherichia coli L-asparaginase 1

Escherichia coli L-asparaginase 2

Escherichia coli L-asparaginase 3

enhanced Green Fluorescent Protein

error prone Polymerase Chain Reaction

Fluorescence Activated Cell Sorting

Flavin Adenine Dinucleotide

human L-asparaginase 1

human L-asparaginase 3

human Aspartyl-GlucosAminidase

HorseRadish Peroxidase

Isopropyl $\beta$-D-1-thiogalactopyranoside

L-Aspartate Oxidase

Layer-by-Layer 
LlASNase

NADH

Ntn-hydrolase

PECs

PhASNase1

QDM1

QDM2

SD

SSM-LIB
Lupinus luteus L-asparaginase

Nicotinamide adenine dinucleotide

$\mathrm{N}$-terminal hydrolase

Poly-Electrolyte Capsules

Pyrococcus horikoshii L-asparaginase 1

QuaDruplet Mutant 1

QuaDruplet Mutant 2

Standard Deviation

Site Saturation Mutagenesis Library 


\section{Introduction}

\subsection{Physiological role and general classification of L-asparaginases}

L-asparaginases (EC 3.5.1.1; L-asparagine amidohydrolase; L-ASNase) are enzymes which primarily catalyze the conversion of L-asparagine (L-Asn) to L-aspartic acid (L-Asp) and ammonia, while some of them are also able to hydrolyze L-glutamine (L-Gln) to L-glutamic acid (L-Glu) and ammonia. These enzymes are present in all kingdoms of life from microbes to higher mammals and play an essential role in the amino acid and nitrogen metabolism [1-3]. L-ASNases are generally classified into two major families of enzymes which are characterized by evolutionarily distinct structural and biochemical properties: the plant-type $[4,5]$ and the bacterial-type L-ASNases [6,7]. Bacterial-type enzymes have attracted notable scientific and medical interest during the last fifty years, because homologs from this family have been extensively used in therapeutic regimens against blood malignacies [8-10]. This wide interest in these enzymes has led to a systematic study of their structural and biochemical characteristics. This superfamily of L-ASNases includes enzymes which exhibit variable affinities against L-Asn covering a $\mathrm{K}_{\mathrm{M}}$ range from micromolar to millimolar, though displaying a high degree of structural homology and sharing similar catalytic mechanisms [11]. Additionally, a particular common property of these enzymes is their tendency to form dimeric and tetrameric states, with the latter being the predominant oligomeric form $[12,13]$. In contrast, the plant-type enzymes have been less studied. Although conceptually their significant role in the nitrogen metabolism of plants has been recognized very early in the 1930s, they have been brought to the center of L-ASNase research only during the last two decades. Literature of the late 1980s [14] classified the plant-type L-ASNases into $\mathrm{K}^{+}-$ dependent and $\mathrm{K}^{+}$-independent subtypes, while the molecular basis for this distinction of catalytic mechanisms is unclear. At this point, it must be underlined that, while bacterial-type homologs have been identified in eukaryotic organisms like mammals and fungi, and they have been biochemically characterized, no similar enzymes have been found in plants. On the contrary, plant-type representatives naturally exist in bacteria and humans [15].

This discovery is of particular importance, since human and bacterial homologs which resemble plant-type L-ASNases belong to the so-called N-terminal nucleophile hydrolase superfamily of enzymes (Ntn-hydrolases) and constitute a relatively well-studied group of 
proteins [16]. Those findings formed the basis for the classification of plant-type L-ASNases into a broader superfamily of enzymes which embraces not only enzymes capable of hydrolyzing L-Asn, but also dipeptidases and proteases. The major common feature of all Ntn-hydrolases is their post-translational autoproteolytic activation as well as their structural fingerprint of an $\alpha \beta \beta \alpha$ motif [17]. In the following sections, the two main superfamilies of LASNases will be presented, and their structural and biochemical properties will be discussed. Maintaining the most recent and widely accepted classification of the plant-type enzymes as a sub-group of the Ntn-hydrolases, they will be explored according to their specific and their common properties as members of this interesting superfamily of enzymes. A schematic illustration of the aforementioned grouping of L-ASNases is shown in Figure 1 below.

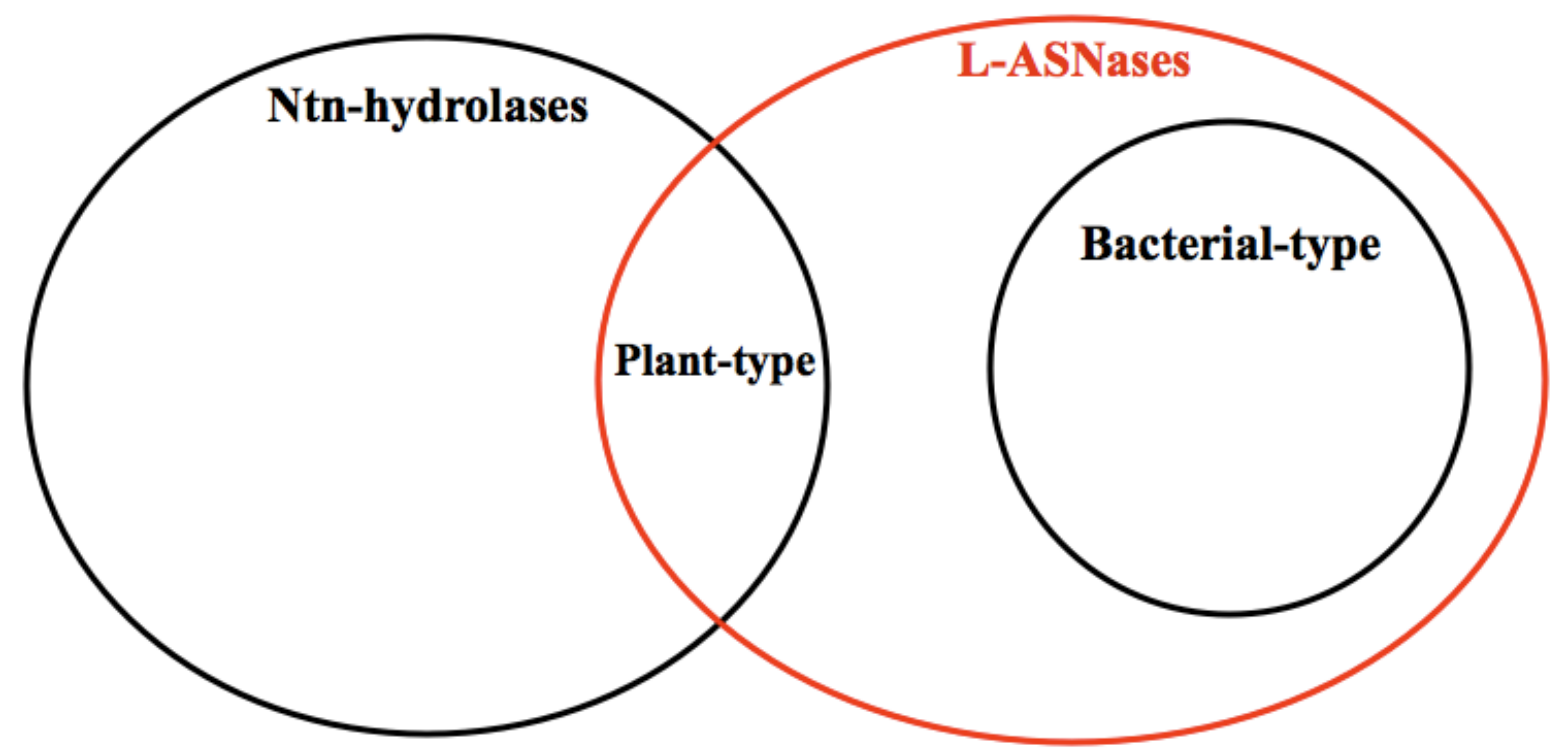

Figure 1. Schematic representation of the different families of L-ASNases. Bacterial-type enzymes constitute a well-studied and characterized superfamily with similar structural characteristics, though distinct biochemical properties. Plant-type L-ASNases can be considered as a sub-group of the broad Ntn-hydrolases superfamily which also includes enzymes other than L-ASNases.

\subsection{N-terminal nucleophile hydrolases}

The N-terminal nucleophile hydrolase superfamily (Ntn-hydrolases) has been defined as a new protein family in 1995 [18]. It includes enzymes which catalyze different enzymatic reactions such as aspartylglucosaminidases [19], penicillin acylases [20], Taspase1 [21], and plant-type L-ASNases [22]. Despite their limited amino acid sequence similarity, the Ntn- 
hydrolases share a common sandwich-like $\alpha \beta \beta \alpha$ folding motif ( $\alpha$ refers to $\alpha$-helix, and $\beta$ to $\beta$ sheet) which is highly conserved among the members of this family. A principal property of this family of enzymes is their expression as inactive precursors that post-translationally undergo an intramolecular autoproteolytic activation process which generates the active enzyme [23]. Therefore, the active enzyme consists of two distinct polypeptide subunits, $\alpha$ and $\beta$, also called protomers, which remain non-covalently and tightly associated during catalysis. This intramolecular cleavage of the catalytically inactive precursor molecule occurs at a scissile peptide bond, usually between a preceding glycine (Gly) residue and a following residue which plays the role of the nucleophile and can be either L-Thr, L-Cys, or L-Ser [24]. Additionally, instead of Gly as preceding amino acid, L-Asp has been identified in some cases [25]. Upon cleavage and generation of the two newly synthesized subunits, the Gly residue will be the last residue of the $\alpha$ subunit at its C-terminus, while the nucleophile (LThr, L-Cys or L-Ser) will be exposed at the N-terminus of the $\beta$ subunit. Such N-terminal exposure of the L-Thr is absolutely crucial for the enzymatic activity, and this has been confirmed by both mutagenesis and structural studies [26]. The nucleophilic character of the catalytic $\mathrm{N}$-terminal residue of the $\beta$ subunit is responsible for the name given to this enzyme family.

\subsubsection{Intramolecular activation mechanism of Ntn-hydrolases}

The autocatalytic mechanism of the Ntn-hydrolases maturation is not yet fully understood, but it can be considered analogous to the activation of serine proteases [27]. It has been proposed that the autoproteolytic mechanism involves a nucleophilic residue (L-Thr, L-Ser or L-Cys) and a general base, which however has not yet been defined, thereby its origins remaining still speculative. It is assumed that this role of the general base can be fulfilled either by an intramolecular residue or another small metabolite molecule. A general model for this process is shown in Figure 2. It is believed that the nucleophile residue is activated by the general base which subtracts the proton from its $-\mathrm{O}(\mathrm{S}) \mathrm{H}$ group (from L-Thr, L-Ser or LCys) while the basicity of this general base could potentially be further intensified by an adjacent acidic residue [28]. 


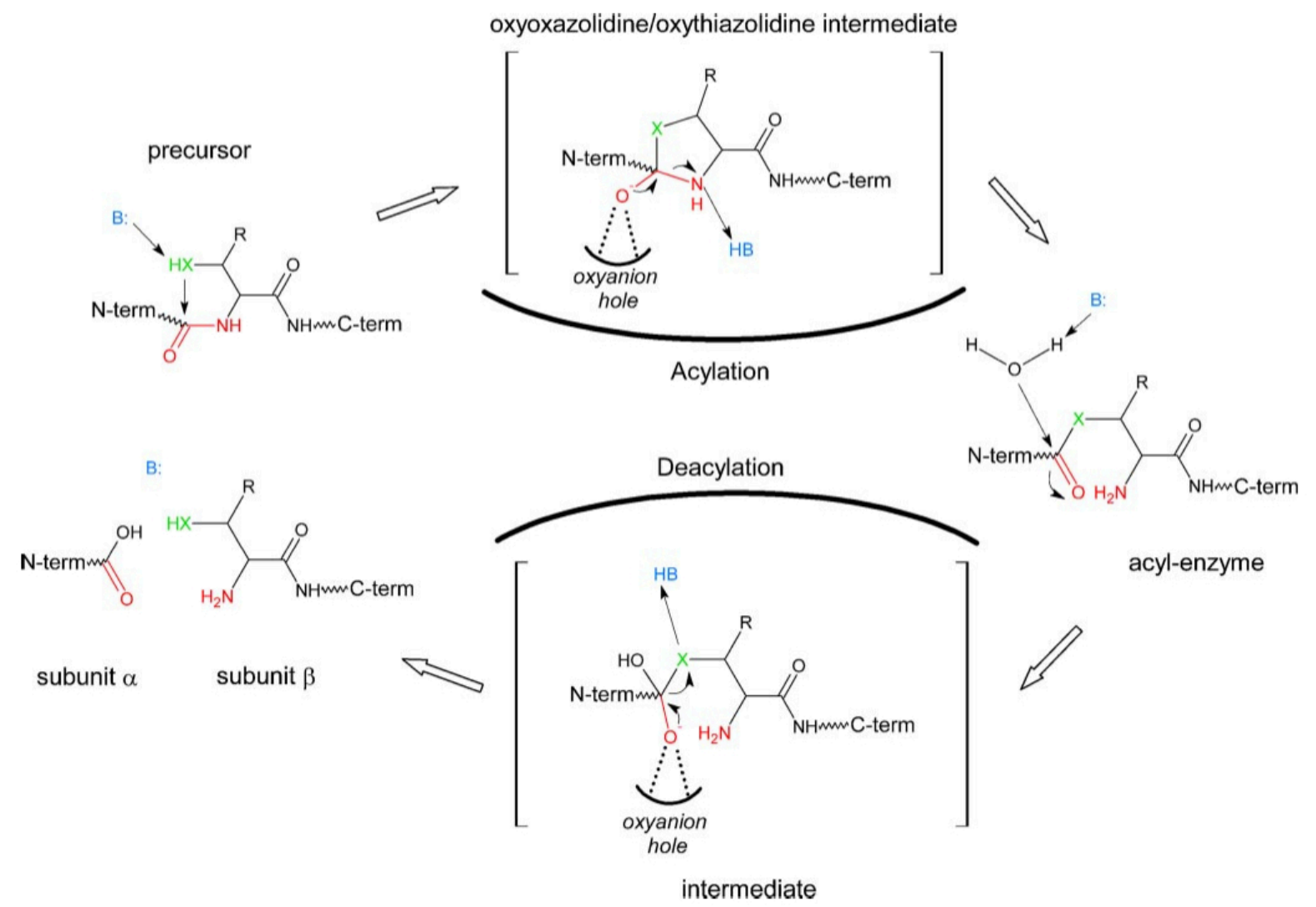

Figure 2. Proposed model for the autoproteolytic activation of Ntn-hydrolases. The general base is denoted as B: in blue, the nucleophile HX is in green, and the peptide bond at which the cleavage takes place is in red. If the nucleophile residue is L-Thr or L-Ser, then HX corresponds to - $\mathrm{OH}$, while in case of L-Cys it is - $\mathrm{SH}$. In addition, in case of L-Cys or L-Ser, the R-group is $-\mathrm{H}$, and $-\mathrm{CH}_{3}$ in case of L-Thr. Adapted from [28].

The deprotonation of the $-\mathrm{O}(\mathrm{S}) \mathrm{H}$ group of the nucleophile residue is a prerequisite for the subsequent first step of the intramolecular activation which includes an $\mathrm{N} \rightarrow \mathrm{O}$ or $\mathrm{N} \rightarrow \mathrm{S}$ acyl shift and the formation of a tetrahedral intermediate (Figure 2; oxyoxazolidine/ oxythiazolidine intermediate), which is stabilized by a so-called oxyanion hole. This oxyanion hole is essential for the stabilization of the acyl/deacyl-intermediates and is formed between the oxygen atom of the former peptide bond and usually a neighboring amide group which points towards this oxygen. The generation of this intermediate is induced upon the nucleophilic attack of either the deprotonated hydroxyl or thiol group on the peptide carbonyl $\mathrm{C}$ atom [28]. In certain representatives of the Ntn-hydrolase superfamily which have been structurally well-studied, such as aspartylglucosaminidases, the role of the base which activates the nucleophile seems to be fulfilled by either an L-His or an L-Asp residue, Nterminal to the scissile peptide bond [29]. Yet, the handicap with this model of the L-His, L- 
Asp, L-Thr (L-Cys or L-Ser) autoactivation triad is that the L-His and L-Asp residues are not conserved throughout the Ntn-hydrolases, and therefore it cannot be considered as a general activation mechanism. Therefore, it appears reasonable to point out that the lack of a consensus sequence profile comprising the autocleavage region of Ntn-hydrolases impedes even further the elucidation and interpretation of the molecular mechanism of autoproteolysis.

\subsubsection{Structural and sequence analysis of Ntn-hydrolases}

A well characterized mammalian member of the Ntn nucleophile hydrolase superfamily is the human lysosomal aspartylglucosaminidase (hAGA), which catalyzes the hydrolysis of glucosylated L-Asn molecules, generated during proteolytic breakdown of glycoproteins [30]. Deficiency of AGA causes the inborn lysosomal storage disease aspartylglucosaminuria [31]. Yet, hAGA is capable of hydrolyzing L-Asn, though with poor catalytic characteristics. The human genome encodes another enzyme of this Ntn hydrolase family, variably termed human asparaginase-like protein 1 (hASRGL1) [32], glial asparaginase (GLIAP) [33], CRASH [34], or hASNase3 due to its high homology with E.coli L-ASNase3 (encoded by the iaaA gene) [35]. Crystal structures of the wild-type form [36] and of a circular permutant version of hASNase3 [37] have been reported, while in a recent study we have shown that its autoproteolytic activation can be drastically and selectively accelerated by the free amino acid glycine, which could potentially play the role of the essential base that is required for the deprotonation of the -OH group of the nucleophile residue [38]. Yet, the generalization of this proposed model needs to be tested for other members of the Ntn hydrolase family of enzymes.

The physiological role of hASNase 3 has not yet been elucidated. It was shown that this enzyme is able to hydrolyze $\beta$-aspartyl-dipeptides (or iso-aspartyl-dipeptides) displaying $K_{M}$ in the sub-millimolar range, as well as L-Asn, though less efficiently ( $\mathrm{K}_{\mathrm{M}}$ in the millimolar range) [32]. Iso-aspartyl-dipeptides are formed upon non-enzymatic transfer of the peptide bond to the side chain of an L-Asn or an L-Asp residue, resulting in crucial protein structural rearrangements and consequently dysfunction [39]. This protein modification can be potentially repaired, or, alternatively, the affected proteins are degraded initially by proteases, and subsequently the iso-aspartyl-dipeptides are further eliminated by enzymes called iso- 
aspartyl-dipeptidases [40]. Hence, based on the catalytic properties of hASNase3, it is believed to play a key role in the cellular clearance of isoaspartate-containing peptides, degradation products of misfolded polypeptides, thereby acting as aspartyl-dipeptidase and secondarily as L-ASNase. This dual catalytic role of hASNase3 has been confirmed for its E.coli homolog (encoded by the iaaA gene) as well as for the $\mathrm{K}^{+}$-independent plant homolog from Lupinus luteus [5]. A sequence alignment between the last three aforementioned enzymes, including hAGA, is shown in Figure 3.

\footnotetext{
hASNase3 $1 \ldots . .$. MN P I VVVHGGGA G P IS - . . - KDRKERVHQGMVRAATVGYG I LREGGSAVDAVEGA 51 EcASNase3 1 ..... MGKAV I A I HGGAGA IS RA QMS LQQELR - Y I EALSA I VETGQKMLEAGESALDVVTEA 56

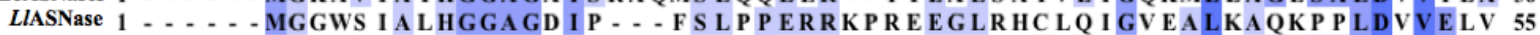
hAGA 1 MARKSNLPVLLVPFLLCQALVR - - C CS PLPLVVNTWPFKNATEAAWRALASGGSALDAVESG 60

52 VVALE - D D P EFNAGCGSVLNTNGEVEMDAS IMDGKDLSAGAVSAVQCIANP I KLARLVMEKTPH 114 57 VRLLE- ECPLFNAG I GAVFTRDETHELDACVMDGNTLKAGAVAGVSHLRNPVLAARLVMEQSPH 119 56 VRELE - N I EHFNAG I GSVLTNSGTVEMEAS IMDGNTMKCGAVS GLSTVLNP ISLARLVMDKTPH 118 61 CAMCER EQCDGS VGFGGS P D LGET T LDAMIMDGTTMDVGAVGDLRR I KNA I GVARKVLEHTTH 124

115 C FLT DQGAA QFAAAMGVPE I PGEKLVTERNKKRLEKE - . . - K KEKGAQKTDCQ - . . - . 163 120 VMM I GEGAENFAFARGMERVSPEIFS TS LRYEQLLAA - . . - RKEGATVLDHSG - . . . 168 119 I Y LAF QGA QDFAKQQGVETVDS S HL I TAENVERLKLA - . . . - I EANRVOVDYS OYNYPEPVK 175 125 TLLVGESATTFAQSMGF INEDLSTSASQALHSDWLARNCQPNYWRNV I PDPSKYCGPYKPPG IL 188 *

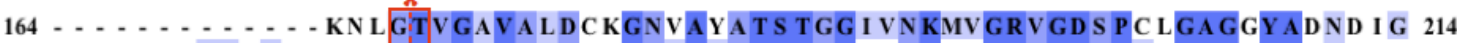
$169 \ldots . . . . A P L D E-K Q K M G T V G A V A L D L D G N L A A A T S T G G M T N K L P G R V G D S$ P LGAGCYANNASV 225 176 D D A E K L P L TNGDS O I GTVGCVAVDS HGN LASATS TGGLVNKMVGR IGDTPLIGAGTYAN - E LC 238 189 KQD I P I HKETEDDRGHDT I GMVVI HKTGH I A AGTS TNG I KFK I HGRVGDS I PGAGAYADDTAG 252

215 AVS TTGHGES I LKVNLAR L T LFH I EQ - GKTVEEAADL S L GYMKSRVKGLGGLIVVSKTGDWVAK 277 226 AVS CTGTGEVF I RA LAAYD I AA LMDYGGLS LA EACERVVMEKLPALGGSGGLIA IDHEGNVALP 289 239 A V S A T GKGEE I I RA TVARDVAA LMEFKGLS LKEAADFVI HERTP - KGTVGL IAVSAAGE IAMP 300 253 AAAATGNGD I LMR F LPSYQAVEYMRRGEDPTIACQKVISR I QKHFPEFFGAVICANVTGSYGAA 316

278 WT S T S MP WA A A KD - GKLH F G I D P D D T T I T D L P 308 290 F N T E GMY RA WGY A GD T P T T G I Y REKGD TVA T Q 321 301 F N T T GMF RACA T EDGYS E I A IWP T T . . . . - 325 317 CNKLSTFT QF S FMVYNS EKN QP TEEKVDC I - - 346
}

Figure 3. Amino acid sequence alignment of Ntn-hydrolases. human L-ASNase3 (hASNase3) (UniProt: Q7L266), E.coli (EcASNase3) (UniProt: E0J5W2), L.luteus (LlASNase) (UniProt: Q9ZSD6) and human lysosomal aspartylglucosaminidase (hAGA) (UniProt: P20933). All four enzymes belong to the Ntn-hydrolase superfamily and undergo the characteristic intramolecular post-translational autoproteolytic activation. The scissile peptide bond which is cleaved resulting in the generation of two subunits, is denoted by a red asterisk and a red frame (dotted red line indicates the cleavage between Gly-Thr) in the figure. Blue-shaded are highly conserved amino acids. Note that the nucleophile in all these cases is L-Thr, but the preceding amino acid is L-Asp in case of hAGA, unlike in the other three enzymes where this residue is Gly. The lack of a consensus sequence flanking the cleavage region makes interpretation of the molecular mechanism of activation difficult. The alignment was performed using CLUSTALW [41] and the image was prepared using JalView [42]. 
The four enzymes which are compared in the sequence alignment above in Figure 3 do not show very high degree of sequence homology, and this holds even for the regions around the scissile peptide bond. Yet, they share a very similar tertiary structure which follows an $\alpha \beta \beta \alpha$ motif, hallmark of the Ntn-hydrolases, and form dimers consisting of two activated monomers. The solved structures of hASNase3 [36,37], EcASNase3 [5], and hAGA [19] are shown below in Figure 4 for direct comparison.
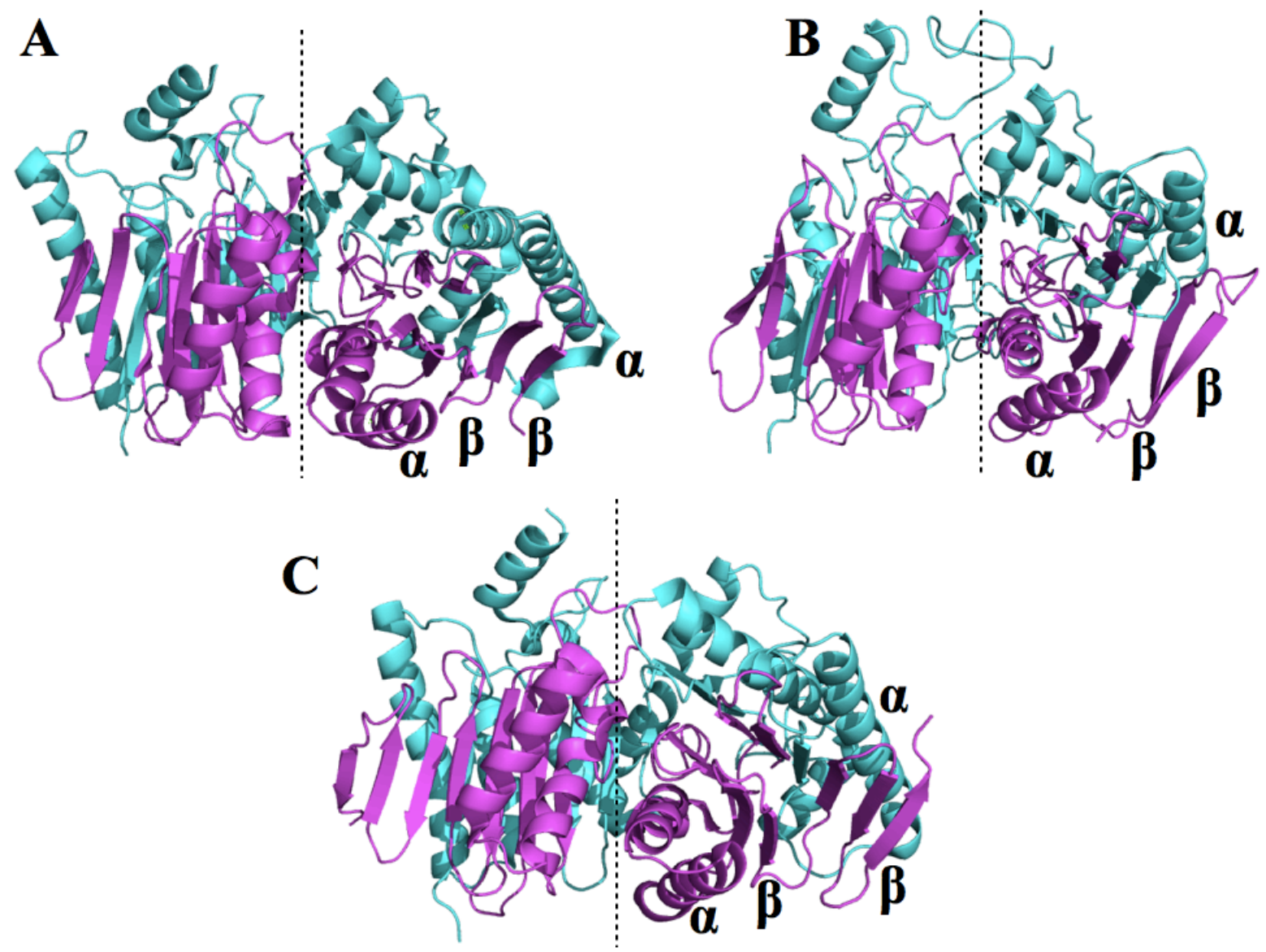

Figure 4. Structure representation of Ntn-hydrolases. (A) EcASNase3 (PDB entry: 2ZAL), (B) hAGA (PDB entry: 1APY), and (C) hASNase3 (PDB entry: 4GDW). The dotted lines indicate the dimer interfaces which consist of two identical, fully-activated monomers. In the figure is also shown the $\alpha \beta \beta \alpha$ folding motif ( $\alpha$ refers to $\alpha$-helix and $\beta$ to $\beta$-sheet) of each monomer for all three enzymes. With cyan are labelled the $\alpha$ subunits, while with magenta are labelled the $\beta$ subunits of the enzymes. The figures were generated by PyMol [43].

The molecular mechanism of the intramolecular activation of the Ntn-hydrolases remains obscured at the moment. The main handicap for the suggestion of a general model is related to the lack of a general, and highly conserved interacting group which would activate 
(deprotonate) the $-\mathrm{OH}$ of the L-Thr, enabling it to perform the nucleophilic attack on the peptide bond formed by the preceding residue. The postulation that this role could be fulfilled by the $\alpha$-amino group which is liberated upon cleavage of the scissile peptide bond faces many limitations and is debatable. The major restriction with this model is that the $\alpha$-amino group of the L-Thr is occupied within the peptide bond, and then the question arises as to what initiates the autoproteolysis. In addition, there is high likelihood that the amino group is itself protonated at physiological $\mathrm{pH}$, thereby being unable to subtract another proton [15].

An important aspect of this intramolecular processing, which is in direct connection with the mechanism behind it, is reflected by the distinct autoactivation rates of the different representatives of the Ntn-hydrolase superfamily of enzymes. For example, recombinant expression of hASNase3 and EcASNase3 using E.coli as expression system, results in a variable degree of processed enzyme species. This is illustrated in Figure 5, which shows an SDS-PAGE of purified hASNase3 and EcASNase3 enzymes. Clearly, in case of hASNase3 the predominant form (90\%) is the full-length inactive precursor, while its E.coli homolog is more than $75 \%$ activated. Further activation of hASNase 3 will take place in a time-dependent manner, but, unlike EcASNase3, it does not reach completion [38]. This highly variable rate of autoproteolysis raises many questions regarding the mechanism which governs this phenomenon, as well as concerning the lack of full activation versus partial activation among different enzymes.

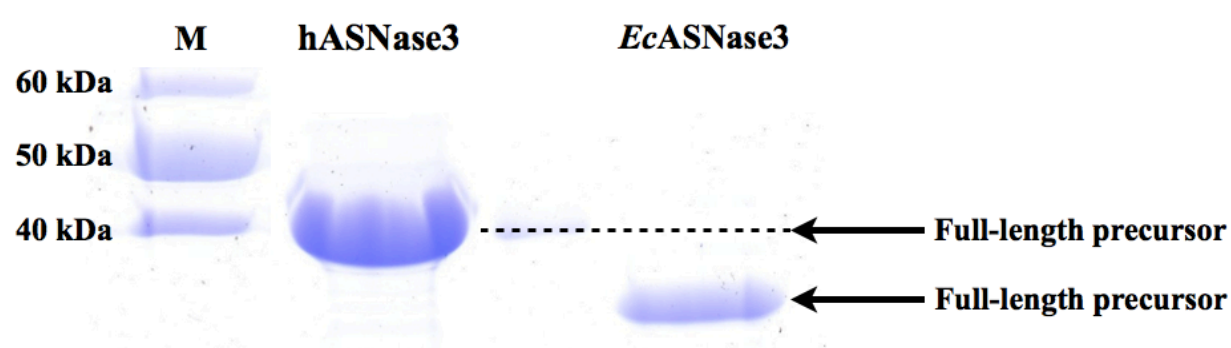

30 kDa

$25 \mathrm{kDa}$

$\boldsymbol{\alpha}$

20 kDa

$\boldsymbol{\alpha}$

$\boldsymbol{\beta}$

$\boldsymbol{\beta}$

15 kDa

Figure 5. SDS-PAGE analysis of purified hASNase 3 and $E c A S N a s e 3$. The proteins were recombinantly expressed in E.coli at $16{ }^{\circ} \mathrm{C}$ and were purified at $4{ }^{\circ} \mathrm{C}$. In the figure, the two 
subunits $\alpha$ and $\beta$ are denoted, and arrows indicate the full-length inactive precursors before autoproteolysis.

\section{Summary}

The plant-type L-ASNases, which have been more specifically discussed in this section, are considered as a subgroup of the Ntn-hydrolase family of enzymes. The particular characteristic of this family is that the enzymes are translated as inactive precursors which undergo an intramolecular activation step resulting in the final active form. The active form consists of two subunits ( $\alpha$ and $\beta$ ) which remain non-covalently associated upon the activation event. All members of this superfamily contain a very highly conserved folding motif, namely $\alpha \beta \beta \alpha$, despite low amino acid sequence identity. The underlined molecular mechanism of the autoactivation step remains unclear, and the proposed models suffer from certain limitations. Plant-type L-ASNases (e.g. plant type hASNase3, EcASNase3) exhibit a dual enzymatic profile, being able to hydrolyze iso-aspartyl-dipeptides more efficiently than L-Asn: They display a $\mathrm{K}_{\mathrm{M}}$ in the submillimolar range, $\sim 0.1 \mathrm{mM}$ for iso-aspartyl-dipeptides, and in the millimolar range, $\sim 3 \mathrm{mM}$ for L-Asn. Therefore, they are also called iso-aspartyldipeptidases (or $\beta$-aspartyl-dipeptidases). Recombinant expression of different plant-type LASNases revealed highly variable degrees of autoactivation, which brings up several questions concerning the intrinsic properties of these enzymes and the general mechanism behind their intramolecular activation.

\subsection{Bacterial-type L-ASNases}

Bacterial-type L-ASNases comprise a large family of enzymes, which are distributed from bacteria to higher mammals. In contrast, no such enzyme homologs have been found in plants up until now. Bacterial-type enzymes are further classified into bacterial-type I and bacterialtype II subgroups, which are characterized by discrete biochemical properties and are localized at different cellular compartments [6,7] (for consistency reasons with the abbreviations of enzymes in published work, we will refer to the type-I as type 1 and for type-II as type 2). Type 1 group enzymes are localized in the cytoplasm (also called cytoplasmic L-ASNases) and display a low affinity against L-Asn ( $\mathrm{K}_{\mathrm{M}}$ in the millimolar range), while type 2 enzymes are post-translationally transported to the periplasm of bacterial 
cells (periplasmic L-ASNases) and demonstrate a high affinity for L-Asn ( $K_{M}$ in the micromolar range). Periplasmic L-ASNases are additionally able to hydrolyze L-Gln $\left(\mathrm{K}_{\mathrm{M}}\right.$ in the millimolar range), while this does not apply to the cytoplasmic enzymes. The two subgroups of bacterial L-ASNases exhibit relatively high sequence identity, yet phylogenetic analyses based on their sequences can distinguish them as separate groups [44]. The active form of type 1 enzymes varies from dimeric to tetrameric assemblies, while type 2 isoforms act predominantly as tetramers [45-47].

\subsubsection{Catalytic mechanism of bacterial-type L-ASNases}

The catalytic mechanism of L-Asn hydrolysis has been much better studied for type 2 enzymes, but it is believed to be shared by both subgroups, given the high degree of homology around their active sites [48]. Similar to the plant-type enzymes, catalysis is initiated by the nucleophilic attack on the amide bond of the substrate. This role is fulfilled by an L-Thr residue whose -OH group is activated by a neighboring interacting group, usually L-Tyr [49]. At this point, it must be underscored that bacterial-type enzymes do not undergo any intramolecular activation mechanism like the plant-type enzymes, yet the principle of the catalysis initiation appears to be highly conserved involving a primary nucleophile residue (L-Thr), whose -OH group requires deprotonation for its activation for the subsequent attack on the carboxylic carbon of the substrate. In addition, unlike the plant-type enzymes whose amino group of the catalytic L-Thr is released upon intramolecular processing, in bacterialtype enzymes the respective amino group of the catalytic L-Thr is still occupied by the preceding peptide bond. The catalytic reaction is thought to cause the formation of a tetrahedral acyl-enzyme intermediate between the substrate and the enzyme upon the first nucleophilic attack, followed by a second nucleophilic attack by a water molecule [50]. This second attack induces the formation of a second tetrahedral intermediate which converts into the final products. This catalytic mechanism has been proposed to be common for all enzymes which exhibit L-ASNase activity, including those which belong to the Ntn hydrolase family. However, even this postulated mechanism remains questionable, with the main handicap being the identity of the group which activates the $-\mathrm{OH}$ of the nucleophilic L-Thr. A schematic representation of the catalytic mechanism described above is shown in Figure 6. 


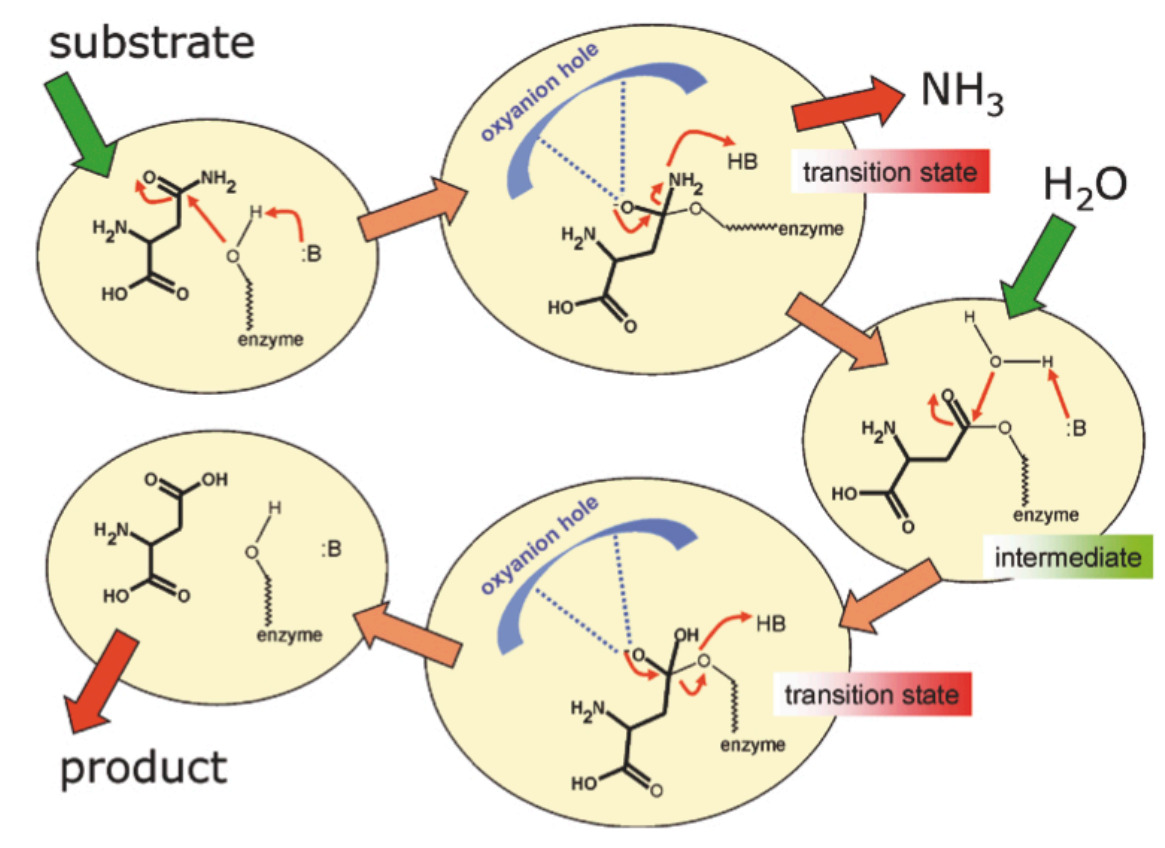

Figure 6. Proposed catalytic mechanism of L-ASNases. The reaction comprises two steps. In the first step, the catalytic nucleophile of the enzyme gets activated by a general base which subtracts its proton and subsequently the activated nucleophile attacks the $\mathrm{C}$ atom of the substrates's amide group. This leads to the generation of a transition state with an acylenzyme intermediate. Similar to the intramolecular activation proposed mechanism of Ntnhydrolases, the formation of an oxyanion hole (as a result of the amide oxygen) is stabilized by neighboring hydrogen-bond donors. The second step involves the attack of an activated water molecule to the $\mathrm{C}$ ester atom of the intermediate playing the role of another nucleophile and the final release of the enzyme and the product. (Adapted from [15]).

\subsubsection{Bacterial-type 1 L-ASNases}

Cytoplasmic L-ASNases have been only recently studied in detail, and structural analysis contributed considerably towards this direction. The crystal structure determination of E.coli L-ASNase1 (EcASNase1; encoded by ansA gene) [51] unveiled that this enzyme is homotetramer. Interestingly, the structural analysis revealed the presence of an additional LAsn binding site within the same monomer, located close to the catalytic site. Further kinetic and mutational characterization identified this second binding as an allosteric site, which modulates the activity of EcASNase1. This is a particular type of allostery where the same compound (in this case L-Asn) plays simultaneously the role of both effector and substrate of the enzyme. These effectors are called homotropic, and particularly in cases like this of EcASNase1 where the enzymatic activity increases with the concentration of this effector, they are called positive homotropic effectors [52]. EcASNase1 was the first bacterial-type L- 
ASNase to be identified as an enzyme which shows positive cooperativity. Unfortunately, in the absence of crystal structures from other cytoplasmic L-ASNases, it is difficult to conclude whether those cytoplasmic enzymes are allosteric in general, or whether this is a specific case only for EcASNase1. The only other available crystal structure of type-1 L-ASNase is from the archaeon Pyrococcus horikoshii (PhASNase1) [53]. Unlike EcASNase1, the PhASNase1 appeared as a dimer in the crystal structure, while its putative allosteric site is characterized by significant architectural differences as compared to the EcASNase1 enzyme. The lack of additional biochemical data which would further support or disprove the allosteric behavior of this enzyme, makes it difficult to draw a safe conclusion about its properties.

EcASNase1 and PhASNase1 share 37\% sequence identity, which is sufficient to classify them in the same subgroup of type-I enzymes. A cytoplasmic L-ASNase has been also identified based on sequence similarities in case of Saccharomyces cerevisiae (ScASNase1), which is encoded by the ASP1 gene [54]. Despite the fact that the structure of this enzyme is unknown, it appears to behave as tetramer in size-exclusion chromatography experiments, as well as to exhibit weak positive cooperative kinetics (our unpublished data). Therefore, there are good reasons to believe that this is another cytoplasmic L-ASNase which shows similar biochemical properties with the well-studied EcASNase1, and it has eukaryotic origin. Interestingly, such homologous enzymes have been also identified in mammalian cells. The first report which suggested the existence of bacterial-type 1 L-ASNase in rat cells appeared fifteen years ago [55]. This study demonstrated the isolation of a cDNA from rat liver which codes for a two-domain enzyme designated 60-kDa lysophospholipase. Sequence analysis showed that this enzyme consists of an N-terminal domain which significantly resembles the EcASNase1 ( 47\% identity) and a C-terminal ankyrin repeat-like domain. The authors assigned three distinct enzymatic activities to this enzyme, namely lysophospholipase, acetylhydrolase, and L-asparaginase, though their measurements relied on cell extracts rather than on purified enzyme [55]. Almost ten years later, another group reported on the human homolog of the $60-\mathrm{kDa}$ rat lysophospholipase [56], which also contains an $\mathrm{N}$-terminal domain very similar to EcASNase1 ( 47\% identity) and a C-terminal ankyrin repeat-like domain. This study confirmed the lipase activities of the human enzyme, yet without assigning with certainty any L-ASNase activity. In contrast, recently we showed that the Nterminal domain of the human $60-\mathrm{kDa}$ lysophospholipase, which appears to be highly homologous to the EcASNase1 enzyme holds L-ASNase activity, that is allosterically 
regulated by the substrate L-Asn [57]. However, no lysophospholipase activity was detected when the N-terminal domain was assayed [57]. Figure 7 shows a sequence alignment of five different type-1 L-ASNases and highlights important residues which are involved in catalysis and allosteric binding. Furthermore, Figure 8 shows the tertiary structures of EcASNase 1 and PhASNase1, the only two available structures of type-1 L-ASNases.

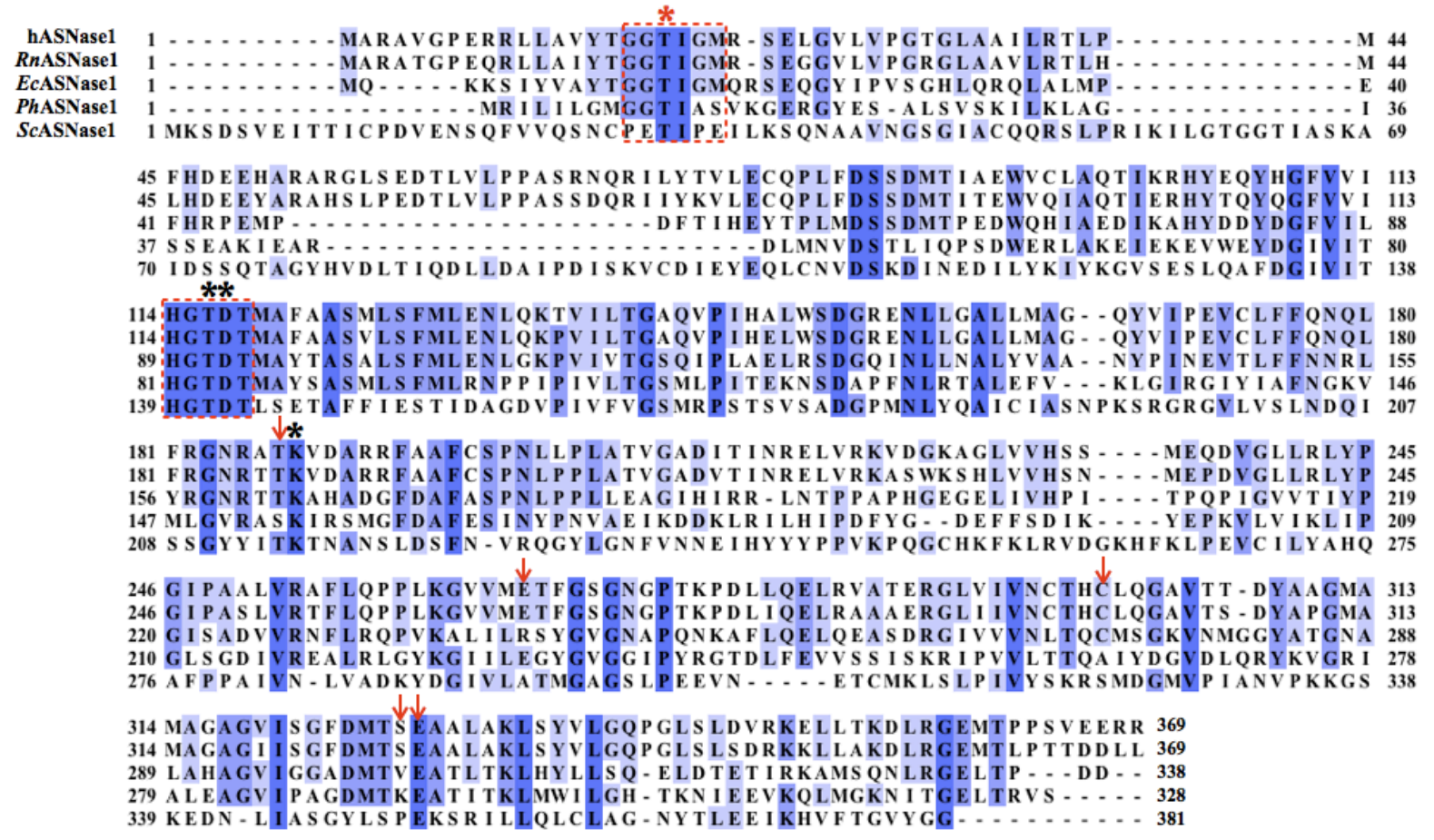

Figure 7. Sequence alignment of bacterial type-1 L-ASNases. Human ASNase 1 (hASNase1)corresponds to the N-terminal domain of the $60-\mathrm{kDa}$ lysophospholipase enzyme (UniProt: Q86U10), R.norvegicus (RnASNase1) corresponds to the N-terminal domain of the 60-kDa R.norvegicus lysophospholipase enzyme (UniProt: O88202), E.coli (EcASNase1) (UniProt: P0A962), P.horikoshii (PhASNase1) (UniProt: O57797) and S.cerevisiae (ScASNase1) (UniProt: P38986). The red asterisk indicates the catalytic L-Thr which is believed to play the role of the primary nucleophilic residue. The three black asterisks denote the so-called catalytic triad residues (Thr-Asp-Lys) of bacterial-type 1 L-ASNases which ensure proper substrate binding. The red arrows point to residues which comprise the allosteric pocket of EcASNase1 and which show only a moderate degree of conservation. The alignment was performed using CLUSTALW [41], and the image was prepared using JalView [42]. 

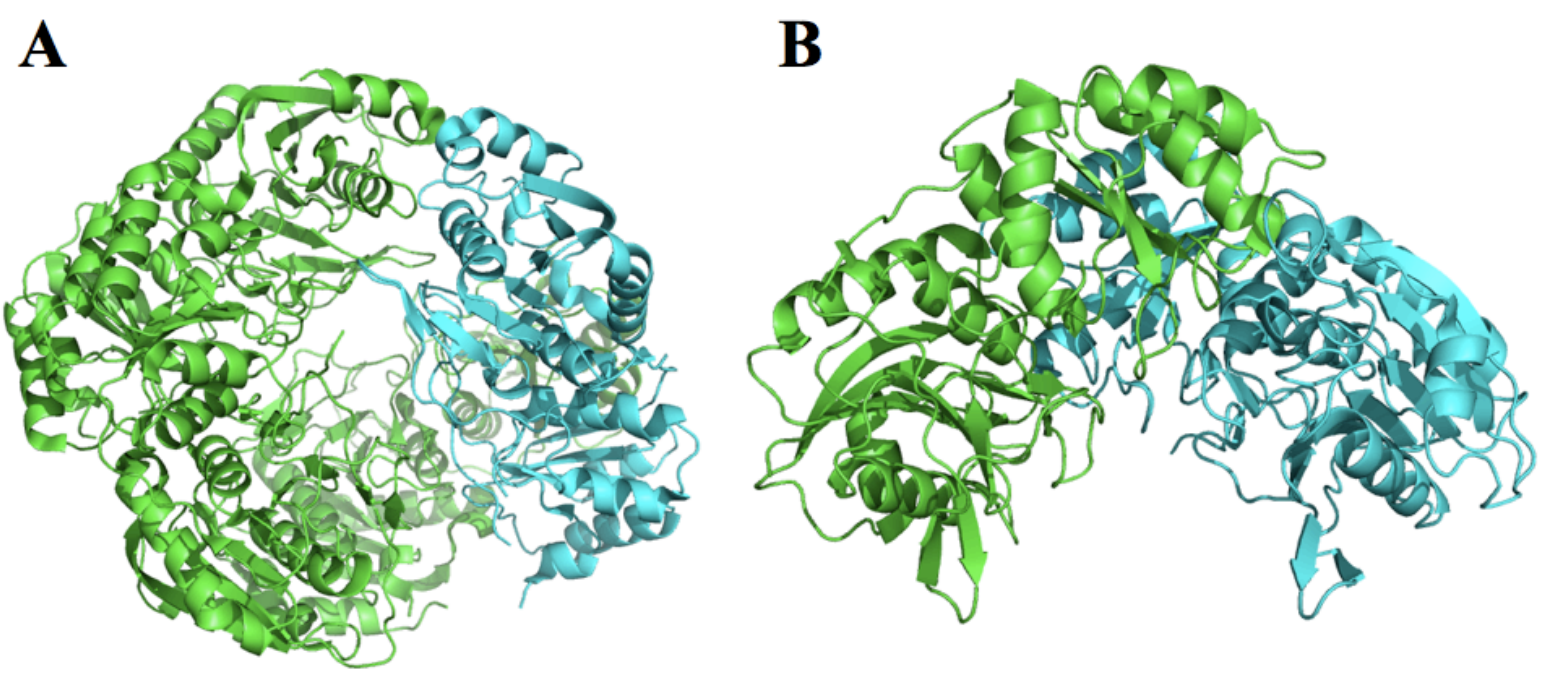

Figure 8. Structural representation of bacterial type-1 L-ASNases. (A) Escherichia coli ASNase1 (PDB entry: 2P2D) and (B) Pyrococcus horikoshii ASNase1 (PDB entry: 1WLS). With cyan is labeled the monomer of each enzyme, while with green are shown the rest identical monomers which form the tetrameric structure for EcASNase1 and the dimeric for PhASNase1, respectively. The figures were generated by PyMol [43].

\section{Summary}

Bacterial cytoplasmic L-ASNases form a subgroup of bacterial L-ASNases which have not yet been extensively studied. The available two structures from E.coli and P.horikoshii revealed a tetrameric and a dimeric assembly, respectively. Biochemical characterization of EcASNase1 showed that the enzyme is allosterically regulated by its own substrate L-Asn displaying a Hill coefficient $\left(\mathrm{n}_{\mathrm{H}}\right) \sim 2.6$, and a relatively high $\mathrm{S}_{0.5}$ of $1 \mathrm{mM}$ [51]. On the contrary, no glutaminase activity was detected for this enzyme. Recalling the kinetic properties of Ntn-hydrolases like hASNase3 or EcASNase3 against L-Asn, one could argue that they are similar to those from EcASNase1, except from the fact that Ntn-hydrolases do not behave allosterically, and their catalytic rates slightly differ $\left(\sim 7 \mathrm{~s}^{-1}\right.$ for EcASNase 1 and 3 $\mathrm{s}^{-1}$ for hASNase3 at $\left.37{ }^{\circ} \mathrm{C}\right)[32,38,51]$. The mammalian homologs of this subgroup of enzymes, i.e. hASNase 1 and RnASNase1, have attracted particular attention since they seem to be part of larger enzymes which catalyze different activities; further structural and biochemical studies are required to shed more light on the properties of those L-ASNase domains. 


\subsubsection{Bacterial-type 2 L-ASNases}

Periplasmic bacterial L-ASNases have been extensively structurally and biochemically studied over the last five decades, and therefore more detailed information is available as compared to the cytoplasmic enzymes. Yet, there are still debatable arguments with respect to their exact catalytic mechanism (see section 1.3.1 above). This subgroup includes enzymes which are exclusively active as tetramers and are localized in the periplasm of bacterial species. They are expressed in the cytoplasm, and subsequently they are transported to the periplasm through the bacterial translocation machinery. This is achieved through the recognition of a peptide-leader amino acid sequence at the $\mathrm{N}$-terminus of these enzymes (usually 20-23 long) by the translocation machinery's protein factors [58]. It has been shown that, unlike the cytoplasmic enzymes, the type-2 L-ASNases are not constitutively expressed in the cell, but only upon nitrogen starvation [59]. Additionally, periplasmic enzymes show a very high affinity to L-Asn, and this is reflected by their low $\mathrm{K}_{M}$ which falls within the micromolar range $(10-30 \mu \mathrm{M})$, while they are capable of hydrolyzing less efficiently L-Gln $\left(\mathrm{K}_{\mathrm{M}} \sim 3-5 \mathrm{mM}\right)$ [60]. Moreover, none of the extensively studied L-ASNase2 from different bacterial species has shown allosteric regulation or non-Michaelis-Menten kinetics.

Eukaryotic homologs of type-2 enzymes have been only identified up to the level of Saccharomyces cerevisiae (ASP3 gene) [61]. Astonishingly, there are four copies of the gene, designated ASP3-1, ASP3-2, ASP3-3 and ASP3-4 respectively, which codes for the periplasmic $S c$ ASNase2, and they are all located in tandem in a stretch of $20 \mathrm{~kb}$ [62]. In contrast, in higher organisms like plants or mammals no such homologs have been discovered to date. Figure 9 shows a sequence alignment of four type-2 L-ASNases, three from bacterial species (E.coli, Wolinella succinogenes, Erwinia chrysanthemi), and one from Saccharomyces cerevisiae, while Figure 10 represents the tertiary structures of EcASNase2 and ErASNase2. 


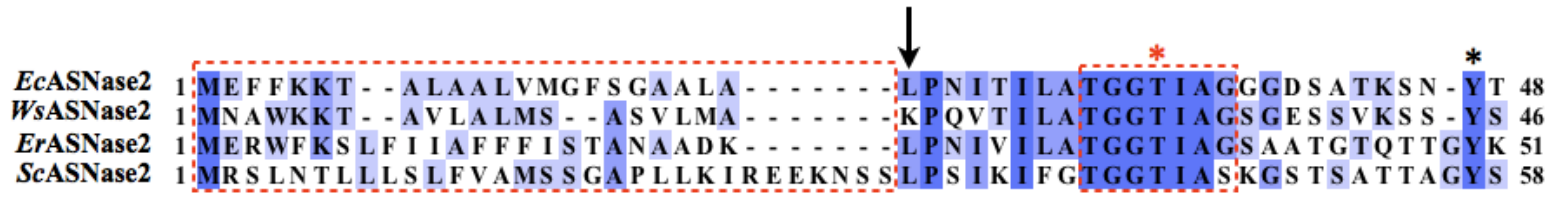

49 VGKVGVENLVNAVPQLKD I ANVKGEQVVN I GS QDMNDNVWLTLAKK IN - - TDCDKTDG 104 47 AGAVTVDKLLAAVPA I NDLAT I KGEO IS S I GS OEMTGKVWLKLAKRVNELLAOKETEA 104 52 AGA LGVDTL INAVPEVKKLANVKGEQFANMASENMTGDVVLKLS QRVNALLARDDVDG 109 59 VGLT - VNDLIEAVPSLAEKANLDYLQVSNVGSNS LNYTHLI PLYHG I S EALAS DDYAG 115 105 FV ITHGTD TME ETAY F LDLTVKCDKPVVMVGAMRPS TS MS A D G F N L Y NAVVTAADKA 162 105 V I THGTDTME TAFF LNLTVKS QKPVVLVGAMRS GSSMS ADGPMN LYNAVNVA INKA 162

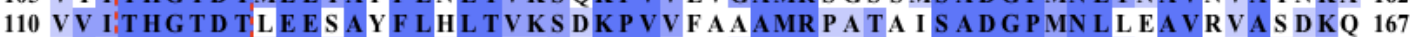
116 AVVTHGTDTME ETAFFLDLTINSEKPVC I AGAMR - ATATSADGPMN LYQAVS IAAS EK 172

163 SANRGVLVVMNDTVLDGRDVTKTNTTDVATFKS NNYGPLGY I HNGK I DYQRTPARKHT 220 163 S TNKGVV I VMNDE I HAAREATKLNTTAVNAFAS PNTGK I GTVYYGKVEYFTQS VRPHT 220 168 SRGRGVLVVLNDR I GS ARY I TKS NASTLDS FRANEEGYLGVV I GNH I YYONRLDKLHT 225 173 SLGRGTMITLNDR IASGFWTTKMNANSLDTFRADEQGYLGYFS NDDVEFYYPPVKPNG 230

221 S D T P F DVSKLN - - - ELPKVG IVYNYA - NAS DLPAKALVDAGYDG IVS AGVGNGNLYKS 274 221 LAS EF D ISK I E - - E LPRVD I LYAHP - DD TDVLVNAA LQA GAKG I I HAGMGNGNPFPL 274 226 NR S V F DVRGLA - - S LPKVDI LYGYQ - DDS EY LY DAA I S HGVKG IVYAGMGAGS VS VR 279 231 - WQFF D I S NLTDP S E I P EV I I LY S Y QG LN P E L I VKAVKD LGA KG IV LA GS GA GS WTA T 287

275 - V F D T LATAAKTGTAVVRS S RVP TGATT QDAEVDDAKYGFVASGTLN PQKARVLLQLA 331 275 - TQNALEKAAKS GVVVARS SRVGS GS T TQEAEVDDKKLGFVATES LN PQKARVLLMLA 331 280 - G I A GMR KA QD KGVVVMR S R TGNG IVPPDEALP - . - GLVA - D S LN PAHAR I L LMLA 331 288 G S IVNEQLYEEYG I P IVHSRRTADGTVP P DD A P EY - - - A I G S GYLN PQKS R ILLQLC 341

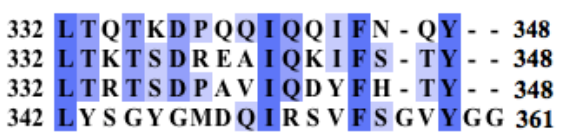

Figure 9. Sequence alignment of bacterial type-2 L-ASNases. E.coli ASNase2 (EcASNase2) (Uniprot: P00805), W.succinogenes (WsASNase2) (Uniprot: P50286), E.chrysanthemi (ErASNase2) (Uniprot: P06608) and S.cerevisiae (ScASNase2) (Uniprot: P0CZ17). The dotted red frame indicates the first 20-25 residues which act as signal-peptide for the translocation to the periplasm. Upon translocation, the signal-peptide is cleaved by aminopeptidases generating the mature enzymes, whose first amino acid is considered to be the one which is denoted by the black arrow. The red asterisk indicates the catalytic L-Thr which is believed to play the role of the primary nucleophilic residue. The two black asterisks show the L-Tyr residue which activates the primary nucleophile by subtracting the proton from the -OH group, and the L-Thr residue which contributes to the proper substrate binding, respectively. The alignment was performed using CLUSTALW [41], and the image was prepared using JalView [42]. 

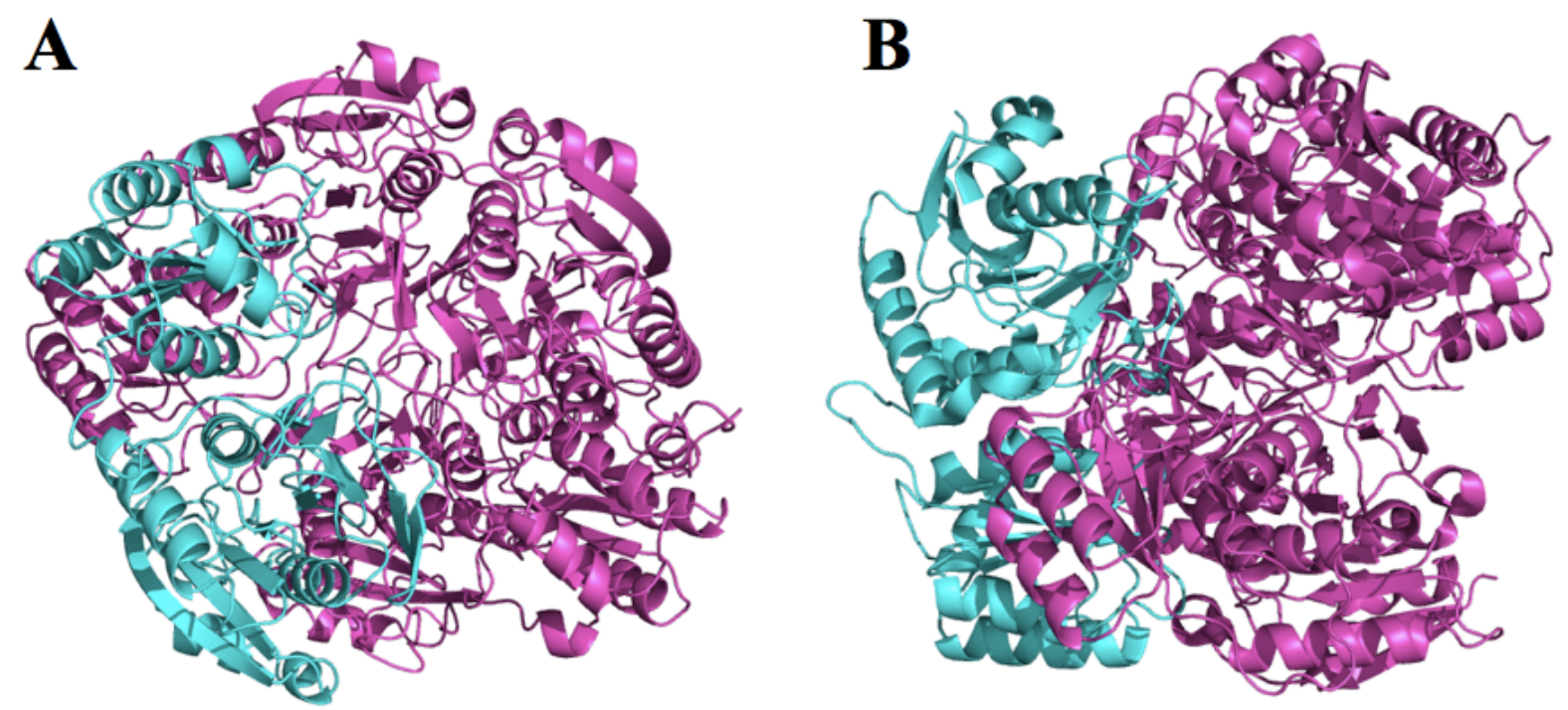

Figure 10. Structural representation of bacterial type-2 L-ASNases. (A) EcASNase2 (PDB entry: 3ECA) and (B) ErASNase2 (PDB entry: 1HFW) enzymes. With cyan is labeled the monomer of each enzyme, while with magenta are shown the rest identical monomers which form the tetrameric structures for both enzymes. Structural representations were generated by PyMol [43].

\section{Summary}

As stated briefly above, periplasmic L-ASNases have been studied more extensively than any other enzymes which possess L-Asn hydrolytic activity. Some of them have been used as drugs for over 50 years for the treatment of Acute Lymphoblastic Leukemia (ALL), a type of blood cancer which most often develops in infants [63-65]. More specifically, L-ASNase2 from E.coli and Erwinia chrysanthemi bacterial species (the ones shown in Figures $9 \& 10$ ) have been approved by FDA for treating humans who suffer from ALL [66-67]. Therefore, both enzymes have been in-depth studied by means of structure, steady-state kinetic, mutagenesis, pharmacodynamic and pharmacokinetic analyses [68-70]. In the next section, the principle of ALL therapy by L-ASNases will be discussed, as well as major limitations which result from this treatment, and current alternative directions aiming at the improvement of ALL therapy. The following Table 1 summarizes the properties of L-ASNases, representatives of the different families and groups analyzed above. 


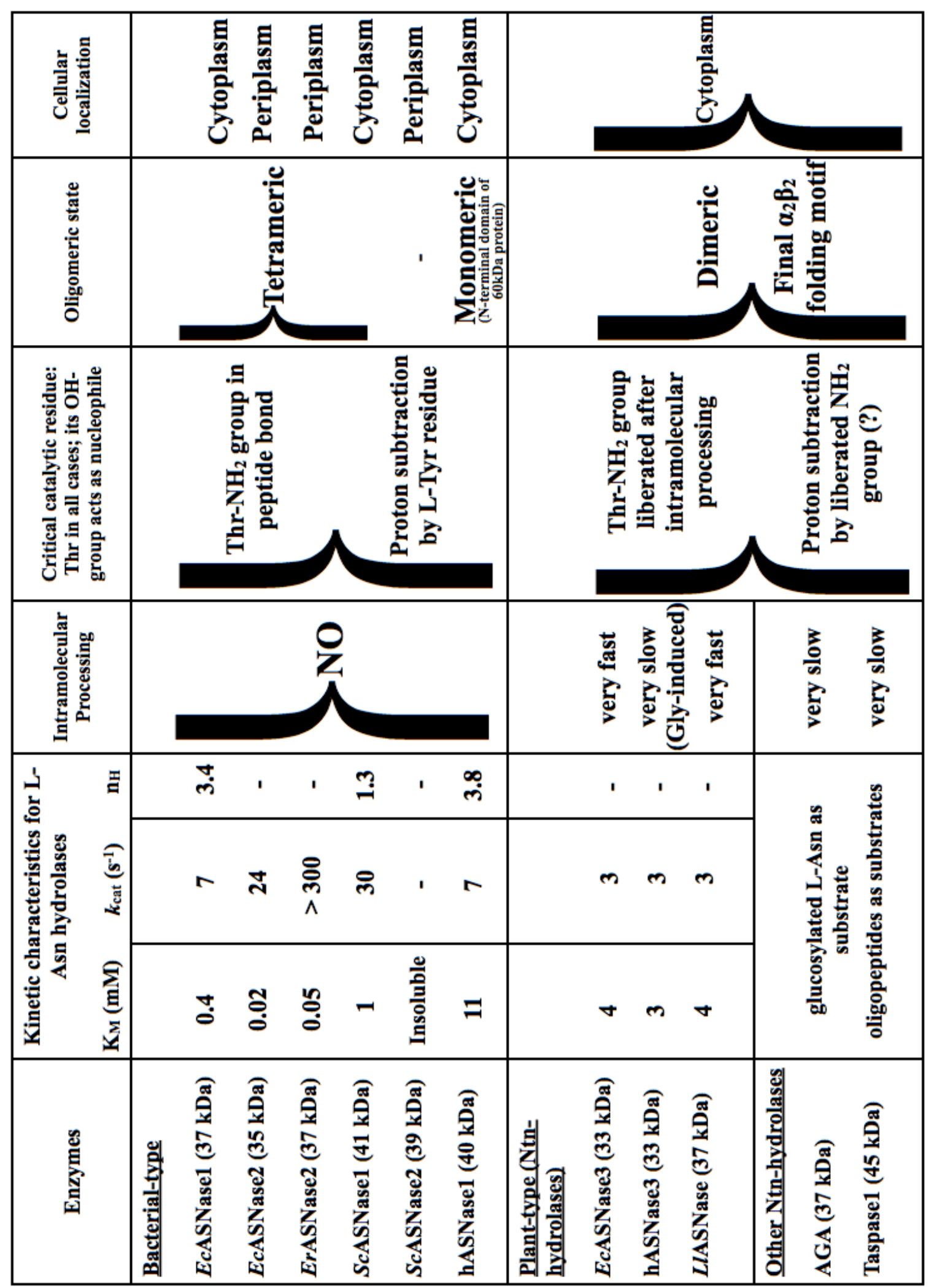




\subsection{Leukemia therapy by L-ASNase}

Acute Lymphoblastic Leukemia (ALL) is a type of leukemia which develops predominantly in children in the age of 1-7 years old. Yet, this does not exclude the possibility to develop in adults as well, though with less likelihood [71]. In principle, ALL is one of the four types of leukemia which have been defined to describe this disease. In order to obtain a better understanding of the different types of leukemia, I give an overview of the different types of cells which exist in blood. Figure 11 illustrates the classification of the different types of blood cells, describing briefly their roles in the body.

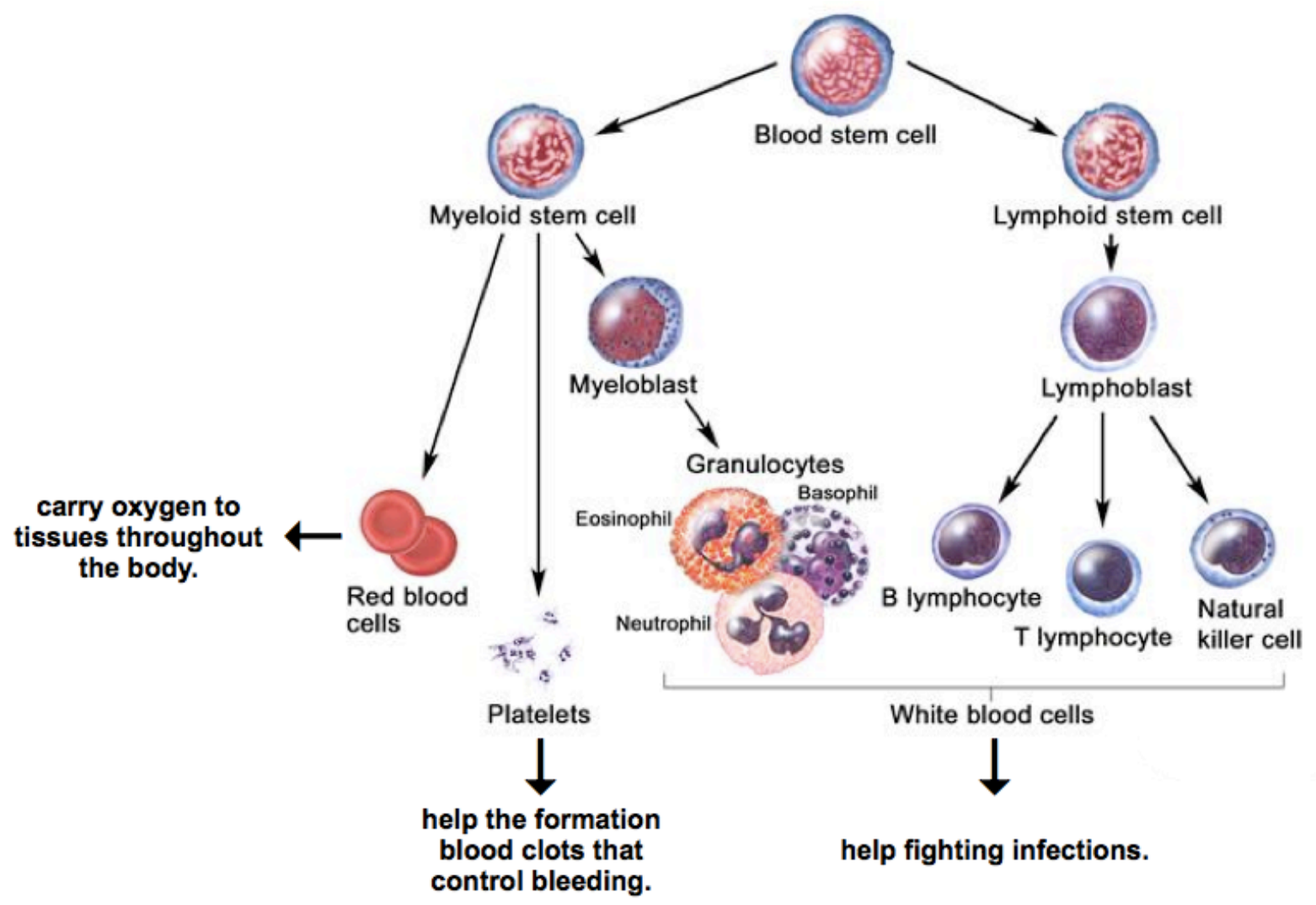

Figure 11. Human blood cells categorization and development. Primary blood stem cells give rise either to myeloid or to lymphoid stem cells, which subsequently differentiate further to other types of cells. Lymphoid stem cells are transformed to the so-called lymphoblasts that can be, in turn, differentiated to B or T lymphocytes or to natural killer (NK) cells. On the other hand, myeloid stem cells can be ultimately converted to red blood cells, to platelets or to granulocytes. As denoted in the figure, the B and T lymphocytes, the NK cells and the granulocytes account for the so-called white blood cells which form the backbone of the immune system. The red blood cells are in charge of carrying and distributing oxygen throughout the whole body, and the platelets contribute to the formation of blood clots which control bleeding. Adapted from National Cancer Institute (NCI). 


\subsubsection{Types of Leukemia}

Leukemia is a type of cancer which affects the blood and the bone marrow. This disease is characterized by an uncontrollable proliferation of, mostly, undifferentiated blood cells in the bone marrow. This results in a progressive displacement of the normal and healthy cells and consequently, the body is fully crowded by dysfunctional blood cells [72,73]. Leukemia is grouped into mainly four types according to the cells which are affected:

- Acute Myeloid Leukemia (AML)

- Chronic Myeloid Leukemia (CML)

- Acute Lymphoblastic Leukemia (ALL)

- Chronic Lymphoblastic Leukemia (CLL)

\subsubsection{Myeloid Leukemia}

In case of myeloid leukemia, myeloblasts (Figure 11) divide unmanageably into abnormal granulocytic white blood cells, which cannot perform their normal functions. Myeloid leukemia is also called granulocytic or non-lymphocytic leukemia. Depending on which subtype of cells are affected, myeloid leukemia can be further grouped into the respective subtype which will define the type of patient's treatment. Furthermore, as implied by the terms, acute myeloid leukemia progresses very fast and necessitates immediate anti-cancer treatment, while the chronic refers to this type of disease which develops slowly. The symptoms of ML among others, include abnormal prolonged nose bleeding, tiredness, night sweats and mild fever events, weight loss and frequent minor infections [74]. Diagnosis can be done by different means such as blood or bone marrow tests that usually includes cell counting, which shows the number of the different subtypes of cells (in case of ML, the number of red cells and platelets is expected to be considerably lower than normal). Moreover, conventional Polymerase Chain Reaction (PCR) can be used to analyze certain genes whose structures are usually affected in case of ML [75]. Once a patient has been diagnosed with ML, he/she enters a phase of chemotherapy treatment which is highly dependent on the type of ML (Acute or Chronic), the age of the patient, the stage and the subtype of the disease. Both types are considered to be curable provided that the diagnosis 
and the treatment will initiate very soon after the development of the disease. Towards this direction, a proper prognosis might be proven to be extremely helpful. Certain risk-factors exist which help to predict the development of such a disease. Those factors include genetic disorders like Down syndrome, Fanconi anemia, having siblings suffering from leukemia, being exposed to ionizing radiation or chemicals like benzene [76].

\subsubsection{Lymphoblastic Leukemia}

In analogy to myeloid leukemia, lymphoblastic leukemia (LL) develops from an uncontrollable proliferation of the white blood cells in the bone marrow and depending on which subtype of white blood cells are affected (B, T or NK) LL can be further classified into additional subtypes, though the most common ones concern the T-cell and B-cell LL [77]. Figure 12 contrasts the bone marrow state under healthy and cancerous conditions. ALL develops very rapidly, thereby causing immediate and severe symptoms, while CLL develops slower. The symptoms of LL do not considerably differ from those of ML, and therefore the type of leukemia cannot be concluded solely based on the symptoms. Even diagnosis can become difficult, requiring a series of tests before drawing a safe conclusion about the type of the disease and the initiation of the treatment. The diagnostic toolbox which is used for the determination of ML also applies for the case of LL, though immunophenotyping of leukemic lymphoblasts by flow cytometry sometimes is essential to determine the correct cell lineage [78]. Prognosis for LL includes certain genetic disorders like translocation of a part of the socalled Philadelphia chromosome to another chromosome [79], Down syndrome, Shwachman syndrome, and Bloom syndrome [80]. 
A

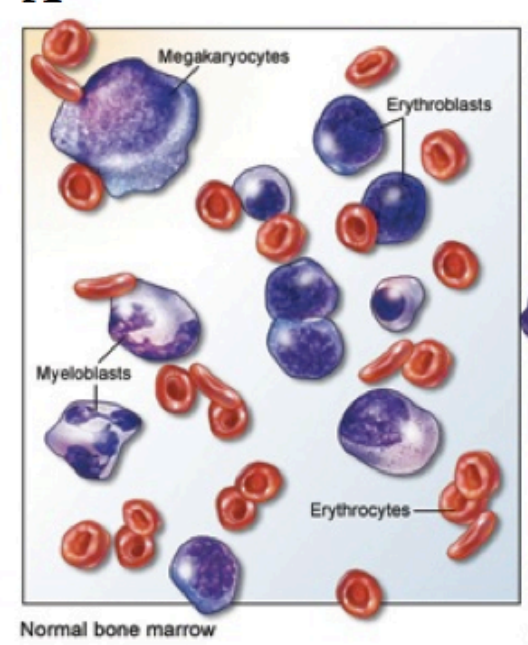

B

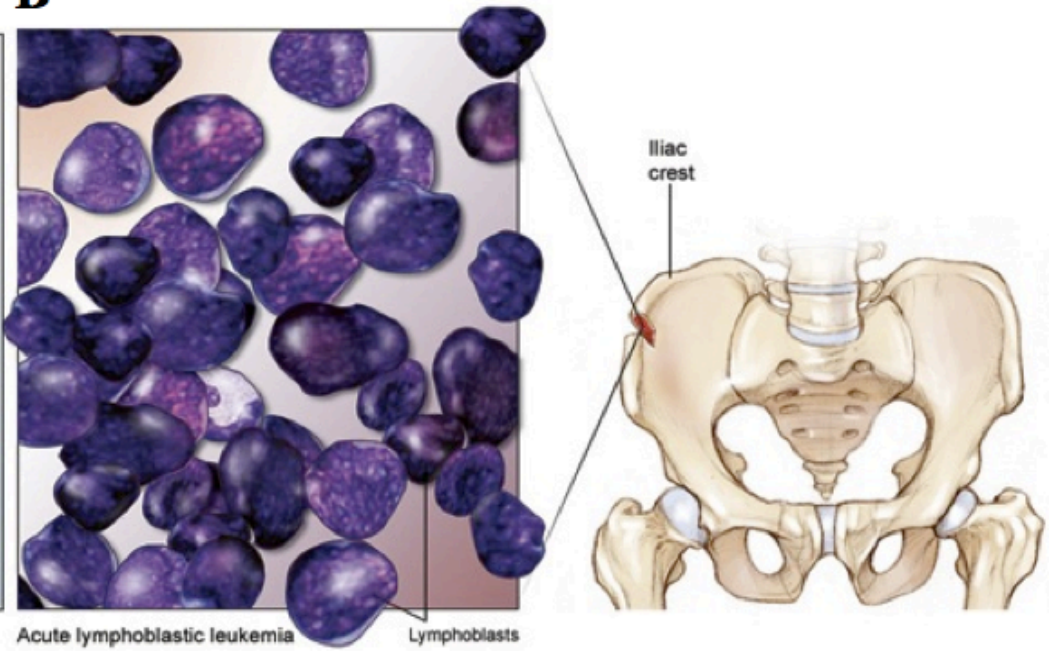

Figure 12. Bone marrow with (A) healthy cells and (B) cancerous cells. In case of ALL, numerous dysfunctional lymphoblasts are crowding out healthy and functional cells (A) resulting in progressive loss of infection-fighting capabilities. Adapted from National Cancer Institute (NCI).

\subsubsection{Acute Lymphoblastic Leukemia treatment with L-ASNase}

The treatment of ALL includes a combination of chemotherapy, radiation and/or chemotherapy with stem cells transplantation, and, in principle, it is divided into three phases: i) the remission induction phase, the ii) consolidation/intensification phase, and the iii) maintenance phase [81]. During the first phase, the goal is to kill as many as possible malignant cells in the bone marrow and the blood. This will lead to the remission of leukemia. The consolidation/intensification phase of treatment commences when the disease is in remission. This phase aims at the elimination of any leukemic cells which possibly might still exist in the body and could potentially cause the so-called relapse (the reappearance of a disease with its characteristic symptoms and signs after a period of improvement). The maintenance phase intends to kill any leukemic cells which may proliferate and cause a relapse. During this phase, the overall treatments are provided to the patients in lower doses as compared to the preceding two phases [82]. This treatment scheme is a general one and applies not only for ALL, but also for all types of leukemia.

Chemotherapy against ALL includes an arsenal of FDA-approved compounds, which most often are given in cocktail doses to the patient [83]. Briefly, some of them are Vincristine Sulfate, Mercaptopurine, Cyclophosphamide, Dexamethasone, Methotrexate, and Clofarabine 
[84]. However, chemotherapy treatments in case of ALL is done using some of the chemotherapeutics above and always in combination with the enzyme L-ASNase. L-ASNase has been a cornerstone in the treatment of ALL and non-Hodgkin lymphoma [85] since the 1960s. The observation that L-ASNase causes death to leukemic cells goes back to the 1950s when Kidd administered serum of guinea pigs into rat and mice, and he found that this leads to regression of the transplanted lymphomas [86]. Almost a decade later, Broome discovered that the factor with the anti-lymphoma properties in guinea pig serum was L-ASNase [87]. Subsequently, in 1966 Yellin and Wriston [88] managed to isolate L-ASNase from guinea pig and demonstrated its anti-leukemic activities; the first clinical use of partially purified guinea pig L-ASNase was reported in the same year and concerned the administration to an eight years old child. Since then, L-ASNase is one of the most essential anti-leukemic agents, and it is always present in the chemotherapeutic treatments during the first remission induction phase. Attempts to exclude L-ASNase from the treatment plan because of the high cost resulted in enormously increased relapse rates [89]. The dogma of therapy relies on the, partial or total, inability of the enzyme asparagine synthetase of the cancerous lymphoblasts to synthesize L-Asn. Therefore, the survival of the malignant cells is exclusively dependent on the exogenous supply of L-Asn from the patient's serum, since they need this amino acid to meet the requirements of their high metabolic rate [90]. Intramuscularly or intravenously administered L-ASNase catalyzes the hydrolysis of L-Asn to L-Asp and ammonia and in principle destroys the available supply for the leukemic cells, which have no longer access to this, essential for them, amino acid [91]. Consequently, they undergo protein-synthesis inhibition and ultimately apoptosis [92]. At this point, it must highlighted the fact that the normal cells are not affected by this treatment because they can synthesize L-Asn. Interestingly, there have been published some reports which demonstrate the significance of depriving L-Gln in parallel with the L-Asn for achieving full anti-leukemic activity of LASNase $[93,94]$. This is happening to a certain extent if we consider the fact that the LASNases which are currently used for the treatment of ALL can hydrolyze L-Gln, though much less efficiently as compared to L-Asn hydrolysis. However, this claim is highly questionable and remains to be further validated or disproved. An important aspect of this principle of therapy is that it is believed to be a common intrinsic property for the majority of cancer types (including organ cancer) that cancerous cells are characterized by certain metabolic enzyme deficiencies, resulting in their total dependency on amino acids from the 
surrounding serum environment [95]. This has formed a solid basis for the development of the so-called "Amino Acid Depletion Cancer Therapy" [96]. Another example of such cancer therapy is the use of the enzyme arginase to treat hepatocellular carcinomas, melanomas and prostate carcinomas [97]. These cancerous cells cannot express the urea cycle enzyme argininosuccinate synthase (ASS) and thus are sensitive to L-Arg depletion [98].

\subsubsection{Side effects as a result of L-ASNase treatment}

One of the primary concerns regarding the treatment of any type of cancer is the immediate treatment which will rapidly kill as many malignant cells as possible and achieve a successful remission. In case of ALL, the use of highly efficient L-ASNase enzymes which could deplete rapidly the serum levels of L-Asn, would play a detrimental role in the success of the treatment. This is the reason that for the treatment of ALL, only enzymes from bacterial origins have been used for the last fifty years. Those enzymes are the periplasmic EcASNase2 and ErASNase2 which were discussed above (section 1.3.3). Both enzymes have a very low $\mathrm{K}_{\mathrm{M}}$ (high affinity) against L-Asn $(\sim 10 \mu \mathrm{M}$ for EcASNase2, and $\sim 50 \mu \mathrm{M}$ for ErASNase2) while ErASNase2 is considerably faster $\left(\sim 300 \mathrm{~s}^{-1}\right)$ in comparison to EcASNase2 $\left(\sim 30 \mathrm{~s}^{-1}\right)$ [99]. Given the fact that the serum L-Asn levels fall within the range of $\sim 100 \mu \mathrm{M}$, it becomes understandable that both aforementioned enzymes can function very efficiently and deplete L-Asn rapidly.

However, treatment rarely occurs without severe side effects which are mainly attributed to the bacterial origins of those enzymes [100]. Side effects vary from hypersensitivity reactions to lethal anaphylactic shocks and immunogenicity. Others include hepatic dysfunctions, pancreatitis (notably in children), central nervous system abnormalities, anorexia, hyperglycemia and thrombosis [101-103]. Certain side effects are thought to be caused by the intrinsic glutaminase activity of bacterial L-ASNases, influencing both the glutamine and glutamic acid levels [104]. Glutamine is the major transport form of amino nitrogen in blood, whereas glutamic acid has a notable impact on the central nervous system, since it plays a key role as a neurotransmitter. This belief is contradictory to what was mentioned above that L-Gln depletion is absolutely necessary for full anti-leukemic activity of L-ASNase. Such contradictions underscore the necessity to further elucidate the involved molecular mechanisms in ALL treatment. However, the major limitation of L-ASNase used as anti- 
cancer agent is related to the so called "silent inactivation" of the enzyme [105]. This inactivation arises from the interaction with specific antibodies which are generated upon recognition of the enzyme as antigen by the patients' immune system. This inactivation of LASNase, which manifests itself in a blood serum half-life of only about 24 hours, necessitates continuous administration and progressively higher doses of the therapeutic enzyme. It has also been shown recently [106] that, apart from silent inactivation, two highly specific lysosomal proteases degrade L-ASNase and therefore are co-responsible for the clearance of the enzyme. Considering all the side effects which arise from the use of bacterial L-ASNases for the treatment of ALL, it appears reasonable to seek for alternative routes which have the potential to result in more friendly medical care and by-pass certain severe side effects, yet with no compromises concerning the success of the therapy. Such avenues will be discussed in the following section.

\subsection{Avenues to improve L-ASNase-based ALL therapy}

Given the wide spectrum of side effects which accompany the administration of bacterial LASNases to humans for treatment of ALL, several attempts have been made to circumvent certain limitations of this medical therapy. One way to cope with the most serious problem of immunogenicity is to switch the administration of one bacterial L-ASNase to the other one [107]. In other words, some patients develop severe immune-reactions when they are treated at the beginning with EcASNase2, while some others can tolerate this treatment better. For those patients who react negatively with EcASNase2, different chemotherapeutic protocols are employed which include ErASNase2 instead of EcASNase2 [108]. This change contributes, usually, positively to the alleviation of the immune reactions. Yet, this is a shortterm "trick" to bypass the immune-reactions but many of the side effects which were mentioned above persist. Below are discussed the two main paths which have been extensively explored to pave the way for improved ALL therapy, followed by additional two that are currently under development and appear to be highly promising. 


\subsubsection{Polyethylene-glycol (PEG) modification of EcASNase2}

One of the main limitations of the use of L-ASNases as anti-leukemic drug is their silent inactivation by antibodies. In addition, it has been shown that a number of proteases specifically recognize and degrade EcASNase2, thereby reducing significantly its half-life in the body [109]. Those observations led to the modification of EcASNase2 by covalently attaching Poly-Ethyleno-Glycol (PEG) molecules on it [110]. This chemical modification prevents the enzyme from being degraded by proteases and results in prolonged half-life in the body in comparison to the native enzyme (from $\sim 24$ hours for the native enzyme, up to 1 week for the modified one) [111]. In addition, it has been so far well-documented through extensive clinical studies that PEG-modified EcASNase2 (commercial name approved by FDA: Pegaspargase) led to decreased immunogenicity in certain cases, as evidenced by the number of patients who developed anti-EcASNase 2 antibodies as compared to those who were treated with native EcASNase2 [112]. This may be attributed to the masked epitopes of the PEG-modified enzyme, which are no longer accessible for recognition by the immune system [113]. On the other hand, it must not be disregarded the fact that PEG-modification influences the catalytic properties of the enzyme and this is reflected by the longer period of time it takes to deplete serum L-Asn, whereas some of the main side effects remain (thrombosis, liver dysfunctions, central nervous system disorders). Moreover, there are patients who still do not react positively against the PEG-modified enzyme and experience severe immune reactions [112]. No PEGylated ErASNase2 is approved for treatment so far.

\subsubsection{De-immunization of bacterial L-ASNases}

It is well documented that the plethora of side effects which arise from the treatment of ALL by L-ASNase are associated with the bacterial origins of those enzymes [100]. A possible approach to circumvent this sort of problem is the so-called de-immunization of bacterial enzymes [114]. This is an effort to reduce the immunogenicity of proteins by mutating amino acid sequences (known as epitopes) which are likely to be recognized by the antibody repertoire (B-cells epitopes) or sequences which are recognized and bound by the major histocompatibility complex II (MHC-II), thereby inducing T-cell immune responses (T-cell epitopes) [115]. However, the determination and elimination of B-cell epitopes is an 
exceptionally tedious and complicated process due to our limited knowledge regarding their conformational profile and how they differ among different human populations [116]. On the contrary, the removal of T-cell epitopes is much better well-studied and understood as evidenced by certain successful modifications in some "humanized" therapeutic proteins which resulted in reduced antibody responses [117]. In principle, T-cell receptors on CD4+ cells recognize peptides which are part from the antigen (usually 15-25mers) and are presented in complex with the MHC-II on the surface of antigen-presenting-cells [118]. MHC-II is characterized by four binding pockets which recognize and accommodate the side chains of different antigenic residues, and this is what defines the binding affinity and specificity [119]. The identification of such putative antigenic T-cell epitopes which bind to various MHC-II alleles, has been additionally aided by in silico analyses in some cases. At this point it must be underlined the difficulty of not only determining putative T-cell epitopes within an antigen, but also the impact on the catalytic activity which may be caused upon disruption and modification of certain residues. It is well-established that catalysis can be seriously influenced by amino acid mutations which do not only occur close to the catalytic centre of the enzyme, but also by those that are distant from it [120]. Therefore, the major challenge of de-immunization enterprises is the removal of T-cell epitopes coupled with retainability of the initial enzymatic function and activity. High-throughput screening of combinatorial libraries has proven to be a valuable tool for such experimental endeavors [121].

\subsubsection{Replacement of the bacterial enzymes by engineered human homologs}

Recalling the numerous complications which are associated with the use of EcASNase 2 and ErASNase2 against ALL, despite several efforts to circumvent them, it is plausible to direct our thinking towards alternative drug developments. A major step forward would be the replacement of the current bacterial enzymes by others from human origins. However, as stated and analyzed in detail above, the human genome codes for two enzymes that belong to those L-ASNase families which show poor catalytic properties against L-Asn hydrolysis $\left(\mathrm{K}_{M}\right.$ in the millimolar range). The first example is hASNase3 that belongs to the Ntn-hydrolase superfamily, characterized by the particular, post-translational autoproteolysis activation step [36-38]. The second example includes the N-terminal domain of the 60-kDa human 
lysophospholipase whose sequence significantly resembles the cytoplasmic EcASNase1 and presumably exhibits similar catalytic properties [57]. However, although they belong to LASNase families whose members do not hydrolyze efficiently L-Asn, these two human enzymes deserve certainly more attention; and their characterization could lay the basis for the development of L-ASNase enzyme drugs which could function efficiently in the body, eliminating immune reactions given their human origins. Allies towards those efforts can be the different structural determination tools, as well as the well-established directed enzyme evolution schemes, coupled with state-of-the-art high-throughput screening systems for the identification of catalytically improved variants. Availability of an enzyme's crystal structure can help to design rational (defined point mutations), or semi-rational (randomization of certain amino acids and generation of combinatorial libraries) mutagenesis protocols aiming at the generation and isolation of ameliorated variants. However, the bottleneck in such cases of directed evolution experiments is the availability of a highly sensitive and efficient highthroughput screening system. In the following chapters, I will discuss the use and the development of such screening systems and their potential applications in directed evolution experiments. Engineering of hASNase1 and hASNase 3 are excellent enzyme candidates, which would absolutely justify the development of high-throughput screening systems.

\subsubsection{Encapsulation of L-ASNases applying drug delivery technologies}

Over the last decade, there has been an explosive development of multifunctional drug delivery systems with very promising application and high perspectives in cancer therapy and biotechnology [122,123]. Such systems are built of highly customizable materials which meet certain requirements for biomedical applications [124]. Perhaps, the most well-studied drug delivery system is related to the use of the so-called polyelectrolyte nano- and microcapsules [125-128]. These capsules are globular in shape and consist of successive layers of polyelectrolytes (negatively and positively charged), and their fabrication is based on the Layer-by-Layer (LbL) assembly technique [129]. Particular features of such assemblies are their small size (from nanometer to micrometer diameter range), their biodegradability, and their high drug load-capacity [130]. In addition, one of their major advantages is the

possibility to functionalize their surfaces with specific molecules such as antibodies or other recognition chemical groups aiming at their cellular targeting, thereby making them cell- 
specific [131]. Proteins (including antibodies and enzymes) have found very promising applications in cancer therapy during the last decades, and this is supported by several molecules which are currently under clinical trials, whereas others have been approved for medical treatment [132]. The potency of those drugs might be considerably improved if they could be combined with certain protein delivery systems. For example, the packaging of LASNases in polyelectrolyte microcapsules could potentially protect the enzyme from proteases' attacks, and consequently, prolonging their half-life in the body. In addition, the enzyme encapsulation could alleviate the immunogenicity problems by masking certain epitopes and preventing them from getting in contact with the patient's immune system. However, in order to develop such a protein system, it is absolutely crucial that the protein retains its initial (or at least sufficient) biological activity during the process of encapsulation. Proteins are very labile molecules, with inherent instabilities and their tendencies to denature, aggregate or degrade being major hurdles in drug delivery systems [133]. Yet, the advantages which might arise from successful development of such protein delivery systems certainly are worth the efforts towards these directions.

\section{Summary}

In the last section, I discussed several approaches which could improve the treatment of ALL using L-ASNases. The first two approaches have already been employed (PEG-modification of EcASNase2 and de-immunization of T-cell epitopes), though not very successfully. From one side, while the PEG-modified EcASNase2 is cleared from the body in longer periods of time than the native enzyme, still some patients react negatively upon administration of this drug [112]. On the other hand, application of de-immunization protocols coupled with high throughput screening schemes, can result in mutants which appear be less immunogenic $[113,117]$. However, given the high complexity of the antigenic epitopes and their recognition by the human immune system, it is not clear whether those mutants will significantly alleviate the immune reactions in patients as well. The last two approaches which were shortly introduced above (1.5.3 and 1.5.4), refer to the engineering of human L-ASNases envisioning replacement of the current bacterial enzymes and the development of protein delivery systems. Both define a great challenge. Considering the poor catalytic properties of hASNase 1 and hASNase3, the route of protein engineering is inevitable in these cases. In 
addition, the development of protein delivery systems requires very careful handling of the enzymes avoiding their total loss of catalytic activity.

In the next two chapters, I will discuss different protein engineering approaches and the development of high-throughput screening systems for directed evolution experimental set ups; as well as the use of polyelectrolyte microcapsules as drug delivery systems and how they could potentially be used in case of L-ASNases.

\subsection{Development of high-throughput screening platforms for protein directed evolution applications}

The progressively increased use of enzymes for biomedical, therapeutic, chemical and industrial applications has led to the necessity for development of new catalysts with improved properties of interest such as stability, ligand binding, substrate specificity and catalysis. In the modern era of molecular biology, researchers are fully supported by the availability of state-of-the-art toolboxes which help them towards the aforementioned goal. Two different but complementary approaches have been applied to accomplish this goal: i) rational design, and ii) directed evolution methods [134].

\subsubsection{Rational design}

Rational design is a structure-based approach aiming at the identification of amino acid residues which are crucial for the enzymatic activity and potential substitution could modify certain properties of interest [135]. This approach is highly dependent on the availability of a crystal structure or at least of a model of the protein of interest based on the structure of another homolog. Additionally, detailed knowledge of precise function and catalytic mechanism are required, in order to design precise amino acid changes which can be ultimately incorporated by means of site-directed mutagenesis [136]. Therefore, the success of this approach has been predominantly demonstrated and is mainly reserved for proteins which have been extensively studied and characterized [137]. 


\subsubsection{Directed evolution}

In contrast, the directed evolution approach does not require knowledge of a structure and how it relates to a function. It mimics the natural process of evolution which is, in principle, based on the selection of the fittest sequences [138]. This technique employs the generation of random mutations throughout the whole sequence of a protein or the randomization of certain regions of the protein sequence, followed by selection or screening for the identification of variants with the desired properties [139]. The whole process is repeated in iterative cycles until the final improvement is met. Several molecular biology techniques have been developed for generating protein diversity in directed evolution experiments. They can in principle be divided into two basic parts: the single gene manipulation techniques (the most widely used are error-prone Polymerase Chain Reaction (epPCR) [140] and SiteSaturation Mutagenesis (SSM) [141]), and recombination-based techniques (DNA-Shuffling [142] and homology-independent crossovers [143]). All those techniques have been, individually and/or in combination, applied with great success in certain cases, and new variants were generated with improved properties [144-146]. However, it must be underlined the fact that the bottleneck of a directed evolution experimental set up is the availability of an efficient screening system which can identify ameliorated variants. To illustrate this better, let's consider an example of a relatively small protein consisting of 100 residues. Even for this small protein, the so-called protein fitness landscape [147] includes $20^{100}\left(\sim 10^{130}\right)$ possible sequences. It becomes immediately understandable that it is impossible to experimentally address the whole sequence space of a protein due its vast size. If we randomize just five codons of the gene which codes for the aforementioned protein allowing all four nucleotides $(\mathrm{A}, \mathrm{T}, \mathrm{G}, \mathrm{C})$ to be incorporated with equal probability at each position, we end up having $\sim 10^{10}$ mutants on the DNA level and $3 * 10^{6}$ (less, due to the degeneration of the genetic code) mutants on the amino acid level. In order to screen a library of this size, achieving a $95 \%$ coverage, one would need to screen at least $10^{7}$ individual mutants which means the use of $10^{5} 96$-well plates! There is an excellent report which describes the statistics of random and site-saturation mutagenesis libraries and the effort required for their screening coverage [148].

Statistically, random mutations have the tendency to be deleterious causing considerable loss of protein function [149]. As concluded from the outcome of numerous directed evolution 
experiments, $30-50 \%$ of single amino acids have a significantly negative impact on the proteins' function, $50-70 \%$ are neutral, and only around $0.1-1 \%$ are beneficial [150]. Those numbers point to a situation where one could say that searching for an improved mutant in a library is like "searching a needle in the haystack". Screening of a library plays a pivotal role in the identification of improved variants and therefore, it must ensure high coverage and sensitivity. Most of the conventional screening tools rely on the development of novel enzymatic assays which are applied in 96-well plates to monitor the activity of several enzyme mutants [151]. However, considering the screening effort which is required even for the analysis of a single, relatively small library, it becomes apparent that this is an extremely costly and time-consuming process on a laboratory scale. The screening effort can be enormously decreased and advanced by the development of high-throughput screening systems which can analyze several of hundreds of mutants per unit of time. In the last decade, the field of directed evolution has substantially benefited from the involvement of flow cytometry and droplet-based microfluidic setups in the screening of mutant libraries $[152,153]$. Both technologies capitalize on the power of fluorescence and allow the analysis of up to $10^{5}$ events per second, thereby enabling the researchers to screen very big libraries within a daily experimental setup. In the next sections, I will discuss the development of high-throughput screening platforms based on those two technologies and connect them with the engineering of hASNases for the isolation of variants with improved catalytic properties.

\subsubsection{Fluorescence Activated Cell Sorting (FACS)-based high-throughput screening systems}

\subsubsection{Introduction to flow cytometry instrumentation}

Flow cytometry is a powerful method to analyze multiple parameters of individual cells in a population of cells. It is widely used as diagnostic tool in medical applications where different cell populations need to be characterized [154]. Its use is progressively growing in microbiology during the recent years, and this is evidenced by the increasing number of publications reporting on novel directed evolution studies [155-158]. The principle of flow cytometry is based on the forward and right-angle scattering of laser light when it is directed onto a stream of fluid which contains the cell population of interest. Additionally, several 
fluorescence parameters can be detected simultaneously. A flow cytometer can be divided into three major parts: i) the fluidics, ii) the optics, and iii) the electronics part. The fluidic part includes appropriate instrumentation which introduces the cell sample into a fluid, called sheath fluid that is forced through an orifice resulting in the generation of a stream governed by laminar-flow [159]. This process is called hydrodynamic focusing, and the primary goal is to produce a single stream of particles (or cells), or in other words, to allow the cells to pass the light source in a single manner. A schematic representation of this process is given in Figure 13. Once the conditions of the flow stream have been adjusted and the cells are flowing in a single mode, light generated by one or more lasers (depending on the equipment) is focused on the stream at a defined point. This will cause different forward and side light scattering depending on the properties of the particle which flows through the stream (Figure 14). Additionally, if the particle is characterized by specific fluorescence properties (due to fluorescent dyes or intrinsic fluorescence), the emitted light can also be detected [160].

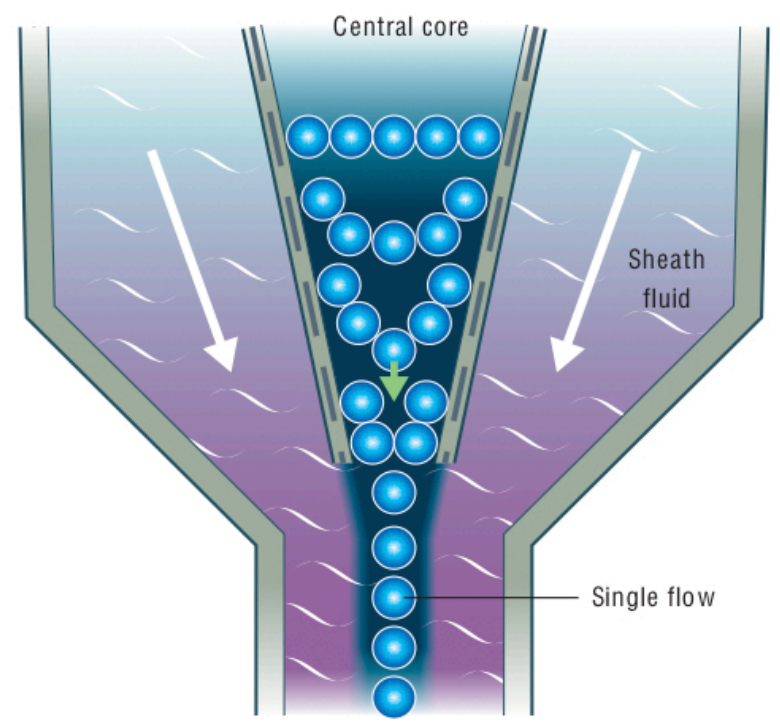

Figure 13. Hydrodynamic focusing process of a flow cytometer. From a central core, the cells (or any particles to be analyzed) are directed downwards to the carrier fluid, termed sheath fluid, which is forced to generate a stream. The pressure of the system (usually $50-100$ psi) matches the orifice size $(50-100 \mu \mathrm{m})$, and this results in the generation of laminar-flow in the centre of the stream, allowing the cells to flow in a single manner. In most of the cases, the sheath fluid as well as the fluid which contains the cells or particles is phosphate-buffered saline (PBS).(Adapted from "Introduction to Flow Cytometry" by Misha Rahman). 


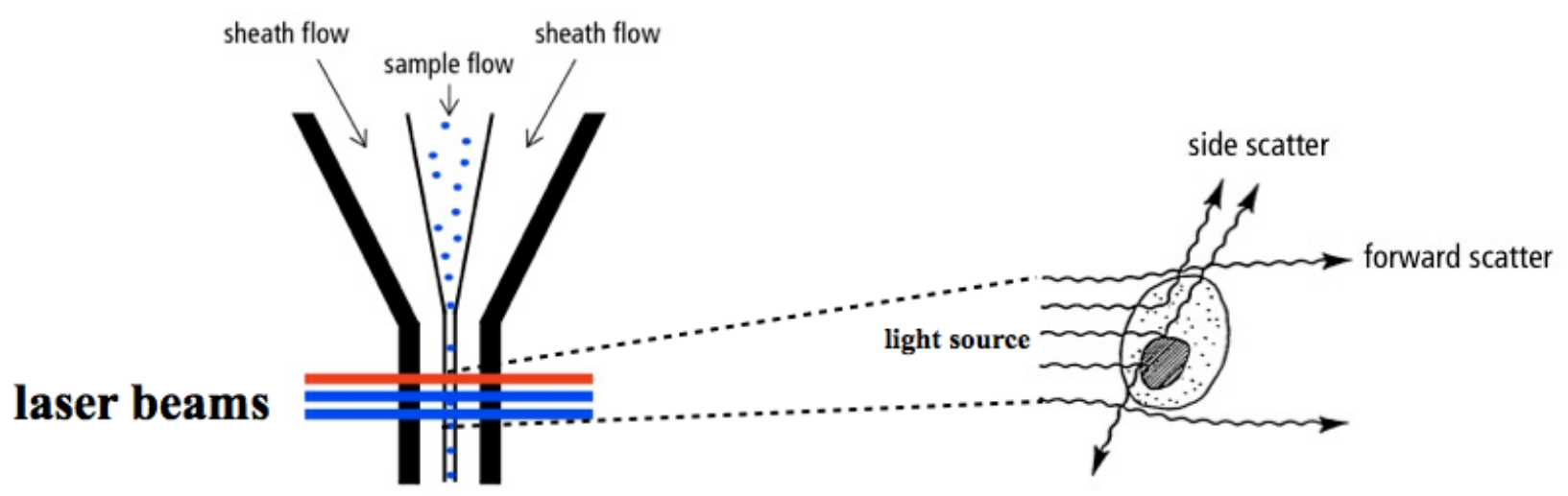

Figure 14. Application of light generated usually by lasers onto the flow stream. The side and forward scattering as well as several fluorescence parameters of each particle are recorded by the optics part of the instrument for further analysis. (Figure licensed and modified from BD).

The scattered and emitted light is detected by the so-called detector arrays which consist of photomultiplier tubes (PMTs). In principle, PMTs enhance the light signals, and this is the point where they are digitalized. Furthermore, bandpass filters are used in combination with the PMTs for measuring the emission from specific fluorophores. The output signal of the PMTs is recorded for each cell, and the final data are stored on a computer for further analysis.

Up to this point, this was a description of a conventional flow cytometer. However, a very important upgrade of this system is cell sorting. This enables the user of a flow cytometer to sort and isolate individual cells from the analyzed cell population based on certain properties which are met and imposed by the researcher. For example, the availability of a cell-sorting system allows the identification and isolation of a subpopulation of cells, which show higher fluorescence intensity as compared to the rest of the cells within a mixture. This cell-sorting part is considered to be an extension of a standard flow cytometer equipment, and very often it adds an additional high cost to the already initial high cost of the flow cytometer (it also depends on the brand of the instrument). This particular feature of flow cytometers is very essential for microbiology and directed evolution experiments since it allows the isolation and separation of individual clones based on their properties; otherwise it would be only possible to acquire general informations for a cell population (e.g. the mean fluorescence intensity of the cells) without having means of separating them. In the instruments which accommodate such sorting parts (also called sorters) the stream is divided into drops by applying vibrational pulses with a defined frequency. The system can be adjusted such that there is no high probability of more than one cell per drop [161]. Through the computer 
settings, we can define how the cells will be sorted before the formation of the drops at the end of the stream (for example sorting cells with high and discard cells with low fluorescence intensity, and vice versa). Subsequently, based on the measured fluorescence intensity signal of the individual cells, it is decided whether a cell will be sorted or not, and an electrical charge is applied to the stream resulting in the charge of the newly formed drop. The charged drops can be deflected and further directed by the so-called electrostatic deflection plates. The electrostatic deflection plates are negatively and positively charged metals which will interact with the charged drops and direct them into collection tubes [162]. A schematic representation of a sorting process is shown in Figure 15.

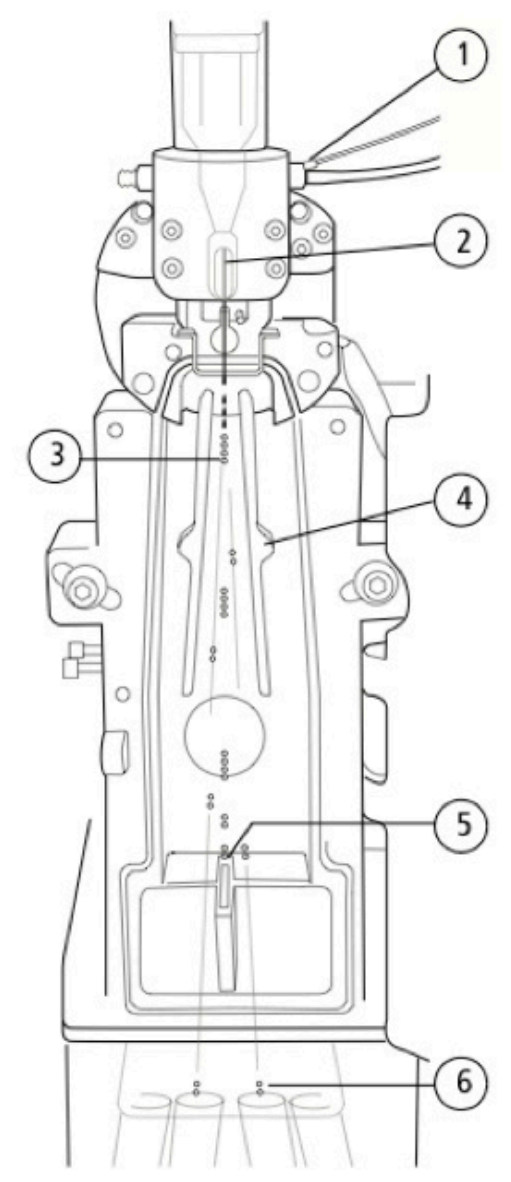

\section{1: Charge is applied via the stream-charging wire in the barb.}

2: Sample generates light scatter and fluorescent signal; signal is analyzed.

3: The charged drop breaks off.

4: Deflection plates attract or repel charged drops

5: Uncharged drops pass to waste.

6. Charged drops containing cells of interest are collected.

Figure 15. Schematic representation of the sorting process. After the signal analysis (point 2), the drops which contain the cells are charged, followed by the separation from the main stream (drops break-off; point 3). Finally, by passing through the electrostatic deflection plates (point 4), they are directed to collection tubes (point 6), while uncharged drops which do not meet the sorting requirements are discarded (point 5). (Figure licensed and modified from BD). 


\subsubsection{Applications of flow cytometry and cell sorting in screening mutant libraries}

Flow cytometry coupled with cell sorting (FACS) has proven to be an invaluable tool for the screening of protein mutant libraries. This is largely dependent on the extremely high sensitivity provided by the fluorescence that allows the quantitative analysis of different parameters at the single cell level. FACS has been very successfully used for screening a wide variety of antibody, polypeptide and enzyme mutant libraries, thereby offering the possibility to study both binding and catalytic phenomena and linking the mutant genotypes with the respective phenotypes [163]. The vast majority of the reported assays rely on fluorescence as read-out signal. Over the years, mainly two kinds of fluorescence have dominated the development of high-throughput screening assays: i) the use of fluorescent polypeptide reporters, and ii) the use of fluorogenic chemical compounds [164].

Fluorescent polypeptide reporters generally refer either to autofluorescent proteins like the green fluorescent protein (GFP), or to peptide tags which can fluoresce upon treatment of the cells with specific chemical compounds [165]. Both types of fluorescent polypeptide reporters have been extensively used, and their main advantage is that they provide very fast real-time quantitative resolution. On the other hand, the use of fluorogenic compounds have been mostly used in cases where intracellular enzymatic activities are to be determined, or binding phenomena between antibodies and antigens [166]. Yet, it must be underscored that the use of such fluorogenic compounds in many cases necessitates cell disruption since they are bulky organic molecules and cannot diffuse inside the cells to reach the target molecule. In addition, another drawback might be the possibility of generation of a diffusible product which leaks out of the cell, resulting in low resolution quantitative analysis and measuring artifacts. By capitalizing on the wide availability of fluorescent probes described above, it is possible to record the fluorescent profile which is interconnected with different phenomena taking place within individual cells. Subsequently, we can isolate them according to our needs. By generating fusions between autofluorescent proteins and proteins of interest, it has become feasible to follow several genetic events like gene expression and regulation, protein localization in the cells $[167,168]$, as well as protein-protein interactions using pairs of autofluorescent proteins that exhibit FRET [169]. An excellent review which highlights the 
use of different fluorescent probes for single-cell FACS applications has been recently published [170].

\subsubsection{Fluorescent screens for enzymatic activity determination by FACS}

One of the most challenging applications is the development of fluorescent screens for monitoring enzymatic activities. It is often very difficult and demanding to develop a fluorescent assay which enables the detection and the quantitative profiling of enzymatic catalysis at the single-cell level for subsequent analysis with flow cytometry. There are several requirements which must be fulfilled and impede such developments. Cells must be permeable to the reaction substrate(s) and this can be difficult in cases where the substrate(s) is (are) big molecules whose diffusion is limited. On the other hand, the enzymatic reaction must yield a product which does not diffuse out of the cell and simultaneously it needs to be adequately fluorescent for detection and single-cell analysis. If the latter is the case, then library screening by FACS can become awkward. A potential solution to this handicap is the compartmentalization of the cells in water-in-oil emulsions which can entrap the cells, thereby maintaining a physical link between the genotype and phenotype (product of the reaction) which is crucial for the recovery of mutants of interest. Such setups will be discussed in the following section of droplet-based microfluidic technology. Yet, several studies have been published reporting on single-cell fluorescent assays for screening mutant and directed evolution libraries for novel enzymatic activities of glutathione-S-transferases [171], esterases [172], proteases [173], and aminoacyl-tRNA-synthetases [174].

A promising alternative to the use of fluorogenic substrates is the use of GFP or other autofluorescent proteins as activity reporters. During the last years, there have been developed many new approaches relying on the use of GFP to study the functions of other proteins as well as to evaluate the activities of target enzymes intracellularly. Worthmentioning examples include the involvement of GFP as a reporter protein to study the function of the chaperonin GroEL which accepts GFP as substrate. GroEL was randomly mutated and the library was transformed into E.coli cells expressing GFP under the regulation of an inducible promoter [175]. The impact of the different mutations on GroEL was reflected by the ability of the chaperonin to fold GFP, and, consequently, the GFP fluorescence (which depends on the protein's correct folding) provided a means of evaluating the GroEL mutants' 
folding activity. Another example involves the use of GFP to study the site-specificity of Cre recombinase [176]. This enzyme is widely used to introduce mutations and substitute genes since it catalyzes the recombination of two identical double-stranded DNA sites of a defined sequence mostly known as loxP. One of the limitations of this recombination system is related to its dependency on the strict positioning of the loxP sites. Therefore, the generation and identification of Cre variants with the ability to recombine artificially introduced recombination sites would be of prime interest. Santoro et al. [176] developed a FACS-based genetic screening approach which is based on the co-expression of two plasmids, one coding for Cre mutant enzymes and the other containing two autofluorescent proteins (GFP and eYFP) cloned in anti-parallel orientations, and two loxP sites upstream of each autofluorescent protein. Depending on the activity of the Cre recombinase, the orientation of the autofluorescent proteins is rearranged resulting in different expression patterns which are reflected by the particular fluorescence of GFP and eYFP proteins. The best Cre isolated mutant exhibited $a \sim 6^{*} 10^{4}$-fold improved specificity against a novel variant loxP site as compared to wildtype Cre. In a comparable case, the substrate specificity of an amino-acyltRNA synthetase was changed making possible the incorporation of unnatural amino acids into a protein of interest [177]. Furthermore, GFP as reporter protein has been used in different ways in cases of directed evolution of hydrolases. An interesting example is the use of a $\mathrm{pH}$-sensitive GFP mutant, called pHluorin, whose excitation spectrum alters according to the surrounding environment's $\mathrm{pH}$. The hydrolytic activity of certain enzymes usually releases protons which consequently change the $\mathrm{pH}$. Those $\mathrm{pH}$ changes were sensed by the $\mathrm{pH}$-sensitive GFP, and this provided a means of correlating the hydrolytic activity of mutants and the excitation ratio of the pHluorin [178]. As model enzyme for this screening setup, an esterase from Geobacillus stearothermophilus was used [178]. All these examples are schematically represented by the following two Figures 16 and 17. 


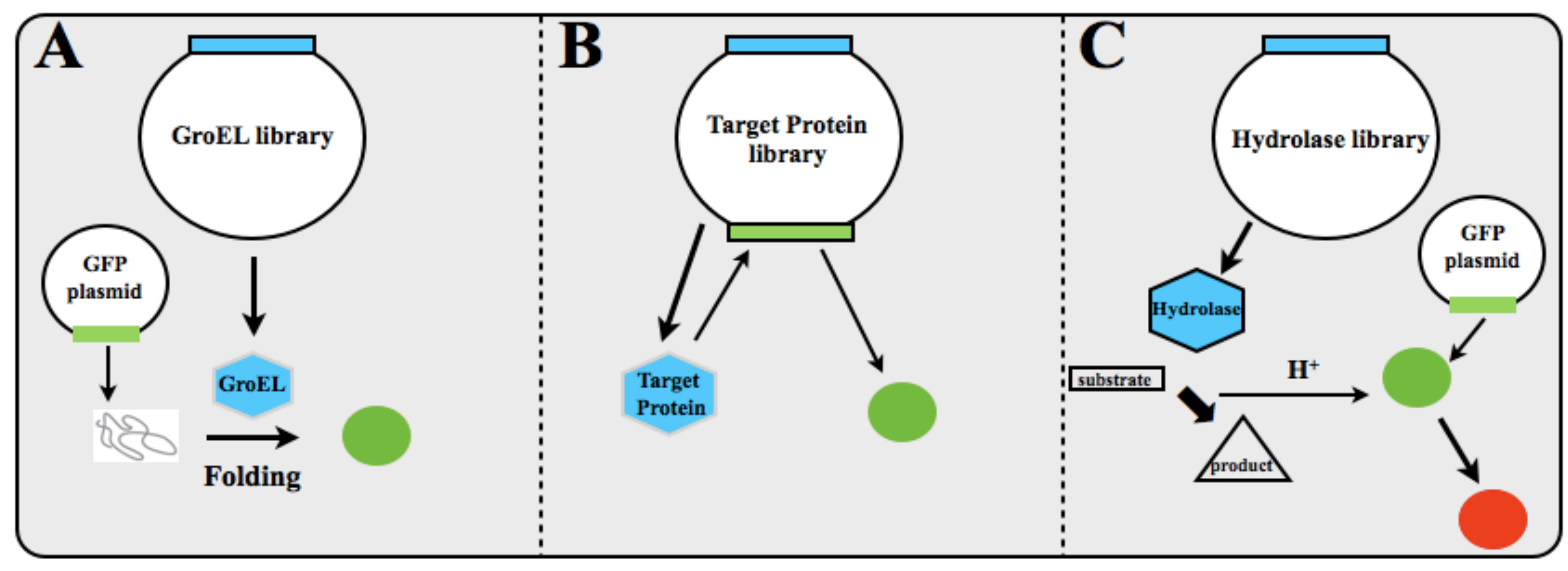

Figure 16. Different FACS-screening approaches which involve GFP as reporter protein. (A) GFP can be used as helper protein to follow the folding activity of GroEL, (B) GFP can also be used as a reporter protein to indicate the expression of another protein of interest whose expression induces the GFP expression, and (C) a pH-sensitive GFP variant senses intracellular $\mathrm{pH}$ variations resulted from hydrolases' activities. Figure was prepared using Keynote.

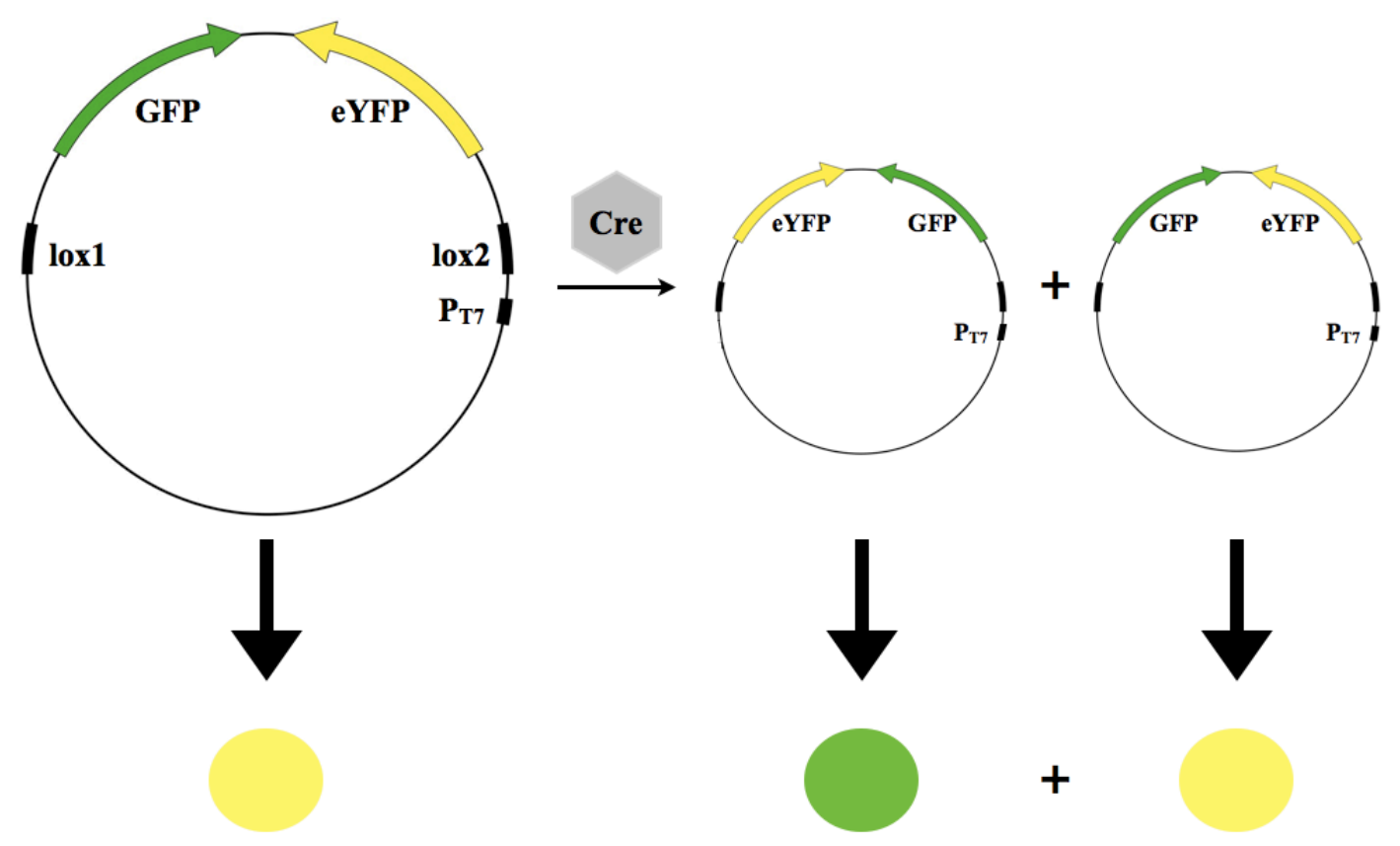

Figure 17. Schematic representation of the Cre recombinase FACS-based screening system. Cells without Cre recombinase activity express only eYFP. In contrast, active Cre enzyme induces equal expression of GFP and eYFP. Figure was prepared using XPlasMap for the construction of the plasmids and Keynote for the final layout.

Perhaps the most relevant for L-ASNases engineering example of a FACS-based highthroughput screening system which relies on the use of GFP as reporter protein, is the study 
from Cantor et al. [179]. In this study, the goal was not the identification and isolation of catalytically improved EcASNase2 variants, but variants which were showing similar catalytic properties as the wildtype upon mutation of specific regions acting as putative T-cell epitopes. This was an effort for de-immunization of the EcASNase2 enzyme and discussed briefly in a previous section. The screening setup was based on an E.coli genetic complementation assay, coupled with the co-expression of GFP as reporter protein. Sitesaturation mutant libraries were constructed using the gene of EcASNase2 whose expression was controlled by a constitutive tet-promoter. The mutant plasmids were transformed in a five-gene-deletion E.coli strain harboring additionally a second GFP-plasmid under the regulation of an inducible lac-promoter. The five gene deletions of the used E.coli strain concerned genes which encode the following enzymes: L-ASNase1, L-ASNase2, LASNase3, L-Aspartate Aminotransferase, and L-Tyrosine Aminotransferase. All these enzymes contribute to the biosynthesis of L-Asp in E.coli. Consequently, this particular strain cannot grow without the amino acid L-Asp in the growth medium. This L-Asp biosynthesis deficiency could be rescued by the expression of L-ASNase because the product of the LASNase activity is L-Asp. Therefore, the availability of L-Asp is directly proportional to the L-ASNase activity of the expressed mutants in the cells. Co-expression of GFP enabled another level of quantification of the L-ASNase activities, since the availability of L-Asp can be correlated with the production of GFP, and consequently the fluorescence intensity of each individual cell reflects semi-quantitatively the L-ASNase activity. Figure 18 depicts schematically the principle of this screening system, which is of particular interest for the engineering of hASNases, though the experimental conditions differ because of certain properties of hASNase3 (autoproteolytic activation step) which require specific growth conditions. In addition, in case of hASNases in general, the ultimate goal is to isolate catalytically improved variants and not those which appear to be equal to wildtype. More details about the application of this screening system to the engineering of hASNase 3 will be discussed in the Results chapter 5. 


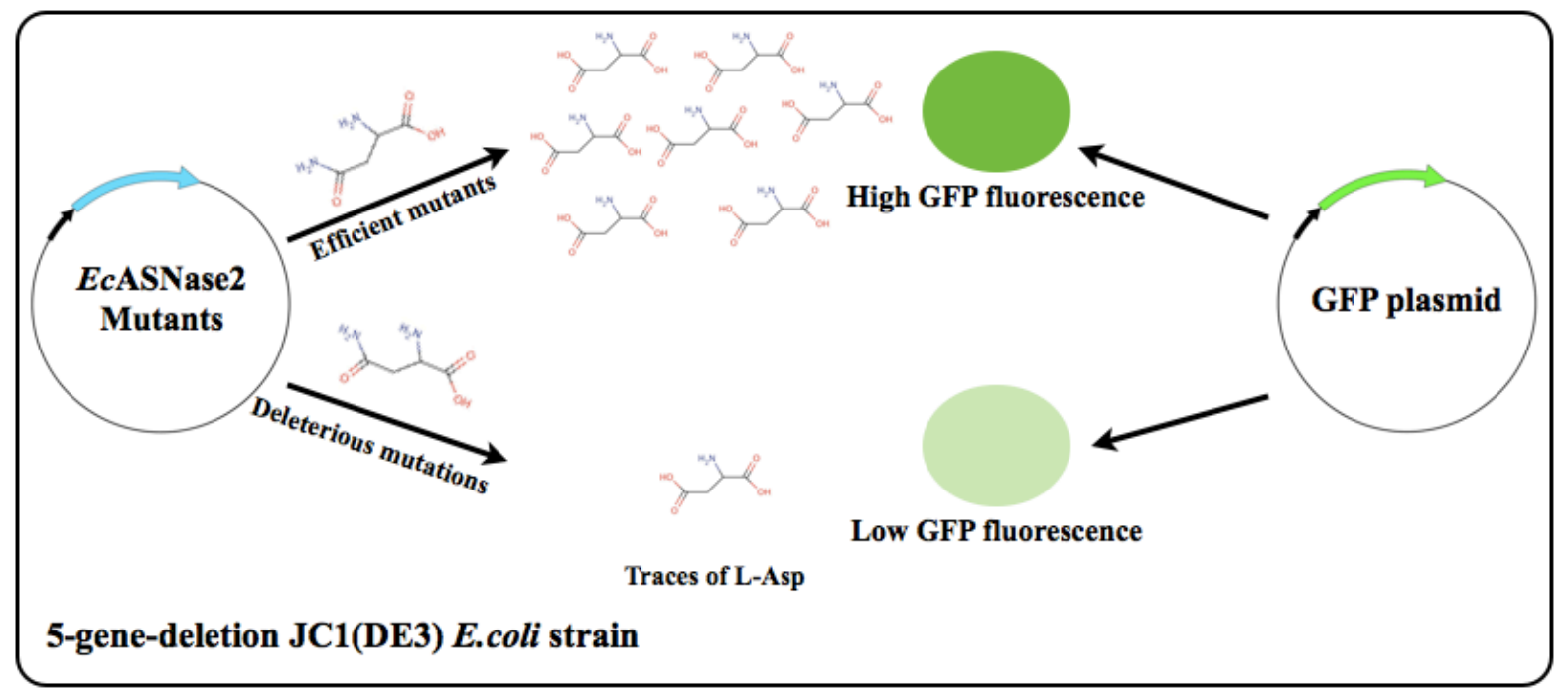

Figure 18. Principle of FACS-based high-throughput screening system for EcASNase2 using GFP as reporter protein. An L-Asp-biosynthesis-deficient E.coli strain is cotransformed with two plasmids which express L-ASNase mutants and GFP, respectively. Beneficial or neutral mutations result in active enzymes which hydrolyze L-Asn efficiently, while deleterious mutations impair the enzymatic activity providing only traces of L-Asp in the cellular environment. The higher the availability of L-Asp, the higher expression levels of GFP can be achieved, while lack of L-Asp results in protein synthesis inhibition since this E.coli strain cannot synthesize L-Asp. Therefore, the activity of EcASNase2 mutants is proportional to the fluorescence intensity, and cells of interest can be analyzed and sorted by FACS for further analysis. Figure was generated using XPlasMap for the plasmids, MarvinSketch for the L-Asn and L-Asp molecules and Keynote for the final layout.

\section{Summary}

The present section focused on the contribution of flow cytometry, and more specifically of FACS, to the development of high-throughput screening systems for directed evolution experimental designs. FACS-based screening systems have been successfully employed to identify and isolate enzyme variants with improved properties of interest, and certain notable examples from the literature were cited above [171-174]. One of the major practical limitations of FACS is the lack of its wide availability because of high instrumentation costs, and the requirement of a certain level of expertise for the operation of the machine, though with the latest user-friendly advanced software, it has become more accessible to researchers with different scientific backgrounds.

As with all high-throughput screening systems, the link between genotype and phenotype must be maintained, and this can be experimentally demanding in some cases. Furthermore, 
the screening of experimental setups which center on the study of enzymatic activity and catalysis is more challenging as compared to those which focus on binding phenomena. For cases where the screening criterion is enzymatic activity, the involvement of GFP as a reporter protein has proven to be extremely beneficial in numerous cases [176-179]. In the present study, we capitalized on the use of FACS for the engineering of hASNase 3 aiming at the generation and identification of catalytically improved variants. We made use of an E.coli strain with five gene deletions which totally impaired the L-Asp biosynthesis in the bacterial cells. Co-expression of GFP as reporter protein provided a means of linking the intracellular L-ASNase activity with GFP fluorescence and subsequent sorting of the most highly fluorescent cells. This screening protocol is a modification of the one which was used by Cantor et al. for the de-immunization of EcASNase2 and is described in Figure 18 [179]. More details on the application of this system are included in the Results chapter 5.

In the next section, I will discuss the use of droplet-based microfluidic setups as highthroughput screening systems. This technology can be considered as complementary to the FACS-based systems, and despite its only recent development it appears to be highly promising, solving some limitations, such as product diffusion out of the cells, which might arise from the other systems.

\subsubsection{Droplet-based microfluidic setups as high-throughput screening systems}

\subsubsection{Introduction to droplet-based microfluidics}

Droplet-based microfluidics is a subsection of the broader field of microfluidics, which includes two more main groups of continuous-flow and digital microfluidics [180]. Dropletbased microfluidics has emerged as a powerful and immensely promising tool for a number of different applications notably in biotechnology [181]. Those setups rely on the formation of droplets upon mixing of two phases consisting of two immiscible fluids, one aqueous and one oil as shown in Figure 19. The continuous phase is often mineral or fluorinated oil, and drops are formed when the oil phase interrupts the stream of the water [182]. The subsequent movement of the drops is dictated by laminar flow, similar to drops in case of FACS that was described in the previous section. The typical size of the formed droplets is in the range of 
$10-100 \mu \mathrm{m}$, and this is dependent on the diameter of the channel within which the two phases flow. Additionally, by adjusting the flow rates of the phases, different numbers of drops can be produced per unit of time [183]. There

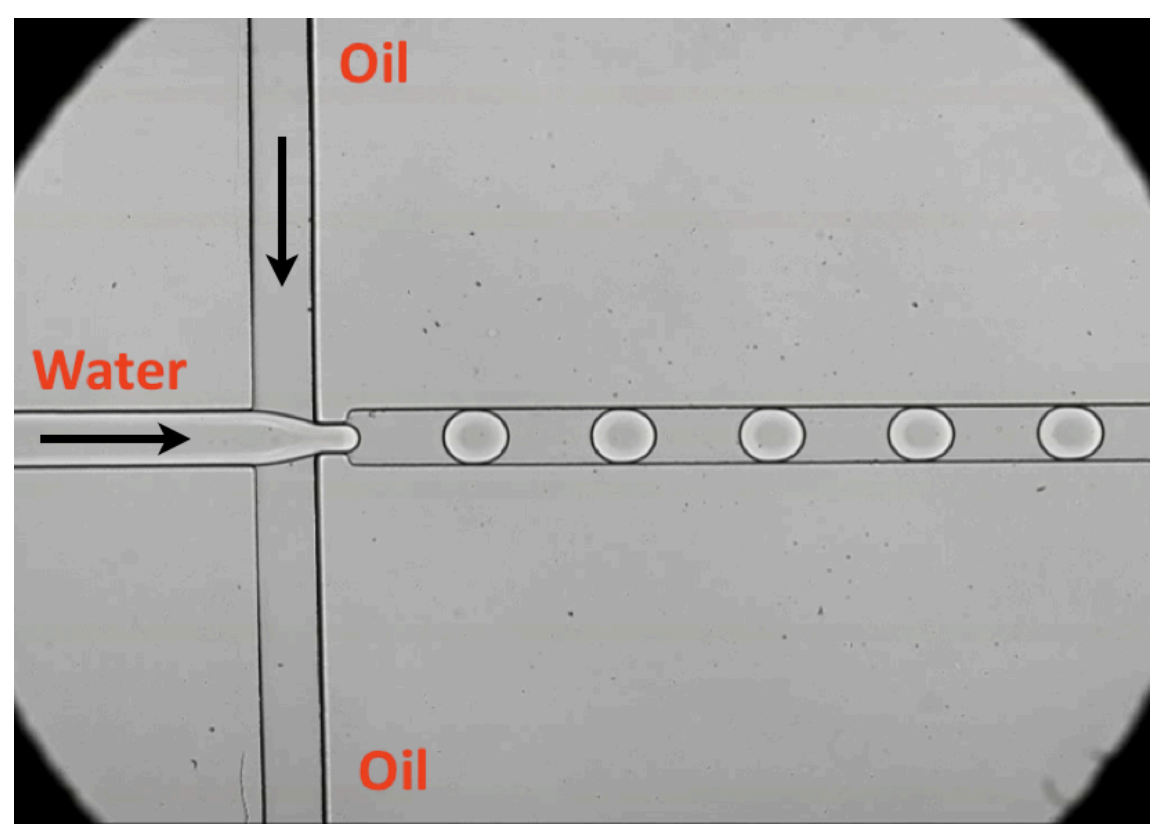

Figure 19. Snapshot of droplet formation process. The figure represents a "T-junction" type of droplet formation. The oil phase is running perpendicular to the aqueous phase and at the point where they meet, the formation of monodisperse emulsions takes place. The two black arrows indicate the direction of the flow of the two phases (aqueous and oil). The frame shows a subsection of a chip. (Image is a kind offer from Dr. Jean-Christophe Baret's lab).

are different types of oils which are used for the production of droplets, and these mainly include silicon-oil, hydrocarbon-oil, and fluorinated-oil [184]. Each type of oil is characterized by distinct properties, and their use is based on the different experimental setup. The formation of the droplets takes place in specific chips which are manufactured usually by using polydimethylsiloxane (PDMS) (Figure 20) [185]. These chips consist of a network of channels within which the water, the oil and the droplets flow. The geometric pattern of channels for each chip is unique and is constructed by means of photolithography [186]. Details of the process of photolithography and the chip construction are beyond the scope of the present study, though excellent references and reviews can be found $[187,188]$. 


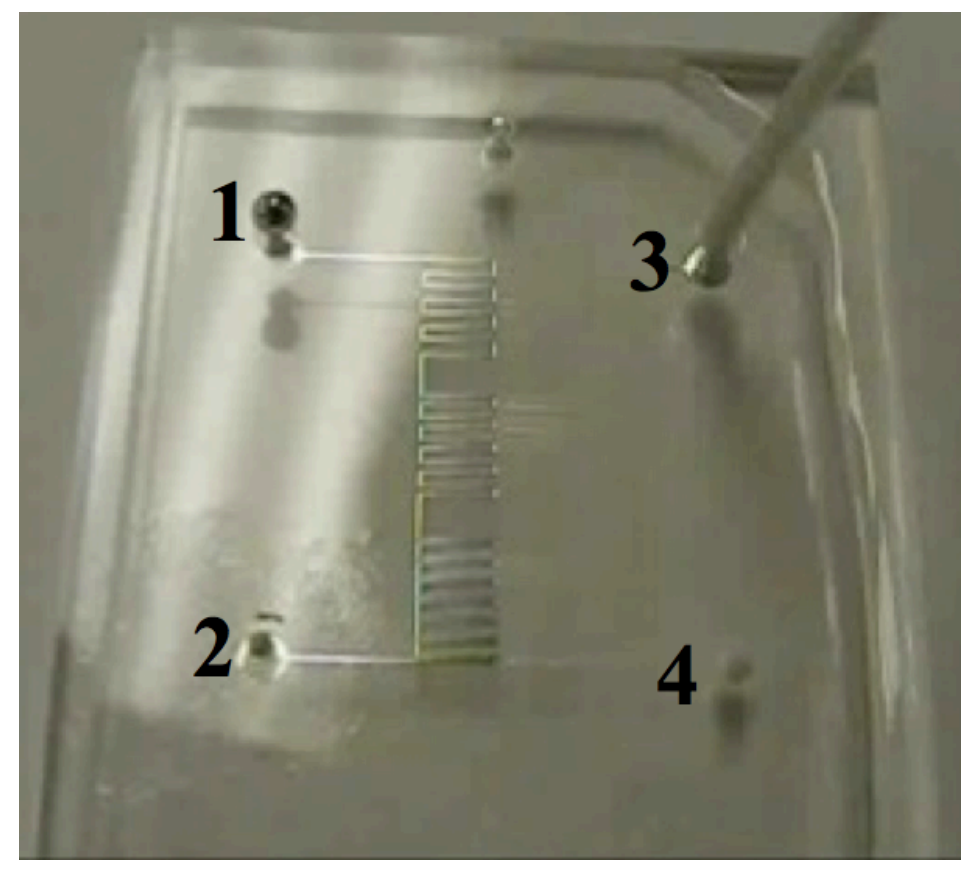

Figure 20. Home-made chip used in droplet-based microfluidic setups. In the center of the figure, it can be seen a geometric pattern which has been constructed by means of photolithography and consists of channels within which the droplets are moving under laminar flow conditions. Positions $1 \& 2$ correspond to inlet points for tubing connections. Positions 3, and 4 are available for electrode connections for the application of electric field. (Image is a kind offer from Dr. Jean-Christophe Baret's lab).

\subsubsection{Key characteristics of droplet-based microfluidic platforms}

In the past, the initial schemes of experiments in droplets were suffering from a major limitation due to the polydisperse character of the emulsions [189]. As a result, the experiments were taking place in different volumes under uncontrollable conditions. However, the contribution of different scientific disciplines such as engineering, chemistry and physics to the droplet-based microfluidic field, enabled the design and fabrication of microfluidic chips suitable for the formation of monodisperse droplets as well as the achievement of very small sizes (down to the fL range) [190]. Major contribution towards this accomplishment was the use of improved surfactants which stabilize the formed droplets and prevent them from coalescence inside the devices [191]. Droplets of well-defined, homogeneous and small volumes brought the concept of compartmentalization of chemical reactions to a more advanced level. Monodisperse emulsions can be considered as microreactors within which complex chemical or enzymatic reactions can take place, thereby serving as excellent screening systems for biological and chemical compounds [192]. The currently available systems allow a number of different manipulations of the droplets within the chips including electrocoalescence which allow the fusion of two distinct droplets, 
collection of several droplets in tubes and incubation for desired periods of reaction times, entrapment of cells, as well as sorting of droplets according to their properties applying dielectrophoretic force [193]. All these possibilities provide an exceptionally interesting toolbox for applications in biotechnology and directed evolution experiments. Figure 21 summarizes schematically all the aforementioned manipulations of droplets. At this point, it must be accentuated the fact that, the different manipulations of the droplets mentioned above require the integration of a number of distinct modules in a very controlled manner, and this can be technologically challenging. Such modules include electrodes which apply dielectrophoretic forces for sorting applications, high-speed cameras coupled with highresolution microscopes for the visualization of the droplets inside the devices (it is very essential to inspect the flow of the droplets since different leaking events might occur) and specific syringes and tubing [194].

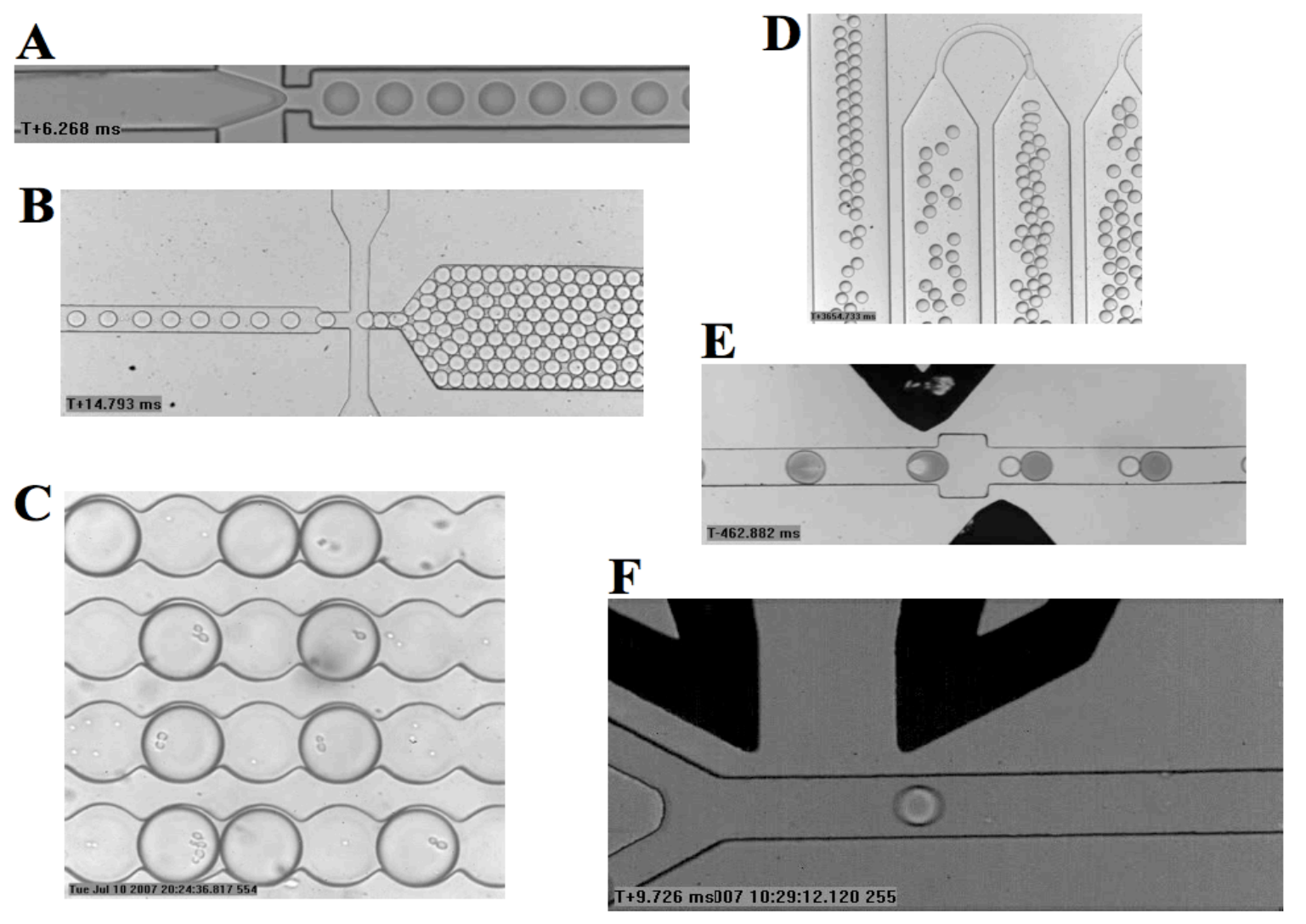

Figure 21. Overview of the most common droplet manipulation procedures. (A) droplet formation, (B) droplet re-injection from a reservoir, (C) cell entrapment into droplets, (D) droplet incubation in channels, (E) droplet fusion, and (F) droplet sorting. (Images are a kind offer from Dr. Jean-Christophe Baret's lab). 


\subsubsection{Applications of droplet-based microfluidic platforms}

The particular features of droplet-based microfluidic setups as they have been presented in the previous section, make such systems highly customizable and adaptable to a wide range of applications. They provide a compartment in which many reactions can take place, they are monodisperse and consequently quantitative measurements can be done, and they offer the possibility to work with extremely small volumes in which single-cells or singlemolecules can be entrapped [195]. Over the last five years a great number of studies have been published highlighting the wide applicability of droplet-based microfluidics and have defined a new field in the biological applied sciences namely lab-on-a-chip [196]. The use of such systems particularly for biotechnology applications has proven to be exceedingly promising as evidenced by a series of novel studies which have been reported and the future appears to be brighter.

The possibility of compartmentalization, which is one of the major limitations of conventional FACS, has set the basis for the individual encapsulation of cells. The loading of cells in droplets can be done in a controlled way, and given the fact that the droplet's content can be modified by fusion with other droplets containing different reagents, it enables the researchers to carry out assays at the single-cell level [197]. In addition, the well-defined volume and the monodisperse character of the droplets maintain the individuality of each microreactor, thereby offering the possibility to analyze them separately and quantitatively in a continuous manner and preserve the link between the genotype and phenotype in one compartment. Most experiments which have been reported relied on the use of bacterial [198] and yeast cells [199], though interesting studies using mammalian cells have also been published [200]. The power of such setups has been very successfully demonstrated in certain cases of directed evolution experiments, although it must be mentioned that up until now, mainly model enzymes like horseradish peroxidase and $\beta$-galactosidase have been at the center of those studies. Baret et al. [201] introduced the term "FADS" (FluorescenceActivated Droplet Sorting) in analogy to the term FACS in their study in 2009, where they reported on the development of an efficient droplet-based microfluidic system for the sorting of droplets based on the measuring of endogenous $\beta$-galactosidase activities of E.coli cells. In the same year, Agresti et al. [202] evolved the enzyme horseradish peroxidase by applying directed evolution approaches and sorted the most highly active variants by using a yeast 
surface display system upon compartmentalization of one yeast cell per droplet. Recently, Kintses et al. published their work on the evolution of a sulfatase by screening directed evolution-generated libraries using microfluidic droplet compartments [198]. They expressed the mutant enzymes in the cytoplasm of E.coli cells and upon encapsulation in droplets, the cells were lysed using commercially available lysing reagents, which were mixed with cells and the enzymatic assay reagents during the formation of the droplets. Finally, they sorted droplets which exhibited the highest enzymatic activities and used the plasmid DNA from the lysed cells, which was still present in the droplets, to recover the genotype of the improved variants.

Considerable progress has also been made in the field of directed evolution using cell-free components. A completely in-vitro ultrahigh-throughput droplet-based microfluidic screening system was developed by Fallah-Araghi et al. [203]. In this study, single genes of $\beta$ galactosidase were compartmentalized in aqueous droplets and amplified by PCR. Subsequently, the droplets containing multiple copies of the parental gene were fused with other droplets containing a cell-free coupled transcription-translation system and the appropriate reagents for a fluorescent assay. Ultimately, droplets with desired enzymatic activity were sorted applying electrocoalescence. Another example of in-vitro expression system was reported by Dittrich et al. [204]. This study centered on the expression of GFP, while it was further advanced by Courtois et al. [205], who stored $\sim 10^{6}$ monodisperse droplets in a reservoir and followed the protein expression from single GFP gene copies. It is worth mentioning that despite the reported progress in the field of directed evolution, dropletbased microfluidic setups have been used as cancer diagnostic tools, being able to detect mutated oncogenes from a mixture containing 200,000-fold excess of wildtype DNA, upon compartmentalization in monodisperse emulsions [206]. Other studies have demonstrated the development of an ELISA-like format derived from a droplet-based microfluidic setup aiming at the quantitative detection of a low-abundance enzyme biomarker which can serve as diagnostic element [207].

The vast majority of droplet-based microfluidic platforms depend on fluorescence measurements for the analysis of the droplets' content. This might be a handicap in some cases of enzymes for which no fluorescent assay is available. Absorption-based spectrophotometric assays cannot be used due to their limited sensitivity since the volumes of droplets are minimized to $1 \mathrm{~nL}$ (or fL) and consequently the light path lengths are scaled 
down to $1 \mu \mathrm{m}$. Additionally, considerable attention must be given to the development of fluorescent assays because certain compounds have the tendency to diffuse out of droplets, where the enzymatic reaction took place, entering empty droplets and thus causing erroneous measurements. In contrast, the availability of a sensitive fluorescent assay can offer great possibilities for the study of enzymatic activities, performing both steady-state and transient kinetic experiments very rapidly and accurately [208]. Other methods which have been integrated in droplet-based microfluidic setups include Raman and mass spectroscopy [209], as well as electrochemical detection modules [210], but they are all less universal and widely used as compared to fluorescence analysis.

\section{Summary}

Droplet-based microfluidics has emerged as a powerful tool for high-throughput applications. They combine a number of highly attractive features which are unique to this technique. Among others, those include the very small volumes in which a great number of different reactions can take place demanding extremely small amounts of compounds, and their compartmentalization properties which circumvent several problems not easily solvable with conventional FACS-based systems. At this point, though, it must be underscored the possibility of using in-vitro compartmentalization approaches with FACS, but those are characterized by three major limitations: i) double emulsions must be generated which are complex, ii) they are polydisperse limiting quantitative analysis, and iii) there is restricted chances for post-formation droplet manipulation, e.g. fusion of droplets [211]. The field of directed evolution has hugely benefited from droplet-based microfluidics so far, and still there is much potential for more progress. Perhaps the major limitation of this rising field is related to its very exceptional know-how which requires bringing together joint forces from different scientific disciplines like engineering, chemistry, and biology. The lack of a readyto-use mainstream commercial instrument limits the wide availability of droplet-based microfluidic setups. This is the main advantage of FACS-based systems which can be obtained commercially and operated by a wide range of researchers due to their simplified and user-friendly interface. On the other hand, the exponentially emerging interest for the droplet-based microfluidic applications provides good reasons to believe that, in the future the availability of such setups will become wider. Table 2 might partially explain why. The 
present study focused on the development of a droplet-based microfluidic high-throughput screening system for the directed evolution of hASNases. The unavailability of a sensitive fluorescent assay for monitoring L-ASNase activity prompted us to develop and validate such an assay whose sensitivity and applicability was demonstrated in both macroscopic (cuvette) and microscopic (droplets) volumes using recombinant purified EcASNase2. Additionally, we studied the system quantitatively up to the single-cell level by displaying the EcASNase2 in the inner membrane of E.coli cells, thereby forming a solid basis for the screening of hASNase 3 mutant libraries aiming at the isolation of catalytically improved variants.

Table 2. Comparison of cost and time required for screening, using traditional robotic microtiter plates and droplet-based microfluidic setups. (Data adapted from [202]).

\begin{tabular}{|l|c|c|}
\hline & $\begin{array}{c}\text { Robot-microtiter plate } \\
\text {-based screening }\end{array}$ & $\begin{array}{c}\text { Droplet-based microfluidic } \\
\text { screening }\end{array}$ \\
\hline Total reactions & $5 \times 10^{7}$ & $5 \times 10^{7}$ \\
\hline Reaction volume & $100 \mu \mathrm{L}$ & $150 \mu \mathrm{L}$ \\
\hline Total volume & $5,000 \mathrm{~L}$ & $1 \times 10^{8}$ \\
\hline Reactions/day & 73,000 & 2 \\
\hline Total time & 260,000 & $\$ 1.00$ \\
\hline Number of plates/devices & $\$ 520,000$ & $\$ 0.30$ \\
\hline Cost of plates/devices & $\$ 10$ million & $\$ 1.70$ \\
\hline Cost of tips & $\$ 280,000$ & $\$ 0.25$ \\
\hline Amortized cost of instruments & $\$ 4.75$ million & $\$ 2.50$ \\
\hline Substrate & $\$ 15.81$ million & \begin{tabular}{c} 
years \\
\hline Total cost
\end{tabular} \\
\hline
\end{tabular}

In summary, it is very important to point out that both FACS and droplet-based microfluidic setups can become two complementary platforms for the high-throughput screening of mutant libraries derived from directed evolution experiments. Each approach has its own 
advantages and disadvantages as described above in the respective sections. However, the fortunate situation of the availability of both techniques can form a firm basis for success in the challenging and multi-step field of directed evolution (Figure 22).

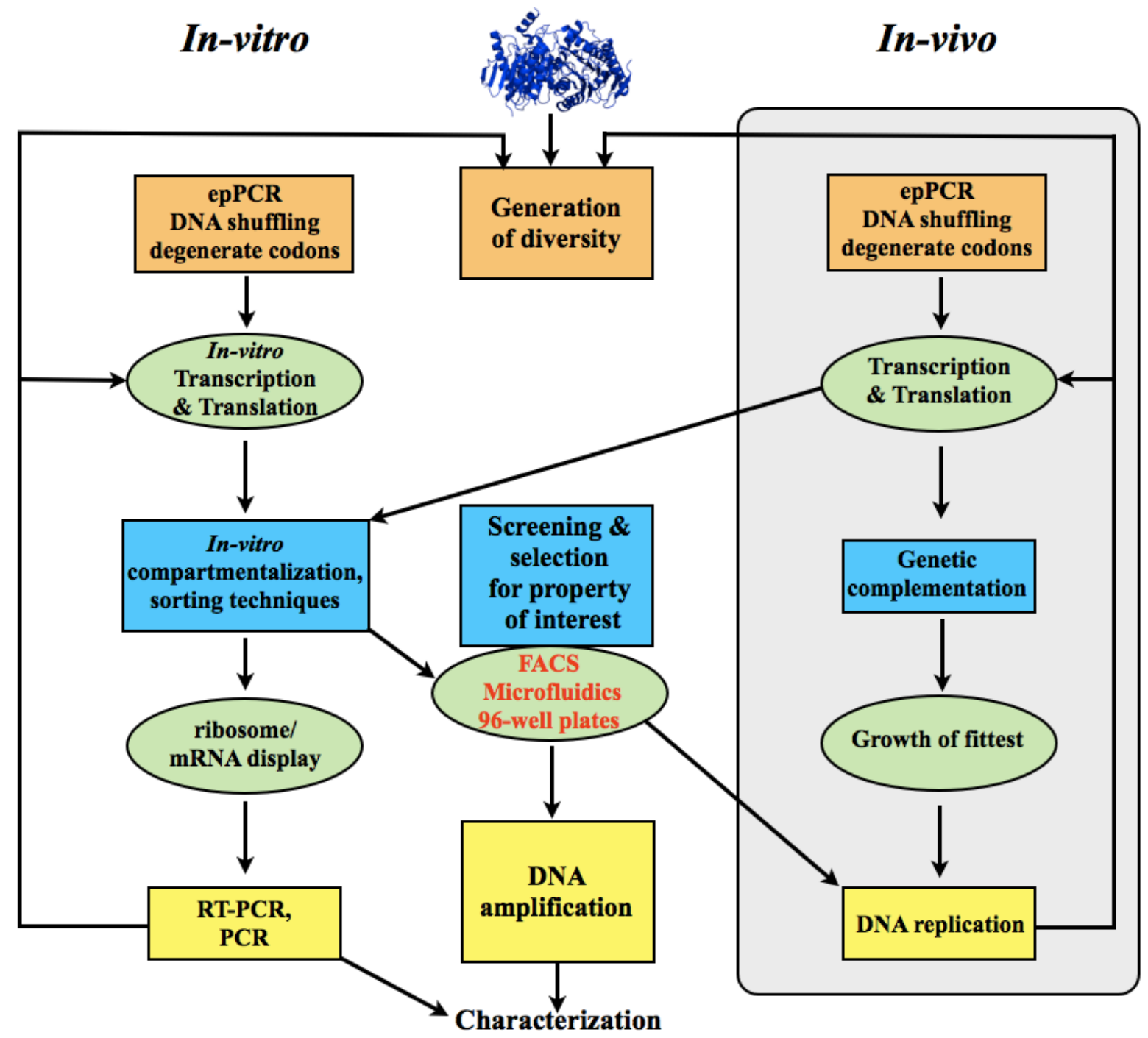

Figure 22. Schematic representation of the major processes, strategies and techniques of directed evolution. A great number of methods are available for generating diversity, screening for the fittest mutants and further characterizing them by biophysical and biochemical means. Figure was prepared using Keynote. 


\subsection{Polyelectrolyte capsules for drug delivery applications}

Drug delivery is a scientific field which centers on the development of customizable systems that can deliver therapeutic compounds quantitatively to a specific target in a controllable manner in the body [212]. Several carrier systems have been developed during the last fifteen years, and those include: i) liposomes [213], ii) solid lipid nanoparticles [214], iii) dendrimers [215], iv) magnetic nanoparticles [216], and v) carbon nanotubes [217]. However, perhaps the most extensively and well-studied class of drug vehicles is the polyelectrolyte capsules (PECs) [218-220]. Conceptually, they were introduced by Möhwald and his team in 1998 [221], and since their invention, PECs have influenced several areas of science, with the most notable impact on biomedicine and more specifically the therapeutic applications.

\subsubsection{Formation of polyelectrolyte microcapsules}

The fabrication of PECs is based on a process called Layer-by-Layer (LbL) assembly and includes the successive adsorption of positively and negatively charged polyelectrolyte layers on an initial sacrificial template, mostly composed of calcium carbonate spheres [222]. The polyelectrolytes can be synthetic such as polystyrene sulfonate (PSS) and allylamine hydrochloride $(\mathrm{PAH})$, or biocompatible, which are preferred for biomedical applications, such as poly-dextran sulfate (PDS) and poly-arginine hydrochloride (PArH). A schematic representation of the fabrication process of PECs is shown in Figure 23. The initial template which is also known as core, is responsible for the final shape, morphology and properties of the polymeric structures [223]. Polystyrene (PS), $\mathrm{SiO}_{2}$ and $\mathrm{CaCO}_{3}$ are the most widely used cores, whereas $\mathrm{MgCO}_{3}, \mathrm{CdCO}_{3}$, and melamine formaldehyde (MF) are less popular. Each template material is characterized by different properties, and the choice depends on the application. For biomedical applications, $\mathrm{CaCO}_{3}$ is by far the most preferable due to its biocompatibility and biodegradability [224]. In addition, spherical structures of $\mathrm{CaCO}_{3}$ are highly porous and have a large surface area, thereby enabling the adsorption of high amounts of biomolecules and chemical compounds which serve as drugs [225]. 


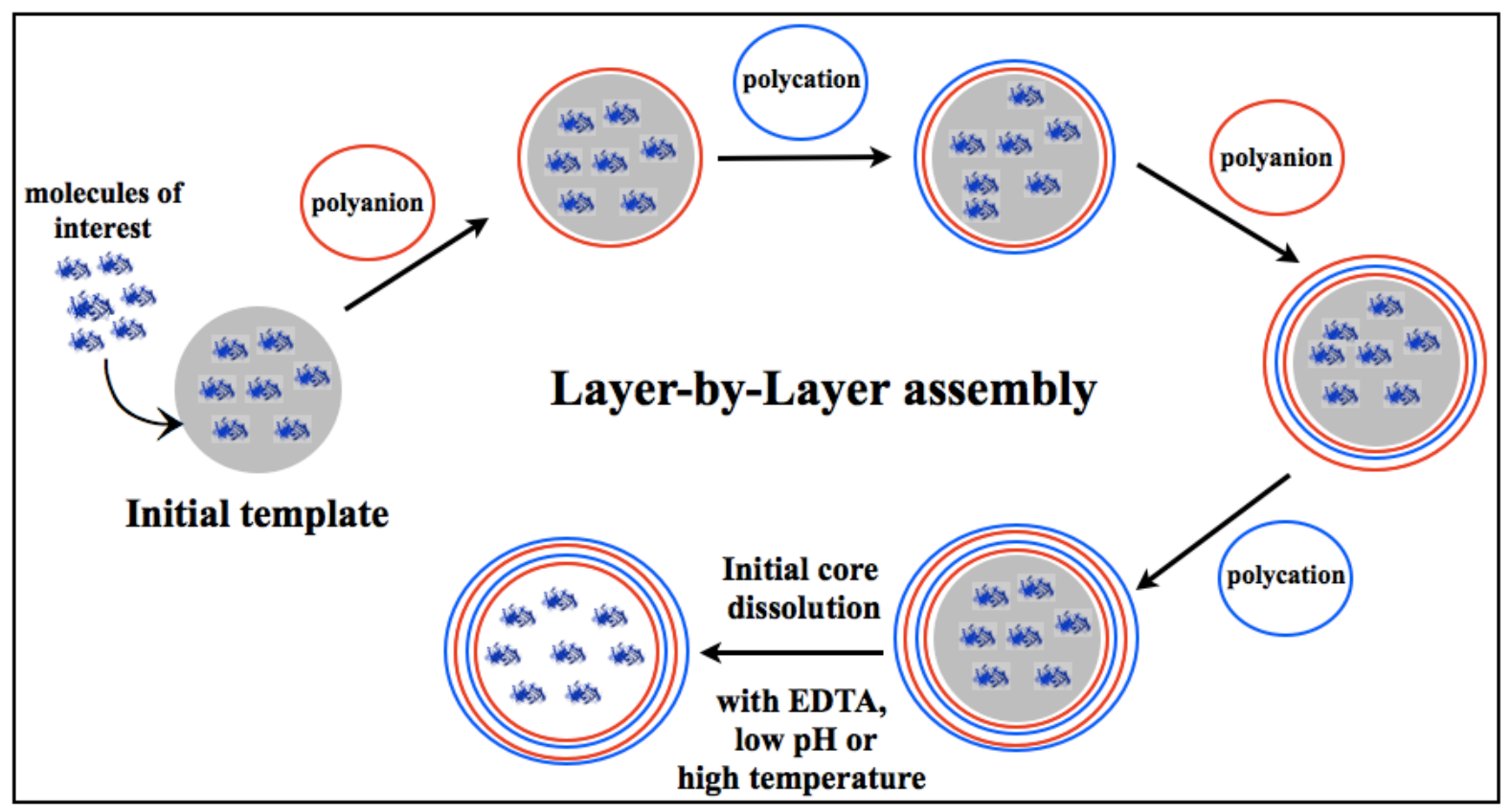

Figure 23. Layer-by-Layer (LbL) assembly for polyelectrolyte microcapsules (PECs) formation. The process commences with the loading (see different approaches below) of the molecules of interest onto an initial template. Subsequently, poly-ionic polyelectrolytes are adsorbed through electrostatic interactions onto the surface of the template. The coating can begin with either polyanions or polycations depending on the properties of the initial template in combination with those of the loaded molecule. After the deposition of the desirable number of polyelectrolyte bilayers, the initial sacrificial template is dissolved by different means, resulting in the entrapment of the molecules in the interior space of the final hollow polyelectrolyte capsules. Figure was prepared using Keynote.

Another interesting biologically-derived example of template is the erythrocytes (red blood cells). Their main advantage is related to their absolute biocompatibility, though their major disadvantage is that they need special handling which results in high cost [226]. Depending on the subsequent application, different sizes of templates can be formed ranging from nanometers to micrometers diameter [227]. The size and the morphology of the initial core will have a direct impact on the final formed capsules. Nanometer- capsules are usually targeted for intracellular uptake, while micrometer-sized assemblies can accommodate higher amounts of drugs and are useful for extracellular or in-vitro applications [228].

The loading of the drug onto the capsules can be done by various approaches, and in principle there is no a straightforward protocol which would have to be followed at the beginning. The encapsulation of a drug highly depends on the properties of the compound to be loaded, and this is a trial-and-error process. There are basically three main methods for encapsulation: i) 
encapsulation by co-precipitation, adsorption or solvent exchange, ii) reversible shrinking/ expansion triggered by $\mathrm{pH}$ and light, and iii) irreversible shrinking induced by salt and temperature. Encapsulation by co-precipitation is called the process in which the molecules of interest (e.g. drug) are combined with the primary compounds which will form the template upon mixing. For example, $\mathrm{CaCO}_{3}$ cores are produced upon mixing equal molar amounts of $\mathrm{CaCl}_{2}$ and $\mathrm{Na}_{2} \mathrm{CO}_{3}$. During co-precipitation, a chemical compound will be mixed together with $\mathrm{CaCl}_{2}$ and $\mathrm{Na}_{2} \mathrm{CO}_{3}$ resulting in its entrapment in the interior space of $\mathrm{CaCO}_{3}$ cores [229]. In contrast, adsorption is called the method in which the molecules of interest are added to already preformed cores, driven by diffusion and electrostatic phenomena [230]. It is plausible to point out that, in case of co-precipitation higher loading efficiencies can be achieved as compared to the adsorption approach; however quite often the co-precipitation of molecules results in cores, and consequently in capsules, of bigger size [231]. The solventexchange is based on the different solubilities of molecules in different buffers, and this is a less common approach of loading [232].

The other two methods for loading are applied once the final capsules have been fabricated. The $\mathrm{pH}$ approach is based on the observation that PECs expand by increasing the $\mathrm{pH}$ of the solution, and this results in the opening of pores which allow the penetration of molecules [233]. The $\mathrm{pH}$ increase and the loading of the molecules are followed by $\mathrm{pH}$ decrease which induces the closing of the pores, thereby entrapping the molecules inside the capsules. Lightinduced loading is somewhat more complex than the $\mathrm{pH}$-induced process. It relies on the use of certain types of molecules (e.g. azo-benzene) which undergo cis-trans configurations upon light exposure, which ultimately open and close the pores of the capsules for encapsulation of molecules of interest. In the simplest case, UV-light can be applied to transiently open pores to the capsules [234]. Irreversible shrinking by temperature and salt depends on the particular intrinsic properties of PECs to undergo an irreversible shrinking or even expansion upon overcoming a certain temperature and ionic strength threshold [235]. This approach can lead to mechanically more robust capsules, but it employs quite harsh and aggressive loading conditions (extreme ionic strength and temperature values) which are not tolerated by biomolecules such as enzymes. At this point it must pointed out that, the method which will be followed for the loading of the molecules of interest onto the initial cores or the final capsules, depends on several parameters, and they must be taken into account during the experimental design. Those parameters are mainly associated with the conditions which are 
imposed by each approach and whether they are tolerable by the molecules of interest. Figure 24 summarizes schematically the three main encapsulation methods described above.
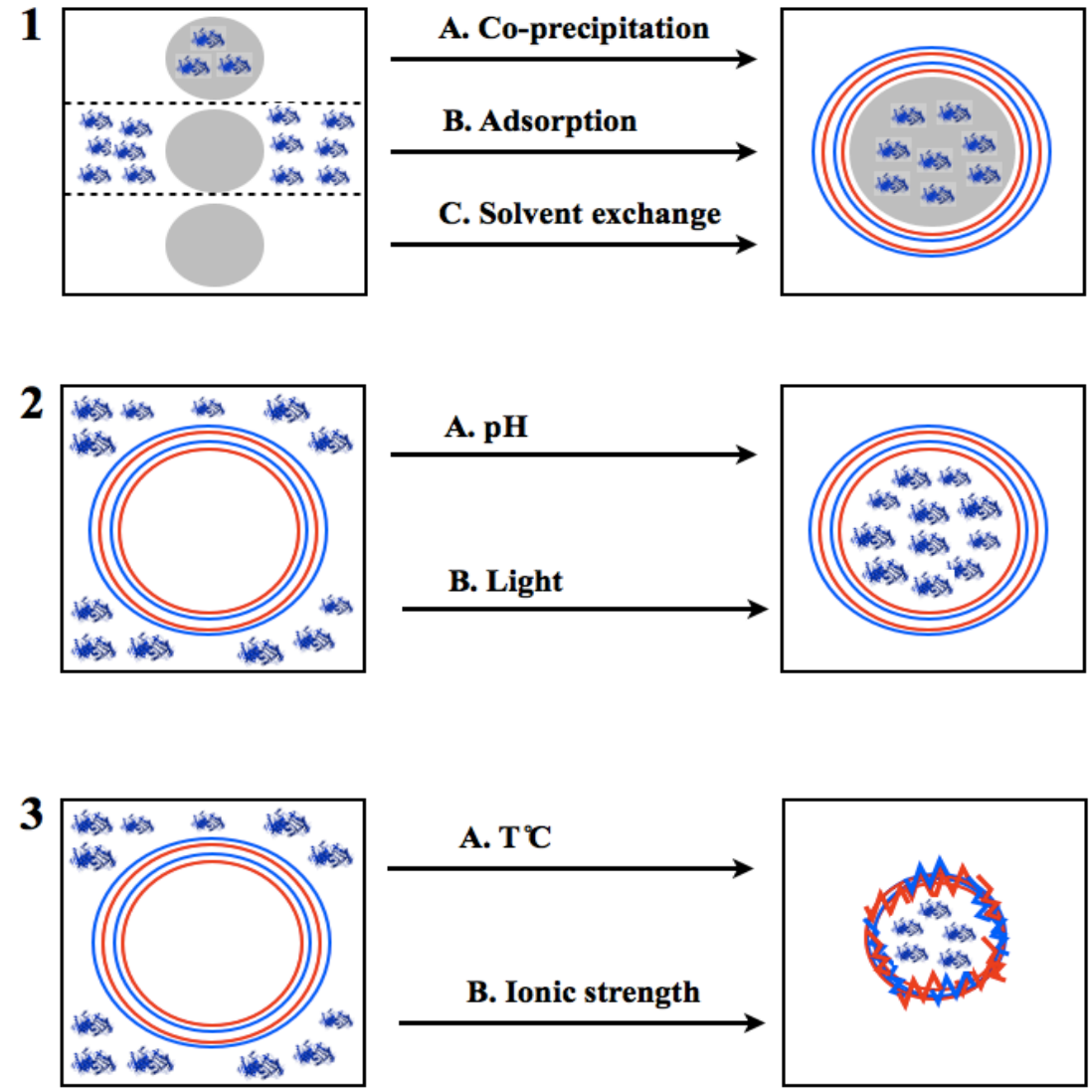

Figure 24. Different encapsulation methods. (1A) co-precipitation, (1B) adsorption, and (1C) solvent exchange. Reversible shrinking/expansion of capsules by (2A) $\mathrm{pH}$ and (2B) light. Irreversible shrinking by (3A) temperature, and $3 \mathrm{~B}$ ) salt. Figure was prepared using Keynote.

\subsubsection{Targeting and content release of PECs}

One of the most attractive properties of PECs for biomedical applications is the possibility to functionalize their surface, predominantly using antibodies, and ultimately target them to specific cells [236]. There have been reported several studies focusing on the properties of PECs upon functionalization with antibodies or other targeting agents (e.g. affibodies) [237], since the attachment of such molecules on the surface of the capsules affects their size, their mobility as well as the activity of the encapsulated bioactive molecules [238]. In the vast majority of delivery applications, the so-called delivery scheme consists of the three following steps: i) targeting and directing of PECs to specific cellular parts, ii) internalization 
of the capsules by the cells, and iii) release of the contained compound which acts as a drug or pro-drug [239]. Internalization typically takes place through phagocytic events, and there are different studies that have shown this [240,241]. Regarding the release of the capsules' content, numerous methods have been developed which in principle, can be classified into three categories depending on the nature of the stimuli that trigger the release: i) physical, ii) chemical and iii) biological [242]. Figure 25 describes a number of different release methods from all three categories mentioned above.

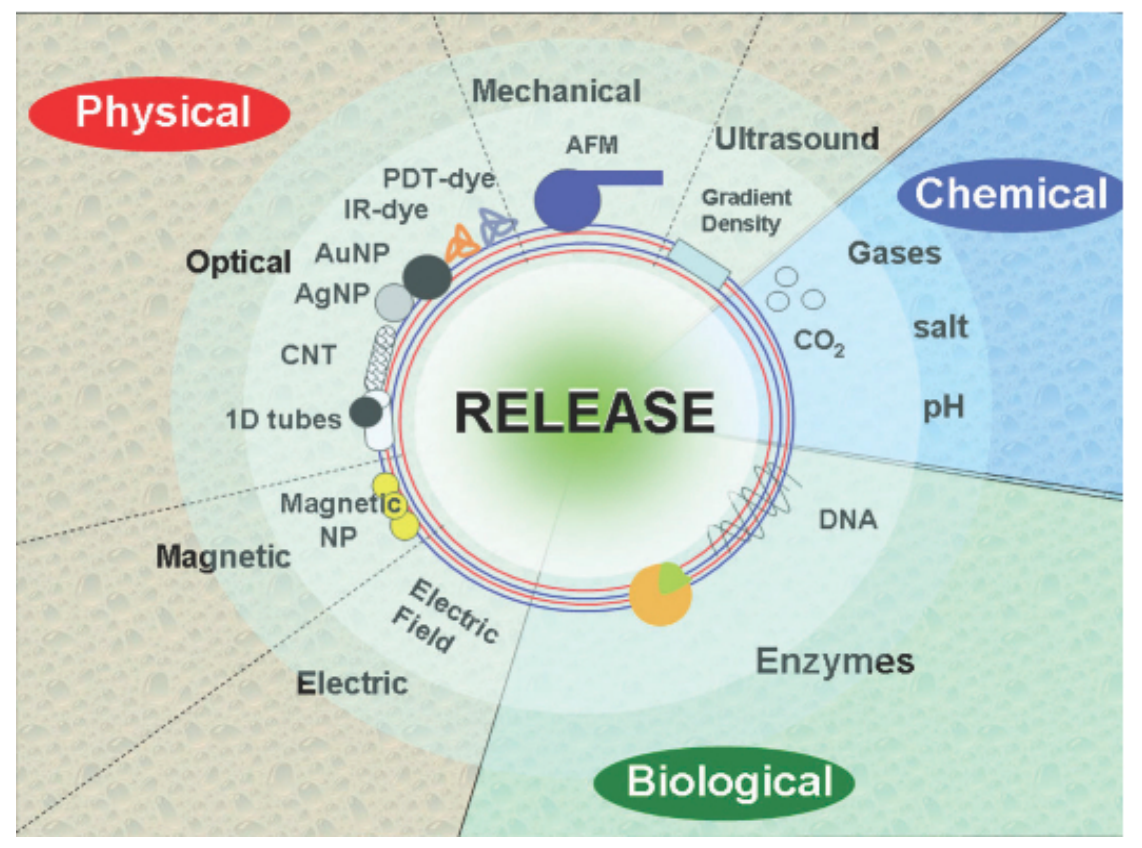

Figure 25. Main three categories of release. Biological, chemical and physical methods are further classified into specific release approaches as depicted in the figure. (Adapted from [242]).

Some of the most interesting and well-studied release approaches include the use of light as a source of electromagnetic field [243], application of magnetic and electric fields [244], ultrasounds [245], enzymatic degradation [246], and chemical stimuli such as $\mathrm{pH}$ and salt [247]. When light is used, the mechanism involves a localized temperature increase on the polymeric shell of metal nanoparticles, resulting in the generation of pores, thereby releasing the content [248]. A similar principle governs the approach of the magnetic field application, with the only variation that magnetic nanoparticles are used in this case. Ultrasound waves have also been used successfully to induce release of capsules' content in certain studies [249]. Enzymatic degradation-based release relies on the degradation of the outer polymeric 
shell of the PECs, thus liberating the encapsulated compound [250]. Low $\mathrm{pH}$ and high concentration of salt are two parameters which can additionally induce release (they were mentioned as methods of encapsulation as well), though with restricted applicability in the biomedical applications.

Evidently, the release methods are exceedingly diverse, and each of them is characterized by certain advantages and disadvantages. The decision about which method must be followed depends on the specific application we are interested in, as well as the properties of the encapsulated molecules since their function could be irreversibly influenced by the applied parameters for achieving release (low $\mathrm{pH}$, heating).

\subsubsection{Applications of PECs}

The use of PECs covers a really wide spectrum of applications in manifold areas. There are many outstanding reports which highlight and analyze the use of PECs in various fields such as biosensor development [251], dentistry [252], mucosal irritation of slugs [253], antireflection coating development [254]. However, the most relevant application for the present study is the contribution of those assemblies to the area of biomedicine and more specifically the cancer treatment. Current chemotherapy suffers from certain limitations which can be very drastic in some cases of treatment. Major impediments of the currently used anticancerous regimens are the lack of specificity and selectivity against the cancer cells [255]. Typically, they do not affect only malignant cells but also healthy ones, resulting in several side effects and general cytotoxicity. In addition, usually quite high amounts of the drugs must be used in order to achieve a considerable treatment level and this leads to more dosedependent side effects [256]. Another important limitation, which is also related directly to leukemia treatment, is the high degradation susceptibility of the used anticancer compounds. Drugs, especially enzymes, are getting degraded rapidly in the body by proteases and other clearance mechanisms (reticuloendothelial system), thus seriously restricting their efficacy [257].

Polyelectrolyte capsules and nanotechnological drug delivery systems in general, can be used to overcome the aforementioned limitations which arise from conventional cancer treatment schemes. Principal advantages of those systems over the free drug administration are i) their small dimensions, ii) the possibility of specific cell targeting, iii) the protection of the drug 
molecules through their encapsulation, thereby increasing their stability and consequently their half-life, and iv) the possibility of combination of more than one drug at once, which could offer a synergistic anticancer effect [258]. Current preliminary results at least on the invitro level concerning the efficacy of drug-loaded PECs against cancer cells are very promising [259]. The same holds for some in vivo-model experiments using mice [260]. However, still some hurdles need to be addressed and further investigated for the improvement of such systems.

\section{Summary}

The invention of polyelectrolyte capsules has undoubtedly defined a new challenging field of research, with a vast potential for applications in many disciplines of material science and biosciences. Particularly in biomedicine, the use of PECs appears to be highly promising especially for the advancement of cancer treatment [236,240]. Given the complex and still uncovered properties of cancer cells, it becomes apparent that the development of "smart" delivery systems for the treatment of cancer will be an exceptionally demanding and challenging task. Many of the aforementioned possibilities concerning the cell targeting and release have been demonstrated only on the laboratory level and their applicability in real schemes is still missing. However, considering the great interest which has been generated over the last years towards the development of such systems, as well as the first promising preliminary results, it is almost certain that further progress will be accomplished in the near future. The present study addressed the possible contribution of PECs to the treatment of ALL. The encapsulation of different L-ASNases using biodegradable and biocompatible polyelectrolyte microcapsules was investigated. As stated above, of great importance in case of drug delivery systems, is the maintenance of the functionality of the loaded drug during the preparation of the capsules. This is particularly true for enzymes whose activity can be irreversibly affected by different materials, or by reaction conditions required for capsule fabrication. Those factors were investigated for EcASNase2 and ScASNase1. We developed an efficient protocol for the successful encapsulation of labile biomacromolecules, allowing them to maintain their functionality upon encapsulation. In addition, the thermal stability, the stability at the physiological temperature $\left(37^{\circ} \mathrm{C}\right)$, the long-term storage stability and the proteolytic resistance against certain proteases which degrade the enzyme during ALL 
treatment, of the encapsulated enzyme were investigated. Ultimately, we tested the encapsulated EcASNase2 and ScASNase1 using two leukemic cell lines aiming at the evaluation of their efficacy under in-vitro conditions.

\subsection{Aims of the present work and Overview}

The present work aimed at the biochemical and biophysical characterization of different LASNases, as well as the development and utilization of high-throughput screening platforms for the directed evolution of L-ASNases. Primary attention was drawn to the study of two human enzymes which possess L-ASNase activity, namely hASNase1 and hASNase3, envisioning future replacement of the bacterial enzymes which are currently used in antileukemic regimens. Chapter 2 discusses the discovery related to the property of hASNase3 to be activated by the free amino acid glycine. This is demonstrated by biochemical means and by the crystal structure determination in complex with glycine, which forms the basis for proposing a mechanistic model that underlies this activation. Chapter 3 focuses on the development of an E.coli co-expression system for the two $\alpha$ and $\beta$ subunits, which form the final active state of hASNase3. This work provides an alternative to circumvent the need of hASNase 3 activation by glycine, thereby skipping many experimental steps for the preparation of the enzyme. The circular permutant-like enzyme produced by this method exhibits the same specific activity as the enzyme which is activated by glycine. Subsequently, chapter 4 describes the characterization of a novel three-step, coupled-enzyme assay for L-ASNase which can be employed in both fluorescence and absorbance mode. The novelty of this assay arises from the fact that, it is the first fluorescence assay for L-ASNase activity determination which solely depends on its natural substrate L-Asn. A number of available fluorescent assays for monitoring L-ASNase activity are based on the use of substrate analogs, thus not being suitable for screening L-ASNase mutant libraries for the identification of variants improved for L-Asn hydrolysis. This assay might be also useful in clinics for the determination of L-ASNase activities from L-ASNase-treated patients who are suffering from acute lymphoblastic leukemia. Chapter $\mathbf{5}$ is devoted to unpublished results which account for a considerable percentage of this work. The chapter is divided into two major parts with the first one centering on the screening of hASNase 3 mutant libraries and the identification of catalytically improved variants; and the second describing the 
development of a new droplet-based microfluidic high-throughput screening system for directed evolution applications. Chapter 6 discusses the first biochemical characterization of a second human L-ASNase, designated hASNase1, which is a mammalian homolog of bacterial-type 1 L-ASNases. A particular property of this enzyme is its high degree of allosteric regulation by its substrate L-Asn despite its monomeric state under assay conditions. This enzyme resides at the N-terminal domain of a $60-\mathrm{kDa}$ enzyme, reported to act as lysophospholipase, though our biochemical data for the $\mathrm{N}$-terminal truncated domain do not support these findings. Structural characterization of hASNase1 in complex with different ligands such as L-Asn or lysophospholipids could shed more light on the interesting biochemical features of this enzyme. The experimental section ends with chapter 7 which describes our work on the encapsulation of L-ASNase1 from Saccharomyces cerevisiae and L-ASNase2 from Escherichia coli by capitalizing on the Layer-by-Layer assembly technology. We established an easy and widely applicable protocol for the fabrication of polyelectrolyte microcapsules filled with active enzyme, which caused killing of leukemic cells upon incubation in-vitro. Eventually, chapter 8 summarizes and discusses the most significant findings of the present work, correlates them with published work, addresses certain weak points which could be further improved, and suggests potential experimental directions that may form the basis for extending this work in the future. 


\title{
2. Free glycine accelerates the autoproteolytic activation of human asparaginase.
}

\author{
Su Y. ${ }^{1}$, Karamitros C.S. ${ }^{2}$, Nomme J. ${ }^{1}$, McSorley T. ${ }^{2}$, Konrad M. ${ }^{2}$, Lavie A. ${ }^{1}$ \\ (2013) \\ ${ }^{1}$ Department of Biochemistry and Molecular Genetics, University of Illinois at Chicago, \\ Chicago, IL 60607, USA \\ ${ }^{2}$ Enzyme Biochemistry Group, Max Planck Institute for Biophysical Chemistry, Göttingen \\ D-37077, Germany \\ Chemistry and Biology \\ http://www.cell.com/chemistry-biology/abstract/S1074-5521(13)00089-6 \\ doi: http://dx.doi.org/10.1016/j.chembiol.2013.03.006
}

\section{$\underline{\text { Author contributions }}$}

C.S.K., M.K. and A.L. conceived the project and designed research; C.S.K., T.M., Y.S. and J.N. performed research; C.S.K., M.K., and A.L. analyzed data and wrote the paper.

\section{Detailed author contributions of C.S.Karamitros}

- Experimental work: cloning, expression, purification and biochemical characterization of hASNase3. Incubation with different metabolites and activity measurements. Cell culture and western blot experiments with T.M. All crystallography work was done by Y.S., J.N. and A.L.

- Data analysis: excluding crystallographic data, all data were analyzed by C.S.K. and M.K.

- Preparation of the manuscript: C.S.K., M.K., and A.L. wrote the paper.

\section{Copyright notice:}

This material is reproduced by permission from the Cell Press in accordance to their copyright and licensing regulations. 


\title{
Free Glycine Accelerates the Autoproteolytic Activation of Human Asparaginase
}

\author{
Ying Su, ${ }^{1}$ Christos S. Karamitros, ${ }^{2}$ Julian Nomme, ${ }^{1}$ Theresa McSorley, ${ }^{2}$ Manfred Konrad, ${ }^{2}$ and Arnon Lavie ${ }^{1, *}$ \\ ${ }^{1}$ Department of Biochemistry and Molecular Genetics, University of Illinois at Chicago, Chicago, IL 60607, USA \\ ${ }^{2}$ Max Planck Institute for Biophysical Chemistry, 37077 Goettingen, Germany \\ ${ }^{*}$ Correspondence: lavie@uic.edu \\ http://dx.doi.org/10.1016/j.chembiol.2013.03.006
}

\section{SUMMARY}

Human asparaginase 3 (hASNase3), which belongs to the $\mathrm{N}$-terminal nucleophile hydrolase superfamily, is synthesized as a single polypeptide that is devoid of asparaginase activity. Intramolecular autoproteolytic processing releases the amino group of Thr168, a moiety required for catalyzing asparagine hydrolysis. Recombinant hASNase 3 purifies as the uncleaved, asparaginase-inactive form and undergoes self-cleavage to the active form at a very slow rate. Here, we show that the free amino acid glycine selectively acts to accelerate hASNase 3 cleavage both in vitro and in human cells. Other small amino acids such as alanine, serine, or the substrate asparagine are not capable of promoting autoproteolysis. Crystal structures of hASNase 3 in complex with glycine in the uncleaved and cleaved enzyme states reveal the mechanism of glycine-accelerated posttranslational processing and explain why no other amino acid can substitute for glycine.

\section{INTRODUCTION}

The human genome codes for at least three enzymes capable of hydrolyzing the amino acid asparagine to aspartate and ammonia. The most-studied enzyme is the lysosomal aspartylglucosaminidase (AGA), whose function is to remove carbohydrate groups linked to asparagine, as the final step in the degradation of cell-surface glycoproteins (Oinonen et al., 1995). Defects in AGA are the cause of aspartylglucosaminuria, an inborn lysosomal storage disease (Saito et al., 2008). A second enzyme is called $60 \mathrm{kDa}$ lysophospholipase, which has an N-terminal domain homologous to the $E$. coli type I asparaginase (Sugimoto et al., 1998). The third enzyme, and the focus of this work, is called L-asparaginase (also known as hASRGL1/ ALP [Bush et al., 2002] or CRASH [Evtimova et al., 2004]). Due to the sequence and structural homology of this enzyme with the $E$. coli type III asparaginase, we refer to this human asparaginase as hASNase3. A member of the $\mathrm{N}$-terminal nucleophile (Ntn) family of hydrolases (Brannigan et al., 1995), this 308 residue enzyme is produced as an inactive single polypeptide that must undergo a peptide-bond break between residues Gly167 and Thr168 to attain asparaginase activity. Cleavage releases the amino group of Thr168, and this endows the enzyme with catalytic activity. This mechanism of protease activation is different from that which occurs in proenzymes (zymogens) such as trypsinogen, pepsinogen, thrombin, or caspases, where the inactivating protein region is cleaved off, either through another protease or through autoproteolysis, and then separates from the now-active enzyme (Kassell and Kay, 1973; Wolan et al., 2009). Importantly, the fold of Ntn family members is unchanged after the cleavage reaction, with the $\mathrm{N}$-terminal (referred to as the $\alpha$ chain) and C-terminal ( $\beta$ chain) parts remaining tightly associated to build a single functional unit. The cleavage reaction of Ntn hydrolases occurs autocatalytically, without a need for proteases (Brannigan et al., 1995; Xu et al., 1999).

Expression of hASNase3 in normal human tissue, observed in all developmental stages except neonate (based on the expressed sequence tags database), is restricted to a few organs that include the testis, brain, esophagus, prostate, and proliferating endometrium (Bush et al., 2002; Weidle et al., 2009). Interestingly, it has also been detected in several human tumors (Weidle et al., 2009), but the implication or the role of hASNase3 in cancer biology is unknown. To increase our understanding of this enzyme, we recombinantly expressed hASNase3, which purified as the uncleaved form. Since the uncleaved enzyme is catalytically inactive, we sought conditions that would promote the transformation to the cleaved and active state. We report that the free amino acid glycine highly selectively acts to promote the autocleavage reaction of hASNase 3 in a concentration-, temperature-, and time-dependent manner. In addition, we present crystal structures of hASNase 3 in complex with glycine that provide a molecular basis for the glycine-induced autocleavage of the enzyme. We propose that glycine-dependent activation of hASNase 3 is related to the altered metabolic profile of cells characterized by increased glycolysis and reduced flux through the tricarboxylic acid (TCA) cycle. Since de novo synthesis of aspartate requires the TCA cycle intermediate oxaloacetate, such cells can instead convert asparagine to aspartate using hASNase3. Glycine, synthesized from the glycolysis metabolite 3-phosphoglycerate via serine, would act as the sensor that regulates cellular aspartate concentrations via hASNas3 activation.

\section{RESULTS}

Correlation between Glycine and hASNase 3 Cleavage Upon bacterial expression and purification, hASNase 3 exhibited a single $\sim 40 \mathrm{kDa}$ band on SDS-PAGE corresponding to the uncleaved form (Figure 1A). In contrast, when we purified the homologous E. coli type III asparaginase, the predominant form was the cleaved version that presents itself as two 


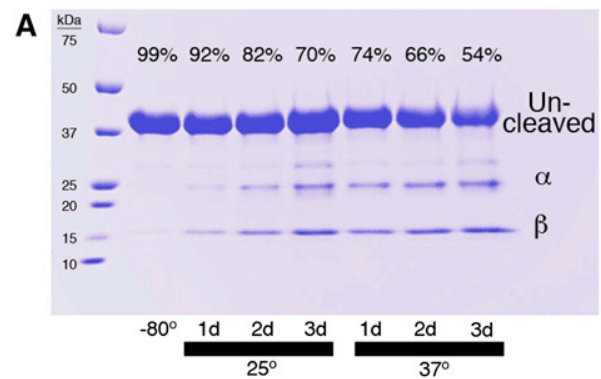

B uncleaved
C

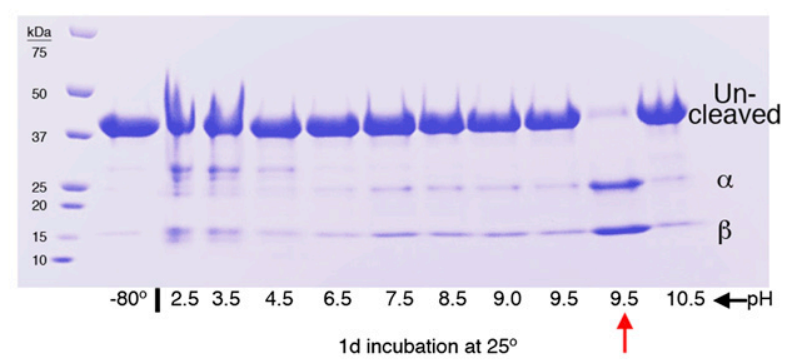

A.

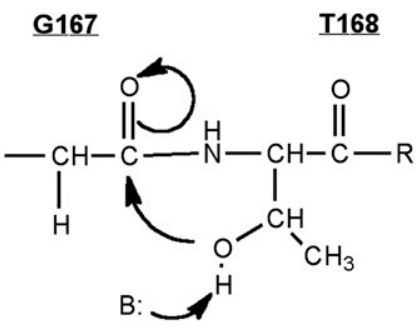<smiles>C[C@H]1[CH]C1</smiles><smiles>[R]C(=O)C1NCC2([C@@H]([R])[2H])C[C@H]1CO2</smiles>

$\mathrm{BH}$

B.

D.

Freed amino group<smiles>[R]C(=O)C(N)C(C)O</smiles>

$\alpha$-subunit<smiles>[R]C([2H])C(=O)O</smiles>

cleaved $\beta$-subunit<smiles>[R]C(=O)C(N)C(C)O[C@H]1CC[C@H](C([R])C)[C@@H](CC)C1=O</smiles>

Figure 1. Self-Cleavage of hASNase 3 Is Slow in the Absence of Glycine

(A) hASNase $3(4 \mathrm{mg} / \mathrm{ml})$ was incubated in storage buffer at $25^{\circ} \mathrm{C}$ or $37^{\circ} \mathrm{C}$, and samples were taken daily. Even after 3 days, the majority of the enzyme is still uncleaved (top band). Cleaved protein runs as two separate bands of lower molecular weight, representing the $\alpha$ and $\beta$ subunits. Percentage denotes uncleaved band intensity relative to the sum of all forms (uncleaved $+\alpha+\beta$ ).

(B) Schematic of the proposed cleavage reaction. A base (shown as B) would accept the proton from the hydroxyl group of Thr168. The identity of this base in the case of hASNase3 was not known prior to this work.

(C) hASNase 3 was incubated for 1 day at $25^{\circ} \mathrm{C}$ at different $\mathrm{pH}$ values using appropriate buffers: citrate, $\mathrm{pH} 2.5-6.5$; Tris, $\mathrm{pH} 7.5$ and 8.5 ; Bicine, $\mathrm{pH} 9.0$; TAPS, $\mathrm{pH}$ 9.5; glycine, pH 9.5 (red arrow); CAPS, $\mathrm{pH} 10.5$. All buffers were at $100 \mathrm{mM}$. The enzyme sample prior to incubation is labeled $-80^{\circ} \mathrm{C}$ in all figures.

See also Figures $\mathrm{S} 1$ and $\mathrm{S} 2$.

lower-molecular-weight bands on a gel (data not shown). The proportion of cleaved hASNase3 did increase gradually over time, but even after 3 days, the uncleaved form constituted $\sim 70 \%$ of the protein incubated at $25^{\circ} \mathrm{C}$ and $\sim 54 \%$ of the protein incubated at $37^{\circ} \mathrm{C}$ (Figure $1 \mathrm{~A}$; Figure $\mathrm{S} 1$ available online). The measured asparaginase activity of hASNase 3 was proportional to the amount of cleaved enzyme, indicating that only the cleaved state was catalytically competent (data not shown). The extremely slow and incomplete self-cleavage of hASNase3 is consistent with a previous report (Cantor et al., 2009), but is different from observations made with bacterial (Borek and Jaskólski, 2000; Borek et al., 2004) and plant (Michalska et al., 2006) asparaginases, which showed efficient autoproteolysis in vitro even at $4^{\circ} \mathrm{C}$, with the bacterial enzyme being fully cleaved in the first purification steps.
Analogy to other studied Ntn family members (Michalska et al., 2008) suggested that self-cleavage commences with the side chain of Thr168 acting as a nucleophile that attacks the carbonyl of Gly167 (Figure 1B, subpanel A). Note that the essential residue Thr168 of hASNase3 plays a dual role. First, its side chain is required for the cleavage reaction. Second, with the break of the peptide bond between Gly167 and Thr168, the freed amino group of Thr168 participates in catalyzing the hydrolysis of asparagine. A base that would accept the proton from the Thr168 hydroxyl group would accelerate the first step of the cleavage reaction. In several Ntn enzymes, the side chain of an aspartic acid residue prior to the scissile bond acts as this base (Qian et al., 2003), but in hASNAse3 the residue preceding Thr168 is a glycine. When replacing this glycine with aspartate in hASNase3, no cleavage was detected (data not shown). 
A

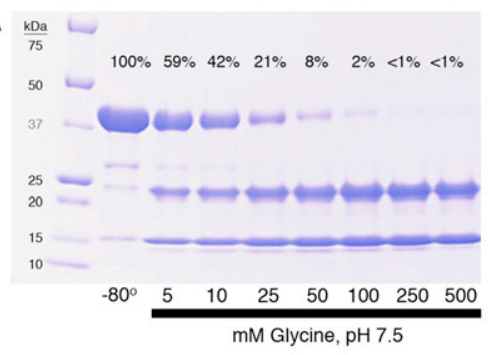

B

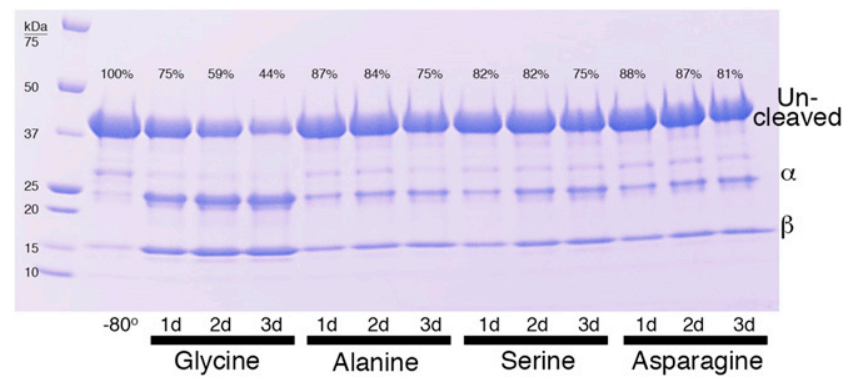

Figure 2. Increase in Extent of hASNase3 Cleavage Is Specifically Dependent on Glycine

(A) hASNase 3 was incubated at $25^{\circ} \mathrm{C}$ for $42 \mathrm{hr}$ in storage buffer containing increasing glycine concentrations at pH 7.5. There is a glycine-dose-dependent increase in the extent of cleavage. Percentage reflects quantification of uncleaved band intensity, with enzyme prior to incubation set at $100 \%$.

(B) hASNase 3 was incubated at $25^{\circ} \mathrm{C}$ in storage buffer supplemented with $10 \mathrm{mM}$ glycine, alanine, serine, or asparagine, and samples were taken daily for 3 days. Quantification as in (A). In all conditions, the amount of cleaved hASNase3 slowly increases due to the intrinsic rate of autoproteolysis. However, only glycine accelerates autocleavage beyond the intrinsic rate.

See also Figures S3, S4, S8, and S9.

Interestingly, the $E$. coli type III enzyme, like the human enzyme, has a glycine residue preceding the cleavage site, yet it undergoes self-cleavage efficiently (Borek et al., 2004). This points to divergence in the mechanism of cleavage between Ntn family members. After building the tetrahedral intermediate, water is thought to act to complete the hydrolysis reaction (Figure 1B, subpanel C). Due to the observation of very slow cleavage of hASNase3 at physiological $\mathrm{pH}$, we reasoned that a high $\mathrm{pH}$ would promote deprotonation of the Thr168 hydroxyl, making it more nucleophilic. However, incubation of hASNase 3 in three different buffers spanning the $\mathrm{pH}$ range of 9 to 10 showed no increase in the cleaved form of the enzyme (Figure S2). When repeating the $\mathrm{pH}$-scanning experiment in a wider $\mathrm{pH}$ range (from 2.5 to 10.5), all but one of the ten $\mathrm{pH}$ conditions tested showed the single band of the uncleaved enzyme, whereas at $\mathrm{pH} 9.5$, using a glycine buffer, we observed essentially complete cleavage of hASNase3 (Figure 1C, red arrow). The two lowermolecular-weight bands of about 22 and $15 \mathrm{kDa}$ observed with glycine buffer correspond to residues 1-167 ( $\alpha$ chain) and 168308 ( $\beta$-chain), respectively, of the cleaved enzyme. This finding was surprising since we had already tested this $\mathrm{pH}$ range and saw no increase in cleavage (Figure S2). Indeed, the lane adjacent to the glycine-treated sample (Figure 1C) contained TAPS buffer at the same $\mathrm{pH}$ of 9.5 , yet showed very little cleavage. This suggested that glycine, rather than simply the $\mathrm{pH}$, must play a critical role in promoting cleavage of hASNase3, potentially as the base depicted in Figure 1B.

Next, we assessed the dependence of self-cleavage on the glycine concentration and $\mathrm{pH}$. Indeed, the extent of cleavage was glycine-dose dependent (Figure 2A), and even the lowest glycine concentration $(5 \mathrm{mM}$ ) promoted $40 \%$ cleavage, a cleavage amount requiring $\sim 11$ days in a buffer lacking glycine (Figure S1). Significantly, this glycine acceleration of hASNase3 cleavage occurs at the physiological $\mathrm{pH}$ of 7.5 nearly as efficiently as at $\mathrm{pH} 9.5$ (Figure S3). We wondered whether other amino acids could function as cleavage accelerators. Since this would presumably require the binding of the amino acid to the active site of hASNase3, we reasoned that amino acids with small side chains would have a greater chance of replacing glycine. However, the addition of alanine or serine did not in- crease the proportion of cleaved enzyme above that obtained by intrinsic cleavage (Figure 2B). Moreover, the substrate asparagine also did not accelerate the cleavage rate beyond its intrinsic rate. Likewise, small-molecule metabolites, such as glycolate, glyoxylate, sarcosine, oxalate, choline, or ethanolamine, failed to trigger or inhibit the cleavage reaction (Figure S4).

We also examined the effects of these metabolites on the asparaginase activity of the cleaved enzyme. Indeed, the cleavage-activator glycine, as well as some of these compounds at relatively high concentration, inhibited the activated form of the enzyme. While glycine at either 10 or $50 \mathrm{mM}$ reduced asparaginase activity by about $50 \%$, glycolate, glyoxylate, oxalate, L-aspartate, and serine showed a concentration-dependent effect, lowering the catalytic activity by up to 5 -fold at nonphysiological $50 \mathrm{mM}$ (Figure S5). This demonstrates that several of the tested compounds have the ability to bind to the enzyme (at least to the cleaved form), but only glycine has the ability to promote the cleavage reaction.

\section{Structural Analysis of hASNase 3}

To understand the mechanism behind the glycine-induced cleavage reaction, we solved the crystal structure of uncleaved hASNase3 in complex with glycine. Our previous work on hASNase3 (Nomme et al., 2012) revealed that, depending on the age of our crystals, we can obtain the structure of either the uncleaved (using fresh crystals) or cleaved state (using crystals grown several months earlier). In those structures, we observed a precipitant molecule (e.g., malonate, sulfate) occupying the asparagine-binding site. In fact, due to competition between the precipitant and any added amino acid, in order to obtain the hASNase3-ASP complex, we had to transfer the crystals out of the precipitant and into a highly concentrated ASP solution (Nomme et al., 2012). Based on this experience, to obtain the uncleaved hASNase3-glycine complex, we used freshly prepared crystals, which were transferred to a $3 \mathrm{M}$ glycine solution. The high glycine concentration was required to stabilize the crystal in the absence of the precipitant and to supply the glycine for complex formation. Despite using fresh crystals that were exposed only shortly to a $\mathrm{pH} 7.5$ glycine solution, diffraction data revealed full hASNase3 cleavage. Based on the cleavage 


\begin{tabular}{|c|c|c|}
\hline Complex & GLY, pH 4.9 & GLY, pH 3.3 \\
\hline Protein Data Bank codes & $4 \mathrm{HLP}$ & $4 \mathrm{HLO}$ \\
\hline \multirow[t]{2}{*}{ X-ray source and detector } & SERCAT BM & SERCAT ID \\
\hline & MARCCD 225 & MARCCD 300 \\
\hline Wavelength $(\AA)$ & 1.0 & 1.0 \\
\hline Temperature $(\mathrm{K})$ & 100 & 100 \\
\hline Resolution $^{\mathrm{a}}(\AA)$ & $1.91(1.91-2.02)$ & $1.95(1.95-2.0)$ \\
\hline \multicolumn{3}{|l|}{ Number of reflections } \\
\hline Observed & 144,084 & 425,284 \\
\hline Unique & 45,006 & 43,500 \\
\hline Completeness (\%) & $95.7(81.7)$ & $98.2(90.0)$ \\
\hline $\mathrm{R}_{\text {sym }}(\%)$ & $5.5(37.9)$ & $12.5(51.2)$ \\
\hline $1 / \sigma(I)$ & $12.40(1.55)$ & $12.70(2.12)$ \\
\hline Space group & $\mathrm{P} 6_{5}$ & $\mathrm{P} 6_{5}$ \\
\hline \multicolumn{3}{|l|}{ Unit cell $(\AA)$} \\
\hline$a=b$ & 59.74 & 59.94 \\
\hline c & 301.53 & 301.10 \\
\hline Refinement program & REFMAC5 & REFMAC5 \\
\hline Twinning fraction & 0.514 & 0.505 \\
\hline \multicolumn{3}{|l|}{ Refinement statistics } \\
\hline $\mathrm{R}_{\text {cryst }}(\%)$ & 18.4 & 16.7 \\
\hline $\mathrm{R}_{\text {free }}(\%)$ & 22.4 & 20.9 \\
\hline Resolution range $(\AA ̊)$ & $30-1.91$ & $30-1.95$ \\
\hline Protein molecules per a.u. & 2 & 2 \\
\hline \multicolumn{3}{|l|}{ Number of atoms } \\
\hline Protein & $2,158,2,158$ & $2,196,2,199$ \\
\hline Glycine & $5 \times 4$ & $5 \times 5$ \\
\hline Water & 181 & 176 \\
\hline \multicolumn{3}{|l|}{ Rmsd } \\
\hline Bond length $(\AA)$ & 0.009 & 0.011 \\
\hline Bond angles $\left({ }^{\circ}\right)$ & 1.116 & 1.354 \\
\hline \multicolumn{3}{|l|}{ Average B-factors $\left(\AA^{2}\right)$ /chain } \\
\hline Protein & 31,32 & 24,24 \\
\hline Glycine & 34 & 33 \\
\hline Water molecules & 32 & 23 \\
\hline \multicolumn{3}{|l|}{ Ramachandran plot } \\
\hline Most favored regions (\%) & 92.2 & 88.5 \\
\hline Additionally allowed regions (\%) & 7.2 & 10.5 \\
\hline Generously allowed regions (\%) & 0.2 & 0.6 \\
\hline Disallowed regions (\%) & 0.4 & 0.4 \\
\hline
\end{tabular}

a Last shell in parentheses.

mechanism (Figure 1B), we reasoned that a lower $\mathrm{pH}$ and an even shorter soak time would allow us to trap the enzyme in its precleaved state, the state relevant for probing the glycine-induced cleavage reaction. Yet again, a data set (1.91 A resolution; Table 1) collected from a crystal soaked in $3 \mathrm{M}$ glycine $(\mathrm{pH} 4.9)$ for $<1 \mathrm{~min}$ showed full protein cleavage (Figure 3A). In this postcleavage state of hASNase3, glycine bound at the active site very similarly to the product aspartate (Nomme et al., 2012), forming interactions with the conserved residues Arg196 via its carboxylic acid moiety and Asp199 via its amino group (Figure 3B). The sole interaction with Thr168 occurs between the glycine amino group and the hydroxyl moiety of Thr168 (3.3 $\AA$ ). This glycine-binding mode failed to clarify how the amino acid would act to accelerate peptide cleavage between Gly167 and Thr168.

The observation of a fully cleaved enzyme state in the $\mathrm{pH} 4.9$ soaked crystal prompted us to test an even lower $\mathrm{pH}$. Indeed, by soaking a crystal of hASNase3 in a glycine solution of $\mathrm{pH}$ 3.3, we successfully obtained the complex in the precleaved enzyme state (Figure $3 \mathrm{C}$ ). For the uncleaved enzyme, we could model the entire polypeptide chain except four residues that span Gln158 and Gln163. The electron density of the scissile bond between Gly167 and Thr168 clearly shows an intact peptide bond (Figure 3D). In this complex structure, solved at $1.95 \AA$ resolution, we again observed the previously mentioned glycine molecule at the substrate-binding site, making very similar interactions as noted in the $\mathrm{pH} 4.9$ structure (labeled GLY1 in Figure 3C). Unexpectedly, an additional glycine molecule occupied the active site (labeled GLY2 in Figures $3 \mathrm{C}$ and S6), this time spanning the conserved threonines at positions 168 and 219. Notably, the carboxylic acid group of this second glycine is at only $2.6 \AA$ distance to the hydroxyl of the catalytically essential Thr168. This type of interaction would allow GLY2 to act as the base that accepts the proton from the Thr168 hydroxyl group, thereby activating it to attack the carbonyl group of the preceding residue, Gly167. We analyzed the binding site of GLY2 in the hASNase3 active site in order to understand why no other amino acid could substitute for glycine in promoting cleavage (Figure 2B). Modeling of alanine in place of GLY2 reveals a steric clash between the methyl side-chain and mainchain atoms of hASNase3 (Figure S7). This rationalizes why even the next-smallest amino acid, alanine, would be sterically excluded from the GLY2 binding site, and hence the cleavagepromoting specificity of glycine. It is of interest to mention here that the dipeptide glycylglycine does not induce (Figure S4) or inhibit autocleavage (Figure S8) or affect the asparaginase activity of the activated enzyme (Figure S5), suggesting that glycylglycine lacks the ability to bind to the hASNas3 active site.

\section{Glycine Promotes hASNase 3 Cleavage in Human Cells}

We next asked whether glycine-accelerated cleavage, and hence activation, also occurs in mammalian cells. HEK293 cells were transiently transfected with the hASNase3 gene, and hASNase 3 expression level and molecular state were monitored using western blot analysis (Figure 4). Cells grown in regular media, without glycine supplementation, predominantly expressed uncleaved hASNase3 (Figure 4). Note that the media used for the cell culture experiments contained $135 \mu \mathrm{M}$ glycine, not taking into account any glycine coming from the fetal bovine serum. This explains the low, but not absent, cleavage of hASNase 3 in this condition. The addition of an extra $1 \mathrm{mM}$ glycine to the media resulted in a more than $25 \%$ reduction of uncleaved protein with a concomitant increase in the cleaved forms. Higher concentrations of glycine promoted progressively increased protein cleavage, such that at $5 \mathrm{mM}$ added glycine, less than $10 \%$ remains in the uncleaved state, with approximately complete cleavage occurring at higher glycine concentrations after the same incubation time. 

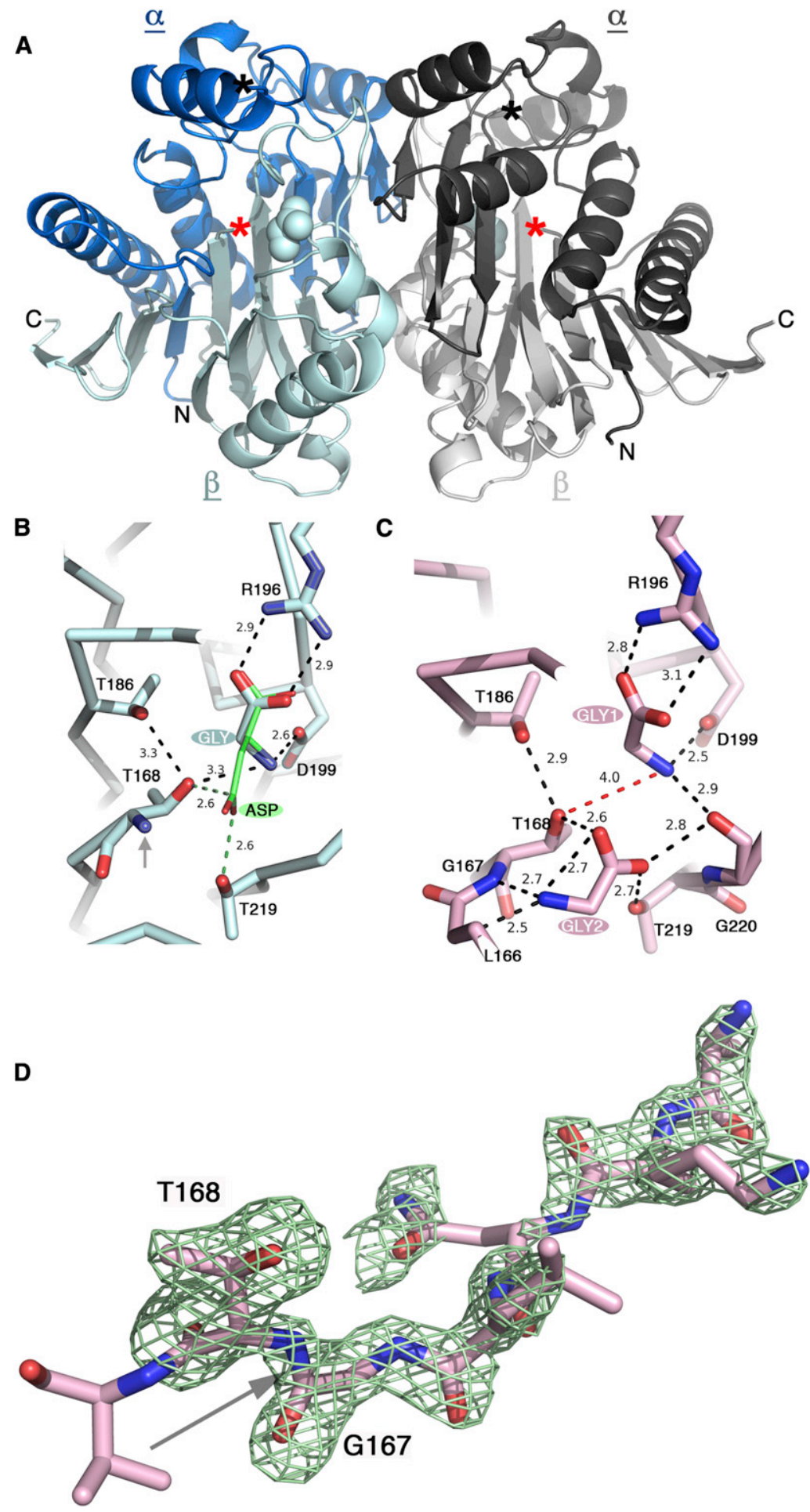

Figure 3. hASNase3 Structure in Complex with Glycine

(A) The cleaved state of the homodimeric enzyme, obtained from crystals soaked in a $3 \mathrm{M}$ glycine solution, $\mathrm{pH} 4.9$, is depicted. One protomer is colored in dark and light blue, and the other in dark and light gray. The dark and light shades depict the $\alpha$ and $\beta$ chains, respectively. The glycine bound at the active site is depicted as a space-filling object. The black star denotes the most C-terminal residue of the $\alpha$ chain with traceable electron density, and the red star denotes the $\mathrm{N}$-terminal residue of the $\beta$ chain, which is Thr168.

(B) Active-site residues in the cleaved hASNase3-GLY complex structure at $\mathrm{pH}$ 4.9. Gray arrow denotes the free amino group of Thr168 resulting from cleavage. Glycine binds in a similar fashion to aspartate (green; as seen in the hASNase3-ASP complex structure; Protein Data Bank code $4 G D W)$. Distances are in angstroms.

(C) The uncleaved hASNase3-GLY complex structure at $\mathrm{pH}$ 3.3. Two free glycine molecules are present at the active site. Glycine 1 (GLY1) binds in the same position as the glycine in (B), and glycine 2 (GLY2) binds in proximity to the scissile bond. Importantly, GLY2 interacts directly with the Thr168 OH group via its carboxylic acid moiety $(2.6 \AA)$. It is this interaction that can serve to activate Thr168 to attack the carbonyl group of Gly 167 .

(D) The Fo-Fc omit map contoured at the 3 sigma level displays the uncleaved peptide bond. To avoid model bias, residues 163 to 168 were omitted from the model, which then underwent multiple refinement rounds. The difference electron density map reveals the conformation of Thr168 and of the residues that precede it. This region, despite being uncleaved, seems to have increased mobility relative to the core structure. As a result, some side-chain densities (e.g., Leu166, Asn165) are not contiguous but clearly present. The increased mobility of this region was also observed in the structures of hASNase3 mutants that cannot undergo cleavage (data not shown). A gray arrow points to the peptide bond that breaks as hASNase 3 is cleaved into the $\alpha$ and $\beta$ chains.

See also Figures S6 and S7.

pending on the tissue examined) have been measured in vivo (Pitkänen et al., 2003). Remarkably, the most recent work, which examined the role of the pyruvate kinase isoform M2 (PKM2) in regulating glycolytic flux in cancer cells and the dependence of PKM2 activity on serine and glycine biosynthesis, revealed intracellular accumulation of free glycine of up to $10 \mathrm{mM}$ (Chaneton et al., 2012), a concentration that would promote complete cleavage of hASNase3 (Figure 4). Glycine did not activate PKM2 in cells or in vitro, yet this work demonstrated serine as the only amino acid to do so in vitro, with this finding being further substantiated by structural analyses of

\section{DISCUSSION}

Here, we report that physiologically relevant glycine concentrations can dramatically accelerate, and drive to completion, the weak intrinsic self-cleavage reaction of hASNase3. While we do find that millimolar glycine levels are required for efficient cleavage (Figure 4), concentrations between 3 and $8 \mathrm{mM}$ (de-
PKM2-serine complexes (Chaneton et al., 2012). In the case of hASNase3, we discovered a specific role of glycine in activating the enzyme both in vitro and in cells. This prompts the following questions: What is the relevance of glycine levels in cells to hASNase3's in vivo function, specifically to any potential role of glycine as a regulator of hASNase3 activity? Can we rationalize its expression pattern in normal tissue and in several 
A

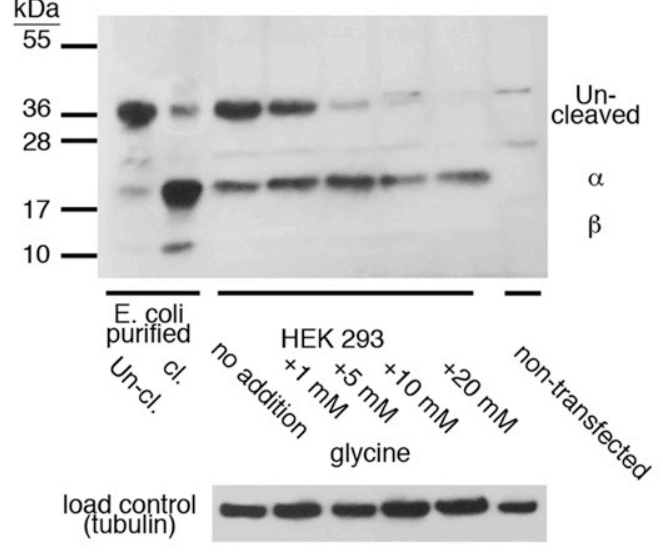

B

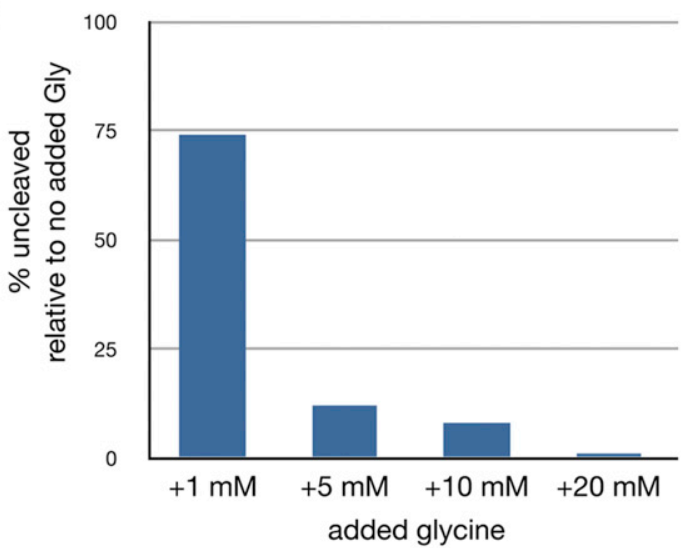

Figure 4. Glycine Promotes hASNase 3 Cleavage in Human Cells

(A) Western blot of HEK293 cells transfected with the gene for hASNase3, grown in regular media or in media supplemented with the noted glycine levels for 2 days. Detection of the hASNase 3 state was done using polyclonal antibodies raised against the full-length protein. Antibodies detect well the uncleaved enzyme and the $\alpha$ subunit, but give only a weak signal for the $\beta$ subunit. The uncleaved (Un-cl) and cleaved (cl) human enzyme purified from $E$. coli is shown for reference. Protein loading control is shown below.

(B) Quantification of the uncleaved band, with the condition of no glycine supplementation set at $100 \%$.

human tumors? We note that hASNase3 has dual asparaginase and isoaspartyl peptidase activities (isoaspartyl peptide linkages are a common source of protein damage; Aswad et al., 2000), with seemingly similar kinetic efficiencies (Cantor et al., 2009). This causes ambiguity as to the precise physiological function of hASNase3. In this context, it is worth recalling that human AGA also has dual catalytic function, hydrolyzing $\beta$-aspartyl-glucosamine linkages in glycoproteins and various $\beta$-aspartyl-amides, including asparagine itself. Moreover, the threonine aspartase Taspase1 acts as an endopeptidase that cleaves the mixed-lineage leukemia protein (Hsieh et al., 2003), the basal transcription factor TFIIA (Hsieh et al., 2003), and other intracellular substrates after an aspartate residue in the recognition sequence (Bier et al., 2011). Like hASNase3, these enzymes belong to the Ntn-hydrolase protein family where the full-length enzymes undergo posttranslational autoproteolytic processing, liberating the $\mathrm{N}$-terminal threonine of the $\beta$ subunit as the catalytic nucleophile. This threonine residue has been shown by mutational analyses to act as the nucleophile for both autoproteolysis and hydrolase activity. While several residues critical for self-cleavage have been identified in human AGA (Bier et al., 2011), no regulatory, or triggering, factor is known for this intramolecular activation step. In contrast, in the case of hASNase3, our work reveals that free glycine very selectively triggers this activation process, which suggests a link to physiological situations of increased glycine levels.

In the physiological context, one intriguing possibility relates to situations where intracellular glycine levels are increased with a concomitant decrease in aspartate levels. This metabolic imbalance is observed in cancer cells where altered gene expression increases the flux through glycolysis (Warburg, 1956). One enzyme implicated in increased glycolysis is PKM2, which is detected in cancer cells and replaces the catalytically more efficient M1 isoform (Vander Heiden et al., 2009). The consequence of reduced pyruvate kinase activity is closure of the spigot connecting the glycolytic pathway to the TCA cycle.
This allows for the diversion of glycolysis intermediates for the synthesis of essential metabolites. In fact, it was recently shown that a small-molecule activator of PKM2 induces serine auxotrophy, an amino acid whose synthesis relies on the glycolytic intermediate 3-phosphoglycerate (Kung et al., 2012). A second enzyme involved in diverting glycolytic intermediates into serine biosynthesis is phosphoglycerate dehydrogenase, and this activity has also been proposed to contribute to cancer cell proliferation (Locasale et al., 2011). As glycine is generated from serine, increased glycolytic flux would increase the glycine levels, as demonstrated by studies of a cancer cell line (Locasale et al., 2011) and brain tumors in vivo (Maher et al., 2012). A recent metabolite-profiling study of $\mathrm{NCl}-60$ cancer cell lines correlated glycine levels with proliferation rates, showcasing the important role of this amino acid in rapidly dividing cells (Jain et al., 2012).

At the same time, increased glycolytic flux reduces the flow of metabolites to the TCA cycle. Reduced TCA flux limits the synthesis of molecules that originate from TCA intermediates. One specific example of such a molecule is the amino acid aspartate, whose de novo synthesis requires the TCA intermediate oxaloacetate. Indeed, a marked decrease in the level of the amino acid aspartate was observed in rat brain gliomas (Ziegler et al., 2001). The data we present here suggest that one mechanism used by cells to counter low TCA flux is to activate the enzyme hASNase3, which then provides the required aspartic acid by catalyzing asparagine hydrolysis. Thus, activation of hASNase3 by glycine would compensate for the lack of aspartate by converting asparagine to aspartate.

Thus, we suggest a model where cancer cells usurp a regulatory mechanism (i.e., dependency of hASNase3 activation on glycine levels) that pre-exists in noncancer cells (see below) that normally express hASNase3 for the purpose of maintaining the required metabolic balance. This model, which relates the activity of metabolic enzymes to metabolite levels in order to compensate for the altered metabolic requirements of rapidly dividing cells, implies that inhibitors of such enzymes (e.g., 
hASNase3) could be explored as potential cancer therapeutics. In fact, the targeting of enzymes that supply asparagine, in order to limit cancer cell proliferation, is currently being explored by inhibiting asparagine synthetase (Richards and Kilberg, 2006). We examined additional metabolites other than glycine that are increased in glycolytic cells, such as pyruvate and lactate, but these failed to recapitulate glycine's ability to accelerate hASNase3 cleavage (Figure S9), highlighting the glycine specificity of this process.

Finally, the cancer implications of hASNase 3 raise questions on its role in normal cells and the properties of these normal cells vis-à-vis glycine and aspartate concentrations. Consistent with our model, it has been reported that normal brain (Coles et al., 2000) and testis (Liu et al., 2011), two organs with high hASNase3 expression, have increased glycolytic activity. Additionally, protein analyses of sperm extracts (Bush et al., 2002) revealed hASNase3 in the predominately cleaved form, thereby correlating the cleaved state of the enzyme with high glycine levels. Further studies that probe the levels of amino acids in normal and diseased states, and the cellular response to these changes, are warranted.

\section{SIGNIFICANCE}

Human asparaginase 3 is an Ntn-family hydrolase requiring cleavage between Gly167 and Thr168 for enzymatic activity. Autoproteolysis of recombinantly produced hASNase3 is very slow. We discovered that the free amino acid glycine dramatically increases the rate and extent of cleavage. Other amino acids or various small-molecule metabolites tested in vitro are not capable of promoting self-cleavage. The crystal structure of uncleaved hASNase3 in complex with glycine explains the ability of the amino acid to promote this intramolecular reaction. We observe two glycine molecules bound at the active site. The first binds at the same position as the substrate asparagine. The second glycine is positioned such that its carboxylic acid moiety is in close proximity to the Thr168 hydroxyl group. The latter binding mode would allow glycine to act as a base that activates the hydroxyl group to attack the carbonyl group of the preceding Gly167, thereby initiating the cleavage reaction. The enzyme has limited expression in normal tissue, being confined mainly to the testis and brain. Notably, it has also been detected in several cancers. A unifying feature of tissues that express hASNase 3 is high glycolytic level. Increased flux through glycolysis would promote glycine synthesis, as it is generated from the glycolysis metabolite 3-phosphoglycerate via serine. At the same time, aspartate levels would be decreased, as de novo synthesis of this amino acid requires the TCA cycle intermediate oxaloacetate. Together, this implies that the function of hASNase 3 is to increase the level of aspartate as compensation for its reduced synthesis and that the enzyme's activity is regulated by the glycine concentration. The requirement of highly glycolytic cells, such as those found in cancers, to generate sufficient levels of key metabolites, such as aspartate, suggests that molecules inhibiting hASNase3 may act to reduce cancer cell proliferation.

\section{EXPERIMENTAL PROCEDURES}

Cloning, Expression, and Purification of Human ASNase3

The cloning and expression of hASNase3 have been reported previously (Nomme et al., 2012). In short, E. coli BL21(DE3) C41 carrying a modified $\mathrm{pET} 14 \mathrm{~b}$ to include a SUMO tag was grown at $37^{\circ} \mathrm{C}$. Upon reaching an optical density of $0.6-0.8$, the temperature was reduced to $18^{\circ} \mathrm{C}$ and $0.5 \mathrm{mM}$ IPTG was added to induce expression. After cell lysis by sonication, the enzyme was purified using a metal affinity column and the SUMO tag was cleaved by SUMO protease, followed by gel filtration. The fractions containing hASNse 3 were concentrated to $38 \mathrm{mg} / \mathrm{ml}$, aliquoted, and stored at $-80^{\circ} \mathrm{C}$.

Crystallization of hASNase 3

A total of $1 \mu \mathrm{l}$ of hASNase 3 at $38 \mathrm{mg} / \mathrm{ml}$ (in $25 \mathrm{mM}$ Tris [pH 7.5], $200 \mathrm{mM} \mathrm{NaCl}$, $2 \mathrm{mM}$ DTT) was mixed with $1 \mu \mathrm{l}$ of the reservoir solution $(2.2 \mathrm{M}$ sodium malonate) on a glass coverslip and left to undergo vapor diffusion using the hanging drop method at $20^{\circ} \mathrm{C}$. To form the glycine complex, a crystal was transferred to a $3 \mathrm{M}$ glycine, $\mathrm{pH} 7.5$ solution. Diffraction data on this crystal revealed the cleaved state of the enzyme and a glycine molecule at the active site. To obtain a complex with the uncleaved enzyme, glycine solutions of lower $\mathrm{pH}$ were tested. At pH 4.9, crystals of the cleaved form were obtained (identical to those soaked at $\mathrm{pH}$ 7.5). However, soaking at $\mathrm{pH} 3.3$ yielded crystals with the uncleaved protein.

Data Collection and Structure Solution of hASNase3

Diffraction data were collected at the Advanced Photon Source (APS) located at Argonne National Laboratory, using the SERCAT beamlines (see Table 1 for data collection and refinement statistics). The structures were refined with Refmac5 (Murshudov et al., 1997). All crystals were perfectly twinned, with the true space group being $\mathrm{P}_{5}$ (apparent space group, $\mathrm{P}_{5} 22$ ) and contained two copies of hASNase 3 (that is, an $\alpha \beta$ dimer) in the asymmetric unit. Refinement using the twin option in Refmac5 showed a twin domain fraction of $\sim 50 \%$. Data collected for freshly grown crystals showed the uncleaved state of the enzyme, whereas in older crystals the enzyme was in the cleaved state. Attempts to soak in the product aspartate (ASP) or glycine (GLY), even at a concentration of $100 \mathrm{mM}$, failed to reveal electron density for these amino acids. We interpret this as being due to competition by the precipitant salt. To obtain the GLY complex, we transferred the crystals to a $3 \mathrm{M}$ glycine solution. The rationale was that a high concentration of glycine would act as a precipitant to keep the crystals stable (attempts to transfer the crystals to a nonsalt condition such as PEG were unsuccessful) and would also supply the GLY molecule bound at the active site. Diffraction data collected on a crystal soaked in $3 \mathrm{M} \mathrm{GLY} \mathrm{pH} 7.5$ revealed the fully cleaved state (data not shown). The same result was obtained with a crystal soaked in $3 \mathrm{M} \mathrm{GLY,} \mathrm{pH} \mathrm{4.9.} \mathrm{In}$ contrast, we observed the uncleaved state in a crystal soaked in $3 \mathrm{M}$ GLY, $\mathrm{pH} 3.3$.

For the cleaved pH 4.9 hASNase3-GLY complex (described here), we could model residues 1 to 153 of the $\alpha$ chain (no traceable density for residues 154-167) and 168-308 of the $\beta$ chain. For the uncleaved pH 3.3 GLY complex, we could model all residues except 158-163. The electron density for the residues immediately preceding the cleavage site was unambiguous (Figure 3D). All structure figures were made with PyMol.

Western Blot Experiments

HEK293 cells, grown in RPMI 1640 supplemented with $10 \%$ fetal bovine serum, were transfected to express hASNase3, and cell lysates were analyzed by western blots. All SDS-PAGE gel band intensities were quantified using ImageJ. Polyclonal antibodies against hASNase3 were generated by immunizing rabbits with $0.6 \mathrm{mg}$ of SDS-PAGE-purified full-length protein according to the Eurogentec (Seraing, Belgium) custom antibody production protocol. For expressing and analyzing the enzyme in a human cell line, the gene coding for hASNase3 was transferred from the pET14b vector to a pcDNA3.1(-) (Invitrogen) with the addition of a Kozak sequence. HEK293 cells $\left(3 \times 10^{6}\right)$ were grown to $90 \%-95 \%$ confluency and then transfected using Lipofectamine 2000 (Invitrogen). At this point, glycine at various concentrations was added to the media. Cells were then incubated at $37^{\circ} \mathrm{C}$ with $5 \% \mathrm{CO}_{2}$. After 2 days, cells were harvested and lysates analyzed by western blots. Loading controls were done using a tubulin antibody. 


\section{ACCESSION NUMBERS}

The structures of hASNase3 have been deposited in the Protein Data Bank under accession codes 4HLP (GLY pH 4.9) and 4HLO (GLY pH 3.3).

\section{SUPPLEMENTAL INFORMATION}

Supplemental Information includes nine figures and can be found with this article online at http://dx.doi.org/10.1016/j.chembiol.2013.03.006.

\section{ACKNOWLEDGMENTS}

We thank A. Mankin for his comments on the manuscript, J. Kaplan and M. Caffrey for discussions, U. Welscher-Altschäffel for technical assistance, and C. Gemski for editing advice. Diffraction data were collected at Southeast Regional Collaborative Access Team beamlines at the Advanced Photon Source, Argonne National Laboratory. Use of the Advanced Photon Source was supported by the US Department of Energy, Office of Science, Office of Basic Energy Sciences, under contract no. W-31-109-Eng-38. This work was supported by National Institutes of Health grant R21 CA155424, the Max Planck Society, and the Deutsche Forschungsgemeinschaft (to T.M.).

Received: October 18, 2012

Revised: February 25, 2013

Accepted: March 4, 2013

Published: April 18, 2013

\section{REFERENCES}

Aswad, D.W., Paranandi, M.V., and Schurter, B.T. (2000). Isoaspartate in peptides and proteins: formation, significance, and analysis. J. Pharm. Biomed. Anal. 21, 1129-1136.

Bier, C., Knauer, S.K., Klapthor, A., Schweitzer, A., Rekik, A., Krämer, O.H., Marschalek, R., and Stauber, R.H. (2011). Cell-based analysis of structurefunction activity of threonine aspartase 1. J. Biol. Chem. 286, 3007-3017.

Borek, D., and Jaskólski, M. (2000). Crystallization and preliminary crystallographic studies of a new L-asparaginase encoded by the Escherichia coli genome. Acta Crystallogr. D Biol. Crystallogr. 56, 1505-1507.

Borek, D., Michalska, K., Brzezinski, K., Kisiel, A., Podkowinski, J., Bonthron, D.T., Krowarsch, D., Otlewski, J., and Jaskolski, M. (2004). Expression, purification and catalytic activity of Lupinus luteus asparagine beta-amidohydrolase and its Escherichia coli homolog. Eur. J. Biochem. 271, 3215-3226.

Brannigan, J.A., Dodson, G., Duggleby, H.J., Moody, P.C., Smith, J.L., Tomchick, D.R., and Murzin, A.G. (1995). A protein catalytic framework with an N-terminal nucleophile is capable of self-activation. Nature 378, 416-419. Bush, L.A., Herr, J.C., Wolkowicz, M., Sherman, N.E., Shore, A., and Flickinger, C.J. (2002). A novel asparaginase-like protein is a sperm autoantigen in rats. Mol. Reprod. Dev. 62, 233-247.

Cantor, J.R., Stone, E.M., Chantranupong, L., and Georgiou, G. (2009). The human asparaginase-like protein $1 \mathrm{hASRGL} 1$ is an Ntn hydrolase with beta-aspartyl peptidase activity. Biochemistry 48, 11026-11031.

Chaneton, B., Hillmann, P., Zheng, L., Martin, A.C., Maddocks, O.D., Chokkathukalam, A., Coyle, J.E., Jankevics, A., Holding, F.P., Vousden, K.H., et al. (2012). Serine is a natural ligand and allosteric activator of pyruvate kinase M2. Nature 491, 458-462.

Coles, J.A., Véga, C., and Marcaggi, P. (2000). Metabolic trafficking between cells in nervous tissue. Prog. Brain Res. 125, 241-254.

Evtimova, V., Zeillinger, R., Kaul, S., and Weidle, U.H. (2004). Identification of CRASH, a gene deregulated in gynecological tumors. Int. J. Oncol. 24, 33-41. Hsieh, J.J., Cheng, E.H., and Korsmeyer, S.J. (2003). Taspase1: a threonine aspartase required for cleavage of MLL and proper HOX gene expression. Cell 115, 293-303.

Jain, M., Nilsson, R., Sharma, S., Madhusudhan, N., Kitami, T., Souza, A.L., Kafri, R., Kirschner, M.W., Clish, C.B., and Mootha, V.K. (2012). Metabolite profiling identifies a key role for glycine in rapid cancer cell proliferation. Science 336, 1040-1044.

Kassell, B., and Kay, J. (1973). Zymogens of proteolytic enzymes. Science 180, 1022-1027.

Kung, C., Hixon, J., Choe, S., Marks, K., Gross, S., Murphy, E., DeLaBarre, B., Cianchetta, G., Sethumadhavan, S., Wang, X., et al. (2012). Small molecule activation of PKM2 in cancer cells induces serine auxotrophy. Chem. Biol. 19, 1187-1198.

Liu, F., Jin, S., Li, N., Liu, X., Wang, H., and Li, J. (2011). Comparative and functional analysis of testis-specific genes. Biol. Pharm. Bull. 34, 28-35.

Locasale, J.W., Grassian, A.R., Melman, T., Lyssiotis, C.A., Mattaini, K.R., Bass, A.J., Heffron, G., Metallo, C.M., Muranen, T., Sharfi, H., et al. (2011). Phosphoglycerate dehydrogenase diverts glycolytic flux and contributes to oncogenesis. Nat. Genet. 43, 869-874.

Maher, E.A., Marin-Valencia, I., Bachoo, R.M., Mashimo, T., Raisanen, J., Hatanpaa, K.J., Jindal, A., Jeffrey, F.M., Choi, C., Madden, C., et al. (2012). Metabolism of [U-13 C]glucose in human brain tumors in vivo. NMR Biomed. 25, 1234-1244.

Michalska, K., Bujacz, G., and Jaskolski, M. (2006). Crystal structure of plant asparaginase. J. Mol. Biol. 360, 105-116.

Michalska, K., Hernandez-Santoyo, A., and Jaskolski, M. (2008). The mechanism of autocatalytic activation of plant-type L-asparaginases. J. Biol. Chem. 283, 13388-13397.

Murshudov, G.N., Vagin, A.A., and Dodson, E.J. (1997). Refinement of macromolecular structures by the maximum-likelihood method. Acta Crystallogr. D Biol. Crystallogr. 53, 240-255.

Nomme, J., Su, Y., Konrad, M., and Lavie, A. (2012). Structures of apo and product-bound human L-asparaginase: insights into the mechanism of autoproteolysis and substrate hydrolysis. Biochemistry 51, 6816-6826.

Oinonen, C., Tikkanen, R., Rouvinen, J., and Peltonen, L. (1995). Threedimensional structure of human lysosomal aspartylglucosaminidase. Nat. Struct. Biol. 2, 1102-1108.

Pitkänen, H.T., Oja, S.S., Kemppainen, K., Seppä, J.M., and Mero, A.A. (2003). Serum amino acid concentrations in aging men and women. Amino Acids 24, 413-421.

Qian, X., Guan, C., and Guo, H.C. (2003). A dual role for an aspartic acid in glycosylasparaginase autoproteolysis. Structure 11, 997-1003.

Richards, N.G., and Kilberg, M.S. (2006). Asparagine synthetase chemotherapy. Annu. Rev. Biochem. 75, 629-654.

Saito, S., Ohno, K., Sugawara, K., Suzuki, T., Togawa, T., and Sakuraba, H. (2008). Structural basis of aspartylglucosaminuria. Biochem. Biophys. Res. Commun. 377, 1168-1172.

Sugimoto, H., Odani, S., and Yamashita, S. (1998). Cloning and expression of cDNA encoding rat liver $60-\mathrm{kDa}$ lysophospholipase containing an asparaginase-like region and ankyrin repeat. J. Biol. Chem. 273, 12536-12542.

Vander Heiden, M.G., Cantley, L.C., and Thompson, C.B. (2009). Understanding the Warburg effect: the metabolic requirements of cell proliferation. Science 324, 1029-1033.

Warburg, O. (1956). On the origin of cancer cells. Science 123, 309-314.

Weidle, U.H., Evtimova, V., Alberti, S., Guerra, E., Fersis, N., and Kaul, S. (2009). Cell growth stimulation by $\mathrm{CRASH}$, an asparaginase-like protein overexpressed in human tumors and metastatic breast cancers. Anticancer Res. 29, 951-963.

Wolan, D.W., Zorn, J.A., Gray, D.C., and Wells, J.A. (2009). Small-molecule activators of a proenzyme. Science $326,853-858$.

Xu, Q., Buckley, D., Guan, C., and Guo, H.C. (1999). Structural insights into the mechanism of intramolecular proteolysis. Cell 98, 651-661.

Ziegler, A., von Kienlin, M., Décorps, M., and Rémy, C. (2001). High glycolytic activity in rat glioma demonstrated in vivo by correlation peak $1 \mathrm{H}$ magnetic resonance imaging. Cancer Res. 61, 5595-5600. 


\section{Supplemental Information}

\section{Free Glycine Accelerates the Autoproteolytic Activation of Human Asparaginase}

Ying Su, Christos S. Karamitros, Julian Nomme, Theresa McSorley, Manfred Konrad, and Arnon Lavie

\section{Inventory of Supplemental Information}

Figure S1, related to Figure 1: hASNase3 has a very slow intrinsic cleavage rate.

Figure S2, related to Figure 1: High pH by itself does not accelerate cleavage.

Figure S3, related to Figure 2: Cleavage of hASNase3 is glycine dose-dependent and occurs also at physiological pH.

Figure S4, related to Figure 2: Promotion of hASNase3 cleavage is specific to glycine.

Figure S5, related to the main text section titled "Structural analysis of hASNase3": Effect of small metabolites on hASNase3-catalyzed asparagine hydrolysis.

Figure S6, related to Figure 3: Fo-Fc map contoured at 3 sigma around GLY1 and GLY2.

Figure S7, related to Figure 3: A model of an alanine bound in the same position as GLY2.

Figure S8, related to Figure 2: Promotion of hASNase3 cleavage by glycine is not inhibited by di-glycine, aspartate, or serine.

Figure S9, related to Figure 2: Small-molecule metabolites other than glycine that are expected to be present in glycolytic cells do not duplicate glycine's ability to accelerate cleavage. 


\section{Supplemental Figures}

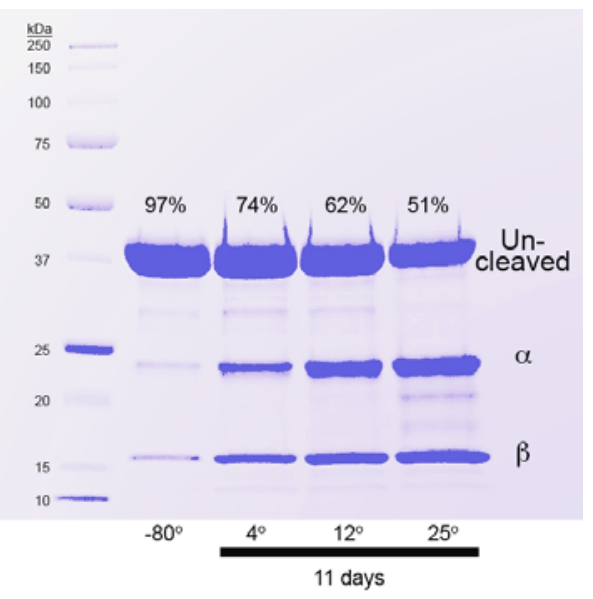

Figure S1. hASNase 3 has a very slow intrinsic cleavage rate. Samples were incubated in storage buffer for 11 days, at either 4,12 , or $25^{\circ} \mathrm{C}$. These temperatures were selected since the enzyme is not stable for more than 3 days at $37^{\circ} \mathrm{C}$. Percentage denotes precursor band strength relative to the sum of all three forms (precursor $+\alpha+\beta$ ). 


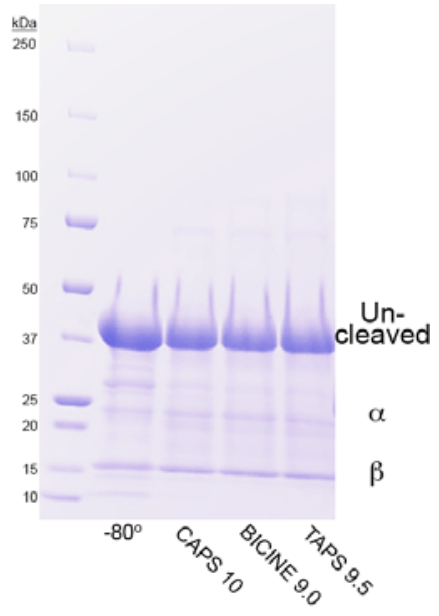

Figure S2. High pH by itself does not accelerate cleavage. hASNase 3 (freezer-stored original sample labeled $-80^{\circ}$ ) was incubated in 25 mM CAPS, pH 10.0, BICINE pH 9.0, or TAPS, $\mathrm{pH} 9.5$ for 42 hours. Note that the enzyme remained predominantly in its uncleaved form. 
A

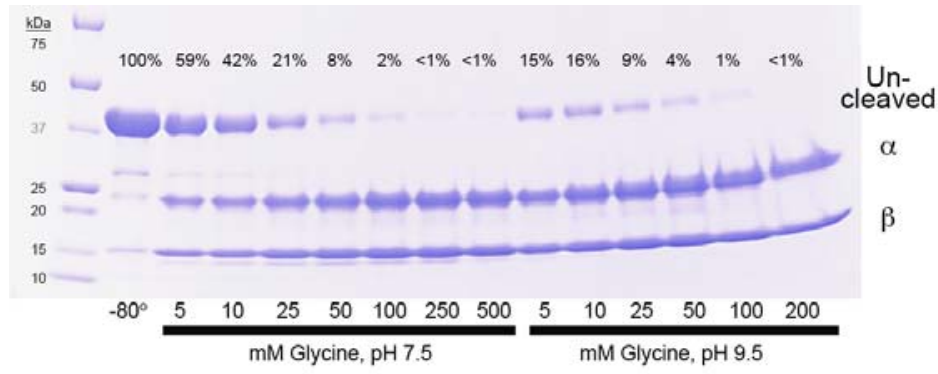

B

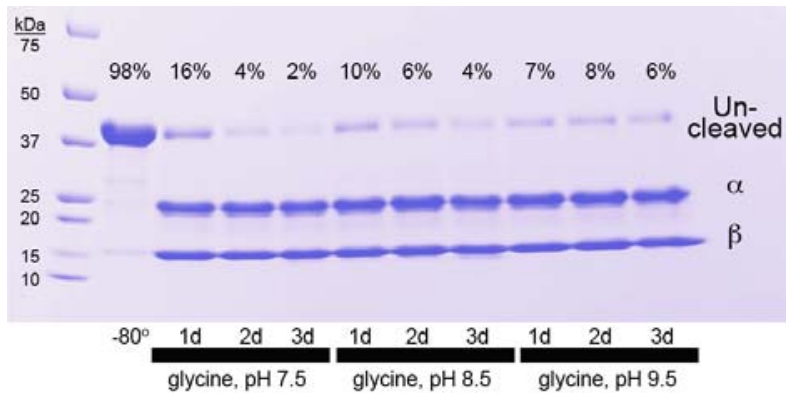

Figure S3. Cleavage of hASNase 3 is glycine dose-dependent and occurs also at physiological pH. (A) Left panel; same as Figure 2a; right part, at pH 9.5. Cleavage rate is better at the higher $\mathrm{pH}$, but still significant at the physiological $\mathrm{pH}$ of 7.5. (B) hASNase3 was incubated in storage buffer plus $100 \mathrm{mM}$ glycine at $\mathrm{pH} 7.5,8.5$, and 9.5. Samples were taken after 1,2 , and 3 days incubation at $25^{\circ} \mathrm{C}$. The state of the starting material as taken from the freezer is shown in the lane labeled $-80^{\circ}$. Percentage denotes precursor band strength relative to the sum of all three forms (precursor $+\alpha+\beta$ ). 

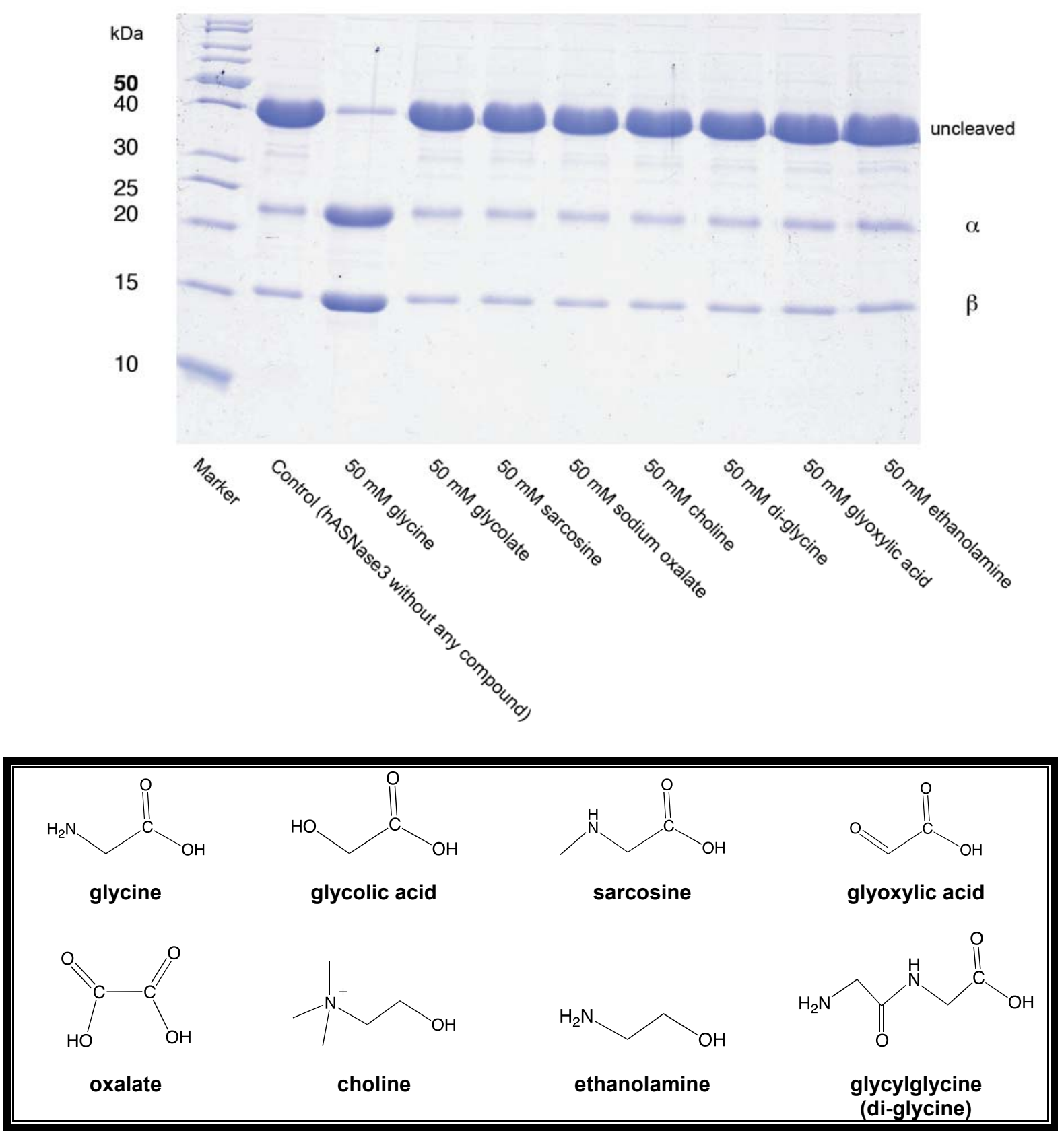

Figure S4. Promotion of hASNase 3 cleavage is specific to glycine. The enzyme hASNase 3 at a concentration of $3.5 \mathrm{mg} / \mathrm{ml}$ was incubated with the indicated molecules at $37^{\circ} \mathrm{C}$ in $50 \mathrm{mM}$ sodium phosphate, $\mathrm{pH} 8$, and $0.5 \mathrm{M}$ sodium chloride for 24 hours. The control lane displays the very limited intrinsic cleavage of hASNase3. Addition of glycine, but not of the other molecules tested, promoted the cleavage reaction. This demonstrates the selectivity of glycine for triggering hASNase3 cleavage. 
a.

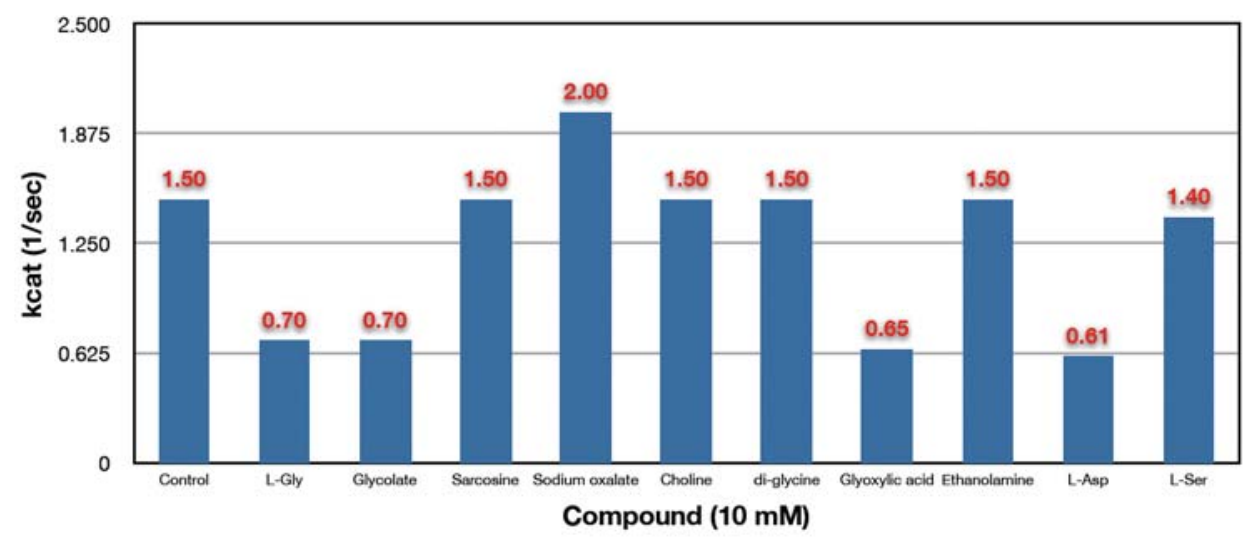

b.

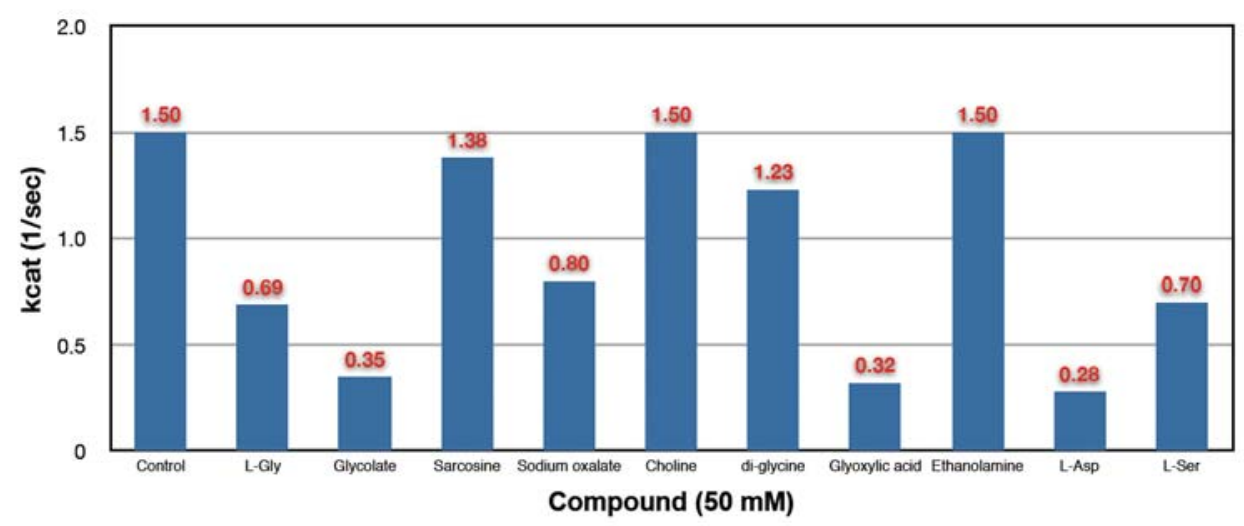

Figure S5: Effect of small metabolites on hASNase3-catalyzed asparagine hydrolysis. The enzyme was fully activated during production in E. coli using $200 \mathrm{mM}$ glycine. After purification, cleaved hASNase 3 was dialyzed against $50 \mathrm{mM}$ sodium phosphate, $\mathrm{pH} 8,0.5 \mathrm{M}$ sodium chloride. The asparaginase activity was measured applying the conventional NADH dependent assay with a L-Asn concentration of $3 \mathrm{mM}$. 


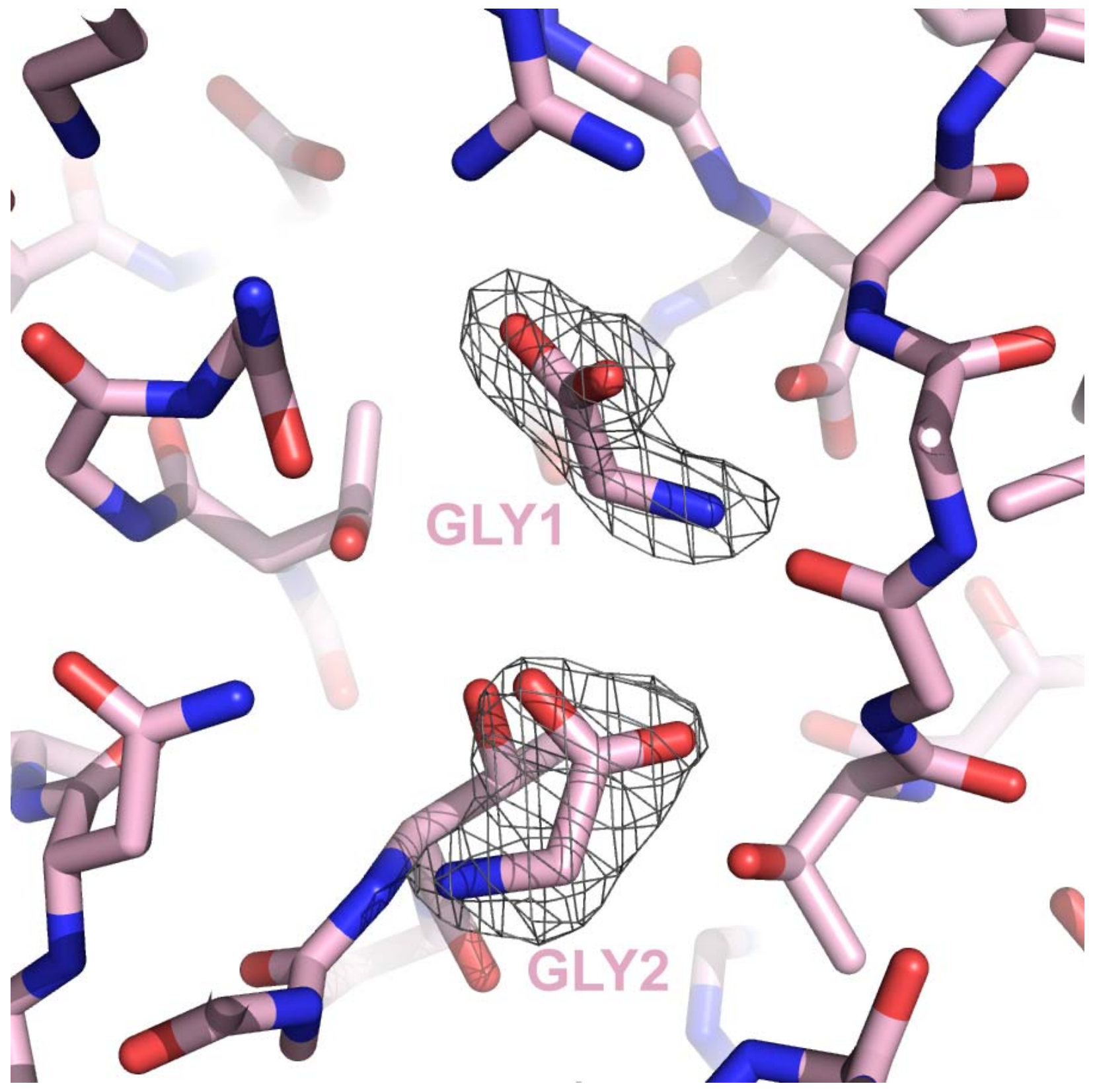

Figure S6. Fo-Fc map contoured at 3 sigma around GLY1 and GLY2. The glycine molecules were removed from the model that then underwent several rounds of refinement to eliminate model bias. The resulting Fo-Fc map clearly shows the presence of the two glycine molecules, labeled GLY1 and GLY2. 


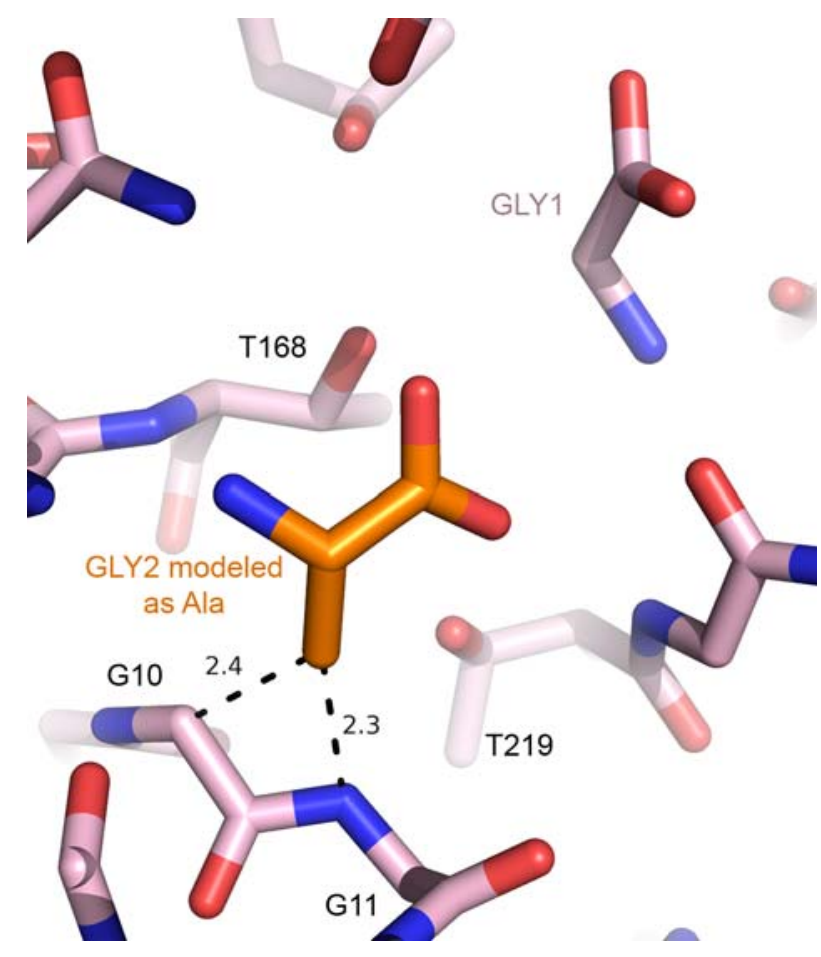

Figure S7. A model of an alanine bound in the same position as GLY2. An alanine (orange) was modeled into the same position observed for GLY2 in the pH 3.3 structure. This reveals a steric clash between the alanine side chain and the enzyme, explaining the glycinespecificity of the accelerated cleavage reaction. For orientation purposes, GLY1 is also shown. 


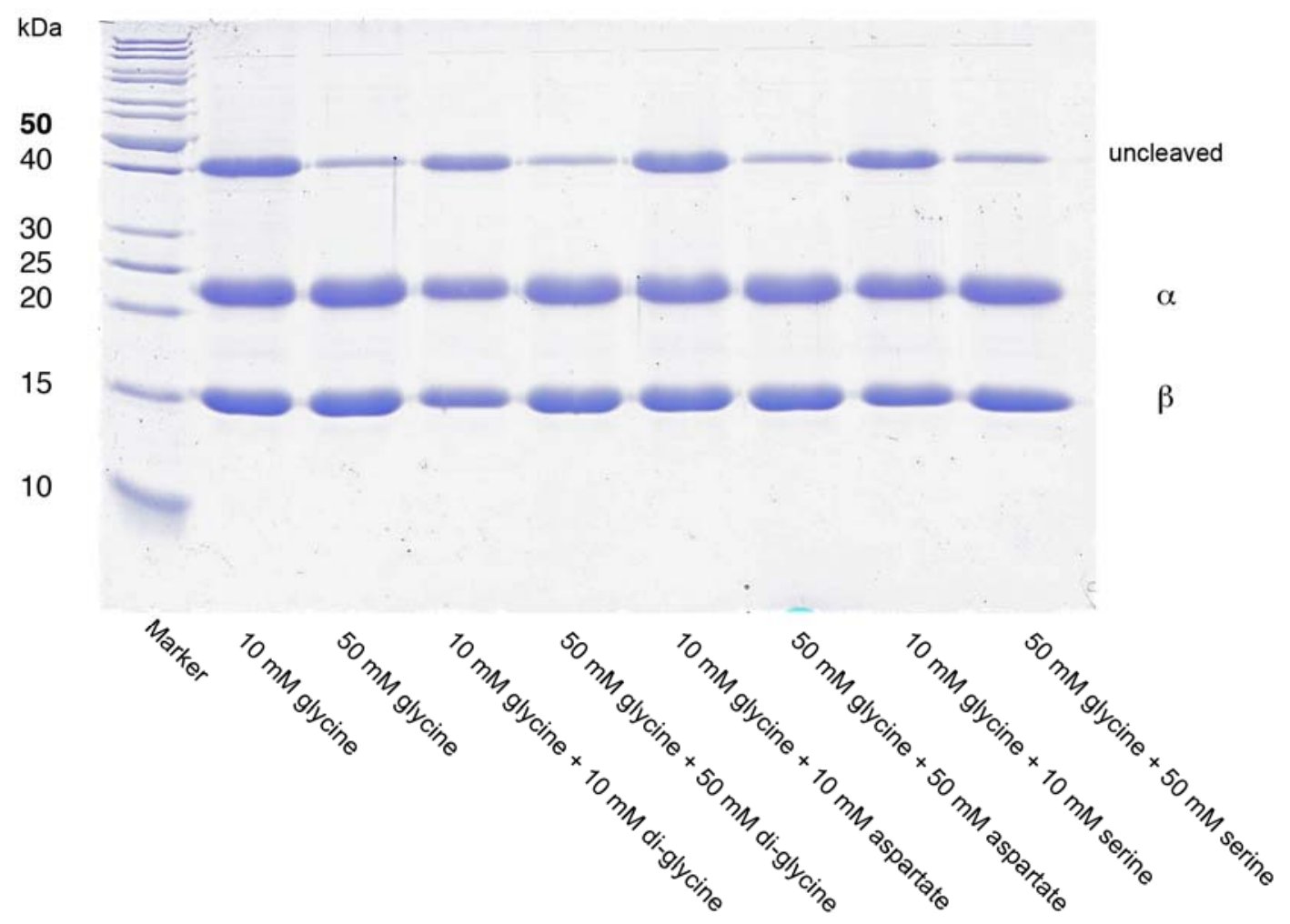

Figure S8. Promotion of hASNase 3 cleavage by glycine is not inhibited by di-glycine, aspartate, or serine. The enzyme hASNase 3 at a concentration of $3.5 \mathrm{mg} / \mathrm{ml}$ was incubated with the indicated molecules at $37^{\circ} \mathrm{C}$ in $50 \mathrm{mM}$ sodium phosphate, $\mathrm{pH}$ 8, and $0.5 \mathrm{M}$ sodium chloride for 24 hours. Addition of di-glycine, aspartate, or serine did not reduce the amount of cleaved protein promoted by $10 \mathrm{mM}$ or $50 \mathrm{mM}$ glycine. 
a.

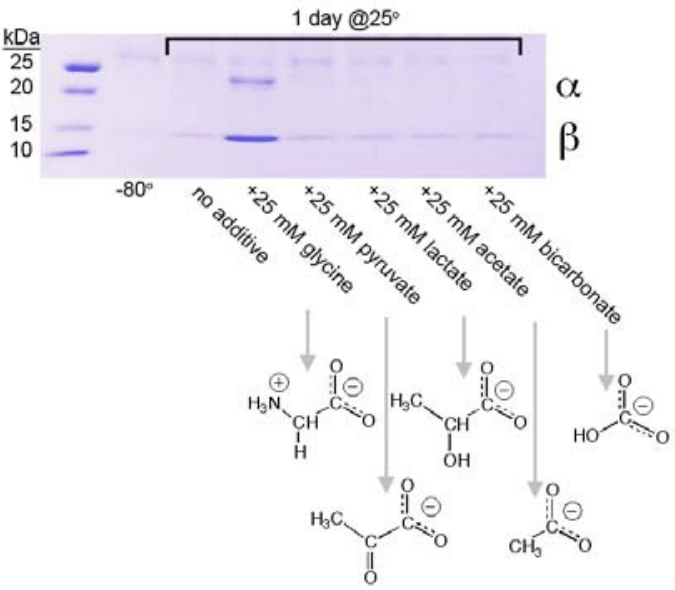

b.

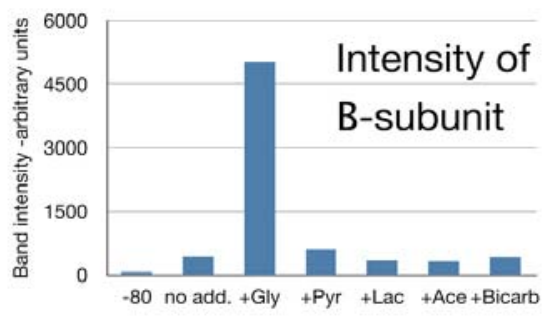

Figure S9. Small-molecule metabolites other than glycine that are expected to be present in glycolytic cells do not duplicate glycine's ability to accelerate cleavage. (A)

Recombinant hASNase 3 was incubated for 1 day at $25^{\circ} \mathrm{C}$ in Storage Buffer with no additives, or with $25 \mathrm{mM}$ of glycine, pyruvate, lactate, acetate, or bicarbonate. Only the enzyme in the presence of glycine showed a significant increase in the amount of the cleaved form, as demonstrated by the increase of the $\alpha$ - and $\beta$-chains. The structure of the various additives is shown below the gel, demonstrating the structural similarity of the molecules. (B) Quantification of the $\beta$-chain band intensity shows the very specific effect of glycine on cleavage. 


\title{
3. Bacterial co-expression of the $\alpha$ and $\beta$ protomers of human $1-$ asparaginase-3: Achieving essential $\mathrm{N}$-terminal exposure of a catalytically critical threonine located in the $\beta$-subunit.
}

\author{
Karamitros C.S. ${ }^{1}$ and Konrad M. ${ }^{1}$ (2014) \\ ${ }^{1}$ Enzyme Biochemistry Group, Max Planck Institute for Biophysical Chemistry, Göttingen \\ D-37077, Germany \\ Protein Expression and Purification \\ http://www.sciencedirect.com/science/article/pii/S1046592813002118 \\ doi: http://dx.doi.org/10.1016/j.pep.2013.10.007
}

\section{$\underline{\text { Author contributions }}$}

C.S.K. and M.K. conceived the project and designed research. C.S.K. performed research. C.S.K. and M.K. analyzed data and wrote the paper.

Copyright notice:

This material is reproduced by permission from Elsevier in accordance to their copyright and licensing regulations. 


\section{hASNase 3}

$\stackrel{\beta}{\beta}$ subunit

|

Gly167

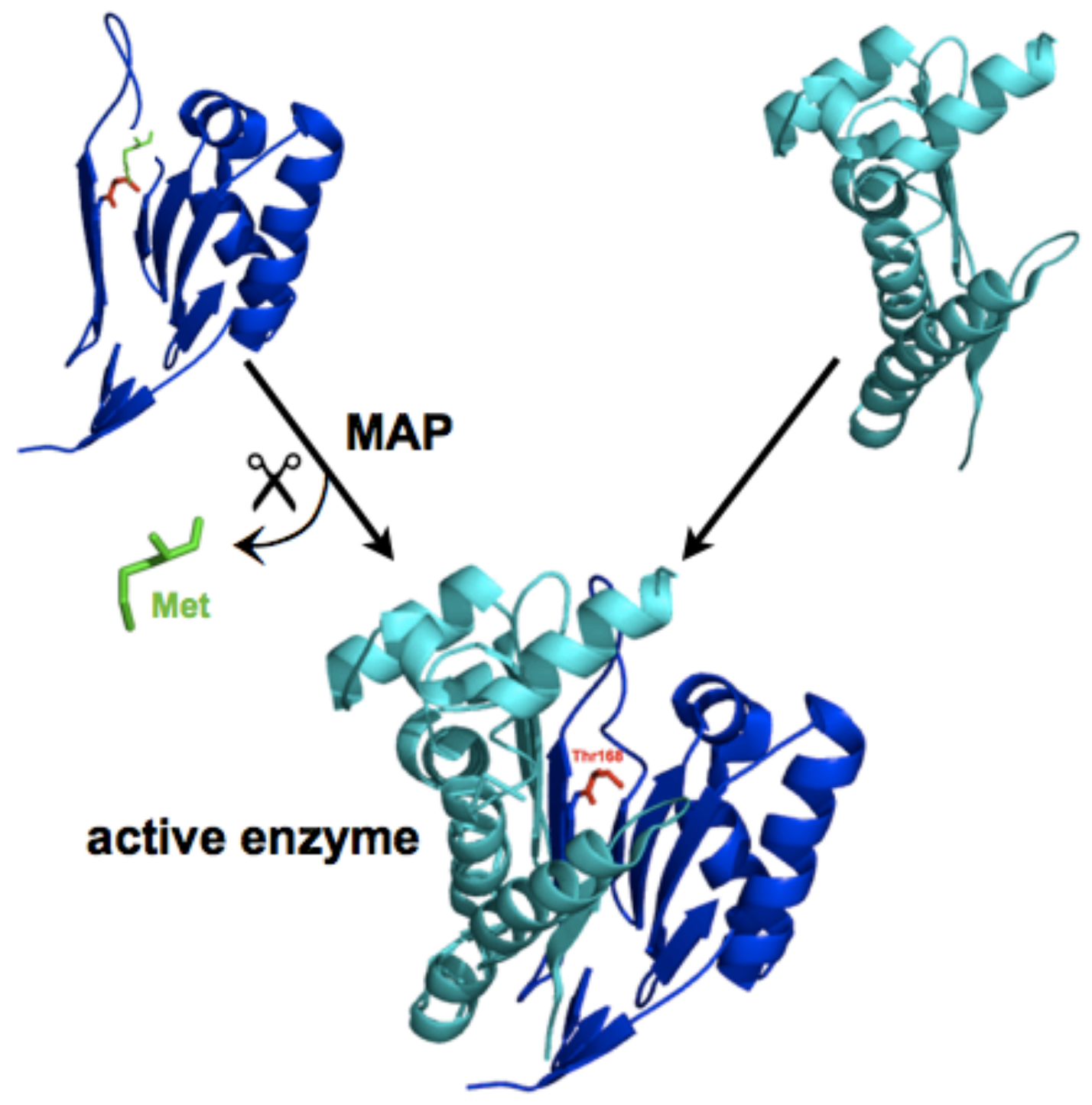




\title{
Bacterial co-expression of the $\alpha$ and $\beta$ protomers of human L-asparaginase-3: Achieving essential N-terminal exposure of a catalytically critical threonine located in the $\beta$-subunit
}

\author{
Christos S. Karamitros, Manfred Konrad* \\ Enzyme Biochemistry Group, Max Planck Institute for Biophysical Chemistry, Göttingen D-37077, Germany
}

\section{A R T I C L E I N F O}

\section{Article history:}

Received 26 September 2013

and in revised form 11 October 2013

Available online 22 October 2013

\section{Keywords:}

Human L-asparaginase-3

$\mathrm{N}$-terminal hydrolase

$\alpha / \beta$ Protomers

Catalytic threonine

Co-expression

\begin{abstract}
A B S T R A C T
L-asparaginases hydrolyze L-asparagine to L-aspartic acid and ammonia. Enzymes of bacterial origin are used as therapeutic agents for the treatment of acute lymphoblastic leukemia. Recently, the structure of a human homolog, hASNase3, which possesses L-asparaginase activity, was solved setting the basis for the development of an anti-leukemic protein drug of human origin. Being an N-terminal hydrolase, hASNase3 undergoes intramolecular self-cleavage generating two protomers (subunits $\alpha$ and $\beta$ ) which remain non-covalently associated and constitute the catalytically active form of the enzyme. However, recombinant expression of full-length hASNase3 in Escherichia coli results in only partial processing towards the active enzyme. We developed a co-expression system for the two subunits that allowed production of the $\beta$-subunit complexed to the $\alpha$-subunit such that the $\mathrm{N}$-terminal methionine is removed by endogenous methionine aminopeptidase to expose the catalytically essential threonine residue at the $\mathrm{N}$-terminus of the $\beta$-subunit. The enzyme produced by this co-expression strategy is fully active, thus obviating the necessity of self-activation by slow autoproteolytic cleavage.
\end{abstract}

(c) 2013 Elsevier Inc. All rights reserved.

\section{Introduction}

L-asparaginases (L-ASNase) ${ }^{1}$ catalyze the conversion of L-asparagine (L-Asn) to L-aspartic acid (L-Asp) and ammonia. These enzymes have been extensively used for the treatment of Acute Lymphoblastic Leukemia (ALL) since more than four decades [1-4]. Cancerous lymphoblasts are unable to synthesize L-Asn due to a deficiency in their asparagine synthetase. Therefore, their survival is exclusively dependent on the free amino acid L-Asn available from the blood. Exhaustive depletion of L-Asn by L-ASNase administered to the patient causes protein synthesis inhibition in the tumor cells which ultimately undergo apoptosis [5]. However, the use of bacterial enzymes for human therapy causes numerous side effects during treatment. As an alternative to currently used bacterial enzymes, it is desirable to develop L-ASNase-like enzymes of human origin into an efficient protein drug. Our recent studies [6] elucidated the structure of the first human enzyme which is able to hydrolyze L-Asn, albeit with poor catalytic properties. Because of high structural homology (sequence identity 38\%) with the previously described Escherichia coli L-ASNase3 [7], we named the human enzyme hASNase3 [8].

\footnotetext{
* Corresponding author. Tel.: +49 551 2011706; fax: +49 5512011074.

E-mail address: mkonrad@gwdg.de (M. Konrad).

1 Abbreviations used: L-ASNase, L-asparaginases; L-Asn, L-asparagine; ALL, Acute Lymphoblastic Leukemia; RBS, ribosome-binding site; MAP, Methionine Amino Peptidase; MCS, multiple cloning site.
}

Human ASNase3, as well as its bacterial homologs, belong to the $\mathrm{N}$-terminal hydrolase family of enzymes [9]. These enzymes are produced as inactive single-polypeptide chain precursors that post-translationally undergo an intramolecular cleavage at a scissile peptide bond (autoproteolysis) resulting in the generation of two tightly associated protomers (subunits $\alpha$ and $\beta$ ) [10]. This intramolecular processing is essential for the conversion of the pro-enzymes to the catalytically active forms of these enzymes, since it liberates the amino group of an amino acid (usually threonine) acting as a nucleophile at the N-terminus of the newly synthesized $\beta$-subunit, enabling nucleophilic attack on the substrate L-Asn. In the case of hASNase3 (308 amino acids long), this autocleavage occurs between residues G167 and T168 [6,9]. However, recombinant expression of hASNase3 in E. coli results in production of a mixture of processed and unprocessed enzyme molecules. Although ways have been described to circumvent this problem, either by adding free glycine which acts as activation inducer for hASNase3 [8], or by using a circular permutant version of this enzyme [11], the possibility of co-expressing the two protomers $\alpha$ and $\beta$, thus generating the active enzyme, has not yet been investigated. While the use of glycine $(>10 \mathrm{mM})$ to induce self-processing of the enzyme is an efficient in vitro tool for the activation of hASNase3, it involves additional steps of incubation of the purified protein, which must be subsequently subjected to dialysis to remove the glycine that inhibits the enzymatic reaction, and this glycine-induced intramolecular processing may not go to 
completion [8]. On the other hand, the circular permutant version of the protein [11] contains an additional long hydrophilic linker that spans the distance between the $\mathrm{N}$ and $\mathrm{C}$ termini, and such a construct may not adequately reflect the structure and the catalytic features of the wild-type enzyme. Therefore, it has been of great interest to investigate novel strategies for the production of hASNase 3 by co-expressing the two subunits in a way which would result in not only the formation of intact $\alpha$ and $\beta$ subunits, but also generate the catalytically active enzyme carrying the free threonine residue at the $\mathrm{N}$-terminus of the $\beta$ subunit.

The co-expression of two or more coding regions in E. coli can be achieved by using either a single plasmid, or by transforming one bacterial cell with two or more different plasmids. In case of single-plasmid constructs, one can follow different strategies: One of these strategies includes the use of a single promoter in one plasmid, linking the two genes by a ribosome-binding site (RBS) similarly to genomic E. coli operons. This gene arrangement is also called bicistronic (two genes) or polycistronic (more than two genes) as termed in other studies [12-14]. This will yield one single transcript containing the information for the genes to be transcribed. Another approach for co-expressing two gene units relies on tandem coding regions involving two promoters [15], where in general the number of promoters is the same as the number of genes transcribed. While the latter case of co-expression might offer better yields in recombinant protein production, because transcription is not coupled, this might lead to imbalanced ratios of the expressed polypeptides [16]. Co-expression from separate plasmids in E. coli is an alternative to operon-like constructs, often requiring less cloning steps, notably when many combinations of individual constructs need to be tested [12]. In principle, for achieving efficient co-expression from two separate plasmids in E. coli, ideally one should use two different origins of replication (ori), as well as two different antibiotic resistance markers, in order to stably maintain the plasmids in the host cells. However, it has been shown that two plasmids with two different antibiotic markers, yet having the same ori, can co-exist in the cells by simply using higher concentrations of antibiotics [17].

In the present study, we applied the basic approaches described above for co-expressing the two subunits of hASNase3. We emphasize that, in this particular case of co-expression, the removal of the $\beta$-subunit's N-terminal methionine by endogenous Methionine Amino Peptidase (MAP) is of critical importance since the L-ASNase activity depends on the free threonine residue of the $\beta$-subunit. In other words, successful co-expression includes not only production of the two subunits in soluble form, but also removal of the $\mathrm{N}$-terminal methionine. Our results highlight several factors that play key roles for efficient co-expression and protein production, and for removal of the $\mathrm{N}$-terminal methionine. Among these critical factors are tags present at the N-terminus of the genes to be transcribed, the type of vectors used, and the order of the genes coding for the subunits. This work may be of general interest to studies where co-expression is required for the production of interacting proteins or protein domains.

\section{Materials and methods}

\section{E. coli strains, plasmids, cDNA, and chemicals}

Plasmids pET-14b and pET-29a were purchased from Novagen. Plasmid pJC20 [18,19] carries the ampicillin resistance marker and the ColE1-based origin of replication, and occurs at $\sim 100$ copies/ cell. Both pET14b and pET29a have the same origin of replication (pBR322) and are medium copy number plasmids ( 30 copies/ cell), but they have different antibiotic resistance markers (ampicillin and kanamycin, respectively). A modified version of the
pET14b plasmid, designated pET14b-SUMO [6], was used to include the SUMO tag (derived from the Saccharomyces cerevisiae smt3 gene) between the $\mathrm{N}$-terminal $\mathrm{His}_{6}$-tag and the multiple cloning site (MCS). The E. coli host strain C41(DE3) was obtained from Lucigen/BioCat, BL21(DE3)pLysS from Invitrogen, and Rosetta (DE3) from Novagen. The XL-1 Blue strain was purchased from Stratagene. All restriction and DNA-modifying enzymes were from New England Biolabs. The oligonucleotides used in this study were obtained from IBA (Goettingen, Germany). Yeast extract, peptone, agar, ampicillin, kanamycin, chloramphenicol and Isopropyl-betaD-thiogalactoside (IPTG) were purchased from Applichem (Gatersleben, Germany). All PCR reactions were performed with KAPA HiFi polymerase purchased from PeqLab (Erlangen, Germany). Gel extraction and PCR purification kits, and nickel resin for affinity purification of poly-histidine-tagged proteins was from Macherey Nagel (Düren, Germany), plasmid purification kit from Fermentas (Thermo Fisher Scientific, Germany). The hASNase3 open reading frame was amplified using as template cDNA from a human skin and meninges library (Source Bioscience, UK). All cloning steps involving PCR amplifications were confirmed by sequencing (SeqLab, Goettingen, Germany).

\section{Construction of the pET14b-hASNase $[\alpha+\beta]$ vector}

Using as template the full length cDNA of hASNase3, the gene fragments corresponding to the two subunits were PCR-amplified independently. The $\alpha$-subunit DNA-fragment (501 bp) codes for amino acid residues M1-G167, while the $\beta$-subunit fragment (426 bp) codes for T168-P309 (Scheme S1). A singular NdeI site was incorporated at the $5^{\prime}$-end of the $\alpha$-subunit, while its $3^{\prime}$-end contains the sequence for the ribosomal binding site (RBS; identical to the one present in pET14b), followed by unique KpnI and BamHI sites. For directional cloning, KpnI and BamHI restriction sites were incorporated at the $5^{\prime}$ - and $3^{\prime}$-ends of the $\beta$-subunit, respectively. The vector was constructed stepwise: First, the $\alpha$ subunit was ligated via the NdeI and BamHI sites, and then the $\beta$-subunit was ligated via KpnI and BamHI (Scheme 1). The final construct was sequence-verified, and then used to transform chemically competent $E$. coli cells for expression analysis. For the PCR reactions, the following oligonucleotide primers were used (restriction enzyme recognition sites are underlined in italics, while the RBS is in bold):

Protomer $\alpha$

Forward $(\alpha)$ : 5'-GGAATTCCATATGAATCCCATC GTAG TGGTCCACG-3'

Reverse ( $\alpha)$ : 5'-CGCGGATCCGGAGGT ACCGGTATATCTCCTTCTTA A AGTTA AACA AAAT T ATTT CTAGA GTTATCCC AAGTTTT TTTG A CAATC-3'

\section{Protomer $\beta$}

Forward ( $\beta)$ : 5'-CGGGGTACCATGACCGTGGGTGCTGTTGCCTTG-3' Reverse ( $\beta$ ): 5'-CGCGGATCCTTAGGGAAGGTCGGTGATAGTAGT-3'

Construction of the pET14b-SUMO-hASNase3[ $\alpha+\beta]$, pJC20-hASNase3$[\alpha+\beta]$, and pJC20-SUMO-hASNase $3[\alpha+\beta]$ vectors

To generate the hASNase $3[\alpha+\beta]$ construct, carrying at its $\mathrm{N}$-terminus the SUMO tag fused to the $\alpha$-subunit (Scheme S2), the DNA fragment encoding the two subunits was digested with NdeI and BamHI, gel-purified, and subcloned into the pET14bSUMO vector. For the preparation of both pJC20 constructs (Schemes S3 \& S4), the pET14b-hASNase3[ $\alpha+\beta]$ and pET14bSUMO-hASNase $3[\alpha+\beta]$ were digested with NcoI and BamHI, and the excised inserts $(1 \mathrm{~kb})$ containing the $\mathrm{His}_{6}$ and the SUMO tag 


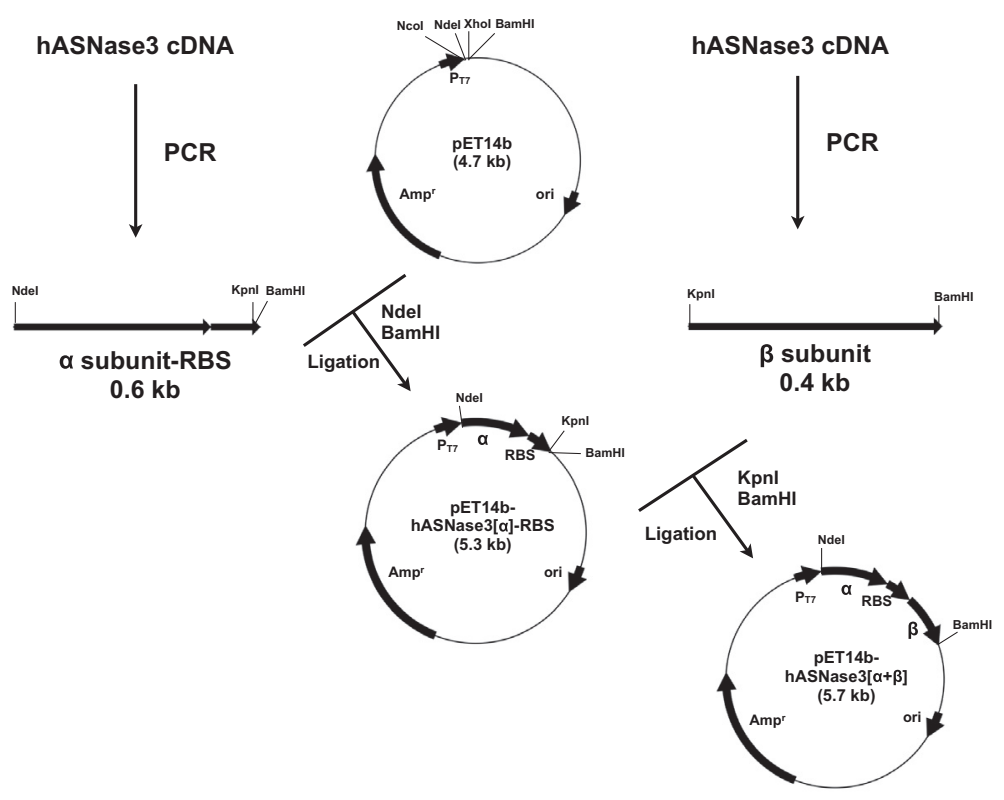

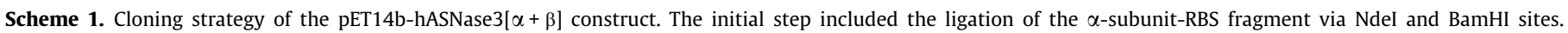
Subsequently, the $\beta$-subunit was cloned downstream of the RBS via KpnI and BamHI, resulting in the final operon-like construct.

were ligated into the pJC20 vector which shares with the pET14b vector a similar multiple cloning site (MCS).

\section{Construction of pET14b-hASNase3[ $\alpha$ ] and pET29a-hASNase3[ $\beta$ ]} vectors

To co-express the two hASNase3 subunits from two different vectors, we cloned the $\alpha$-subunit in pET14b-SUMO and the $\beta$-subunit in pET29a via NdeI and BamHI sites (Scheme 2). These plasmids carry the same origin of replication (ori from pBR322), but they contain different antibiotic markers: pET14b confers resistance to ampicillin, while pET29a to kanamycin. For cloning
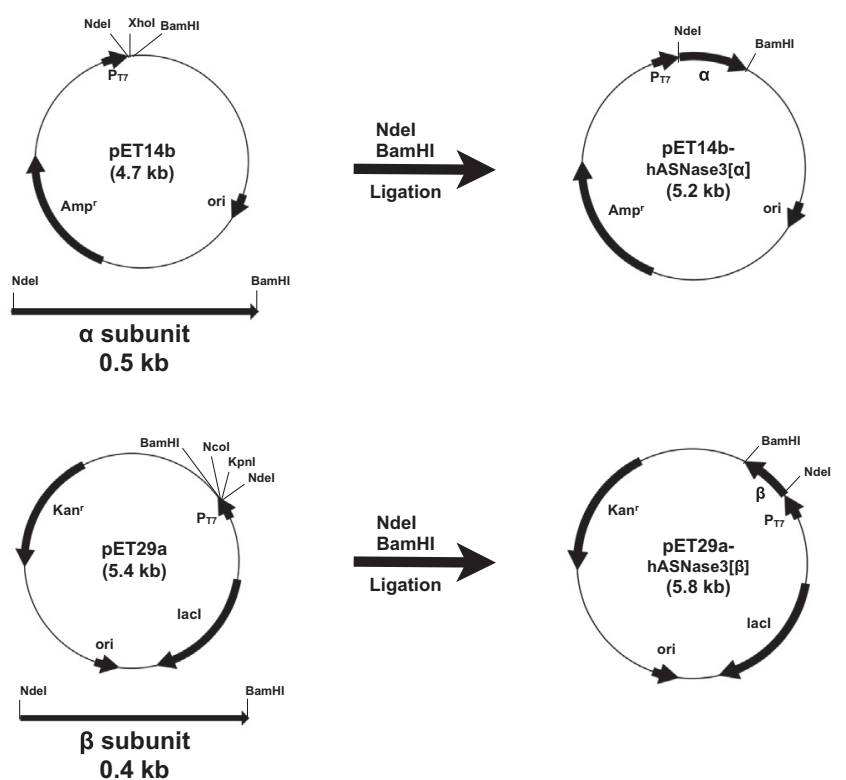

Scheme 2. Description of the pET14b-hASNase3[ $\alpha]$ and pET29-hASNase3[ $\beta]$ constructs. Both units were cloned via NdeI and BamHI sites as described in the Methods section. of the $\beta$-subunit, the NdeI site was chosen at the $5^{\prime}$-end harboring the starting codon. Thus, after translation, the second amino acid is threonine, and after removal of the $\mathrm{N}$-terminal methionine by endogenous $E$. coli methionine aminopeptidase (MAP), the first amino acid is the critical threonine whose amino group is free for catalysis. Furthermore, expression of the $\beta$-subunit from pET29a yields a protein fragment without $\mathrm{His}_{6}$ tag that could be co-purified via strong non-covalent interaction with the $\alpha$-subunit; tight association of the protomers has been evidenced in our previous work [8]. The following primers were used to generate these constructs (restriction sites underlined in italics):

Protomer $\alpha$

Forward $(\alpha):$ 5'-GGAATTCCATATGAATCCCATCGTAGTGGTCCA CG-3'

Reverse $(\alpha)$ : 5'-CGCGGATCCTTATCCCAAGTTTTTTTGACAATCTG$3^{\prime}$

Protomer $\beta$

Forward $(\beta)$ : 5'-GGAATTCCATATGACCGTGGG TGCTGTTGCCT TGG-3'

Reverse ( $\beta$ ): 5'-CGCGGATCCTTAGGGAAGGTCGGTGATAGTAGT-3'

Construction of the pET14b-hASNase3[ $\beta+\alpha]$ and pJC20-hASNase3$[\beta+\alpha]$ vectors

To generate a construct with the gene fragments arranged to encode the two subunits in reversed order (i.e. instead of having $\alpha+\beta$, we now have $\beta+\alpha$ ), a circular permutant-like version of the hASNase3 gene was designed. However, unlike circular permutant constructs of various other proteins studied in the past $[20,21]$, in the present analysis of hASNase3 we introduce no linker sequence to connect the two new termini. In order to produce this construct, a modified version of the pET14b vector was developed by applying the QuikChange site-directed mutagenesis technique (Stratagene). The unique Ncol site located within the initiation codon was mutated to $\mathrm{NdeI}_{1}$ which served as the $5^{\prime}$-cloning site for the $\beta$-subunit. In this construct, the $\beta$-subunit was cloned first via NdeI and BamHI carrying at its 3 '-end the ribosomal binding site (RBS), as well as another KpnI site for the subsequent ligation 
A

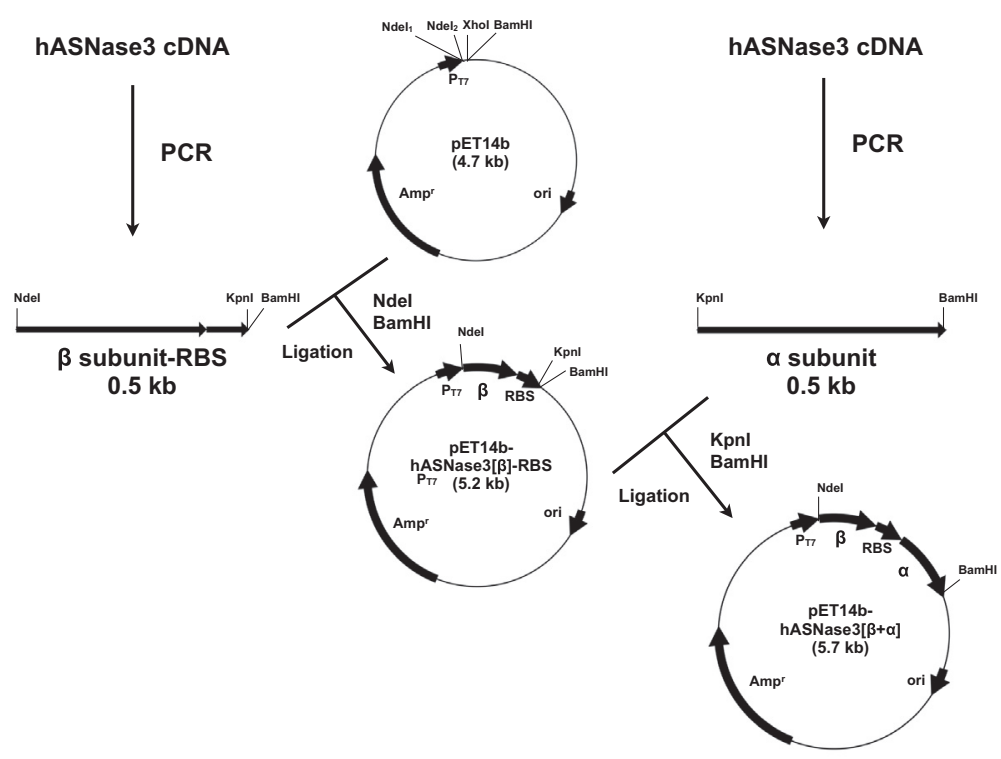

B

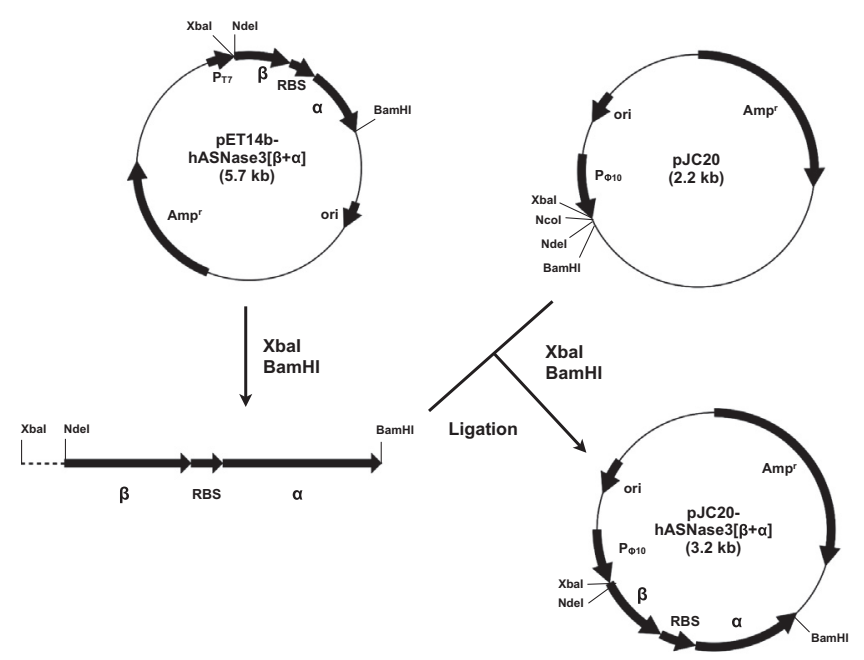

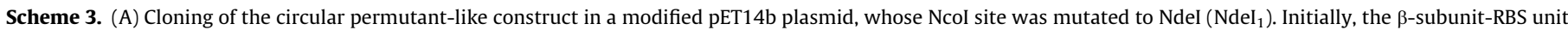

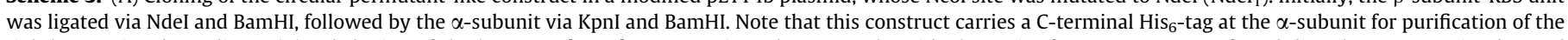

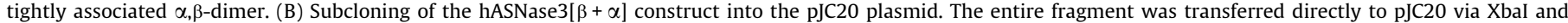
BamHI sites, leaving the overall unit unchanged.

of the $\alpha$-subunit via KpnI and BamHI (Scheme 3A). Another property of this construct is that the $\mathrm{His}_{6}$-tag was fused to the $\mathrm{C}$ terminus of the $\alpha$-subunit to facilitate purification, since it is essential to obtain the $\beta$-subunit which carries the catalytically critical Thr-residue at the $\mathrm{N}$-terminus after endogenous removal of methionine. The pJC20-hASNase $3[\beta+\alpha]$ plasmid was generated by simple transfer of the Xbal-BamHI-fragment from the final pET14b-hASNase3 $[\beta+\alpha]$ construct (Scheme 3B). The following primers were used for generating these constructs (restriction sites are underlined in italics and the $\mathrm{His}_{6}$-encoding sequence is in bold):

Vector modification

Forward NcoI $\rightarrow$ NdeI: 5'-GAAGGAGATATACCATATGCAGCAGCCATCATC-3

Reverse NcoI $\rightarrow$ NdeI: 5'-GATGATGGCTGCTGCATATG GTATATCT CCTTC-3'
Protomer $\beta$

Forward $(\beta)$ : 5'-GGGAATTCCATATGACCGTGGGTGCTGTTGCCTT GGACTGC-3

Reverse $(\beta)$ : 5'-CGCGGATCCGGAGGTACCGGTATATCTCCTTCTAAA GTTTAGGGAAGGTCGGTGATAGTAGTATCGTC-3'

\section{Protomer $\alpha$}

Forward $(\alpha)$ : 5'-CGGGGTACCATGAATCCCATCGTAGTGGTCCAC-3' Reverse $(\alpha)$ : $5^{\prime}$-CGCGGATCCTTAGTGATGATGATGATGATGTCCCAA GTTTTTTTGACAATCTGTTTTCTG-3'

\section{Protein expression and purification}

After sequence verification of the final vectors, they were used to transform several chemically competent $E$. coli expression strains [C41 (DE3), BL21(DE3)pLysS, Rosetta (DE3)] to test for optimum conditions in terms of protein yield and solubility. In case of 

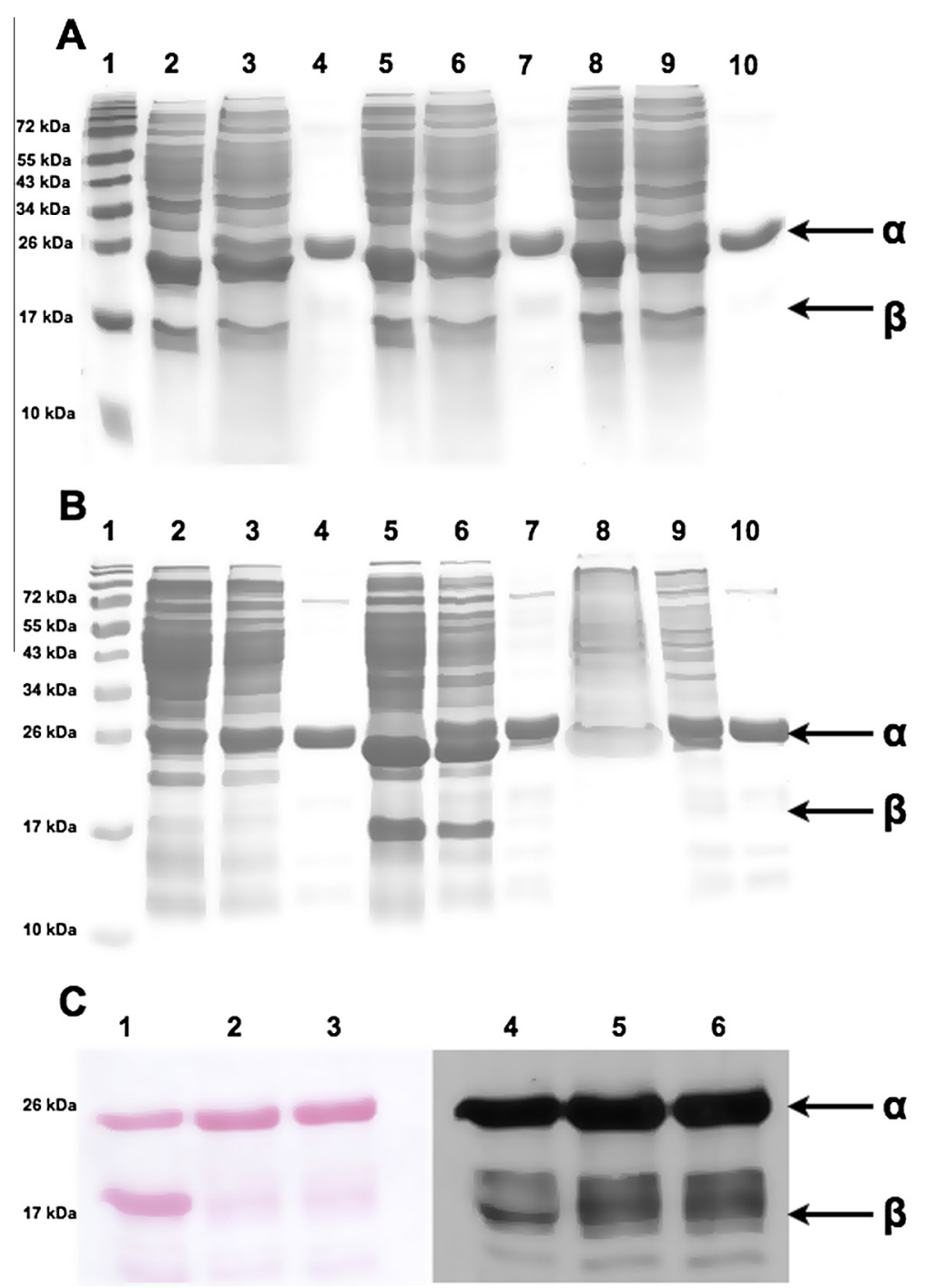

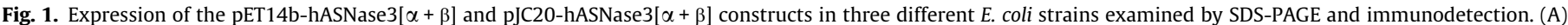

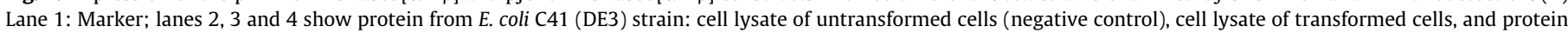

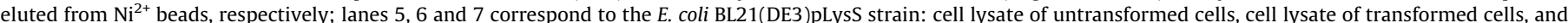

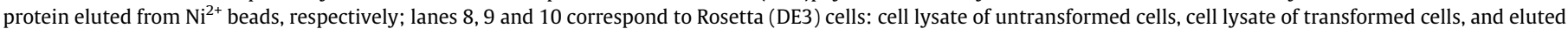

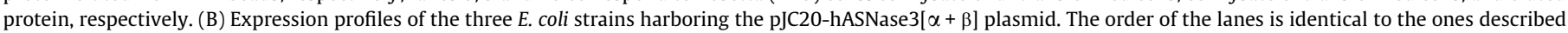

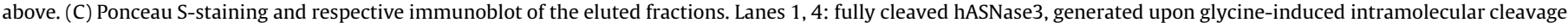
of the full-length protein (control); lanes 2,5 \& 3,6: eluted protein expressed from pET14b-hASNase3[ $\alpha+\beta]$ and pJC20-hASNase3[ $\alpha+\beta]$ constructs, respectively.

co-expression using two different plasmids, the cells were first transformed with one plasmid, grown, and were then made competent for the transformation with the second plasmid. The transformation mixtures were plated onto LB plates containing the respective antibiotic and incubated at $37^{\circ} \mathrm{C}$ overnight. Single colonies were used to inoculate $50 \mathrm{~mL}$ cultures in LB-medium. After overnight incubation at $37^{\circ} \mathrm{C}$, these cultures were used to inoculate $1 \mathrm{~L}$ of $2 \mathrm{xYT}$ or TB medium (dilution 1:50). When O.D.600 reached $0.5-0.7$, protein expression was induced by adding IPTG to a final concentration of $1 \mathrm{mM}$. After $6-8 \mathrm{~h}$ of incubation at $37^{\circ} \mathrm{C}$, the cultures were centrifuged at $4000 \mathrm{~g}$ for $20 \mathrm{~min}$; the cells were resuspended in buffer $\mathrm{A}\left(50 \mathrm{mM} \mathrm{Na} \mathrm{HPO}_{4}, 0.5 \mathrm{M} \mathrm{NaCl}\right.$, $10 \mathrm{mM}$ imidazole, $\mathrm{pH} \mathrm{8}$ ), and lysed by ultrasonication. The cell lysate was centrifuged ( $45 \mathrm{~min}$ at $17,200 \mathrm{~g}$ ), the resulting supernatant mixed with pre-equilibrated nickel agarose beads, and incubated at $4{ }^{\circ} \mathrm{C}$ for $2 \mathrm{~h}$ in a rotating beaker. The slurry mixture was filled in $12 \mathrm{~mL}$ polypropylene columns, dried by gravity, and the agarose beads were then washed with 25 bed volumes of buffer B $(50 \mathrm{mM}$ $\mathrm{Na}_{2} \mathrm{HPO}_{4}, 1 \mathrm{M} \mathrm{NaCl}, 20 \mathrm{mM}$ imidazole, 10\% glycerol, pH 8). Bound protein was eluted from the column by applying $300 \mathrm{mM}$ imidazole in buffer A. All purification steps were performed at $4{ }^{\circ} \mathrm{C}$ in a cold room. Eluted fractions were mixed, buffer was exchanged against $50 \mathrm{mM} \mathrm{Na} 2 \mathrm{HPO}_{4}, 100 \mathrm{mM} \mathrm{NaCl}, \mathrm{pH} 7.5$ using a PD-10 column (GE Healthcare), the protein-containing fractions analyzed by SDS-PAGE [22], and tested for L-ASNase activity.

\section{Western blots for identifying hASNase3 full-length protein and subunits}

Immunoblotting was performed according to standard protocols. The protein concentration of the samples was determined by the Bradford dye-binding method [23] using as standard bovine serum albumin (BSA). Upon immunoblotting, bands were detected using an enhanced chemiluminescence (ECL) kit (Amersham Pharmacia, Piscataway, USA). Polyclonal anti-hASNase 3 antibodies were generated by immunizing a rabbit with overall $0.6 \mathrm{mg}$ of SDSPAGE-purified full-length protein according to Eurogentec's (Seraing, Belgium) standard protocols. Polyclonal rabbit anti-hASNase3 

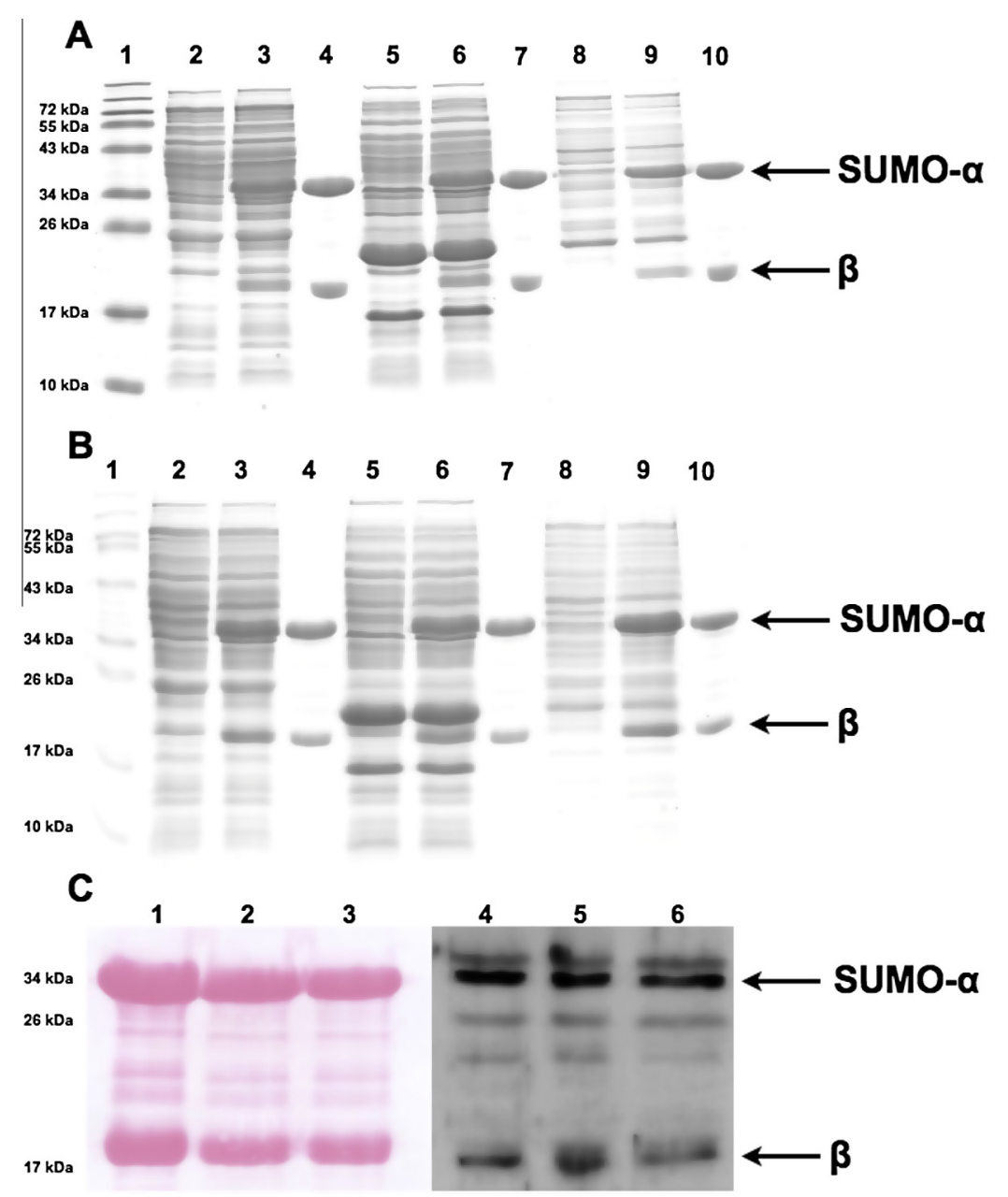

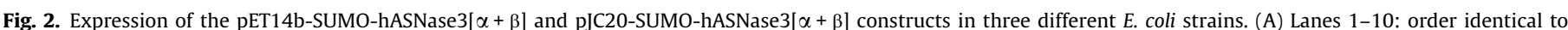

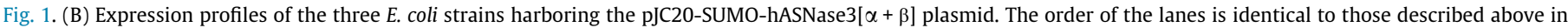

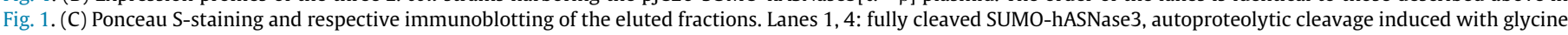
using the full-length protein (control); Lanes $2,5 \& 3,6$ : eluted protein expressed from pET14b-SUMO-hASNase3[ $\alpha+\beta]$ and pJC20-SUMO-hASNase3[ $\alpha+\beta]$, respectively.

antibodies were enriched from serum samples by affinity chromatography purification using recombinant hASNase3 as ligand as described [24].

\section{L-asparaginase assay}

To determine L-ASNase activity after expression and purification of hASNase3 constructs, we followed the Nesslerization method as reported before [25]. Briefly, the purified enzyme was incubated with the substrate L-Asn in $50 \mathrm{mM} \mathrm{Na}{ }_{2} \mathrm{HPO}_{4}, 300 \mathrm{mM}$ $\mathrm{NaCl}, \mathrm{pH} 7.5$, under substrate saturation conditions ( $5 \mathrm{mM} \mathrm{L}-\mathrm{Asn}$ ) at $37^{\circ} \mathrm{C}$ for $20 \mathrm{~min}$. In parallel, to detect ammonium generated in the absence of L-ASNase activity, a second identical sample was incubated at $37^{\circ} \mathrm{C}$ for $20 \mathrm{~min}$, in which the enzymatic reaction was quenched at time zero by adding trichloroacetic acid (TCA) to a final concentration of $10 \%$. After TCA precipitation, both samples were centrifuged at $16,100 \mathrm{~g}$ for $2 \mathrm{~min}$. The supernatant was mixed with Nessler's reagent, and the absorbance was monitored spectrophotometrically at $\sim 440 \mathrm{~nm}$ after $10 \mathrm{~min}$ incubation at room temperature. The concentration of ammonia produced by the enzymatic reaction was determined from a reference curve using ammonium chloride as standard. An enzymatic unit is defined as the amount of enzyme that catalyzes the conversion of $1 \mu \mathrm{mol}$ of L-asparagine/min under the aforementioned conditions.

\section{$N$-terminal amino acid sequencing}

In order to analyze structural reasons for the lack of enzymatic activity we observed in case of the production of both subunits from certain constructs, we sequenced the N-terminus of the copurified $\beta$-subunit. SDS-PAGE was used to separate the two non-covalently but tightly associated subunits. The band corresponding to the $\beta$-subunit was cut out of the gel, blotted onto a PVDF membrane, and stained with Coomassie blue. The stained protein band was cut out, and analyzed by standard methods on a Procise 491 protein sequencer from Applied Biosystems, run in the Bioanalytical Mass Spectrometry service department of the MPIbpc, Goettingen.

\section{Results and discussion}

Expression of hASNase $3[\alpha+\beta]$ and SUMO-hASNase $3[\alpha+\beta]$ constructs in $p E T 14 b$ and $p J C 20$ vectors

The first trials for co-expressing the two subunits of hASNase3 were based on an operon-like construct in which the $\alpha$-subunit (residues M1-G167; theoretical $M_{r}$ : $17.5 \mathrm{kDa}$ but it appears to run slower at $24 \mathrm{kDa}$ on SDS-PAGE) is located at the $5^{\prime}$-end, followed by a ribosome binding site (RBS), and the $\beta$-subunit 

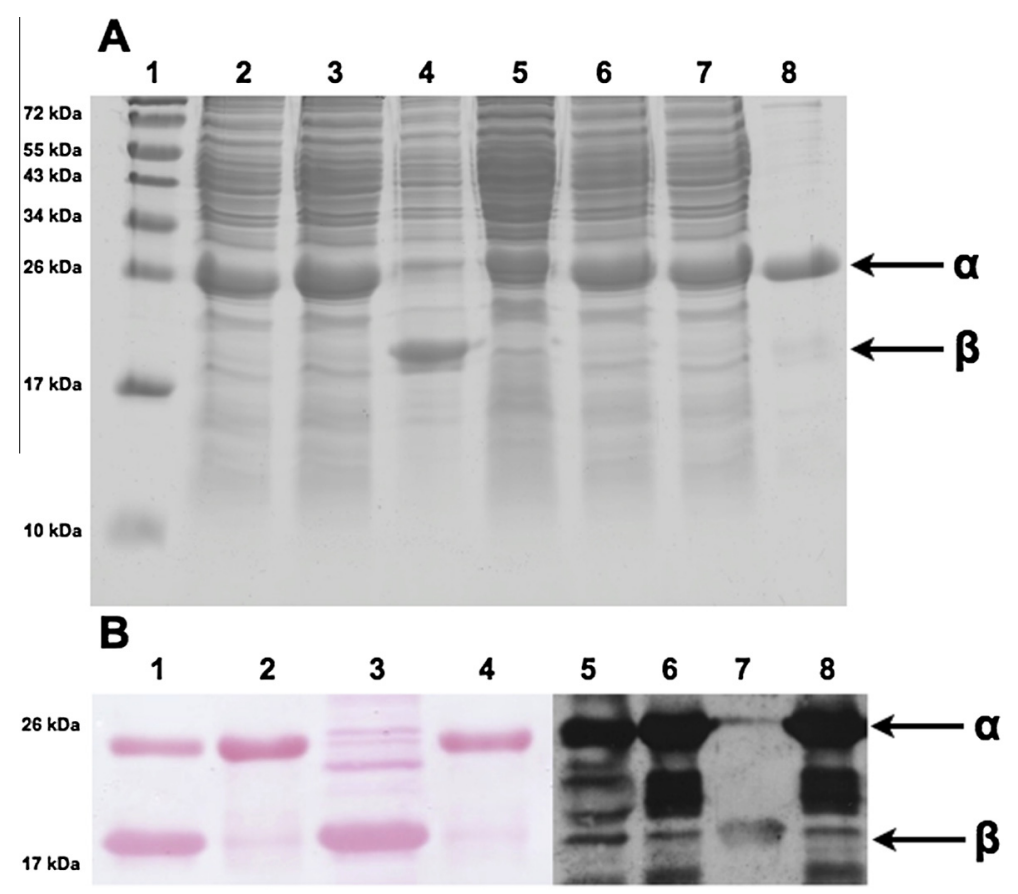

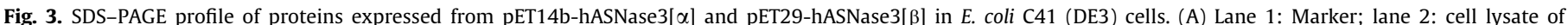

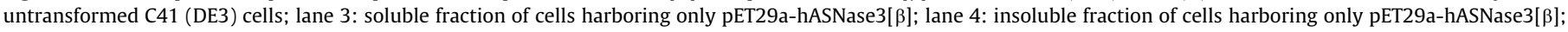

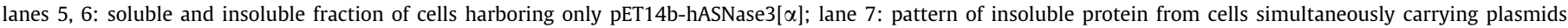

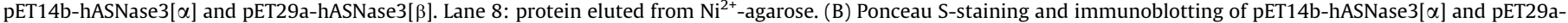

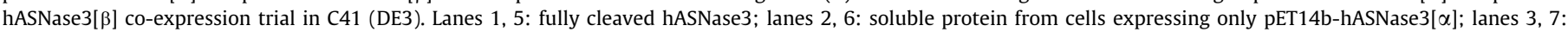
insoluble protein from cells expressing only pET29a-hASNase3[ $\beta$; lanes 4, 8: eluted protein fraction from lysate of cells expressing both plasmids.

(residues T168-P309; theoretical $M_{r}: 14.5 \mathrm{kDa}$ and apparent $M_{r}$ $\sim 18 \mathrm{kDa}$ on SDS-PAGE) (Scheme 1). The RBS sequence is identical to the one which is present in the pET14b vector; it was included in the reverse primer used for PCR-amplification of the $\alpha$-subunit (oligonucleotide primers described in the Methods section). Although the expression of this construct yielded high amounts of the $\alpha$-subunit (>8 mg/L 2xYT medium), the $\beta$-subunit was only poorly produced as evidenced by SDS-PAGE and immunoblot analysis (Fig. 1A and C). In addition, to detect very low levels of the $\beta$ subunit, which might associate with the $\alpha$-subunit to form a functional enzyme, we tested for enzymatic activity. However, this was not observed upon production in all three E. coli expression strains tested, indicating that this unexpected expression profile was strain-independent. A reason for this weak production of $\beta$-subunit could be the less-than-optimal intermediate RBS sequence, and/or the presence of rare codons (Arg, Pro, Leu, Iso) at the N-terminus of the $\beta$-subunit, since the presence of rare codons right after the first Met had earlier been shown to play a detrimental role in the expression of certain genes in E. coli [26]. However, inspection of the cDNA sequence of hASNase 3 did not reveal any rare codon within the stretch of the first 35 codons at the $5^{\prime}$-end of the $\beta$-subunit. Instead, there are four rare codons (Pro2 and 14, and Arg22 and 29) within the first 35 codons at the $5^{\prime}$-end of the $\alpha$-subunit, without affecting high-level expression of this protomer. Similar negative results were obtained when the high-copy number pJC20 vector was used instead of pET14b (Fig. 1B and C).

Interestingly, when the hASNase $3[\alpha+\beta]$ construct was transferred into both pET14b-SUMO and pJC20-SUMO vectors (Schemes S2, S4), which expressed the $\alpha$-subunit as fusion with the N-terminal SUMO-tag (SUMO-tag- $\alpha$-subunit theoretical $M_{r}$ : $30 \mathrm{kDa}$ but it appears to run at $\sim 35 \mathrm{kDa}$ on SDS-PAGE), the $\beta$-subunit was successfully produced (Fig. $2 \mathrm{~A}$ and $\mathrm{B}$ ). Immunoblot detection of the two subunits is shown in Fig. 2C: Ponceau S-staining indicates a 1:1 ratio of the two subunits, but polyclonal antibodies recognize the $\beta$-subunit only weakly. Thus, the presence of the SUMO-tag was proven to be beneficial for the production of the $\beta$-subunit from either vector.

Nevertheless, despite the production of both subunits in soluble form using the latter SUMO-fusion constructs, the SUMO-tagged and the tag-free (after cleavage with SUMO protease) purified enzymes were inactive when tested for L-ASNase activity. Provided that the two subunits were properly folded and had adopted the same tertiary structure as when produced as full-length intact polypeptide chain, followed by intramolecular cleavage to form the two protomers $[6,8,9]$, the most likely explanation for the lack of activity would be the presence of the initiating methionine at the $\mathrm{N}$-terminus of the $\beta$-subunit. If the $\mathrm{N}$-terminal methionine was not removed from the $\beta$-subunit by endogenous MAP, then the critical threonine-residue which follows this methionine would not catalyze the reaction. The reason is that its $\alpha$-amino group resides in the peptide bond formed with the preceding methionine, and, therefore, cannot perform the nucleophilic attack on the substrate L-Asn. According to the reported catalytic mechanism of Ntn-hydrolases, it is only the free $\alpha$-amino group of Thr, Ser, or Cys that can fulfill this role [27]. To verify this assumption, we analyzed the $\beta$-subunit by mass spectrometry and sequenced its $\mathrm{N}$-terminus. Indeed, the $\mathrm{N}$-terminal methionine was not removed from the recombinantly produced $\beta$-subunit, resulting in totally inactive hASNase3.

\section{Co-expression of hASNase $\alpha$ and $\beta$ subunits using two different vectors}

Since production of the two subunits upon expression from an operon in the pET14b-SUMO-hASNase3 $[\alpha+\beta]$ vector resulted in an inactive enzyme, we decided to co-express the two subunits using two different vectors. Therefore, the $\alpha$-subunit was cloned into the pET14b vector (pET14b-hASNase3[a]) which confers 

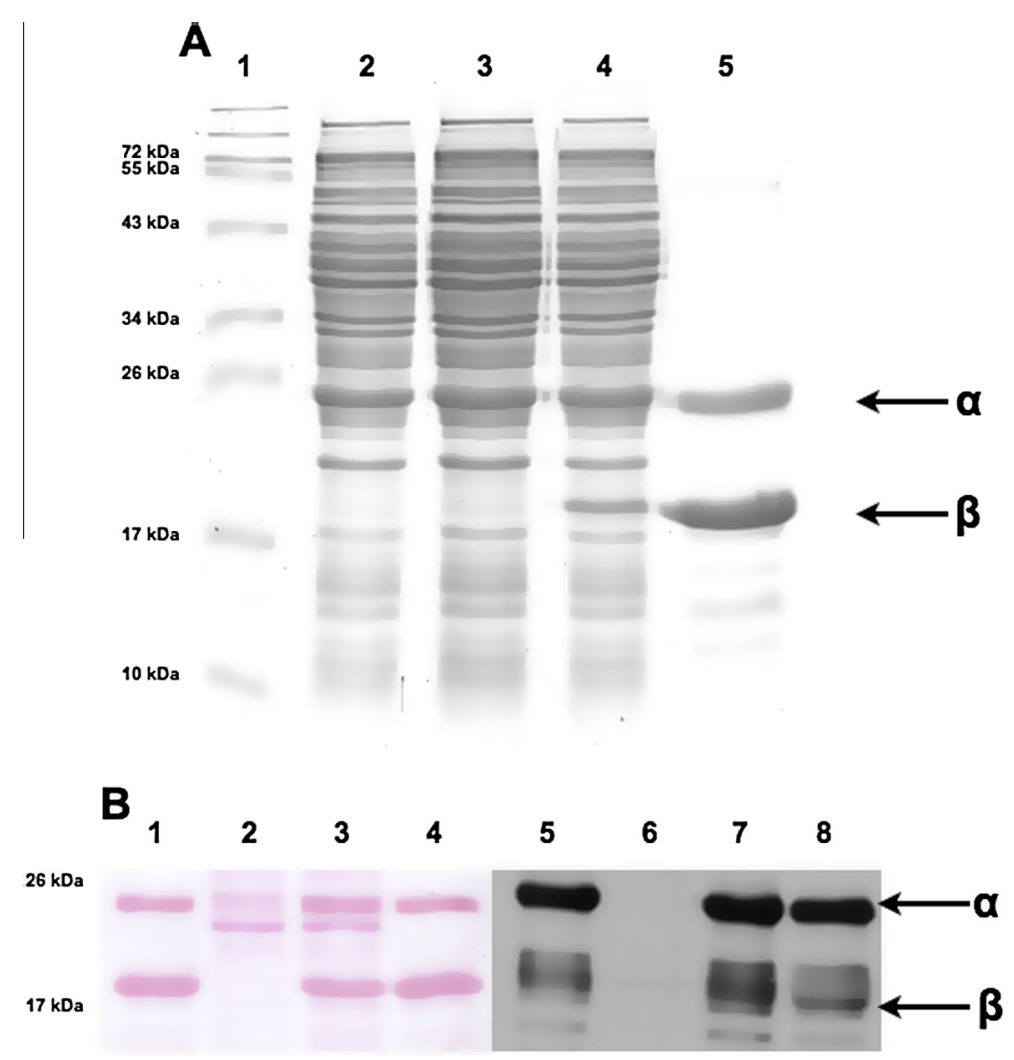

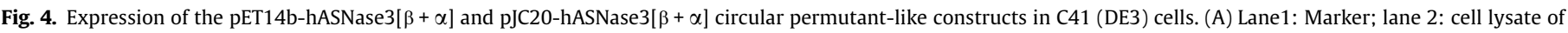

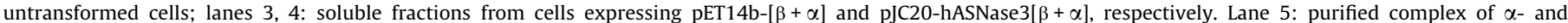

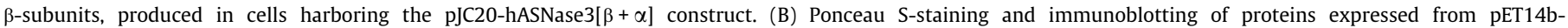

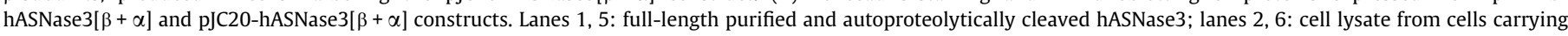

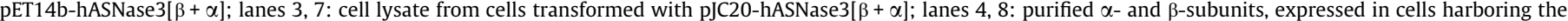
pJC20-hASNase $3[\beta+\alpha]$ plasmid.

ampicillin resistance, and the $\beta$-subunit was cloned into the pET29a (pET29a-hASNase3[ $\beta]$ ) vector containing the kanamycin resistance marker. Though the two vectors share the same origin of replication (derived from pBR322), the different antibiotic markers allow them to co-exist in the same bacterial cell producing different recombinant proteins [17]. Initially, we investigated whether the two subunits could be produced separately, and, therefore, E. coli C41 (DE3) cells were transformed with either pET14b-hASNase3 $[\alpha]$ or pET29a-hASNase3[ $\beta]$. As shown in Fig. $3 \mathrm{~A}$, either subunit was produced, the $\alpha$-subunit was soluble, whereas the $\beta$-subunit polypeptide was totally insoluble. It had been shown by others that upon separate expression of two protein domains, misfolding and insolubility problems might arise [28-30]. However, although either domain was produced when expressed separately, co-transformation of both plasmids led to production of the $\alpha$-subunit only (Fig. 3A and B). Loss, or elimination, of the plasmid from the cell can be excluded since resistance against kanamycin was preserved as evidenced by the growth of co-transformed cells in medium containing both antibiotics throughout the whole period tested for expression. Thus, we observed that, similarly to the operon-like construct, co-expression from two plasmids also yielded solely the $\alpha$-subunit.

\section{Expression of hASNase $3[\beta+\alpha]$ construct in $p E T 14 b$ and $p J C 20$ vectors}

As an alternative to the previous strategies for co-expressing the two subunits of hASNase 3 aiming to produce the soluble and active enzyme, we generated another circular permutant-like construct (Scheme 3). In this case, the coding regions for the two subunits were arranged in an operon-like construct, but the order of the DNA-fragments was reversed: In this case, the $\beta$-subunit encoding region is located at the $5^{\prime}$-end, followed by the RBS sequence, and then the $\alpha$-subunit at the $3^{\prime}$-end. Unlike the circular permutant-arrangement of previously described two-subunit proteins $[19,20]$, whose domains were connected with a linker, in our case, the two subunits are not linked. Since the $\beta$-subunit is translated first, before production of, and association with, the $\alpha$ subunit, this could facilitate access of the endogenous MAP to the $\mathrm{N}$-terminal methionine and its removal, resulting in the exposure of threonine as the first amino acid of the $\beta$-chain. Particularly, in this construct, the $\mathrm{His}_{6}$-tag was fused to the C-terminus of the $\alpha$-subunit, since attaching it either to the C-terminus of the $\beta$-chain or to the $\mathrm{N}$-terminus of the $\alpha$-chain, the tag would be masked inside the dimeric protein structure [6].

Despite multiple attempts to express the hASNase $3[\beta+\alpha]$ construct using the pET14b vector under various conditions (different media, temperatures, induction periods, E. coli strains), no protein production was detected (Fig. 4); we did not analyze transcription of the two open reading frames. In contrast, when this construct was transferred to the pJC20 vector and tested for expression, the two subunits were equally well produced. It is worth mentioning that expression from pJC20-hASNase $3[\beta+\alpha]$ yielded lower amounts of protein ( $\sim 2 \mathrm{mg} / \mathrm{L}$ of TB medium) as compared to the pJC20-SUMO-hASNase3 $[\alpha+\beta]$ version $(\sim 8 \mathrm{mg} / \mathrm{L}$ 2XYT medium). Potentially, the use of an E. coli codon-optimized sequence for the hASNase 3 cDNA could improve protein yields [31]. Most importantly, our initial assumption that in this gene arrangement, efficient removal of the $\mathrm{N}$-terminal methionine from 
the $\beta$-subunit would occur, was confirmed by enzymatic activity measurements. The purified enzyme exhibited specific L-ASNase activity $(\sim 0.5 \mathrm{U} / \mathrm{mg}$ ) which was similar to the one we obtained when we assayed the same amount of wild-type full-length protein whose intramolecular cleavage between residues Gly167 and Thr168 was triggered by glycine [8].

In summary, these findings suggest that the production of the two subunit-enzyme using the particular construct with reversed order of the two subunit-encoding DNA fragments yielded soluble and catalytically fully active hASNase3. Moreover, the highcopy-number pJC20 vector, as compared to the widely used pET14b plasmid, aided co-production of the two subunits at equal levels.

\section{Conclusions}

Production of two or more proteins, subunits, or domains in $E$. coli can be achieved by different co-expression strategies. Often, there is no straightforward way to decide which approach is the most promising one. The present study focused on the coexpression of the two subunits ( $\alpha$ and $\beta$, also termed protomers) of the human L-Asn-hydrolyzing enzyme hASNase3 following several strategies as summarized in Table 1. Successful co-expression of these protomers is of particular interest because they must not only be produced in soluble form and subsequently associate to form dimers, but removal of the $\beta$-subunit's N-terminal methionine by endogenous $E$. coli MAP must occur efficiently. Cleavage of this translation initiation methionine is of critical importance for the activity of the enzyme since the catalytic threonine's $\alpha$-amino group (residue Thr168 of the full-length protein) located at the $\mathrm{N}$-terminus of the $\beta$-subunit needs to be free for nucleophilic attack on the substrate L-Asn.

Our findings suggest that several factors play a pivotal role in order to achieve successful co-expression. Such factors include the presence of different tags at the $\mathrm{N}$-terminus of the constructs of interest. In our case, the presence of the N-terminal SUMO-tag ultimately resulted in efficient translation of the $\beta$-subunit, which was very poorly detected when the same construct carried only the histidine-tag at the $\mathrm{N}$-terminus of the $\alpha$-subunit. We found that the order of the open reading frame units constituting the operon determines the production of active enzyme, and this is directly related to the action of endogenous MAP. Therefore, in such particular cases where the removal of the N-terminal methionine is essential for enzymatic activity of the recombinantly produced protein, the order of the involved coding units must be considered.

Table 1

Constructs described in the present study and their properties.

\begin{tabular}{|c|c|c|c|c|c|}
\hline \multirow[t]{2}{*}{ Construct } & \multicolumn{2}{|c|}{ Expression } & \multicolumn{2}{|c|}{ Solubility } & \multirow[t]{2}{*}{ Activity } \\
\hline & $\alpha$ & $\beta$ & $\alpha$ & $\beta$ & \\
\hline pET14b-hASNase3 $[\alpha+\beta]\left(N-\alpha-\text { His }_{6}\right)^{\mathrm{a}}$ & $+++^{\mathrm{b}}$ & + & + & + & n.d. ${ }^{c}$ \\
\hline pET14b-SUMO-hASNase $3[\alpha+\beta]\left(\mathrm{N}-\alpha-\mathrm{His}_{6}\right)$ & +++ & +++ & + & + & n.d. \\
\hline pJC20-hASNase $3[\alpha+\beta]\left(\mathrm{N}-\alpha-\mathrm{His}_{6}\right)$ & +++ & + & + & + & n.d. \\
\hline pJC20-SUMO-hASNase $3[\alpha+\beta]\left(\mathrm{N}-\alpha-\mathrm{His}_{6}\right)$ & +++ & +++ & + & + & n.d. \\
\hline pET14b-hASNase3[ $\alpha]\left(\mathrm{N}-\alpha-\mathrm{His}_{6}\right)$ & +++ & & + & & \\
\hline pET29a-hASNase3[ $\beta]$ & & +++ & & - & \\
\hline $\begin{array}{l}\text { pET14b-hASNase3 }[\alpha]\left(\mathrm{N}-\alpha-\mathrm{His}_{6}\right) / \mathrm{pET} 29 \mathrm{a}- \\
\text { hASNase3 }[\beta]\end{array}$ & +++ & - & + & - & n.d. \\
\hline pET14b- $\beta-\alpha\left(C-\alpha-\mathrm{His}_{6}\right)^{\mathrm{a}}$ & - & - & - & - & n.d. \\
\hline pJC20- $\beta-\alpha\left(C-\alpha-\mathrm{His}_{6}\right)$ & ++ & ++ & + & + & $\begin{array}{l}0.5 \mathrm{U} / \\
\mathrm{mg}\end{array}$ \\
\hline
\end{tabular}

\footnotetext{
${ }^{a}\left(\mathrm{~N}-\alpha-\mathrm{His}_{6}\right)$ and $\left(\mathrm{C}-\alpha-\mathrm{His}_{6}\right)$ indicate the location of the histidine-tag at either the amino- or the carboxy-terminus of the $\alpha$-subunit.

$\mathrm{b}$ Indicates the expression levels (+++, high; ++, moderate; +, low) or the solubility $(+$, soluble; - , insoluble) of the two subunits.

c Indicates non-detectable levels of activity.
}

Unexpectedly, the vector system used for expression was also found important for successful co-production of the two hASNase3 subunits. The high-copy-number pJC20 plasmid allowed to achieve satisfying expression levels of both subunits when the order of the two gene units was reversed (pJC20-hASNase3 $[\beta+\alpha]$, instead of pJC20-hASNase3 $[\alpha+\beta]$ ), while the plasmid pET14b-hASNase3 $[\beta+\alpha]$ showed no expression at all. We conclude that the two subunit-expression analysis of the present study might help to find solutions if co-expression via standard vectors fails.

\section{Acknowledgments}

We are thankful to U. Welscher-Altschaeffel for technical assistance, as well as Professor H. Urlaub and U. Plessmann for mass spectrometry analyses.

\section{Appendix A. Supplementary data}

Supplementary data associated with this article can be found, in the online version, at http://dx.doi.org/10.1016/j.pep.2013.10.007.

\section{References}

[1] A.L. Stecher, P.M. de Deus, I. Polikarpov, J. Abrahao-Neto, Stability of Lasparaginase: an enzyme used in leukemia treatment, Pharm. Acta. Helv. 74 (1999) 1-9.

[2] M. Duval, S. Suciu, A. Ferster, X. Rialland, B. Nelken, P. Lutz, Y. Benoit, A. Robert, A.M. Manel, E. Vilmer, J. Otten, N. Philippe, Comparison of Escherichia coliasparaginase with Erwinia-asparaginase in the treatment of childhood lymphoid malignancies: results of a randomized European Organization for Research and Treatment of Cancer-Children's Leukemia Group phase 3 trial, Blood 99 (2002) 2734-2739.

[3] B.L. Asselin, D. Ryan, C.N. Frantz, S.D. Bernal, P. Leavitt, S.E. Sallan, H.J. Cohen, In vitro and in vivo killing of acute lymphoblastic leukemia cells by Lasparaginase, Cancer Res. 49 (1989) 4363-4368.

[4] I.J. Ertel, M.E. Nesbit, D. Hammond, J. Weiner, H. Sather, Effective dose of Lasparaginase for induction of remission in previously treated children with acute lymphoblastic leukemia: a report from Childrens Cancer Study Group, Cancer Res. 39 (1979) 3893-3896.

[5] A. Holleman, M.L. den Boer, K.M. Kazemier, G.E. Janka-Schaub, R. Pieters, Resistance to different classes of drugs is associated with impaired apoptosis in childhood acute lymphoblastic leukemia, Blood 102 (2003) 4541-4546.

[6] J. Nomme, Y. Su, M. Konrad, A. Lavie, Structures of Apo and Product-Bound Human L-Asparaginase: Insights into the Mechanism of Autoproteolysis and Substrate Hydrolysis, Biochemistry 51 (2012) 6816-6826.

[7] D. Borek, K. Michalska, K. Brzezinski, A. Kisiel, J. Podkowinski, D.T. Bonthron, D. Krowarsch, J. Otlewski, M. Jaskolski, Expression, purification and catalytic activity of Lupinus luteus asparagine $\beta$-amidohydrolase and its Escherichia coli homolog, Eur. J. Biochem. 271 (2004) 3215-3226.

[8] Y. Su, C.S. Karamitros, J. Nomme, T. McSorley, M. Konrad, A. Lavie, Free Glycine Accelerates the Autoproteolytic Activation of Human Asparaginase, Chem. Biol. 20 (2013) 533-540.

[9] J.R. Cantor, E.M. Stone, L. Chantranupong, G. Georgiou, The Human Asparaginase-like Protein 1 hASRGL1 Is an Ntn Hydrolase with $\beta$-Aspartyl Peptidase Activity, Biochemistry 48 (2009) 11026-11031.

[10] K. Michalska, M. Jaskolski, Structural aspects of L-asparaginases, their friends and relations, Acta Biochimica Polonica 53 (2006) 627-640.

[11] W. Li, J.R. Cantor, S.D. Yogesha, S. Yang, L. Chantranupong, J.Q. Liu, G. Agnello, G. Georgiou, E.M. Stone, Y. Zhang, Uncoupling Intramolecular Processing and Substrate Hydrolysis in the N-Terminal Nucleophile Hydrolase hASRGL1 by Circular Permutation, Chemical Biology 7 (2012) 1840-1847.

[12] O.K. Dzivenu, H.H. Park, H. Wu, General co-expression vectors for the overexpression of heterodimeric protein complexes in Escherichia coli, Protein Expression and Purification 38 (2000) 1-8.

[13] S. Tan, A Modular Polycistronic Expression System for Overexpressing Protein Complexes in Escherichia coli, Protein Expression and Purification 21 (2001) 224-234.

[14] Y. Liu, Q. Li, X. Hu, J. Yang, Construction and co-expression of polycistronic plasmid encoding D-hydantoinase and D-carbamoylase for the production of D-amino acids, Enzyme and Microbial Technology 42 (2008) 589-593.

[15] N.H. Tolia, L.J. Tor, Strategies for protein coexpression in E.coli, Nature Methods 3 (2006) 55-64.

[16] A.L. Szymczak, D.A.A. Vignali, Development of 2A peptide-based strategies in the design of multicistronic vectors, Expert Opin. Biol. Ther. 5 (2005) 627-638.

[17] K. Graham-Siegenthaler, S. Gauthier, P.L. Davies, J.S. Elce, Active recombinant ratcalpain II. Bacterially produced large and small subunits associate both in vivo and in vitro, J. Biol. Chem. 269 (1994) 30457-30460. 
[18] J. Clos, S. Brandau, PJC20 and pJC40-Two High-Copy-Number Vectors for T7 RNA Polymerase-Dependent Expression of Recombinant Genes in Escherichia coli, Protein Expression and Purification 5 (1994) 133-137.

[19] M. Konrad, Cloning and expression of the essential gene for guanylate kinase from yeast, J. Biol. Chem. 267 (1992) 25652-25655.

[20] Y. Yu, S. Lutz, Circular permutation: a different way to engineer enzyme structure and function, Trends in Biotechnology 29 (2011) 18-25.

[21] S. Bliven, A. Prlic, Circular Permutation in Proteins, PLoS Comput. Biol. 8 (2012) e1002445.

[22] U.K. Laemmli, Cleavage of structural proteins during the assembly of the head of bacteriophage T4, Nature 227 (1970) 680-685.

[23] M.A. Bradford, A rapid and sensitive method for the quantitation of microgram quantities of protein utilizing the principle of protein-dye binding, Anal. Biochem. 72 (1976) 248-254.

[24] S.T. Wei Cun, M.T. Wong, L.L. Few, M. Konrad, Highly specific antibodies for codetection of human choline kinase alpha 1 and alpha 2 isoforms, PLoS One 5 (2010) e12999.

[25] C. Derst, J. Henseling, K.H. Rohm, Probing the role of threonine and serine residues of E.coli asparaginase II by site-specific mutagenesis, Protein Eng. 5 (1992) 785-789.
[26] J.F. Kane, Effects of rare codon clusters on high-level expression of heterologous proteins in Escherichia coli, Curr. Opin. Biotechnol. 6 (1995) 494-500.

[27] J.A. Brannigan, G. Dodson, H.J. Duggleby, P.C. Moody, J.L. Smith, D.R. Tomchick, A.G. Murzin, A protein catalytic framework with an N-terminal nucleophile is capable of self-activation, Nature 378 (1995) 416-419.

[28] M. Enari, H. Sakahira, H. Yokoyama, K. Okawa, A. Iwamatsu, S. Nagata, A caspase-activated DNase that degrades DNA during apoptosis and its inhibitor ICAD, Nature 391 (1998) 43-50.

[29] N. Inohara, T. Koseki, S. Chen, M.A. Benedict, G. Nunez, Identification of regulatory and catalytic domains in the apoptosis nuclease DFF40/CAD, J. Biol. Chem. 274 (1999) 270-274.

[30] H. Sakahira, A. Iwamatsu, S. Nagata, Specific chaperone-like activity of inhibitor of caspase-activated DNase for caspase-activated DNase, J. Biol Chem. 275 (2000) 8091-8096.

[31] N.A. Burgess-Brown, S. Sharma, F. Sobott, C. Loenarz, U. Oppermann, O. Gileadi, Codon optimization can improve expression of human genes in Escherichia coli: A multi -gene study, Protein Expression and Purification 59 (2008) 94102. 


\section{Supplementary Information}

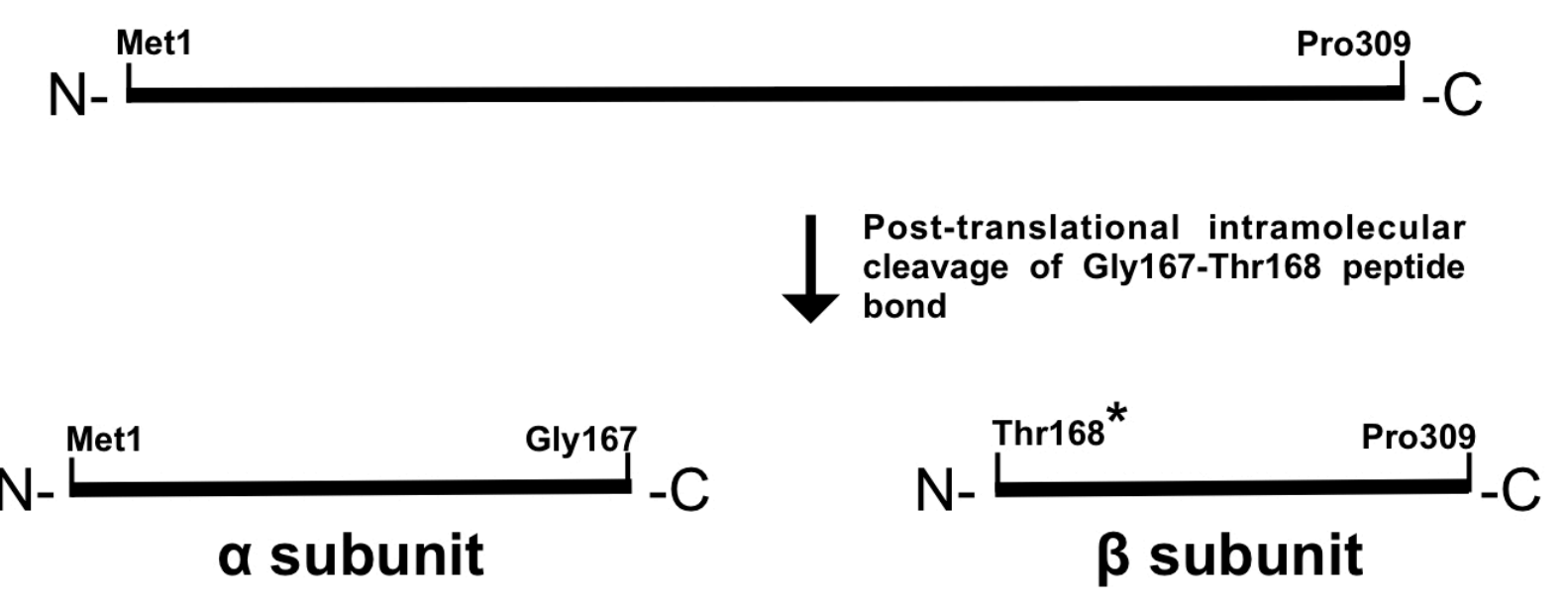

Scheme S1. Schematic representation of hASNase3 intramolecular cleavage by autoproteolysis. The initial, L-ASNase-inactive full-length polypeptide chain undergoes post-translationally an intramolecular cleavage at the Gly167-Thr168 peptide bond, generating two subunits (also called protomers) which remain tightly associated. The asterisk on Thr168 indicates the catalytically critical residue whose amino group must be free at the very $\mathrm{N}$-terminus of the $\beta$-subunit. 


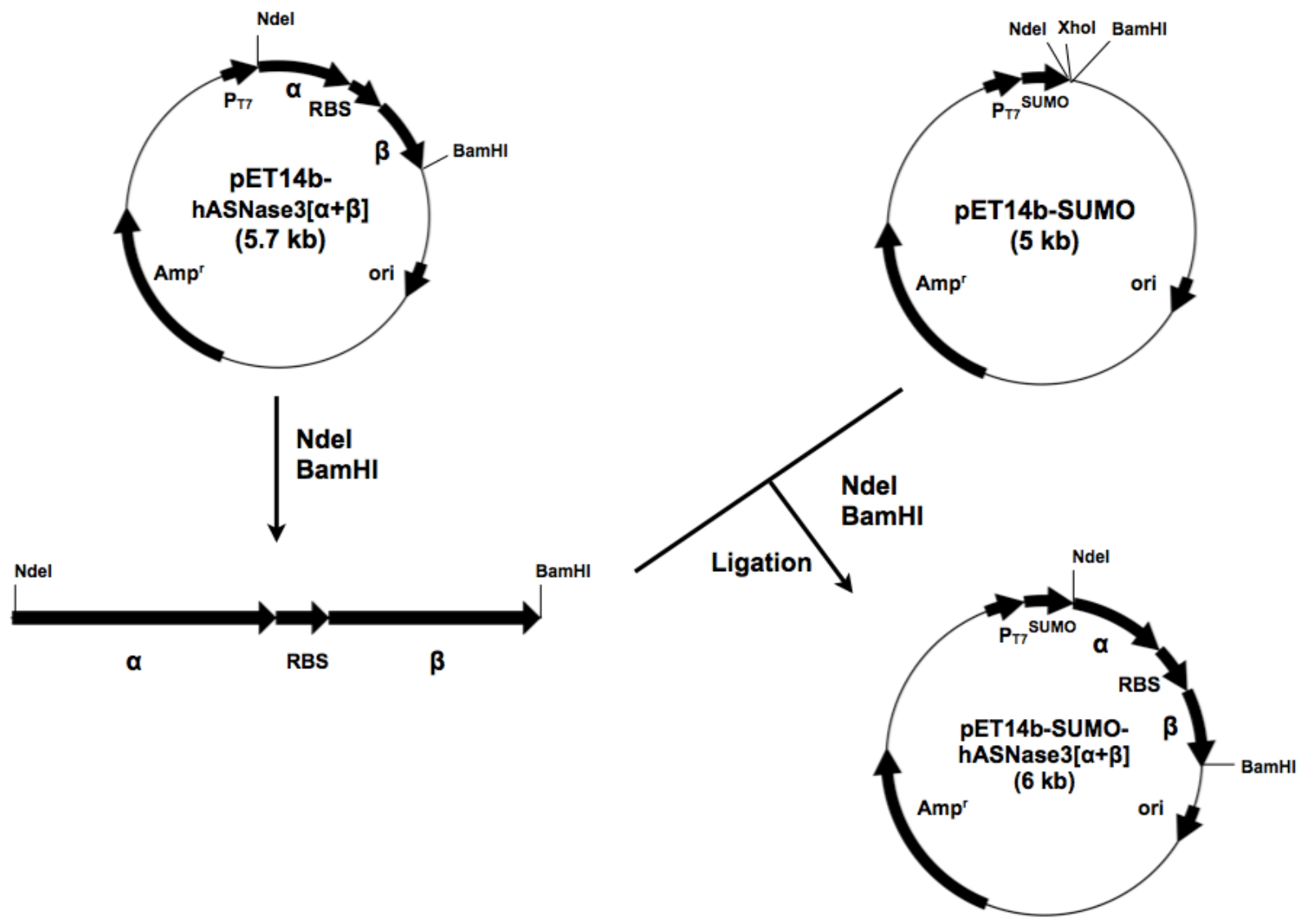

Scheme S2. Subcloning of the hASNase $[\alpha+\beta]$ fragment into the pET14b-SUMO vector. The initial $p E T 14 b-h A S N a s e 3[\alpha+\beta]$ plasmid was digested with $\mathrm{Ndel}$ and $\mathrm{BamHI}$, and the isolated insert was then transferred to the pET14b-SUMO plasmid. 

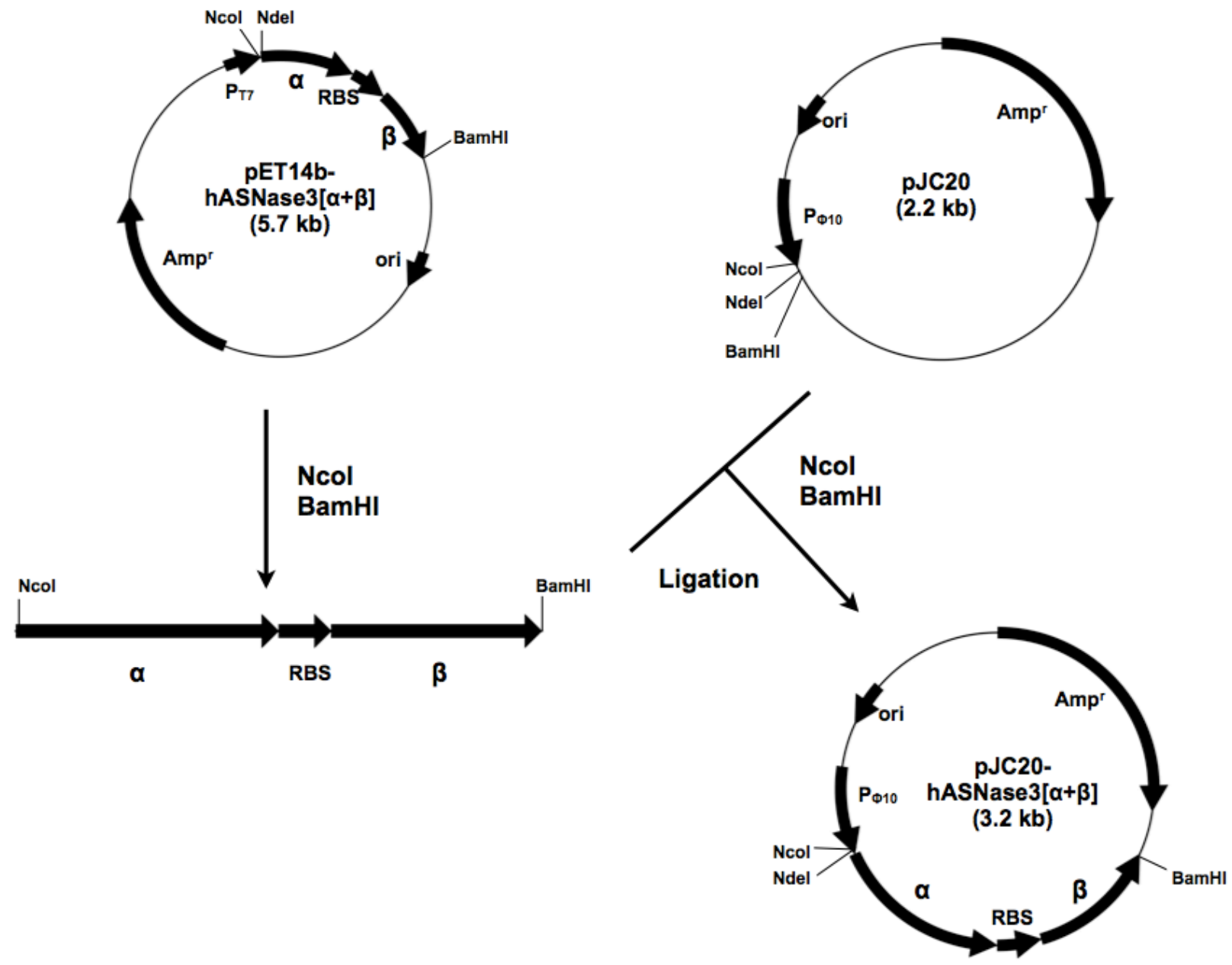

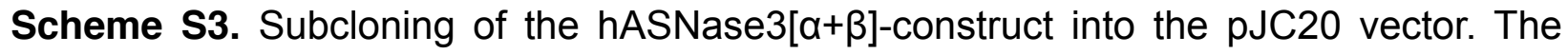

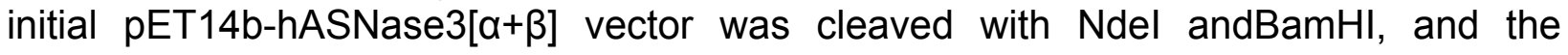
isolated insert was then ligated into the pJC20 plasmid whose MCS is similar to that of pET14b. 

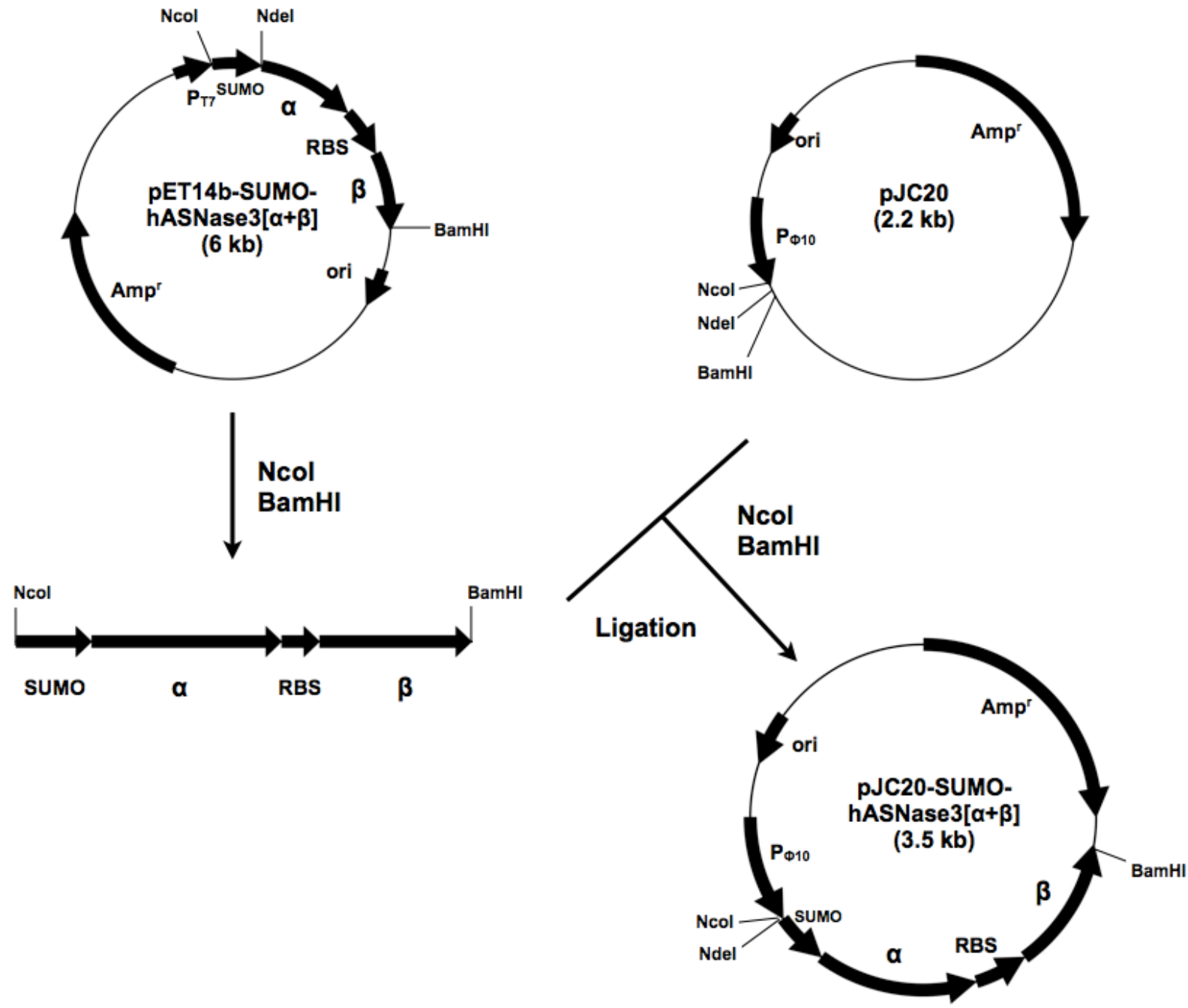

Scheme S4. Subcloning of the SUMO-hASNase3[a+ $\beta]$ construct into the pJC20 vector. The initial pET14b-SUMO-hASNase $3[\alpha+\beta]$ vector was digested with $\mathrm{Ncol}$ and $\mathrm{BamHI}$, and the isolated insert was then subcloned into the pJC20 plasmid. 


\title{
4. An Amplex Red-based fluorometric and spectrophotometric assay for L-asparaginase using its natural substrate.
}

\author{
Karamitros C.S. ${ }^{1}$ Lim J. ${ }^{2}$ and Konrad M. ${ }^{1}$ (2014) \\ ${ }^{1}$ Enzyme Biochemistry Group, Max Planck Institute for Biophysical Chemistry, Göttingen \\ D-37077, Germany \\ ${ }^{2}$ Droplets, Membranes and Interfaces Group, Max Planck Institute for Dynamics and Self- \\ Organization, D-37077 Goettingen, Germany
}

\section{Analytical Biochemistry}

http://www.sciencedirect.com/science/article/pii/S0003269713004661

doi: http://dx.doi.org/10.1016/j.ab.2013.09.028

\section{$\underline{\text { Author contributions }}$}

C.S.K. and M.K. conceived the project and designed research. C.S.K. performed research (J.L. repeated some of the experiments). C.S.K. and M.K. analyzed data and wrote the paper.

Copyright notice:

This material is reproduced by permission from Elsevier in accordance to their copyright and licensing regulations. 
Notes \& Tips

\title{
An Amplex Red-based fluorometric and spectrophotometric assay for L-asparaginase using its natural substrate
}

\author{
Christos S. Karamitros a , Jiseok Lim ${ }^{\mathrm{a}, \mathrm{b}}$, Manfred Konrad ${ }^{\mathrm{a}, *}$ \\ a Max Planck Institute for Biophysical Chemistry, D-37077 Goettingen, Germany \\ ${ }^{\mathrm{b}}$ Max Planck Institute for Dynamics and Self-Organization, D-37077 Goettingen, Germany
}

\section{A R T I C L E I N F O}

\section{Article history:}

Received 7 June 2013

Received in revised form 5 September 2013

Accepted 24 September 2013

Available online 7 October 2013

\section{Keywords:}

L-Asparaginase

Leukemia

L-Aspartate oxidase

Coupled enzyme assay

Amplex Red

\begin{abstract}
A B S T R A C T
We report on the development of a sensitive real-time assay for monitoring the activity of L-asparaginase that hydrolyzes L-asparagine to L-aspartate and ammonia. In this method, L-aspartate is oxidized by Laspartate oxidase to iminoaspartate and hydrogen peroxide $\left(\mathrm{H}_{2} \mathrm{O}_{2}\right)$, and in the detection step horseradish peroxidase uses $\mathrm{H}_{2} \mathrm{O}_{2}$ to convert the colorless, nonfluorescent reagent Amplex Red to the red-colored and highly fluorescent product resorufin. The assay was validated in both the absorbance and the fluorescence modes. We show that, due to its high sensitivity and substrate selectivity, this assay can be used to measure enzymatic activity in human serum containing L-asparaginase.
\end{abstract}

(c) 2013 Elsevier Inc. All rights reserved.
The enzyme L-asparaginase (EC 3.5.1.1, L-asparagine amidohydrolase, L-ASNase), ${ }^{1}$ which predominantly occurs in microorganisms and plants, catalyzes the hydrolysis of L-asparagine (L-Asn) to L-aspartic acid (L-Asp) and ammonia [1,2]. Escherichia coli L-ASNase has been used extensively as a therapeutic enzyme in the frontline treatment of lymphoblastic malignancies, such as acute lymphoblastic leukemia (ALL) and non-Hodgkin lymphoma [3,4], since the 1960s. In light of the significance of $\mathrm{L}-\mathrm{ASN}$ ase as a therapeutic protein, various methods have been developed for measuring the enzyme's activity. Those assays can be used in either absorbance mode [5-10] or fluorescence mode $[11,12]$. However, these assays suffer from certain disadvantages that are primarily related to the use of substrate analogs instead of the natural substrate L-Asn $[6,7,11,12]$. Such assays are not suitable for in vitro evolution of L-asparaginases that aims to select variants showing improved catalytic efficiency and selectivity for the physiological substrate. Moreover, the limited sensitivity of absorption-based spectrophotometric assays $[5-7,9,10]$ is a major handicap to significantly reducing reaction volumes. A noteworthy example of such a case is droplet-based microfluidics, which has emerged as a powerful tool for high-throughput screening in directed protein evolution $[13,14]$. In these experimental setups, the assay volume is minimized to $1 \mathrm{nl}$, to $1 \mathrm{fl}$, scaling down light path lengths to $1 \mu \mathrm{m}$.

\footnotetext{
* Corresponding author. Fax: +495512011074.

E-mail address: mkonrad@gwdg.de (M. Konrad).

1 Abbreviations used: L-ASNase, L-asparaginase; L-Asn, L-asparagine: L-Asp, L-aspartic acid; ALL, acute lymphoblastic leukemia; L-AspOx, L-aspartate oxidase; $\mathrm{H}_{2} \mathrm{O}_{2}$, hydrogen peroxide; HRP, horseradish peroxidase; AR, Amplex Red; FAD, flavin adenine dinucleotide; PBS, phosphate-buffered saline; LOD, limit of detection; NADH, nicotinamide adenine dinucleotide, reduced form; $\mathrm{GDH}$, glutamate dehydrogenase.
}

Here, we report on the development of a novel L-ASNase assay that can be used in either the fluorescence or absorbance mode and relies solely on the use of the physiological substrate L-Asn. In this three-step coupled enzyme system (Fig. 1), L-Asp, which is one of the two products of the L-ASNase reaction, is oxidized by L-aspartate oxidase ( $\mathrm{L}-\mathrm{AspOx})$, resulting in the formation of iminoaspartate and hydrogen peroxide $\left(\mathrm{H}_{2} \mathrm{O}_{2}\right)$; the latter product is used by horseradish peroxidase (HRP) to oxidize the nonfluorescent compound Amplex Red (AR) to resorufin, which exhibits excellent fluorescence as well as absorbance properties.

For establishing and quantitatively evaluating the assay, we cloned and recombinantly produced E. coli L-AspOx [15] (see Fig. S1 in online supplementary material) and periplasmic mature E. coli L-ASNase [16] (Fig. S2), which is the current approved antineoplastic enzyme drug for ALL treatment. The assay was optimized in both the absorbance and fluorescence modes using, for our measurements, a Uvikon 943 double beam UV/VIS (ultraviolet/visible) spectrophotometer and a Jasco FP 8300 spectrofluorometer, respectively. One of the most important criteria to be fulfilled in assays relying on a coupled enzyme system like the one described here is that activity of the auxiliary enzymes always exceeds the enzyme under study. We verified that the rate-determining step of absorption or fluorescence changes in our system was indeed the initial L-ASNase reaction by varying the concentration of L-ASNase. In this context, it is worth underlining that the three enzymes that form this reaction system are characterized by large differences in their kinetic constants, notably in their turnover numbers, with L-AspOx being the least efficient one (steady-state kinetics of L-AspOx is shown in Fig. S3 of the supplementary material) (respective $K_{\mathrm{m}}$ 


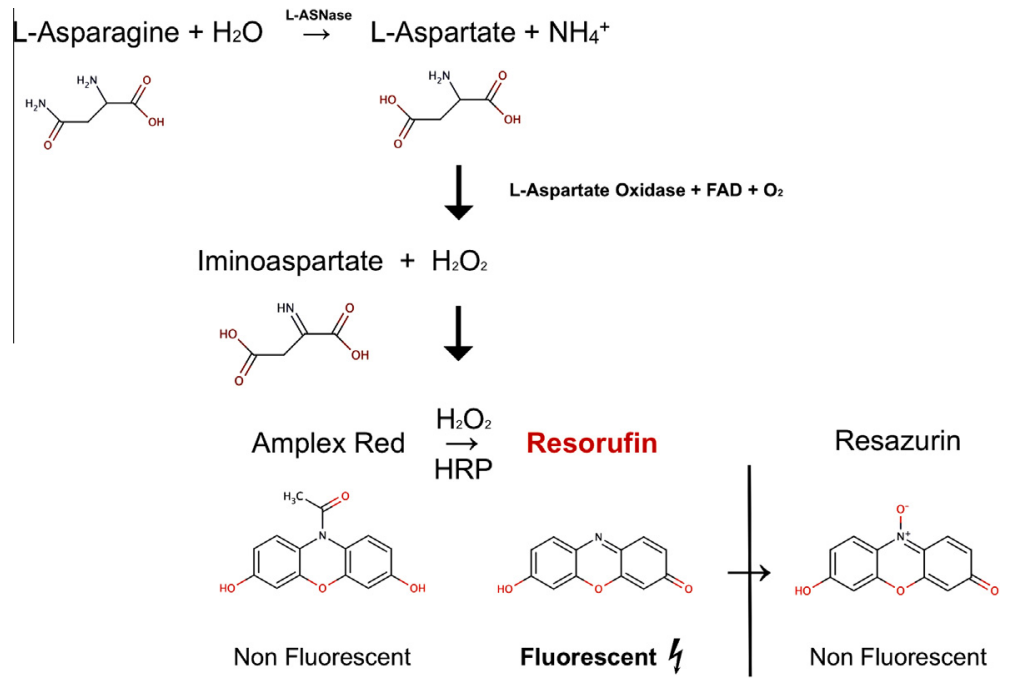

Fig.1. Schematic representation of the AR-dependent L-ASNase coupled-enzyme assay.

and $k_{\text {cat }}$ values: $20 \mu \mathrm{M}$ and $12 \mathrm{~s}^{-1}$ for E. coli L-ASNase; $1.5 \mathrm{mM}$ and $0.5 \mathrm{~s}^{-1}$ for E. coli $\mathrm{L}$-AspOx; $0.1 \mathrm{mM}$ and $560 \mathrm{~s}^{-1}$ for HRP) [17,18].

For standardizing the assay in the fluorescence mode, the final reaction mixture contained 3.5 $\mu \mathrm{M}$ L-AspOx, $10 \mu \mathrm{M}$ flavin adenine dinucleotide (FAD), $100 \mathrm{nM} \mathrm{HRP,} 50 \mu \mathrm{M}$ AR, 4.6 nM L-ASNase ( $16 \mathrm{ng} / 0.1 \mathrm{ml}$ ), and different amounts of $\mathrm{L}$-Asn in a final volume of $100 \mu \mathrm{l}$ using phosphate-buffered saline (PBS) as buffer. Samples were incubated in dark Eppendorf tubes at room temperature. Fluorescence signals were recorded (excitation at $568 \mathrm{~nm}$, emission at $584 \mathrm{~nm}$, bandwidths at $2.5 \mathrm{~nm}$ each) and ultimately corrected by subtracting the control values (no L-Asn) from all samples. Aiming at the optimization of the assay in the fluorescence mode, two critical parameters were further investigated: the concentration of AR in the reaction mixture and the incubation time before recording fluorescence signals. The nonfluorescent AR reacts with $\mathrm{H}_{2} \mathrm{O}_{2}$ in a stoichiometric $1: 1$ ratio, catalyzed by HRP, to form the highly fluorescent product resorufin $[19,20]$. However, as has been shown by others [20], at excess levels of $\mathrm{H}_{2} \mathrm{O}_{2}$ resorufin can be further oxidized to the nonfluorescent compound resazurin. Therefore, the concentration of AR should be kept at least five times higher than that of $\mathrm{H}_{2} \mathrm{O}_{2}$. We tested five different AR concentrations in the range of 10 to $60 \mu \mathrm{M}$ using $1 \mu \mathrm{M} \mathrm{L}$-Asn. Fig. S4 of the supplementary material shows that signals obtained under these conditions are similar and reproducible. In subsequent experiments, we kept AR constant at $50 \mu \mathrm{M}$. We realized that the incubation time prior to activity measurements can be a critical variable that must be optimized in these fluorescent assays. As shown in Fig. S5, fluorescence increases linearly during the first $20 \mathrm{~min}$ and then saturates and remains stable for up to $60 \mathrm{~min}$ after incubation. However, the best signal-to-noise ratio (factor of $\sim 25$ ) was obtained after $30 \mathrm{~min}$ of incubation. Therefore, in all reactions, we incubated the mixture for $30 \mathrm{~min}$ at room temperature before monitoring fluorescence. Alternatively, the reaction can be monitored in a continuous manner for direct kinetic measurements if necessary (Fig. S6). As Fig. 2A shows, the fluorescence signals increase proportionally with the L-ASNase concentration in the range of 0.1 to $4.6 \mathrm{nM}(0.4-$ $16 \mathrm{ng} / 0.1 \mathrm{ml})$. In subsequent kinetic experiments, we used $16 \mathrm{ng}$ of L-ASNase in $0.1-\mathrm{ml}$ reaction mixtures. Importantly, this proportionality also holds in the case of L-ASNase-supplemented human serum samples; therefore, this assay can be used in clinical analyses of L-ASNase activity (Fig. S7). The limit of detection (LOD) of this fluorescent assay was determined to be $100 \mathrm{nM} \mathrm{L}$-Asn based on the standard deviation $(\sigma)$ of the blank samples and the slope $(m)$ of the calibration curve, according to the following equation: $\operatorname{LOD}=(3 \times \sigma) / \mathrm{m}$. Moreover, this concentration could be reliably traced with high reproducibility ( $Z$ ' factor $~ 0.77$; see supplementary material). Fig. 2B demonstrates the linearity of the assay in the concentration range of 0.1 to $10 \mu \mathrm{M}$ L-Asn.
As mentioned above, one of the major advantages of this assay is the fact that it can be used not only in fluorescence mode but also in absorbance mode due to the favorable absorption properties of the final product resorufin showing a maximum at approximately $570 \mathrm{~nm}$ with $\varepsilon=5.4 \times 10^{4} \mathrm{M}^{-1} \mathrm{~cm}^{-1}$ [21]. Similar to optimized conditions for fluorescence detection, absorbance measurements were performed at $25^{\circ} \mathrm{C}$ for a time period of $10 \mathrm{~min}$ in a final volume of $1 \mathrm{ml}$ of PBS buffer containing $3.5 \mu \mathrm{M}$ L-AspOx, $10 \mu \mathrm{M}$ FAD, $100 \mathrm{nM}$ HRP, and $50 \mu \mathrm{M}$ AR. To assess quantitative features and general validity of the new L-ASNase assay, we performed steady-state kinetic analyses to determine characteristic catalytic parameters. First, we determined the level of L-ASNase, up to which the velocity of the reaction remains linear. Fig. $2 \mathrm{C}$ shows that the response is linear in the AR-dependent assay when using 0.68 to $34 \mathrm{nM}(24-1200 \mathrm{ng} / \mathrm{ml}) \mathrm{L}-\mathrm{ASN}$ ase. Based on this result, and to meet conditions required for initial rate measurements, $30 \mathrm{ng}$ of enzyme per milliliter ( $0.85 \mathrm{nM})$ was used for subsequent kinetic experiments covering substrate concentrations in the range of 0 to $15 K_{\mathrm{m}}$. The enzyme followed Michaelis-Menten kinetics (Fig. 2D). Kinetic constants were calculated by nonlinear regression, yielding $K_{\mathrm{m}}=22 \pm 1.2 \mu \mathrm{M}, k_{\text {cat }} \sim 12.1 \pm 0.5 \mathrm{~s}^{-1}$, and $k_{\text {cat }} / K_{\mathrm{m}}=5.5 \times 10^{5} \mathrm{M}^{-1} \mathrm{~s}^{-1}$.

To validate this AR-dependent assay, we compared our data with kinetic constants reported before by making use of the nicotinamide adenine dinucleotide (NADH)-dependent assay [9]. Briefly, the produced ammonia serves as substrate for glutamate dehydrogenase (GDH) that converts $\alpha$-ketoglutarate to L-glutamic acid (L-Glu) with simultaneous oxidation of NADH. The disappearance of NADH is reflected by a decrease in absorption at $340 \mathrm{~nm}$, with $\varepsilon=6.22 \times 10^{3}$ $\mathrm{M}^{-1} \mathrm{~cm}^{-1}$ (at $\mathrm{pH} 7.5$ ). Thus, the oxidation of $\mathrm{NADH}$ is directly proportional to the amount of ammonium generated by L-ASNase activity. Similar to the L-AspOx-dependent spectrophotometric assay, reactions were performed at $25^{\circ} \mathrm{C}$ in a final volume of $1 \mathrm{ml}$ of PBS containing $0.25 \mathrm{mM} \mathrm{NADH}, 0.25 \mathrm{mM} \alpha$-ketoglutarate, and $35 \mathrm{nM} \mathrm{GDH}$ from bovine liver. The L-ASNase concentration was $7 \mathrm{nM}(\sim 250 \mathrm{ng} /$ $\mathrm{ml}$ ). Fig. S6 shows the resulting Michaelis-Menten plot. The calculated kinetic constants are $21 \pm 1.5 \mu \mathrm{M}$ for $K_{\mathrm{m}}, 11.5 \pm 0.4 \mathrm{~s}^{-1}$ for $k_{\text {cat }}$, and $5.4 \times 10^{5} \mathrm{M}^{-1} \mathrm{~s}^{-1}$ for $k_{\mathrm{cat}} / K_{\mathrm{m}}$. The values obtained by these two assays (summarized in Table S1 of the supplementary material) are in excellent agreement, indicating that the newly developed fluorescence assay is a reliable and more sensitive alternative to the NADHdependent assay, requiring approximately 10 -fold less L-ASNase.

In summary, here we reported on the development of a highly sensitive assay for measurement of L-ASNase activity. This L-ASNase assay comprises three coupled enzymatic reactions. It relies on the use of L-AspOx in the second reaction step, which generates $\mathrm{H}_{2} \mathrm{O}_{2}$ that, in the presence of HRP, oxidizes the nonfluorescent AR agent to the highly 

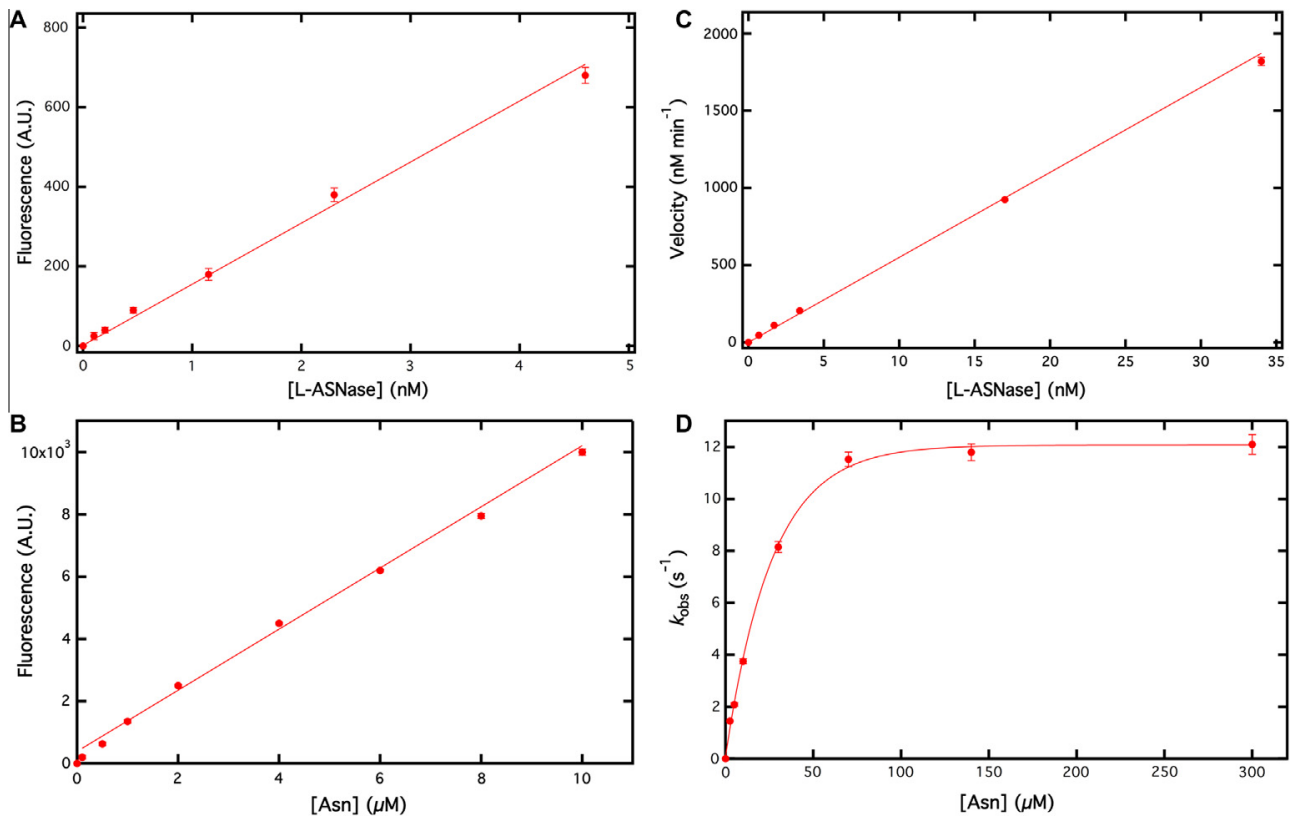

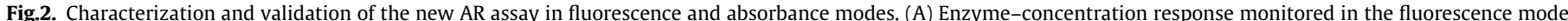

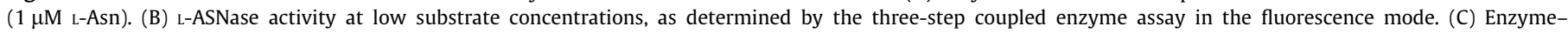

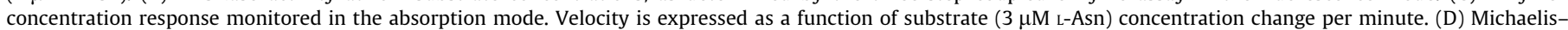

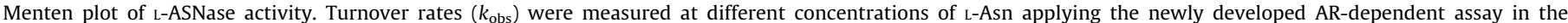
absorbance mode. All data points are the means \pm standard deviations of triplicate measurements.

red fluorescent resorufin product. Advantages of this assay are that it is homogeneous, allowing adjustment of the assay conditions, such as temperature and buffer composition, according to specific requirements, and that it can in principle be applied in both the absorbance and fluorescence modes. Taking advantage of resorufin's excellent fluorescence and absorption properties, we showed that this assay can reliably be used to detect low amounts of $\mathrm{L}$-Asn and monitor low levels of $\mathrm{L}-$ ASNase activity not only when purified enzyme is used but also when its activity is to be determined in human serum. Notably, the enzyme can be kinetically characterized using its natural substrate and requiring 10 -fold less protein compared with other widely used assays.

\section{Acknowledgments}

This work was supported by the Max Planck Society and by a National Research Foundation of Korea fellowship to J.L. (NRF2011-357-D00011). We thank Ursula Welscher-Altschäffel for technical assistance with protein preparation and thank Claudia Höbartner for helpful comments on the manuscript.

\section{Appendix A. Supplementary data}

Supplementary data associated with this article can be found, in the online version, at http://dx.doi.org/10.1016/j.ab.2013.09.028.

\section{References}

[1] K. Aghaiypour, A. Wlodawer, J. Lubkowski, Structural basis for the activity and substrate specificity of Erwinia chrysanthemi L-asparaginase, Biochemistry 40 (2001) 5655-5664.

[2] M. Kozak, D. Borek, R. Janowski, M. Jaskolski, Crystallization and preliminary crystallographic studies of five crystal forms of Escherichia coli L-asparaginase II (Asp90Glu mutant), Acta Crystallogr. D 58 (2002) 130-132.

[3] A.L. Stecher, P.M. de Deus, I. Polikarpov, J. Abrahao-Neto, Stability of L-asparaginase: an enzyme used in leukemia treatment, Pharm. Acta Helv. 74 (1999) 1-9.

[4] M. Duval, S. Suciu, A. Ferster, X. Rialland, B. Nelken, P. Lutz, Y. Benoit, A. Robert, A.M. Manel, E. Vilmer, J. Otten, N. Philippe, Comparison of Escherichia coliasparaginase with Erwinia-asparaginase in the treatment of childhood lymphoid malignancies: results of a randomized european organization for research and treatment of cancer-children's leukemia group phase 3 trial, Blood 99 (2002) 2734-2739.
[5] H.A. Campbell, L.T. Mashburn, E.A. Boyse, L.J. Old, Two L-asparaginases from Escherichia coli B: their separation, purification, and antitumor activity, Biochemistry 6 (1967) 721-730.

[6] D. Cooney, R. Handschumacher, L-Asparaginase and L-asparagine metabolism, Annu. Rev. Pharmacol. 10 (1970) 421-440.

[7] Y.Z. Frohwein, M. Friedman, J. Reizer, N. Grossowicz, Sensitive and rapid assay for L-asparaginase, Nat. New Biol. 230 (1971) 158-159.

[8] C. Lanvers, J.P.V. Pinheiro, G. Hempel, G. Wuerthwein, J. Boos, Analytical validation of a microplate reader-based method for the therapeutic drug monitoring of $\mathrm{L}$ asparaginase in human serum, Anal. Biochem. 309 (2002) 117-126.

[9] V.M. Balcão, C. Mateo, R. Fernandez-Lafuente, F.X. Malcata, J.M. Guisa, Structural and functional stabilization of L-asparaginase via multisubunit immobilization onto highly activated supports, Biotechnol. Prog. 17 (2001) 537-542.

[10] A. Credali, M. Garcia-Calderon, S. Dam, J. Perry, A. Diaz-Quintana, M. Parniske, T.L. Wang, J. Stougaard, J.M. Vega, A.J. Marquez, The $\mathrm{K}^{+}$-dependent asparaginase, NSE1, is crucial for plant growth and seed production in Lotus japonicus, Plant Cell Physiol. 54 (2013) 107-118.

[11] I.T. Mononen, V.M. Kaartinen, J.C. Williams, A fluorometric assay for glycosylasparaginase activity and detection of aspartylglycosaminuria, Anal. Biochem. 208 (1993) 372-374.

[12] P. Ylikangas, I. Mononen, A fluorometric assay for L-asparaginase activity and monitoring of L-asparaginase therapy, Anal. Biochem. 280 (2000) 42-45.

[13] J.C. Baret, O.J. Miller, V. Taly, M. Ryckelynck, A. El-Harrak, L. Frenz, C. Rick, M.L. Samuels, J.B. Hutchison, J.J. Agresti, D.R. Link, D.A. Weitzc, A.D. Griffiths, Fluorescence-activated droplet sorting (FADS): efficient microfluidic cell sorting based on enzymatic activity, Lab Chip 9 (2009) 1850-1858.

[14] J. Lim, P. Gruner, M. Konrad, J.C. Baret, Micro-optical lens array for fluorescence detection in droplet-based microfluidics, Lab Chip 13 (2013) 1472-1475.

[15] R. Flachmann, N. Kunz, J. Seifert, M. Gutlich, F.J. Wientjes, A. Laufer, H.G. Gassen, Molecular biology of pyridine nucleotide biosynthesis in Escherichia coli, Eur. J. Biochem. 175 (1988) 221-228.

[16] D.T. Bonthron, L-Asparaginase II of Escherichia coli K-12: cloning, mapping, and sequencing of the ansB gene, Gene 91 (1990) 101-105.

[17] G. Tedeschi, S. Ronchi, T. Simonic, C. Treu, A. Mattevi, A. Negri, Probing the active site of L-aspartate oxidase by site-directed mutagenesis: role of basic residues in fumarate reduction, Biochemistry 40 (2001) 4738-4744.

[18] B.D. Howes, N.C. Brissett, W.A. Doyle, A.T. Smith, G. Smulevich, Spectroscopic and kinetic properties of the horseradish peroxidase mutant T171S: evidence for selective effects on the reduced state of the enzyme, FEBS J. 272 (2005) 5514-5521.

[19] J.G. Mohanty, J.S. Jaffe, E.S. Schulman, D.G. Raible, A highly sensitive fluorescent micro-assay of $\mathrm{H}_{2} \mathrm{O}_{2}$ release from activated human leukocytes using a dihydroxyphenoxazine derivative, J. Immunol. Methods 202 (1997) 133-141.

[20] A. Zhu, R. Romero, H.R. Petty, A sensitive fluorimetric assay for pyruvate, Anal. Biochem. 396 (2010) 146-151.

[21] M. Zhou, Z. Diwu, N. Panchuk-Voloshina, R.P. Haugland, A stable nonfluorescent derivative of resorufin for the fluorometric determination of trace hydrogen peroxide: applications in detecting the activity of phagocyte NADPH oxidase and other oxidases, Anal. Biochem. 253 (1997) 162168. 


\section{Supplementary Material}

\section{Chemicals and reagents}

L-asparagine (L-Asn), flavine adenine dinucleotide (FAD), nicotinamide adenine dinucleotide (NADH), horseradish peroxidase (HRP type X, $386 \mathrm{U} / \mathrm{mg}, M_{r} \sim 44 \mathrm{kDa}$ ), glutamate dehydrogenase from bovine liver (GDH, $\left.44 \mathrm{U} / \mathrm{mg}, M_{r} \sim 56 \mathrm{kDa}\right), a-$ ketoglutarate, o-dianisidine, L-aspartate (L-Asp), and human serum (from male $A B$ plasma) were obtained from Sigma Aldrich (St. Louis MO, USA). Amplex Red (AR) was purchased from Cayman chemicals (Michigan, USA). Yeast extract, peptone, agar, ampicillin, and Isopropyl-beta-D-thiogalactoside (IPTG) were purchased from Applichem (Gatersleben, Germany). KAPA HiFi polymerase was obtained from PeqLab (Erlangen, Germany), restriction enzymes and T4 DNA ligase from NEB (Ipswich, MA, USA). Gel extraction and PCR purification kits, and nickel agarose for affinity purification of polyhistidine-tagged proteins were from Macherey Nagel (Düren, Germany), plasmid purification kit from Fermentas (Thermo Fisher Scientific, Germany), genomic DNA preparation kit from Qiagen (Hilden, Germany), gel filtration chromatography column Superdex 200 from Pharmacia/GE Health Care Life Sciences (Uppsala Sweden). Coomassie Brilliant Blue G-250 (Bradford reagent) for protein assays was from Roth (Karlsruhe, Germany). 


\section{Cloning, expression and purification of L-aspartate oxidase and periplasmic L- asparaginase from E.coli.}

Since L-aspartate oxidase (L-AspOx) is commercially not available, we cloned the E.coli gene $(n a d B)$ and produced milligram amounts of the enzyme recombinantly. The open reading frame (ORF) of L-AspOx consisting of $1623 \mathrm{bp}$, was amplified by PCR using as template genomic DNA isolated from the E.coli XL1-Blue strain. Unique Ndel and BamHI restrictions sites (underlined below) were incorporated at the 5'- and 3'- ends of the respective oligonucleotides (5'-GGAATTCCATATGAATACTCTCCCTGAACATTC-3', and 5'-CGCGGATCCTTATCTGTTTATGTAATGATTGC-3'). The PCR mixture (50 $\mu$ L) consisted of $1 \mu \mathrm{g}$ genomic DNA, oligonucleotide mix (10 pmol each), KAPA high fidelity buffer, dNTPs (0.2 mM each), and 1 Unit KAPA HiFi DNA polymerase. The reaction was performed at initial temperature of $95{ }^{\circ} \mathrm{C}$ for $3 \mathrm{~min}$, followed by 25 cycles of the next steps: at $98^{\circ} \mathrm{C}$ for $20 \mathrm{~s}, 60^{\circ} \mathrm{C}$ for $60 \mathrm{~s}$, and $72{ }^{\circ} \mathrm{C}$ for $30 \mathrm{~s}$. The reaction was terminated after a $3^{\prime}$-end polishing step of $5 \mathrm{~min}$ at $72{ }^{\circ} \mathrm{C}$. The PCR product was agarose gelpurified, digested with $\mathrm{Ndel}$ and $\mathrm{BamHI} \mathrm{HF}$, and ligated overnight at $16{ }^{\circ} \mathrm{C}$ into a pET14b-SUMO vector [1] using T4 DNA ligase. The ligation mixture was used to transform DH5a E.coli cells, and positive clones were first identified by colony PCR screening. Extracted plasmid DNA was digested with Ndel and BamHI HF to verify correct size of the cloned fragment, and finally the gene insert was sequenced (SeqLab, Goettingen, Germany). 
The expressed protein construct includes an $\mathrm{N}$-terminal six-histidine tag, followed by a yeast SUMO (ㅁmall U Ubiquitin-related MOdifier) tag which has proven to ameliorate heterologous protein solubility and stability [2]. E.coli BL21(DE3) C41 cells (Lucigen, Madison, USA) were cultured overnight at $37^{\circ} \mathrm{C}$ in LB medium supplemented with 200 $\mu \mathrm{g} / \mathrm{ml}$ ampicillin. A fraction of this culture was used to inoculate fresh $2 \times Y T$ culture medium (dilution 1:100) supplemented with $200 \mu \mathrm{g} / \mathrm{ml}$ ampicillin. When O.D.600 reached $\sim 0.5-0.7$, expression was induced by adding IPTG to a final concentration of $1 \mathrm{mM}$. After incubation at $37^{\circ} \mathrm{C}$ for 8 hours, the cells were harvested by centrifugation (20 min at 5,000g), resuspended in nickel agarose binding buffer $\mathrm{A}\left(50 \mathrm{mM} \mathrm{Na} 2 \mathrm{HPO}_{4}, 0.5 \mathrm{M}\right.$ $\mathrm{NaCl}, 10 \mathrm{mM}$ imidazole, $\mathrm{pH}$ 8), and ultimately lysed by ultrasonication. The cell lysate was centrifuged (45 $\mathrm{min}$ at $17,200 \mathrm{~g}$ ), the resulting supernatant mixed with preequilibrated nickel agarose beads, and incubated at $4{ }^{\circ} \mathrm{C}$ for 3 hours in a rotating beaker. The slurry mixture was filled in $5 \mathrm{~mL}$ polypropylene columns, dried by gravity, and the agarose beads were then washed with 25 bed volumes of buffer $B(50 \mathrm{mM}$ $\mathrm{Na}_{2} \mathrm{HPO}_{4}, 1 \mathrm{M} \mathrm{NaCl}, 25 \mathrm{mM}$ imidazole, $\mathrm{pH}$ 8). Bound protein was eluted from the column by applying $300 \mathrm{mM}$ imidazole in buffer B. All purification steps were performed at $4{ }^{\circ} \mathrm{C}$ in a cold room. Eluted fractions were mixed, and buffer was exchanged against $50 \mathrm{mM} \mathrm{Na}_{2} \mathrm{HPO}_{4}, 100 \mathrm{mM} \mathrm{NaCl}, \mathrm{pH}$ 7.5, using a PD-10 column (GE Healthcare). Eluted protein was incubated with yeast SUMO protease (molar ratio $1: 100$ ) at $30{ }^{\circ} \mathrm{C}$ for 2 hours to cleave the $\mathrm{N}$-terminal tag. This treatment resulted in the final intact L-AspOx with predicted $M_{r} \sim 60.3 \mathrm{kDa}$ (apparent $M_{r}$ on SDS-PAGE $\sim 60 \mathrm{kDa}$ ). The protein solution was concentrated using ultrafiltration tubes, 10,000 MWCO (Sartorius, Goettingen, Germany). In the final purification step, the enzyme was passed through a 
Superdex 200 column to remove the cleaved tag, SUMO protease, and impurities remaining after the affinity purification step. Protein purity, as evaluated by SDS-PAGE, was more than $95 \%$. The protein was aliquoted, mixed with glycerol (25\% final concentration), and stored at $-20{ }^{\circ} \mathrm{C}$ until further use. The overall protein yield for LAspOx was estimated to be approximately $40 \mathrm{mg}$ per $\mathrm{L}$ of $2 \mathrm{xYT}$ growth medium with a specific activity $\sim 0.05 \mathrm{U} / \mathrm{mg}$ as determined by applying the o-dianisidine assay described elsewhere [3], which was also used for the kinetic characterization of this enzyme (Fig. S3).

Periplasmic mature E.coli L-ASNase (ansB) was cloned using the primer pair 5'-

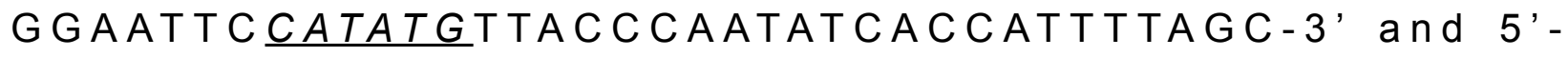
CGCGGATCCTTAGTACTGATTGAAGATCTGCTGG-3', expressed, and purified in a similar way. The protein amount obtained out of $1 \mathrm{~L} 2 \mathrm{xYT}$ medium was $\sim 15 \mathrm{mg}$ with a specific activity of $1 \mathrm{U} / \mathrm{mg}$ as determined by the NADH-dependent assay. The predicted $M_{r}$ of the mature E.coli L-ASNase is $34.5 \mathrm{kDa}$, while on SDS-PAGE it appears to run somewhat faster $(\sim 32 \mathrm{kDa})$. 


\section{Figures}

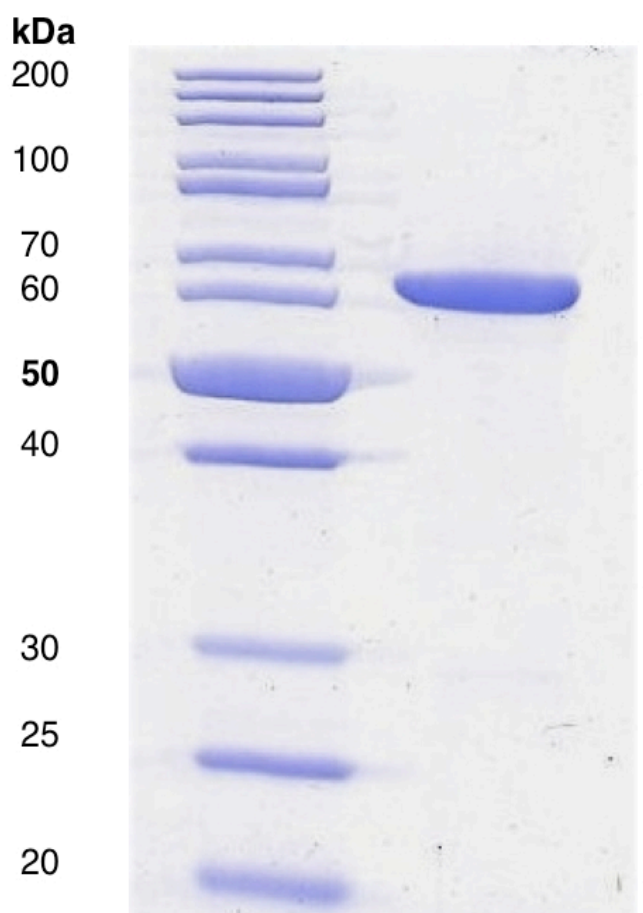

Figure S1. SDS-PAGE (15\%) analysis of purified E.coli L-AspOx (calculated $M_{r}: 60.3$ $\mathrm{kDa})$. 


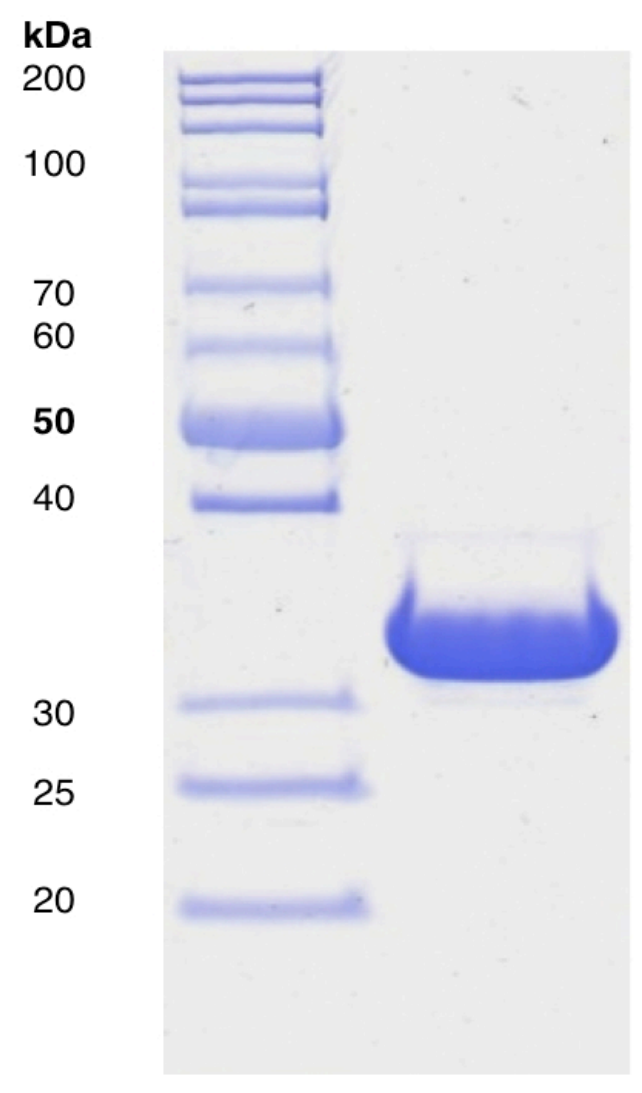

Figure S2. SDS-PAGE (15\%) analysis of purified mature E.coli L-ASNase (calculated $\left.M_{r}: 34.5 \mathrm{kDa}\right)$. 


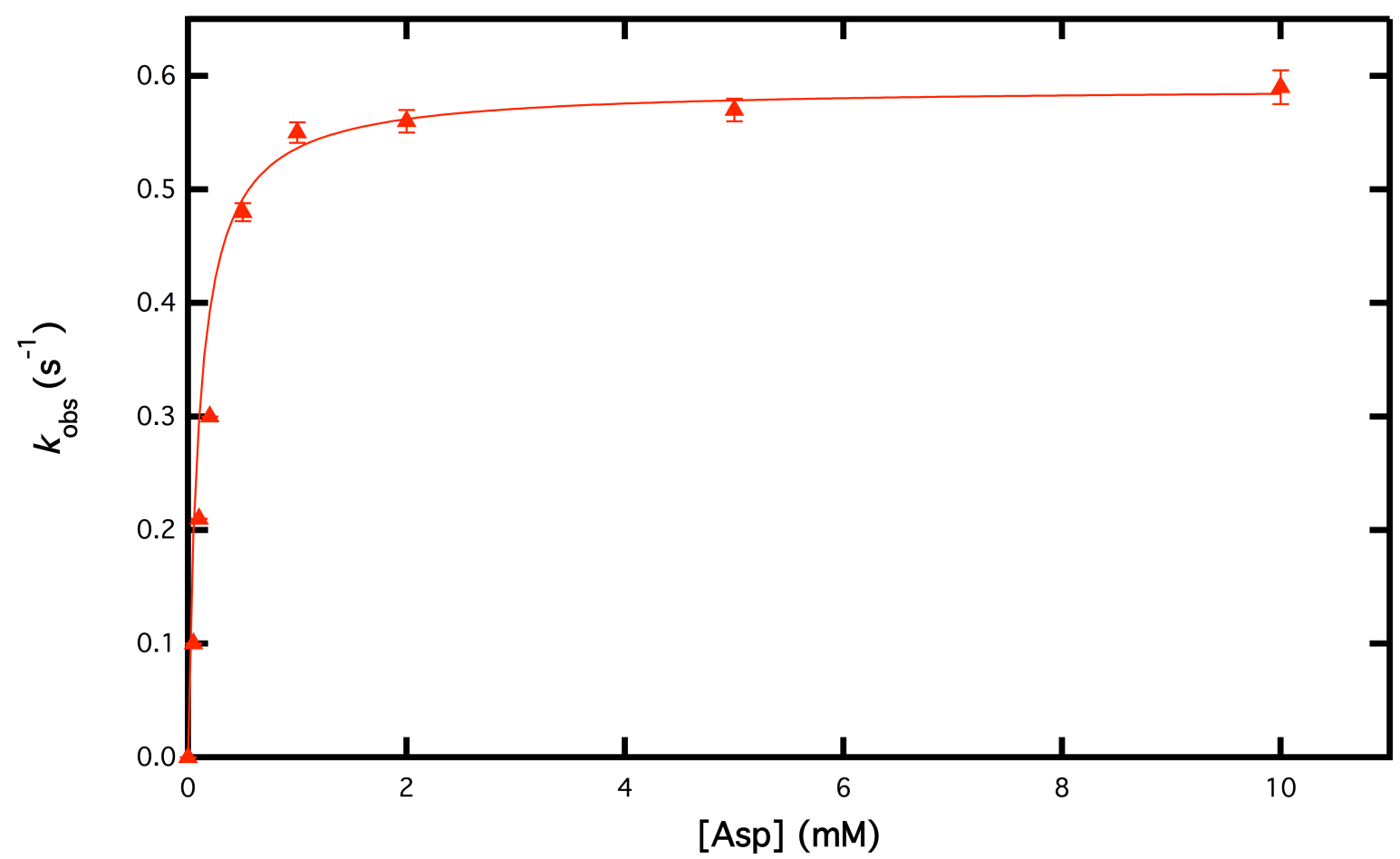

Figure S3. Steady-state turnover rates ( $k_{\text {obs }}$ ) of L-AspOx determined by applying the chromogenic o-dianisidine-based assay [3]. Reactions were performed at $25^{\circ} \mathrm{C}$ in $1 \mathrm{ml}$ PBS, containing $20 \mu \mathrm{M}$ FAD, $100 \mathrm{nM} \mathrm{HRP}, 10 \mu \mathrm{g} / \mathrm{ml}$ o-dianisidine, $65 \mathrm{nM}(\sim 5 \mu \mathrm{g}) \mathrm{L}-$ AspOx, and variable amounts of substrate. Kinetic constants (see text) were calculated by non-linear regression of the Michaelis-Menten (Igor Pro, Wavemetrics). Measurements were performed in triplicate $(n=3)$, and values are expressed as means $\pm \mathrm{SD}$. 


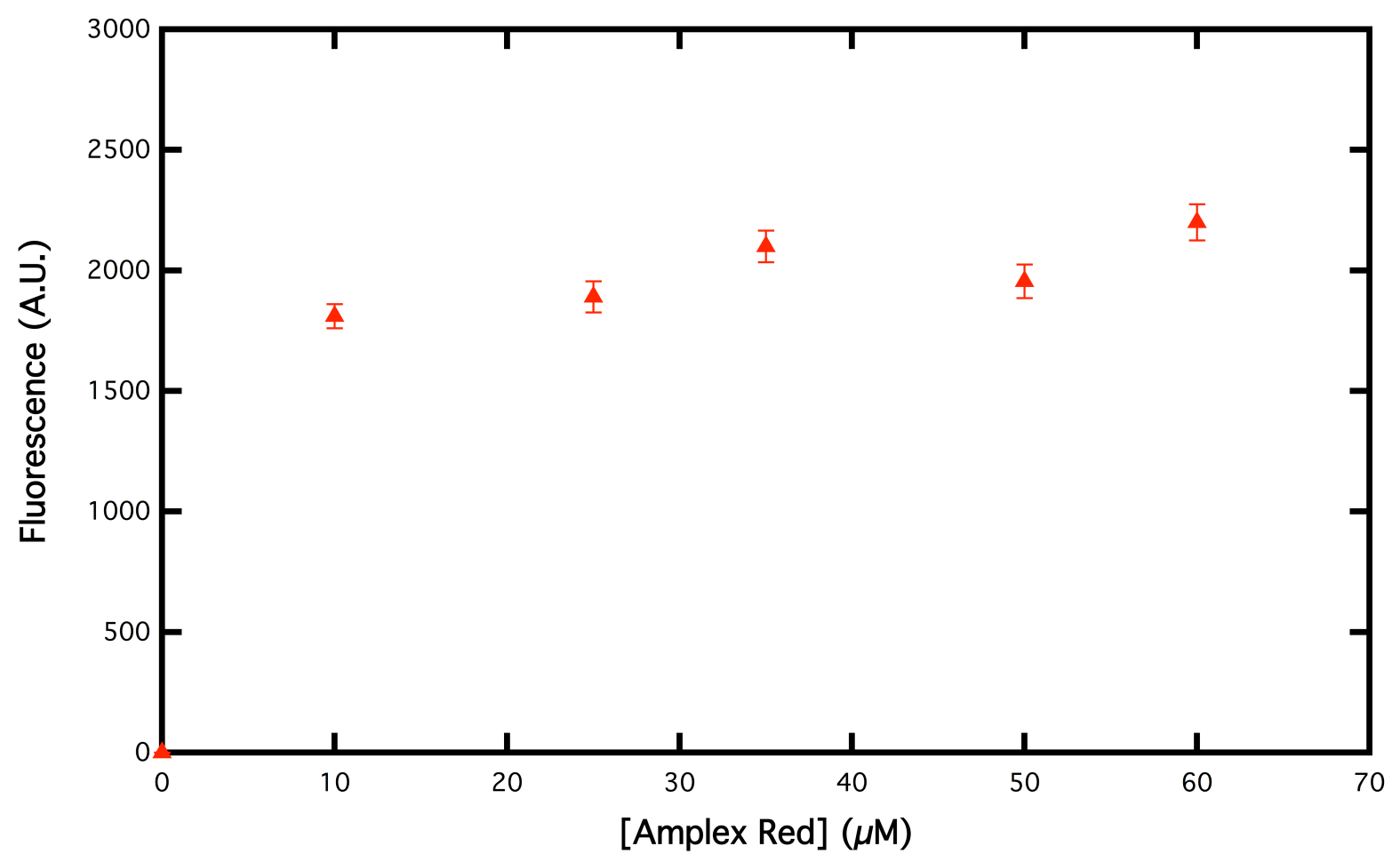

Figure S4. Fluorescence intensity as a function of AR concentration. The L-Asn concentration was kept constant at $1 \mu \mathrm{M}$, while L-ASNase concentration was $\sim 4.6 \mathrm{nM}$. The fluorescence signal was recorded after $30 \mathrm{~min}$ in each case, $(n=3, \pm S D)$ 


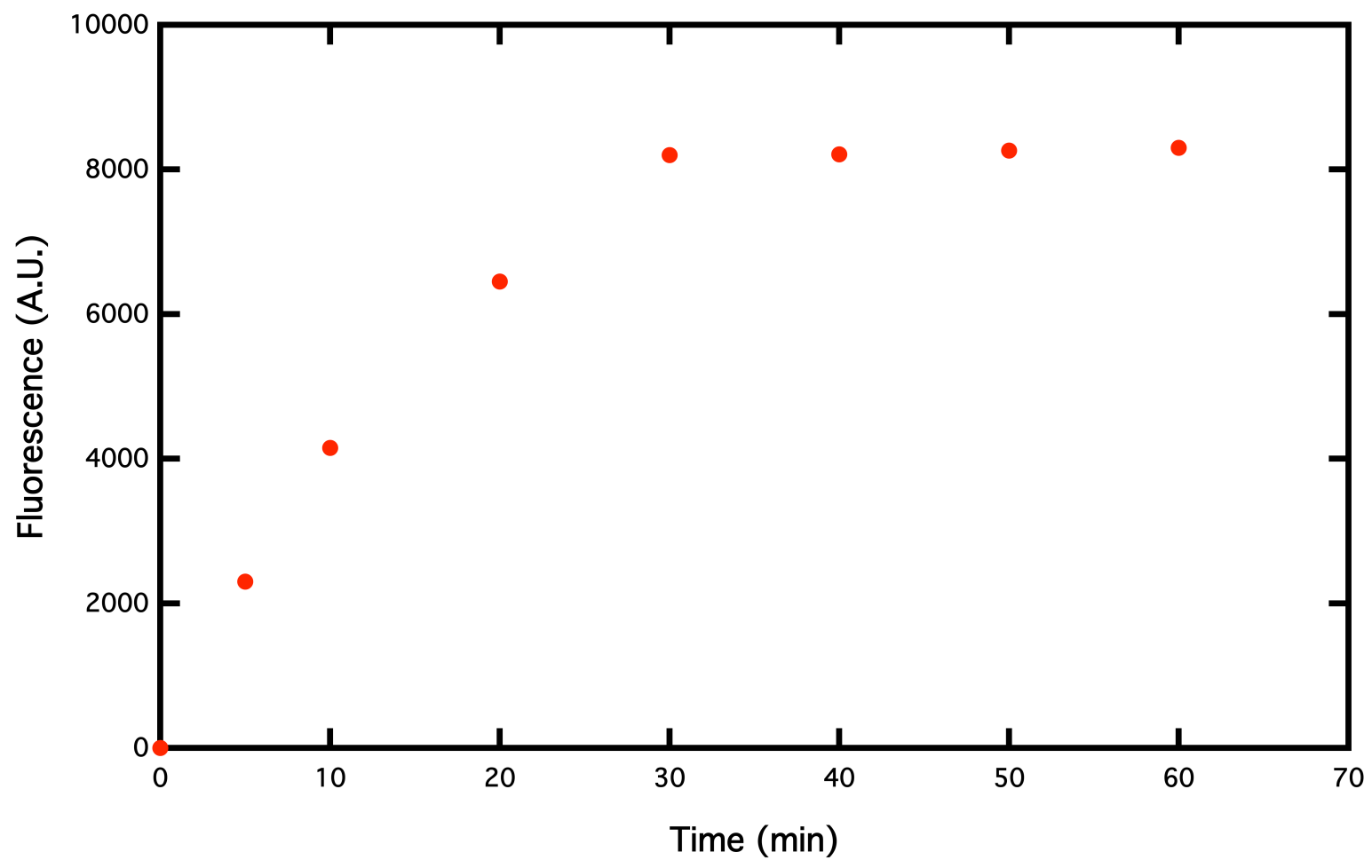

Figure S5. Time course of the L-ASNase reaction monitored at discrete time points using the fluorescence assay. Concentrations: $10 \mu \mathrm{M}$ L-Asn, $50 \mu \mathrm{M} A R, \sim 4.6 \mathrm{nM}$ LASNase, $(n=3, \pm S D)$. Error bars are not shown when they are smaller than the symbols. 


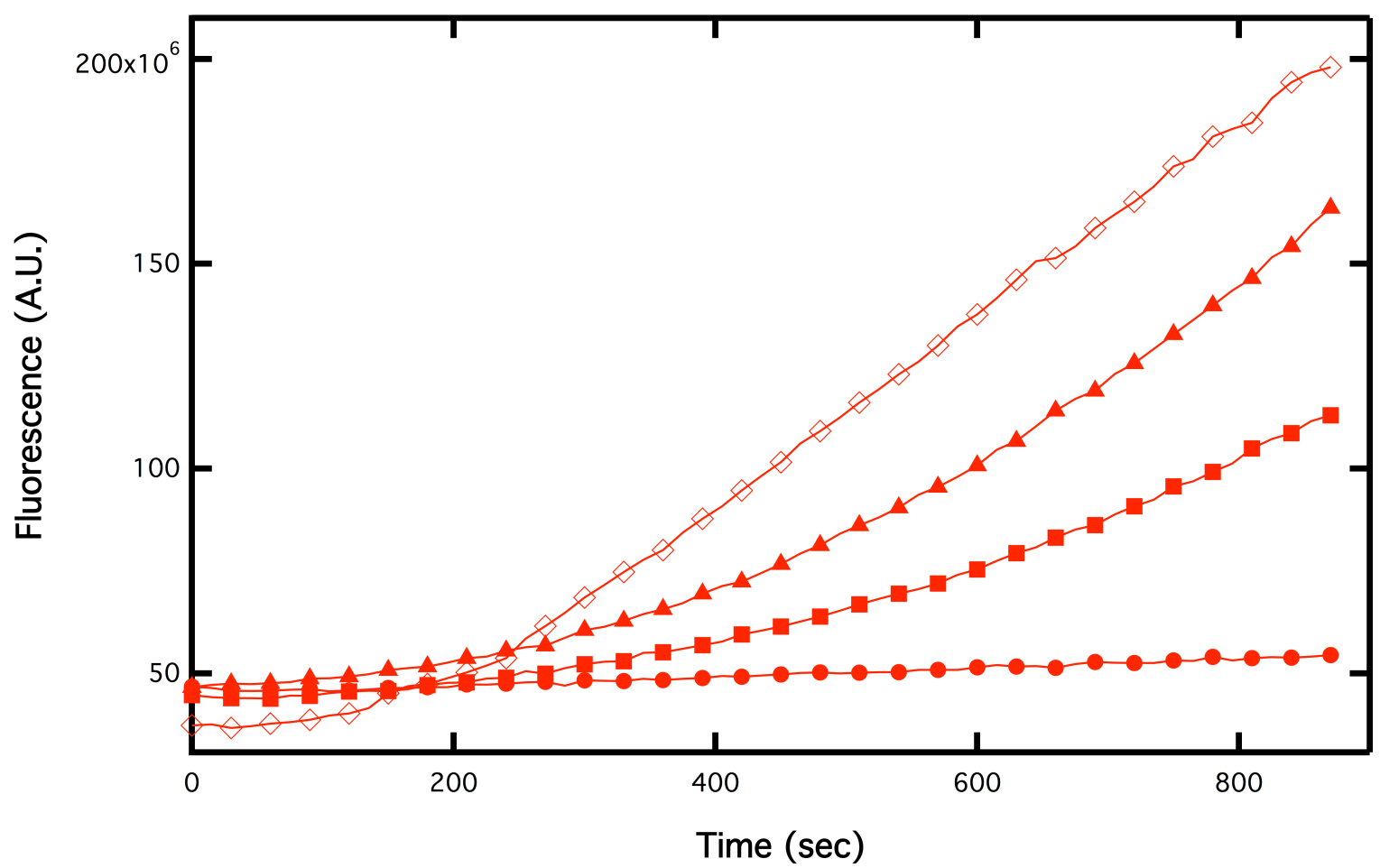

Figure S6. Real-time monitoring of the three-step enzymatic reaction to detect Lasparaginase activity using a plate-reader (Molecular Devices, SpectraMax Paradigm; wavelength settings were $532 \mathrm{~nm}$ for Ex, and $592 \mathrm{~nm}$ for Em). The four reactions took place simultaneously in a 96-well plate in a final volume of $100 \mu \mathrm{L}$ at the following concentrations: $10 \mu \mathrm{M}$ L-Asn, $50 \mu \mathrm{M}$ AR; L-ASNase concentrations were as follows: (•): $0 \mathrm{nM},(\square): 1 \mathrm{nM},(\Delta): 2 \mathrm{nM}$, and $(\diamond): 4 \mathrm{nM}$. 


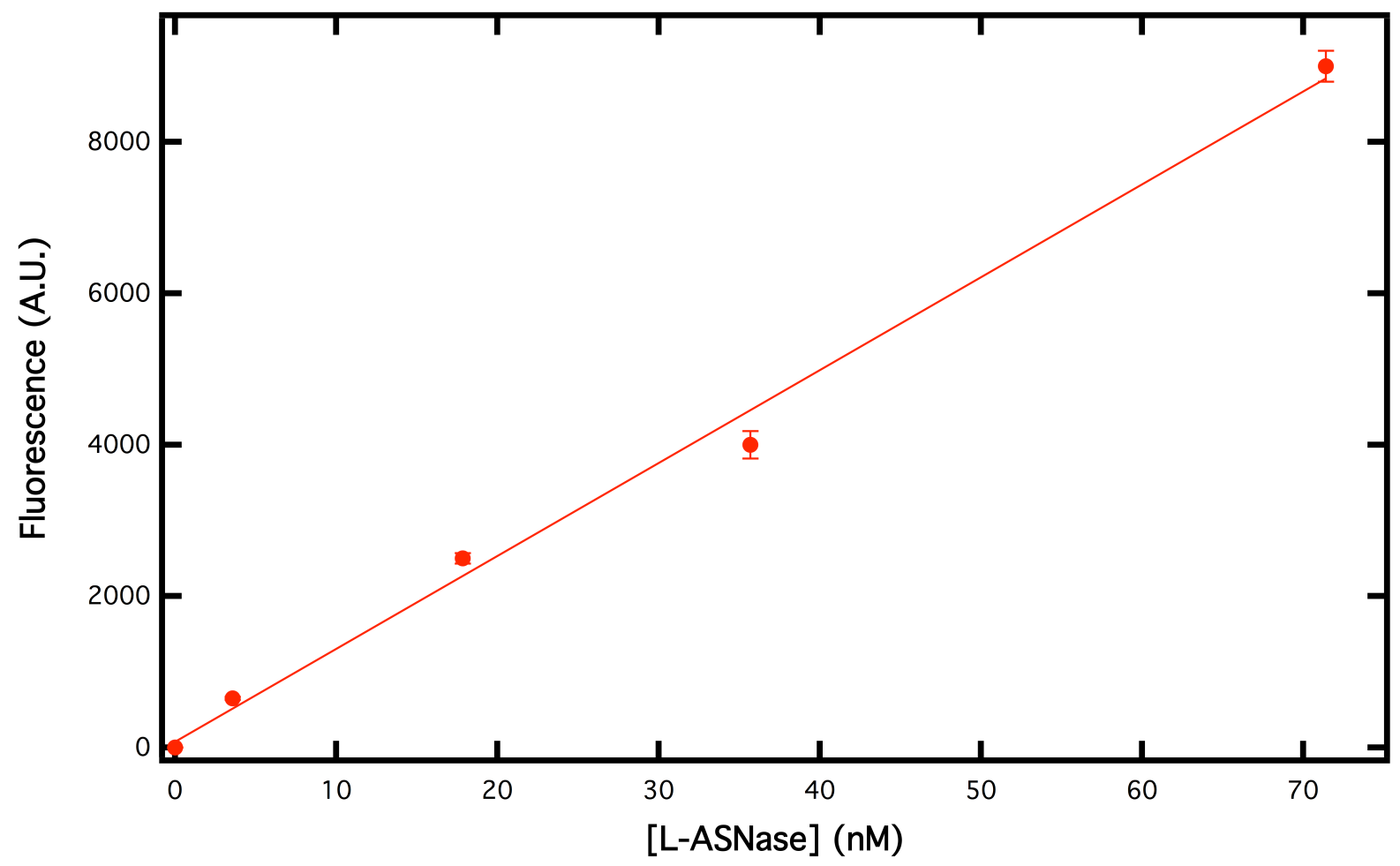

Figure S7. Enzyme-concentration response using human serum supplemented with LASNase. $200 \mu \mathrm{L}$ of human serum were spiked with $50 \mu \mathrm{g}$ L-ASNase previously diluted in PBS, and mixed thoroughly. Subsequently, aliquots were used to detect enzymatic activity in a final volume of $100 \mu \mathrm{L}$ using standardized conditions as described in the main text for the fluorescence mode, except from the following modifications of concentrations and settings: $100 \mu \mathrm{M}$ L-Asn and $500 \mu \mathrm{M}$ AR, Ex. bandwidth at $1 \mathrm{~nm}$, while Em. bandwidth was kept at $2.5 \mathrm{~nm}$. Fluorescence signals were corrected by subtracting the control values (no L-Asn) from all samples, ( $n=3, \pm S D$ ). 


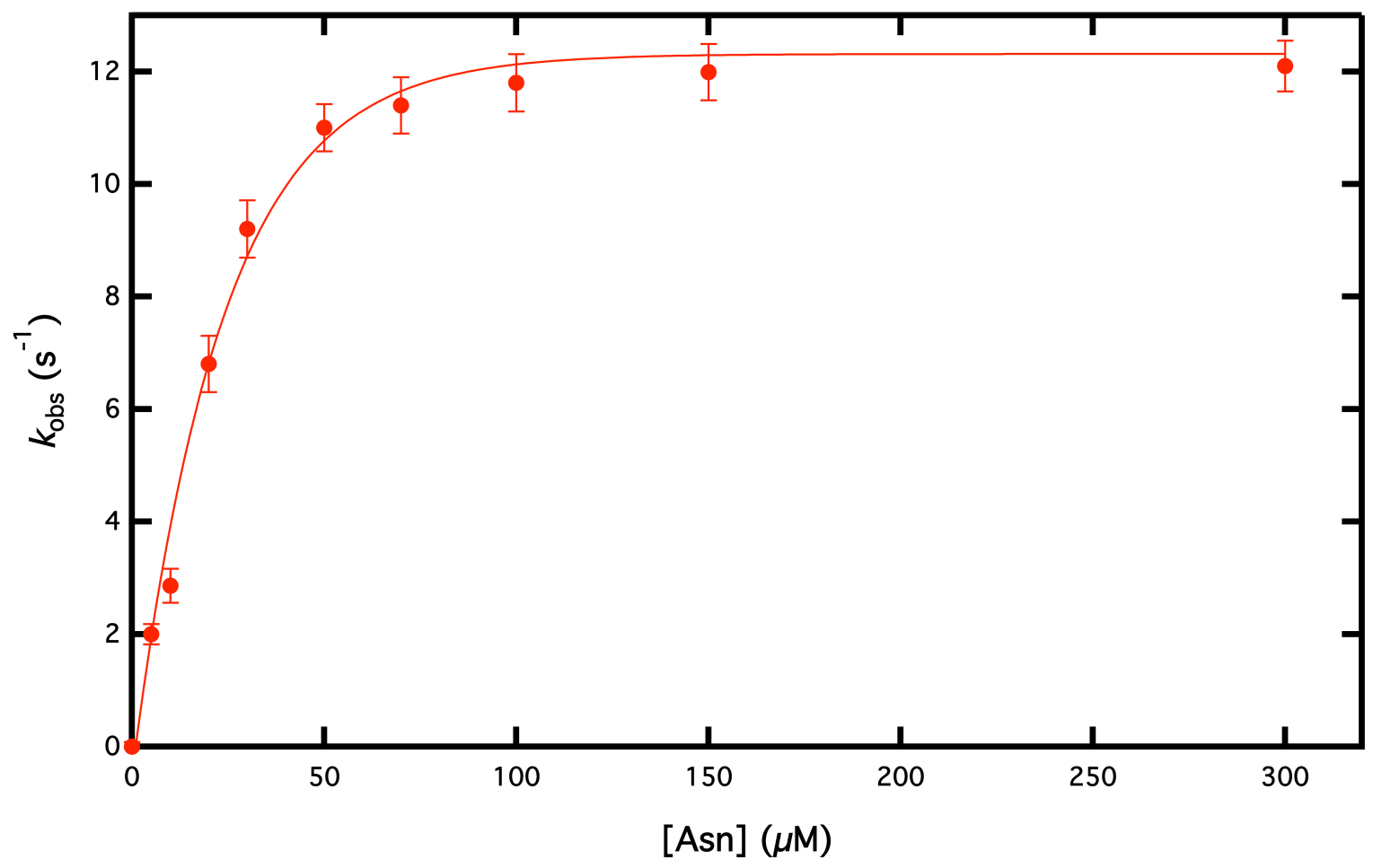

Figure S8. Steady-state kinetics of L-ASNase applying the NADH-dependent assay. Reactions were performed at $25^{\circ} \mathrm{C}$ in $1 \mathrm{ml}$ PBS, containing $0.25 \mathrm{mM} \mathrm{NADH}, 0.25 \mathrm{mM}$ a-ketoglutarate, $35 \mathrm{nM} \mathrm{GDH}$ and $7 \mathrm{nM}(\sim 250 \mathrm{ng} / \mathrm{ml})$ L-ASNase. Data points are represented as means of triplicate measurements, and error bars are standard deviations of three separate reactions. Kinetic constants were calculated by non-linear regression of the Michaelis-Menten equation (Igor Pro, Wavemetrics) and are shown in Table S1. 
Table S1. Steady state-kinetic parameters ( $n=3, \pm$ S.D.) for E.coli L-ASNase determined by applying the AR or the NADH-dependent assay.

\begin{tabular}{ccc}
\hline Kinetic parameter & Amplex Red assay & NADH assay \\
\hline$K_{m}$ & $22 \pm 1.2(\mu \mathrm{M})$ & $21 \pm 1.5(\mu \mathrm{M})$ \\
$k_{\mathrm{cat}}$ & $12.1 \pm 0.5 \mathrm{~s}^{-1}$ & $11.5 \pm 0.4 \mathrm{~s}^{-1}$ \\
$k_{\mathrm{ca}} / K_{\mathrm{m}}$ & $5.5 \times 10^{5} \mathrm{M}^{-1} \mathrm{~s}^{-1}$ & $5.4 \times 10^{5} \mathrm{M}^{-1} \mathrm{~s}^{-1}$ \\
\hline
\end{tabular}

\section{Z' factor determination}

In order to evaluate and validate the newly developed assay for L-ASNase, we determined the $Z^{\prime}$ factor [4] which is defined as follows:

$$
Z^{\prime}=1-\frac{\left(3 \sigma_{c+}+3 \sigma_{c-}\right)}{\left|\mu_{c+}-\mu_{c-}\right|}
$$

where $\mu_{c^{+}}$and $\mu_{c-}$ are the means of the positive and negative control values, respectively, and $\sigma_{\mathrm{c}^{+}}$and $\sigma_{\mathrm{c}-}$ are the standard deviations of the corresponding signals. The statistical parameters were calculated from six samples containing $10 \mu \mathrm{M} \mathrm{L-}$ asparagine for the positive control, and six ones which contained all components except from L-Asn for the negative control. The final reaction solution contained $3.5 \mu \mathrm{M} \mathrm{L-}$ 
AspOx, $10 \mu \mathrm{M}$ FAD, $100 \mathrm{nM}$ HRP, $50 \mu \mathrm{M}$ AR and $4.6 \mathrm{nM}$ L-ASNase ( $16 \mathrm{ng} / 0.1 \mathrm{ml})$.

The $Z^{\prime}$ factor was determined to be 0.77 suggesting that this is a very sensitive and reproducible assay.

\section{Some Notes and Tips}

1) L-AspOx, a monomeric enzyme, has been highly overexpressed recombinantly in E.coli. Instead of IPTG, one could use equally efficiently the cheaper "auto-induction" medium which contains lactose as inducer. We found similar expression yields (>40 $\mathrm{mg} / \mathrm{L}$ 2XYT medium) in both cases, using the pET-14b-SUMO vector. Such yields could be easily achieved not only when BL21(DE3) C41 was used as expression host, but also with other conventional E.coli strains such as BL21(DE3), BL21(DE3) pLysS, or BL21(DE3) Rosetta.

2) $A R$ is a light-sensitive dye, and therefore it is essential to avoid its exposure to light. We observed that the reaction mixture (without L-ASNase to trigger the reaction) remains stable, exhibiting a very low background fluorescence signal, for 3 hours at room temperature in dark Eppendorf tube.

3) FAD is a crucial co-factor for the activity of L-AspOx; it is non-covalently bound to the enzyme with 1:1 stoichiometry. However, it is not tightly bound $(K \sim 1 \mu \mathrm{M})$, and therefore a 3-fold molar excess of FAD provides a more stable environment for the activity of L-AspOx.

4) Fresh preparation of all buffers is advantageous, as this can diminish undesired background activity. A noteworthy example is the L-Asn stock solution. L-Asn, when stored for long periods even at $4{ }^{\circ} \mathrm{C}$, can spontaneously hydrolyze to L-Asp and ammonia. This would cause a considerably higher initial background activity, since the presence of L-Asp would initiate reaction steps two and three of the coupledenzyme system. Furthermore, in a series of experiments, we observed that $A R$ remained stable for 4 months when dissolved in DMSO at a final concentration of 50 $\mathrm{mM}$. 
5) For the purpose of detection of L-ASNase activity in serum, it is preferable to use saturated L-Asn concentrations (for E.coli L-ASNase $>80 \mu \mathrm{M}$ ) and consequently higher AR concentrations (five fold higher than L-Asn). This will decrease the background reaction and improve the signal-to-noise ratio.

\section{References}

[1] J. Nomme, Y. Su, M. Konrad, A. Lavie, Structures of Apo and Product-Bound Human L-Asparaginase: Insights into the Mechanism of Autoproteolysis and Substrate Hydrolysis, Biochemistry 51 (2012) 6816-6826.

[2] T. Panavas, C. Sanders, T. R. Butt, SUMO Fusion Technology for Enhanced Protein Production in Prokaryotic and Eukaryotic Expression Systems, Methods in Molecular Biology, SUMO Protocols 497 (2009) 303-317.

[3] G. Tedeschi, S. Ronchi, T. Simonic, C. Treu, A. Mattevi, A. Negri, Probing the active site of L-Aspartate oxidase by site-directed mutagenesis: Role of basic residues in fumarate reduction, Biochemistry 40 (2001) 4738-4744.

[4] J.H. Zhang, T.D.Y. Chung, K.R. Oldenburg, A simple statistical parameter for use in evaluation and validation of high throughput screening assays, J. Biomol. Screen. 67 (1999) 67-73. 


\section{Chapter 5. FACS-based high-throughput screening analysis of hASNase 3 mutant libraries and developent of a droplet-based high- throughput screening platform.}

The following Chapter 5 is presenting unpublished results regarding the FACSbased high-throughput screening of hASNase3 mutant libraries, as well as the development of a droplet-based microfluidic high-throughput screening system. The chapter is divided into two main parts:

Part 5.1 is focusing on the analysis of a number of hASNase3 mutant libraries using a FACS high-throughput screening platform. Catalytically improved hASNase3 variants were identified and further kinetically characterized. All the FACS measurements were done at the Structure and Dynamics of Mitochondria research group at the MPI-bpc (Prof. Dr. Stefan Jakobs). A manuscript is in preparation for submission to a peer-review Journal.

Part 5.2 describes the preliminary development of a novel droplet-based microfluidic highthroughput screening platform for the analysis of L-ASNase mutant libraries. This project is running in collaboration with Dr. Jean-Christophe Baret, head of the Droplets, Membranes and Interfaces Research Group at Max Planck Institute for Dynamics and Self-Organization. All the experiments and data analysis were performed by Christos S. Karamitros, Jiseok Lim (Postdoctoral researcher) and Philipp Gruner (Ph.D. student) with equal contribution. A manuscript is ready for submission to Journal for peer-review evaluation. 


\subsection{FACS-based screening of hASNase3 mutant libraries}

\subsubsection{Materials}

\subsubsection{Plasmids, E.coli strains, cDNA, and chemicals}

Plasmid pET-14b was purchased from Novagen. It has the pBR322 origin of replication, is medium copy number plasmid ( $\sim 30$ copies per cell) and carries the ampicillin antibiotic resistance marker ampR coding for the enzyme beta-lactamase (BLA). A modified version of the pET14b plasmid, designated pET14b-SUMO [38], was used to include the SUMO tag (derived from the Saccharomyces cerevisiae smt3 gene) between the N-terminal His 6 -tag and the multiple cloning site (MCS). The E.coli host strain C41 (DE3) was obtained from Lucigen/BioCat. The XL1-Blue and DH5 $\alpha$ strains were purchased from Stratagene and Invitrogen, respectively. Plasmid eGFP-pBAD33 was a kind gift from Dr. George Georgiou's lab (University of Texas at Austin, Department of Chemical Engineering and Institute for Cellular and Molecular Biology). pBAD33 is a medium copy number plasmid ( $\sim 25$ copies per cell) and has the chloramphenicol antibiotic resistance marker cmR coding for chloramphenicol acetyltransferase (CAT). The five-gene E.coli deletion strain, designated JC1(DE3), was also a kind gift from Dr. George Georgiou's lab. The deleted genes of this strain are the following: The L-asparaginase genes ansA (encoding L-ASNase1), ansB (encoding L-ASNase2), iaaA (encoding L-ASNase3), the aspartate aminotransferase gene aspC, and the tyrosine aminotransferase gene tyrB. The parental strain which was used for the chromosomal deletions was E.coli $\mathrm{MC} 1061$ having the following genotype $\left[\mathrm{F}^{-} \Delta\right.$ (ara-leu) 7697 [araD139] Br $_{\text {S }} \Delta$ (codB-lacI)3 galK16 galE15 $\lambda^{-}$e14- mcrA0 relA1 rpsL150(strR) spoT1 mcrB1 hsdR2 $\left.\left(\mathrm{r}^{-} \mathrm{m}^{+}\right)\right]$. All restriction and DNA-modifying enzymes and Taq polymerase were from New England Biolabs. Separate solutions of dNTPs (dATP, dCTP, dGTP and dTTP) were purchased from New England Biolabs. The oligonucleotides used in this study were obtained from IBA (Goettingen, Germany). Yeast extract, peptone, agar, ampicillin, chloramphenicol, isopropyl-beta-D-thiogalactoside (IPTG), arabinose and glucose were purchased from Applichem (Gatersleben, Germany). L-asparagine, flavine adenine dinucleotide (FAD), nicotinamide adenine dinucleotide (NADH), horseradish peroxidase (HRP type X, $386 \mathrm{U} / \mathrm{mg}, M_{r} \sim 44 \mathrm{kDa}$ ), glutamate dehydrogenase from bovine liver (GDH, 
$44 \mathrm{U} / \mathrm{mg}, M_{r} \sim 56 \mathrm{kDa}$ ), $\alpha$-ketoglutarate, L-aspartate, all other amino acids used for M9 minimal medium, and the SYPRO Orange dye were obtained from Sigma Aldrich (St. Louis MO, USA). Amplex Red (AR) was purchased from the Cayman Chemical Company (Michigan, USA). The gel filtration chromatography column Superdex 200 was from Pharmacia/GE Health Care Life Sciences (Uppsala Sweden). Coomasie Brilliant Blue G-250 (Bradford reagent) for protein assays was from Roth (Karlsruhe, Germany). All PCR reactions were performed with KAPA HiFi polymerase purchased from PeqLab (Erlangen, Germany). Gel extraction and PCR purification kits, and nickel resin for affinity purification of poly-histidine-tagged proteins was from Macherey Nagel (Düren, Germany), plasmid purification kits from Fermentas (Thermo Fisher Scientific, Germany), kits for genomic DNA preparation from Qiagen (Hilden, Germany). The hASNase3 open reading frame was amplified using as template cDNA from a human skin and meninges library (Source Bioscience, UK). All cloning and mutagenesis steps involving PCR amplifications were confirmed by sequencing (SeqLab, Goettingen, Germany) according to the company's instructions.

Table 1. Oligonucleotides used for the generation of hASNase 3 libraries. The degenerate codons are in bold, and the recognition sites for the restriction endonucleases are in italics (CATATG for NdeI, GGATCC for BamHI).

\begin{tabular}{|l|l|}
\hline \multicolumn{1}{|c|}{ Primer } & \multicolumn{1}{|c|}{ Sequence } \\
\hline FWhASNase3wt & GGAATTCCATATGAATCCCATCGTAGTGGTCCACGGCGGCGG \\
\hline RV $\_$hASNase3wt & CGCGGATCCTTAGGGAAGGTCGGTGATAGTAGTATCGTCAG \\
\hline FW_SSM-LIB1 & $\begin{array}{l}\text { GGAATTCCATATGAATCCCNNSNNSNNSNNSCACGGCGGCGGAG } \\
\text { CCGGTC }\end{array}$ \\
\hline RV: RV hASNase3wt & \\
\hline FW_SSM-LIB2 & TAATAAANNSNNSGGCCGCGTTGGGGACTCACCGNNSNNSGGA \\
\hline RV_SSM-LIB2 & $\begin{array}{l}\text { GCTCCSNNSNNCGGTGAGTCCCCAACGCGGCCSNNSNNTTTATT } \\
\text { A }\end{array}$ \\
\hline FW_SSM-LIB3 & ACCTCCACAGGCGGTNNSNNSAATAAAATGGTCGGCCGC \\
\hline RV_SSM-LIB3 & GCGGCCGACCATTTTATTSNNSNNACCGCCTGTGGAGGT \\
\hline FW_SSM-LIB4 & CTGGTGACAGAGNNSAACAAAAAGNNSCTGGAAAAAGAG \\
\hline RV_SSM-LIB4 & CTCTTTTTCCAGSNNCTTTTTGTTSNNCTCTGTCACCAG \\
\hline
\end{tabular}




\subsubsection{Methods}

\subsubsection{Cloning of hASNase3}

The ORF of hASNase3 was PCR-amplified using as template the full-length cDNA. The cloning process for this ORF was the following: NdeI and BamHI sites were incorporated at the 5'- and 3'-ends of the used oligonucleotides, respectively (primers are shown in Table 1). The PCR reaction mixture consisted of oligonucleotide mix (100 pmol each), KAPA high fidelity buffer, dNTPs (0.25 mM each), template DNA (10 ng), and KAPA HiFi DNA polymerase ( 1 unit). The reaction was initiated at $95{ }^{\circ} \mathrm{C}$ for $3 \mathrm{~min}$, followed by 25 cycles of denaturation at $98{ }^{\circ} \mathrm{C}$ for $20 \mathrm{~s}$, primer annealing at $60{ }^{\circ} \mathrm{C}$ for $30 \mathrm{~s}$, and extension at $72{ }^{\circ} \mathrm{C}$ for $30 \mathrm{~s}$. The amplification reaction was terminated after a $5 \mathrm{~min}$ polishing step at $72{ }^{\circ} \mathrm{C}$. The PCR product was gel-purified, digested with NdeI and BamHI H.F., purified with PCR cleanup kit, and ultimately ligated overnight at $16{ }^{\circ} \mathrm{C}$ into pET14b-SUMO vector (17 ng DNA fragment plus $50 \mathrm{ng}$ plasmid in a final volume of $20 \mu \mathrm{L}$ ) using T4 DNA ligase (400 units). The ligation mixture was used to transform DH5 $\alpha$ E.coli cells. Positive clones were identified by colony PCR screening using one primer specific for the vector (forward) and one primer specific for the insert (reverse), followed by restriction digestion with NdeI and BamHI H.F., and finally sequencing of the cloned DNA insert. The expressed protein construct includes an N-terminal 6-histidine $\left(\mathrm{His}_{6}\right)$ tag, followed by the yeast SUMO ( $\underline{\text { Small }}$ Ubiquitin $\underline{\text { MOdifier) }}$ tag which has proven to improve heterologous protein solubility and stability [261].

\subsubsection{Expression and purification of recombinant enzymes}

Recombinantly produced hASNase3 (wildtype and all mutants) were expressed and purified according to the following protocol (although their expression and purification are described in detail in certain publications, for the sake of consistency of the present unpublished data chapter, they are also included briefly here).

E.coli BL21(DE3) C41 cells containing the plasmid (pET14b-SUMO) with the gene of interest were cultured overnight at $37{ }^{\circ} \mathrm{C}$ in $2 x Y T$ medium supplemented with $200 \mu \mathrm{g} / \mathrm{ml}$ ampicillin. A fraction of this culture was used to inoculate fresh 2xYT culture (dilution 1:100) supplemented with $200 \mu \mathrm{g} / \mathrm{ml}$ ampicillin and $200 \mathrm{mM}$ glycine to accelerate the autoproteolytic processing of hASNase3. When O.D.600 reached $\sim 0.5-0.7$, the expression 
was induced by adding IPTG to a final concentration of $1 \mathrm{mM}$. After incubation at $37^{\circ} \mathrm{C}$ for 6 $\mathrm{h}$, the culture was centrifuged at 4,000g for $30 \mathrm{~min}$, the cells were harvested, resuspended in nickel agarose affinity matrix binding buffer $\left(50 \mathrm{mM} \mathrm{Na} 2 \mathrm{HPO}_{4}, 0.5 \mathrm{M} \mathrm{NaCl}, 10 \mathrm{mM}\right.$ imidazole, $\mathrm{pH}$ 8.0), and ultimately lysed by sonication. The cell lysate was centrifuged at $17,200 \mathrm{~g}$ for $45 \mathrm{~min}$, the resulting supernatant mixed with pre-equilibrated nickel agarose beads, and incubated at $4{ }^{\circ} \mathrm{C}$ for $3 \mathrm{~h}$ under rotation. Subsequently, the mixture was filled in a $5 \mathrm{~mL}$ polypropylene column and dried by gravity. The nickel resin was treated with 25 bed volumes of washing buffer ( $50 \mathrm{mM} \mathrm{Na} \mathrm{HPO}_{4}, 0.5 \mathrm{M} \mathrm{NaCl}, 20 \mathrm{mM}$ imidazole, $\mathrm{pH} 8.0$ ). Finally, the bound protein was eluted from the column by applying $300 \mathrm{mM}$ imidazole, and dropwise collection of fractions. All purification steps were performed at $4{ }^{\circ} \mathrm{C}$. The collected fractions were mixed and incubated with $500 \mathrm{mM}$ glycine for $4 \mathrm{~h}$ at $25^{\circ} \mathrm{C}$. Subsequently, the buffer was exchanged against $50 \mathrm{mM} \mathrm{Na} \mathrm{HPO}_{4}, 0.5 \mathrm{NaCl}$, pH 7.5 using a PD-10 column (GE). The eluted protein fraction was incubated with yeast SUMO protease (molar ratio protease:protein $\sim 1: 100$ ) at $30{ }^{\circ} \mathrm{C}$ for $2 \mathrm{~h}$ in order to cleave the $\mathrm{N}$-terminal 6-His-SUMO tag (alternatively, this was done overnight at $4{ }^{\circ} \mathrm{C}$ ). In a last purification step, the protein was subjected to size exclusion chromatography by passing it through a Superdex 200 column (size: $30 \times 1 \mathrm{~cm}$; Pharmacia/GE) to remove the cleaved tag and remaining impurities. Protein purity was evaluated by SDS-PAGE, the protein samples were aliquoted, mixed with $25 \%$ glycerol, and stored at $-20{ }^{\circ} \mathrm{C}$ until use. Protein concentration was determined by the Bradford method [262], and by using the molar extinction coefficient of hASNase $3(22,460$ $\mathrm{M}^{-1} \mathrm{~cm}^{-1}$ ) as calculated by the online proteomics tools provided by ExPASy (http:// www.expasy.org/). The two methods showed less than $15 \%$ difference, and ultimately the values were averaged.

\subsubsection{Assays for L-asparaginase activity determination and kinetic characterization of the enzyme}

In the present study, three different assays were employed for activity determination and kinetic characterization of L-ASNases: (A) Nesslerization, (B) NADH-dependent spectrophotometric assay, and (C) Amplex Red-dependent fluorescent assay. They are described in the following sections. 
(A) Nesslerization is called the process in which Nessler's reagent is used to detect free ammonium ions. Nessler's reagent is a yellowish solution of potassium tetraiodomercurate which turns into light orange to red (depending on the concentration of ammonia) when it binds to ammonia [263]. It is the most widely used L-ASNase assay mainly because of its simplicity. It is quite sensitive (limit of detection is $\sim 1 \mu \mathrm{M}$ of ammonia produced in $1 \mathrm{~mL}$ reaction volume as determined in the present study), and it can be used to determine LASNase activity in a quantitative manner applying end-point measurements. Briefly, the purified enzyme was incubated with different concentrations of the substrate L-asparagine either in buffer $50 \mathrm{mM} \mathrm{Na}_{2} \mathrm{HPO}_{4}, 300 \mathrm{mM} \mathrm{NaCl}, \mathrm{pH}$ 7.5, or $50 \mathrm{mM}$ Tris-Cl, $100 \mathrm{mM} \mathrm{NaCl}$, $\mathrm{pH} 8$ at $37{ }^{\circ} \mathrm{C}$ for $20 \mathrm{~min}$. In parallel, to detect ammonia generated in the absence of LASNase activity, a second identical sample was incubated at $37{ }^{\circ} \mathrm{C}$ for $20 \mathrm{~min}$, in which the enzymatic reaction was quenched at time zero by adding trichloroacetic acid (TCA) to a final concentration of $10 \%$. After TCA precipitation, both samples were centrifuged at 16,100 g for 2 min. The supernatant $(0.8 \mathrm{~mL})$ was mixed with Nessler's reagent $(0.2 \mathrm{~mL})$, and the absorbance was monitored spectrophotometrically at $\sim 440 \mathrm{~nm}$ after $5 \mathrm{~min}$ incubation at room temperature. The concentration of the enzymatically produced ammonia was calculated based on a standard curve generated with known concentrations of either ammonium sulphate or ammonium chloride. An enzymatic unit is defined as the amount of enzyme that catalyzes the conversion of $1 \mu$ mole of L-asparagine per minute under the aforementioned conditions. This assay was used in the present study only qualitatively, but not for determining steadystate kinetic properties of enzymes. Given the fact that it is colorimetric and can be performed very rapidly, it facilitates the determination of L-ASNase activities from enzyme fractions after purification steps.

(B) The NADH-dependent assay is a continuous spectrophotometric coupled-enzyme assay which monitors the conversion of $\alpha$-ketoglutarate plus ammonia to glutamate in a glutamate dehydrogenase (GDH)-coupled reaction [264]. The disappearance of NADH was monitored continuously at $340 \mathrm{~nm}$ and was directly proportional to the L-ASNase activity. For these measurements, a Jasco UV/VIS V-650 spectrophotometer was used. All enzymes used for the kinetic experiments were free of the His6-SUMO tag which was removed during the last size exclusion chromatographic step (see above). For steady-state kinetic analysis of all hASNase 3 species, L-Asn concentrations were varied in the range of $0-5 \mathrm{~K}_{M}$ in a final volume 
of $1 \mathrm{~mL}$ of $50 \mathrm{mM}$ Tris- $\mathrm{Cl}, 100 \mathrm{mM} \mathrm{NaCl}, \mathrm{pH}$. The final enzyme concentration was typically in the range of $1-1.5 \mu \mathrm{M}(\sim 30-50 \mu \mathrm{g}$ in $1 \mathrm{~mL})$. The obtained $\mathrm{V} / \mathrm{E}$ (velocity/total enzyme concentration) values were plotted against the respective substrate concentrations. The kinetic constants $\mathrm{K}_{\mathrm{M}}$ and $k_{\text {cat }}$ were calculated from the resulting plots by non-linear regression using the Michaelis-Menten model (equation 1) and analyzed by the SoftZymics software (Igor Pro, Wavemetrics):

$$
V=\frac{V_{\max }[S]}{K_{M}+[S]}
$$

(C) The Amplex Red-dependent fluorescent assay was used for the final screening step in 96-well plates of the selected mutants following the last FACS sorting round. Details for the assay development, and its validation in general, can be found in the respective publication [265]. The assay conditions using 96-well plates during the last screening step for the identification of active mutants included: $5 \mu \mathrm{M}(\sim 300 \mu \mathrm{g} / \mathrm{mL})$ L-Aspartate Oxidase (LAspOx), $10 \mu \mathrm{M}$ FAD, $100 \mathrm{nM}(0.1 \mathrm{U} / \mathrm{mL}) \mathrm{HRP}, 50 \mu \mathrm{M}$ Amplex Red, and $1 \mathrm{mM}$ L-Asn in a final volume of $50 \mu \mathrm{L}$ per well. The use of $1 \mathrm{mM}$ as final L-Asn concentration was chosen in order to select mutants stringently for improved $\mathrm{K}_{\mathrm{M}}$ given the fact that wildtype hASNase3 exhibits a $\mathrm{K}_{\mathrm{M}}$ value $\sim 3 \mathrm{mM}$. The reaction kinetics was monitored continuously for $20 \mathrm{~min}$ using a fluorescent plate reader (Molecular Devices, SpectraMax Paradigm, and filter settings Ex. 532, Em. 592).

\subsubsection{Generation of hASNase3 mutant libraries}

Two types of mutant libraries were generated and screened for the evolution of hASNase3. Initially, an error-prone PCR (epPCR) protocol was developed, and in addition four sitesaturation mutagenesis libraries were prepared as described below.

(A) Error-prone PCR library. The error rate of an epPCR depends on a number of parameters such as the \% GC content and the concentration of the gene which is used as template, the concentration of metal ions $\left(\mathrm{MgCl}_{2}\right.$ and $\left.\mathrm{MnCl}_{2}\right)$, dNTPs, the amount of Taq 
polymerase, and the number of reaction cycles [266]. The use of identical mutagenic conditions for two epPCRs for two different genes with distinct characteristics (different \% GC content, different lengths) might yield different final error rates. Therefore, the determination of the appropriate mutagenic conditions for individual genes is fundamental for achieving the desired error rates, rather than following epPCR schemes from protocols. It has been shown that the main mutagenic parameter in epPCR is the concentration of $\mathrm{MnCl}_{2}$ [267]. For the development of epPCR schemes for hASNase3, four different experimental conditions were tested using the concentrations shown in Table 2 (the different tested concentrations of $\mathrm{MnCl}_{2}$ are in bold-red):

Table 2. Different epPCR conditions tested for determining the error rates using as template the pET14b-SUMO plasmid.

\begin{tabular}{|c|c|c|}
\hline Compound & Stock Concentration & Final Concentration \\
\hline dATP & $100 \mathrm{mM}$ & $0.2 \mathrm{mM}$ \\
\hline dGTP & $100 \mathrm{mM}$ & $0.2 \mathrm{mM}$ \\
\hline $\mathrm{dCTP}$ & $100 \mathrm{mM}$ & $1 \mathrm{mM}$ \\
\hline dTTP & $100 \mathrm{mM}$ & $1 \mathrm{mM}$ \\
\hline $\mathrm{MgCl}_{2}$ & $25 \mathrm{mM}$ & $7 \mathrm{mM}$ \\
\hline $\mathrm{MnCl}_{2}$ & $5 \mathrm{mM}$ & $0.01,0.05,0.2,0.5 \mathrm{mM}$ \\
\hline BSA & $10 \mathrm{mg} / \mathrm{mL}$ & $5 \mu \mathrm{g} / \mathrm{mL}$ \\
\hline Template DNA & $100 \mathrm{ng} / \mu \mathrm{L}$ & $\begin{array}{l}50 \mathrm{nM} \text { (calculated based on } \\
\text { the ORF size of hASNase } 3 \text { ) }\end{array}$ \\
\hline Primers & $0.1 \mathrm{nmol} / \mu \mathrm{L}$ & $0.03 \mathrm{nmol} / \mu \mathrm{L}$ \\
\hline Taq buffer & $10 X$ & $1 \mathrm{X}$ \\
\hline Taq Polymerase & $5,000 \mathrm{U} / \mathrm{mL}$ & 2.5 Units \\
\hline Final volume & & $50 \mu \mathrm{L}$ \\
\hline
\end{tabular}

The parameters were identical for all the reactions: Initial denaturation step at $95^{\circ} \mathrm{C}$ for $30 \mathrm{~s}$, followed by 30 cycles of $95{ }^{\circ} \mathrm{C}$ for $30 \mathrm{~s}, 55^{\circ} \mathrm{C}$ for $30 \mathrm{~s}, 68^{\circ} \mathrm{C} 90 \mathrm{~s}$. The reaction was stopped after a 5 min polishing step at $68^{\circ} \mathrm{C}$. The amplicons were agarose-gel purified, digested with 
restriction enzymes (NdeI and BamHI H.F.), PCR-purified and ligated into the pET14bSUMO vector overnight at $16{ }^{\circ} \mathrm{C}$ using T4 DNA ligase and T4 DNA ligase buffer using a molar ratio of 1:3 (vector:insert). In parallel, the same amount of digested plasmid was mixed with T4 DNA ligase and T4 DNA ligase buffer, but without insert in order to determine the background of the ligation reaction; this sample served as negative control.

The ligation mixtures were made salt-free by using a PCR-clean-up kit, and finally $\sim 10 \mathrm{ng}$ of each were used to transform $50 \mu \mathrm{L}$ electro-competent DH5 $\alpha$ E.coli cells using pre-chilled 1mm gap Bio-Rad cuvettes. A Gene Pulser instrument (Bio-Rad electroporation device) was used to apply a pulse on the cuvettes containing the transformation mixtures (cells and ligation reactions) with the following settings: $25 \mu \mathrm{F}, 1.5 \mathrm{kV}, 200 \Omega$. Subsequently, $1 \mathrm{~mL}$ of SOC medium (Super Optimal broth with Catabolite repression; $2 \% \mathrm{w} / \mathrm{v}$ tryptone, $0.5 \% \mathrm{w} / \mathrm{v}$ yeast extract, $10 \mathrm{mM} \mathrm{NaCl}, 2.5 \mathrm{mM} \mathrm{KCl}, 10 \mathrm{mM} \mathrm{MgCl}_{2}, 20 \mathrm{mM}$ glucose) was added to the transformed cells and transferred to a $10-\mathrm{mL}$ Falcon tube. With an additional $1 \mathrm{~mL}$ SOC medium, the electroporation cuvette was rinsed to remove any remaining cells and was combined with the rest of the medium. Cells were grown at $37{ }^{\circ} \mathrm{C}$ under vigorous shaking (250 rpm), and ultimately were plated onto ampicillin plates. The plates were placed at $30{ }^{\circ} \mathrm{C}$ overnight. The next day, the transformation efficiency was determined based on the number of the obtained individual colonies in relation to the used DNA for transformation. The average transformation efficiency was between $2 \times 10^{7}$ and $5 \times 10^{7}$ per $\mu$ g of plasmid DNA. In cases where the background ligation reaction of the negative control (without insert) accounted for $>5 \%$ of the positive control, the plates were discarded and the process was reinitiated from the cloning level. For the determination of the final error rate, 15 colonies of each plate resulting from different mutagenic conditions were cultured overnight at $37^{\circ} \mathrm{C}$ in 5 mL 2xYT medium supplemented with $200 \mu \mathrm{g} / \mathrm{mL}$ ampicillin. Plasmids were extracted using plasmid extraction kit, and an aliquot of about $1 \mu \mathrm{g}$ of plasmid DNA was digested with NdeI/ BamHI H.F. to determine the presence of insert. Usually, more than 13 out of 15 colonies contained the insert. Plasmids from 10 colonies were sequenced, and based on the sequencing results the error rates were determined, serving as an indicator of the quality of the library.

Cells were scraped from the original plates and resuspended in 2xYT medium supplemented with $200 \mu \mathrm{g} / \mathrm{mL}$ ampicillin. A fraction of this suspension was used to inoculate $0.5 \mathrm{~L}$ of 2xYT medium; the remaining cells were pooled and stored at $-80{ }^{\circ} \mathrm{C}$ as glycerol stocks. After overnight growth at $30{ }^{\circ} \mathrm{C}$, the $0.5 \mathrm{~L}$ culture was centrifuged, and the plasmid DNA was 
extracted using a MIDI-PREP kit. Approximately $100 \mathrm{ng}$ of the plasmid mutant library (pET14b-SUMO-hASNase3) were used to transform electro-competent cells of the JC1(DE3) five-gene-deletion strain which already harbors the pBAD33-eGFP plasmid (chloramphenicol resistance). After the transformation, the cells were cultured in $0.5 \mathrm{~L} 2 \mathrm{xYT}$ medium supplemented with $200 \mu \mathrm{g} / \mathrm{mL}$ ampicillin and $35 \mu \mathrm{g} / \mathrm{mL}$ chloramphenicol. When $\mathrm{OD}_{600}$ reached $\sim 2,0.5 \mathrm{~mL}$ aliquots were pooled and stored at $-80{ }^{\circ} \mathrm{C}$ as glycerol stocks. For subsequent screening, a $0.5 \mathrm{~mL}$ aliquot was used to inoculate a culture of $2 \mathrm{xYT}$ medium as described in the next section where the screening process is discussed.

(B) Site-saturation mutagenesis. Site-Saturation-Mutagenesis (SSM) constructs were generated and screened for catalytically improved hASNase3 variants. The libraries were designed using as template the crystal structure model of hASNase3 (PDB entry: 4OSY) and based on a massive amino acid sequence alignment of more than 1000 homologous enzymes from different organisms. Figure 1 shows the first ten consensus residues of this alignment (NPVIAIHGG), highlighting in a red frame the, critical for intramolecular processing and catalytic activity, highly conserved triad His8-Gly9-Gly10 (counting starts from Met1). The alignment served as tool to determine highly conserved residues in the hASNase 3 sequence, thus avoiding mutating them since they could play a pivotal role in the enzyme's activity and stability. Amino acid residues localized around the first, second and third shell of the active site (first shell: residues with $\sim 1-5 \AA$ distance from the active site; second shell: 5-12 $\AA$, and third shell $>12 \AA$ A) were identified and their codons were randomized using degenerate oligonucleotides (Table 1) following the NNS scheme (N: A, T, G, C; S: G, C). For randomization, the NNS-type of codons was chosen in order to minimize the probability of generating stop-codons. This combination of nucleotides allows all the twenty possible amino acids to be encoded, but only one stop-codon (UAG). The residues which were randomized for each library are shown in Figures 2 to 5. Each library is denoted with the abbreviation SSM-LIB (Site-Saturation-Mutagenesis Library). In addition, an image with highlighted residues of the wildtype enzyme's active site is shown for direct comparison with the residues which were mutated. 


\section{Wildtype hASNase3}

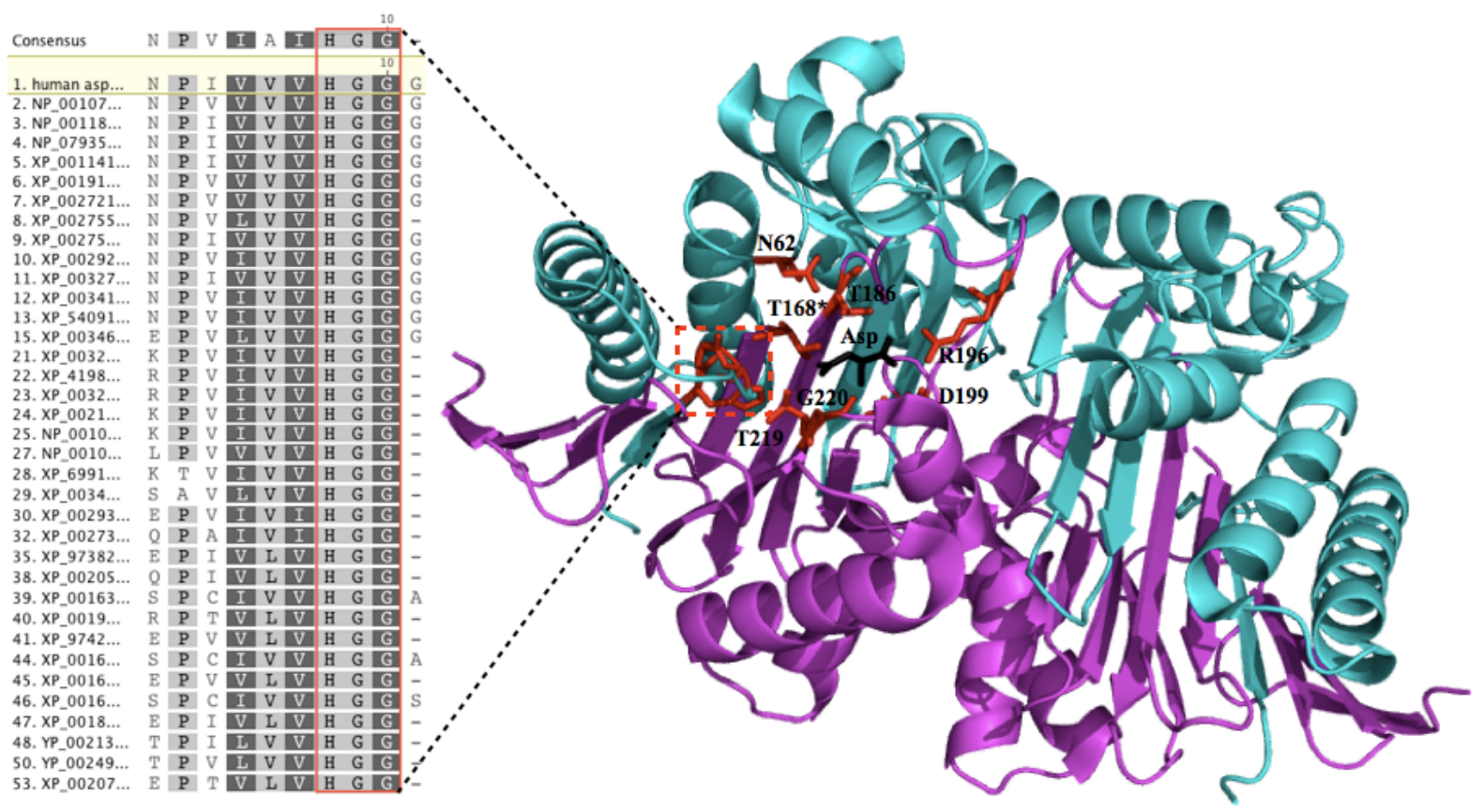

Figure 1. Structure of wildtype hASNase3. With cyan is colored the $\alpha$ subunit and with magenta the $\beta$ subunit. Black-labeled is the product of the enzymatic reaction L-Asp. Redlabeled are the residues which constitute the catalytic centre of the enzyme and are directly involved in the substrate binding. These are: Asn62, Thr186, Arg196, Asp199, Thr219, Gly220, and the catalytic threonine Thr168* which plays the role of the nucleophile (as discussed in the Introduction). In the figure is also shown the alignment of the homologous enzymes from different organisms (1000 in total, here are shown only 50). The first line corresponds to hASNase 3 (number 1), and on the top of the alignment the consensus residues for each amino acid position are shown. In the structure, three amino acids are framed in a red square and represent the amino acid triad His8-Gly9-Gly10. Structural analysis [36-38] has shown that this triad is crucial for the enzyme's autoproteolytic activation and catalysis, and based on the alignment comparison, it is evident that these residues are highly conserved among all homologs from different organisms. The first library was constructed by randomizing residues close to this conserved triad, as shown in the following figure 2 . The alignment was done using the Geneious program, the structural representation was prepared by PyMol, and the whole figure was generated using Keynote. This also applies to all the following figures 2-5. 


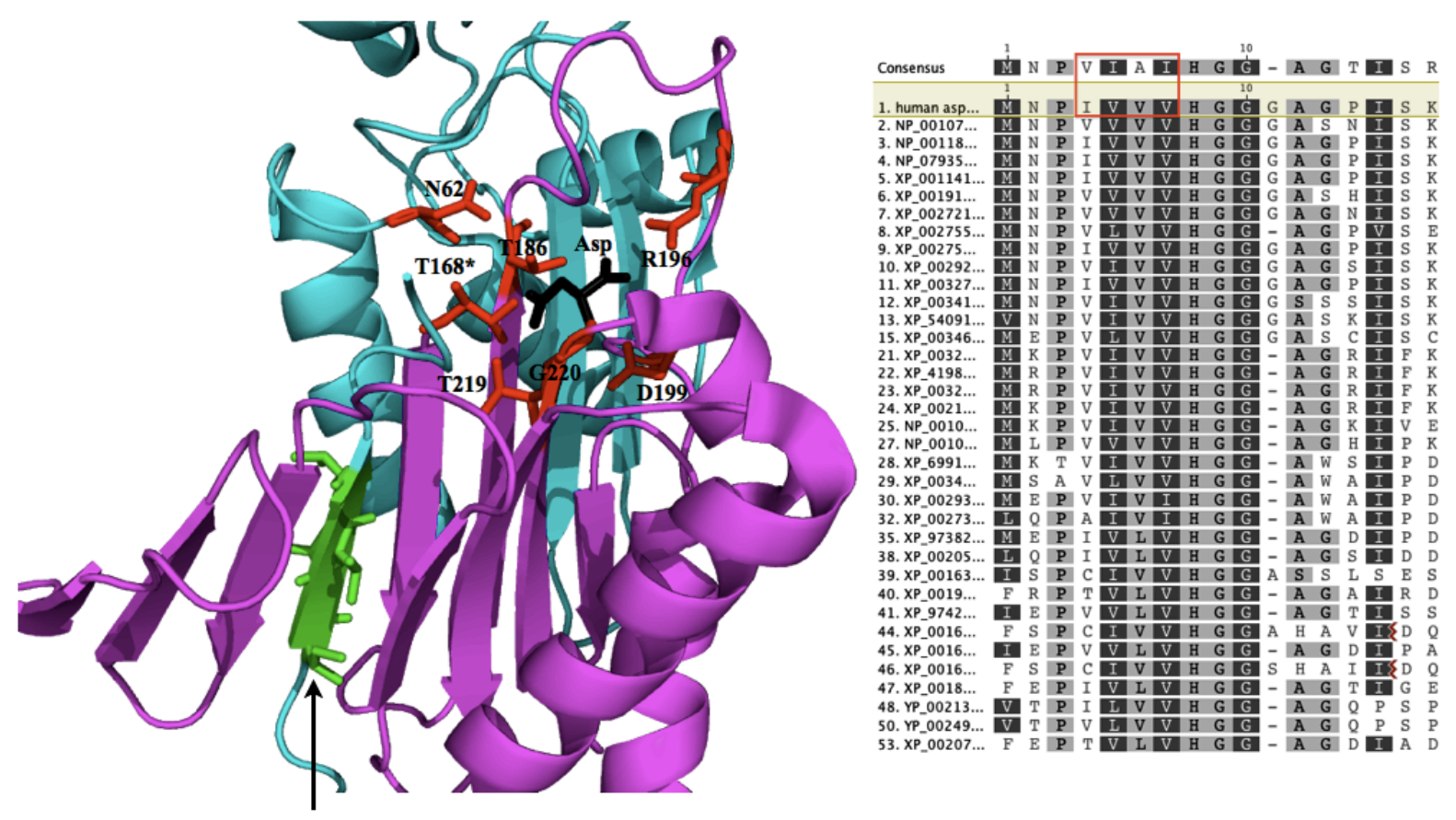

Figure 2. Randomized residues of the SSM-LIB1. The residues which were randomized are green-labeled and are indicated by a black arrow. These are Ile4, Val5, Val6, Val7 and correspond to the four amino acids preceding the critical triad HGG as shown in the alignment at the right of the figure. The distance between the mutated residues and the centre of the active site is $\sim 17 \AA$ as calculated by PyMol. 


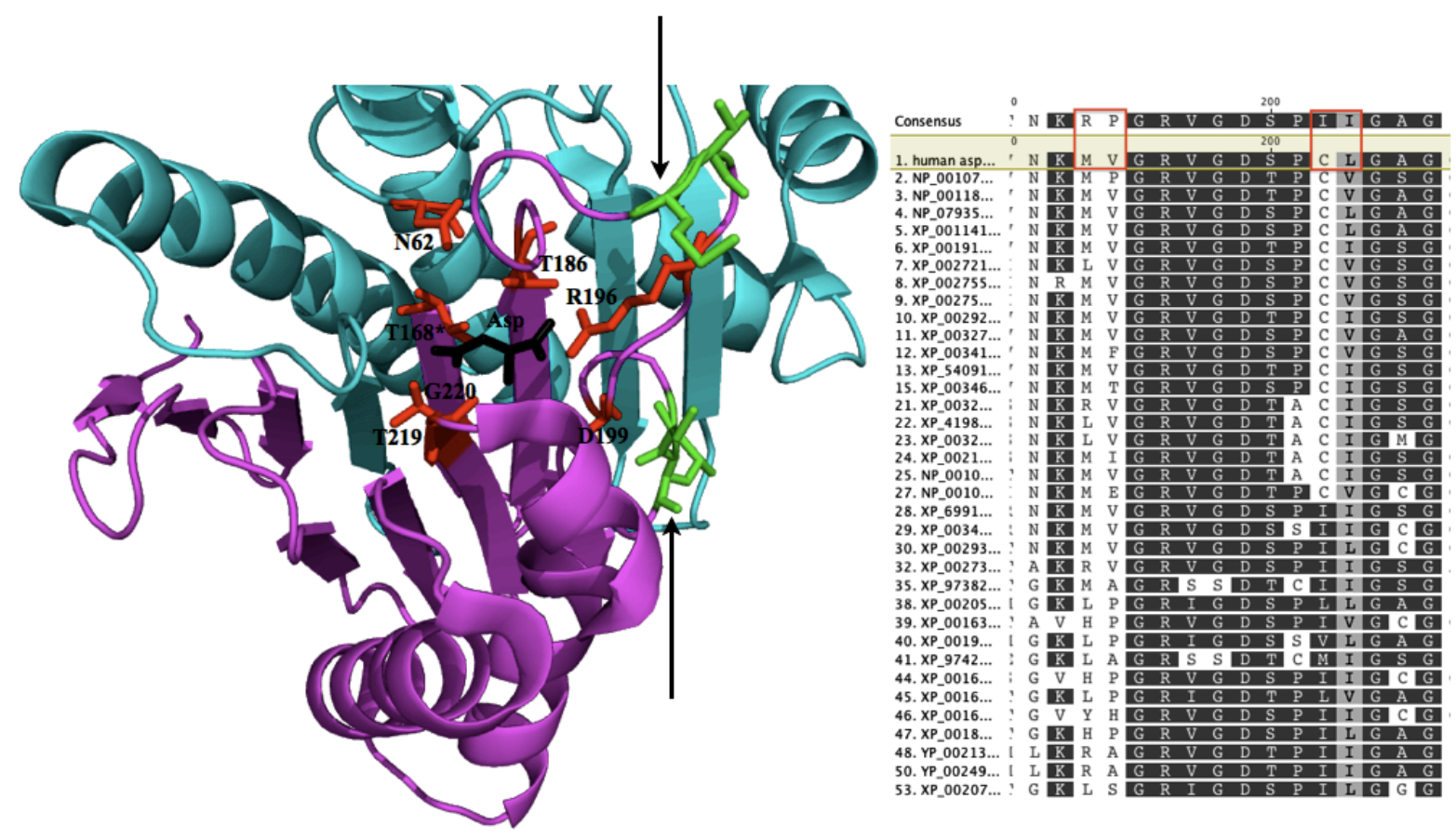

Figure 3. Randomized residues of the SSM-LIB2. The residues which were randomized are green-labeled and are indicated by two black arrows. These are Met193, Val194, Cys202, Leu203, which are not highly conserved as shown in the alignment at the right of the figure. The distance between the Met193-Val194 and the Cys202-Leu203 tandem residue pairs and the centre of the active site is $\sim 14$ and $15 \AA$, respectively, as calculated by PyMol. 

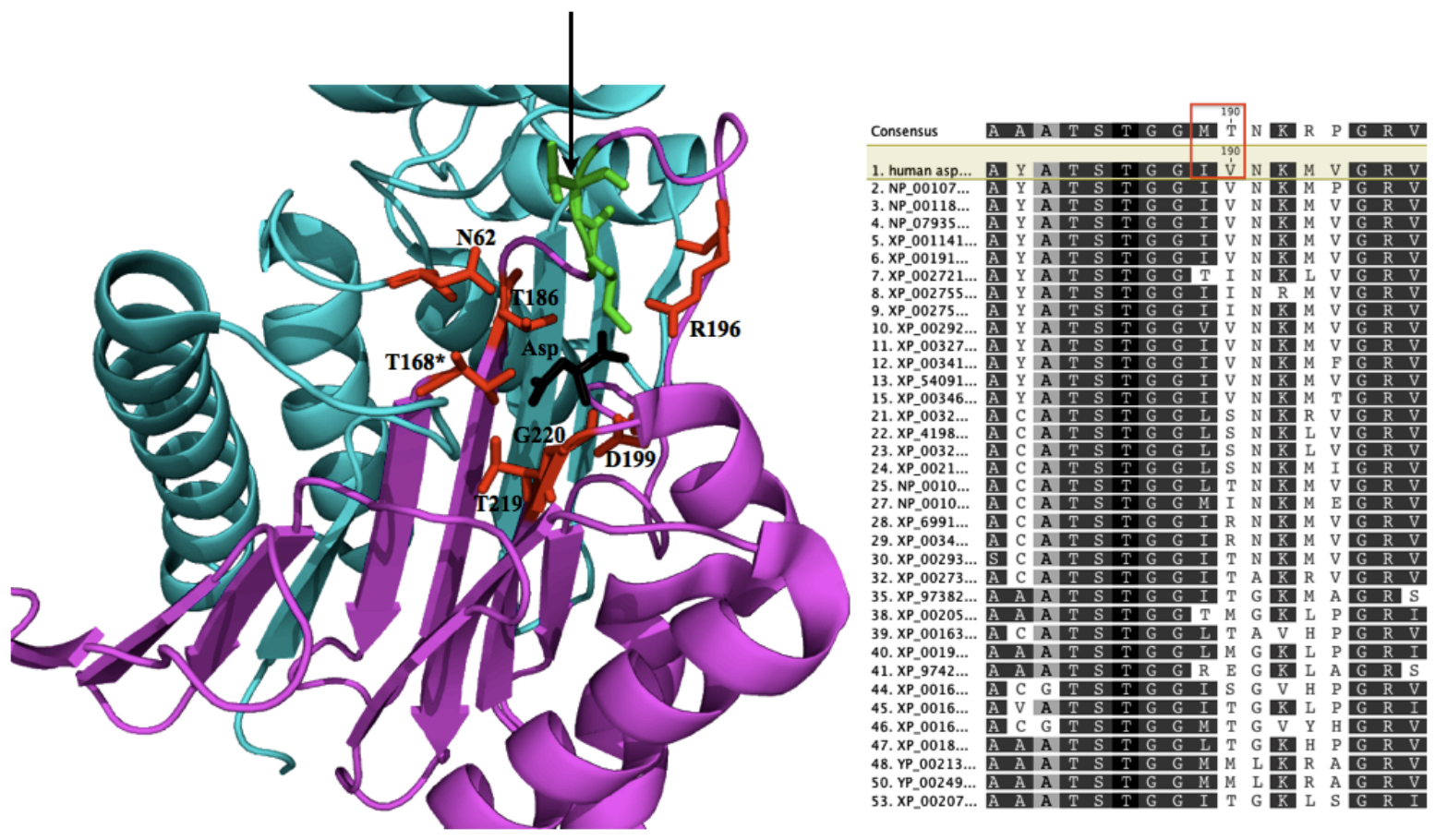

Figure 4. Randomized residues of the SSM-LIB3. The amino acids which were randomized are green-labeled and are indicated by a black arrow. These are Ile189, Val190, which are not highly conserved as shown in the alignment at the right of the figure. The distance between the mutated residues and the centre of the active site is $\sim 5 \AA$ as calculated by PyMol. 


\section{SSM-LIB4}

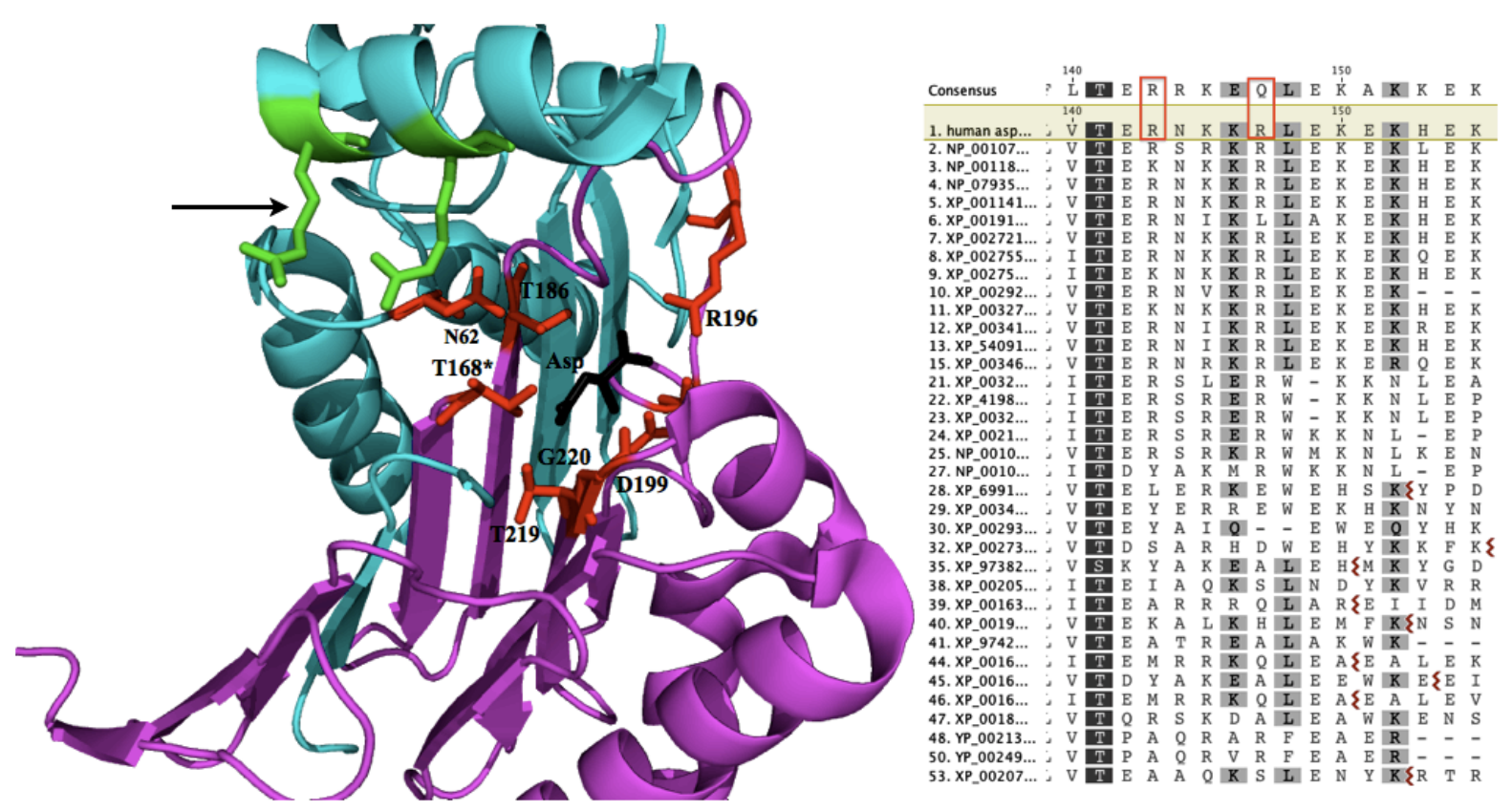

Figure 5. Randomized residues of the SSM-LIB4. The amino acids which were mutated are green-labeled and are indicated by a black arrow. These are Arg143, Arg147, which are not highly conserved as shown in the alignment at the right of the figure. The distance between Arg143 and Arg147 and the centre of the active site is $\sim 17$ and $14 \AA$, respectively, as calculated by PyMol.

The libraries described above were generated applying the overlap extension PCR methodology [268] which is depicted in Figure 6 below. 


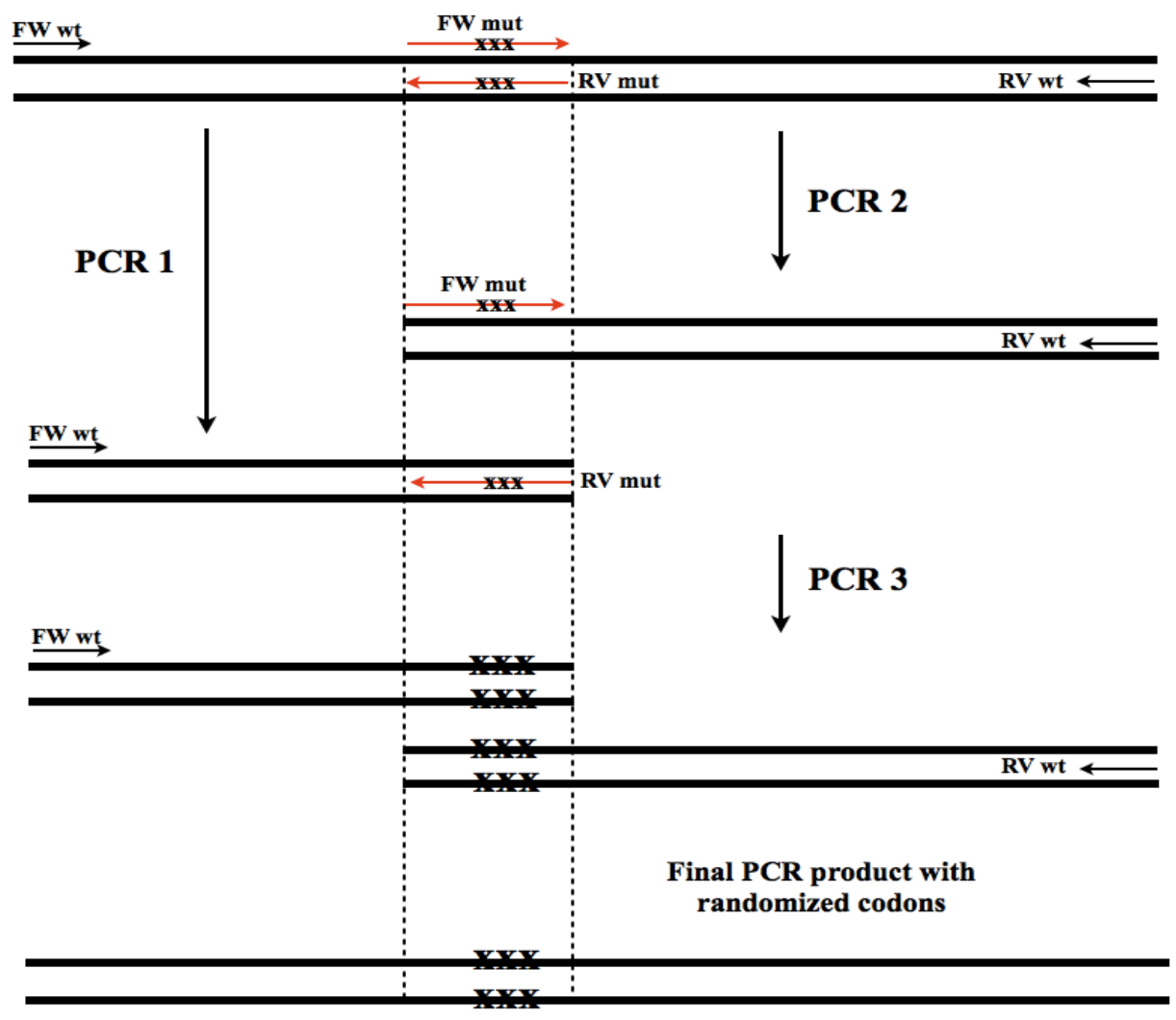

Figure 6. Overlap extension PCR method. This method consists of three successive amplification steps and involves four primers: two external ones which cover the 5'- and 3'ends of the wildtype sequence (designated as FW wt and RV wt, respectively), and two additional ones which carry the desirable degenerate codons (NNS) to be incorporated in the final sequence (indicated by red arrows in the figure; $\mathrm{xxx}$ denotes the nucleotide mismatches). Two independent PCR reactions (PCR 1 and PCR 2) amplify two fragments which overlap at the regions around the mismatches. In a final third step, the two amplified fragments are combined in equal-molar quantities with the initial external wildtype primers and are subjected to the last PCR reaction resulting in the final amplicon carrying the degenerated codons, or point mutations. The number of nucleotides upstream and downstream of the mismatch codons is very critical for the success of this technique, since upon those overhangs relies the annealing of the long fragments generated during the first two PCRs. This number should be at least fifteen nucleotides or longer depending on the number of codons which are randomized. Figure was prepared using Keynote.

The PCRs for the construction of the four SSM-LIBs described above were run in a final volume of $50 \mu \mathrm{L}$ and included: $10 \mathrm{ng}$ of plasmid DNA template (pET14b-SUMOhASNase3), 100 pmoles of each primer, 1X KAPA HiFi buffer, $0.5 \mathrm{mM}$ dNTPs, and 1 Unit KAPA-HiFi DNA polymerase. In the first two PCRs, the reactions were initiated at $95^{\circ} \mathrm{C}$ for 3 min, followed by 30 cycles of denaturation at $98{ }^{\circ} \mathrm{C}$ for $20 \mathrm{~s}$, primer annealing at $60{ }^{\circ} \mathrm{C}$ for 30 $\mathrm{s}$, and extension at $72{ }^{\circ} \mathrm{C}$ for $40 \mathrm{~s}$. The amplification reactions were terminated after a 5-min 
polishing step at $72{ }^{\circ} \mathrm{C}$. The PCR products were gel-purified, and then mixed in equal-molar amounts for the final overlap extension PCR using the external FW wt and RV wt primers. The reaction parameters were identical to the ones described above, except from the annealing step which was done at $65{ }^{\circ} \mathrm{C}$, and the overall cycles were 25 . The final PCR product was gel-purified, digested with NdeI and BamHI H.F., purified with PCR clean-up kit and then ligated overnight at $16{ }^{\circ} \mathrm{C}$ into the pET14b-SUMO vector using T4 DNA ligase (molar ratio 1:3; vector:insert). Quality control of the libraries was done as described above for the epPCR library. Briefly, the ligation mixture was used to transform electrocompetent DH5 $\alpha$ E.coli cells which were streaked onto ampicillin-containing 2 XYT plates and placed at $30{ }^{\circ} \mathrm{C}$ overnight. Positive clones were determined following restriction digestion with NdeI and BamHI H.F., and finally sequencing of the cloned DNA insert to verify proper randomization of the codons at the desired sites.

In case of successful library construction, the cells were scraped from the original plates and resuspended in 2xYT medium supplemented with $200 \mu \mathrm{g} / \mathrm{mL}$ ampicillin. A fraction of this suspension was used to culture $0.5 \mathrm{~L}$ of $2 \mathrm{xYT}$ medium, and the rest cells were pooled and stored at $-80{ }^{\circ} \mathrm{C}$ as glycerol stocks. Following overnight growth at $30^{\circ} \mathrm{C}$, the $0.5 \mathrm{~L}$ culture was centrifuged, and the plasmid DNA was extracted using a MIDI-PREP kit. Approximately 100 ng of the plasmid mutant library (pET14b-SUMO-hASNase3) were used to transform the electro-competent JC1(DE3) five-gene-deletion strain which already harbors the pBAD33eGFP plasmid (chloramphenicol resistance). The transformed cells were cultured in $0.5 \mathrm{~L}$ 2xYT medium supplemented with $200 \mu \mathrm{g} / \mathrm{mL}$ ampicillin and $35 \mu \mathrm{g} / \mathrm{mL}$ chloramphenicol. When $\mathrm{OD}_{600}$ reached $\sim 2,0.5 \mathrm{~mL}$, aliquots were pooled and stored at $-80{ }^{\circ} \mathrm{C}$ as glycerol stocks. For subsequent screening, a $0.5 \mathrm{~mL}$ aliquot was used to inoculate a $50 \mathrm{~mL}$ culture of $2 \mathrm{xYT}$ medium as described in the next section where the screening process is discussed.

\subsubsection{Screening steps of hASNase3 mutant libraries}

(A) Validation of mutant libraries using the JC1(DE3) five-gene-deletion strain. To validate the bacterial screening strategy, the genetic complementation of five different L-ASNases (hASNase3, hASNase1, EcASNase1, EcASNase2, and ScASNase1) using the JC1(DE3) strain was investigated before initiating the main screening process for hASNase 3 libraries. All the genes were cloned into the pET14b-SUMO plasmid, which was used to transform 
chemically competent JC1(DE3) E.coli cells. The transformants were cultured at $37^{\circ} \mathrm{C}$ for $1 \mathrm{~h}$ and ultimately were plated onto M9 minimal plates supplemented with $0.4 \%$ glucose, $3.5 \mu \mathrm{g} /$ $\mathrm{mL}$ thiamine, $1 \mathrm{mM} \mathrm{MgSO} 4,0.1 \mathrm{mM} \mathrm{CaCl}_{2}, 160 \mu \mathrm{g} / \mathrm{mL}$ of L-Tyr, and $80 \mu \mathrm{g} / \mathrm{mL}$ of the remaining 18 amino acids except from L-Asp, $1 \mathrm{mM}$ IPTG and $200 \mu \mathrm{g} / \mathrm{mL}$ ampicillin. In parallel, cells transformed with empty plasmid (no L-ASNase insert) served as negative control. Furthermore, cells transformed with each of the L-ASNases mentioned above were plated onto M9 minimal plates supplemented with L-Asp: These transformants served as positive control in order to evaluate the growth of the cells in the presence of all amino acids and contrast the growth in case of the absence of L-Asp. The principle of this screening strategy relies on the impaired genetic ability of the JC1(DE3) E.coli strain to synthesize LAsp since all genes involve in L-Asp biosynthesis are deleted. Therefore, the survival of the cells and the formation of colonies solely depend on the availability of L-Asp generated by the encoded L-ASNases. Figure 7 schematically represents the principle of this screening system. The same process was repeated with liquid culture (M9 medium lacking L-Asp) and subsequent FACS analysis of JC1(DE3) cells harboring hASNase3 and EcASNase2 (the most active among the L-ASNases) and pBAD33-eGFP plasmids, in order to correlate quantitatively the intracellular fluorescent signal with the distinct enzymatic activities of hASNase3 and EcASNase2.

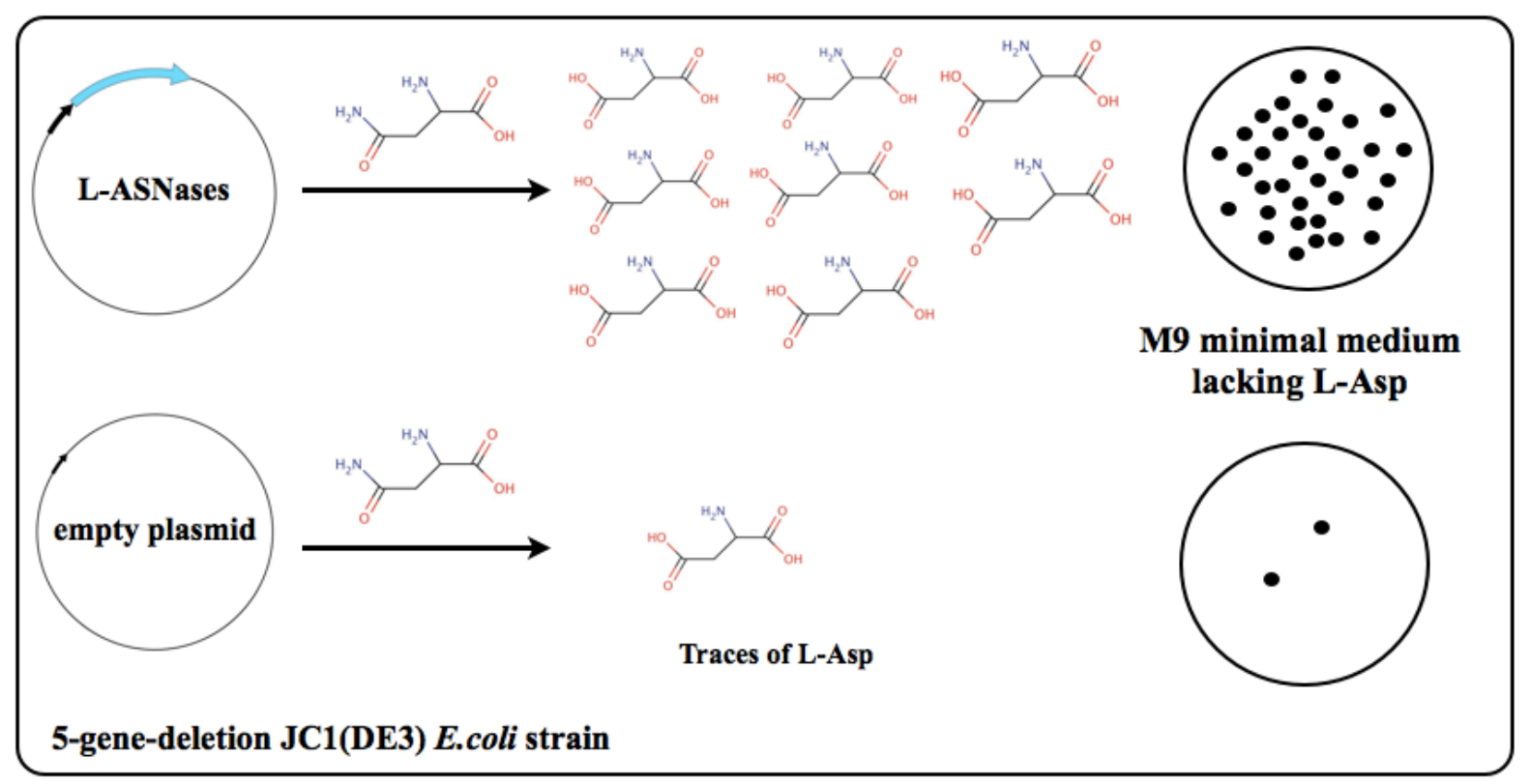

Figure 7. Principle of the genetic complementation screening system. The expressed LASNases provide the free amino acid L-Asp essential for growth of the E.coli cells, resulting 
in the formation of many colonies. On the contrary, the absence of L-ASNase leads to cell death due to unavailability of L-Asp and protein synthesis inhibition. Figure was generated using XPlasMap for the plasmids, MarvinSketch for the L-Asn and L-Asp molecules and Keynote for the final layout (the following Figure 8 was prepared in a similar way).

(B) FACS-based screening of hASNase3 mutant libraries. Frozen aliquots of JC1(DE3) cells co-transformed with either an epPCR or an SSM library and pBAD-eGFP plasmids were used to inoculate $50 \mathrm{~mL} 2 \mathrm{xYT}$ cultures supplemented with $0.4 \%$ glucose, $200 \mu \mathrm{g} / \mathrm{mL}$ ampicillin, $35 \mu \mathrm{g} / \mathrm{mL}$ chloramphenicol, and $50 \mathrm{mM}$ glycine (which induces the intramolecular activation of hASNase3); the starting $\mathrm{OD}_{600}$ was $\sim 0.1$. The cells were grown at $37{ }^{\circ} \mathrm{C}$, and when $\mathrm{OD}_{600}$ reached 1 , the cells were centrifuged for $5 \mathrm{~min}$ at $8,000 \mathrm{~g}$ at $4{ }^{\circ} \mathrm{C}$ and washed $3 \mathrm{X}$ with ice-cold $0.9 \% \mathrm{NaCl}$. Subsequently, the cells were resuspended in M9 minimal medium containing $1 \%$ glycerol, $3.5 \mu \mathrm{g} / \mathrm{mL}$ thiamine, $1 \mathrm{mM} \mathrm{MgSO}_{4}, 0.1 \mathrm{mM} \mathrm{CaCl}_{2}$, $160 \mu \mathrm{g} / \mathrm{mL}$ of L-Tyr, $5 \mathrm{mM} \mathrm{L}-\mathrm{Asn}$, and $80 \mu \mathrm{g} / \mathrm{mL}$ of the remaining 18 amino acids except from L-Asp, $200 \mu \mathrm{g} / \mathrm{mL}$ ampicillin, $35 \mu \mathrm{g} / \mathrm{mL}$ chloramphenicol, $1 \mathrm{mM}$ IPTG, 2\% arabinose and $50 \mathrm{mM}$ glycine. The cultures were placed at $37^{\circ} \mathrm{C}$ under shaking conditions $(250 \mathrm{rpm})$ for $2 \mathrm{~h}$. Next, the cells were centrifuged $5 \mathrm{~min}$ at $8,000 \mathrm{~g}$ at $4{ }^{\circ} \mathrm{C}$ and washed twice with ice-cold PBS. Ultimately they were resuspended in PBS solution at a final $\mathrm{OD}_{600}$ of 0.05 for subsequent FACS analysis.

Flow cytometric analyses were performed with a BD Biosciences Influx FACS instrument using a 488-nm solid-state laser for excitation and a 495-525 bandpass filter for detection. The cells corresponding to the $5 \%$ of the most highly fluorescent cells of the parental population were sorted in a throughput of $\sim 4-5,000$ cells per second in the single-cell mode. Depending on the diversity and the size of each library, the maximum number of the sorted events covered the range of $10^{6}-10^{7}(\sim 2.5 \mathrm{~h})$. The sorted cells were collected in tubes containing 2xYT medium supplemented with $200 \mu \mathrm{g} / \mathrm{mL}$ ampicillin and $35 \mu \mathrm{g} / \mathrm{mL}$ chloramphenicol, and were finally plated onto $2 \mathrm{xYT}$ plates with the respective antibiotics. Following overnight growth, the clones were pooled and stored at $-80{ }^{\circ} \mathrm{C}$ in aliquots for the next sorting round. Figure 8 shows the principle of the FACS screening system using eGFP as reporter protein to correlate the L-ASNase activity with intracellular fluorescence, followed by FACS sorting. 


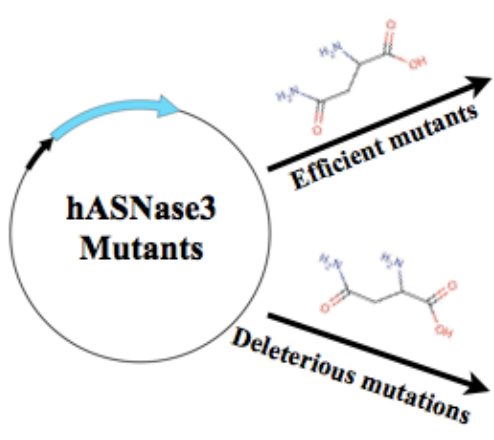

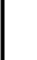
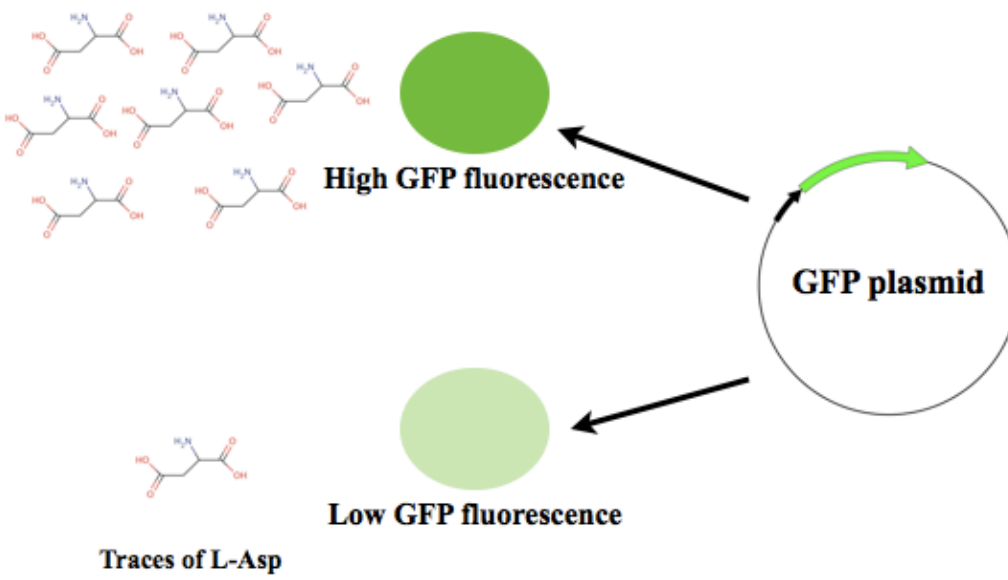

5-gene-deletion JC1(DE3) E.coli strain

Figure 8. Principle of the FACS-based screening system of hASNase3 mutant libraries. Catalytically improved mutants produce higher amounts of L-Asp as compared to mutants which have deleterious mutations. The intracellular L-Asp availability can be quantitatively correlated with eGFP fluorescence since a higher L-Asp concentration will result in higher expression levels of eGFP and, in turn, higher fluorescence.

(C) Identification of catalytically improved hASNase3 mutants. After the last FACS-sorting round of the mutant libraries, the pooled clones were cultured, and their plasmid DNA was extracted (plasmid DNA contains both pET14-SUMO and pBAD33 plasmids). The extracted plasmid DNA was used as template for the amplification of the coding region of hASNase 3 mutants using the primers $\mathrm{FW}_{\mathrm{hASNase}} \mathrm{wt}$ and $\mathrm{RV}_{\mathrm{hASNase}} \mathrm{wt}$. The amplified sequences were gel-purified, digested with NdeI and BamHI H.F., and finally were ligated into the pET14bSUMO plasmid overnight at $16{ }^{\circ} \mathrm{C}$ using T4 DNA ligase. The overnight ligation mixture was made salt-free using a PCR-clean-up kit, and subsequently was electroporated into C41(DE3) electrocompetent cells. The cells were resuspended in $2 \mathrm{~mL}$ SOC. medium and then incubated for $1 \mathrm{~h}$ at $37{ }^{\circ} \mathrm{C}$ for recovery. Finally, they were streaked onto $2 x Y T$ plates supplemented with $200 \mu \mathrm{g} / \mathrm{mL}$ ampicillin, and placed at $30^{\circ} \mathrm{C}$.

Single clones were selected from the plates and used to inoculate wells of a sterile 96-well plate containing $120 \mu \mathrm{L} \mathrm{2xYT} \mathrm{medium} \mathrm{per} \mathrm{well,} \mathrm{with} 200 \mu \mathrm{g} / \mathrm{mL}$ ampicillin, $0.4 \%$ glucose and $50 \mathrm{mM}$ glycine. The plates were placed at $37^{\circ} \mathrm{C}$ under vigorous shaking at $300 \mathrm{rpm}$ for 3 h. In the following step, $100 \mu \mathrm{L}$ were transferred to a second 96-well plated containing 200 $\mu \mathrm{g} / \mathrm{mL}$ ampicillin, $0.4 \%$ glucose, $1 \mathrm{mM}$ IPTG, and $50 \mathrm{mM}$ glycine, while the rest of the cells from the first plate $(\sim 20 \mu \mathrm{L})$ were temporally stored at $4{ }^{\circ} \mathrm{C}$. The second plate was incubated at $37^{\circ} \mathrm{C}$ for further $3 \mathrm{~h}$, followed by centrifugation for $20 \mathrm{~min}$ at 3,000g using a Sigma $4-15 \mathrm{C}$ 
swinging bucket plate-centrifuge. The supernatant culture medium was discarded using a 12channel Eppendorf pipette; the pelleted cells were resuspended in $50 \mathrm{mM} \mathrm{Na} 2 \mathrm{HPO}_{4}, 300 \mathrm{mM}$ $\mathrm{NaCl}, \mathrm{pH} 8$, supplemented with $1 \mathrm{X}$ BugBuster (Novagen) cell lysis reagent and $5 \mathrm{KU} / \mathrm{mL}$ recombinant lysozyme (rLysozyme, Novagen). The plates were incubated at RT for $30 \mathrm{~min}$ under mild shaking (50 rpm), and were then centrifuged for $20 \mathrm{~min}$ at 3,000g. The supernatants were transferred to Ni-NTA-coated HisSorb 96-well plates (Qiagen) and were left overnight at $4{ }^{\circ} \mathrm{C}$ under mild shaking. Ultimately, the supernatants were discarded and the plates were rinsed twice using $50 \mathrm{mM} \mathrm{Na}_{2} \mathrm{HPO}_{4}, 300 \mathrm{mM} \mathrm{NaCl}, 20 \mathrm{mM}$ imidazole, $\mathrm{pH} 8$, before washing them twice with PBS, which was the final assay buffer. Enzymatic activities were determined applying the Amplex Red-dependent fluorescent assay directly in the HisSorb plates containing $300 \mu \mathrm{g} / \mathrm{mL}(5 \mu \mathrm{M})$ L-Aspartate Oxidase (L-AspOx), $12 \mu \mathrm{M}$ FAD, $100 \mathrm{nM}$ HRP $(0.1 \mathrm{U} / \mathrm{mL}), 50 \mu \mathrm{M}$ Amplex Red, and $1 \mathrm{mM}$ L-Asn in a final volume of $50 \mu \mathrm{L}$ per well, using a fluorescent plate reader (Molecular Devices, SpectraMax Paradigm, Ex. 532, Em. 592).

(D) Biochemical characterization of catalytically improved mutants. The most highly active mutants, which were identified after the final activity measurements using the fluorescent plate reader, were traced back to the original 96-well plates where they had grown; they were re-cultured in $50 \mathrm{~mL}$ for plasmid extraction, and aliquots were stored at $-80{ }^{\circ} \mathrm{C}$. In addition, the selected original clones were used for expression and protein purification. The purified enzymes were kinetically characterized applying the NADH-dependent continuous spectrophotometric assay. Both processes of expression-purification and kinetic characterization were performed as described above in the Methods section. Furthermore, the hASNase3 mutants were analyzed by differential scanning fluorimetry (DSF) [269] in order to investigate the impact of mutations on the structural stability of the enzymes. Enzyme samples from $-20{ }^{\circ} \mathrm{C}$ glycerol stocks were dialyzed (Pierce, Slide-A-Lyzer, 10,000 MWCO) against $50 \mathrm{mM}$ Tris-Cl, $0.1 \mathrm{NaCl}, \mathrm{pH} 8$, to remove glycerol, and were mixed with SYPRO Orange (Sigma- Aldrich) in a final volume of $20 \mu \mathrm{L}$. The final concentrations of the enzyme and the dye were $1 \mu \mathrm{M}$ and $10 \% \mathrm{v} / \mathrm{v}$, respectively; the DMSO stock solution of the dye $(5,000 \mathrm{X})$ was pre-diluted in $\mathrm{H}_{2} \mathrm{O}$ giving a $100 \mathrm{X}$ solution, from which aliquots were used according to the experimental needs. The samples were mixed in a 96-well plate suitable for real-time (RT)-PCR measurements, centrifuged at $500 \mathrm{rpm}$ for $30 \mathrm{~s}$, and finally sealed with 
heat-resistant membranes (Microseal B adhesive Sealer) to prevent evaporation. The proteinmelting experiments were performed using a CFX96 RT-PCR machine (Bio-Rad) with the following settings: 2 min pre-warming step at $30{ }^{\circ} \mathrm{C}$, and subsequent temperature gradient between $31-95{ }^{\circ} \mathrm{C}$ with $1{ }^{\circ} \mathrm{C} / \mathrm{min}$ increments. SYPRO Orange fluorescence was monitored using FAMex $(492 \mathrm{~nm})$ and ROXem $(610 \mathrm{~nm})$ filters. Data were exported as Excel-based worksheet and further analyzed by Igor-Pro (Wavemetrics). Melting temperatures $(T \mathrm{~m})$ were obtained by plotting the first derivative $\mathrm{d}(\mathrm{RFU}) / \mathrm{dT}$ of the raw data as a function of temperature increase [270]. Figure 9 shows an overall scheme of the whole screening process starting from the library generation up to the level of the characterization of individual sorted mutants and, in principle, represents each step described in the Methods section above. 


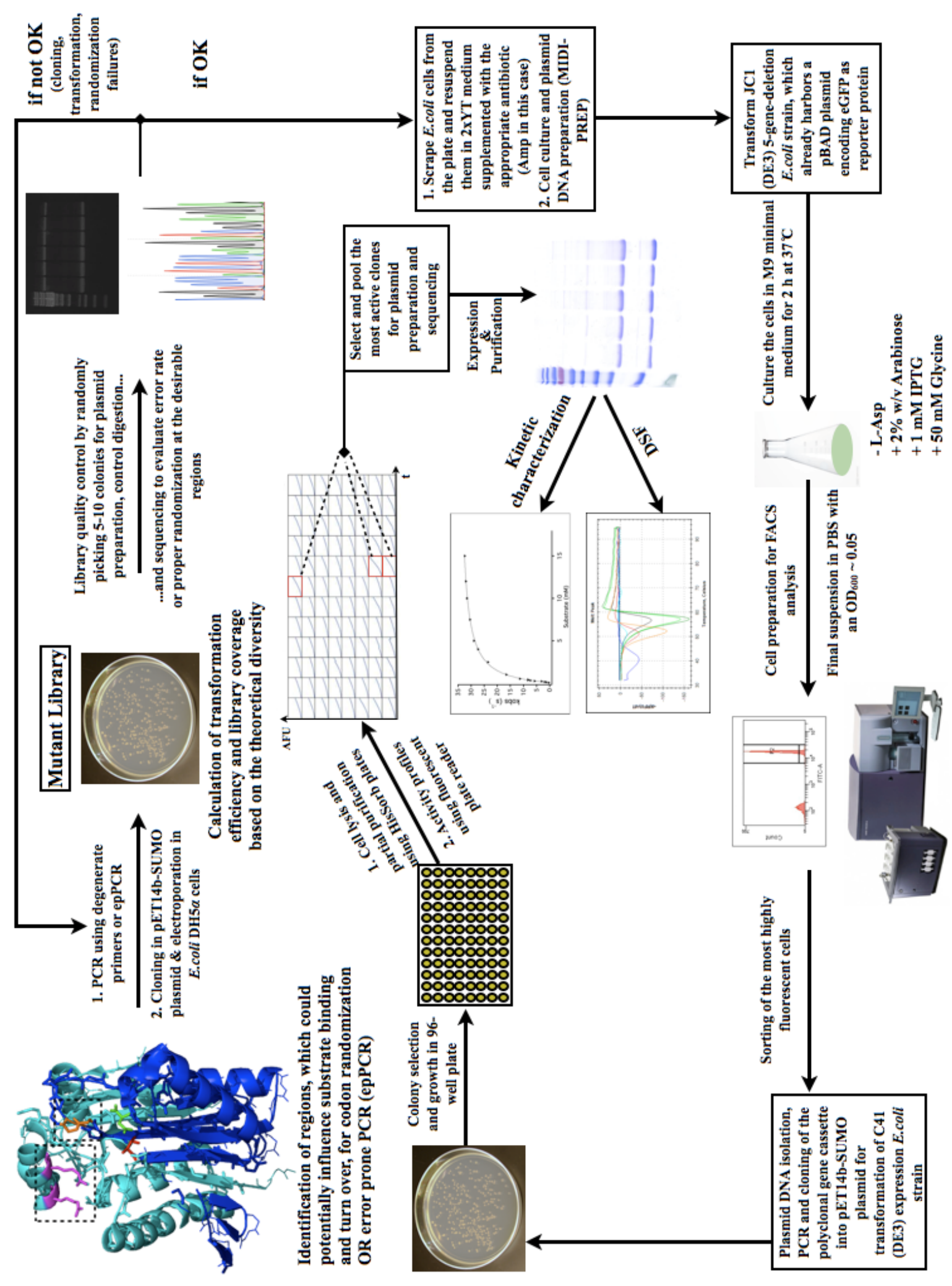




\subsubsection{Results}

\subsubsection{Genetic complementation of L-ASNase deficiency in E.coli}

The genetic complementation of five L-ASNases from human, E.coli and yeast cloned in the plasmid pET14b-SUMO was investigated using the five-gene-deletion strain JC1(DE3). The following enzymes were tested for genetic rescue of L-ASNase deficiency: hASNase1, hASNase3, EcASNase1, EcASNase2, and ScASNase1. The plasmid with no L-ASNaseencoding insert was included in this series of experiments in order to evaluate the background growth of the cells. Cells transformed with equal amounts of DNA were plated onto M9 minimal plates supplemented with all amino acids except from L-Asp, 1mM IPTG to induce the expression of the L-ASNases, and all the other compounds as described in the Methods section, and then placed at $37{ }^{\circ} \mathrm{C}$. Eventually, colony formation was observed in all transformants after $\sim 30 \mathrm{~h}$ of incubation indicating positive genetic complementation. The plates with the negative control (plasmid with no insert) showed very small colonies, possibly due to the presence of L-Asp traces in the stocks of the other amino acids, or due to spontaneous hydrolysis of L-Asn, which provided the basal level of L-Asp allowing for initial cell growth. On the other hand, when the same experiment was done in liquid cultures with M9 minimal medium, after $24 \mathrm{~h}$ of incubation at $37^{\circ} \mathrm{C}$, the negative control did not show any cell growth (non-detectable $\mathrm{OD}_{600}$ increase), while the five positive controls grew normally exhibiting an $\mathrm{OD}_{600} \sim 1$. However, it must be mentioned that this $\mathrm{OD}_{600}$ was reached in different periods of time for each enzyme depending on its kinetic properties. For example cells expressing the EcASNase 2 reached $\mathrm{OD}_{600} \sim 1$ after $\sim 12 \mathrm{~h}$ of incubation at 37 ${ }^{\circ} \mathrm{C}$, while for ScASNase 1, hASNase1 and hASNase3 cells took $~ 16$ and $24 \mathrm{~h}$ (same time for both human enzymes), respectively.

The next validation of the screening strategy included a quantitative comparison between the rescue capacities of wildtype hASNase 3 and EcASNase 2 using the FACS technique before starting screening of the generated hASNase 3 mutant libraries. This experiment aimed to determine the mean fluorescence of the cell population, which can be obtained from cells expressing two enzymes with distinct catalytic properties. Wildtype hASNase 3 displays a $k_{\text {cat }} /$ $\mathrm{K}_{\mathrm{M}} \sim 3 \times 10^{2} \mathrm{M}^{-1} \mathrm{~s}^{-1}$, while EcASNase2 has a value of $\sim 6 \times 10^{5} \mathrm{M}^{-1} \mathrm{~s}^{-1}$ (kinetic constants determined by applying the NADH-dependent assay at $25^{\circ} \mathrm{C}$ ), which means that the E.coli 
enzyme is $\sim 2,000$-fold more efficient. To this end, cells transformed with hASNase3 and EcASNase2, in either case harboring in parallel the pBAD33-eGFP plasmid, were prepared for FACS analysis as described in the Methods section 5.1.2.5 above. Figure 10 shows fluorescence profiles resulting from this experiment.

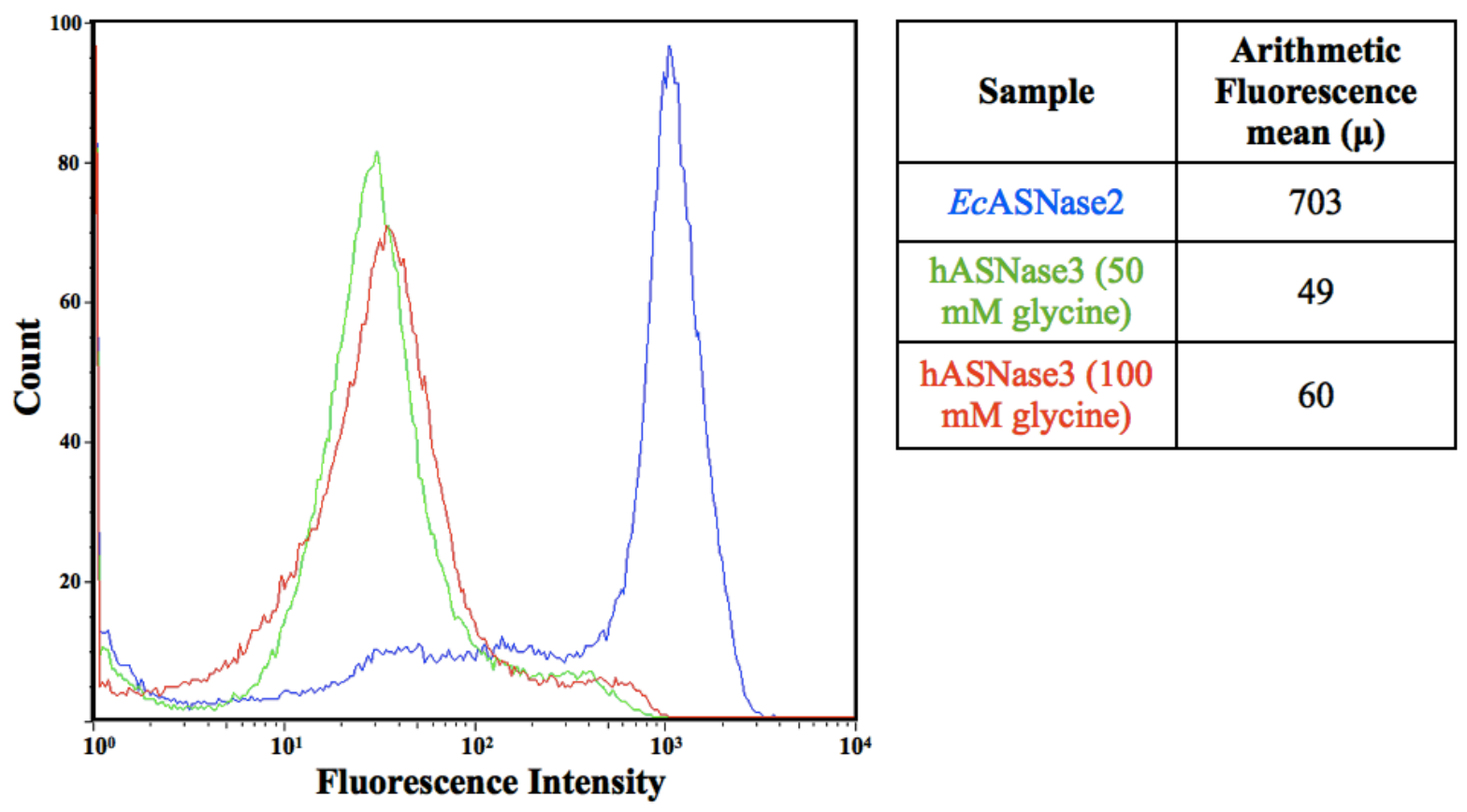

Figure 10. FACS fluorescence profiles obtained for hASNase3- and EcASNase2dependent complementation in the E.coli strain JC1(DE3) which is deficient for Laspartate biosynthesis. Bacterial cells transformed with either hASNase3 or EcASNase2 were analyzed by FACS after incubation in M9 minimal medium for $2 \mathrm{~h}$ lacking L-Asp. The histogram show the fluorescence distribution of 10,000 cells.

The results revealed that while the in-vitro catalytic properties of the two enzymes differ 2,000-fold, the genetic complementation assay using eGFP as reporter protein for metabolic rescue showed only $\sim 20$-fold difference of their fluorescence means. This may be attributed to saturated expression levels of eGFP in the case of EcASNase2 expression, or the utilization of the produced L-Asp for other cellular processes, thereby not reflecting in quantitative terms the real catalytic differences between the two enzymes. Perhaps the fluorescence profile of another L-ASNase considerably more efficient than EcASNase2 would provide more information about the upper limit of the present screening assay In addition, it is very interesting the fact that both treatments with glycine, i.e. using 50 or 100 $\mathrm{mM}$ glycine, respectively, resulted in practically similar fluorescence means. From in-vitro incubation experiments, we have found that the activation of hASNase 3 is induced by glycine 
in a concentration-dependent manner, reaching saturation at glycine concentrations higher than $300 \mathrm{mM}$. Those FACS results could be potentially explained on the basis of equal glycine uptake by the E.coli cells in both tested concentrations. In other words, it could well be that the cells take from their environment a certain amount of glycine, which saturates at concentrations lower than $50 \mathrm{mM}$ that is the lowest which was tested in the present experiment, and anything more than this does not lead to further uptake. Based on the observation that cells grew somewhat slower in the presence of $100 \mathrm{mM}$ glycine, $50 \mathrm{mM}$ was used as standard glycine concentration for the next screening experiments.

\subsubsection{FACS analyses of hASNase 3 mutant libraries}

\section{(A) epPCR library}

Based on the sequencing results obtained for the four epPCRs that were run under different mutagenic conditions (see Methods), an epPCR library was generated for the hASNase3. The per cent-error rates calculated for each treatment are the following: $0.1 \%$ for $0.01 \mathrm{mM}$ $\mathrm{MnCl}_{2} ; 0.1 \%$ for $0.05 \mathrm{mM} ; 0.2 \%$ for $0.2 \mathrm{mM}$, and $0.6 \%$ for $0.5 \mathrm{mM}$. It is generally believed that libraries with higher error rates are more likely to be enriched with improved sequences as compared to libraries with lower error rates [271]. In addition, the generation of more mutations may increase the probability of detecting a positive interaction between mutated residues in a non-additive manner, the so-called positive epistasis [272]. Therefore, an epPCR library was constructed following the protocol which introduces more mutations in the sequence, i.e. using $0.5 \mathrm{mM} \mathrm{MnCl}_{2}$. The final library yielded $\sim 10^{7}$ transformants, while the negative control showed less than $1 \%$ background. Ten randomly chosen clones were cultured, their plasmid DNA was extracted,

and then digested with the restriction enzymes NdeI and BamHI to evaluate cloning efficiency. All ten clones were positive and ultimately were sequenced to verify the expected error rate $(\sim 0.6 \%)$. Eventually, it turned out that out of ten clones sequenced, $80 \%$ had 6 mutations, and the other $20 \%$ had 5 mutations. In addition, out of overall 58 mutations (6 mutations per sequence for 8 sequences +5 mutations per sequence for 2 sequences), 18 were silent (31\%), while the rest varied from neutral (amino acids with similar properties, e.g. leucine to isoleucine) to more drastic mutations (e.g. amino acids with totally distinct 
properties e.g. L-Asp to L-Lys). No frame shifts were observed in this epPCR library, though other clones may contain such modifications since frame shifts are a common phenomenon in epPCR libraries [273]. The library was prepared for FACS analysis, and the results are shown in Figure 11.

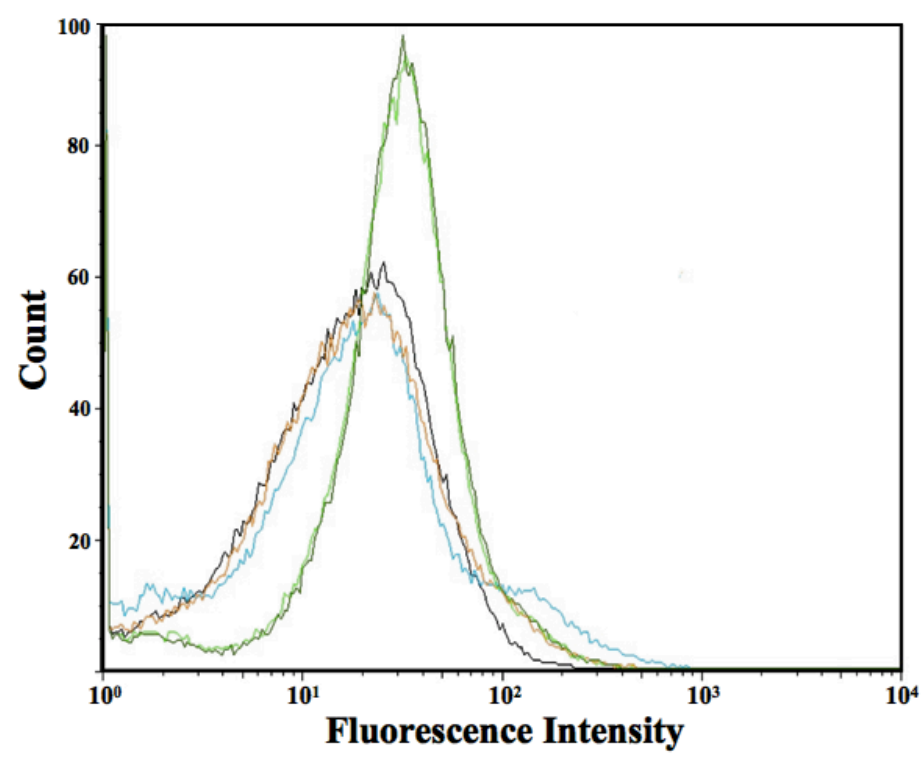

\begin{tabular}{|c|c|}
\hline Sample & $\begin{array}{c}\text { Arithmetic } \\
\text { Fluorescence } \\
\text { mean }(\boldsymbol{\mu})\end{array}$ \\
\hline hASNase3 S0 & 38.2 \\
\hline hASNase3 S1 & 39.1 \\
\hline hASNase3 S2 & 28.2 \\
\hline hASNase3 S3 & 36.5 \\
\hline hASNase3 S4 & 23.0 \\
\hline
\end{tabular}

Figure 11. Fluorescence profiles of the four sorting rounds of the hASNase 3 epPCR library. The histograms show the fluorescence distribution of 10,000 cells. hASNase 3 S0 corresponds to the arithmetic mean fluorescence $(\mu)$ of the initial cell population of the library, S1 is the $\mu$ of the first, S2 of the second, S3 of the third, and S4 of the fourth sorting round, respectively. The sorted cells of each round accounted for the $5 \%$ of the most highly fluorescent cells. The first sorting was done in the purity mode, while the other three were performed in the single-cell mode. After each round of sorting, the cells were plated onto ampicillin/chloramphenicol plates, and grew at $37{ }^{\circ} \mathrm{C}$ overnight. Subsequently, the clones were pooled and stored at $-80^{\circ} \mathrm{C}$ for the next round of FACS analysis.

FACS data obtained for the epPCR library suggest that, even after four rounds of sorting, no fluorescence enrichment was achieved. In the previous analysis (Figure 10), the wildtype hASNase 3 exhibited a $\mu$-value of about 50 , while in case of the epPCR library the initial population did not exceed 40 (hASNase3 S0). The four successive sorting rounds displayed a $\mu$ within the range of $20-40$, with the lowest one being 23 of the last round. These data suggest that the screened epPCR library did not contain improved hASNase 3 variants as evidenced by the lack of fluorescence enrichment throughout the sorting steps. In addition, given the fact that the wildtype enzyme showed a $\mu \sim 50$, while all following values for the library were lower than 50 , it is likely that most of the introduced mutations were neutral or deleterious, assuming that expression levels as well as glycine-induced intramolecular 
activation were similar in these cell populations. In conclusion, the screening of the epPCR library with an average number of 6 mutations per gene was not proven to be beneficial for the evolution of hASNase3. In future work, the generation of more epPCR libraries with different error rates could increase the probability of identifying catalytically improved variants. However, the availability of the hASNase 3 crystal structure prompted us to design structure-based site-saturation mutant libraries following a semi-rational route, thereby exploiting various tools for directed evolution. The screening results of four site-saturation mutagenesis (SSM) libraries are presented and discussed in the following sections.

\section{(B) SSM-LIB1}

The first SSM library was generated by a focused randomization of four sequential Nterminal residues: Ile4-Va15-Val6-Va17. These amino acids, as shown in Figure 2, are part of a $\beta$-sheet on the top of which is located the triad His8-Gly9-Gly10 that is critical for catalysis and for the glycine-dependent autoactivation step. The His8-Gly9-Gly10 triad is a highly conserved structural motif of Ntn-hydrolases which display L-ASNase activity and can be considered as a "fingerprint" for these enzymes. Single-site mutations of these three amino acids totally abolished the intramolecular activation of hASNase3, as well as the enzymatic activity of a circular permutant version of hASNase3 [37]. Based on these findings, we reasoned that amino acids which are located close to this triad could provide a promising target region for randomization and screening. The stretch of these four residues is $\sim 17 \AA$ away from the centre of the hASNase3 catalytic site encompassing residues Asn62, Thr186, Arg196, Asp199, Thr219, Gly220, and the catalytic threonine Thr168. Therefore, one may assume that they are located at the outer third notional shell regarding the distance from the active site. The library was constructed using degenerate primers by randomizing these four residues close to the N-terminus applying the scheme NNS to each codon, covering a theoretical diversity of $\sim 10^{6}$ variants in a population of $\sim 10^{7}$ individual transformants. Quality control and characterization of the library were done, in the present study as for all other libraries, as described in detail in the Methods section. The FACS results after three sorting rounds are shown in Figure 12. 


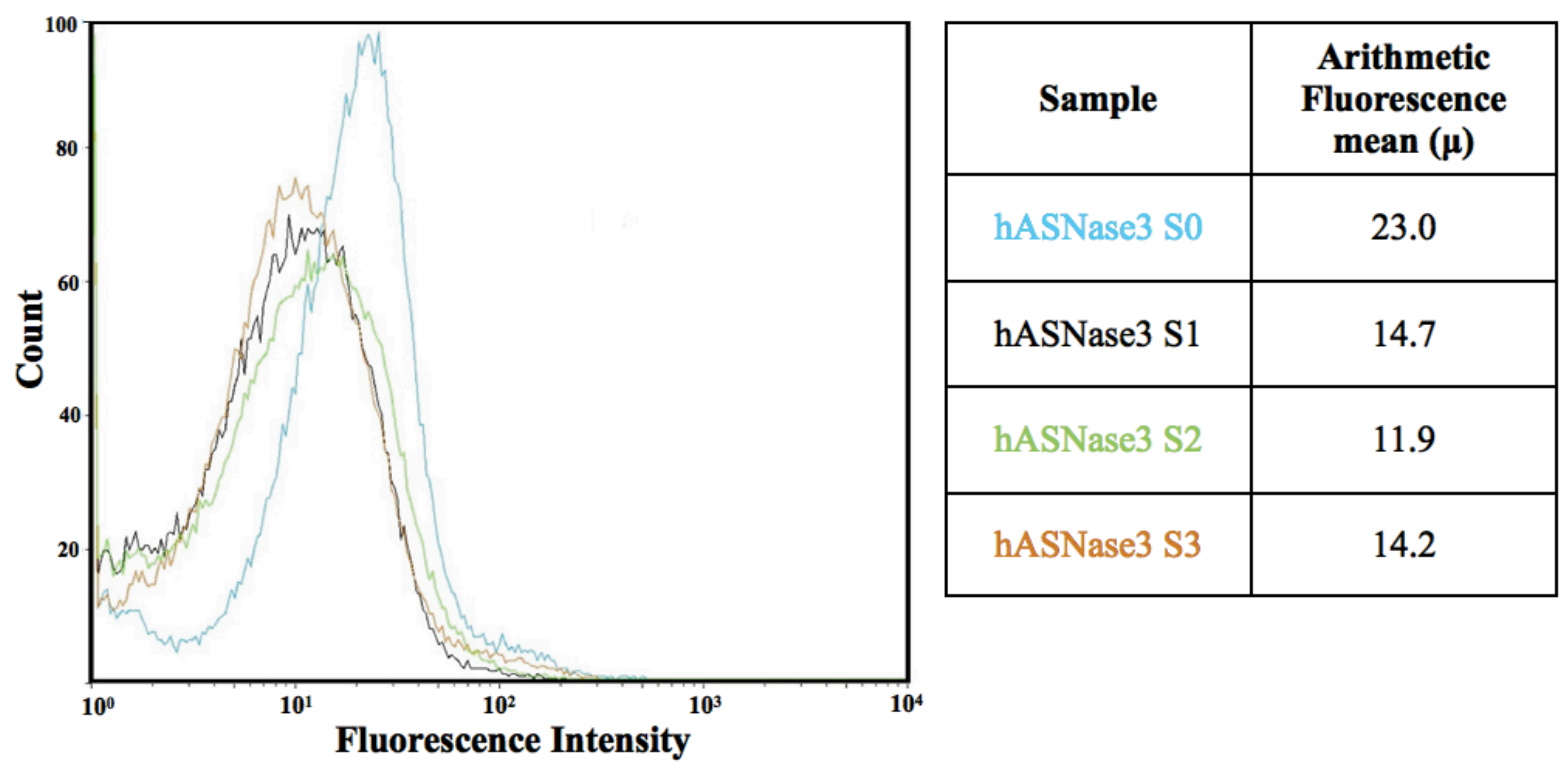

Figure 12. Fluorescence profiles of the three sorting rounds of the hASNase3 SSM-LIB1. The histograms show the fluorescence distribution of 10,000 cells. In each round, the $5 \%$ of the most highly fluorescent cells from the parental population were sorted. The first sorting was done in the purity mode, the two following rounds in the single-cell mode. The sorted cells from each round were collected in tubes containing 2xYT medium supplemented with ampicillin and chloramphenicol, and finally they were plated on $2 \mathrm{xYT}$ plates. The grown colonies were pooled and stored at $-80^{\circ} \mathrm{C}$ until the subsequent FACS analysis.

As evidenced by the mean fluorescence intensities of each of the three sorting rounds of this SSM library, with $\mu$-values being in the range 10-25, mutations in the Ile4-Val5-Val6-Val7 region had a negative impact on hASNase 3 activity, considering that the wildtype enzyme shows a $\mu$-value of around 50. In the light of the structural importance of this region for both autoproteolytic activation and enzymatic activity, it is not easy to rationalize whether randomization of these residues impairs primarily the rate and the degree of intramolecular processing of the enzyme, and secondarily its activity. In order to obtain such information, individual mutants of this library would have to be tested for their intramolecular activation rates, as well as for their L-asparaginase activity profiles. Overall, it appears that this specific hASNase3 region (residues 4 to 7) located close to the His8-Gly9-Gly10 triad (residues 1 to 15) does not tolerate mutations.

\section{(C) SSM-LIB2}

The second SSM library was generated by randomizing the following four residues: Met193Val194-Cys202-Leu203. Residues targeted in this mutant library are located closer in space 
to the active site residues $(\sim 14 \AA$ apart) in comparison to those of the previous SSM-LIB1, and are part of a flexible loop which connects two $\beta$-sheets (residues 180-185 and 205-210, respectively) in close proximity to the active site (Figure 3). In this loop is also located residue Arg196 which participates in the formation of the substrate binding pocket (Figure 1). According to the amino acid alignment, these four residues are not highly conserved among the homologs from different organisms. In contrast, the amino acids upstream and downstream of Met193-Val194 and Cys202-Leu203, respectively, are highly conserved. Similar to the first SSM library, SSM-LIB2 has a theoretical diversity of $10^{6}$, which was covered by the $8 \times 10^{6}$ transformants obtained. The results from three sorting rounds are shown in Figure 13.

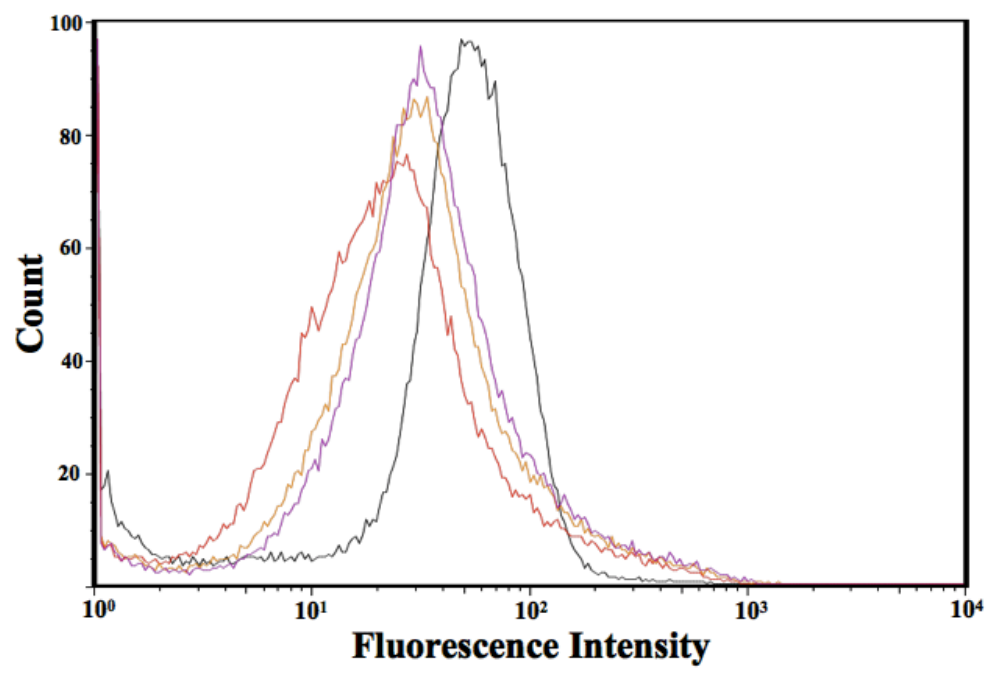

\begin{tabular}{|c|c|}
\hline Sample & $\begin{array}{c}\text { Arithmetic } \\
\text { Fluorescence } \\
\text { mean }(\boldsymbol{\mu})\end{array}$ \\
\hline hASNase3 S0 & 58 \\
\hline hASNase3 S1 & 52 \\
\hline hASNase3 S2 & 41 \\
\hline hASNase3 S3 & 51 \\
\hline
\end{tabular}

Figure 13. Fluorescence profiles of the three sorting rounds of the hASNase3 SSM-LIB2. As with previous cases, the histograms show the fluorescence distribution of 10,000 cells. Cells were sorted as described in the previous figure legends.

The obtained values of the arithmetic fluorescence means of each sorting round showed also in this SSM library, similar to SSM-LIB1, no enrichment. These results strongly suggest that the randomization of the selected residues negatively affected the enzyme's activity. However, it must also be kept in mind that, in case of hASNase3, catalytic activity is not the only parameter which can be influenced in a negative manner. The autoproteolytic activation of the enzyme might also be affected by the different introduced mutations. In summary, the region composed by the four amino acids M193-V194-C202-L203, which are surrounded by 
highly conserved residues and are part of a critical loop close to the substrate binding pocket, does not seem to be a potential target for generating improved hASNase 3 variants.

\section{(D) SSM-LIB3}

The third SSM library was generated by randomizing only two amino acid residues: Ile189Val190 (Figure 4). This tandem pair is located very close to the active site $(\sim 5 \AA)$ and is part of the first notional shell that surrounds it. Particularly, the side-chain of I189 points towards the substrate binding pocket and is in space very near to Thr186 and Arg196 which contribute to the binding of the substrate L-Asn. Despite the fact that they are located very close to the active site being part of a flexible loop, Ile189 and Val190 are not highly conserved (Figure 4), and, therefore, they were thought to be an attractive target for randomization aiming at potential positive impact on the substrate binding and/or turnover. This relatively small library has a theoretical diversity of $\sim 10^{3}$, and thus was covered about $10^{4}$-fold by the yield of $10^{7}$ transformants. The cells were analyzed in only two sorting rounds, since the size of the library is very small. The results are shown in Figure 14.
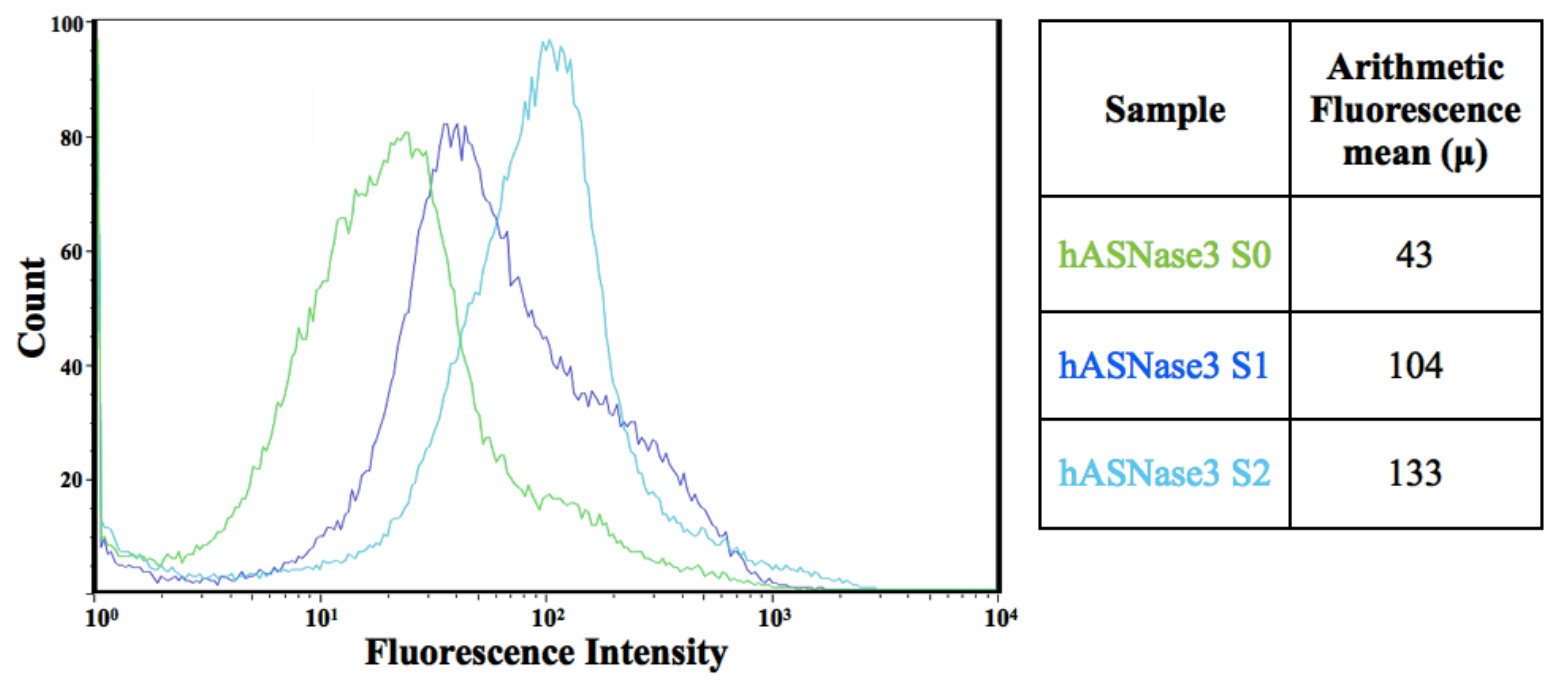

Figure 14. Fluorescence profiles of the two sorting rounds of the hASNase3 SSM-LIB3. The histograms show the fluorescence distribution of 10,000 cells. From each round $\sim 5 \%$ of the most highly fluorescent cells from the parental population were sorted. Both sorting rounds were done in single-cell mode. 
Figure 14 shows the arithmetic mean fluorescence values for the starting cell population (S0) and the two sorting rounds (S1 and S2). Interestingly, unlike the previous larger libraries (4 NNS), significant enrichment was observed through the successive two sorting rounds. Starting from $\mu \sim 43$, the cell population of S1 showed $\mu \sim 104$, and S2 $\sim 133$, indicating that intracellular eGFP fluorescence constantly increased, possibly because the population is enriched with cells harboring catalytically improved hASNase3 variants. The sorted mutants from the last round were pooled, cultured for plasmid DNA extraction and stored at $-80{ }^{\circ} \mathrm{C}$ in aliquots for further screening and analysis.

\section{(E) SSM-LIB4}

The fourth SSM library was constructed by randomizing the two arginine residues R143 and R147 which are located on the surface of hASNase3 (Figure 5), at $\sim 17$ and $14 \AA$ distance from the active site, respectively. Both amino acids point outwardly from the notional direction to the active site and are part of an $\alpha$-helix of the enzyme's $\alpha$ subunit. According to the sequence comparison shown in Figure 5, this region appears to be non-conserved. Similar to the previous SSM-LIB3, SSM-LIB4 is a relatively small library characterized by a theoretical diversity of $\sim 10^{3}$. The number of individual transformants obtained was $\sim 5 \times 10^{6}$. Cells were prepared for FACS analysis similar to the previous libraries and were subjected to two sorting rounds. Figure 15 depicts the results of these experiments.
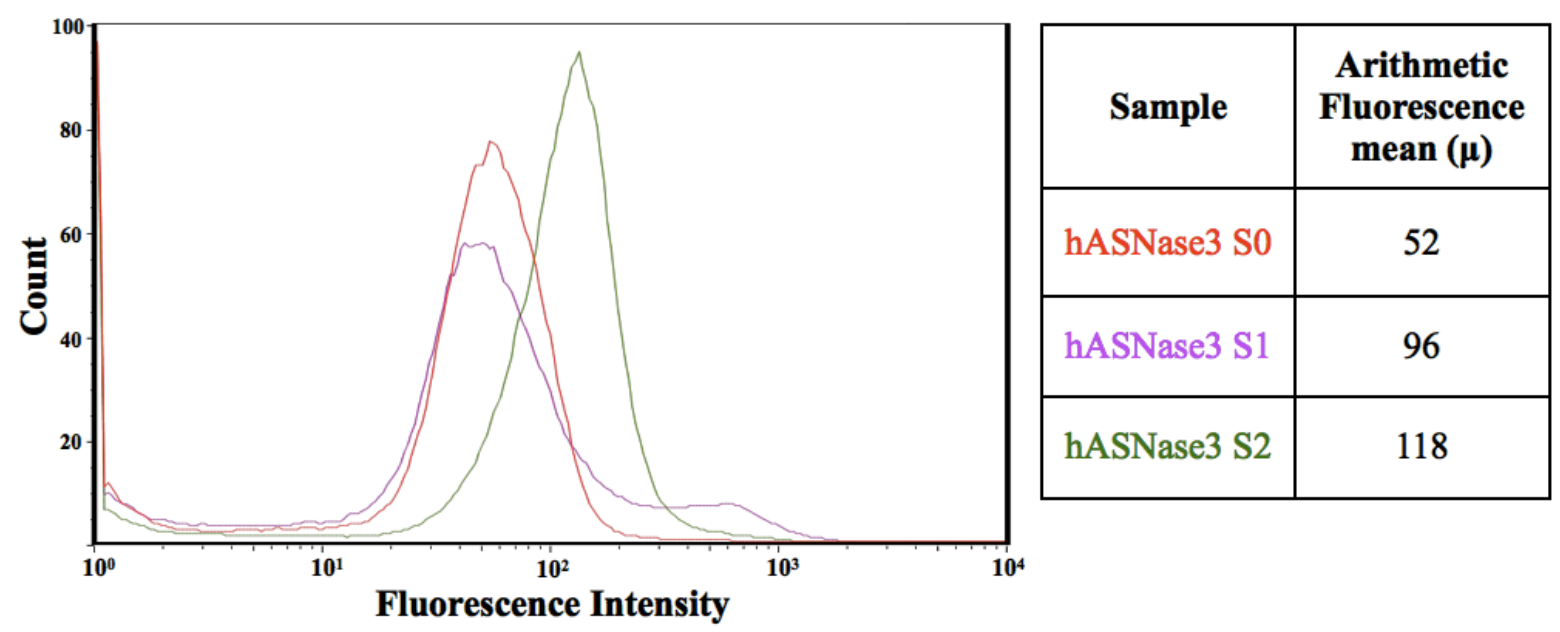

Figure 15. Fluorescence profiles of the two sorting rounds of the hASNase3 SSM-LIB4. The histograms show the fluorescence distribution of 10,000 cells, and from each round $\sim 5 \%$ 
of the most highly fluorescent cells from the parental population were sorted. Both sorting rounds were done in single-cell mode.

As shown in Figure 15, SSM-LIB4 is another library which displayed enrichment after two subsequent sorting rounds. The fluorescence mean increased from $\mu \sim 52$ for the initial population to $\mu \sim 96$ for $\mathrm{S} 1$, and $\mu \sim 118$ for S2, suggesting that this library was enriched with catalytically ameliorated hASNase 3 mutants. The clones from the last sorting round were pulled and stored at $-80^{\circ} \mathrm{C}$ in aliquots for further screening and analysis.

\subsubsection{Identification of catalytically improved mutants from SSM-LIB3 and SSM-LIB4}

Following the final sorting rounds of each of the two libraries, SSM-LIB3 and SSM-LIB4, the plasmid DNA of the clones was extracted, and the coding region of the hASNase3 mutants was amplified using the primer pair $\mathrm{FW}_{\mathrm{hASNase}} \mathrm{wt}$ and $\mathrm{RV}_{\mathrm{hASNase}}$ wt. The amplified polyclonal gene cassette was cloned into the pET14b-SUMO vector and used to transform C41(DE3) cells. Single-clone activity analysis in 96-well plates applying the Amplex Red fluorescent assay revealed that a few clones were more active than the wildtype hASNase 3 which served as control. The most active clones from each library were sequenced in order to determine the mutations at the respective randomized regions. Strikingly, in the case of the SSM-LIB3 library, three clones were identical encoding the mutant Ile189Thr-Val190Ile (Double Mutant 1; DM1), and one encoding the variant Ile189Val-Val190Ile (Double Mutant 2; DM2). Analysis of SSM-LIB4 uncovered three clones which exhibited higher activities than the wildtype enzyme, and all three were identical variants with the mutations Arg143Glu-Arg147Lys (Double Mutant 3; DM3). The discovery of these three distinct mutants motivated us to generate by site-directed mutagenesis two additional variants which would combine the four identified mutations. Therefore, the quadruplet mutants Ile189ThrVal190Ile-Arg143Glu-Arg147Lys (Quadruplet Mutant 1; QDM 1) and Ile189Val-Val190IleR143Glu-Arg147Lys (Quadruplet Mutant 2; QDM 2) were constructed using the oligonucleotide primers shown in Table 1. QDM1 and QDM2 were generated using the same pair of primers which introduced the mutations Arg143Glu-Arg147Lys in the respective templates: DM1 for QDM1, and DM2 for QDM2. The five variants that were obtained 
(DM1, DM2, DM3, QDM1 and QDM2) were recombinantly expressed and purified for enzymatic characterization.

\subsubsection{Expression, purification, and kinetic characterization of five hASNase 3 variants selected from the libraries}

One of the primary concerns regarding the five pulled mutants was their potential failure to undergo the autoproteolytic activation step which is directly related to their catalytic activity, and whether this activation could be accelerated by glycine. Though the three main mutants (DM1, DM2 \& DM3) were pulled from a library growing in medium supplemented by 50 $\mathrm{mM}$ glycine, in principle, their intramolecular processing state (autoproteolytic cleavage) was unknown up to the level of the single-clone activity assay in 96-well plates. The point here was to confirm that the observed improved activity of the mutants was not due to different autoactivation rates, but indeed because of improved catalytic efficiency. Therefore, all five variants, and the wildtype enzyme, were initially produced in C41(DE3) E.coli cells grown in 2xYT medium without glycine, and at low temperature (at $16{ }^{\circ} \mathrm{C}$ overnight) in order to slow down their activation process. After a rapid NTA-affinity purification step at $4{ }^{\circ} \mathrm{C}$ and incubation with SUMO-protease (for $4 \mathrm{~h}$ at $4{ }^{\circ} \mathrm{C} ; 1: 100$ molar ratio of protease:enzyme) to cleave the His6-SUMO tag, the samples were analyzed by SDS-PAGE to evaluate their activation state. The results are shown in Figure 16.

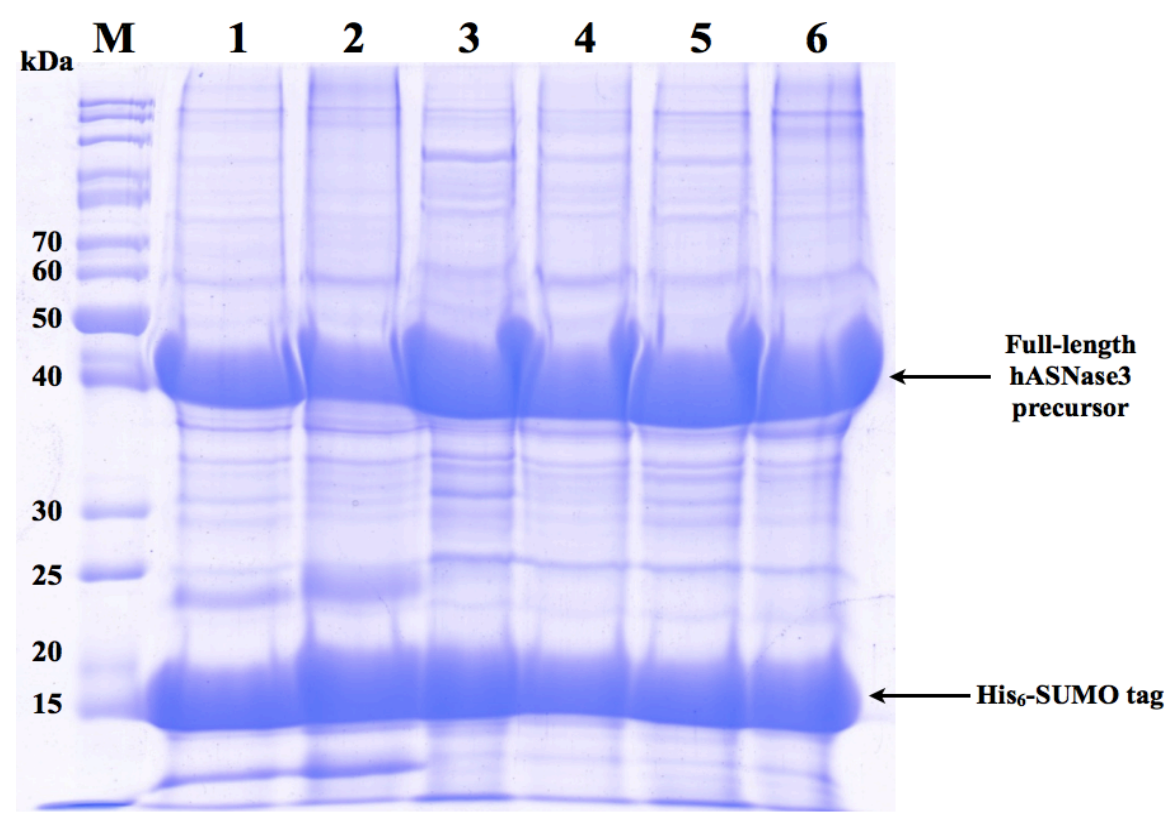


Figure 16. 15\% SDS-PAGE analysis of hASNase 3 wildtype and five mutants without glycine treatment. After a short NTA-affinity purification step, the soluble proteins were analyzed in order to evaluate their autoproteolytic activation. Lane 1: wildtype; Lane 2: DM1; Lane 3: DM2; Lane 4: DM3; Lane 5: QDM1; Lane 6: QDM2. The first arrow indicates the expected molecular weight of the full-length hASNase3 inactive precursor (theoretical $\left.M_{\mathrm{r}}: 33 \mathrm{kDa}\right)$, and the second one shows the His6-SUMO tag $(\sim 13 \mathrm{kDa})$ which was cleaved by SUMO-protease.

As evidenced by SDS-PAGE analysis, all five hASNase3 variants were not cleaved without being treated with glycine, and their electrophoretic profiles were similar to the wildtype enzyme. The next question which was intuitively brought up concerned the possibility of activation of those mutants using glycine similar to the wildtype hASNase3. This time, all five variants were produced following the standard expression protocol which was used for the expression of the wildtype enzyme resulting in totally activated enzyme species. To this end, wildtype and hASNase3 variants were expressed in 2xYT medium supplemented with $200 \mathrm{mM}$ glycine for $6 \mathrm{~h}$ at $37^{\circ} \mathrm{C}$. Subsequently, the enzymes were purified as described above in Methods, incubated with $500 \mathrm{mM}$ glycine at $25^{\circ} \mathrm{C}$ for additional $4 \mathrm{~h}$, and their activation state as well as their purity were analyzed by SDS-PAGE as shown in Figure 17.

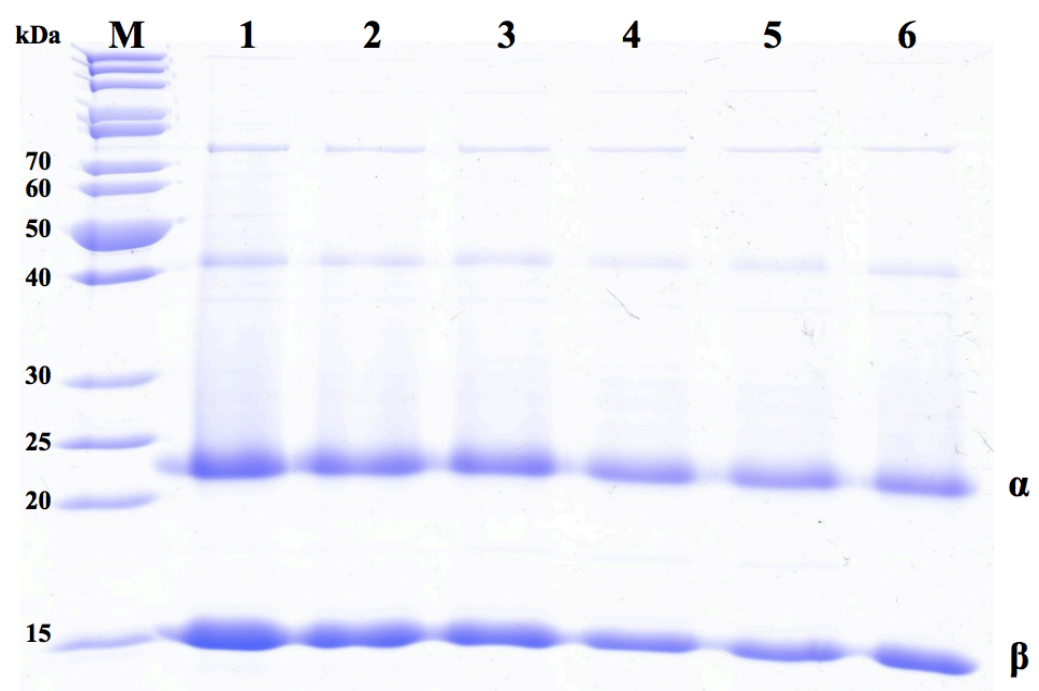

Figure 17. 15\% SDS-PAGE analysis of hASNase 3 mutants. The figure shows the purity and the activation status of wildtype and the five hASNase 3 mutants described above. Lane 1: wildtype; Lane 2: DM1; Lane 3: DM2; Lane 4: DM3; Lane 5: QDM1; Lane 6: QDM2. The two generated subunits $\alpha$ and $\beta$ are the major protein bands. The electrophoretic profiles of the enzymes reflect the final purity obtained after the last gel filtration step for the removal of the His6-SUMO tag. Autoproteolytic processing of the enzyme precursors (weak bands at 
about $40 \mathrm{kDa}$ ) was induced by including $200 \mathrm{mM}$ glycine in the expression medium, as well as upon further treatment with $500 \mathrm{mM}$ glycine immediately after the first affinity purification step.

As shown in Figure 17, all five hASNase3 variants exhibited autoactivation behavior similar to the wildtype enzyme, and glycine treatment accelerated their intramolecular cleavage. This fact indicates that the introduced mutations at these specific regions did not influence the ability of the enzyme variants to undergo the autoproteolytic activation step which is crucial for their activity. The second step addressed the question of whether their catalytic properties were improved. It could well be that the selected DM1, DM2 and DM3 variants were simply expressed at higher levels as compared to the wildtype enzyme and, hence, their apparent intracellular activity was higher. Therefore, wildtype hASNase3 and all mutants were characterized by steady-state kinetics applying the NADH-coupled continuous spectrophotometric assay. The tested substrate concentrations covered the range $0-5 \mathrm{~K}_{\mathrm{M}}$, and the obtained V/E (velocity/total enzyme concentration) values were plotted against the respective substrate concentrations. Kinetic constants $\mathrm{K}_{\mathrm{M}}$ and $k_{\text {cat }}$ were calculated from the resulting plots by non-linear regression using the Michaelis-Menten model and analyzed by the SoftZymics software (Igor Pro, Wavemetrics). Figure 18 shows the steady-state kinetic plots for the wildtype, DM1, DM2 and DM3 enzyme species, and Figure 19 for the QDM1 and QDM2 enzymes. All kinetic data are summarized in Table 3. 

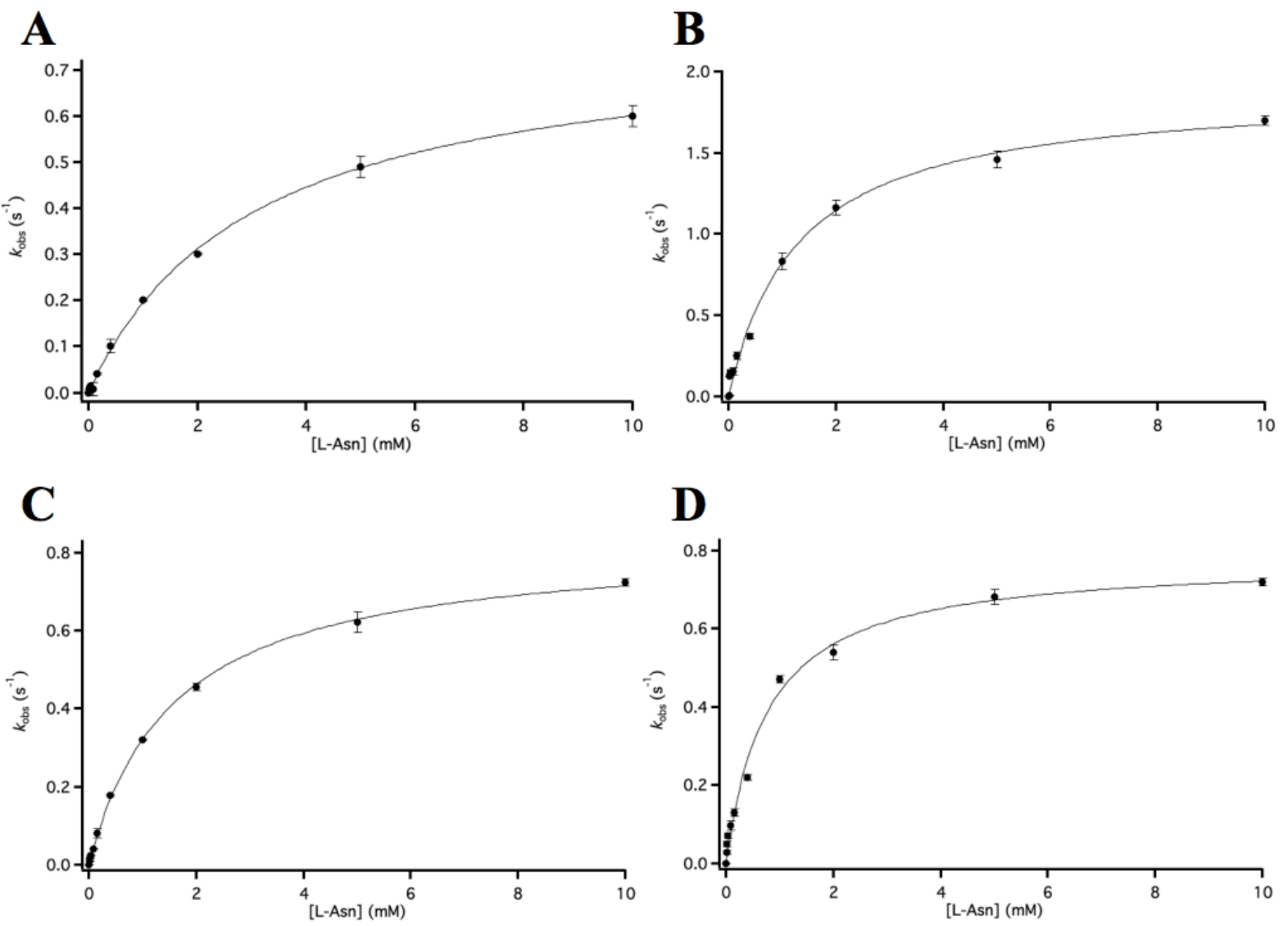

Figure 18. Steady-state kinetic plots for wildtype and mutant hASNase 3 enzymes. V/E versus [L-Asn] plot for (A) wildtype hASNase1, (B) DM1, (C) DM2, and (D) DM3. Activities were measure in $1 \mathrm{~mL} 50 \mathrm{mM}$ Tris-Cl, $100 \mathrm{mM} \mathrm{NaCl}, \mathrm{pH} 8$, at $25{ }^{\circ} \mathrm{C}$, using a final enzyme concentration of $\sim 1 \mu \mathrm{M}\left(\sim 30 \mu \mathrm{g}\right.$ in $1 \mathrm{~mL}$; hASNase $\left.3 M_{\mathrm{r}}: 33 \mathrm{kDa}\right)$. Steady-state turnover rates $\left(\mathrm{s}^{-1}\right)$ are expressed as a function of the substrate concentration. Data points are represented as means \pm SD of duplicate sample measurements. Plots were prepared and analyzed by the SoftZymics software (Igor Pro, Wavemetrics) by non-linear regression using the Michaelis-Menten equation (Section 5.1.2.3, Equation 1).
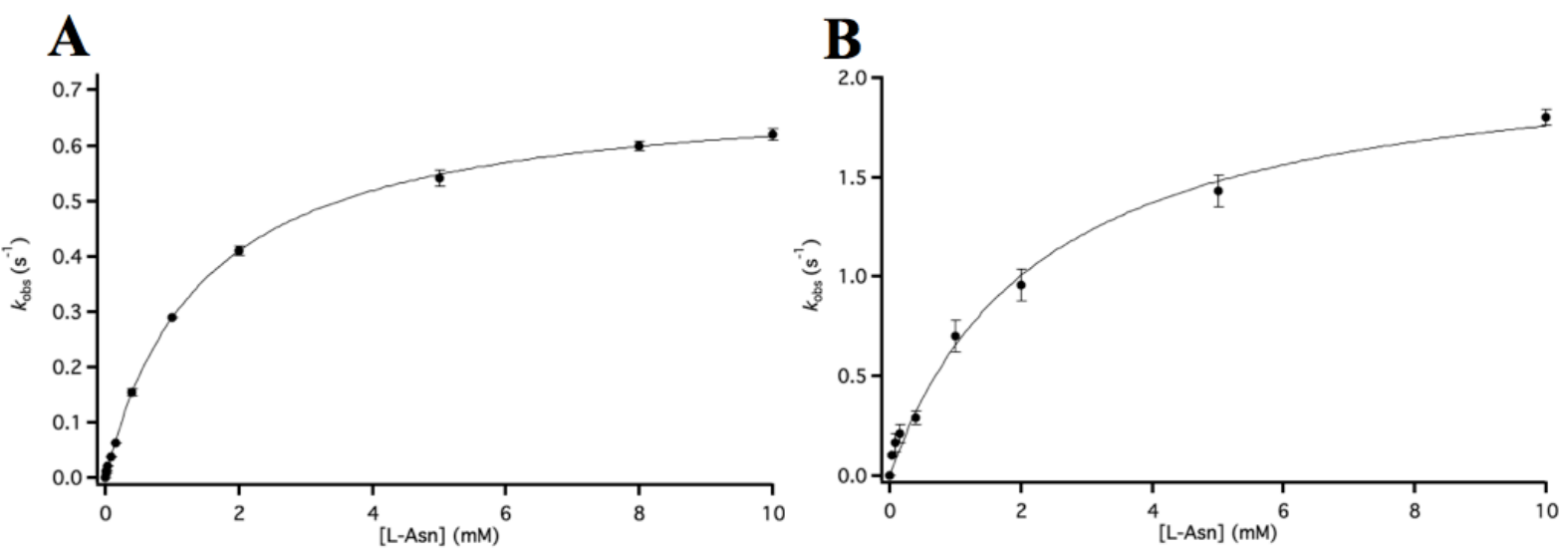

Figure 19. Steady-state kinetic plots for QDM1 and QDM2 hASNase3 enzymes. V/E versus [L-Asn] plot for (A) QDM1 and (B) QDM2. Activity measurements were performed and plots were generated as detailed in Fig. 18 legend. 
Table 3. Steady-state kinetic constants for wildtype hASNase3 and all mutants selected by FACS analyses. The constants were calculated by non-linear regression analysis using the Michaelis-Menten model. The values are represented as means \pm standard deviation of duplicated measurements of a representative experiment.

\begin{tabular}{c|c|c|c}
\hline Enzyme & $\boldsymbol{k}_{\text {cat }}\left(\mathbf{s}^{-1}\right)$ & $\mathbf{K}_{\mathbf{M}}(\mathbf{m M})$ & $\boldsymbol{k}_{\text {cat }} / \mathbf{K}_{\mathbf{M}}\left(\mathbf{M}^{-1} \mathbf{s}^{-1}\right)$ \\
\hline wildtype hASNase3 & $0.78 \pm 0.016$ & $3 \pm 0.16$ & $260 \pm 0.16$ \\
\hline DM1 (Ile189Thr-Val190Ile) & $1.89 \pm 0.07$ & $1.3 \pm 0.16$ & $1450 \pm 0.44$ \\
\hline DM2 (Ile189Val-Val190Ile) & $0.83 \pm 0.01$ & $1.58 \pm 0.06$ & $525 \pm 0.17$ \\
\hline DM3 (Arg143Glu-Arg147Lys) & $0.78 \pm 0.03$ & $0.78 \pm 0.1$ & $1000 \pm 0.3$ \\
\hline $\begin{array}{c}\text { QDM1 } \\
\text { (Ile189Thr-Val190Ile-Arg143Glu- } \\
\text { Arg147Lys) }\end{array}$ & $0.7 \pm 0.01$ & $1.46 \pm 0.03$ & $480 \pm 0.33$ \\
\hline $\begin{array}{c}\text { QDM2 } \\
\text { (Ile189Val-Val190Ile-Arg143Glu- } \\
\text { Arg147Lys) }\end{array}$ & $2.2 \pm 0.11$ & $2.3 \pm 0.33$ & $950 \pm 0.33$ \\
\hline \multicolumn{2}{c}{} & & \\
\hline
\end{tabular}

All five hASNase 3 variants are characterized by improved kinetic constants $\left(k_{\text {cat }}\right.$ and $\left.\mathrm{K}_{\mathrm{M}}\right)$ in comparison to the wildtype enzyme. More specifically, the DM1 variant carrying the Ile189Thr-Val190Ile mutations and pulled from SSM-LIB3, displayed an $\sim$ 6-fold improvement regarding the overall catalytic efficiency $k_{\mathrm{cat}} / \mathrm{K}_{\mathrm{M}}$ as compared to the wildtype. Interestingly, both kinetic constants were improved; the turn-over increased 2.5-fold, and the $\mathrm{K}_{\mathrm{M}}$ value decreased 2.3-fold. In contrast, the second mutant which was isolated from the same library, Ile189Val-Val190Ile, showed a 2-fold improvement with respect the $k_{\text {cat }} / \mathrm{K}_{\mathrm{M}}$. The $k_{\text {cat }}$ of this variant is similar to that of the wildtype enzyme $\left(\sim 0.8 \mathrm{~s}^{-1}\right)$, but the binding affinity against L-Asn was 2-fold increased as evidenced by the lower $\mathrm{K}_{\mathrm{M}}$ value $(1.6 \mathrm{mM}$ versus $3 \mathrm{mM}$ for the wildtype). The DM3 variant, which was isolated from the SSM-LIB4 and which was the only mutant to be identified as catalytically improved from this library, showed approximately 4-times amelioration regarding the $k_{\mathrm{cat}} / \mathrm{K}_{\mathrm{M}}$. As with DM2, the turnover number of DM3 is similar to that from the wildtype, unlike the $\mathrm{K}_{M}$ value, which got improved $\sim 4$-fold $(0.77 \mathrm{mM})$.

On the other hand, the rationally designed quadruplet mutants QDM1 and QDM2, which reflect an attempt to combine the improved characteristics of the pulled double mutants, showed a certain degree of improvement, but overall they did not exceed the highest level of 
advancement that was exhibited by the DM1 variant (6-fold). The QDM1 (Ile189ThrVal190Ile-Arg143Glu-Arg147Lys), which is a combination of DM1 and DM3 variants, inherited almost the same catalytic constant from DM3 $\left(0.7 \mathrm{~s}^{-1}\right)$ and similar $\mathrm{K}_{M}$ value from DM1 (1.45 mM), resulting in a 2-fold $k_{\text {cat }} / \mathrm{K}_{\mathrm{M}}$ overall improvement compared to the wildtype. The second quadruplet mutant QDM2 comprised the two mutations from DM2 (Ile189ValVal190Ile) and the two from DM3 (Arg143Glu-Arg147Lys). Interestingly, this variant showed the highest $k_{\text {cat }}$ value among all the mutants which is $\sim 2.2 \mathrm{~s}^{-1}$. However, simultaneously, it has the highest $\mathrm{K}_{\mathrm{M}}$ among all the variants $(2.3 \mathrm{mM})$ which resembles the one from the wildtype enzyme $(3 \mathrm{mM})$. These kinetic constants of QDM2 rank this variant as the second most improved one together with DM3, having a $k_{\text {cat }} / K_{M} \sim 950 \mathrm{M}^{-1} \mathrm{~s}^{-1}\left(1000 \mathrm{M}^{-1} \mathrm{~s}^{-1}\right.$ for DM3).

In summary, the steady-state kinetic characterization of the individual variants which were pulled from the two SSM libraries (SSM-LIB3 \& SSM-LIB4) confirmed the fluorescence enrichment of the final sorted cell population from the FACS screening. The increase of the fluorescence mean can be attributed to the improved catalytic properties of DM1, DM2 and DM3, which provided higher amounts of L-Asp in the intracellular environment, thereby promoting the expression of eGFP and, in turn, increasing the fluorescent signal. The combination of the best variants' mutations resulted in the generation of two quadruplet enzymes, though without further improvement in comparison to their precursor molecules. In a last characterization step, the impact of the mutations on the stability of the hASNase 3 variants was studied by differential scanning fluorimetry (DSF).

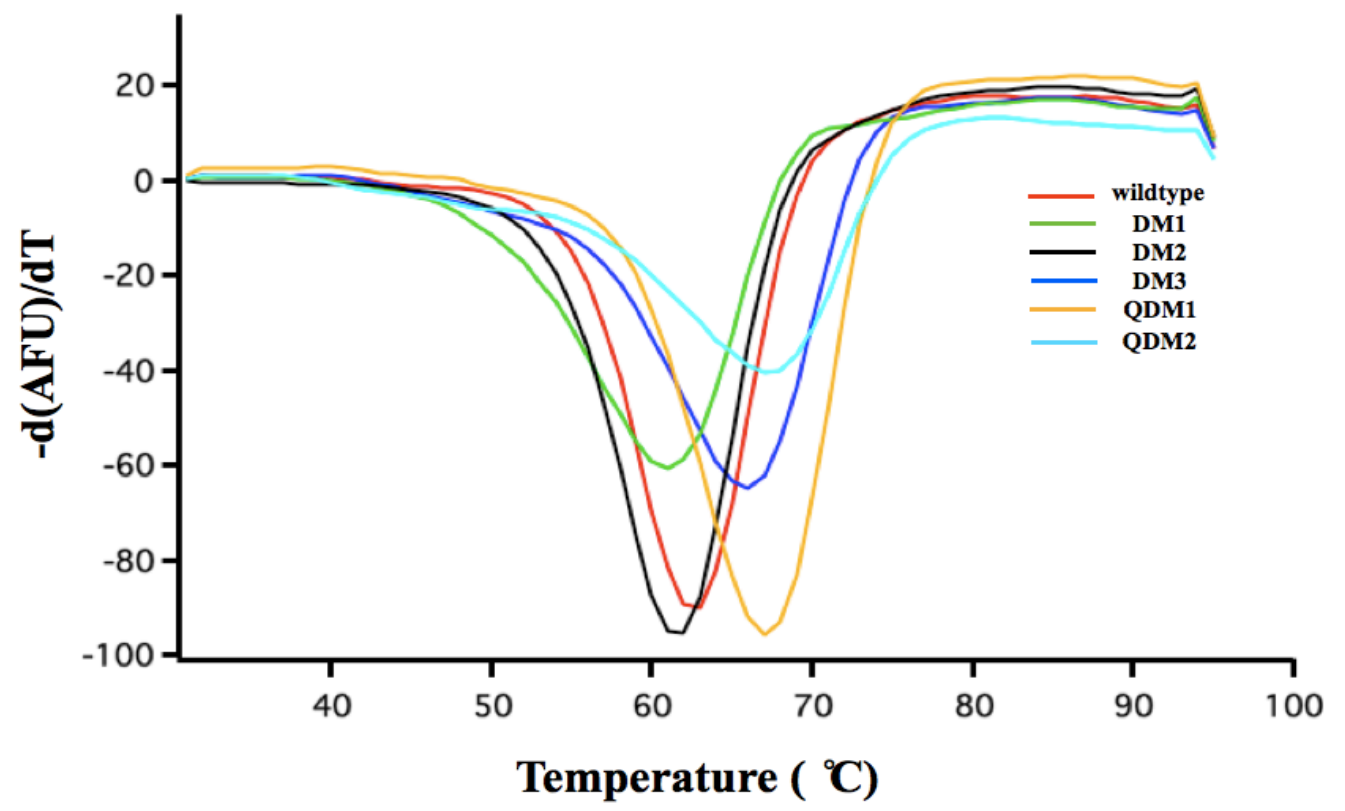


Figure 20. Differential scanning fluorimetry (DSF) melting curves for wildtype and mutant hASNase3 enzymes. Enzyme samples from $-20{ }^{\circ} \mathrm{C}$ glycerol stocks were dialyzed (Pierce, Slide-A-Lyzer, 10,000 MWCO) against $50 \mathrm{mM}$ Tris-Cl, $0.1 \mathrm{NaCl}, \mathrm{pH} 8$, to remove glycerol, and were mixed with SYPRO Orange (Sigma-Aldrich) in a final volume of $20 \mu \mathrm{L}$. The final concentrations of the enzyme and the dye were $1 \mu \mathrm{M}$ and $10 \% \mathrm{v} / \mathrm{v}$, respectively. The enzyme-melting experiments were performed using a CFX96 RT-PCR machine (BioRad) with the following settings: 2 min pre-warming step at $30{ }^{\circ} \mathrm{C}$, and subsequent gradient between $31-95{ }^{\circ} \mathrm{C}$ with $1{ }^{\circ} \mathrm{C} / \mathrm{min}$ increments. SYPRO Orange fluorescence was monitored using FAMex $(492 \mathrm{~nm})$ and ROXem $(610 \mathrm{~nm})$ filters. Data were exported as Excel-based worksheet and further analyzed by Igor-Pro (Wavemetrics). Melting temperatures $(T \mathrm{~m})$ were obtained by plotting the first derivative $\mathrm{d}(\mathrm{AFU}) / \mathrm{dT}$ of the raw data as a function of temperature increase.

The results of the DSF analysis indicated that the different mutations had distinct influence on the stability of hASNase3. As shown in Figure 20, the melting curves for all hASNase3 enzymes were monophasic, with some of them having stabilizing and others destabilizing effects. More specifically among the double mutants, the only one which exhibited a higher melting temperature $\left(T_{\mathrm{m}}\right)$ in comparison to the wildtype is DM3 (Arg143Glu-Arg147Lys) with a $T_{\mathrm{m}}$ value $\sim 66.5^{\circ} \mathrm{C}$ against $62.5^{\circ} \mathrm{C}$ for the wildtype. Both DM1 (Ile189Thr-Val190Ile) and DM2 (Ile189Val-Val190Ile), which were isolated from the same SSM-LIB3, showed lower $T_{\mathrm{m}}$ values, 61 and $61.8^{\circ} \mathrm{C}$, respectively. Strikingly, the quadruplet mutants displayed the highest $T_{\mathrm{m}}$ values which were considerably improved in comparison to the wildtype enzyme: $68{ }^{\circ} \mathrm{C}$ for both of them. These data strongly suggest that the variant with the most improved catalytic properties (DM1) showed the lowest melting temperature $\left(61{ }^{\circ} \mathrm{C}\right)$ and consequently lowest stability; this also holds true for the second mutant isolated from the same library (DM2 with $T_{\mathrm{m}} 61.8$ ), though being catalytically 3-fold less improved. 


\subsection{Development of a droplet-based microfluidic screening system}

\subsubsection{Materials}

\subsubsection{Plasmids, E.coli strains and chemicals}

All the materials and reagents for the microfluidic chip fabrication and the droplet formation (fluorinated oil, surfactants, fluorescein) were contributed from Dr. Jean-Christophe Baret's lab. The materials for the droplet formation include HFE7500 fluorinated oil (Novec, 3M), PFPE-PEG-PFPE surfactant (home-made synthesis [274]), and fluorescein sodium salt (Sigma-Aldrich) which was used as droplet tracer.

All the molecular biology and biochemistry reagents used in this study are described in detail in the description of the previous FACS-based screening system section unless otherwise stated. Additionally, the plasmid APEx1 [275] was used for the EcASNase2 anchoring in the inner membrane of E.coli cells, as well as the pUC8 vector for the cytoplasmic expression of eGFP. APEx1 was a kind giftfrom Dr. George Georgiou's lab (University of Texas at Austin, Department of Chemical Engineering and Institute for Cellular and Molecular Biology). APEx1 is a medium copy number plasmid (range 50-70) and has the chloramphenicol antibiotic resistance marker. It is derived from the plasmid pMoPac1 after replacement of the pelB leader sequence by the leader peptide and the first six amino acids of the mature NlpA E.coli inner membrane lipoprotein [155]. pUC8 is a high copy number plasmid (range of 400-500) with ampicillin antibiotic resistance marker.

Table 1. Oligonucleotides used in this study. The recognition sites for the restriction endonucleases are in italics (SfiI: GGCCCAGCCGGCC; NdeI: CATATG; BamHI: GGATCC), and the His6-tag codons in bold.

\begin{tabular}{|l|l|}
\hline Primer & Sequence \\
\hline FW_APEx1-EcASNase2 & CGA GGCCCAGCCGGCC ATG TTA CCC \\
AAT ATC ACC ATT TTA GCA ACC GGC GGG \\
\hline RV_APEx1-EcASNase2 & CATC GGCCTCGGGGGCC TTA GTA CTG \\
& ATT GAA GAT CTG CTG GAT CTG CTG \\
\hline
\end{tabular}




\begin{tabular}{|l|l|}
\hline Primer & Sequence \\
\hline RV_APEx1-EcASNase2-His6-C & $\begin{array}{l}\text { CATC GGCCTCGGGGGCC } \\
\text { TTAATGATGATGATGATGATGGTACTGAT } \\
\text { TGAAGATCTGCTGGATCTGCTG }\end{array}$ \\
\hline RV_pET14b-SUMO-EcASNase2-His6-C & $\begin{array}{l}\text { CGCGGATCCTTAATGATGATGATGATGAT } \\
\text { GGTACTGATTGAAGATCTGCTGGA }\end{array}$ \\
\hline FW_eGFP & $\begin{array}{l}\text { GGAATTCCATATGGTGAGCAAGGGCGAGG } \\
\text { AGCTG }\end{array}$ \\
\hline RV_eGFP & CGCGGATCCTTACTTGTACAGCTCGTCCAT \\
GCCG
\end{tabular}

\subsubsection{Methods}

\subsubsection{Cloning of EcASNase2 and eGFP}

In the present study, overall five constructs were used: pET14b-SUMO-EcASNase2, pET14b-SUMO-EcASNase2-His6-C, APEx1-EcASNase2, APEx1-EcASNase2-His6-C, and pUC8-eGFP. The plasmid pET14b-SUMO-EcASNase2 is the one which has been used for the recombinant expression and purification of EcASNase2: the construction of this plasmid has been described elsewhere [257]. The plasmid pET14b-SUMO-EcASNase2-His6-C was constructed for the needs of the present study for having the enzyme carrying the His 6 -tag at its $\mathrm{C}$-terminus for immunoblotting analysis using anti-His6- $\mathrm{C}$ tag antibodies. The construction of this plasmid was done similar to the plasmid pET14b-SUMO-EcASNase2 after PCRamplifying the EcASNase2-His6-C insert with a reverse primer containing six additional codons for the His6-tag (Oligonucleotides are shown in Table 1). Plasmids APEx1EcASNase2, APEx1-EcASNase2-His6-C, and pUC8-eGFP were constructed analogously (see Methods section in FACS-based screening system, section 5.1.2.1). APEx1-EcASNase2 expressed untagged $E c$ ASNase2 and was used for all enzymatic activity measurements at the single-cell level in droplets. In contrast, APEx1-EcASNase2-His6-C produces the enzyme with a C-terminal His6-tag and served exclusively as a probe for the anchoring confirmation of EcASNase2 at the inner membrane of E.coli cells by means of immunoblotting. The eGFP protein encoded by pUC8-eGFP does not carry any tag, neither at the N- nor at the C- 
terminus, and the expression of eGFP is under the regulation of the lac promoter, which is inducible by IPTG. This plasmid was used solely for the intracellular expression of eGFP in E.coli cells, which served as a visualization tool in order to follow the number of encapsulated cells in droplets. The plasmids used in this study are summarized in Figure 1, and the used oligonucleotides are shown in Table $\mathbf{1 .}$

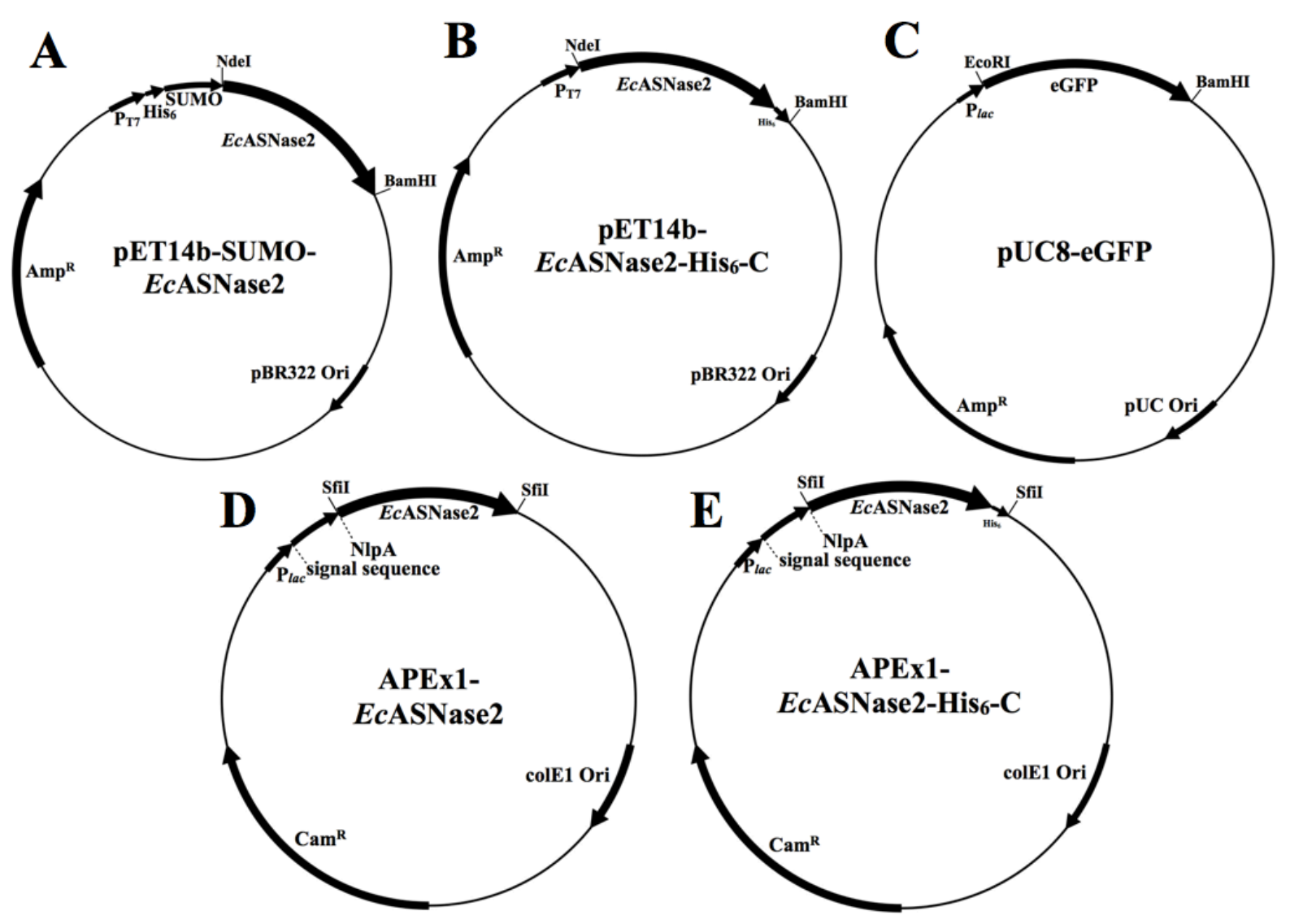

Figure 1. Schemes of plasmids used in this study. (A) pET14b-SUMO-EcASNase2, (B) pET14b-SUMO-EcASNase2-His 6 -C, (C) pUC8-eGFP, (D) APEx1-EcASNase2, and (E) APEx1-EcASNase2-His6-C. The figure was prepared using XPlasMap and Keynote.

\subsubsection{Droplet-based microfluidic system: Preparation and quantitative} analysis of $E c$ ASNase2 activities in drops

The activity of purified EcASNase2 was measured in $600 \mathrm{pL}$ droplets (radius $\sim 50 \mu \mathrm{m}$ ) applying the three-step Amplex Red-based assay as described elsewhere [265]. Briefly, the reaction took place at $25{ }^{\circ} \mathrm{C}$, and the assay mixture contained different amounts of 
EcASNase2, $250 \mu \mathrm{M}$ L-Asn, $\sim 4 \mu \mathrm{M}$ L-AspOx $(\sim 250 \mu \mathrm{g} / \mathrm{mL}), 10 \mu \mathrm{M}$ FAD, $\sim 100 \mathrm{nM}$ HRP $(\sim 0.1 \mathrm{U} / \mathrm{mL})$ and $6.5 \mu \mathrm{M}$ Amplex Red. The droplet experiments were standardized using a lower concentration of Amplex Red, instead of $50 \mu \mathrm{M}$ as described in [265], because the signal was more stable, and unforeseeable background artifacts were observed when higher concentrations were tested. The linearity of the three-step coupled-enzyme reaction as a function of the L-asparaginase concentration was tested using a wide range of EcASNase2 concentrations $(0-250 \mathrm{ng} / \mathrm{mL})$. Subsequently, using an enzyme concentration which falls within the linear range, steady-state kinetic analysis was performed in droplets by varying the concentration of L-Asn in the range of $0-5 \mathrm{~K}_{\mathrm{M}}$. The results were compared to those obtained by carrying out the assay in 96 -well plates (final volume $100 \mu \mathrm{L}$ per well) following the course of the reaction continuously by a fluorescence plate reader (Molecular Devices, SpectraMax Paradigm, Ex 532/Em $592 \mathrm{~nm}$ ). The resulting data were additionally compared to those obtained from the standard spectrophotometric activity determination in a $1 \mathrm{~mL}$ cuvette using a Uvikon 943 double beam (UV/VIS) spectrophotometer [265]. The microfluidic chip which was used for the activity measurements is shown in Figure 2, and the overall experimental setup is shown in Figure 3.

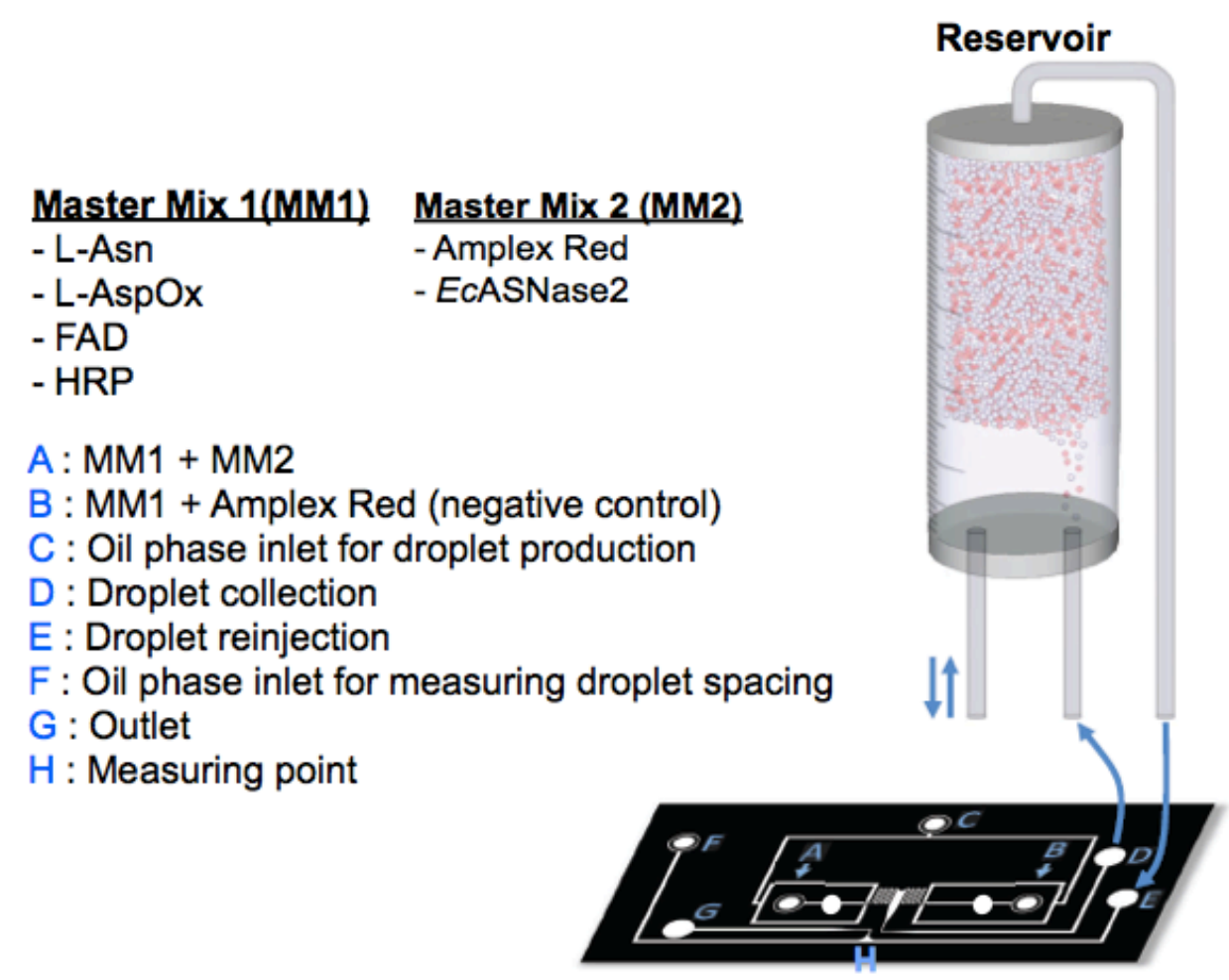

Figure 2. Microfluidic chip $(\sim 1 \times 4 \mathrm{~cm})$ used for the recombinant EcASNase2 activity measurements. Two master mixtures were prepared for monitoring of the enzymatic activity. The two Master Mixtures MM1 and MM2, which represent the aqueous phase, consisted of the assay reagents as shown in the Figure and were mixed in a 1:1 volume ratio (spot A; the 
initial concentration of the compounds was $2 \mathrm{X}$ ending up to $1 \mathrm{X}$ upon mixing the two aqueous phases). The same mixtures without EcASNase 2 served as negative control and represent the second droplet population in the chip (spot B). The droplets were formed upon mixing the aqueous (flow rate $10 \mu \mathrm{L} / \mathrm{min}$ ) and the oil phases (coming from spot $\mathrm{C}$ with flow rate $80 \mu \mathrm{L} /$ min) consisting of fluorinated oil (HFE 7500): The final volume of the water-in-oil droplets was $600 \mathrm{pL}$. To prevent coalescence and prepare stable droplets, $0.5 \% \mathrm{w} / \mathrm{w}$ surfactant [276] was added in the oil phase. Droplets in which the enzymatic reaction took place were labeled with $1 \mu \mathrm{M}$ fluorescein, and the negative control (no enzyme) contained $0.5 \mu \mathrm{M}$ fluorescein, in order to distinguish the two types of droplets by their fluorescence signal amplitudes and follow their integrity in the chip. Approximately $10^{6}$ droplets were produced and were stored in the oil-filled reservoir. Subsequently, the droplets were re-injected in the chip (spot E), and the fluorescent signal was measured (spot H). In order to control the spacing between the droplets, an additional oil phase was applied (spot F). The fluorescence readout frequency was $\sim 100-200$ droplets per second. The fluorescence intensity per droplet was plotted against the droplet population, and the arithmetic fluorescence mean was calculated by a Gaussian distribution fitting [277]. (The chip and the reservoir part of the figure were adapted from [278], while the final figure was prepared using Keynote).
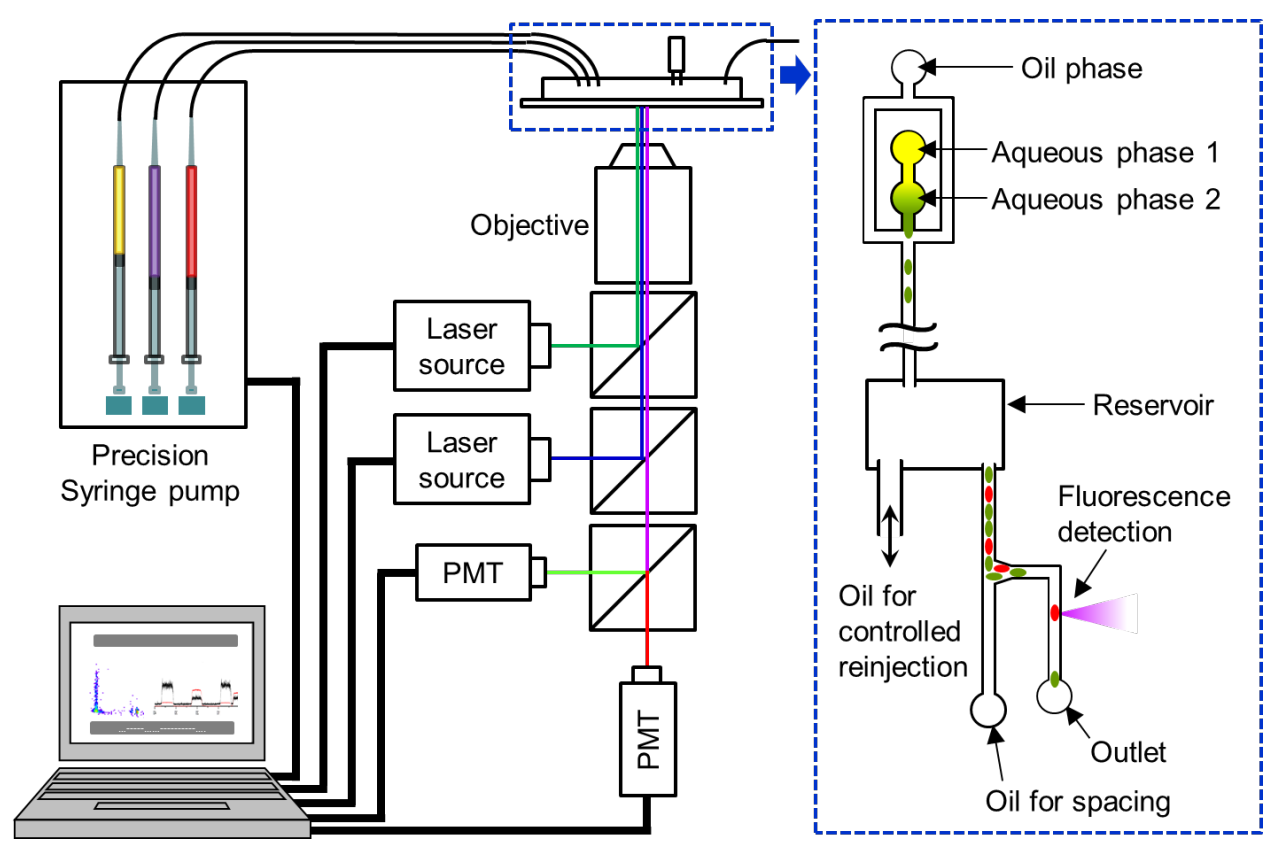

Figure 3. Schematic experimental setup for monitoring the activity of EcASNase2 in droplets. The flow rates of the aqueous and oil phases were adjusted and controlled by precision syringe pumps (Nemesis, Cetoni $\mathrm{GmbH}$ ). Two-color laser-induced fluorescence detectors were used, with 473 and $532 \mathrm{~nm}$ diodes and a photomultiplier (PMT) tube. A 20X objective lens focused the laser on the chip's microchannels. Fluorescent signals were monitored in real-time using Labview software (The figure was kindly provided by Dr. JeanChristophe Baret's lab). 


\subsubsection{Preparation and encapsulation of eGFP-expressing cells}

We used bacterial cells expressing eGFP as visualization means for the determination of the appropriate cell dilution conditions for achieving encapsulation of a defined number of cells per droplet. E.coli C41(DE3) cells were transformed with pUC8-eGFP and were cultured in $50 \mathrm{~mL} 2 \mathrm{xYT}$ medium supplemented with $200 \mu \mathrm{g} / \mathrm{mL}$ ampicillin. When $\mathrm{OD}_{600}$ reached 0.6-0.8, IPTG was added in the culture to a final concentration of $1 \mathrm{mM}$ to induce the expression of eGFP. The culture was grown for additional $4 \mathrm{~h}$ at $37{ }^{\circ} \mathrm{C}$; then, $10 \mathrm{~mL}$ were centrifuged for $10 \mathrm{~min}$ at 5,000g, washed 5X with PBS buffer, and finally were resuspended in PBS at a final $\mathrm{OD}_{600} \sim 1$, which corresponds to $\sim 10^{9}$ cells per $\mathrm{mL}$ as determined by the serial dilution method [279]. A $10 \mu \mathrm{L}$ aliquot of the latter suspension was placed onto a microscope slide and visually inspected to confirm eGFP expression using an Olympus SZX12 stereo fluorescence microscope with the following settings: DFPLFL 1.6X PF objective and 144X magnification using a 10X eyepiece. Once the eGFP expression was confirmed, different dilutions of cells were prepared and encapsulated in droplets, which were "arrested" by modifying the flow rate in special well-like microchannels which allow holding the droplets immobilized; cell-containing droplets were further visualized using a fluorescence microscope to determine the average number of cells per droplet by visual inspection.

\subsubsection{Cytoplasmic expression of EcASNase2 and trials for lysing the cells in droplets for subsequent enzymatic activity determination}

Initially, we encapsulated E.coli cells which were expressing cytoplasmically the EcASNase2 enzyme, considering the fact that cytoplasmic expression offers by far higher expression yields as compared to periplasmic or display approaches. However, this approach necessitates the lysis of the E.coli cells aiming at the release of the intracellularly produced enzyme, in order to make it accessible to the substrate for the subsequent enzymatic reactions to take place. To this end, we tested three types of lysing agents upon cell encapsulation in the droplets, and attempted to measure L-asparaginase activity of EcASNase2 by applying the 3step Amplex Red fluorescence assay [265]: (i) The first trial relied on the use of hen egg lysozyme at a final concentration of $2 \mathrm{mg} / \mathrm{mL}$ in $50 \mathrm{mM}$ Tris-Cl, $2 \mathrm{mM}$ EDTA, $25 \mathrm{mM} \mathrm{NaCl}$, 
$\mathrm{pH}$ 8. In this buffer, which constituted the first aqueous phase, all the assay compounds were added (L-Asn, FAD, HRP and L-AspOx, all at the concentrations described above), while the Amplex Red was mixed with the cells in $50 \mathrm{mM}$ Tris-Cl, $2 \mathrm{mM}$ EDTA, $25 \mathrm{mM} \mathrm{NaCl}, \mathrm{pH}$, forming the second aqueous phase. At this point, it must be emphasized that the upper concentrations refer to the final ones. Since both aqueous phases were mixed in a 1:1 ratio, the initial buffer concentrations were prepared as $2 \mathrm{X}$ solutions. (ii) A second attempt for lysing the cells in the droplets included the lysozyme solution described above in combination with the compound Polymyxin B sulfate (Sigma-Aldrich). Polymyxin B is a cationic polypeptide antibiotic, naturally synthesized by the microbe Bacillus polymyxa, and it is widely used a lysing agent for gram negative bacteria [280]. The final concentration of Polymyxin B was $4 \mathrm{mg} / \mathrm{mL}$. (iii) Finally, as an alternative means of cell lysis, we used the commercially available BugBuster solution (Novagen) which consists of detergents capable of promoting cell lysis. This solution was combined with a special version of commercial lysozyme (rLysozyme, Novagen) which has been shown to be 250 -fold more efficient than the standard hen egg lysozyme (Company's Instructions Manual). The buffer used for this trial consisted of $50 \mathrm{mM}$ Tris-Cl, $100 \mathrm{mM} \mathrm{NaCl}, 0.1 \mathrm{mM}$ EDTA, $1 \mathrm{mM}$ DTT, $0.1 \%$ Triton $\mathrm{X}-100, \mathrm{pH}$ 7.5. The final cell-lysis mixture contained $1 \mathrm{KU}$ (i.e. 1,000 Units based on the supplier's unit definition) rLysozyme, 1X BugBuster protein extraction reagent (provided commercially as $10 \mathrm{X}$ solution), and all the assay compounds at concentrations as described above. The mixtures of cells, lysing agents and assay compounds were incubated for 30-40 min in droplets, followed by fluorescence signal measurement to evaluate the enzymatic activity profiles.

\subsubsection{Anchoring, detection, and quantification of EcASNase2 displayed in the inner membrane of $E$.coli cells.}

(A) Anchoring of EcASNase2 in the inner membrane of E.coli C41(DE3). E.coli cells harboring the pAPEx1-EcASNase2 plasmid were grown overnight at $37^{\circ} \mathrm{C}$ in $2 x Y T$ medium, supplemented with $35 \mu \mathrm{g} / \mathrm{mL}$ chloramphenicol. A fraction of this culture was used to inoculate a fresh $50 \mathrm{~mL} 2 \mathrm{xYT}$ culture (dilution 1:50). When $\mathrm{OD}_{600}$ reached 0.6-0.8, IPTG was added to a final concentration of $1 \mathrm{mM}$, and the culture was placed at $37^{\circ} \mathrm{C}$ under shaking conditions. At different time intervals (up to $10 \mathrm{~h}$ ) after addition of IPTG, 5-mL fractions from the main culture were centrifuged, washed $5 \mathrm{X}$ with PBS, and finally resuspended in 
PBS and left on ice. Whole-cell lysates using $\sim 10^{9}$ cells (this number corresponds to an $\mathrm{OD}_{600} \sim 1$ as calculated by the serial dilution method), were analyzed by SDS-PAGE aiming at the determination of the expression levels of the displayed enzyme. Whole-cell lysates from untransformed cells, and from cells transformed with the pAPEx1-EcASNase2 plasmid, were analyzed in parallel as negative control. Furthermore, in order to qualitatively investigate the successful anchoring of active EcASNase2, a small fraction of the PBSresuspended cells was mixed with $10 \mathrm{mM} \mathrm{L}-A s n$, incubated at $37^{\circ} \mathrm{C}$ for $10 \mathrm{~min}$, followed by the addition of Nessler's reagent which detects free ammonia, one of the products of the Lasparaginase reaction.

(B) Detection of displayed EcASNase2. Aiming at the confirmation of the display of EcASNase2 in the inner membrane, cells expressing the EcASNase2-His6-C protein were fractionated as described elsewhere [281], and the membranes were used for immunoblotting. Briefly, $\sim 10^{9}$ cells were spun down and resuspended in $20 \mu \mathrm{L}$ of ice-cold solution of $0.1 \mathrm{M}$ Tris-Cl, $0.75 \mathrm{M}$ sucrose, $100 \mu \mathrm{g} / \mathrm{mL}$ hen egg lysozyme, $\mathrm{pH}$ 8. Subsequently, $30 \mu \mathrm{L}$ of $1 \mathrm{mM}$ EDTA were added dropwise, and the mixture was incubated on ice for $10 \mathrm{~min}$, followed by the addition of $5 \mu \mathrm{L} 0.5 \mathrm{M} \mathrm{MgCl}_{2}$ for further $10 \mathrm{~min}$ incubation on ice. The treated cells were centrifuged at $16,500 \mathrm{~g}$ for $10 \mathrm{~min}$, and the supernatant (so-called periplasmic fraction) was decanted. The pellet was resuspended in $50 \mu \mathrm{L} \mathrm{ddH}_{2} 0$ and then subjected to 5 freeze-thawing cycles $\left(-20\right.$ to $\left.30{ }^{\circ} \mathrm{C}\right)$. The resulting suspension was centrifuged at 16,500g for $20 \mathrm{~min}$ and the supernatant (intracellular cytoplasmic fraction) was removed. The pellet, which contained the whole membrane fraction was resuspended in $1 \mathrm{X}$ Laemmli buffer, boiled at $95^{\circ} \mathrm{C}$ for $10 \mathrm{~min}$ and analyzed by immunoblotting using monoclonal mouse anti-His ${ }_{6}-\mathrm{C}$ antibodies (Invitrogen), which selectively recognize C-terminal polyhistidine tags with a free carboxyl group ( $\left.\mathrm{His}_{6}-\mathrm{COOH}\right)$.

(C) Quantification of displayed EcASNase2. For the quantification of the displayed EcASNase2 in the inner membrane, the membrane fraction of $\sim 10^{9}$ cells obtained as described in the previous paragraph at different time points after the addition of IPTG was subjected to immunoblotting analysis. In parallel, known amounts of purified recombinant EcASNase2-His6-C were immunoblotted accordingly. All the resulting bands were analyzed 
by ImageJ [282], and based on the band intensities of known amounts of the purified protein, a standard curve was generated from which the amount of the displayed enzyme was estimated. Consequently, considering the number of the used cells $\left(10^{9}\right)$ for the membrane preparation and the calculated overall displayed enzyme amount, the number of the displayed enzyme molecules was estimated using the enzyme's molecular mass and Avogadro's number $\left(6.023 * 10^{23}\right)$.

\subsubsection{Monitoring L-asparaginase activity of EcASNase2 displayed in E.coli cells.}

Once the display of EcASNase2 in the inner membrane of E.coli cells was confirmed, the next step included the encapsulation of single cells in $600 \mathrm{pL}$ droplets and the subsequent determination of activity of the anchored enzyme by applying the Amplex Red-coupled assay. The assay conditions were identical to those which were used for the activity determination of purified EcASNase2 in droplets [250 $\mu \mathrm{M}$ L-Asn, $\sim 4 \mu \mathrm{M}$ L-AspOx $(\sim 250 \mu \mathrm{g} / \mathrm{mL}), 10 \mu \mathrm{M}$ FAD, $100 \mathrm{nM} \mathrm{HRP}(\sim 0.1 \mathrm{U} / \mathrm{mL})$, and $6.5 \mu \mathrm{M}$ Amplex Red]. The cell dilution conditions and the number of formed droplets based on the eGFP-expressed cells visualization experiment were adjusted such that, in average, one cell is present in ten droplets. The number of cells per droplet can be approximated by a Poisson distribution [283]. The time course of the single-cell L-asparaginase reaction was compared to the time course of the reaction using purified enzyme (under the same droplet volume and assay conditions), and based on the immunoblotting quantification experiments, the amount of anchored enzyme per bacterial cell was estimated.

\subsubsection{Results}

\subsubsection{Validation of the Amplex Red-dependent assay in droplets}

The applicability of the Amplex Red assay was tested using a droplet-based microfluidic setup as shown in Figures 2 and 3. The final volume of the formed droplets was $600 \mathrm{pL}$, and the final assay mixture included $\sim 0.6 \mathrm{nM} \mathrm{EcASNase2}(20 \mathrm{ng} / \mathrm{mL}), 250 \mu \mathrm{M}$ L-Asn (saturated 
substrate conditions was determined in other studies [265]), $\sim 4 \mu \mathrm{M} \mathrm{L}-\mathrm{AspOx}(\sim 250 \mu \mathrm{g} / \mathrm{mL})$, $10 \mu \mathrm{M}$ FAD, $\sim 100 \mathrm{nM}$ HRP $(\sim 0.1 \mathrm{U} / \mathrm{mL})$, and $6.5 \mu \mathrm{M}$ Amplex Red. Two populations of droplets were produced: one with all the assay compounds and the enzyme where the enzymatic reaction took place (positive), and one without enzyme which served as negative control. The positive and negative droplet populations were labeled with 1 and $0.5 \mu \mathrm{M}$ fluorescein, respectively, thereby facilitating the inspection of their formation and their stability throughout the entire time course of the reaction by their 2-fold different fluorescence signals. Figure 4 shows the time course of the enzymatic reaction using $20 \mathrm{ng} /$ $\mathrm{mL}$ of purified recombinant $E c \mathrm{ASNase} 2$.
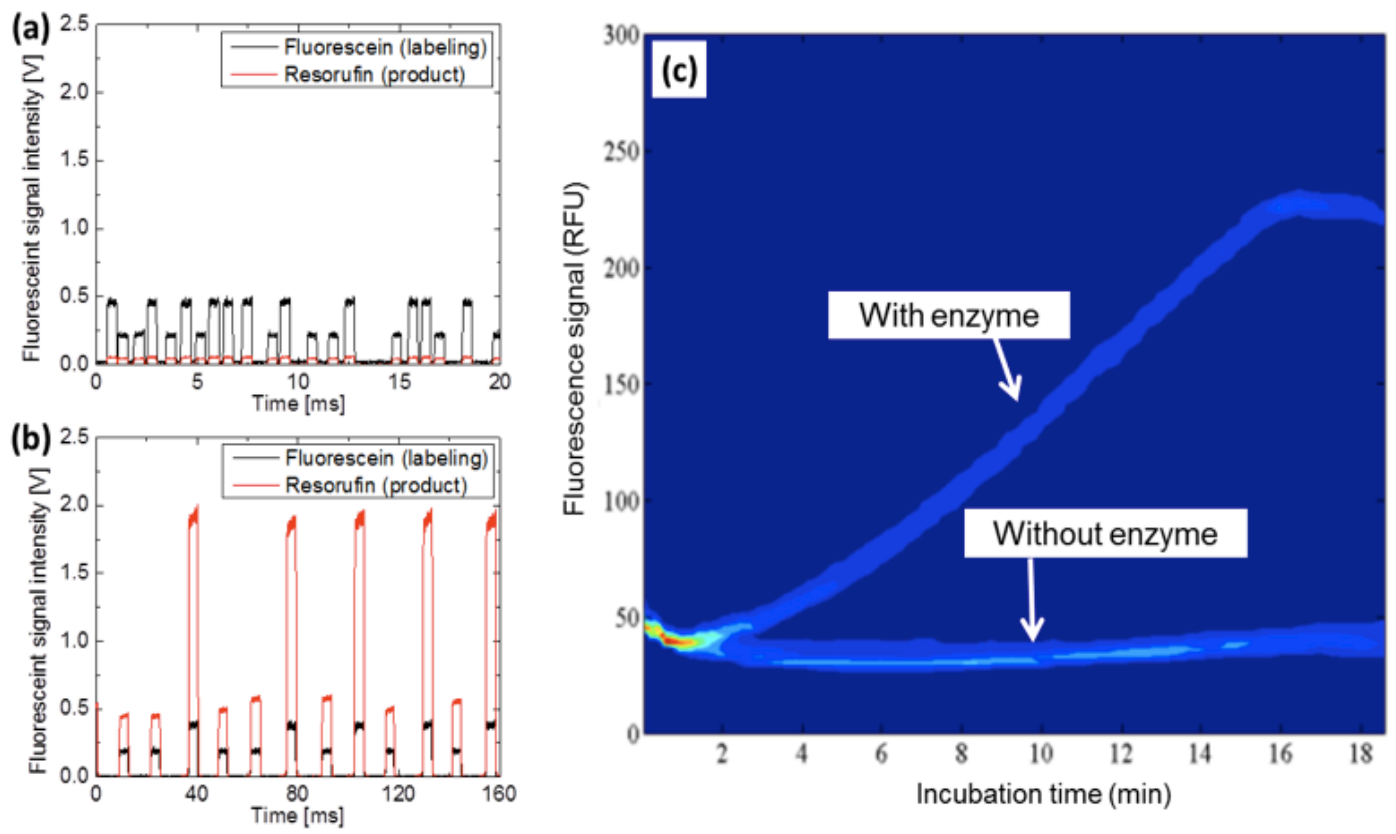

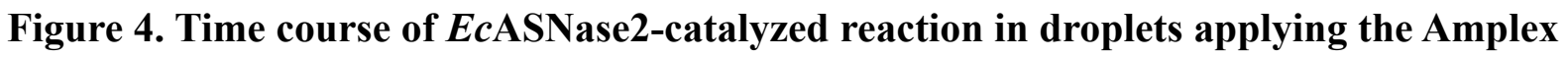
Red assay. Panel (a) shows the fluorescence signal intensity as a function of time after the first $3 \mathrm{~min}$ of the reaction. The black bars correspond to the fluorescein signals of the labeled droplets; the difference of the intensities is due to different fluorescein concentrations which were used for the positive $(1 \mu \mathrm{M})$ and negative $(0.5 \mu \mathrm{M})$ droplets. The red bars reflect the fluorescent signal generated by the product of the enzymatic reaction (Resorufin), which is almost zero after $3 \mathrm{~min}$ of the assay compounds mixing. Panel (b) shows the fluorescence signal intensities $\sim 14$ min after the reaction initiation. This clearly depicts the fluorescent intensity differences between the positive and negative droplets, which are also distinguished by the distinct fluorescein concentrations. The plot in panel (c) shows the overall time course of the reaction containing $0.6 \mathrm{nM} E c$ ASNase $2(20 \mathrm{ng} / \mathrm{mL}), 250 \mu \mathrm{M}$ L-Asn, $\sim 4 \mu \mathrm{M}$ L-AspOx $(\sim 250 \mu \mathrm{g} / \mathrm{mL}), 10 \mu \mathrm{M}$ FAD, $\sim 100 \mathrm{nM}$ HRP $(\sim 0.1 \mathrm{U} / \mathrm{mL})$, and $6.5 \mu \mathrm{M}$ Amplex Red. The reaction took place at $25{ }^{\circ} \mathrm{C}$ and reached saturation $\sim$ after 16 min. Plots (a) and (b) were generated using LabView software, while plot (c) was prepared by using MatLab. 
The next validation steps comprised a linearity test of the assay and the steady-state kinetic characterization of EcASNase 2 in droplets. For the linearity test, the assay components were maintained constant with saturated substrate concentration (250 $\mu \mathrm{M}$ L-Asn), and the EcASNase2 concentration was varied in order to determine the range within which the reaction rate is proportional to the enzyme amount. In parallel, under identical assay conditions, the linearity test was done using a 96-well plate setup, following the reaction in the fluorescent mode with a plate reader. Figure $\mathbf{5 A}$ shows the results of the linearity test in droplets and in a 96-well plate. The data suggest that in both droplets (microscopic volumes) and in a 96-well plate (macroscopic volumes) the system displayed a very similar profile maintaining linearity up to the $200 \mathrm{ng} / \mathrm{mL}$ EcASNase 2 concentration. In contrast, when higher enzyme concentrations were tested, the reaction rate was not increasing further. This can be attributed to limiting amounts of the auxiliary enzymes, notably L-AspOx, and not to HRP, because L-AspOx has poor catalytic properties ( $\mathrm{K}_{\mathrm{M}}$ and $k_{\text {cat }}$ values: $0.5 \mathrm{mM}$ and $0.5 \mathrm{~s}^{-1}$ for L-AspOx against L-Asp; $~ 560 \mathrm{~s}^{-1}$ and $0.1 \mathrm{mM}$ for HRP against hydrogen peroxide at 25 ${ }^{\circ} \mathrm{C}$ ), which cannot follow the catalytic rate of the enzyme which initiates the reaction (EcASNase2 in this case). An increase of the helper enzymes' concentrations can potentially shift the linear trend to higher concentrations of EcASNase2 if necessary.

The concentration of $10 \mathrm{ng} / \mathrm{mL} E c \mathrm{ASNase} 2$ was chosen for subsequent steady-state kinetic analysis of the enzyme in microscopic (droplets) and macroscopic volumes (96-well plate), respectively. The substrate concentration (L-Asn) varied in the range of $0-5 \mathrm{~K}_{\mathrm{M}}$, and the resulted reaction rates (normalized against the maximum rate) were plotted against the substrate concentrations. The data were analyzed by non-linear regression using the Michaelis-Menten model. As shown in Figure 5B, the calculated $\mathrm{K}_{\mathrm{M}}$ from all the experiments is $\sim 20 \mu \mathrm{M}$, and the $k_{\mathrm{cat}} \sim 7 \mathrm{~s}^{-1}$ ( $k_{\mathrm{cat}}$ can be also calculated from plot 5A, where the data are expressed as $\mathrm{nM}$ of product formation per second per concentration of EcASNase2; molecular mass of EcASNase2 $35 \mathrm{kDa}$ ). Both kinetic parameters are very similar to those obtained from previous independent experiments [265]. 

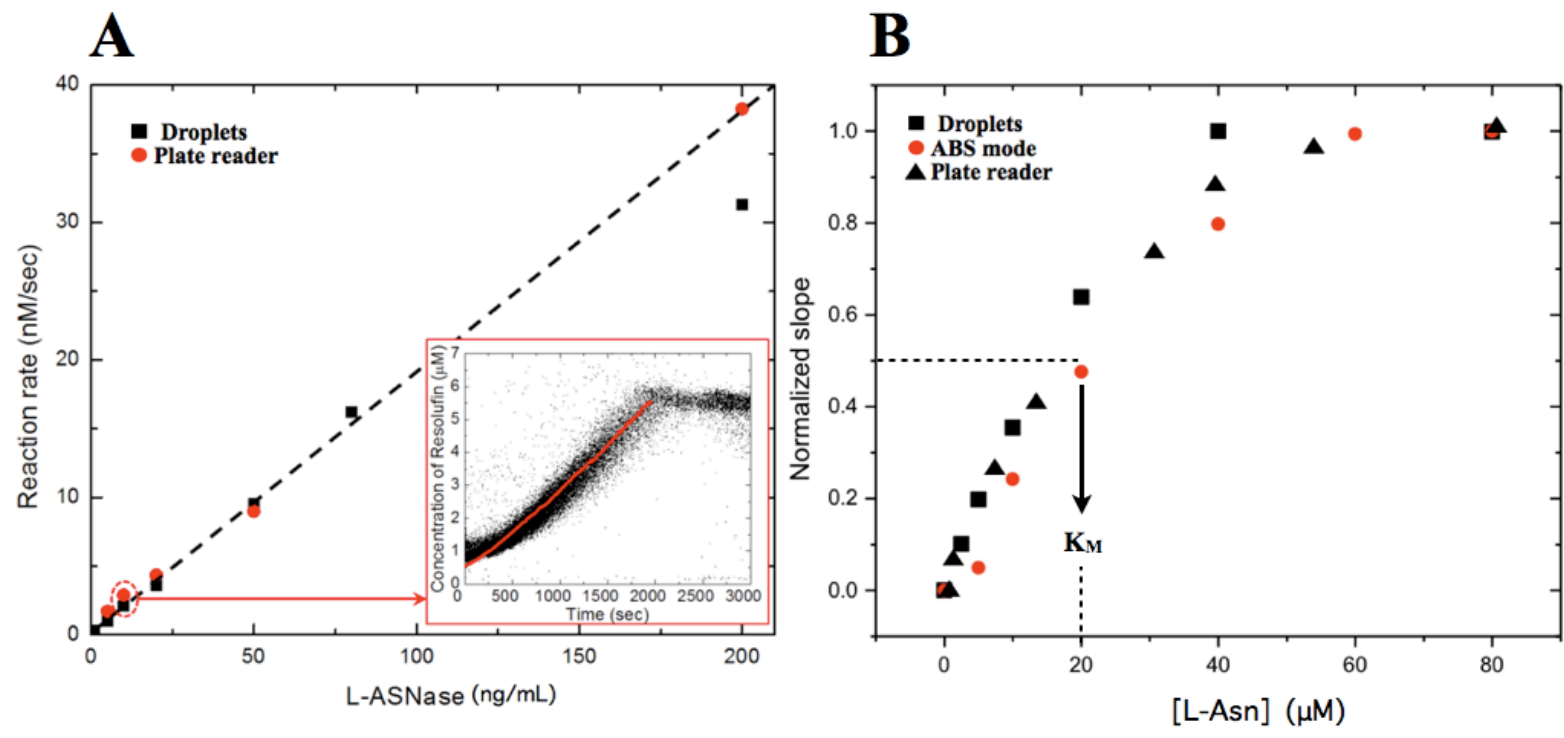

Figure 5. Linearity test (A) and Michaelis-Menten plot (B) for EcASNase2 applying the Amplex Red assay in microscopic and macroscopic volumes. After the investigation of the system's linearity range (plot A), the concentration of $10 \mathrm{ng}$ enzyme $/ \mathrm{mL}$ was chosen for the kinetic characterization of EcASNase2 in droplets and in 96-well plates. The inset of plot A shows the overlap of the time course of the enzymatic reaction in droplets using $10 \mathrm{ng} / \mathrm{mL}$ (accumulated black dots represent data from single droplets), and in a 96-well plate (the red line is the fit of the respective experiment using the same concentration of enzyme in a volume of $100 \mu \mathrm{L}$ per well). Plot B shows the substrate concentration-dependent reaction rates (normalized against the maximum rate) from which the kinetic parameters were determined from the droplet and the plate reader experiments. For comparison, the data points from the assay performed in the absorbance mode (ABS mode) [265] were also included. Plots were generated using Origin 8.5.

Once the fluorescent assay was characterized in droplets using recombinant purified EcASNase2 and the obtained kinetic parameters were found similar to those obtained from macroscopic volume experiments, thus confirming the validity of the assay in microscopic volumes as well, the following step for the development of a high-throughput screening system was undertaken to include the activity determination of EcASNase2 (or any other enzyme of interest) expressed in the host cells (E.coli in our case). Results of these experiments are presented in the following section.

\subsubsection{Encapsulation of single E.coli cells displaying EcASNase2 in droplets and determination of enzymatic activity}

(A) Adjusting the number of cells per droplet. Initially, we wanted to get a rough estimate of the number of cells that must be used in order to ultimately encapsulate $\sim 1$ cell per droplet. 
To this end, we made use of different dilutions of E.coli cells expressing eGFP encapsulating them in the same total number of droplets. Subsequently, the droplets were trapped in specific microchannels in the chip and were visualized by fluorescence microscopy. Figure 6 shows a representative experiment of two dilutions of the eGFP-expressed cells, trapped in microchannels. In Figure $6 \mathrm{~A}$, the number of cells corresponds to $\sim 3 \times 10^{8}$, while in Figure $6 \mathrm{~B}$ the cells are 100 -fold less $\left(\sim 3 \times 10^{6}\right)$. The number of cells was calculated based on the experimentally (serial dilution method) determined equality that an $\mathrm{OD}_{600} \sim 1$ corresponds to $\sim 10^{9}$ cells. Finally, based on the conditions shown in Figure 6B which correspond to OD $_{600} \sim$ 0.002 , we encapsulated the E.coli cells in emulsions with an approximate probability of 1 cell per 10 droplets which results from a Poisson probability distribution based on the number of produced droplets ( 10-fold higher than the number of cells) and the cells which are randomly distributed in the emulsions [284].
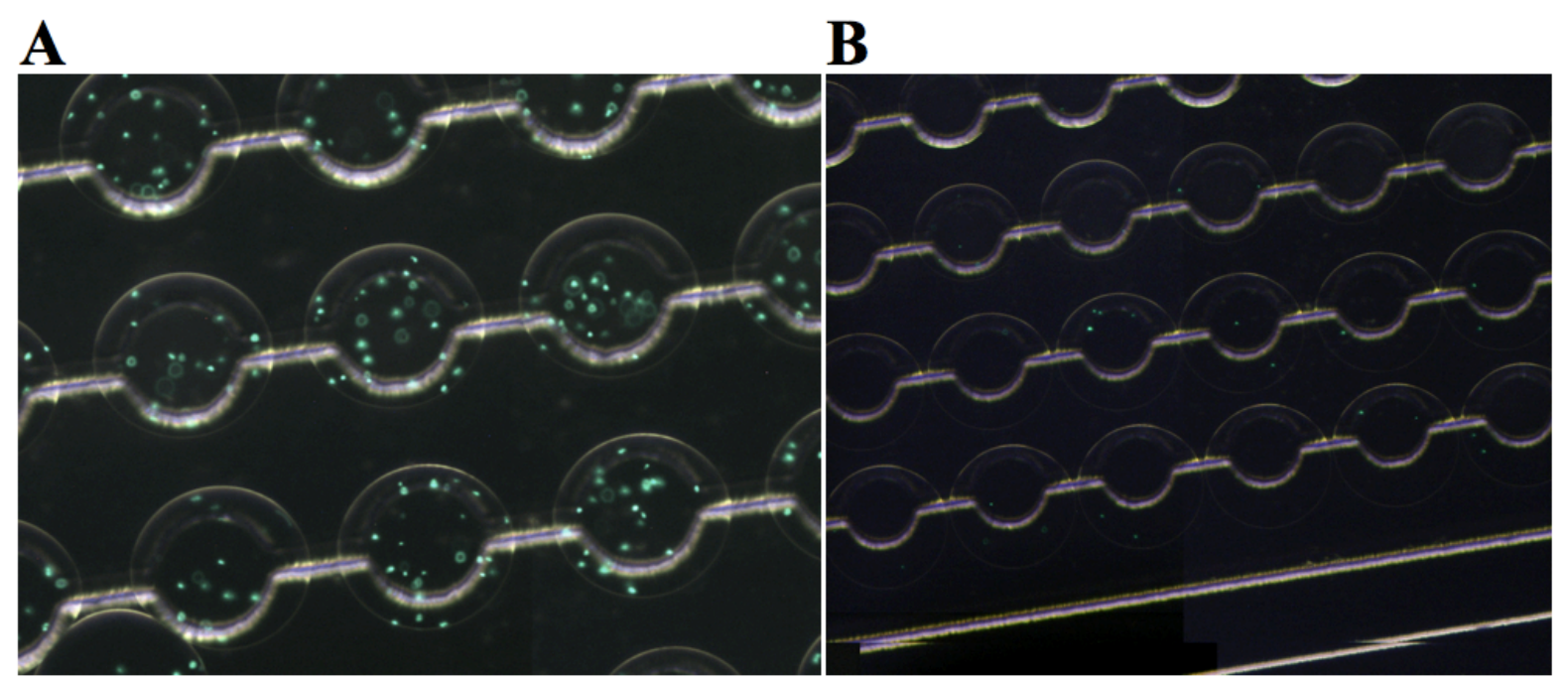

Figure 6. Trapping of eGFP-expressing $\boldsymbol{E}$.coli cells in microchannels. The number of cells correspond to $\sim 3 \times 10^{8}(\mathrm{~A})$ and $\sim 3 \times 10^{6}(\mathrm{~B})$, respectively. Based on the dilution conditions used in (B), the majority of the drops contain either no or only one cell, while some of them contain two or three cells but with low likelihood as can be predicted by a Poisson distribution [284].

\section{(B) Confirmation and quantification of EcASNase2 displayed in the inner membrane of}

E.coli. Our efforts first focused on the determination of enzymatic activity using EcASNase2 cytoplasmically expressed in E.coli cells. Upon compartmentalization in droplets of E.coli cells overexpressing the enzyme, subsequent lysis was attempted for releasing the produced enzyme for the reaction initiation. A number of different lysing agents were used as described in the Methods section. These include hen egg lysozyme, hen egg lysozyme in combination 
with polymyxin $\mathrm{B}$, and commercially available detergents with highly active recombinant lysozyme which was supposed to result in the permeabilization of cell membranes and cell lysis. However, while all those lysing systems work efficiently in macroscopic volumes, in the droplets we totally failed to lyse the cells. Even after long incubation periods of cells with the aforementioned agents in drops, we observed no homogeneous distribution activity profile of the emulsions. In addition, the use of the detergent had an inactivating impact on the helper enzymes which are crucial for the coupled fluorescent assay. This lack of successful lysing of the cells in droplets led us to seek for alternatives to circumvent this hurdle. Therefore, we displayed the EcASNase2 in the inner membrane of E.coli cells by capitalizing on the leader peptide and the first six amino acids of a mature endogenous E.coli lipoprotein, designated NlpA [275].

Membranes are characterized by the presence of a group of proteins, called lipoproteins. The peptide leader sequence of these proteins undergo an amino-terminal lipid modification which ultimately results in their anchoring either in the inner or the outer membrane. They are produced in the cytoplasm as secretory precursors and are exported to the periplasm via the Sec pathway [285]. Once translocated to the periplasm, a cysteine residue which is located at the C-terminus of their peptide leader sequence is modified by the attachment of a diacylglyceride group through a thioether bond. After this modification, the leader sequence is cleaved by signal peptidase II, the protein is fatty acid acylated at the modified cysteine residue, and ultimately the lipophilic fatty acid anchors the protein by inserting it into the membrane. Aiming at the anchoring of EcASNase2 in the inner membrane, we made use of the APEx1 plasmid which expresses the protein of interest as fusion at its N-terminus with the peptide leader sequence and the first six amino acids of the mature NlpA lipoprotein. However, when the whole-cell extract was analyzed on SDS-PAGE to evaluate the expression of the enzyme, no prominent protein band at the expected molecular weight was observed. This result can be explained, considering the fact that the number of available positions for anchoring on the membrane is limited, unlike the intracellular expression where the produced protein can account more than the $25 \%$ of the whole cell's proteins. Figure $7 \mathbf{A}$ shows an SDS-PAGE analysis of cytoplasmically expressed and anchored EcASNase2 in C41(DE3) E.coli cells prepared two, four and six hours after the IPTG addition. The production of the enzyme in case of cytoplasmic expression appears to have reached saturation two hours after the IPTG addition. In contrast, the time-dependent change of 
enzyme production could not be evaluated in case of the displayed enzyme because the expression levels are very low. This fact prompted us to tag the enzyme in order to allow us to evaluate its expression by quantifying it through immunoblot analysis. Therefore, we used the construct APEx1-EcASNase2-His6-C which anchors the enzyme carrying a His6-tag at its C-terminus. The detection of this enzyme upon expression using the membrane fraction after cellular fractionation was done by Western blot using monoclonal mouse anti-His6-C antibodies (Invitrogen), which recognize the $\mathrm{C}$-terminal polyhistidine tag carrying a free carboxyl group (His6-COOH).

In parallel, using the plasmid pET14b-SUMO-EcASNase2-His6-C, the EcASNase2-His6-C version of the enzyme was recombinantly produced and purified. This enzyme served as a probe for immunoblotting known amounts of the protein and preparing a standard curve based on the band intensities which were analyzed by ImageJ [282]. The standard curve was subsequently used to estimate the amount of displayed EcASNase2 per single E.coli cell, given the fact that the membrane fraction which was used for immunoblotting was isolated from $\sim 10^{9}$ cells. Figure 7B shows the Western blots of the recombinant, purified EcASNase2-His6-C which was used in amounts of $0.15,0.75,1.5$, and $2.25 \mu \mathrm{g}$ for the standard curve, and of the membrane fraction isolated from $10^{9}$ cells at 2, 4, 6 and $10 \mathrm{~h}$ after the induction of EcASNase2-His6-C expression. The band intensities of the purified EcASNase2-His6-C were analyzed by ImageJ, and a reference curve was generated which is shown in Figure 7C. In addition, based on the time course of the EcASNase2-His6-C display as shown in Figure 7B, the expression of the enzyme maximizes $\sim 4 \mathrm{~h}$ after the IPTG addition, is maintained constant up to $6 \mathrm{~h}$, and starts declining after $10 \mathrm{~h}$ of incubation. For all the droplet experiments which followed, the cells were induced for $4 \mathrm{~h}$ and next were compartmentalized in emulsions for the activity measurements. The intensity of the band which corresponds to $4 \mathrm{~h}$ after the expression induction was analyzed by ImageJ, and the value was plugged into the equation resulted from linear regression analysis of the EcASNase2-His6-C standards, in order to calculate the amount of the anchored EcASNase2. This final calculated value of the overall amount out of $10^{9}$ cells which is displayed in the inner membrane was found to be $1.2 \mu \mathrm{g}$. Dividing this number by $10^{9}$, we obtain $\sim 1.2 \mathrm{fg}$ of displayed EcASNase2-His6-C per cell. Going a step further, using the molecular weight of EcASNase2 which is $35 \mathrm{kDa}$ and the Avogadro number $\left(6.023 \times 10^{23}\right)$, we can calculate the number of EcASNase2 individual molecules which are anchored per cell, which is $\sim 20,000$. 
This number is $\sim 2$-fold bigger than the calculated number of anchored antibodies in another study [265; Dr. George Georgiou, personal communication]. However, it must be highlighted the fact that the number of the displayed molecules is highly dependent on the properties of the molecules which are anchored, and a 2-fold difference between two distinct molecules (antibodies and enzymes) is within an acceptable range.

$\mathbf{A}$

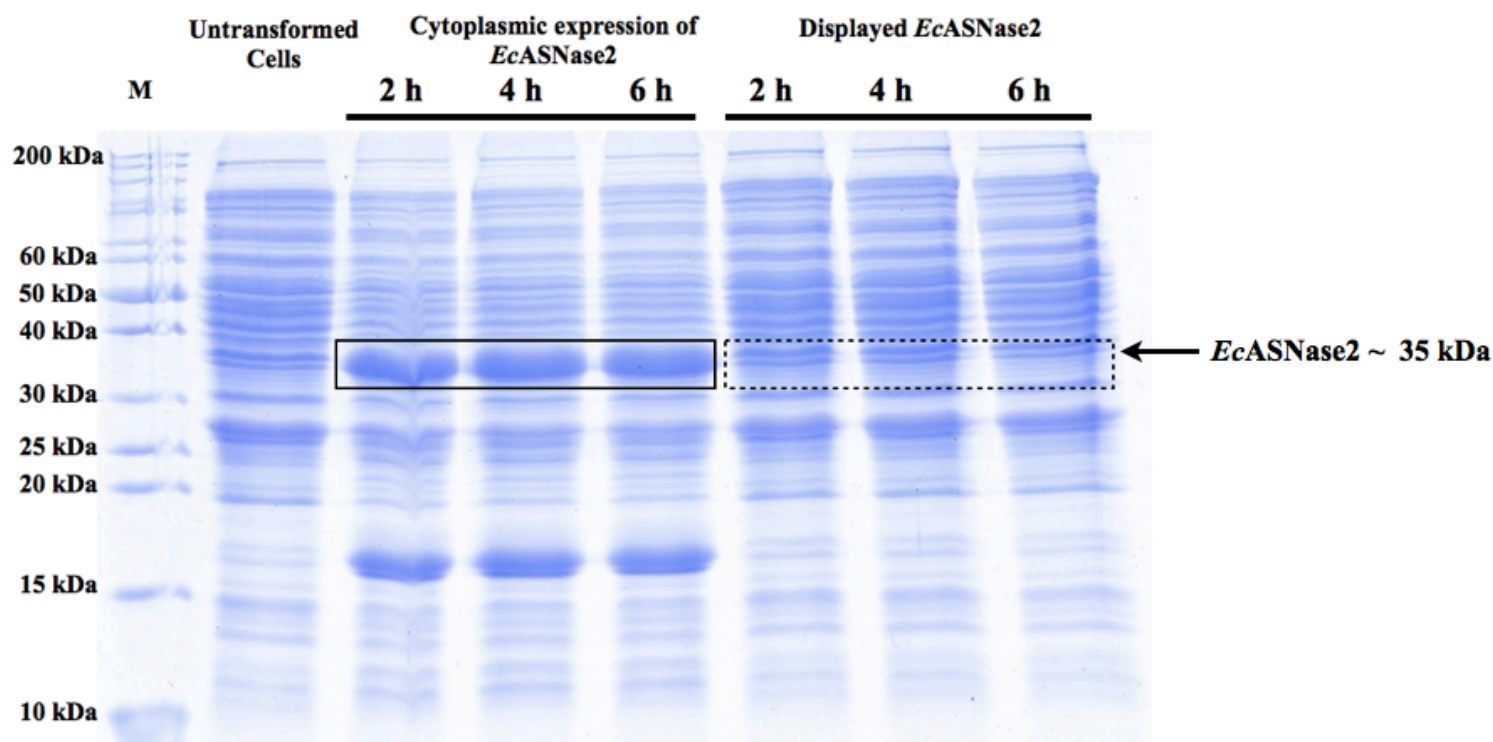

B

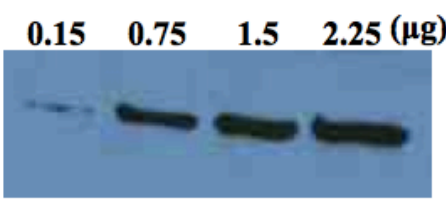

C

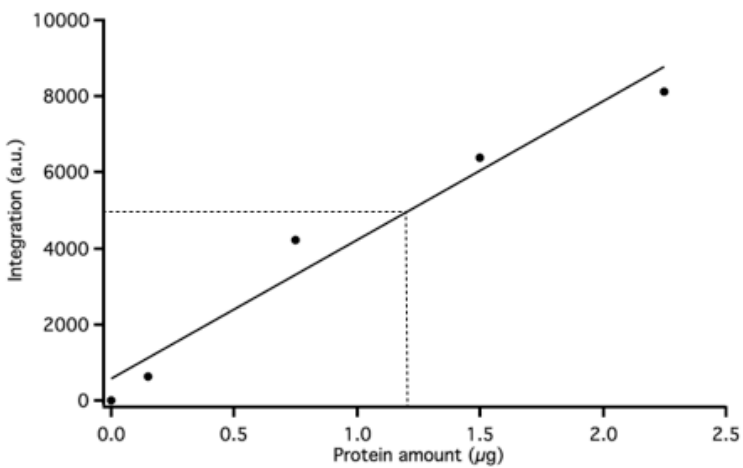

Figure 7. Comparison of expression levels of cytoplasmically expressed and inner membrane-anchored EcASNase2, immunoblotting analysis of anchored EcASNase2His $_{6}-\mathrm{C}$ and purified $E c A S N a s e 2-H_{1}{ }_{6}-C_{\text {, }}$ and standard curve generated based on the band intensities of known amounts of immunoblotted EcASNase2-His6-C. The solid rectangular frame (panel A) in SDS-PAGE shows the overexpressed EcASNase2 in C41 (DE3) cells using the pET14b-SUMO plasmid, while the dotted frame on the right shows the respective expression using the APEx1 plasmid in the same host strain. Panel B shows two Western blots. The upper one shows the bands of $0.15,0.75,1.5$, and $2.25 \mu \mathrm{g}$ of purified EcASNase2-His6-C, and the lower blot depicts the cellular membrane fraction containing anchored EcASNase2-His6-C 2, 4, 6 and $10 \mathrm{~h}$ after the induction of the expression. Panel $\mathrm{C}$ illustrates the standard curve for protein quantification resulting from the band intensities of 
different amounts of purified EcASNase2-His6-C (panel B, upper blot). The dotted line in the plot represents the linear correlation between the value of the band intensity and the respective protein amount detected after $4 \mathrm{~h}$ of EcASNase2-His 6 - $\mathrm{C}$ expression (panel B, lower blot). The linear regression analysis resulted in the curve $y=570+3642 x\left(R^{2}=0.955\right)$, while the band intensity of the unknown protein amount was 4896 (a.u.). This value was used to substitute the $y$ value of the upper linear formula, from which ultimately the value of 1.2 $\mu \mathrm{g}$ (amount of protein) was calculated. The plot was generated using Igor Pro (Wavemetrics), and the figure was prepared using Keynote.

(C) Activity determination of EcASNase2 displayed in the inner membrane of E.coli. The anchoring of mainly antibodies using the plasmid APEx1 has been successfully employed in a number of studies $[265,275]$. In those cases, the outer membrane was permeabilized by EDTA and lysozyme, thereby allowing the antigen to interact directly with the inner membrane-anchored antibody. In our case, the treatment of the cells with lysozyme and EDTA resulted in considerable decrease of the enzymatic activity, which is mainly attributed to the inhibitory effect of EDTA on L-ASNases [257]. Therefore, the outer membrane could not be permeabilized for the activity measurements using standard procedures for permeabilization. In principle, the permeabilization of the outer membrane would not have been absolutely essential in our case, given the fact that both the substrate L-Asn and the product L-Asp are small molecules and therefore can easily diffuse into, and out of, the periplasmic space, thus being accessible to the anchored EcASNase2 for the enzymatic reaction. Figure 8 shows the distribution of the droplet population as a function of the detected fluorescence signal intensity in case of cytoplasmic expression where the cells were incubated with lysozyme and polymyxin B, as well as in case of the displayed EcASNase2. Based on these results, it is evident that upon cytoplasmic expression of the enzyme, even after $\sim 40 \mathrm{~min}$ of incubation with the lysing agents, the fluorescent signal was not homogeneously distributed in the droplet population. In contrast, a two-population distribution was observed (Figure 8A) after $\sim 40 \mathrm{~min}$. This might be attributed to distinct degrees of lysis of the individual E.coli cells resulting in liberation of different amounts of EcASNase2, and, thus, in different observed activity rates. Indeed, when we tried to lyse eGFP-expressing cells with lysozyme and polymyxin B aiming at the visual inspection of the fluorescence distribution in cells in a time-course manner, we observed that cells had released different amounts of eGFP in the emulsions' volume, while in other drops some cells were totally unaffected as evidenced by the continuing flagellar movement of the E.coli cells. 

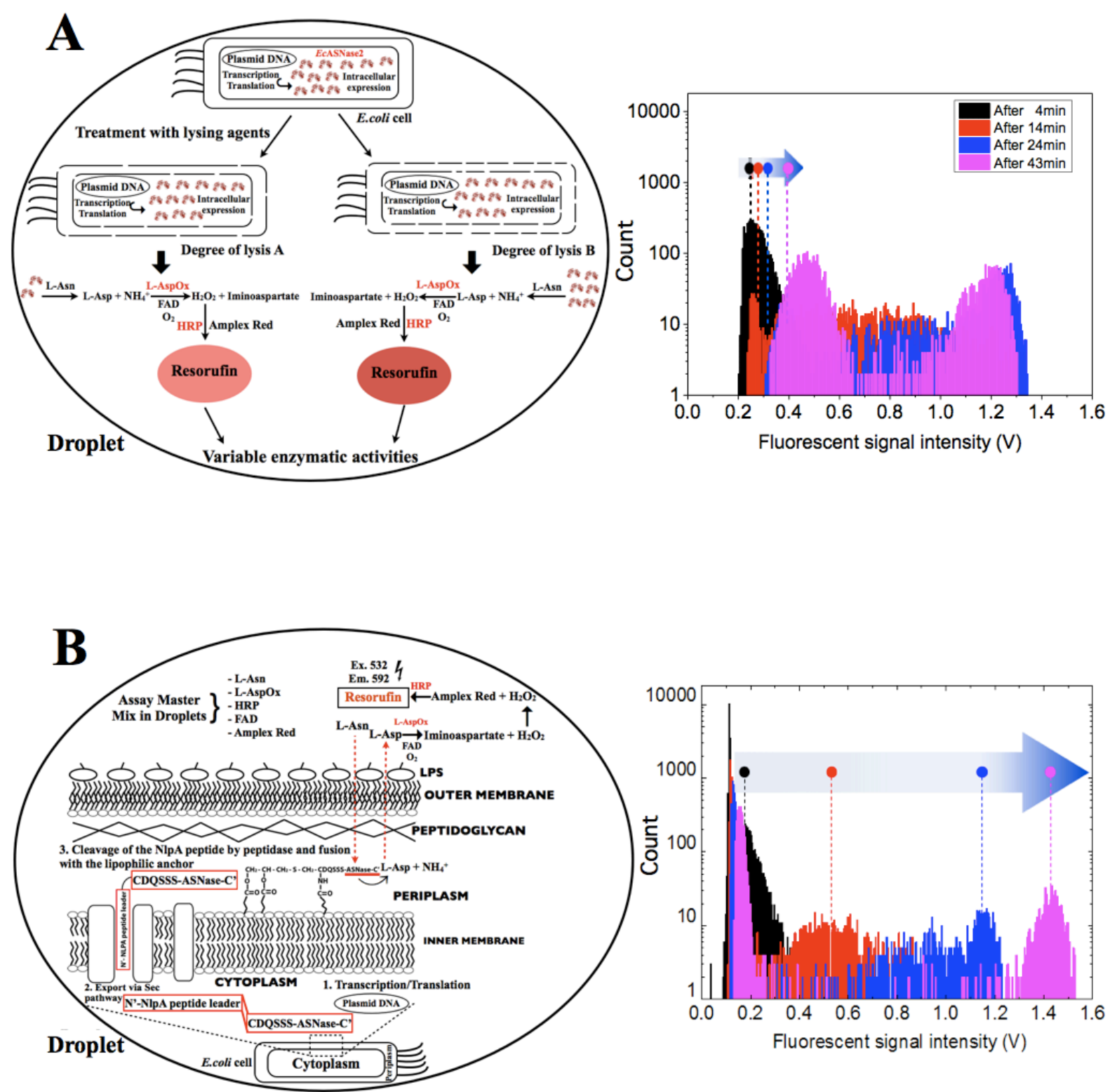

Figure 8. EcASNase2 activity determination in case of (A) cytoplasmic expression, and (B) display of the enzyme in the inner membrane. The reaction was monitored for $43 \mathrm{~min}$ in both cases, and the fluorescent intensity was analyzed at different time points $(4,14,24$, and $43 \mathrm{~min}$, respectively). The lysis of the cells was attempted by using $2 \mathrm{mg} / \mathrm{mL}$ lysozyme in combination with $4 \mathrm{mg} / \mathrm{mL}$ polymyxin B which were added in the aqueous phase together with all the assay reagents as described in the Methods section. The intracellular expression and the display of EcASNase2 were done using the pET14b-SUMO and APEx1 plasmids, respectively, as described in the Methods. The schemes which accompany the histograms above describe the potential partial lysis of E.coli cells in the droplets, and the anchoring process of EcASNase2 in the inner membrane of the cells, respectively. The histograms were generated by Origin 8.5, the schemes were prepared by Adobe Illustrator, and the figures overall were prepared by Keynote. 
As shown in Figure 8B, in case of single-cell level experiments using the anchored EcASNase2, it took $\sim 43 \mathrm{~min}$ for the reaction to be completed. Based on the activity measurements using a well-defined concentration of purified enzyme in droplets, we calculated the amount of the enzyme which is displayed, given the $43 \mathrm{~min}$ time it took to complete the reaction under the same conditions. Figure 5A shows that the use of $10 \mathrm{ng} / \mathrm{mL}$ enzyme completed the reaction in $\sim 2,000 \mathrm{~s}$ which correspond to $\sim 33 \mathrm{~min}$. Considering the volume of the droplets in that case $(600 \mathrm{pL})$, this concentration corresponds to $6 \mathrm{fg}$ of enzyme in such a droplet. Taking into account that for the single-cell anchored enzyme experiment (Figure $8 \mathrm{~B}$ ) it took $43 \mathrm{~min}$, it can be estimated from the linearity of the enzymatic reaction that the amount of the enzyme which contributes to the reaction is $\sim 4 \mathrm{fg}$. This number is rather close to the experimentally determined number of $1.2 \mathrm{fg}$ obtained above in the quantification paragraph. This difference could potentially be attributed to the fact that what was used for the immunoblotting detection was only the membrane fraction, excluding the most likely existing cytoplasmic and periplasmic fractions. Here, it should be recalled that during the anchoring process the enzyme is translocated to the periplasm, followed by the attachment of a diacylglyceride group through a thioether bond at a cysteine residue, and it is ultimately fatty-acylated, thereby becoming anchored in the inner membrane. This implies that before the anchoring event, a certain amount of the enzyme is present in the periplasm during the aforementioned protein modifications step. This fraction of enzyme was excluded from the immunoblotting analysis, since only the membrane fraction was analyzed aiming exclusively at the detection of the displayed EcASNase2. However, the fraction which is located in the periplasm before the final anchoring is expected to also be accessible for the enzymatic reaction. This could explain the deviation between the two different quantification approaches, i.e. the one based on the correlation of the purified enzyme activity measurements with the single-cell activity profile, and the one resulting from immunoblotting analysis. 


\title{
6. Human 60-kDa Lysophospholipase contains an N-terminal L- asparaginase domain which is allosterically regulated by $\mathrm{L}$ - asparagine.
}

\author{
Karamitros C.S. ${ }^{1}$ and Konrad M. ${ }^{1}(2014)$ \\ ${ }^{1}$ Enzyme Biochemistry Group, Max Planck Institute for Biophysical Chemistry, Göttingen \\ D-37077, Germany \\ Journal of Biological Chemistry \\ http://www.jbc.org/content/early/2014/03/22/jbc.M113.545038 \\ doi: http://www.jbc.org/cgi/doi/10.1074/jbc.M113.545038
}

\section{$\underline{\text { Author contributions }}$}

C.S.K. and M.K. conceived the project. C.S.K. designed and performed research. C.S.K. and M.K. analyzed data and wrote the paper.

Copyright notice:

This material is reproduced by permission from the Journal of Biological Chemistry in accordance to their copyright and licensing regulations. 
Enzymology:

Human 60-kDa Lysophospholipase

Contains an N-terminal l-Asparaginase

Domain That Is Allosterically Regulated by

l-Asparagine

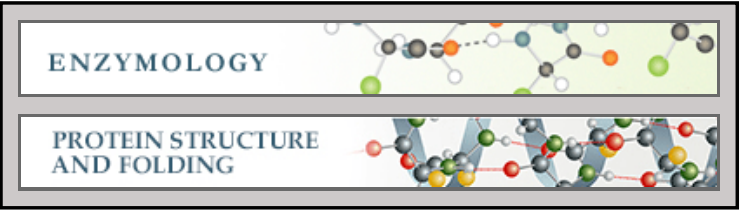

Christos S. Karamitros and Manfred Konrad

J. Biol. Chem. 2014, 289:12962-12975.

doi: 10.1074/jbc.M113.545038 originally published online March 22, 2014

Access the most updated version of this article at doi: $10.1074 / \mathrm{jbc}$ M113.545038

Find articles, minireviews, Reflections and Classics on similar topics on the JBC Affinity Sites.

Alerts:

- When this article is cited

- When a correction for this article is posted

Click here to choose from all of JBC's e-mail alerts

Supplemental material:

http://www.jbc.org/content/suppl/2014/03/22/M113.545038.DC1.html

This article cites 65 references, 18 of which can be accessed free at http://www.jbc.org/content/289/19/12962.full.html\#ref-list-1 


\title{
Human 60-kDa Lysophospholipase Contains an N-terminal L-Asparaginase Domain That Is Allosterically Regulated by L-Asparagine *[S $^{\text {L }}$
}

Received for publication, December 20, 2013, and in revised form, March 2, 2014 Published, JBC Papers in Press, March 22, 2014, DOI 10.1074/jbc.M113.545038

\author{
Christos S. Karamitros and Manfred Konrad
}

From the Enzyme Biochemistry Group, Max Planck Institute for Biophysical Chemistry, Göttingen D-37077, Germany

Background: The $60-\mathrm{kDa}$ human lysophospholipase comprises an N-terminal domain with predicted, yet uncharacterized L-asparaginase activity and a C-terminal ankyrin repeat-like domain.

Results: The N-terminal domain, termed hASNase1, was identified as a functional structural unit possessing catalytic activity. Conclusion: hASNase1 is an allosterically regulated bacterial-type cytoplasmic L-asparaginase.

Significance: Domains of multifunctional human proteins harbor homologs of prokaryotic enzymes displaying similar structural and kinetic features.

The structural and functional characterization of human enzymes that are of potential medical and therapeutic interest is of prime significance for translational research. One of the most notable examples of a therapeutic enzyme is L-asparaginase, which has been established as an antileukemic protein drug for more than four decades. Up until now, only bacterial enzymes have been used in therapy despite a plethora of undesired side effects mainly attributed to the bacterial origins of these enzymes. Therefore, the replacement of the currently approved bacterial drugs by human homologs aiming at the elimination of adverse effects is of great importance. Recently, we structurally and biochemically characterized the enzyme human L-asparaginase 3 (hASNase3), which possesses L-asparaginase activity and belongs to the $\mathrm{N}$-terminal nucleophile superfamily of enzymes. Inspired by the necessity for the development of a protein drug of human origin, in the present study, we focused on the characterization of another human L-asparaginase, termed hASNase1. This bacterial-type cytoplasmic L-asparaginase resides in the $\mathrm{N}$-terminal subdomain of an overall 573 -residue protein previously reported to function as a lysophospholipase. Our kinetic, mutagenesis, structural modeling, and fluorescence labeling data highlight allosteric features of hASNase1 that are similar to those of its Escherichia coli homolog, EcASNase1. Differential scanning fluorometry and urea denaturation experiments demonstrate the impact of particular mutations on the structural and functional integrity of the L-asparaginase domain and provide a direct comparison of sites critical for the conformational stability of the human and $E$. coli enzymes.

L-Asparaginases (EC 3.5.1.1; L-asparagine amidohydrolase; L-ASNase ${ }^{2}$ ) are enzymes that primarily catalyze the conver-

* This work was supported by the Max Planck Society (to C. S. K. and M. K.) and the Göttingen Graduate School for Neurosciences, Biophysics, and Molecular Biosciences (to C. S. K.).

5 This article contains a supplemental table.

${ }^{1}$ To whom correspondence should be addressed. Tel.: 49-551-2011706; Fax: 49-551-2011074; E-mail: mkonrad@gwdg.de.

${ }^{2}$ The abbreviations used are: L-ASNase, L-asparaginase; hASNase1, human L-asparaginase 1; ECASNase1, E. coli L-asparaginase 1; PhASNase1, P. horikoshii sion of L-asparagine (L-Asn) to L-aspartic acid (L-Asp) and ammonia, although some of them are able to also hydrolyze L-glutamine (L-Gln) to L-glutamic acid (L-Glu) and ammonia. These enzymes are present in bacteria to mammals and play pivotal roles in amino acid metabolism $(1,2)$. Enzymes with L-asparaginase activity can be generally classified into two evolutionary distinct families: the bacterial-type and the plant-type L-asparaginases, which are characterized by different structural and biochemical features $(3,4)$. The bacterial-type enzymes have been further grouped into type I and type II depending on their cellular localization. Type I includes cytosolic enzymes that exhibit low affinity (millimolar $K_{m}$ ) for L-Asn, whereas type II enzymes are localized in the periplasm and show considerably higher affinity (micromolar $K_{m}$ ) for L-Asn (5). These enzymes have been studied extensively over the last 40 years mainly because two of the type II isoforms (L-ASNases from Escherichia coli and Erwinia chrysanthemi encoded by the ansB genes) serve as therapeutics for the treatment of acute lymphoblastic leukemia $(6-8)$. Conversely, the less studied plant-type $\mathrm{L}$-asparaginases belong to the so-called $\mathrm{N}$-terminal nucleophile (Ntn) hydrolases, which were defined as a new protein structural family in 1995 (9). A major characteristic of this Ntn hydrolase superfamily is a post-translational processing step that generates the active enzyme. The enzymes are expressed as inactive precursors that undergo a slow intramolecular autoproteolytic cleavage reaction at a specific site resulting in two tightly associated subunits, $\alpha$ and $\beta$, also called protomers. The catalytic residue acting as the nucleophile is exposed at the very $\mathrm{N}$ terminus of the newly generated $\beta$-subunit, which remains complexed with the $\alpha$-subunit during catalysis $(10-12)$. A well characterized mammalian member of the Ntn nucleophile hydrolase superfamily is the human lysosomal aspartylgluco-

L-asparaginase 1; RnASNase1, R. norvegicus L-asparaginase 1; SCASNase1, S. cerevisiae $L$-asparaginase 1 ; ans $A$, gene name for cytoplasmic bacterial L-asparaginases; ans $B$, gene name for periplasmic bacterial L-asparaginases; Ntn, N-terminal nucleophile; palmitoyl-lysoPC, 1-palmitoyl-sn-glycero-3phosphocholine; lysoPI, lysophosphatidylinositol; ADIFAB, acrylodated intestinal fatty acid-binding protein; SUMO, small ubiquitin modifier; $\mathrm{Ni}-$ NTA, nickel-nitrilotriacetic acid; CAPSO, 3-(cyclohexylamino)-2-hydroxy-1propanesulfonic acid. 
saminidase, which catalyzes the hydrolysis of glucosylated L-Asn molecules generated during proteolytic breakdown of glycoproteins $(13,14)$. The human genome encodes another enzyme of this Ntn hydrolase family, variably termed human asparaginase-like protein 1 (15), glial asparaginase (16), CRASH (17), or hASNase3 because its high homology with E. coli LASNase3 (encoded by the iaaA gene) (18). Crystal structures of the wild-type form and of a circular permutant version of hASNase3 have been reported recently (19-22).

The two aforementioned human enzymes and some of their microbial and plant homologs have been characterized as representatives of the Ntn hydrolase superfamily. In contrast, little is known about bacterial-type L-asparaginases expressed in mammalian tissues. An interesting example of such an enzyme is the $60-\mathrm{kDa}$ lysophospholipase first isolated from rat liver (23). This enzyme comprises two domains: an $\mathrm{N}$-terminal domain that is homologous to the E. coli cytoplasmic-type I L-asparaginase (EcASNase1; encoded by the ansA gene) and a C-terminal ankyrin repeatlike domain. This $60-\mathrm{kDa}$ lysophospholipase from rat liver was shown to exhibit three distinct enzymatic activities (lysophospholipase, L-asparaginase, and platelet-activating factor acetylhydrolase) (23); however, no further studies have been reported on this protein, and little is known about its human homolog. In fact, the human 60-kDa lysophospholipase is a poorly characterized protein that shows $79 \%$ overall amino acid sequence identity with its rat homolog. In addition, although it has been demonstrated (24) that the protein holds lysophospholipase activity in vitro and plays a key role in cell proliferation and regulation of membrane channels, there are no supporting data to assign L-asparaginase activity to this human two-domain protein.

The present study focuses on the bacterial expression, purification, and biochemical characterization of the $\mathrm{N}$-terminally located L-asparaginase domain (residues 1-369) of the 60$\mathrm{kDa}$ (573-residue) human lysophospholipase. Because of high sequence similarity of this domain with EcASNase1 (47\% identity), we designate it as hASNase1. Lysophospholipase activity was detected neither for the hASNase1 enzyme nor for the bacterial homolog. We show that the purified enzyme exhibits characteristics similar to those of bacterial type I L-asparaginase. Our kinetic and mutagenesis studies on hASNase1 unveil strong positive allosteric modulation as a function of L-Asn concentration through the action of a second L-Asn binding site, which we probed by cysteine-specific fluorescence labeling of the allosteric site. These results are in line with a previous crystallographic and mechanistic study on the E. coli cytoplasmic homolog demonstrating L-Asn-dependent allosteric regulation of the bacterial enzyme in vitro (25). Interestingly, we observed a substrate-inducing effect on the oligomeric state of hASNase1; however, the predominant state of the enzyme at assay conditions is monomeric. Our thermodynamic analysis of hASNase 1 suggests conformational features and stability distinct from its E. coli counterpart. By mutational analysis, we highlight critical residues located at its putative allosteric and catalytic sites.

\section{EXPERIMENTAL PROCEDURES}

Materials-Yeast extract, peptone from casein, $\mathrm{NaCl}$, Nessler's reagent, urea, SYPRO Orange, L-asparagine, L-glutamine, L-aspartic acid, glutamate dehydrogenase, $\alpha$-ketoglutarate, NADH, synthetic 1-palmitoyl-sn-glycero-3-phosphocholine (palmitoyl-lysoPC), lysophosphatidylinositol (lysoPI) from Glycine max (highly polyunsaturated soybean oil; mixture of different fatty acids and enriched with stearic and palmitic acids), phospholipase $\mathrm{A}_{2}$ from bovine pancreas, $\mathrm{L}$ - $\alpha$-phosphatidylcholine from soybean (mixture of different fatty acids), and palmitic acid were purchased from Sigma-Aldrich-Fluka. The acrylodated intestinal fatty acid-binding protein (ADIFAB) assay kit was obtained from FFA Sciences (San Diego, CA). Dialysis membranes and Coomassie Brilliant Blue G-250 (Bradford reagent) were from Roth (Karlsruhe, Germany). Slide-A-Lyzer was from Pierce. Oligonucleotides were synthesized by IBA GmbH (Goettingen, Germany). Restriction enzymes and T4 DNA ligase were obtained from New England Biolabs (Ipswich, MA). KAPA HiFi polymerase and all PCR reagents were from PeqLab (Erlangen, Germany). Gel extraction and PCR product purification kits as well as nickel-agarose (Ni-NTA) for protein purification were purchased from Macherey Nagel (Düren, Germany). Plasmid purification kits were from Fermentas (Thermo Fisher Scientific, Germany). Genomic DNA preparation kits were from Qiagen (Hilden, Germany).

Cloning of hASNase1, EcASNase1, and Chaperonin 10-The cDNA region coding for the $\mathrm{N}$-terminal domain (termed hASNase1) of the full-length 60-kDa human lysophospholipase was PCR-amplified using as template a cDNA clone of the ASPG gene (human L-asparaginase homolog, NCBI Reference Sequence NM_001080464; GenBank ${ }^{\mathrm{TM}}$ accession number BC035836) isolated from female ovarian tissue (Source Bioscience, UK). Four different C-terminal truncation constructs of hASNase1 were generated and subsequently tested for solubility and enzymatic activity as described in the following sections. NdeI and BamHI sites, respectively, were incorporated in the $5^{\prime}$-ends of the amplifying oligonucleotides (primers are listed in the supplemental table). The PCR mixture in a 50- $\mu$ l final volume consisted of $50 \mathrm{ng}$ of template plasmid DNA, oligonucleotide mixture (10 pmol each), KAPA high fidelity buffer, dNTPs (0.2 mM each), and 1 unit of KAPA HiFi DNA polymerase. The PCR fragment was gel-purified and subjected to additional overlap extension PCRs aiming at the elimination by silent mutation of an internal BamHI restriction site (nucleotide sequence position 740) whose presence would limit the unique cleavage at the $3^{\prime}$-end. The final PCR product was digested with NdeI and BamHI and ultimately ligated overnight at $16^{\circ} \mathrm{C}$ into the pET14b-SUMO vector (10) using T4 DNA ligase. The ligation mixture was used to transform DH $5 \alpha$ E. coli cells. Plasmid DNA isolated from single colonies was digested with NdeI and BamHI to identify positive clones, some of which were sequence-verified. The final constructs include an $\mathrm{N}$-terminal six-histidine tag $\left(\mathrm{His}_{6}\right)$ followed by the small ubiquitin modifier (SUMO; Smt3p protein from yeast; 101 residues; molecular mass, $11.2 \mathrm{kDa}$ ) tag, which has been shown to improve heterologous protein solubility and stability (26). For bacterial expression, the E. coli BL21(DE3) pLysS strain was transformed with 
each of the four hASNase1 constructs. Expression vectors for EcASNase1 and Chaperonin 10 (GroES; encoded by the Cpn 10 gene) were constructed analogously.

Mutagenesis of hASNase1 and EcASNase1-All hASNase1 single site mutants (T19A, T116A, T187S, T187A E266R, E266S, and C299S) and EcASNase1 mutants (R240E and C273S) were generated by applying the QuikChange methodology (Stratagene) using as templates the cloned wild-type genes and KAPA HiFi DNA polymerase. Mutants T187S and T187A were generated in a single PCR using the same pair of primers by making use of the wobbletype oligonucleotides $(\mathrm{G} / \mathrm{T})$ at the first nucleotide of the T187 codon (ACC). Mutants E266R and E266S were constructed in a similar way (the primers are listed in the supplemental table). Sitespecific mutations were confirmed by sequencing the entire coding regions (Seqlab, Goettingen, Germany). All hASNase1 mutants were expressed and purified following the protocol described below for the wild-type enzyme.

Protein Expression and Purification of hASNase1, EcASNase1, and GroES-E. coli BL21(DE3) pLysS cells containing the hASNase 1 plasmid were cultured overnight at $37^{\circ} \mathrm{C}$ in $\mathrm{LB}$ medium supplemented with $200 \mu \mathrm{g} / \mathrm{ml}$ ampicillin and 35 $\mu \mathrm{g} / \mathrm{ml}$ chloramphenicol. A fraction of this culture (dilution 1:100) was used to inoculate fresh $2 \times$ YT medium ( $1 \%$ yeast extract, $1.6 \%$ Tryptone, $0.5 \% \mathrm{NaCl}$ ) supplemented with 200 $\mu \mathrm{g} / \mathrm{ml}$ ampicillin and $35 \mu \mathrm{g} / \mathrm{ml}$ chloramphenicol. When the bacterial culture reached an $A_{600}$ of $\sim 0.5-0.7$, its temperature was lowered to $22^{\circ} \mathrm{C}$, and protein expression was induced by adding isopropyl 1-thio- $\beta$-D-galactopyranoside to a final concentration of $0.25 \mathrm{~mm}$. After incubation at $16{ }^{\circ} \mathrm{C}$ for $18 \mathrm{~h}$, the cells were centrifuged at $4,000 \times g$ for $30 \mathrm{~min}$, resuspended in Ni-NTA-agarose affinity matrix binding buffer A $(50 \mathrm{~mm}$ $\mathrm{Na}_{2} \mathrm{HPO}_{4}, 0.3 \mathrm{M} \mathrm{NaCl}, 10 \mathrm{~mm}$ imidazole, $2 \%$ glycerol, $1 \mathrm{~mm}$ PMSF, pH 8.0), and ultimately lysed by sonication. The cell lysate was centrifuged at $17,200 \times g$ for $45 \mathrm{~min}$, and to the cleared supernatant was added a fraction (1:10 dilution) of $10 \times$ ATP buffer B ( $50 \mathrm{~mm} \mathrm{Na}_{2} \mathrm{HPO}_{4}, 0.3 \mathrm{M} \mathrm{NaCl}, 100 \mathrm{~mm}$ ATP, 200 $\mathrm{mM} \mathrm{MgCl}_{2}, 500 \mathrm{~mm} \mathrm{KCl}, \mathrm{pH}$ 8.0). The combined mixture was incubated in a $37{ }^{\circ} \mathrm{C}$ water bath for $15 \mathrm{~min}$, mixed with preequilibrated Ni-NTA-agarose beads, and incubated at $4{ }^{\circ} \mathrm{C}$ for $3 \mathrm{~h}$ under rotation. Subsequently, the mixture was transferred to a $12-\mathrm{ml}$ polypropylene column and dried by gravity. To remove unspecifically adsorbed material, the nickel resin was first washed with 25 bed volumes of washing buffer C $(50 \mathrm{~mm}$ $\mathrm{Na}_{2} \mathrm{HPO}_{4}, 0.5 \mathrm{M} \mathrm{NaCl}, 20 \mathrm{~mm}$ imidazole, $2 \%$ glycerol, $\mathrm{pH}$ 8.0). The resin was then mixed with a 10-fold diluted buffer B supplemented with GroES at a final concentration of $50 \mu \mathrm{M}$. The mixture was incubated at $4{ }^{\circ} \mathrm{C}$ for $2 \mathrm{~h}$ under rotation, dried by gravity, and then further washed with 10 bed volumes of buffer B without GroES. Finally, the bound $\mathrm{His}_{6}-\mathrm{SUMO}-\mathrm{hASNase1}$ protein was eluted from the column by applying $300 \mathrm{~mm}$ imidazole in buffer A, and fractions were collected dropwise and tested for L-asparaginase activity. All purification steps were performed at $4{ }^{\circ} \mathrm{C}$. The fractions that contained active hASNase 1 were mixed, buffer was exchanged against buffer D (20 mM Tris-Cl, 5\% glycerol, pH 8) using a PD-10 column (GE Healthcare), and then the sample was subjected to DEAE Sephacel anion exchange chromatography. Elution was performed by applying an $\mathrm{NaCl}$ gradient $(0-250 \mathrm{~mm})$ in buffer $\mathrm{D}$. Fractions were analyzed by SDS-PAGE, those containing $>90 \%$ pure hASNase1 were pooled, and buffer was exchanged against buffer E ( $50 \mathrm{~mm}$ Tris-Cl, $100 \mathrm{~mm} \mathrm{NaCl}, \mathrm{pH}$ 8). The protein was incubated with yeast SUMO protease (molar ratio of protease: protein, $\sim 1: 100$ ) at $4{ }^{\circ} \mathrm{C}$ overnight (alternatively $2 \mathrm{~h}$ at $30^{\circ} \mathrm{C}$ ) to cleave the $\mathrm{N}$-terminal $\mathrm{His}_{6}-\mathrm{SUMO}$ tag. In the last purification step, the protein was subjected to size exclusion chromatography by passing it through a Superdex 200 column (size, $30 \times 1$ $\mathrm{cm}$; GE Healthcare) to remove the cleaved tag and remaining impurities. Protein purity was evaluated by SDS-PAGE and was estimated to exceed $95 \%$. The protein sample was aliquoted, mixed with $25 \%$ glycerol, and stored at $-20{ }^{\circ} \mathrm{C}$ at a final concentration of $2 \mathrm{mg} / \mathrm{ml}$ until use. The aforementioned purification protocol was followed for the purification of wild-type hASNase 1 as well as for all mutants described in this study. The final average yield of the purified human enzymes was $\sim 0.3$ $\mathrm{mg} / \mathrm{liter}$ of $2 \times$ YT medium. EcASNase 1 and GroES were purified following the protocol described for hASNase1 excluding the washing steps with ATP and GroES as well as the intermediate anion exchange treatment. EcASNase1 and GroES purifications yielded $\sim 10$ and $2 \mathrm{mg} /$ liter of $2 \times$ YT medium, respectively. Protein quantification was performed by using the Bradford method (27) and by the calculated molar absorption coefficient of each enzyme $\left(23,295 \mathrm{M}^{-1} \mathrm{~cm}^{-1}\right.$ for hASNase1; $26,360 \mathrm{M}^{-1} \mathrm{~cm}^{-1}$ for EcASNase1; $1,490 \mathrm{M}^{-1} \mathrm{~cm}^{-1}$ for GroES) at $280 \mathrm{~nm}$ based on the amino acid sequence (28). The two quantification methods showed less than $10 \%$ difference, and ultimately the values were averaged.

L-Asparaginase and Lysophospholipase Activity Assays-We applied two different methods for monitoring L-asparaginase activity: a single step discontinuous method and a two-step continuous assay for real time measurements of the enzymatic activity. The discontinuous method, the so-called Nesslerization procedure that quantifies ammonia liberated upon deamidation of L-asparagine, has been described previously (29). The continuous assay, which monitors the NADH-dependent conversion of $\alpha$-ketoglutarate plus ammonia to glutamate in a glutamate dehydrogenase-coupled reaction (30), was applied for the kinetic characterization of the enzymes using a Uvikon 943 double beam UV/visible spectrophotometer. All enzymes used for the activity measurements and the kinetic experiments were free of the $\mathrm{His}_{6}$-SUMO tag, which was removed during the last size exclusion chromatographic step (see above). For steadystate kinetic analysis, L-Asn concentrations in the range of $0-10 K_{m}$ were tested in a final volume of $1 \mathrm{ml}$ of $50 \mathrm{~mm}$ Tris-Cl, $100 \mathrm{~mm} \mathrm{NaCl}, \mathrm{pH} 8$ at $37^{\circ} \mathrm{C}$. The final enzyme concentration was $\sim 0.5 \mu \mathrm{M}(20 \mu \mathrm{g}$ in $1 \mathrm{ml})$. The obtained $V / E$ (velocity/total enzyme concentration) values were plotted against the respective substrate concentrations. Kinetic constants $S_{0.5}$ and $k_{\text {cat }}$ were calculated from the resulting plots by non-linear regression using the Hill equation (Equation 1) and analyzed by the SoftZymics software (Igor Pro, Wavemetrics).

$$
V=\frac{V_{\max }[S]^{n}}{[S]_{0.5}^{n}+[S]^{n}}
$$

Here, $V$ is the initial velocity of the enzymatic reaction, $V_{\max }$ is the maximal velocity, $[S]$ is the substrate concentration, $\left[S_{0.5}\right]$ is 
the substrate concentration that yields half-maximal velocity, and $n$ is the Hill coefficient.

Potential lysophospholipase activity of hASNase1 was tested using a continuous fluorescence assay as described previously (31). Briefly, this assay detects free fatty acid molecules, which are released upon hydrolysis of lysophospholipids by lysophospholipases; free fatty acid then is complexed with the intestinal fatty acid-binding protein conjugated with the fluorescent probe acrylodan, commercially known as a free fatty acid indicator and abbreviated as ADIFAB. Free fatty acid binding to $A D I F A B$ induces a fluorescence signal decrease due to changes of the position of the acrylodan fluorophore, which is located close to the free fatty acid binding pocket of intestinal fatty acid-binding protein. In the present study, we tested palmitoyllysoPC and lysoPI as candidate substrates of hASNase1. The assay was performed in a quartz cuvette according to the manufacturer's instructions using final concentrations of $100 \mu \mathrm{M}$ for each substrate, $1 \mu \mathrm{M}$ ADIFAB, and $1.8 \mu \mathrm{m}$ enzyme $(15 \mu \mathrm{g})$ in $200 \mu \mathrm{l}$ of $50 \mathrm{~mm}$ HEPES, $140 \mathrm{~mm} \mathrm{NaCl}, 5 \mathrm{~mm} \mathrm{KCl,} 1 \mathrm{~mm}$ $\mathrm{Na}_{2} \mathrm{HPO}_{4}, \mathrm{pH} 7.5$. Fluorescence intensity was recorded continuously for $10 \mathrm{~min}$ at $25^{\circ} \mathrm{C}$ using a Jasco FP 8300 spectrofluorometer in the high sensitivity mode (excitation at $386 \mathrm{~nm}$ and emission at $432 \mathrm{~nm}$ with $2.5-\mathrm{nm}$ bandwidths). The assay was initially standardized by testing the fluorescence signal decrease upon binding of palmitic acid (one of the expected products of substrate hydrolysis; lysoPI contains a mixture of fatty acid derivatives; see "Materials") with the ADIFAB sensor protein as well as by measuring the activity of phospholipase $\mathrm{A}_{2}$ against $\mathrm{L}$ - $\alpha$-phosphatidylcholine (also provided as a mixture of different fatty acid derivatives). Both hASNase 1 and EcASNase1 were tested for lysophospholipase activity.

Homology Modeling of hASNase 1-A homology model of the $\mathrm{N}$-terminal L-asparaginase domain of the 60 -kDa human lysophospholipase based on the recently determined structure of the homologous EcASNase1 enzyme (25) was obtained from the protein structure prediction service Phyre (32) (Job code 77beadcf4ff $84 d f c$, Fold Library ID c2p2dA, identity of 47\%, estimated precision of $100 \%)$. The resulting hASNase1 structure was overlaid on the EcASNase1 crystal structure (Protein Data Bank code 2HIM) using PyMOL (33). The putative allosteric and catalytic sites of hASNase1 were analyzed for the presence of critical residues previously identified in EcASNase1.

Probing the Putative Allosteric Site of hASNase1 by Fluorescence Labeling of Cys ${ }^{299}$ - Cysteine residue Cys ${ }^{299}$ of the catalytically inactive T19A mutant of hASNase1 was labeled using the compound Atto 465-maleimide as a fluorescent dye (Sigma-Aldrich). Maleimides show excellent reactivity with thiol groups, and therefore, they can serve as site-specific labeling probes in cases where reduced cysteines are available. Labeling was done in the following way. Highly purified enzyme (>95\% pure as determined by SDS-PAGE) in $20 \mathrm{~mm}$ Tris-Cl, $100 \mathrm{~mm} \mathrm{NaCl}, \mathrm{pH} 7.2$ was mixed under stirring with a 10 -fold molar excess of the dye (dissolved in DMSO). The final concentrations in the reaction mixture $(0.5 \mathrm{ml})$ were $20 \mu \mathrm{M}$ enzyme and $200 \mu \mathrm{M}$ dye. The mixture was incubated at $22{ }^{\circ} \mathrm{C}$ for $2 \mathrm{~h}$ in the dark, and subsequently $1 \mathrm{~mm}$ of glutathione (GSH) was added to remove excess thiol-reactive dye. The labeled enzyme mixture was dialyzed against $50 \mathrm{~mm}$ Tris-Cl, $100 \mathrm{~mm} \mathrm{NaCl}, \mathrm{pH}$
8. The labeling of the enzyme was confirmed by SDS-PAGE analysis applying $5 \mu \mathrm{g}$ each of unlabeled enzyme and of the final enzyme-dye conjugate because it has been shown (34) that labeled proteins migrate more slowly than unlabeled species.

For measuring interactions of hASNase1 with L-Asn, L-Asn in the concentration range of $0.1-40 \mathrm{~mm}$ was incubated with labeled enzyme ( $1.5 \mu \mathrm{M}$ final concentration) in a final volume of $100 \mu \mathrm{l}$. The samples were left for $2 \mathrm{~h}$ on ice to equilibrate. Fluorescence intensity was recorded using a Jasco FP 8300 spectrofluorometer in the high sensitivity mode (excitation at $463 \mathrm{~nm}$ and emission at $508 \mathrm{~nm}$ with $2.5-\mathrm{nm}$ bandwidths). All measurements were done in triplicate. Because hASNase1 contains overall 6 cysteines, the mutant C299S was also labeled and served as a negative control (background fluorescence) for signal changes upon L-Asn binding. The resulting saturation binding curve was fitted using SoftZymics software (Igor Pro, Wavemetrics) according to Equation 2,

$$
F=F_{0}-\frac{F_{\max }[S]}{K_{d}+[S]}
$$

where $F$ is the fluorescence intensity, $F_{0}$ is the fluorescence intensity at zero concentration of ligand, $F_{\max }$ is the plateau fluorescence intensity, $K_{d}$ is the dissociation constant, and $[S]$ is the ligand concentration.

Thermodynamic Characterization of hASNase1 Conformational Stability-The conformational stability of hASNase1 and $E c$ ASNase 1 was studied by monitoring changes in intrinsic fluorescence of the stepwise urea-denatured proteins $(35,36)$. The final urea concentrations ranged from 0.5 to $8 \mathrm{M}$ using a stock solution of $10 \mathrm{M}$ urea dissolved in $50 \mathrm{~mm}$ Tris-Cl, $100 \mathrm{~mm}$ $\mathrm{NaCl}, \mathrm{pH}$ 8. The final concentration of the enzymes was adjusted to $50 \mu \mathrm{g} / \mathrm{ml}(\sim 1.25 \mu \mathrm{M}$ for both enzymes) in a final volume of $100 \mu \mathrm{l}$. The two tryptophan residues of hASNase1 $\left(\operatorname{Trp}^{93}\right.$ and $\operatorname{Trp}^{150}$ ) exhibited intrinsic fluorescence upon excitation at $295 \mathrm{~nm}$ (this wavelength was chosen to reduce excitation of tyrosines), showing an emission maximum at $343 \mathrm{~nm}$. The same excitation and emission maxima were obtained for EcASNase1 containing a single tryptophan residue $\left(\operatorname{Trp}^{68}\right)$. Before measurements, equilibrium of the unfolding reactions was confirmed by monitoring the fluorescence signals at different time points until no further change was observed. Data were analyzed assuming a two-state model for reversible protein unfolding. Isothermal urea-induced unfolding experiments were carried out at $10,15,20,25,30,35$, and $40^{\circ} \mathrm{C}$. For each temperature, data analysis using the linear extrapolation method (37) yielded three parameters: (i) the difference of Gibbs free energy between the native and the unfolded state of the protein $\left(\Delta G_{\mathrm{H} 2 \mathrm{O}}\right)$; (ii) the dependence of $\Delta G_{\mathrm{H} 2 \mathrm{O}}$ on denaturant concentration (parameter $m$, which is the slope of the equation $\Delta G=\Delta G_{\mathrm{H} 2 \mathrm{O}}-m$ [urea]), which can be thought of as a measure for the sensitivity of the protein toward the unfolding agent; and (iii) the concentration of urea at which the protein is half-unfolded $\left(C_{1 / 2}\right)$. Subsequently, the calculated $\Delta G_{\mathrm{H} 2 \mathrm{O}}$ values (free energy change at zero concentration of urea) resulting from each temperature were fitted to the Gibbs-Helmholtz equation (Equation 3) (37) as a function of temperature to obtain the $\Delta C_{p}$ values. $T_{m}$ and $\Delta H_{m}$ values as fitting parameters were 
calculated from temperature-induced denaturation experiments (range, $15-85^{\circ} \mathrm{C}$ ) following the intrinsic tryptophan fluorescence changes of protein similar to urea experiments.

$$
\Delta G(T)=\Delta H_{m}\left(1-T / T_{m}\right)-\Delta C_{p}\left[\left(T_{m}-T\right)+T \ln \left(T / T_{m}\right)\right]
$$

$\Delta G(T)$ is the Gibbs free energy difference between the folded and unfolded state at temperature $T, T_{m}$ is the melting temperature, $\Delta H_{m}$ is the enthalpy difference at $T_{m}$, and $\Delta C_{p}$ the heat capacity difference of the folded and unfolded states at constant pressure.

Thermal Stability Determination of Wild-type and Mutant hASNase1 Enzymes by Differential Scanning Fluorometry-The effect of point mutations on thermal stability of hASNase1 was studied by applying differential scanning fluorometry techniques $(38,39)$. Enzyme samples were dialyzed (Slide-A-Lyzer, Pierce; 10,000 molecular weight cutoff) against $50 \mathrm{~mm}$ Tris-Cl, $0.1 \mathrm{NaCl}, \mathrm{pH} 8$ to remove glycerol and subsequently mixed with SYPRO Orange (Sigma-Aldrich) in a final volume of $20 \mu \mathrm{l}$. The final concentrations of the enzyme and the dye were $2 \mu \mathrm{M}$ and $10 \%(\mathrm{v} / \mathrm{v})$, respectively; the DMSO stock solution of the dye $(5,000 \times)$ was prediluted in $\mathrm{H}_{2} \mathrm{O}$, giving a $100 \times$ solution, from which aliquots were used according to the experimental needs. The samples were mixed in a 96-well plate suitable for real time PCR measurements, centrifuged at $500 \mathrm{rpm}$ for $30 \mathrm{~s}$, and finally sealed with heat-resistant membranes (Microseal B adhesive sealer) to prevent evaporation. The enzyme melting experiments were performed using a CFX96 real time PCR machine (Bio-Rad) with the following settings: 2-min prewarming step at $30{ }^{\circ} \mathrm{C}$ and subsequent gradient between 31 and $95{ }^{\circ} \mathrm{C}$ with $1{ }^{\circ} \mathrm{C} /$ min increments. SYPRO Orange fluorescence was monitored using the filters provided with the machine: FAM (492 $\mathrm{nm})$ for excitation and ROX $(610 \mathrm{~nm})$ for emission. Data were exported as an Excel-based worksheet and further analyzed by Igor Pro (Wavemetrics). Melting temperatures $\left(T_{m}\right)$ were obtained by plotting the first derivative $\mathrm{d}(\mathrm{AFU}) / \mathrm{d} T$ ( where AFU represents arbitrary fluorescence units) of the raw data as a function of temperature increase (40).

Effects of $p H$, Divalent Metal Ions, and DTT on Enzyme Activity and Stability-Effects of $\mathrm{pH}$ on enzymatic activity and stability were assessed in a broad range of $\mathrm{pH}$ values using several buffers: sodium acetate ( $\mathrm{pH} 3-5)$, sodium phosphate ( $\mathrm{pH} 6-7)$, Tris- $\mathrm{Cl}$ (pH 7-8.5), and CAPSO (pH 9-10), all at $50 \mathrm{~mm}$ concentration in the presence of $100 \mathrm{~mm} \mathrm{NaCl}$. For the investigation of the $\mathrm{pH}$ effect on the activity of the enzyme, $\sim 50 \mu \mathrm{g}$ of enzyme were assayed in a final volume of $1 \mathrm{ml}$ at saturating substrate concentration (20 mM L-Asn) applying the Nesslerization method. The discontinuous method was preferred to the $\mathrm{NADH}$-dependent continuous assay because the different $\mathrm{pH}$ values could influence the activity of the auxiliary glutamate dehydrogenase enzyme, resulting in unforeseeable artifacts. The stability of hASNase1 under various $\mathrm{pH}$ conditions was studied by incubating the enzyme (at $0.1 \mathrm{mg} / \mathrm{ml}$ final concentration) in several buffers for $12 \mathrm{~h}$ at $4{ }^{\circ} \mathrm{C}$. Aliquots of these mixtures were assayed for residual L-asparaginase activity by Nesslerization using as standard buffer the one at which the enzyme showed the highest activity based on the previous activ- ity analysis. To study metal ion effects, purified hASNase1 (0.1 $\mathrm{mg} / \mathrm{ml}$ ) was preincubated with a final concentration of $1 \mathrm{~mm}$ for each of several divalent metal ions, EDTA, and DTT for $3 \mathrm{~h}$ at $4{ }^{\circ} \mathrm{C}$. Subsequently, aliquots were tested for residual activity by the Nesslerization method.

\section{RESULTS}

Expression and Purification of the hASNase1 Domain-Four different C-terminal truncations (numbered 1, 2, 3, and 4; see Fig. $2 A$ ) of full-length human lysophospholipase (gene code ASPG; asparaginase homolog) were designed based on sequence alignments of the $\mathrm{N}$-terminal putative L-asparaginase domain and different procaryotic (E.coli, EcASNase1, and Pyrococcus horikoshii, PhASNase1) and eucaryotic (Rattus norvegicus, RnASNase1, and Saccharomyces cerevisiae, ScASNase1) cytoplasmic L-asparaginases (Fig. 1). All four constructs were tested for protein expression in E. coli and solubility under a spectrum of different conditions (culture medium, E.coli strain, isopropyl 1-thio- $\beta$-D-galactopyranoside concentration, and temperature). Interestingly, truncation 3 , which appears to fit best to the bacterial cytoplasmic enzymes according to the amino acid sequence comparison, was not soluble, and trials to purify the enzyme under denaturation/renaturation conditions (unfolding by up to $8 \mathrm{~m}$ urea and refolding by dialysis) ultimately resulted in a catalytically inactive protein. Similarly, truncations 1 and 2 failed to produce soluble, active hASNase1. In contrast, truncation 4 (369 amino acid residues), containing 5 additional amino acids (VEERR) downstream of truncation 3, produced the protein in soluble form (Fig. $2 B$ ), and most importantly, the protein purified under native conditions exhibited L-asparaginase activity.

However, when the protein purity was analyzed by SDSPAGE after the last gel filtration step (see "Experimental Procedures"), a prominent band with an apparent molecular mass of $\sim 60 \mathrm{kDa}$ and an estimated stoichiometric 1:1 ratio to the hASNase1 band was constantly observed (Fig. 3). Multiple attempts to remove this associated protein species by altering standard purification protocols were unsuccessful. This prompted us to analyze this protein band by mass spectrometry by which it was finally identified as the endogenous $E$. coli chaperone GroEL. Indeed, contamination by co-purified chaperones can be a hurdle in recombinant protein expression and purification setups (41). Although it has been described previously (42) that incubation of the crude cell extract with $10 \mathrm{~mm}$ ATP at $37^{\circ} \mathrm{C}$ and washing of the affinity column with an ATPcontaining buffer facilitate the removal of GroEL, this approach did not prove beneficial in our case. Moreover, attempts to use an engineered E. coli strain (43) (a kind gift from Prof. Ulrich Hartl's laboratory, Max Planck Institute for Biochemistry, Munich, Germany) carrying the GroEL/ES operon under an arabinose-inducible promoter, which allows tuned regulation of the expression levels of these chaperones, failed to produce the hASNase1 protein (data not shown). Ultimately, we managed to remove the co-purified chaperone by including in the washing buffer the natural binding partner of this protein, GroES, at $\sim 10$-fold excess over GroEL (44). It appears reasonable to assume that the presence of ATP- $\mathrm{Mg}^{2+}$-GroES weakened the binding between GroEL and hASNase1, thus facilitat- 


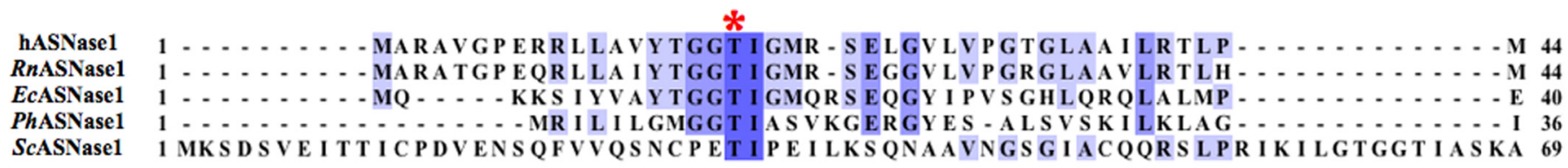

45 FHDEEHARARGLSEDTLVLPPASRNQR I LYTVLECQPLFDSS DMTIAEWVCLAQTIKRHYEQYHGFVVI 113 45 LHDEEYARAHSLPEDTLVLPPAS S DQR I I KVLECQPLFDS S MT I TEWVQ IAQT IERHYTQYQGFVVI 113 41 FHR P EMP . . . . . . . . . . . . . D F T I HEY T P LMD S S DMT P E DWQH I A E I KA HYDDYDGFVIL 88

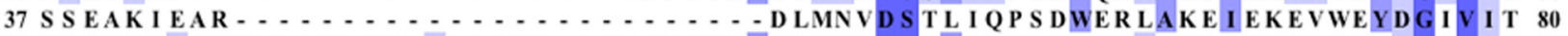
70 IDSS QTAGYHVDLT IQDLLDA I PDISKVCD I EYEQLCNVDSKDINEDILYKIYKGVSESLQAFDGIVIT 138

114 HGTDTMAFAASMLS FMLENLQKTV I LTGAQVP I HALWS DGRENLLGALLMAG - - QYV I PEVCLFFQNQL 180

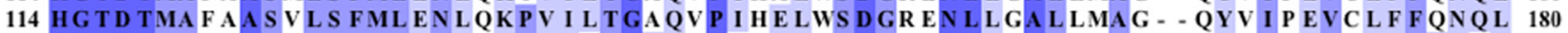
89 HG TD TMAYTASA LS FMLENLGKPVIVTGS I PLAELRS DGQ INLLNALYVAA - NYP INEVTLFFNNRL 155 81 HGTD TMAYSA SMLS FMLRNPP I P IVLTGSMLP ITEKNS DAPFNLRTALEFV - - KLG IRG IYIAFNGKV 146 139 HGTDTLS ETAFF I EST I DAGDVPIVFVGSMRPSTSVSADGPMNLYQA I IASNPKSRGRGVLVS LNDQ I 207 181 FRGNRATKVDARRFAAFCSPNLLPLATVGAD ITINRELVRKVDGKAGLVVHS S - - MEQDVGLLRLYP 245 181 FRGNRT TKVDARRFAAFCS PNLPPLATVGADVTINRELVRKAS WKS HLVVHS - - - MEPDVGLLRLYP 245 156 YRGNRT TKA HADGFDAFASPNLPPLLEAG I H IRR - LNTPPAPHGEGELIVHPI- - T PQP IGVVTIYP 219 147 MLGVRASK I R S MGFDAFES INYPNVAE I KDDKLR I LH I PDFYG - D EFF D I K - - - Y EPKVLVIKL I P 209 208 S S GYY ITKTNANS LDSFN-VRQGYLGNFVNNE IHYYYPPVKPQGCHKFKLRVDGKHFKLPEVCILYAHQ 275 246 G I PAA LVRA F LQP P LKGVVMETFGSGNGPTKP D LLQELRVATERGLVIVNCTHCLQGAVTT-DYAAGMA 313 246 G I PAS LVRT F LQP P LKGVVMETFGSGNGPTKPDL I QELRAAAERGL I IVNCTHCLQGAVTS - DYAPGMA 313 220 G I S A DVVR F LRQPVKAL I LRS YGVGNA P QNKA FLQELQEASDRGIVVVNLTQCMSGKVNMGGYATGNA 288 210 GLS GD IVREALRLGYKG I I LEGYGVGG I PYRGTDLFEVVS S I S KR I PVVLTTOA I YDGVDLORYKVGR I 278 276 A F P P A IVN - LVADKYDG IVLATMGAGS LPEEVN - . - E TCMKLS LPIVYSKRSMDGMVP I ANVPKKGS 338

314 MAGA GVIS GFDMT ÜAALAKLSYVLGQPGLS LDVRKELLTKDLRGEMTPPSVEERR 369 314 MAGA G I I GFDMTS EAALAKLSYVLGQPGLS LSDRKKLLAKDLRGEMTLPTTDDLL 369 289 LAHA GV I GGA DMTVEATLTKLHYLLS Q - ELDTET IRKAMS QNLRGELTP - - DD - - 338 279 A LEA GV I PAGDMTKEAT I TKLMWI LGH - TKN IEEVKQLMGKN I TGELTRVS - - - - 328 339 KEDN - LI I S GYLS PEKS R I LLQLCLAG - NYTLEE IKHVFTGVYGG - - . - . - . - 381

FIGURE 1. Alignment of the amino acid sequences of hASNase1 (UniProt accession number Q86U10), R. norvegicus (UniProt accession number 088202), E. coli (UniProt accession number P0A962), P. horikoshii (UniProt accession number O57797), and S. cerevisiae (UniProt accession number P38986) L-asparaginases type I. The displayed sequence of hASNase1 corresponds to truncation 4, which showed the best expression pattern of the four protein constructs produced in this work (see Fig. 2). Asterisks indicate the two threonine residues that are critical for L-asparaginase activity, and red arrows indicate residues that are located close to the allosteric sites. The alignment was performed using ClustalW (67), and the graph was generated using JalView (68). Blue shading indicates highly conserved amino acid residues.

ing their separation upon exhaustive washing (Fig. 3). It is worth mentioning that the bound GroEL did not affect the activity of hASNase1 because activity measurements on both enzyme preparations (with and without bound GroEL) yielded similar kinetic constants. We emphasize that all hASNase1 variants produced in this work were expressed at levels similar to the wild-type enzyme and showed no aggregation or precipitation tendency at any purification step. GroEL contamination was present during purification of all mutant enzymes as well.

Size exclusion chromatography experiments allowed us not only to obtain a highly pure protein as evidenced by SDSPAGE analysis (Fig. 3) but also to gain insight into the oligomeric state of hASNase1. Strikingly, when we first analyzed native hASNase1, we observed that the enzyme $(\sim 3 \mu \mathrm{M})$ was running as a monomer (molecular mass, $\sim 40 \mathrm{kDa}$ ) on a Superdex 200 gel filtration column (Fig. 4C). As the shift between oligomeric states of enzymes can be drastically influenced by their substrates and/or other interacting partners $(45,46)$, we decided to perform gel filtration analysis in the presence of the substrate of hASNase1. Interestingly, with $20 \mathrm{~mm}$ L-Asn in the running buffer, we witnessed a slightly shifted shoulder peak of the previously observed chromatographic peak assigned to the 40-kDa hASNase1 monomer, indicative of a higher molecular weight species, although the monomer remained the predominant form (Fig. 4D). This finding suggested that L-Asn could trigger the association of monomeric hASNase1 molecules and consequently induce the formation of dimers and even tetramers, which are the characteristic molecular species reported for EcASNase1 (25) as we confirmed in the present study (Fig. $4 B)$. However, at enzymatic assay conditions, hASNase1 $(\sim 0.5$ $\mu \mathrm{M})$ is expected to be monomeric because this concentration is 6 -fold lower than the concentration of the eluted monomeric protein shown in Fig. 4 (0.5 mg of enzyme loaded on the gel filtration column eluted in a final volume of $\sim 4 \mathrm{ml}$ of buffer, i.e. $\sim 3 \mu \mathrm{M}$ final concentration of the enzyme).

The hASNase1 Enzyme Shows Non-Michaelis-Menten Kinetics for L-Asn Hydrolysis Similar to Its E. coli Homolog but Lacks Lysophospholipase Activity-The N-terminal domain of human 60-kDa lysophospholipase, which we have designated as hASNase1, shares $47 \%$ sequence identity with E. coli cytoplasmic L-asparaginase (encoded by the ansA gene; EcASNase1). The crystal structure of EcASNase1 has been reported recently (25), shedding light on distinct structural features associated with L-Asn binding to the catalytic site and to the site responsible for allosteric regulation. Based on this structure, we modeled the hASNase1 structure using the Phyre program. Fig. 5 shows the active and the allosteric sites in the predicted hASNase1 structure overlaid by the homologous regions of EcASNase1. Residues critical for catalysis in the E. coli enzyme, such as $\mathrm{Thr}^{14}$, $\mathrm{Thr}^{91}$, $\mathrm{Asp}^{92}$, and Lys ${ }^{163}$, overlay with high accuracy with those of hASNase1, which has identical residues at these positions $\left(\mathrm{Thr}^{19}{ }^{1}\right.$ Thr $^{116}$ Asp $^{117}$, and Lys ${ }^{188}$ ) (Fig. 5A). Importantly, resi- 

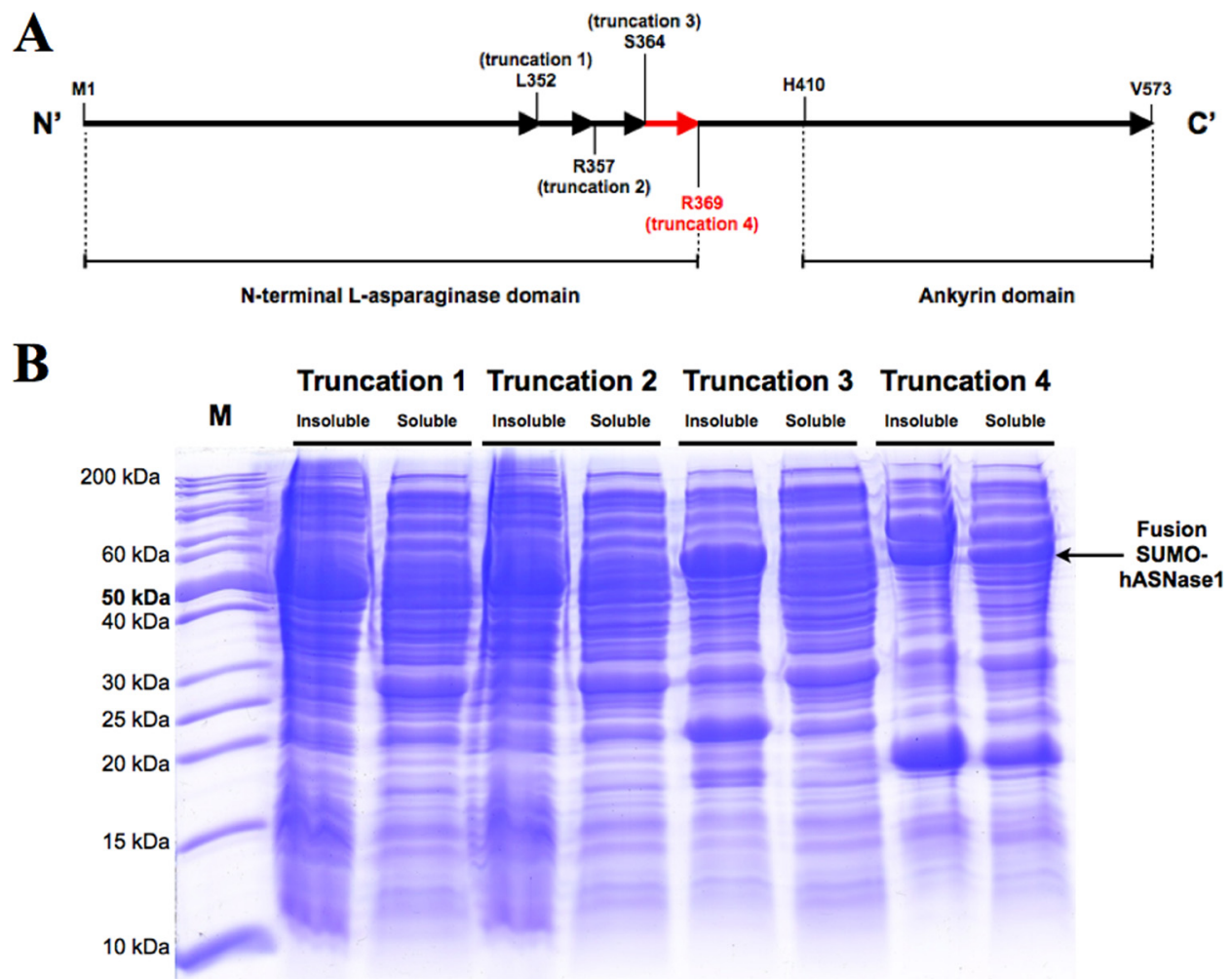

FIGURE 2. A, schematic representation of the full-length 60-kDa human lysophospholipase $\left(\mathrm{Met}^{1}-\mathrm{Val}{ }^{573}\right.$ ). Shown are the four different truncations that were generated and tested for protein solubility and L-asparaginase activity. Residues Leu ${ }^{352}$, $\mathrm{Arg}^{357}, \mathrm{Ser}^{364}$, and $\mathrm{Arg}^{369}$ indicate the last amino acid of the respective truncations. Red labeling indicates truncation 4 of the hASNase1 domain, which showed the best solubility and activity. Downstream of the L-asparaginase domain, the sequence region from $\mathrm{His}^{410}$ to Val ${ }^{573}$ indicates the putative ankyrin domains as also predicted in the rat homolog (23). B, SDS-PAGE analysis of all four expressed truncations of hASNase1. Insoluble and soluble fractions are included for each preparation. The arrow indicates the fusion SUMO-hASNase1 of truncation 4, which resulted in the production of soluble enzyme. The predicted molecular masses for each truncation as a fusion with SUMO are as follows: truncation 1, $50.5 \mathrm{kDa}$; truncation 2, $51.2 \mathrm{kDa}$; truncation 3, $51.9 \mathrm{kDa}$; and truncation 4, 52.5 kDa. Lane M, molecular mass markers.

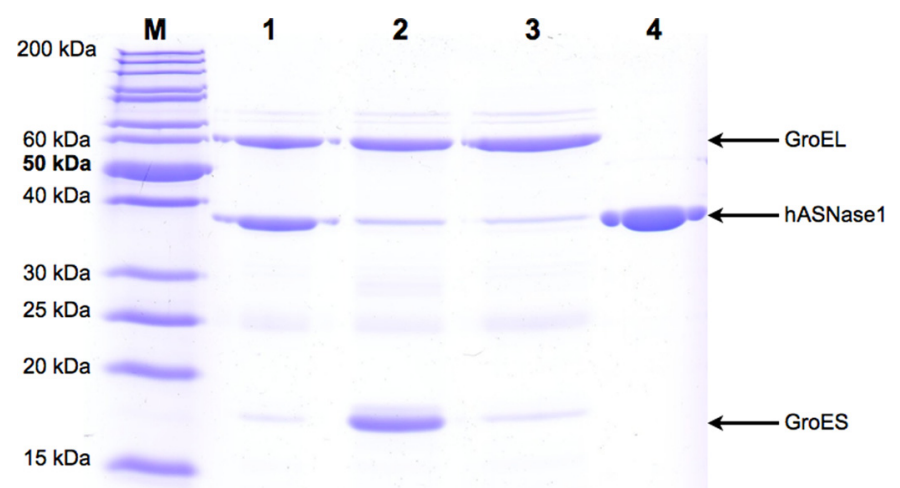

$10 \mathrm{kDa}$

FIGURE 3. Purification of hASNase1. Lane 1, purified enzyme associated with GroEL; lane 2, removal of GroEL by complexation with externally added GroES in the Ni-NTA column; lane 3, washed GroEL fraction from DEAE anion exchange purification step; lane 4, final purity of hASNase1; lane M, molecular mass markers.

dues $\mathrm{Thr}^{14}$ and $\mathrm{Thr}^{91}$ of the bacterial enzyme are considered to be the primary nucleophiles for the attack on the substrate L-asparagine (25). The presence of threonine residues at equivalent positions in the human enzyme is in favor of the view that the primary nucleophile in bacterial L-asparaginases (both cytoplasmic and periplasmic) is a threonine residue (47). This prompted us to investigate this assumption for hASNase 1 by mutagenesis studies. Indeed, when point mutants T19A and T116A of hASNase1 were assayed, no L-asparaginase activity was detected (Table 1), thus indicating the critical catalytic role of these threonine residues in hASNase1.

Steady-state kinetic characterization of wild-type hASNase1 using L-Asn as substrate (there was no detectable L-glutaminase activity) revealed that the enzyme did not follow MichaelisMenten kinetics. Instead, it exhibited a pronounced sigmoidal kinetic behavior, which is a hallmark of allosteric enzymes. The kinetic data were fitted using the Hill equation (Equation 1 under "Experimental Procedures") from which we estimated a Hill coefficient $\left(n_{\mathrm{H}}\right)$ of 3.9 and an $S_{0.5}$ value of $11.5 \mathrm{~mm}$ (Fig. 6). The modeled structure of the allosteric site is shown in Fig. $5 B$ in direct comparison with that of EcASNase1. Significant differences between the two allosteric sites are seen in two key residues that directly interact with the substrate L-Asn: $\mathrm{Glu}^{266}$ and $\mathrm{Ser}^{327}$ in hASNase1 as opposed to $\mathrm{Arg}^{240}$ and $\mathrm{Val}^{302}$ in the bacterial enzyme. Aiming at further characterization of the allosteric sites of both enzymes, we generated a series of mutants and expressed, purified, and tested them for catalytic activity. Strikingly, hASNase1 tolerated none of the mutations introduced in the predicted allosteric site as evidenced by the total lack of activity of the mutant enzymes (Table 1). Given the high structural homology between hASNase1 and EcASNase1, we reasoned that the mutation E266R could potentially lower the $S_{0.5}$ value of the human enzyme by mimicking the $\mathrm{Arg}^{240}$ 

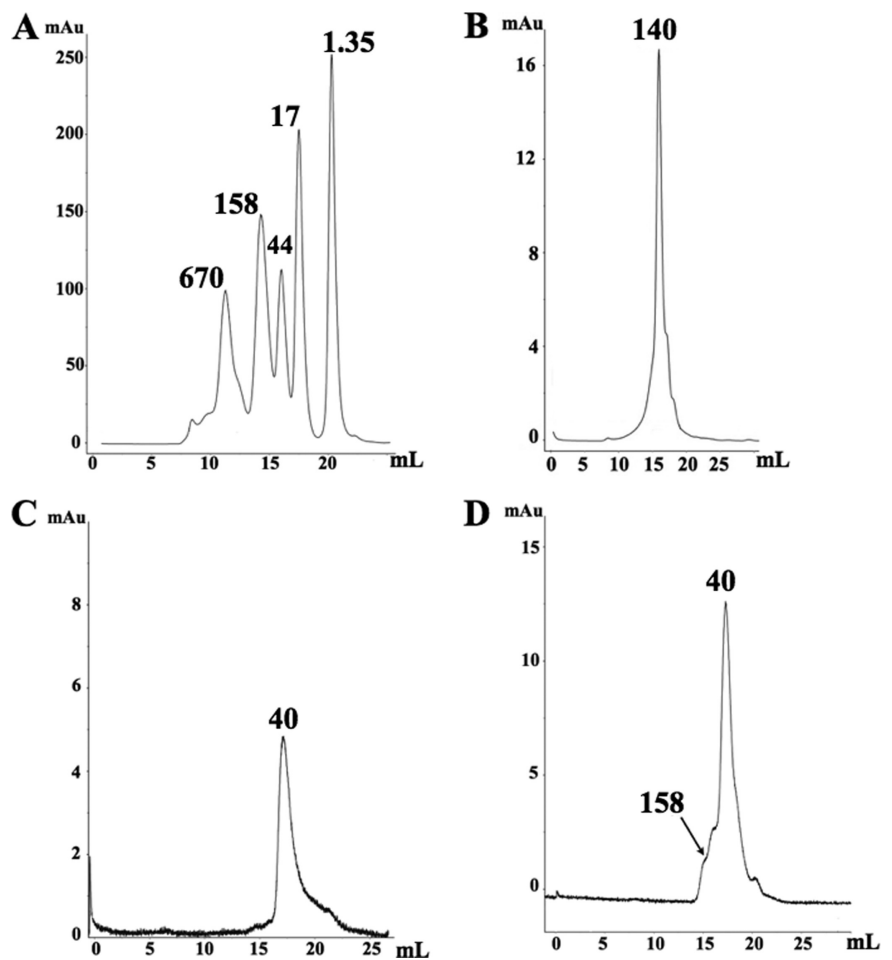

FIGURE 4. Size exclusion chromatography profiles upon separation using Superdex 200 column (GE Healthcare). $A$, standard gel filtration marker (Bio-Rad catalog number 151-1901). B, EcASNase1 chromatogram in $50 \mathrm{~mm}$ Tris-Cl, $100 \mathrm{~mm} \mathrm{NaCl}, \mathrm{pH}$ 8. C, profile of hASNase1 in the absence of L-Asn. Before sample loading, the column was equilibrated with $50 \mathrm{~mm}$ Tris- $\mathrm{Cl}, 100$ $\mathrm{mm} \mathrm{NaCl}, \mathrm{pH}$ 8. D, hASNase1 in the presence of $20 \mathrm{~mm}$ L-Asn. The column was equilibrated with $50 \mathrm{~mm}$ Tris-Cl, $100 \mathrm{~mm} \mathrm{NaCl}, 20 \mathrm{~mm}$ L-Asn, pH 8. In each case $(B, C$, and $D), \sim 0.5 \mathrm{mg}$ of enzyme was loaded on the column. $m A u$, milliabsorbance units.

site of the bacterial homolog. However, this mutation totally inactivated hASNase1. Other mutations introduced around the putative allosteric site of hASNase1, such as E266S, C299S, T187S, and T187A, also abolished activity (Table 1). Unlike the human enzyme, the E. coli enzyme tolerated mutations of its allosteric site. Mutation R240E resulted in an $\sim 5$-fold increase of $S_{0.5}$ and 2-fold increase of $k_{\text {cat }}$ while simultaneously lowering the Hill coefficient $n_{\mathrm{H}}$ from 3.55 to 1.9. The C273S mutation increased even further the $S_{0.5}$ value (7-fold with respect to wild type), whereas the $n_{\mathrm{H}}$ value slightly decreased (Fig. 6). All kinetic data of wild-type hASNase1 and EcASNase1 as well as of mutants generated in this work are summarized in Table 1. Taken together, these results suggest that despite the high similarity of sites predicted to be critical for their activities these two enzymes display different degrees of tolerance toward mutations of residues at these sites.

We additionally tested for potential hydrolysis of palmitoyllysoPC and lysoPI by hASNase1 and EcASNase1. We chose these two substrates because the rat 60-kDa lysophospholipase has been reported to hydrolyze palmitoyl-lysoPC (23), whereas lysoPI could serve as a substrate for the human homolog (24). However, we were unable to detect any activity of either hASNase 1 or EcASNase1 using these two substrates under conditions where the control enzyme phospholipase $\mathrm{A}_{2}$ hydrolyzed $\mathrm{L}-\alpha$-phosphatidylcholine. The rate of the fluorescence decrease in the ADIFAB assay was the same for the blank (ADIFAB plus substrate without enzyme) and the sample (ADIFAB plus sub-

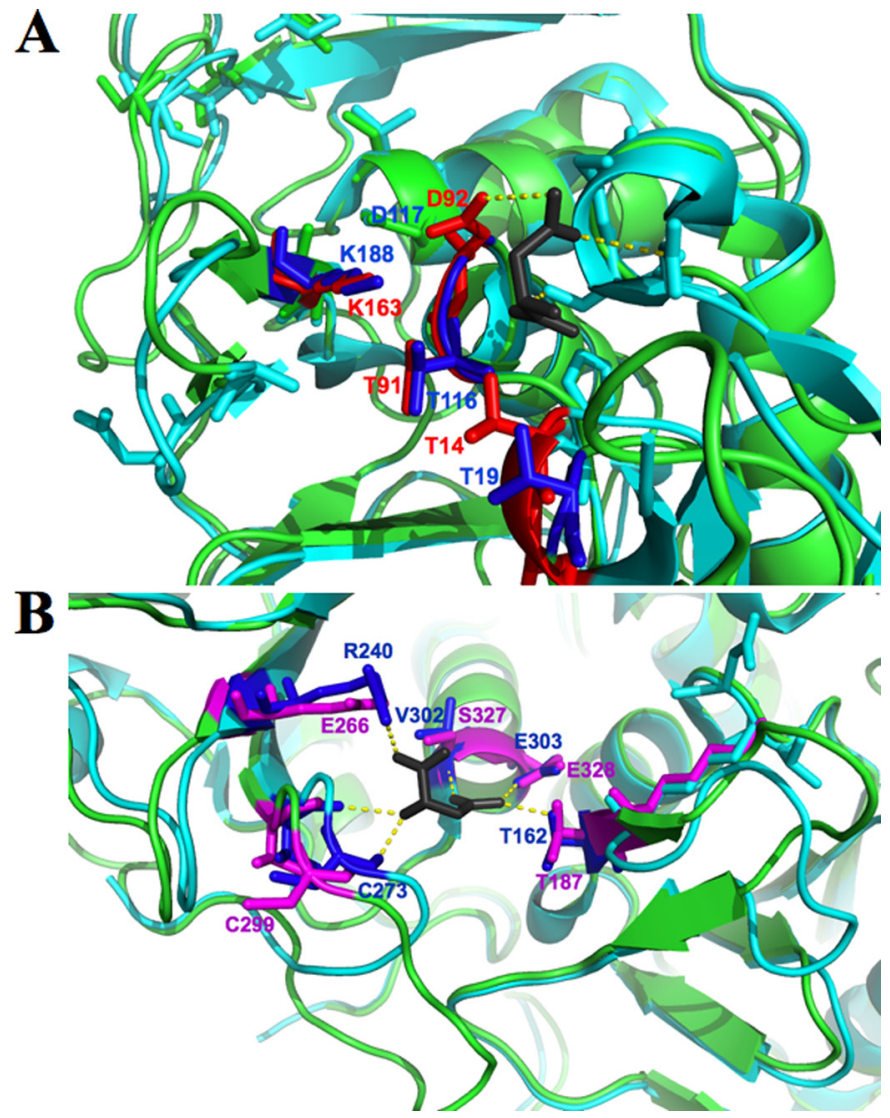

FIGURE 5. A, modeled active site of hASNase1 overlaid by the active site of EcASNase1. Red highlights amino acids that are critical for activity of bacterial L-asparaginases: the catalytic triad formed by residues $\mathrm{Thr}^{91}, \mathrm{Asp}^{92}$, and Lys ${ }^{163}$ that ensure proper orientation of the substrate L-Asn (dark gray) and Thr ${ }^{14}$, which is responsible for subsequent nucleophilic attack $(23,69)$. Blue highlights the respective amino acids of hASNase1: Thr ${ }^{116}, \mathrm{Asp}^{117}$, Lys $^{188}$, and $\mathrm{Thr}^{19}$. B, modeled allosteric site of hASNase1 (magenta residues) overlaid by the EcASNase1 allosteric site (blue residues). Key differences in homologous sites of the human enzyme are residues Glu ${ }^{266}$ versus $\mathrm{Arg}^{240}$ and $\mathrm{Ser}^{327}$ versus $\mathrm{Val}^{302}$. Other residues of the allosteric sites of the two enzymes are highly similar. Also shown is an L-Asn molecule (dark gray), which interacts with allosteric site residues. The interactions between L-Asn and the human enzyme were predicted based on the structure of the bacterial enzyme (Protein Data Bank code 2HIM).

\section{TABLE 1}

Kinetic data on wild-type and mutants of hASNase 1 and EcASNase 1

Steady-state kinetic analysis was performed by applying a continuous NADH-dependent assay (30). Enzyme concentrations were $\sim 0.5 \mu \mathrm{M}$ in a final volume of $1 \mathrm{ml}$ of $50 \mathrm{~mm}$ Tris- $\mathrm{Cl}, 100 \mathrm{mM} \mathrm{NaCl}, \mathrm{pH} 8$ at $37^{\circ} \mathrm{C}$; the tested substrate concentrations covered the range of $0-10 K_{m}$. Parameters were calculated from non-linear regression of a VIE versus [L-Asn] plot using the software SoftZymics (Igor Pro, Wavemetrics). ND, non-detectable; the limit of detection for the applied assays in the present study is $0.5 \mu \mathrm{M}$ of ammonia produced in a $1-\mathrm{ml}$ reaction volume. Data are shown as mean values \pm S.D. of triplicate measurements.

\begin{tabular}{llll}
\hline \multicolumn{1}{c}{ Enzyme } & \multicolumn{1}{c}{$\boldsymbol{k}_{\text {cat }}$} & \multicolumn{1}{c}{$\boldsymbol{S}_{\mathbf{0 . 5}}$} & \multicolumn{1}{c}{$\boldsymbol{n}_{\mathrm{H}}$} \\
\hline Wild-type hASNase1 & \multicolumn{1}{c}{$\boldsymbol{s}^{-1}$} & \multicolumn{1}{c}{$m$ m } & \\
hASNase1 T19A & ND & 11.5 \pm 0.8 & $3.9 \pm 0.2$ \\
hASNase1 T116A & ND & ND & \\
hASNase1 E266R & ND & ND & \\
hASNase1 E266S & ND & ND & \\
hASNase1 T187A & ND & ND & \\
hASNase1 T187S & ND & ND & \\
hASNase1 C299S & ND & ND & \\
Wild-type EcASNase1 & $7.4 \pm 0.3$ & $0.40 \pm 0.05$ & $3.5 \pm 0.3$ \\
EcASNase1 R240E & $14.5 \pm 0.5$ & $1.8 \pm 0.2$ & $1.9 \pm 0.2$ \\
EcASNase1 C273S & $16.8 \pm 0.4$ & $2.8 \pm 0.3$ & $2.7 \pm 0.2$ \\
\hline
\end{tabular}



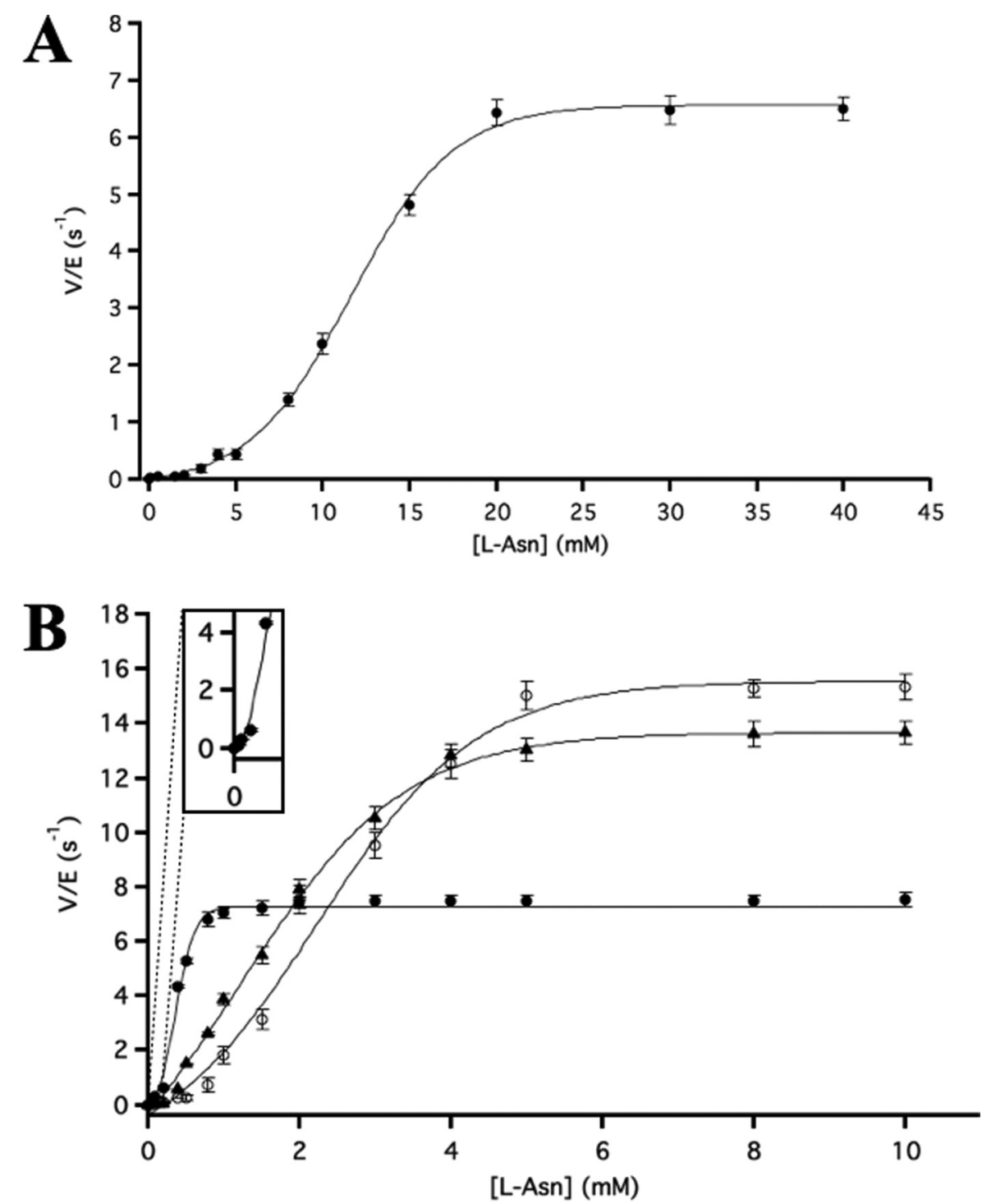

FIGURE 6. L-Asparaginase activities as a function of substrate concentrations of hASNase1 and EcASNase1. A, V/E versus [L-Asn] plot for wild-type hASNase1. Steady-state turnover rates $\left(\mathrm{s}^{-1}\right)$ are expressed as a function of the substrate concentration. Data points are represented as means \pm S.D. of triplicate sample measurements. B, V/E versus [L-Asn] plots for C273S (O) and R240E ( $\mathbf{\Delta})$ mutants and wild type (O) ECASNase1. The inset shows a zoomed frame of the plot at low substrate concentrations for wild-type EcASNase1. Data points are mean values \pm S.D. of triplicate measurements. All steady-state kinetic curves resulted from non-linear regression analysis using the Hill equation (Equation 1 under "Experimental Procedures") in SoftZymics software (Igor Pro, Wavemetrics). Error bars represent S.D.

strate and enzyme) and accounted for $\sim 10$ arbitrary fluorescence units/min (data available upon request). This background activity is in line with another study (31), which also reported a constant slight fluorescence signal decrease in the absence of enzyme. In conclusion, our results demonstrate that the N-terminal domain of the 60-kDa full-length human lysophospholipase can exist as a distinct folding unit that resembles the bacterial-type I L-asparaginases, lacking the capacity to hydrolyze substrates other than L-Asn.

Fluorescence Labeling of hASNase1-Cysteine labeling allowed us to investigate the presence of an L-Asn binding site distinct from the catalytic site in the monomer. This additional binding site could play the role of an allosteric site of hASNase1, and its existence is supported not only by the sigmoidal kinetic behavior of enzyme described in the previous paragraph but also by our results obtained from substrate titration to a fluorescence-labeled, catalytically inactive version of the protein.
Binding of the substrate L-Asn to the putative allosteric site was monitored by fluorescence signal changes of the Atto dye-labeled enzyme. The dye was covalently attached to cysteine residue Cys $^{299}$, which according to the structural model built on the $E$. coli enzyme directly interacts with L-Asn bound to the allosteric site (Fig. 5B). The labeling of hASNase1 was confirmed by SDS-PAGE analysis as shown in Fig. 7 based on the observation that labeled proteins migrate more slowly than the unlabeled species (34). Fig. 8 shows a pronounced fluorescence signal decrease upon incubation of the catalytically inactive T19A mutant of hASNase 1 with L-Asn in the concentration range of 0.1-40 $\mathrm{mm}$. We did not label the wild-type enzyme because upon titration of L-Asn notably at high concentrations it would hydrolyze the substrate without allowing us to solely evaluate binding phenomena (the T19A mutant is assumed to maintain intact its allosteric site similarly to the wild-type enzyme). From the resulting binding curve, we estimated a $K_{d}$ 


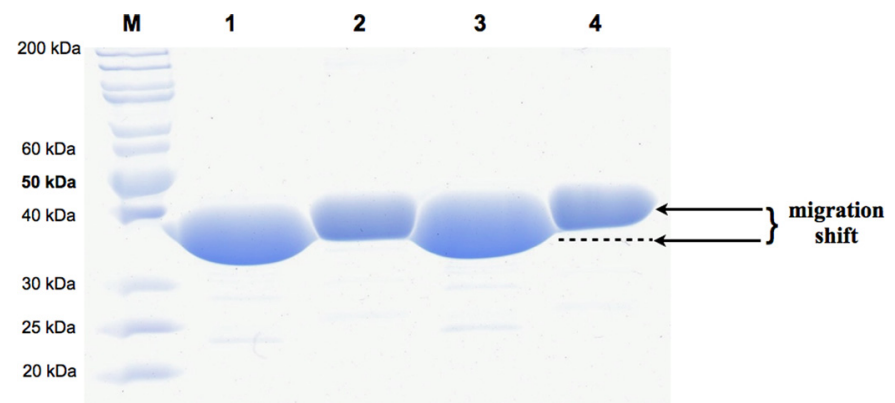

$15 \mathrm{kDa}$

FIGURE 7. SDS-PAGE analysis of unlabeled and labeled T19A and C299S mutants. Lane 1, unlabeled T19A; lane 2, labeled T19A; lane 3, unlabeled C299S; lane 4, labeled C299S; lane M, molecular mass markers. The electrophoretic pattern clearly shows the migration difference between the labeled and unlabeled enzyme species.

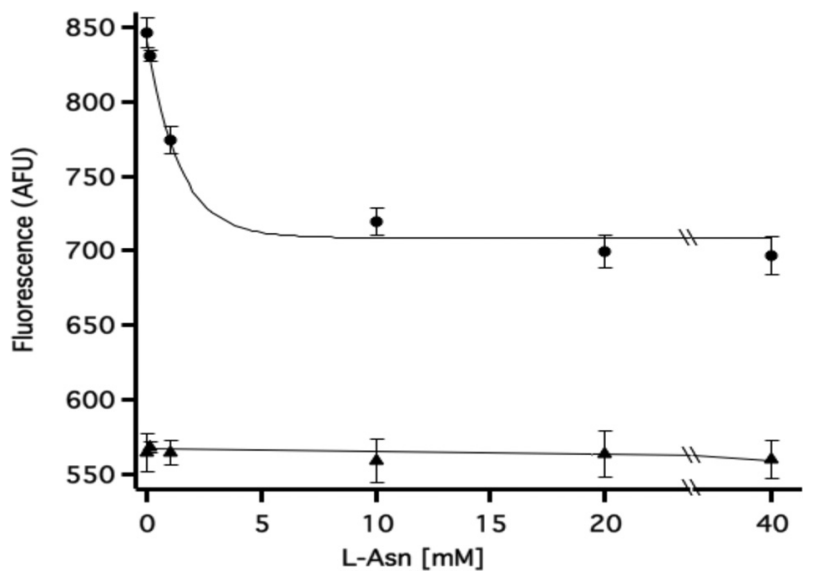

FIGURE 8. Plot of fluorescence signal change of Atto 465-labeled T19A and C299S hASNase1 mutants as a function of L-Asn concentration. Shown are the fluorescence signal decreases in the case of T19A indicating an induced conformational change upon binding of L-Asn to either the allosteric or the active site. In contrast, the C299S mutant, which is not labeled at the putative allosteric site, showed no signal changes upon L-Asn titration; constant background fluorescence results from labeling of other cysteines. The saturation binding curve for the T19A mutant was fitted to a hyperbolic function (Equation 2 in text) as described under "Experimental Procedures." In the case of C299S, where no fluorescence change was observed, the points were fitted to a straight line. Error bars represent S.D. AFU, arbitrary fluorescence units.

of $\sim 1 \mathrm{mM}$, which is about 10 -fold lower than the $S_{0.5}(\sim 11 \mathrm{~mm})$. Unlike T19A, the C299S mutant, which served as a negative control for labeling with the Atto dye, yielding a constant background fluorescence (due to labeling of other cysteines), showed no fluorescence signal changes upon incubation with L-Asn. This indicates that signal changes observed for T19A can be attributed to direct interaction of the substrate L-Asn with either $\mathrm{Cys}^{299}$ or other neighboring residues located within the allosteric cavity. Conversely, we cannot exclude potential interactions between L-Asn and the active site residues leading to conformational alterations that are transmitted to the allosteric site and reflected by fluorescence signal changes. Incubation of labeled enzyme with aspartate induced no fluorescence signal change (data not shown).

Thermodynamic Characterization of hASNase1 and EcASNase1-The free energy difference $\Delta G_{\mathrm{H} 2 \mathrm{O}}$ of folded and unfolded states, which is a measure of protein stability, was

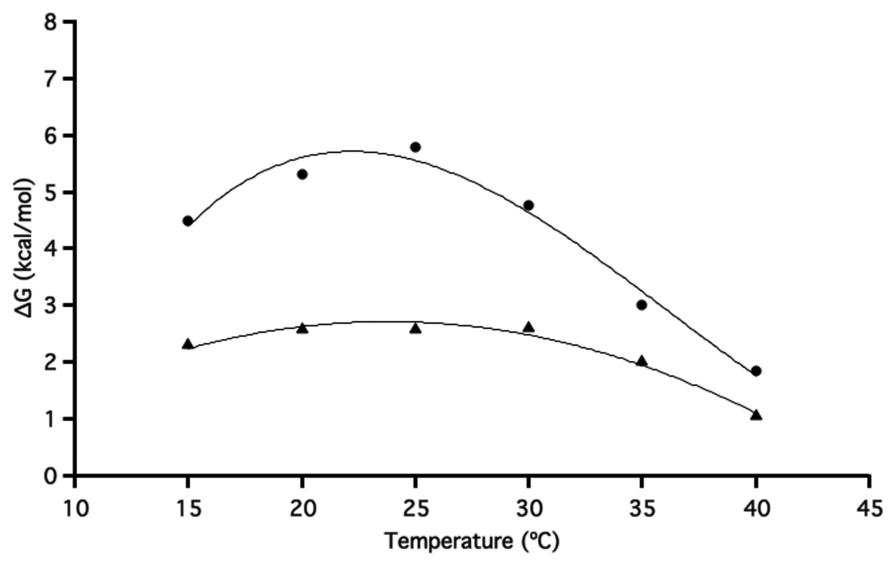

FIGURE 9. Temperature dependence of free energy change during unfolding of human and bacterial ASNase1. Shown is a plot of $\Delta G_{\mathrm{H} 2 \mathrm{O}}$ as obtained at various temperatures versus the respective temperatures for wild-type hASNase1 ( $\mathbf{\Delta})$ and EcASNase1 (O). Data were fitted to the GibbsHelmholtz equation (Equation 3 in text) after determining $T_{m}$ and $\Delta H_{m}$ by thermal denaturation experiments (see "Experimental Procedures"). The standard deviation for $\Delta G_{\mathrm{H} 2 \mathrm{O}}$ (free energy change at zero concentration of urea), evaluated from three independent determinations on the wild-type enzymes at $25^{\circ} \mathrm{C}$, was found to be $0.3 \mathrm{kcal} / \mathrm{mol}$.

\section{TABLE 2}

Thermodynamic parameters for wild-type hASNase 1 and EcASNase1

The $\Delta C_{p}$ values were calculated using the Gibbs-Helmholtz equation (see text) upon plotting the experimentally determined $\Delta G(T), T_{m}, \Delta S_{m}$, and $\Delta H_{m}$ values. $\Delta S_{m}, T_{m}$, and $\Delta H_{m}$ were determined from linear regression analysis of the thermal denaturation of either enzyme. Parameters are represented as means \pm S.D. of three measurements.

\begin{tabular}{ccccc}
\hline Enzyme & $\boldsymbol{\Delta} \boldsymbol{C}_{\boldsymbol{p}}$ & $\boldsymbol{T}_{\boldsymbol{m}}$ & $\boldsymbol{\Delta} \boldsymbol{H}_{\boldsymbol{m}}$ & \multicolumn{1}{c}{$\boldsymbol{\Delta} \boldsymbol{S}_{\boldsymbol{m}}$} \\
\hline & $\mathrm{kcal} / \mathrm{mol} \times K$ & ${ }^{\circ} \mathrm{C}$ & $\mathrm{kcal} / \mathrm{mol}$ & $\mathrm{call} / \mathrm{mol} / \mathrm{K}$ \\
hASNase1 & $5.47 \pm 0.20$ & $58.40 \pm 0.60$ & $104.2 \pm 1.5$ & $314 \pm 2$ \\
EcASNase1 & $3.10 \pm 0.15$ & $59.50 \pm 0.45$ & $89.7 \pm 0.9$ & $270 \pm 1.5$ \\
\hline
\end{tabular}

determined for both enzymes from urea denaturation experiments at different temperatures (37). Non-linear regression analysis was applied to the experimentally obtained $\Delta G_{\mathrm{H} 2 \mathrm{O}}$ values using the Gibbs-Helmholtz equation as a function of temperature, which allows the calculation of the $\Delta C_{p}$ parameter (48). Fig. 9 shows the stability curves for both enzymes studied in this work for direct comparison of their characteristic features under identical conditions. At $25^{\circ} \mathrm{C}$, the bacterial enzyme has a 2 -fold higher $\Delta G_{\mathrm{H} 2 \mathrm{O}}$ in comparison with the human enzyme, although this difference appears to decline at higher temperatures $\left(35\right.$ and $40{ }^{\circ} \mathrm{C}$ ) as has also been observed in other proteins (49). Table 2 summarizes thermodynamic parameters obtained for hASNase1 and EcASNase1. Interestingly, the predicted $\Delta C_{p}$ values are considerably different for the two enzymes, possibly pointing to variations in the surface area that is exposed upon denaturation (50) and distinct mechanisms of enthalpy and entropy changes occurring upon unfolding (51). The two enzymes exhibited similar melting temperatures $T_{m}$ with hASNase1 showing higher $\Delta \mathrm{H}_{m}$ and $\Delta S_{m}$ values, indicating higher enthalpy changes upon unfolding and a higher degree of disorder as compared with the E. coli enzyme (52).

Differential Scanning Fluorometry of hASNase 1-To analyze whether allosteric and active site mutations influenced the thermal stability of hASNase1, several of these hASNase1 mutants were studied by differential scanning fluorometry. The melting curves were all monophasic (Fig. 10). The melting tem- 


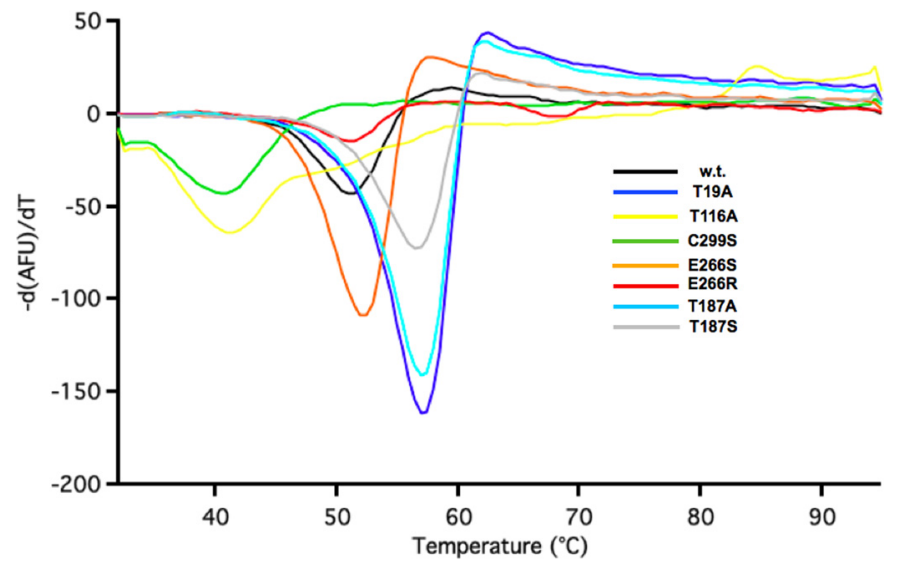

FIGURE 10. Melting curves for wild-type and mutant hASNase 1 as determined by differential scanning fluorometry. Melting temperatures were calculated by plotting the first derivative d(AFU)/dT (where AFU represents arbitrary fluorescence units) of the raw data as a function of the temperature range tested (see "Experimental Procedures").

\section{TABLE 3}

Melting temperatures of wild-type and mutant hASNase1 as determined by differential scanning fluorometry

Samples of $2 \mu \mathrm{M}$ highly purified enzyme solution were mixed with a $10 \%(\mathrm{v} / \mathrm{v})$ final concentration of SYPRO Orange in a final volume of $20 \mu \mathrm{l}$ in a 96-well plate. Enzyme melting experiments were performed using a CFX96 RT-PCR machine (Bio-Rad) and the following settings: 2 -min prewarming step at $30{ }^{\circ} \mathrm{C}$ and subsequent gradient between 31 and $95^{\circ} \mathrm{C}$ with $1{ }^{\circ} \mathrm{C} / \mathrm{min}$ increments. SYPRO Orange fluorescence was monitored using FAM excitation $(492 \mathrm{~nm})$ and ROX emission $(610 \mathrm{~nm})$ filters. Melting temperatures $\left(T_{m}\right)$ were calculated by plotting the first derivative $\mathrm{d}(\mathrm{AFU}) / \mathrm{d} T$ (where AFU represents arbitrary fluorescence units) of the raw data as a function of temperature increase (40).

\begin{tabular}{cc}
\hline Enzyme & $T_{m}$ \\
\hline & ${ }^{\circ} \mathrm{C}$ \\
Wild-type hASNase1 & 51.3 \\
T19A & 58.1 \\
T116A & 41 \\
C299S & 40 \\
E266S & 52.5 \\
E266R & 51 \\
T187A & 57.9 \\
T187S & 57.6 \\
\hline
\end{tabular}

perature $\left(T_{m}\right)$ values are summarized in Table 3. Our results suggest that three point mutations (T19A, T187A, and T187S) had a significant stabilizing effect on the enzyme In contrast, mutations T116A and C299S considerably lowered protein stability as compared with wild type, whereas the two mutations (E266S and E266R) introduced at the allosteric site $\mathrm{Glu}^{266}$ had almost no effect on the stability of hASNase 1.

Dependence of hASNase1 Activity on $\mathrm{pH}$ and Metal IonsThe optimum activity of hASNase1 was investigated in the $\mathrm{pH}$ range from 2.5 to 10 . Fig. $11 A$ shows that the enzyme exhibited the highest activity in a relatively narrow alkaline $\mathrm{pH}$ range as evidenced by a sharp increase from $\mathrm{pH} 7$ to 8 followed by an immediate decline from $\mathrm{pH} 8.5$ to 10 . In addition, the $\mathrm{pH}$ dependence for stability of the enzyme showed a maximum peak at $\mathrm{pH} 8$ (Fig. 11B). These results suggest that protonation of certain residues that are involved in catalysis might partially impair the activity of hASNase1. Furthermore, certain divalent metal ions, such as $\mathrm{Cd}^{2+}$ and $\mathrm{Cu}^{2+}$ at $1 \mathrm{~mm}$, totally inhibited hASNase1 activity, whereas DTT, $\mathrm{Co}^{2+}, \mathrm{Ca}^{2+}, \mathrm{Ni}^{2+}, \mathrm{Mg}^{2+}$, $\mathrm{Mn}^{2+}, \mathrm{Fe}^{2+}$, and $\mathrm{Li}^{+}$only weakly influenced the hydrolysis of L-Asn. Such metal-selective inhibition by $\mathrm{Cd}^{2+}$ and $\mathrm{Cu}^{2+}$ possibly indicates an interaction between these metal ions and a
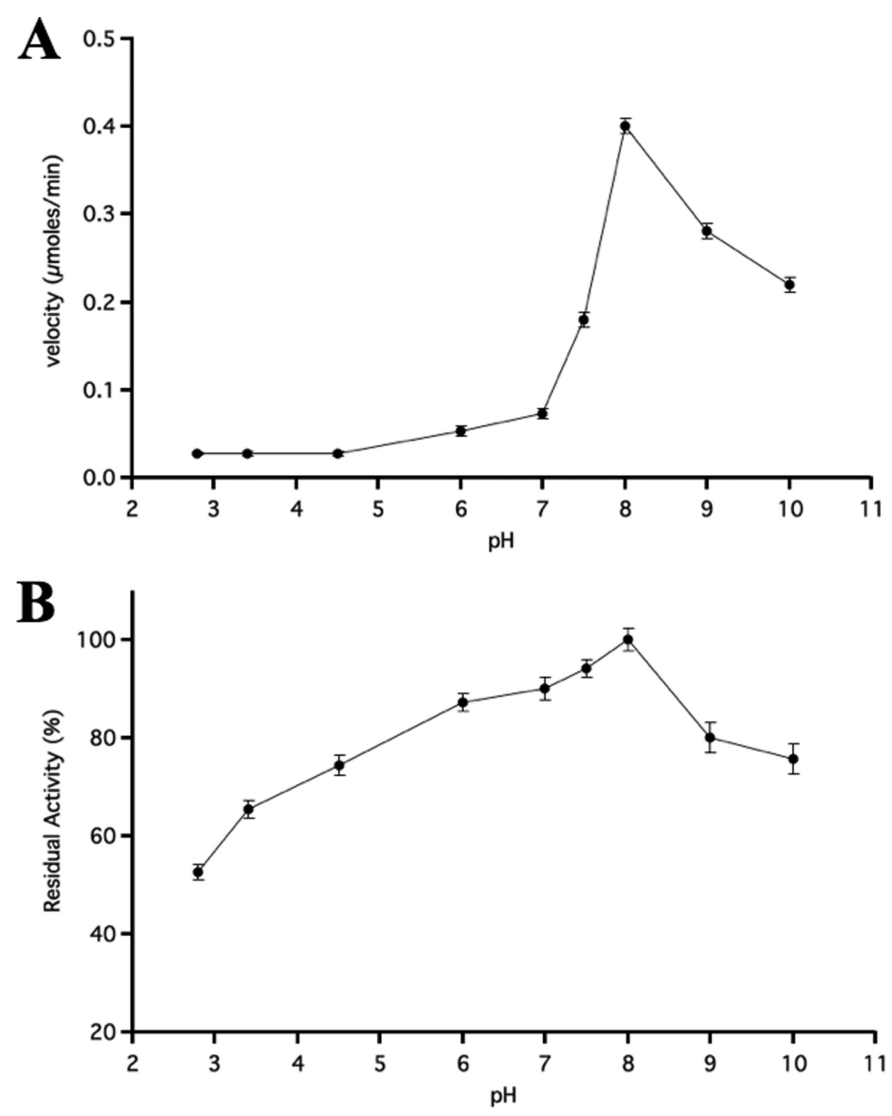

FIGURE 11. Effect of pH on hASNase1 activity $(A)$ and stability (B). Buffers used were: sodium acetate $(\mathrm{pH} 3-5)$, sodium phosphate $(\mathrm{pH} 6-7)$, Tris- $\mathrm{Cl}(\mathrm{pH}$ 7-8.5), and CAPSO (pH 9-10), all at $50 \mathrm{~mm}$ concentration in $100 \mathrm{~mm} \mathrm{NaCl}$. Enzymatic activities were determined as described under "Experimental Procedures" applying the Nesslerization assay for determining ammonia generated by L-asparagine hydrolysis. Error bars represent S.D.

\section{TABLE 4}

Effect of different salts, EDTA, and DTT on the activity of hASNase1

Purified hASNase1 $(0.1 \mathrm{mg} / \mathrm{ml})$ in $1 \mathrm{ml}$ was preincubated with a final concentration of $1 \mathrm{~mm}$ each of several divalent metal ions, EDTA, and DTT for $3 \mathrm{~h}$ at $4{ }^{\circ} \mathrm{C}$. Subsequently, aliquots were tested for residual L-asparaginase activity by the Nesslerization method (29). Data are shown as mean values \pm S.D. of triplicate measurements.

\begin{tabular}{ll}
\hline Compound $(1 \mathrm{mM})$ & \multicolumn{1}{c}{$\%$} \\
\hline & $100 \pm 0.6$ \\
EDTA & $64 \pm 4.1$ \\
$\mathrm{DTT}$ & $90 \pm 4.0$ \\
$\mathrm{NiSO}_{4}$ & $68 \pm 4.0$ \\
$\mathrm{CoCl}_{2}$ & $84 \pm 3.5$ \\
$\mathrm{CaCl}_{2}$ & $89 \pm 4.0$ \\
$\mathrm{MgCl}_{2}$ & 0 \\
$\mathrm{CdCl}_{2}$ & $89 \pm 1.8$ \\
$\mathrm{MnSO}_{4}$ & $73 \pm 2.8$ \\
$\mathrm{Fe}_{2} \mathrm{SO}_{4}$ & $64 \pm 3.8$ \\
$\mathrm{LiCl}_{\mathrm{CuCl}}$ & 0 \\
\hline
\end{tabular}

thiol group pivotal for activity (53). A candidate might be the $\mathrm{Cys}^{299}$ residue, which is located at the allosteric site of the enzyme and plays an important role in L-Asn binding. In addition, EDTA at concentrations up to $1 \mathrm{~mm}$ had no noticeable negative effect on the activity of hASNase1 (Table 4).

\section{DISCUSSION}

The present study focuses on the biochemical analysis of the N-terminal domain of a human $60-\mathrm{kD}$ a protein designated as lysophospholipase. The full-length version of this two-do- 
main protein has been only poorly characterized in one report (24). Our primary motivation to produce and functionally characterize the $\mathrm{N}$-terminal domain of this protein, which structurally and according to its catalytic in vitro properties significantly resembles E. coli cytoplasmic L-asparaginase (ansA; EcASNase1), originated from our previous work on human enzymes that possess L-asparaginase activity $(10,19,21)$. The discovery, molecular engineering, and in vitro evolution of catalytically efficient human L-asparaginases are thought to lay the basis for the replacement of bacterial L-asparaginases presently used as antileukemia therapeutics despite adverse side effects mainly attributed to their bacterial origins (54).

An early study on the rat $60-\mathrm{kDa}$ lysophospholipase (23) assigned three distinct activities to this enzyme acting as lysophospholipase, L-asparaginase, and acetylhydrolase on the platelet-activating factor. Several years later, a report on the human 60-kDa lysophospholipase (24) revealed a specific role of this enzyme as an interacting partner of the serum- and glucocorticoid-induced serine/threonine protein kinase Sgk1, an enzyme involved in various cell proliferation pathways. In contrast to its rat homolog, the human enzyme was not reported to bear L-asparaginase activity (24). However, in both these studies, the distinct enzymatic activities were determined only qualitatively using lysates from cells overexpressing the respective genes rather than purified enzyme preparations.

Here, we show that the $\mathrm{N}$-terminal L-asparaginase domain (residues 1-369) of the 573-residue human protein, that we termed hASNase1, can be produced in soluble form in E. coli. This protein showed L-asparaginase activity with a maximum catalytic rate of about $7 \mathrm{~s}^{-1}$, which is very similar to that of EcASNase1, implying that this $\mathrm{N}$-terminal domain forms a stable and functional folding unit in the absence of the $\mathrm{C}$-terminal putative ankyrin repeat domain. Kinetic and mutational characterization of hASNase1 revealed strong positive allosteric modulation in the velocity versus substrate plot similar to its $E$. coli homolog. To validate our experimental strategy for characterizing this human counterpart of the cytosolic E. coli L-asparaginase, we also recombinantly produced the bacterial protein to directly compare the two enzymes under identical assay conditions. Our kinetic data obtained on the bacterial enzyme are consistent with a previous study that centered on its structural analysis and allosteric regulation (25).

Given the pronounced sigmoidal kinetic behavior that we observed in steady-state kinetics of L-asparagine hydrolysis, we hypothesized that hASNase1 possesses an allosteric site that could act as "sensor" for the presence of substrate. Our dye labeling approach targeting the $\mathrm{Cys}^{299}$ residue indicated fluorescence signal changes upon binding of L-Asn, thus strengthening the idea of the existence of such an allosteric site that can be regulated by the substrate L-Asn. In the absence of information on the three-dimensional structure of hASNase1, which could provide more detailed insight into the catalytic mechanism and allosteric regulation of the enzyme, we referred to a structural homology-model based on the EcASNase1 crystal structure, which allowed us to define the active site and an allosteric site of the human enzyme. The $S_{0.5}$ value of $11.5 \mathrm{~mm}$ determined for hASNase1 is about 10-fold higher than that reported for the E. coli enzyme, raising questions about the physiological role of the full-length protein and its $\mathrm{N}$-terminal domain displaying L-asparaginase activity as shown in this work. The relatively high $S_{0.5}$ value determined for the human enzyme falls well within the range of free concentrations of intracellular amino acids $(>10 \mathrm{~mm})$, including $\mathrm{L}-\mathrm{Asn}$, reported for mammalian cells $(55,56)$, thus making the enzyme operate efficiently, particularly at elevated substrate concentrations.

The homo-oligomeric EcASNase1 displays sigmoidal kinetics, which can be explained by positive cooperativity induced by the substrate L-Asn. In contrast, hASNase1, which shows nonMichaelis-Menten kinetics similar to its E. coli homolog, is monomeric under the conditions of activity measurements. In fact, a number of monomeric enzymes exhibiting allosteric behavior have been reported of which the most thoroughly characterized is human glucokinase $(57,58)$. However, glucokinase (also called hexokinase IV), unlike L-asparaginase, is a twosubstrate (ATP plus glucose) enzyme and displays a moderate degree of allostery (59). However, monomeric enzymes with single binding sites, like hASNase1, can also show allostery (60). Two basic models have been put forward aiming at the mechanistic explanation of monomeric allostery: the mnemonic model (61) and the ligand-induced slow transition model (62). Both models assume the existence of two different enzyme conformations that are characterized by distinct affinities for the substrate (low and high affinity states). Depending on the substrate concentration, certain conformational changes may occur, thereby perturbing the equilibrium of the two states in favor of the high affinity state and an increased catalytic activity. Based on the observed sigmoidal kinetic behavior and the monomeric state of hASNase1, it appears plausible to assume that this is another example of a monomeric enzyme exhibiting positive allosteric regulation. Importantly, given the fact that L-Asn plays a dual role of being both substrate and regulator of hASNase1, L-Asn can be considered as a homotropic allosteric effector of this enzyme, adding to the steadily growing number of allosterically regulated proteins (63).

The role of the putative ankyrin repeat structure located in the C-terminal part of the full-length protein remains unknown at present. In numerous other proteins, ankyrin repeats were shown to mediate protein-protein interactions, and therefore, they may play crucial roles in cellular signaling events $(64-66)$. The study of the kinetics of the full-length $60-\mathrm{kDa}$ human lysophospholipase could provide information on the potential influence of the ankyrin repeat domain on the L-asparaginase activity predicted to reside in the N-terminal part. On the other hand, when we tested palmitoyl-lysoPC and lysoPI as potential substrates for hASNase1, we detected no lipase activity. Our biochemical data clearly support the view that the $\mathrm{N}$-terminal domain bears L-asparaginase activity that exhibits positive allosteric regulation by the substrate L-Asn. A particular characteristic of hASNase 1 is its monomeric state in conjunction with its pronounced sigmoidal steady-state kinetics. We would like to emphasize that the distinct enzymatic activities assigned to rat and human $60-\mathrm{kDa}$ lysophospholipases warrant further analyses at the cellular level to elucidate the physiological role of these two-domain proteins. Our studies on L-asparaginase activity inherent to the $\mathrm{N}$-terminal domain of this $60-\mathrm{kDa}$ protein expands the basis of our work aiming at the identification 
and molecular engineering of enzymes of human origin $(10,21)$ that might become suitable for replacing bacterial enzymes as approved therapeutics in the treatment of leukemias.

Acknowledgments-We thank Henning Urlaub and his team for expert mass spectrometry analyses and protein identification, Ursula Welscher-Altschäffel for technical assistance, and Claudia Höbartner for helpful comments on the manuscript.

\section{REFERENCES}

1. Credali, A., García-Calderón, M., Dam, S., Perry, J., Díaz-Quintana, A., Parniske, M., Wang, T. L., Stougaard, J., Vega, J. M., and Márquez, A. J. (2013) The $\mathrm{K}^{+}$-dependent asparaginase, NSE1, is crucial for plant growth and seed production in Lotus japonicus. Plant Cell Physiol. 54, 107-118

2. Willis, R. C., and Woolfolk, C. A. (1975) L-Asparagine uptake in Escherichia coli. J. Bacteriol. 123, 937-945

3. Michalska, K., and Jaskolski, M. (2006) Structural aspects of L-asparaginases, their friends and relations. Acta Biochim. Pol. 53, 627-640

4. Hejazi, M., Piotukh, K., Mattow, J., Deutzmann, R., Volkmer-Engert, R., and Lockau, W. (2002) Isoaspartyl dipeptidase activity of plant-type asparaginases. Biochem. J. 364, 129-136

5. Srikhanta, Y. N., Atack, J. M., Beacham, I. R., and Jennings, M. P. (2013) Distinct physiological roles for the two L-asparaginases isozymes of Escherichia coli. Biochem. Biophys. Res. Commun. 436, 362-365

6. Stecher, A. L., de Deus, P. M., Polikarpov, I., and Abrahão-Neto, J. (1999) Stability of L-asparaginase: an enzyme used in leukemia treatment. Pharm. Acta Helv. 74, 1-9

7. Duval, M., Suciu, S., Ferster, A., Rialland, X., Nelken, B., Lutz, P., Benoit, Y., Robert, A., Manel, A. M., Vilmer, E., Otten, J., and Philippe, N. (2002) Comparison of Escherichia coli-asparaginase with Erwinia-asparaginase in the treatment of childhood lymphoid malignancies: results of a randomized European Organization for Research and Treatment of CancerChildren's Leukemia Group phase 3 trial. Blood 99, 2734-2739

8. Asselin, B. L., Ryan, D., Frantz, C. N., Bernal, S. D., Leavitt, P., Sallan, S. E., and Cohen, H. J. (1989) In vitro and in vivo killing of acute lymphoblastic leukemia cells by L-asparaginase. Cancer Res. 49, 4363-4368

9. Brannigan, J. A., Dodson, G., Duggleby, H. J., Moody, P. C., Smith, J. L., Tomchick, D. R., and Murzin, A. G. (1995) A protein catalytic framework with an N-terminal nucleophile is capable of self-activation. Nature 378, $416-419$

10. Karamitros, C. S., and Konrad, M. (2014) Bacterial co-expression of the $\alpha$ and $\beta$ protomers of human L-asparaginase-3: achieving essential $\mathrm{N}$-terminal exposure of a catalytically critical threonine located in the $\beta$-subunit. Protein Expr. Purif. 93, 1-10

11. Borek, D., Michalska, K., Brzezinski, K., Kisiel, A., Podkowinski, J., Bonthron, D. T., Krowarsch, D., Otlewski, J., and Jaskolski, M. (2004) Expression, purification and catalytic activity of Lupinus luteus asparagine $\beta$-amidohydrolase and its Escherichia coli homolog. Eur. J. Biochem. 271, 3215-3226

12. Guan, C., Liu, Y., Shao, Y., Cui, T., Liao, W., Ewel, A., Whitaker, R., and Paulus, H. (1998) Characterization and functional analysis of the cis-autoproteolysis active center of glycosylasparaginase. J. Biol. Chem. 273, 9695-9702

13. Oinonen, C., Tikkanen, R., Rouvinen, J., and Peltonen, L. (1995) Threedimensional structure of human lysosomal aspartylglucosaminidase. Nat. Struct. Biol. 2, 1102-1108

14. Saito, S., Ohno, K., Sugawara K., Suzuki, T, Togawa, T., and Sakuraba, H. (2008) Structural basis for aspartylglucosaminuria. Biochem. Biophys. Res. Commun. 377, $1168-1172$

15. Cantor, J. R., Stone, E. M., Chantranupong, L., and Georgiou, G. (2009) The human asparaginase-like protein 1 hASRGL1 is an Ntn hydrolase with $\beta$-aspartyl peptidase activity. Biochemistry 48, 11026-11031

16. Dieterich, D. C., Landwehr, M., Reissner, C., Smalla, K. H., Richter, K., Wolf, G., Böckers, T. M., Gundelfinger, E. D., and Kreutz, M. R. (2003) Gliap: a novel untypical L-asparaginase localized to rat brain astrocytes. J. Neurochem. 85, 1117-1125
17. Weidle, U. H., Evtimova, V., Alberti, S., Guerra, E., Fersis, N., and Kaul, S. (2009) Cell growth stimulation by CRASH, an asparaginase-like protein overexpressed in human tumors and metastatic breast cancers. Anticancer Res. 29, 951-963

18. Michalska, K., Brzezinski, K., and Jaskolski, M. (2005) Crystal structure of isoaspartyl aminopeptidase in complex with L-aspartate. J. Biol. Chem. 280, 28484-28491

19. Nomme, J., Su, Y., Konrad, M., and Lavie, A. (2012) Structures of apo and product-bound human L-asparaginase: insights into the mechanism of auto-proteolysis and substrate hydrolysis. Biochemistry 51, 6816-6826

20. Li, W., Cantor, J. R., Yogesha, S. D., Yang, S., Chantranupong, L., Liu, J. Q., Agnello, G., Georgiou, G., Stone, E. M., and Zhang, Y. (2012) Uncoupling intramolecular processing and substrate hydrolysis in the N-terminal nucleophile hydrolase hASRGL1 by circular permutation. ACS Chem. Biol. $7,1840-1847$

21. Su, Y., Karamitros, C. S., Nomme, J., McSorley, T., Konrad, M., and Lavie, A. (2013) Free glycine accelerates the autoproteolytic activation of human asparaginase. Chem. Biol. 20, 533-540

22. Lowenson, J. D., Kim, E., Young, S. G., and Clarke, S. (2001) Limited accumulation of damaged proteins in L-isoaspartyl (D-aspartyl) $\mathrm{O}$-methyltransferase-deficient mice. J. Biol. Chem. 276, 20695-20702

23. Sugimoto, H., Odani, S., and Yamashita, S. (1998) Cloning and expression of cDNA encoding rat liver $60-\mathrm{kDa}$ lysophospholipase containing an asparaginase-like region and ankyrin repeat. J. Biol. Chem. 273, $12536-12542$

24. Menniti, M., Iuliano, R., Föller, M., Sopjani, M., Alesutan, I., Mariggiò, S., Nofziger, C., Perri, A. M., Amato, R., Blazer-Yost, B., Corda, D., Lang, F., and Perrotti, N. (2010) $60 \mathrm{kDa}$ lysophospholipase, a new Sgk1 molecular partner involved in the regulation of ENaC. Cell Physiol. Biochem. 26, 587-596

25. Yun, M. K., Nourse, A., White, S. W., Rock, C. O., and Heath R. J. (2007) Crystal structure and allosteric regulation of the cytoplasmic Escherichia coli L-asparaginase I. J. Mol. Biol. 369, 794-811

26. Peroutka, R. J., 3rd, Orcutt, S. T., Strickler, J. E., and Butt, T. R. (2011) SUMO fusion technology for enhanced protein expression and purification in prokaryotes and eukaryotes. Methods Mol. Biol. 705, 15-30

27. Bradford, M. M. (1976) A rapid and sensitive method for the quantitation of microgram quantities of protein utilizing the principle of protein-dye binding. Anal. Biochem. 72, 248-254

28. Noble, J. E., and Bailey, M. J. A. (2009) in Methods in Enzymology (Burgess, R. R., and Deutscher, M. P., eds), pp. 73-95, Academic Press, Waltham, MA

29. Derst, C., Henseling, J., and Röhm, K. H., (1992) Probing the role of threonine and serine residues of E.coli asparaginase II by site-specific mutagenesis. Protein Eng. 5, 785-789

30. Balcão, V. M., Mateo, C., Fernández-Lafuente, R., Malcata, F. X., and Guisán, J. M. (2001) Structural and functional stabilization of L-asparaginase via multisubunit immobilization onto highly activated supports. Biotechnol. Prog. 17, 537-542

31. She, H. S., Garsetti, D. E., Steiner, M. R., Egan, R. W., and Clark, M. A. (1994) The substrate specificities of four different lysophospholipases as determined by a novel fluorescence assay. Biochem. J. 298, 23-29

32. Kelley, L. A., and Sternberg, M. J. (2009) Protein structure prediction on the Web: a case study using the Phyre server. Nat. Protoc. 4, 363-371

33. DeLano, D. W. (2002) The PyMOL Molecular Graphics System, Schrödinger, LLC, New York

34. Modesti, M. (2011) in Methods in Molecular Biology (Peterman, E. J. G., and Wuite, G. J. L., eds), pp. 101-120, Humana Press Inc., New York

35. Grimsley, G. R., Trevino, S. R., Thurlkill, R. L., and Scholtz, J. M. (2013) Determining the conformational stability of a protein from urea and thermal unfolding curves. Curr. Protoc. Protein Sci. 71, 28.4.1-28.4.14

36. Shirley B. A. (1995) in Methods in Molecular Biology (Shirley, B. A., ed), pp. 177-190, Humana Press Inc., New York

37. Baskakov, I. V., and Bolen, D. W., (1999) The paradox between m values and $\Delta C$ p's for denaturation of ribonuclease $\mathrm{T} 1$ with disulfide bonds intact and broken. Protein Sci. 8, 1314-1319

38. Vedadi, M., Niesen, F. H., Allali-Hassani, A., Fedorov, O. Y., Finerty, P. J., Jr., Wasney, G. A., Yeung, R., Arrowsmith, C., Ball, L. J., Berglund, H., Hui, 


\section{Allosterically Regulated Human L-Asparaginase}

R., Marsden, B. D., Nordlund, P., Sundstrom, M., Weigelt, J., and Edwards, A. M. (2006) Chemical screening methods to identify ligands that promote protein stability, protein crystallization, and structure determination. Proc. Natl. Acad. Sci. U.S.A. 103, 15835-15840

39. Matulis, D., Kranz, J. K., Salemme, F. R., and Todd, M. J. (2005) Thermodynamic stability of carbonic anhydrase: measurements of binding affinity and stoichiometry using ThermoFluor. Biochemistry 44, 5258-5266

40. Boivin, S., Kozak, S., and Meijers, R. (2013) Optimization of protein purification and characterization using Thermofluor screens. Protein Expr. Purif. 91, 192-206

41. Rial, D. V., and Ceccarelli, E. A. (2002) Removal of DnaK contamination during fusion protein purifications. Protein Expr. Purif. 25, 503-507

42. Rohman, M., and Harrison-Lavoie, K. J. (2000) Separation of copurifying GroEL from glutathione-S-transferase fusion proteins. Protein Expr. Purif. 20, 45- 47

43. Calloni, G., Chen, T., Schermann, S. M., Chang, H. C., Genevaux, P., Agostini, F., Tartaglia, G. G., Hayer-Hartl, M., and Hartl, F. U. (2012) DnaK functions as a central hub in the E.coli chaperone network. Cell Rep. 1, 251-264

44. Thain, A., Gaston, K., Jenkins, O., and Clarke, A. R. (1996) A method for the separation of GST fusion proteins from co-purifying GroEL. Trends Genet. 12, 209-210

45. O’Brien, J. R., Schuller, D. J., Yang, V. S., Dillard, B. D., and Lanzilotta, W. N. (2003) Substrate-induced conformational changes in Escherichia coli taurine $/ \alpha$-ketoglutarate dioxygenase and insight into the oligomeric structure. Biochemistry 42, 5547-5554.

46. Ereño-Orbea, J., Majtan, T., Oyenarte, I., Kraus, J. P., and Martínez-Cruz, L. A. (2013) Structural basis of regulation and oligomerization of human cystathionine $\beta$-synthase, the central enzyme of transsulfuration. Proc. Natl. Acad. Sci. U.S.A. 110, E3790-E3799

47. Swain, A. L., Jaskólski, M., Housset, D., Rao, J. K., and Wlodawer, A. (1993) Crystal structure of Escherichia coli L-asparaginase, an enzyme used in cancer therapy. Proc. Natl. Acad. Sci. U.S.A. 90, 1474-1478

48. Talla-Singh, D., and Stites, W. E. (2008) Refinement of noncalorimetric determination of the change in heat capacity, $\Delta C_{p}$, of protein unfolding and validation across a wide temperature range. Proteins 71, 1607-1616

49. Pace, C. N., Grimsley, G. R., Thomas, S. T., and Makhatadze, G. I. (1999) Heat capacity change for ribonuclease A folding. Protein Sci. 8, $1500-1504$

50. Spolar, R. S., Livingstone, J. R., and Record, M. T., Jr. (1992) Use of liquid hydrocarbon and amide transfer data to estimate contributions to thermodynamic functions of protein folding from the removal of nonpolar and polar surface from water. Biochemistry 31, 3947-3955

51. Greene, R. F., Jr., and Pace, C. N. (1974) Urea and guanidine hydrochloride denaturation of ribonuclease, lysozyme, $\alpha$-chymotrypsin, and $\beta$-lactoglobulin. J. Biol. Chem. 249, 5388-5393

52. Baryshnikova, E. N., Melnik, B. S., Finkelstein, A. V., Semisotnov, G. V., and Bychkova, V. E. (2005) Three-state protein folding: experimental determination of free-energy profile. Protein Sci. 14, $2658-2667$

53. Bandyopadhyay, D., Chatterjee, A. K., and Datta, A. G. (1997) Effect of cadmium, mercury and copper on partially purified hepatic flavokinase of rat. Mol. Cell. Biochem. 167, 73-80

54. Pieters, R., Hunger, S. P., Boos, J., Rizzari, C., Silverman, L., Baruchel, A., Goekbuget, N., Schrappe, M., and Pui, C. H. (2011) L-Asparaginase treatment in acute lymphoblastic leukemia: a focus on Erwinia asparaginase. Cancer 117, $238-249$

55. Chaneton, B., Hillmann, P., Zheng, L., Martin, A. C., Maddocks, O. D., Chokkathukalam, A., Coyle, J. E., Jankevics, A., Holding, F. P., Vousden, K. H., Frezza, C., O'Reilly, M., and Gottlieb, E. (2012) Serine is a natural ligand and allosteric activator of pyruvate kinase M2. Nature 491, $458-462$

56. Hansen, H. A., and Emborg, C. (1994) Extra- and intracellular amino acid concentrations in continuous Chinese hamster ovary cell culture. Appl. Microbiol. Biotechnol. 41, 560-564

57. Kamata, K., Mitsuya, M., Nishimura, T., Eiki, J., and Nagata, Y. (2004) Structural basis for allosteric regulation of the monomeric allosteric enzyme human glucokinase. Structure 12, 429-438

58. Zhang, J., Li, C., Chen, K., Zhu, W., Shen, X., and Jiang, H. (2006) Conformational transition pathway in the allosteric process of human glucokinase. Proc. Natl. Acad. Sci. U.S.A. 103, $13368-13373$

59. Cárdenas, M. L. (2013) Michaelis and Menten and the long road to the discovery of cooperativity. FEBS Lett. 587, 2767-2771

60. Porter, C. M., and Miller, B. G. (2012) Cooperativity in monomeric enzymes with single ligand-binding sites. Bioorg. Chem. 43, 44-50

61. Ricard, J., Meunier, J. C., and Buc, J. (1974) Regulatory behavior of monomeric enzymes. 1. The mnemonical enzyme concept. Eur. J. Biochem. 49, 195-208

62. Cárdenas, M. L., Rabajille, E., and Niemeyer, H. (1984) Suppression of kinetic cooperativity of hexokinase $\mathrm{D}$ (glucokinase) by competitive inhibitors. A slow transition model. Eur. J. Biochem. 145, 163-171

63. Huang, Z., Mou, L., Shen, Q., Lu, S., Li, C., Liu, X., Wang, G., Li, S., Geng, L., Liu, Y., Wu, J., Chen, G., and Zhang, J. (2014) ASD v2.0: updated content and novel features focusing on allosteric regulation. Nucleic Acids Res. 42, D510-D516

64. Li, J., Mahajan, A., and Tsai, M. D. (2006) Ankyrin repeat: a unique motif mediating protein-protein interactions. Biochemistry 45, 15168-15178

65. Bennett, V., and Chen, L. (2001) Ankyrins and cellular targeting of diverse membrane proteins to physiological sites. Curr. Opin. Cell Biol. 13, 61-67

66. Voronin, D. A., and Kiseleva, E. V. (2007) Functional role of proteins containing ankyrin repeats. Tsitologiia 49, 989-999

67. Larkin, M. A., Blackshields, G., Brown, N. P., Chenna, R., McGettigan, P. A., McWilliam, H., Valentin, F., Wallace, I. M., Wilm, A., Lopez, R., Thompson, J. D., Gibson, T. J., and Higgins, D. G. (2007) Clustal W and Clustal X version 2.0. Bioinformatics 23, 2947-2948

68. Waterhouse, A. M., Procter, J. B., Martin, D. M., Clamp, M., and Barton, G. J. (2009) Jalview Version 2: a multiple sequence alignment editor and analysis workbench. Bioinformatics 25, 1189-1191

69. Lubkowski, J., Palm, G. J., Gilliland, G. L., Derst, C., Röhm, K. H., and Wlodawer, A. (1996) Crystal structure and amino acid sequence of Wolinella succinogenes L-asparaginase. Eur. J. Biochem. 241, 201-207 


\section{Supplementary Table (Primers used in this study)}

FW hASNase 1: 5'-GGAATTCCATATGGCGCGCGCGGTGGGGCCCG-3'

RV truncation 4: 5'-CGCGGATCCTTACCGGCGCTCTTCCACCGAGGG-3'

RV truncation 3: 5'-CGCGGATCCTTACGAGGGTGGCGTCATCTCCCC-3'

RV truncation 2: 5'-CGCGGATCCTTACCGAAGGTCCTTGGTCAGCAGC-3'

RV truncation 1: 5'-CGCGGATCCTTACAGCAGCTCCTTCCTGACATC-3'

FW BamHI elim.: 5'-CTCTACCCTGGCATCCCTGCCGCC-3'

RV BamHI elim.: 5'-GGCGGCAGGGATGCCAGGGTAGAG-3'

FW T19A: 5'-CTACACCGGCGGCGCCATTGGCATGCGG-3'

RV T19A: 5'-CCGCATGCCAATGGCGCCGCCGGTGTAG-3'

FW T116A: 5'-GTGGTCATCCACGGCGCCGACACCATGGCC-3'

RV T116A: 5'-GGCCATGGTGTCGGCGCCGTGGATGACCAC-3'

FW T187A/S: 5'-GGGGCAACCGGGCAKCCAAGGTAGACGCTCG-3' (where K: either T or G each with $50 \%$ probability)

RV T187A/S: 5'-CGAGCGTCTACCTTGGMTGCCCGGTTGCCCC-3' (where M: either A or C each with $50 \%$ probability)

FW E266R/S: 5'-GGGCGTGGTCATGMGCACCTTCGGTTCAGGG-3' (where M: either A or

C each with $50 \%$ probability)

RV E266R/S: 5'-CCCTGAACCGAAGGTGCKCATGACCACGCCC-3' (where K: either T or G each with $50 \%$ probability)

FW C299S: 5'-CAACTGTACCCACAGCCTCCAGGGGGCTG-3'

RV C299S: 5'-CAGCCCCCTGGAGGCTGTGGGTACAGTTG-3'

FW EcASNase 1: 5'-GGAATTCCATATGCAAAAGAAATCAATTTACGTTGC-3'

RV EcASNase1: 5'-CGCGGATCCTTAATCATCCGGCGTCAGTTCGCC-3'

FW EcASNase1 R240E: 5'-GAAAGCATTGATTCTGGAATCCTATGGCGTGGG -3'

RV EcASNase1 R240E: 5'-CCCACGCCATAGGATTCCAGAATCAATGCTTTC -3'

FW EcASNase1 C273S: 5'-GGTCAACCTGACACAAAGCATGTCCGGTAAAG -3'

RV EcASNase1 C273S: 5'-CTTTACCGGACATGCTTTGTGTCAGGTTGACC -3'

FW GroES: 5'-GGAATTCCATATGAATATTCGTCCATTGCATGATCG-3'

RV GroES: 5'-CGCGGATCCTTACGCTTCAACAATTGCCAGAATG-3' 


\title{
7. Preserving catalytic activity and enhancing biochemical stability of the therapeutic enzyme asparaginase by biocompatible multilayered polyelectrolyte microcapsules.
}

\author{
Christos S. Karamitros ${ }^{1}$, Alexey M. Yashchenok ${ }^{2}$, Helmuth Möhwald ${ }^{2}$, Andre G. \\ Skirtach $^{2,3}$ and Manfred Konrad ${ }^{1}$ (2013) \\ ${ }^{1}$ Enzyme Biochemistry Group, Max Planck Institute for Biophysical Chemistry, Göttingen \\ D-37077, Germany \\ ${ }^{2}$ Department of Interfaces, Max Planck Institute of Colloids and Interfaces, Potsdam, D-14424, \\ Germany \\ ${ }^{3}$ Department of Molecular Biotechnology, Centre for Nano-Biophotonics, Ghent University, \\ Ghent, B-9000, Belgium \\ Biomacromolecules \\ http://pubs.acs.org/doi/abs/10.1021/bm401341k \\ doi: $10.1021 / \mathrm{bm} 401341 \mathrm{k}$
}

\section{$\underline{\text { Author contributions }}$}

C.S.K. and M.K. conceived the project and designed research. C.S.K. and partially A.M.Y. performed research; C.S.K. and M.K. analyzed data and wrote the paper.

\section{Detailed author contributions of C.S.Karamitros}

- Experimental work: All, excluding the Figure 1B,C,D.

- Data analysis: All data were analyzed by C.S.K. and M.K.

- Preparation of the manuscript: C.S.K. and M.K. wrote the paper.

\section{Copyright notice:}

This material is reproduced by permission from the American Chemical Society in accordance to their copyright and licensing regulations. 


\title{
Preserving Catalytic Activity and Enhancing Biochemical Stability of the Therapeutic Enzyme Asparaginase by Biocompatible Multilayered Polyelectrolyte Microcapsules
}

\author{
Christos S. Karamitros, ${ }_{\dagger}^{\dagger}$ Alexey M. Yashchenok, ${ }^{\ddagger}$ Helmuth Möhwald, ${ }^{\ddagger}$ Andre G. Skirtach, ${ }^{\ddagger}$, \\ and Manfred Konrad* ${ }^{*} \dagger$ \\ ${ }^{\dagger}$ Enzyme Biochemistry Group, Max-Planck Institute for Biophysical Chemistry, Göttingen, D-37077, Germany \\ ${ }^{\ddagger}$ Department of Interfaces, Max-Planck Institute of Colloids and Interfaces, Potsdam, D-14424, Germany \\ ${ }^{\S}$ Department of Molecular Biotechnology, Centre for Nano-Biophotonics, Ghent University, Ghent, B-9000, Belgium
}

\section{Supporting Information}

ABSTRACT: The present study focuses on the formation of microcapsules containing catalytically active L-asparaginase (LASNase), a protein drug of high value in antileukemic therapy. We make use of the layer-by-layer ( $\mathrm{LbL}$ ) technique to coat protein-loaded calcium carbonate $\left(\mathrm{CaCO}_{3}\right)$ particles with two

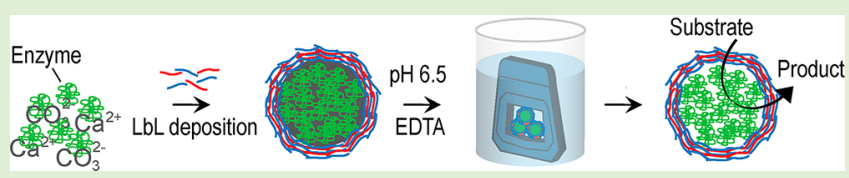
or three poly dextran/poly-L-arginine-based bilayers. To achieve high loading efficiency, the $\mathrm{CaCO}_{3}$ template was generated by coprecipitation with the enzyme. After assembly of the polymer shell, the $\mathrm{CaCO}_{3}$ core material was dissolved under mild conditions by dialysis against $20 \mathrm{mM}$ EDTA. Biochemical stability of the encapsulated L-asparaginase was analyzed by treating the capsules with the proteases trypsin and thrombin, which are known to degrade and inactivate the enzyme during leukemia treatment, allowing us to test for resistance against proteolysis by physiologically relevant proteases through measurement of residual L-asparaginase activities. In addition, the thermal stability, the stability at the physiological temperature, and the longterm storage stability of the encapsulated enzyme were investigated. We show that encapsulation of L-asparaginase remarkably improves both proteolytic resistance and thermal inactivation at $37^{\circ} \mathrm{C}$, which could considerably prolong the enzyme's in vivo half-life during application in acute lymphoblastic leukemia (ALL). Importantly, the use of low EDTA concentrations for the dissolution of $\mathrm{CaCO}_{3}$ by dialysis could be a general approach in cases where the activity of sensitive biomacromolecules is inhibited, or even irreversibly damaged, when standard protocols for fabrication of such LbL microcapsules are used. Encapsulated and free enzyme showed similar efficacies in driving leukemic cells to apoptosis.

\section{INTRODUCTION}

The catabolic enzyme L-asparaginase (L-asparagine amidohydrolase, EC 3.5.1.1; L-ASNase) catalyzes the hydrolysis of the amino acid asparagine to aspartic acid and ammonia. It is present in organisms ranging from bacteria to mammals, though its activity rarely is essential for cell viability. In humans, beyond its metabolic role, L-ASNase serves as a therapeutic enzyme that is used in combination with other drugs, such as vincristine and prednisone for the treatment primarily of acute lymphoblastic leukemia (ALL) and secondarily of non-Hodgkin lymphoma. ${ }^{1}$ ALL is the most common childhood blood cancer, with a long-term survival rate around $80 \%{ }^{2,3}$ The antiproliferating properties of L-ASNase were first identified and characterized in human clinical trials in the 1970s. ${ }^{4-7}$ Since then, this enzyme has become a milestone in the treatment of ALL. The principle of its effect as a protein drug relies on the fact that cancerous lymphoblasts cannot synthesize sufficient levels of asparagine due to down-regulation of their asparagine synthetase. ${ }^{8,9}$ Therefore, contrary to normal cells, asparagine is an essential amino acid for malignant cells whose survival is exclusively dependent on the availability of extracellular asparagine from the bloodstream. Intravenously or intramuscularly administered L-ASNase depletes the available free asparagine from the extracellular fluid, ${ }^{10}$ resulting in protein and nucleic acid synthesis inhibition, which ultimately leads to cell apoptosis. ${ }^{11}$

Bacterial L-ASNase is the only nonhuman enzyme that has been approved by the American Food and Drug Administration (FDA) for treatment of ALL. ${ }^{12}$ The isoforms that are currently used are recombinantly produced proteins derived from Escherichia coli and Erwinia chrysanthemi L-ASNases. Many severe toxic side effects have been attributed to the bacterial origins of these L-ASNases, the most important one being immunogenicity; others include hepatic dysfunctions, pancreatitis, central nervous system abnormalities, anorexia, hyperglycemia, thrombosis, and quite frequently, lethal hypersensitivity reactions. ${ }^{13-15}$ Certain side effects are thought to be caused by the intrinsic glutaminase activity of bacterial LASNases, influencing both the glutamine and glutamic acid levels. ${ }^{16}$ Glutamine is the major transport form of amino nitrogen in blood, and glutamic acid has a notable impact on the central nervous system, since it plays a key role as a

Received: September 6, 2013

Revised: October 17, 2013

Published: October 21, 2013 
neurotransmitter. However, the major limitation of L-ASNase used as an anticancer agent is related to the so-called "silent inactivation" of the enzyme. ${ }^{17}$ This inactivation arises from the interaction with specific antibodies which are generated upon recognition of the enzyme as antigen by the patient's immune system, and this is usually not accompanied by any clinical symptoms of hypersensitivity. ${ }^{18,19}$ This inactivation of LASNase, which manifests itself in a blood serum half-life of only about $24 \mathrm{~h}$, necessitates continuous administration and progressively higher doses of the therapeutic enzyme. ${ }^{19}$ It has also been shown recently ${ }^{20}$ that, apart from silent inactivation, two highly specific lysosomal proteases degrade L-ASNase and therefore are coresponsible for the clearance of the enzyme.

The search for biochemical strategies to increase the half-life of the enzyme has prompted considerable efforts to enhance the stability of L-ASNase, by protecting it through surface modifications and packaging into nanocapsules ${ }^{21}$ and nanospheres. ${ }^{22}$ Encapsulation potentially prevents exposure of the enzyme to the immune system. Furthermore, the enzyme would be protected from degradation by proteases resulting in extended serum half-life. A promising example for success in such efforts was the development of the FDA-approved polyethyleneglycol-modified (PEGylated) form of E. coli LASNase, which diminished the immunogenicity, and raised the in vivo half-life of the enzyme considerably. ${ }^{19,23-25}$ Another study showed that polysialylation of the enzyme ${ }^{26}$ gradually reduced the antigenicity of the enzyme depending on the degree of lysine side-chain modification by polysialic acid, whereas no immune response was elicited against the conjugated agent. The most recent advance toward improving the therapeutic potential of L-ASNase relies on the use of intact erythrocytes ${ }^{27}$ as carriers of the therapeutic enzyme. Although the first preliminary clinical results are quite promising, the whole process is rather time-consuming, complex, and expensive, requiring patient-specific strategies of personalized medicine.

Herein, we report on a novel approach for encapsulation of asparaginase using glutaminase-free Saccharomyces cerevisiae cytoplasmic L-asparaginase I (ScASNaseI) as model enzyme. We capitalize on the use of the layer-by-layer ( $\mathrm{LbL}$ ) method of microcapsule formation. ${ }^{28-32}$ This technique allows biomolecules to be efficiently adsorbed onto a colloidal core particle, which serves as template for the consecutive adsorption of several layers of oppositely charged polymers. The last step of capsule formation includes the dissolution of the template material, which ultimately gives rise to hollow capsules that contain the initially adsorbed biomolecule. The polymers used for therapeutic proteins ideally are biocompatible. Numerous examples of successful encapsulation of proteins, ${ }^{33-35}$ polymers, ${ }^{36}$ and nucleic acids ${ }^{37}$ using this technique have been reported.

In the present study, the encapsulation of L-asparaginase was performed using calcium carbonate particles as templates for protein adsorption, which subsequently were coated with cationic poly-L-arginine and anionic dextran sulfate layers. ${ }^{32}$ The capsules were then treated with EDTA, resulting in the removal of $\mathrm{CaCO}_{3}$ and formation of the final polyelectrolyte capsules. As L-asparaginase is a protein drug with considerable interest for further improvement of its use in blood cancer therapy, we aimed to investigate whether biocompatible encapsulation could be applied to the packaging of this enzyme in order to enhance its biochemical stability and ultimately prolong its ability to maintain catalytic activity in an in vivo situation. We chose as model enzyme the yeast L-asparaginase ScASNaseI, which is a glutaminase-free L-asparaginase with promising catalytic properties. In cell culture experiments, encapsulated and free enzyme displayed similar potency in inducing apoptotic cell death. Thus, our work suggests that efficient encapsulation of therapeutic proteins, in combination with successful enzyme engineering, could set the basis for novel ways for alternative treatment of blood cancers. This view is supported by recent reports on advances in encapsulation of enzymes using various types of polymers ${ }^{38,39}$ for medical applications of nanocontainers as carriers of bioactive molecules. ${ }^{40,41}$

\section{MATERIALS AND METHODS}

Materials. Ethylenediamine-tetraacetic acid disodium salt (EDTA), calcium chloride $\left(\mathrm{CaCl}_{2}\right)$, sodium carbonate $\left(\mathrm{Na}_{2} \mathrm{CO}_{3}\right)$, dextran sulfate sodium salt $\left(M_{\mathrm{w}} \sim 10 \mathrm{kDa}\right)$, poly-L-arginine hydrochloride $\left(M_{\mathrm{w}}>70 \mathrm{kDa}\right)$, yeast extract, peptone from casein, $\mathrm{NaCl}$, and Nessler's reagent were purchased from Sigma-Aldrich-Fluka (St. Louis, MO). Dialysis membranes and Coomassie Brilliant Blue (Bradford) reagent were from ROTH (Karlsruhe, Germany). Oligonucleotides were purchased from IBA GmbH (Goettingen, Germany). Restriction enzymes, T4 DNA ligase and Trypsin Ultra isolated from bovine pancreas were obtained from New England Biolabs (Ipswich, MA). Thrombin from bovine plasma was from Serva (Heidelberg, Germany). KAPA HiFi Polymerase and all PCR reagents were purchased from PeqLab (Erlangen, Germany). Gel extraction and PCR purification kit as well as nickel agarose for protein purification were purchased from Macherey Nagel (Düren, Germany). Plasmid purification kit was from Fermentas (Thermo Fisher Scientific, Germany). Genomic DNA preparation kit was obtained from Qiagen (Hilden, Germany). RPMI 1640 cell culture medium was from PAA (Cölbe, Germany) and WST-1 proliferation kit was purchased from Roche (Mannheim, Germany).

Cloning, Expression and Purification of ScASNasel, EcASNasell, and eGFP-SCASNasel Fusion Protein. The preparation of ScASNaseI, EcASNaseII, and eGFP-ScASNaseI fusion protein was done as described in the Supporting Information.

Preparation of ScASNasel-Loaded Calcium Carbonate Particles. $\mathrm{CaCO}_{3}$ microparticles were prepared by mixing equal molar amounts of $\mathrm{Na}_{2} \mathrm{CO}_{3}$ and $\mathrm{CaCl}_{2}$ under stirring at room temperature at working concentrations of $0.15 \mathrm{M}$ each dissolved in $\mathrm{ddH}_{2} \mathrm{O}$. For the loading of protein, the coprecipitation approach was followed. To obtain calcium carbonate cores loaded with ScASNaseI, we added $2 \mathrm{~mL}$ of protein solution $\left(1.5 \mathrm{mg} / \mathrm{mL}\right.$ in $50 \mathrm{mM} \mathrm{Na}_{2} \mathrm{HPO}_{4}$, $0.5 \mathrm{M} \mathrm{NaCl}, \mathrm{pH} 7.5$ ) to $2 \mathrm{~mL}$ of $\mathrm{Na}_{2} \mathrm{CO}_{3}$ prior to mixing with $2 \mathrm{~mL}$ of $\mathrm{CaCl}_{2}$. Following mixing (for $60 \mathrm{~s}$ at $1000 \mathrm{rpm}$ ) after addition of $\mathrm{CaCl}_{2}$ and the formation of $\mathrm{CaCO}_{3}$ microparticles, the enzyme was entrapped in the porous structure of the templates. Subsequently, the particles were spun down (at $400 \mathrm{~g}$ for $5 \mathrm{~min}$ ) and washed three times with $50 \mathrm{mM} \mathrm{Na}_{2} \mathrm{HPO}_{4}, 0.5 \mathrm{M} \mathrm{NaCl}, \mathrm{pH}$ 7.5. The number of formed $\mathrm{CaCO}_{3}$ particles was determined by using a hemocytometer (BrightLine).

To obtain calcium carbonate particles loaded with eGFPScASNasel, we used the same protocol as mentioned above with minor changes, that is, $1 \mathrm{~mL}(0.8 \mathrm{mg} / \mathrm{mL})$ of eGFP-labeled protein was added to $2 \mathrm{~mL}$ of $\mathrm{CaCl}_{2}$ prior to mixing with $2 \mathrm{~mL}$ of $\mathrm{Na}_{2} \mathrm{CO}_{3}$. Then the mixture was stirred for $1 \mathrm{~min}$ at $1000 \mathrm{rpm}$ after adding of $\mathrm{Na}_{2} \mathrm{CO}_{3}$.

Determination of Protein Loading Efficiency in Calcium Carbonate Particles. To evaluate the loading efficiency of ScASNaseI after the precipitation reaction, the formed $\mathrm{CaCO}_{3}$ particles were centrifuged, washed, and counted. The amount of the enzyme present in the supernatant after coprecipitation, as well as after each washing step, was determined spectrophotometrically by the dyebinding method according to Bradford ${ }^{42}$ using BSA fraction $\mathrm{V}$ as protein standard. The loading efficiency is expressed as amount of enzyme per $\mathrm{CaCO}_{3}$ particle. The encapsulation efficiency was similarly 
estimated by subtracting the loss of protein after centrifugation and washing steps from the total enzyme amount initially used for coprecipitation.

Formation of Hollow Multilayer Polyelectrolyte Microcapsules. The layer-by-layer (LbL) technique was applied in order to prepare multilayer polyelectrolyte microcapsules. Briefly, the protein-loaded $\mathrm{CaCO}_{3}$ particles were first suspended in $1 \mathrm{~mL}$ of 1 $\mathrm{mg} / \mathrm{mL}$ dextran sulfate dissolved in $0.5 \mathrm{M} \mathrm{NaCl}, \mathrm{pH} 6.5$ (adjusted with $\mathrm{HCl} / \mathrm{NaOH}$ ), and the sample was shaken at $1200 \mathrm{rpm}$ at $25^{\circ} \mathrm{C}$ for 20 $\mathrm{min}$. After the attachment of the first polyelectrolyte layer, the particles were centrifuged at $400 \mathrm{~g}$ for $5 \mathrm{~min}$, washed 3 times with washing buffer ( $\left.50 \mathrm{mM} \mathrm{Na}_{2} \mathrm{HPO}_{4}, 0.5 \mathrm{M} \mathrm{NaCl}, \mathrm{pH} 7.5\right)$, and then dispersed in a solution containing $1 \mathrm{mg} / \mathrm{mL}$ poly-L-arginine. In the present work, overall 2 or 3 polyelectrolyte bilayers were incorporated. The fractions after each washing step were collected to determine the loss of protein. To obtain hollow multilayer polyelectrolyte microcapsules loaded with functional L-asparaginase, we modified the widely used standard protocol by dissolving the template material by dialysis under mild conditions, rather than by direct addition of the calcium-chelating agent EDTA, as outlined in Scheme 1. To this end, the initial batch

Scheme 1. Schematic Illustration of the Preparation of ScASNaseI-Filled Polyelectrolyte Capsules ${ }^{a}$

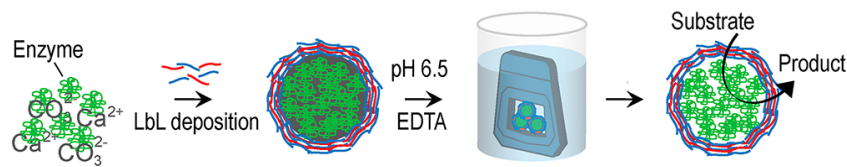

${ }^{a}$ After coating with the outermost polyelectrolyte layer (here: poly-Larginine), the suspension was subjected to overnight-dialysis yielding the final homogeneous hollow capsules containing active L-ASNase.

preparation was divided into smaller volumes which were dialyzed overnight at $4{ }^{\circ} \mathrm{C}$ (volume ratio $1: 1000$ ) against dialysis buffer (50 $\mathrm{mM} \mathrm{Na}_{2} \mathrm{HPO}_{4}, 0.5 \mathrm{M} \mathrm{NaCl}, 20 \mathrm{mM}$ EDTA, $\mathrm{pH}$ 6.5). Following this protocol, we managed to dissolve the $\mathrm{CaCO}_{3}$ cores resulting in the formation of microcapsules containing catalytically active ScASNaseI.

Zeta Potential Measurements. The zeta $(\mathrm{Z})$ potential of the microparticles was measured before adsorbing the first layer to the $\mathrm{CaCO}_{3}$ cores, and then after each polyelectrolyte adsorption step, in order to determine the surface charge. The measurements were performed on a Zetasizer Nano Series analyzer (Malvern). Samples were prepared by dispersing $50 \mu \mathrm{L}$ aliquots of coated microparticles in $1 \mathrm{~mL}$ of $\mathrm{ddH}_{2} \mathrm{O}$. Zeta potential values were determined according to the manufacturer's instructions; measurements on each sample were repeated three times.

Light Microscopy. The formation of $\mathrm{CaCO}_{3}$ particles and of the final microcapsules was followed by light microscopy. Therefore, aliquots of proper dilutions of the batch preparation were placed on a cover glass, and particles were analyzed for spherical homogeneity using an Olympus SZX12 stereo microscope with the following settings: DFPLFL 1.6X PF objective and 144× magnification using a $10 \times$ eyepiece.

Confocal Laser-Scanning Fluorescence Microscopy (CLSM). Confocal micrographs were recorded with a Leica TCS SP confocal scanning microscope (Leica, Germany) in the inverted microscope mode, using a $100 \times$ oil immersion objective with the numerical aperture of 1.4. The excitation wavelength was $488 \mathrm{~nm}$ for eGFPlabeled fusion protein.

Scanning Electron Microscopy (SEM). Scanning electron microscopy images of the capsules filled with active ScASNaseI were taken on samples prepared as follows: capsules in suspension were dropped on silica wafer and were left to dry at room temperature. The wafer was then immobilized on an aluminum stage by using an intermediate carbon layer. Finally, the sample was sputtered with gold film, and was analyzed using a Philips XL30 at $3 \mathrm{kV}$.

L-Asparaginase Activity Assay. The enzymatic activity of ScASNaseI was determined by the Nesslerization method detecting ammonia, as described elsewhere. ${ }^{43}$ Briefly, the encapsulated enzyme was incubated with the substrate L-asparagine in $50 \mathrm{mM} \mathrm{Na}_{2} \mathrm{HPO}_{4}, 0.5$ $\mathrm{M} \mathrm{NaCl}, \mathrm{pH} 7.5$, under saturation conditions ( $5 \mathrm{mM}$ asparagine) at 37 ${ }^{\circ} \mathrm{C}$ for $20 \mathrm{~min}$. In parallel, a second identical sample was incubated at $37{ }^{\circ} \mathrm{C}$ for $20 \mathrm{~min}$, in which the enzymatic reaction was quenched at time zero by adding trichloroacetic acid (TCA) to a final concentration of $10 \%$. After TCA precipitation, both samples were centrifuged at $16100 \mathrm{~g}$ for $2 \mathrm{~min}$. The supernatant was mixed with Nessler's reagent, which is a solution of potassium tetraiodomercurate that forms a yellowish complex with ammonia. The absorbance was monitored spectrophotometrically at $\sim 440 \mathrm{~nm}$ after 10 min incubation at room temperature. The concentration of ammonia produced by the enzymatic reaction was determined from a reference curve using ammonium chloride as standard. An enzymatic unit is defined as the amount of enzyme that catalyzes the conversion of $1 \mu$ mole of $\mathrm{L}$ asparagine per minute under the aforementioned conditions.

Proteolytic Resistance of Encapsulated ScASNasel. Microcapsules filled with $S c$ ASNaseI $\left(\sim 4 \times 10^{7} / \mathrm{mL}\right)$, suspended in $50 \mathrm{mM}$ $\mathrm{Na}_{2} \mathrm{HPO}_{4}, 0.5 \mathrm{M} \mathrm{NaCl}, \mathrm{pH} 7.5$, were aliquoted in $1 \mathrm{~mL}$ samples, mixed independently with either $10 \mu \mathrm{g}$ trypsin (0.02 total units) or 10 $\mu \mathrm{g}$ thrombin (10 total units) and then incubated at $37{ }^{\circ} \mathrm{C}$. Trypsin stock solution $(2 \mathrm{mg} / \mathrm{mL})$ was stored in $50 \mathrm{mM}$ Tris- $\mathrm{HCl}, 20 \mathrm{mM}$ $\mathrm{CaCl}_{2}, \mathrm{pH}$ 8.0; lyophilized thrombin was dissolved in $50 \mathrm{mM}$ Tris$\mathrm{HCl}, 140 \mathrm{mM} \mathrm{NaCl}, 2 \mathrm{mM} \mathrm{CaCl}$, pH $8.0(2 \mathrm{mg} / \mathrm{mL})$. After different periods of time, residual asparaginase activity was measured. Free enzyme, exhibiting initial volumetric activity similar to the encapsulated sample, was incubated in parallel at $37{ }^{\circ} \mathrm{C}$ using the same concentration of proteases. Its residual activity was also evaluated at different time intervals.

Thermal Stability of Free and Encapsulated ScASNasel. Irreversible thermal inactivation of free and encapsulated enzyme was followed by activity measurements. Both preparations resuspended in $50 \mathrm{mM} \mathrm{Na}_{2} \mathrm{HPO}_{4}, 0.5 \mathrm{M} \mathrm{NaCl}, \mathrm{pH} 7.5$, were incubated for $10 \mathrm{~min}$ at different temperatures covering the range $4-65^{\circ} \mathrm{C}$ and then placed on ice for an additional $10 \mathrm{~min}$ to recover. Subsequently, equal volumes of the samples were assayed for activity. Residual activity is expressed as relative (\%) activity in comparison to the initial activity $(\mathrm{U} / \mathrm{mL})$, which was equal for control and samples. The midinactivation temperature $T_{\mathrm{m}}$ is defined as the temperature at which the enzyme retains $50 \%$ of its initial activity and was calculated by plotting the relative residual activity against the temperature.

The rate of isothermal inactivation of the free and encapsulated enzyme was studied at $37{ }^{\circ} \mathrm{C}$ in $50 \mathrm{mM} \mathrm{Na} \mathrm{HPO}_{4}, 0.5 \mathrm{M} \mathrm{NaCl}, \mathrm{pH}$ 7.5 buffer. Samples with equal initial activities were incubated at $37^{\circ} \mathrm{C}$, and their residual activities were measured at defined time intervals over $30 \mathrm{~h}$. The first order inactivation rate constants $k$ were determined from the slopes of the inactivation time courses according to the equation $\ln \left(A_{t} / A_{0}\right)=-k t$, where $A_{0}$ is the initial enzymatic activity, and $A_{t}$ is the activity after time $t$. Rate constants $k$ were estimated by linear regression analysis using the Igor Pro data analysis software (Wavemetrics).

Storage Stability of Free and Encapsulated ScASNasel. Preparations of free and encapsulated $S c$ ASNaseI in $50 \mathrm{mM} \mathrm{Na}_{2} \mathrm{HPO}_{4}$, $0.5 \mathrm{M} \mathrm{NaCl}, \mathrm{pH} 7.5$, exhibiting similar initial volume activities were stored at $4{ }^{\circ} \mathrm{C}$ in the absence of protein stabilizers (such as glycerol). Residual activities of both samples were monitored every week for a total period of 3 months.

In Vitro Killing of Cancerous Lymphocytes by Encapsulated ScASNasel. To assess the effect of encapsulated ScASNaseI on cancerous lymphocytes, we performed cell culture experiments using the two leukemic cell lines SD1 and MOLT-4. Both cell lines were obtained from DSMZ (German Collection of Microorganisms and Cell Cultures, Braunschweig). SD1 cells belong to the $\mathrm{B}$ lymphoblastoid type of cells and have a doubling time of $\sim 30 \mathrm{~h}$, while MOLT-4 cells are derived from T leukemic cells and have a doubling time of $\sim 40 \mathrm{~h}$. The SD1 cell line was cultured at $37{ }^{\circ} \mathrm{C} / 5 \%$ $\mathrm{CO}_{2}$ in RPMI 1640 medium (PAA) with $10 \%$ FBS, while MOLT-4 cells were cultured at $37{ }^{\circ} \mathrm{C} / 5 \% \mathrm{CO}_{2}$ in RPMI 1640 with $20 \%$ FBS. Media were supplemented with a final concentration of $1 \%$ penicillin/ streptomycin. 
For the L-asparaginase treatment of these two cell lines, we used as reference enzyme EcASNaseII which is the currently approved antileukemic enzyme drug. Recombinant production and purification of the E. coli enzyme were done similar to ScASNaseI (see Supporting Information). Capsules containing either EcASNaseII or ScASNaseI were prepared as described above. Before mixing the capsules with the cells, they were made contaminant-free by exposing them to lowintensity UV-light for $10 \mathrm{~min}$, while the free enzymes were passed through a $0.2 \mu \mathrm{m}$ filter (Sartorius). Subsequently, the enzymatic activities were determined as described above, and equal volume activities of the encapsulated and the free enzyme were used to treat the leukemic cells.

For cell proliferation studies, $\sim 10^{6}$ MOLT -4 and $10^{5}$ SD1 cells in a final volume of $200 \mu \mathrm{L}$ in a 96-well plate, were mixed with five units of either encapsulated or free EcASNaseII or ScASNaseI. To test whether encapsulation of the enzyme protected it against trypsin and thrombin degradation under cell culture conditions, the same number of cells were mixed with five units of $\mathrm{L}$-asparaginase in the presence of both proteases. Trypsin was added in a final amount of $0.5 \mu \mathrm{g}$, and thrombin at $20 \mu \mathrm{g}$ per a $200 \mu \mathrm{L}$ volume, to mimic the physiological (presence of thrombin) and the pancreatitis (release of trypsin) conditions in serum. ${ }^{44,45}$ As control, cells were treated with empty capsules and proteases independently to investigate whether they affected the cells in the absence of enzyme. The cell proliferation status after $72 \mathrm{~h}$ of incubation under the aforementioned conditions was assessed using the WST-1 assay (Roche), which quantifies the activity of mitochondrial dehydrogenases, according to the manufacturer's instructions. Absorbance was measured at $450 \mathrm{~nm}$ against a background control (culture medium without cells). All treatments and measurements were done in triplicates. The proliferation state of the treated cells is expressed as relative survival (\%) against the untreated cells.

\section{RESULTS AND DISCUSSION}

Characterization of $\mathrm{CaCO}_{3}$ Particles and Polyelectrolyte Microcapsules. Enzyme-loaded microparticles formed by rapid mixing of $\mathrm{Na}_{2} \mathrm{CO}_{3}$ and $\mathrm{CaCl}_{2}$ in the presence of ScASNaseI exhibited an average size of $1-2 \mu \mathrm{m}$. We observed that microparticles of more homogeneous size and spherical morphology (Figure S5) were obtained when lower than suggested ${ }^{28,30}$ concentrations of $\mathrm{Na}_{2} \mathrm{CO}_{3}$ and $\mathrm{CaCl}_{2}$ were used. Therefore, the initial concentrations of $\mathrm{Na}_{2} \mathrm{CO}_{3}$ and $\mathrm{CaCl}_{2}$ were $0.15 \mathrm{M}$, rather than $1 \mathrm{M}$ or $0.33 \mathrm{M}$, as reported before. Additionally, we chose the coprecipitation method for protein loading, because multiple attempts to load the protein onto preformed microparticles resulted in very low loading efficiency. ${ }^{46}$ On the contrary, in the case of simultaneous mixing of enzyme with $\mathrm{Na}_{2} \mathrm{CO}_{3}$ and $\mathrm{CaCl}_{2}$ (coprecipitation method), almost the total amount of added protein was bound by the $\mathrm{CaCO}_{3}$ microparticles, such that under conditions described in Materials and Methods, $3 \mathrm{mg}$ of enzyme could be loaded with negligible loss $(\sim 0.06 \mathrm{mg})$. When trying to load higher amounts $(4 \mathrm{mg})$, we detected considerable loss of protein.

To quantify the average amount of protein bound per particle, the formed particles were counted using a hemocytometer. Under the aforementioned conditions, we found that the loaded enzyme was distributed in $\sim 10^{8}$ particles, thus, estimating that each particle contained approximately $30 \mathrm{pg}$ ScASNaseI. At this point, it is worth mentioning that ScASNaseI has a calculated isoelectric point $(\mathrm{pI})$ of $\sim 5.3$, indicating that, at the working $\mathrm{pH}$ of 7.5 , the enzyme is negatively charged, while on the other hand, $\mathrm{CaCO}_{3}$ particles are positively charged at $\mathrm{pH}$ values below 9.0. ${ }^{30}$ Therefore, one may assume that high loading efficiency can be attributed to favorable electrostatic interactions between the protein and the calcium carbonate template, in addition to the high degree of adsorption of the enzyme to interior cavity surfaces of the template during the formation of the cores. When loading the enzyme following the same procedure at $\mathrm{pH} 6$ (closer to the enzyme's pI), we observed a 5-fold higher protein loss in the coprecipitation as well as in the washing steps (Figure S6). The enzyme-loaded particles were subjected to stepwise polyelectrolyte coating, starting with dextran sulfate, followed by poly-Larginine. We chose these two polyelectrolytes because of their biocompatibility properties, envisioning future in vivo studies. During the adsorption process, the electrophoretic mobility (zeta potential) was measured after deposition of each polyelectrolyte layer (Figure S7).

Prior to LbL-coating, $\mathrm{CaCO}_{3}$ cores exhibited a positive zeta potential, and therefore, coating was started with dextran sulfate (negatively charged) as the inner layer. After coating with two bilayers, hollow protein-containing capsules were obtained by dialyzing the material against $50 \mathrm{mM} \mathrm{Na}_{2} \mathrm{HPO}_{4}, 0.5 \mathrm{M} \mathrm{NaCl}$, 20 mM EDTA, pH 6.5 (volume ratio 1:1000). We established this modified protocol for dissolution of the $\mathrm{CaCO}_{3}$ template as we observed an inactivating effect of the chelating agent on ScASNaseI when using EDTA at concentrations $\sim 0.1 \mathrm{M}$ or higher. Therefore, the standard method of immersing the coated particles in 0.1 M EDTA was not ideal for this particular enzyme. By testing the inhibitory effect on enzymatic activity using free enzyme (Figure S8), we found that concentrations up to $20 \mathrm{mM}$ EDTA were tolerated by this enzyme. Thus, by dialyzing the coated particles against buffer containing $20 \mathrm{mM}$ EDTA, we managed to dissolve the core material thereby preserving the activity of the enzyme. This is of great importance for encapsulation of therapeutic proteins; most likely, this protocol must be adapted to milder conditions of template removal if less robust proteins of human origin are to be packaged in microcapsules (unpublished data). Moreover, we point out that the dialysis method for template dissolution can be used equally efficiently when more than two bilayers of polyelectrolytes are used for coating. In this work, we focused on the characterization of capsules with two bilayers, instead of three or more, since we observed a delay of reaching the saturation point of the enzymatic reaction in the case of more bilayers, possibly due to rate-limiting substrate or product diffusion through the polymer shell (Figure S9). Figure 1 shows two confocal as well as two scanning electron microscopy images of the final hollow capsules (with two bilayers) loaded with active ScASNaseI or eGFP-ScASNaseI (green fluorescence). The average diameter of the generated capsules was $\sim 1-2 \mu \mathrm{m}$.

The total number of protein-loaded capsules was determined by again using a hemocytometer; this number was found to be $\sim 8 \times 10^{7}$, which is roughly equal to the initial number of particles. However, while it is possible to determine the amount of enzyme loaded on $\mathrm{CaCO}_{3}$ and calculate the approximate amount of enzyme per particle, such quantitative protein data are more difficult to obtain for the capsules. The reason is that when using poly amino acids, such as poly-L-arginine, for capsule formation, these polymers interfere with protein determination assays. ${ }^{32}$ Moreover, it has been shown ${ }^{32}$ that during the last step of $\mathrm{CaCO}_{3}$ core removal, a significant fraction of either adsorbed or coprecipitated protein is released. This release seems to directly correlate with the size of the entrapped macromolecule, while physicochemical properties and the number of bilayers also are important parameters. ${ }^{47}$ Accurate measurements of protein entrapped in the final 

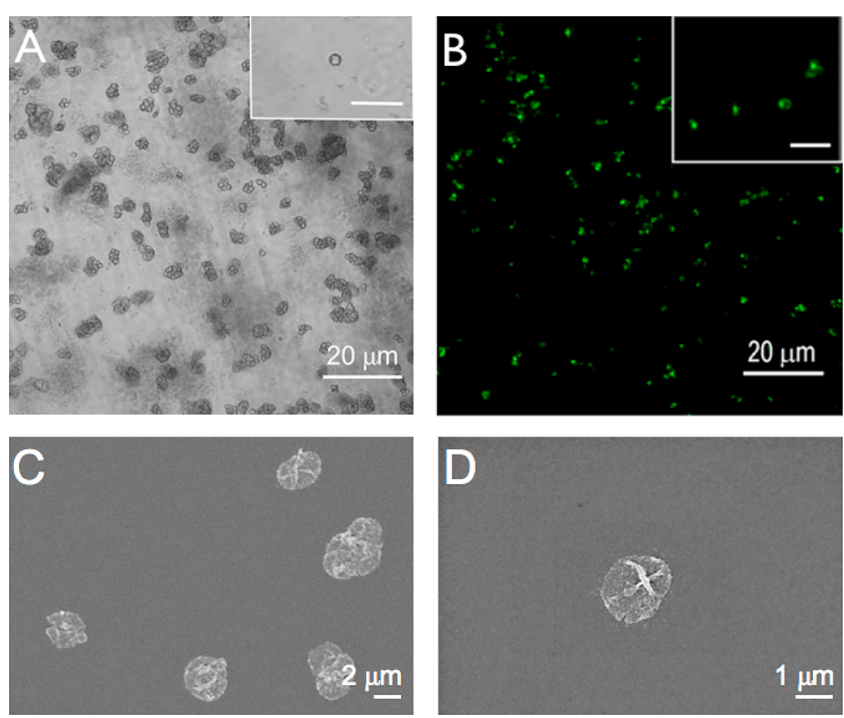

Figure 1. (A) Shown are two-bilayer-microcapsules loaded with ScASNaseI, after EDTA dissolution by dialysis. Images were taken using a confocal laser scanning microscope as described in Materials and Methods. The inset shows a capsule zoomed from the main image (scale bar corresponds to $5 \mu \mathrm{m}$ ). (B) Confocal fluorescence image of microcapsules loaded with eGFP-ScASNaseI fusion protein. Inset is the zoomed area of the main image (scale bar corresponds to $3 \mu \mathrm{m}$ ). (C, D) SEM images of polyelectrolyte microcapsules after removal of the $\mathrm{CaCO}_{3}$ template by dialysis.

capsules may necessitate the use of radiolabeled proteins, but this method is not applicable in all cases. In future work, it will be of general interest to establish a more direct and accurate, radio-label-free method for the determination of protein load in microcapsules. As a compromise in the present study, we compare volume activities of the free and encapsulated enzyme rather than specific activities, because the amount of unlabeled enzyme per capsule cannot be determined.

Resistance of Encapsulated ScASNasel to Proteolysis. A critical complication occurring during treatment of ALL with bacterial L-ASNase is acute pancreatitis, ${ }^{48}$ causing the release of the protease trypsin in the patient's blood serum and inactivation of the administered enzyme. In addition, it has been shown recently, that a dyad of lysosomal proteases degrades specifically E. coli L-ASNase. Therefore, it is of great interest to assess the resistance of encapsulated enzyme to proteolytic degradation. Besides trypsin, we tested the serine protease thrombin which has a key role in blood coagulation reactions. ${ }^{49}$ Equal amounts of both proteases, yet with different specific and total activities (see Materials), were mixed with free and encapsulated ScASNaseI exhibiting similar volume activities. Samples were incubated at $37{ }^{\circ} \mathrm{C}$, and residual activity was measured at successive time points (Figures 2 and 3) for up to $7 \mathrm{~h}$. Our results show that encapsulated L-ASNase is considerably more resistant to trypsin and thrombin degradation. The free enzyme was very sensitive to trypsin treatment, as evidenced by the fact that, after $1 \mathrm{~h}$ incubation, its remaining activity was below 5\%. In contrast, encapsulated LASNase resisted significantly better to trypsin exposure, maintaining up to $\sim 20 \%$ activity even after $7 \mathrm{~h}$.

In contrast to trypsin, thrombin was better tolerated by both free and encapsulated ASNase. Analysis of the primary structure provides a potential explanation for this higher resistance against thrombin. ScASNaseI amino acid sequence analysis

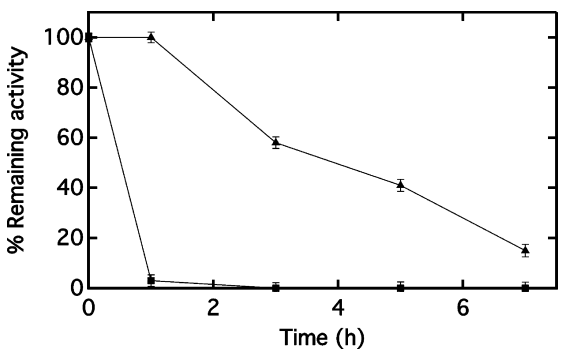

Figure 2. Residual activities of free ( $\boldsymbol{\square}$ ) and encapsulated ( $\boldsymbol{\Delta}$ ) ScASNaseI incubated with trypsin at $37^{\circ} \mathrm{C}$. Activities were measured every $2 \mathrm{~h}$ upon incubation with the protease. Results are means $\pm \mathrm{SD}$ of two independent experiments.

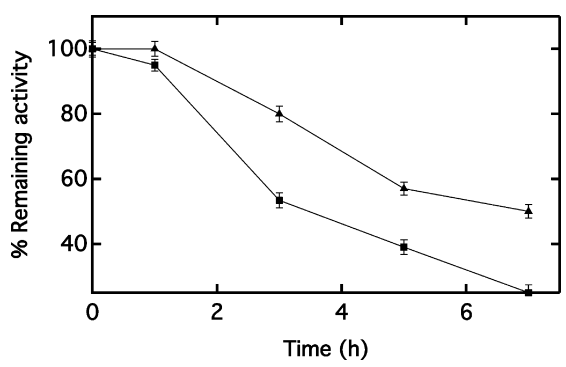

Figure 3. Residual activities of free ( $\boldsymbol{\square})$ and encapsulated ( $\boldsymbol{\Delta}$ ) ScASNaseI incubated with thrombin at $37{ }^{\circ} \mathrm{C}$. Similarly to trypsin treatment, activities were measured every $2 \mathrm{~h}$ upon incubation with the protease. Results are means \pm SD of two independent experiments.

(using Peptide Cutter, Expasy) revealed 31 possible recognition sites for trypsin, but only one for thrombin. Taking into account the molecular sizes of proteins such as trypsin (native $\mathrm{MW} \sim 23.6 \mathrm{kDa})$ and thrombin $(\sim 36 \mathrm{kDa})$, it can be assumed that they should be unable to diffuse into the capsules, though under specific temperature and ionic strength conditions, this may happen to some extent.

Additionally, one may assume that the encapsulated enzyme is mostly localized close to the inner surface due to favorable electrostatic interactions, rather than being homogeneously distributed in the capsule's volume; in that way, the interaction with proteases would be facilitated. Indeed, during $\mathrm{LbL}$ formation of the polyelectrolyte shell around the rough calcium carbonate surface, a polyelectrolyte complex can be formed inside the porous $\mathrm{CaCO}_{3}$. This might induce binding of the protein to such a complex after core decomposition. ${ }^{30}$

Thermal and Storage Stability of Free and Encapsulated ScASNasel. Aiming at further evaluation of the structural stability of the encapsulated L-ASNase, we performed irreversible thermal inactivation experiments using the free enzyme as reference. The two enzyme preparations (free and encapsulated) were incubated at different temperatures and were then assayed for residual activity (Figure 4). The corresponding $T_{\mathrm{m}}$ values are summarized in Table 1 . We noted that the free enzyme showed a relatively high $T_{\mathrm{m}}(51.6$ ${ }^{\circ} \mathrm{C}$ ), considering its origin from yeast which grows at $30{ }^{\circ} \mathrm{C}$. A potential explanation derives from the fact that this enzyme contains 10 cysteines, which might form disulfide bonds. It is known that disulfide bonds usually stabilize the overall protein structure making it more rigid and resistant against harsh conditions such as high temperatures. ${ }^{50}$ Yet, encapsulation of this enzyme enhanced the thermostability of ScASNaseI slightly. Our observations on thermostability of L-asparaginase encapsulated by using biocompatible material and reaction 


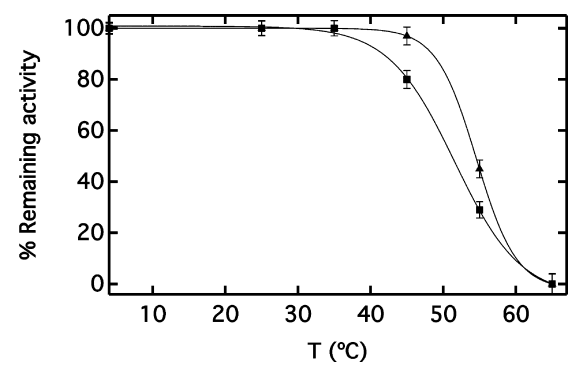

Figure 4. Irreversible thermal inactivation of free $(\boldsymbol{\square})$ and encapsulated ( $\mathbf{\Delta})$ L-ASNase. Residual activities were measured after heat treatment at different temperatures for $10 \mathrm{~min}$. $T_{\mathrm{m}}$ values were calculated by nonlinear regression to a sigmoid function using Igor Pro (Wavemetrics) software. Results are means \pm SD of two independent experiments.

Table 1. Mid-Inactivation Temperatures and Inactivation Rate Constants \pm SD for Free and Encapsulated ScASNaseI

\begin{tabular}{lcc} 
& $T_{\mathrm{m}}\left({ }^{\circ} \mathrm{C}\right)$ & $k_{\text {in }}\left(\times 10^{-3} \mathrm{~h}^{-1}\right)$ \\
free enzyme & $51.6 \pm 0.88$ & $120 \pm 4.72$ \\
encapsulated enzyme & $54.6 \pm 0.47$ & $10 \pm 1.2$ \\
\hline
\end{tabular}

conditions, as compared to stability of the free enzyme, are in good qualitative agreement with an early report on enzyme encapsulation. ${ }^{51}$ In that work, $\alpha$-chymotrypsin was used as a model enzyme for entrapment in hollow microcapsules consisting of four double-layers of sodium poly(styrenesulfonate) (PSS) and poly(allylamine hydrochloride)(PAH) assembled on commercially available micrometer-sized melamine formaldehyde particles, and stability was measured in the temperature range from 20 to $48{ }^{\circ} \mathrm{C}$.

The time course of isothermal inactivation of the two enzyme preparations was evaluated at the physiologically relevant temperature of $37{ }^{\circ} \mathrm{C}$. Kinetic analysis yielded linear plots (Figure 5) from which the first order inactivation rate

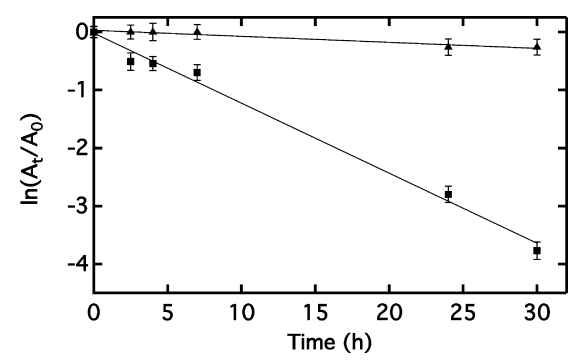

Figure 5. Plot of isothermal inactivation kinetics for free $(\boldsymbol{\square})$ and encapsulated $(\mathbf{\Delta})$ enzyme. The enzyme preparations were incubated at $37^{\circ} \mathrm{C}$, and their residual activities were measured for a period of 30 h. First order inactivation rate constants $k_{\text {in }}$ were calculated by linear regression using the Igor Pro (Wavemetrics) software. The experiment was performed twice.

constants were calculated according to eq 1 of ref 52 . We found a considerably lower inactivation rate constant (12-fold lower) for the encapsulated enzyme in comparison to the free enzyme (Table 1). We hypothesize that the microenvironment which is formed upon encapsulation protects the enzyme from different chemical and physical factors that could affect protein folding and catalytic activity. As it is generally believed that enhanced protein thermal stability is predominantly dependent on an increased number of hydrogen bonds and salt links, as well as better van der Waals' packing ${ }^{53,54}$ in the capsules' microenvironment, the latter two factors might explain the improved thermal stability of encapsulated L-ASNase at $37^{\circ} \mathrm{C}$.

The storage stability of the encapsulated enzyme was also significantly improved in comparison to the free species. As shown in Figure 6, after storing the encapsulated L-asparaginase

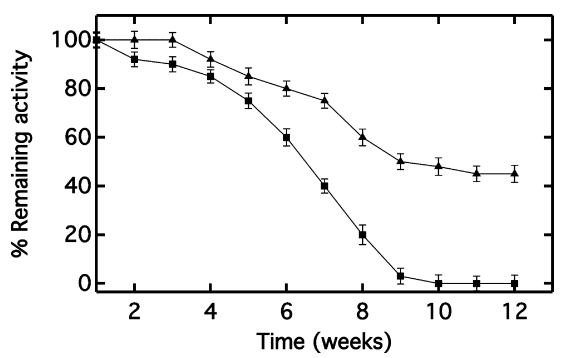

Figure 6. Plot of the storage stability for free $(\boldsymbol{\square})$ and encapsulated (A) L-ASNase. Samples were incubated at $4{ }^{\circ} \mathrm{C}$ in the absence of stabilizers. Their residual activities were measured for a total period of 3 months. The results are means \pm SD of independent measurements on three samples from the same capsule preparation.

for over 3 months at $4{ }^{\circ} \mathrm{C}$, the enzyme kept about $50 \%$ of its initial activity. In contrast, the free enzyme showed total inactivation after 2 months when stored at the same temperature. As microcapsules consist of ionic polymers such as hydrophilic polyelectrolytes (dextran sulfate and poly-Larginine in the present case), our findings are supported by previous work, ${ }^{55}$ which showed that hydrophilic polymers have a particular hydration effect on proteins similar to polyhydric alcohols (polyethylene-glycol, lactitol, sucrose) which also enhance their stability. ${ }^{49}$ Furthermore, it was shown that mixing of an enzyme with soluble linear-chain high molecular weight polymers can promote its stability, suggesting that the interaction between polymers and enzyme molecules forms a network which imposes a mechanical restriction on the protein unfolding process. ${ }^{56}$ Thus, we believe that increased storage stability of encapsulated L-ASNase can be attributed to favorable electrostatic interactions between the enzyme and the polymers.

Treatment of Leukemic Cells Using Free and Encapsulated ScASNasel. Encapsulated and free ScASNaseI were used to treat two different leukemic cell lines in order to evaluate the efficacy of both enzyme preparations on these cells. Free and encapsulated EcASNaseII were included in this series of experiments for comparative purposes, because this enzyme has been used for many decades for the treatment of ALL. In all cases, five units of each enzyme preparation (either encapsulated or free) were added once to the cells which were incubated for $72 \mathrm{~h}$. Subsequently, the proliferation state of the cells was evaluated based on the WST-1 assay.

The results of the different treatments of the two cell lines are presented in Figures 7 and 8. Figure 7 shows the relative survival of the cells which were exposed to different preparations of ScASNaseI. These data suggest that free proteases and empty capsules had no negative impact on the cells, and therefore cell death can be attributed solely to the effect of the L-ASNases. This effect on leukemic cells is due to the depletion of the available levels of L-Asn in the medium, since lack of L-Asn causes protein synthesis arrest in the cancerous cells which ultimately undergo apoptosis and cell death. $^{11}$ Figure 7 shows that the use of either free or 


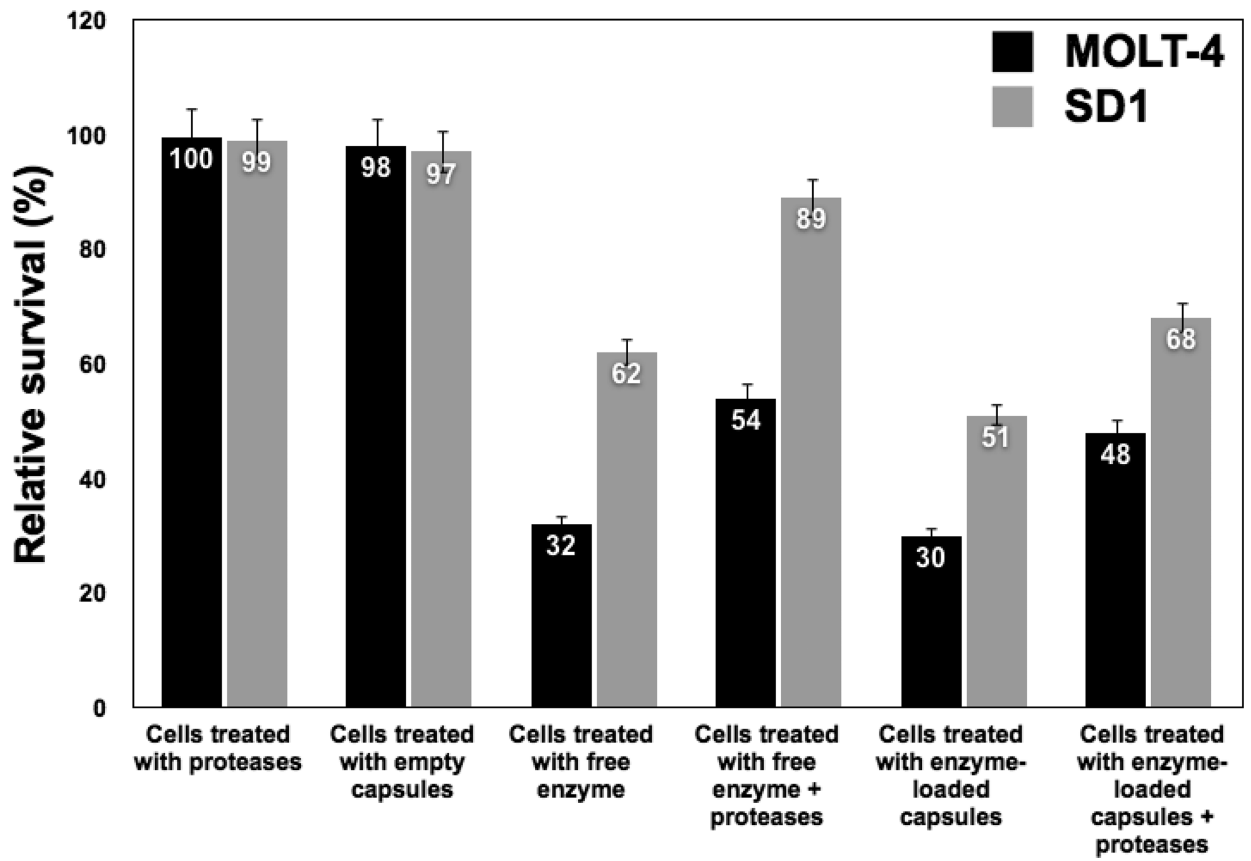

Figure 7. Viability of MOLT-4 and SD1 leukemic cells treated with ScASNaseI preparations. The proliferation state of the cells is expressed as relative survival (\%) against the control (untreated cells). Data are shown as means \pm SD of triplicate measurements.

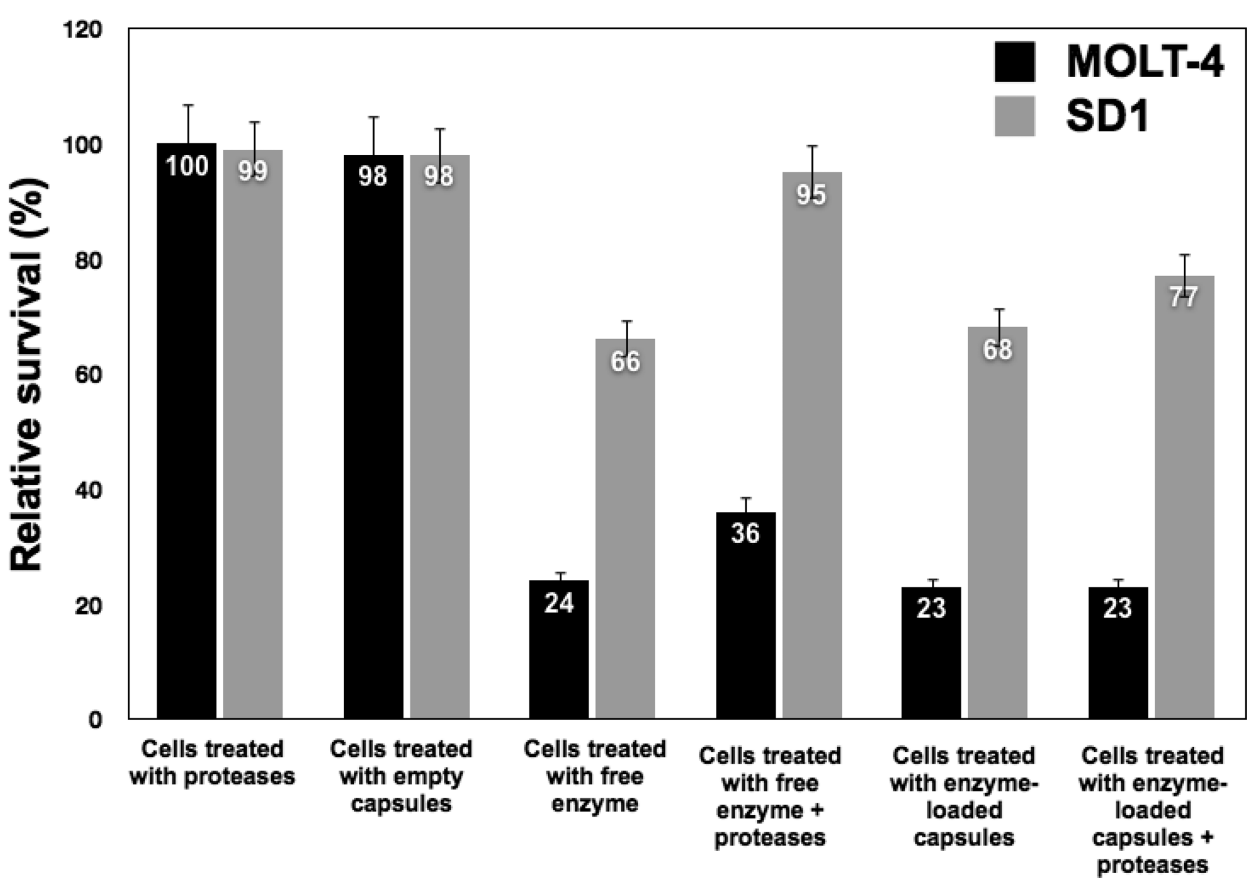

Figure 8. Viability of MOLT-4 and SD1 cells treated with EcASNaseII, expressed as relative survival (\%) against the control (untreated cells). Data are means $\pm \mathrm{SD}$ of triplicate measurements.

encapsulated ScASNaseI inhibited the MOLT-4 and SD1 proliferation after $72 \mathrm{~h}$ of treatment to a relatively similar extent. However, when the cells were incubated with free enzyme in the presence of trypsin and thrombin, the proliferation inhibition was less pronounced, indicating inactivation of ScASNaseI by the proteases. In contrast, when the cells were treated with encapsulated ScASNaseI and proteases, the proliferation of SD1 cells was inhibited similarly to the treatment with free enzyme in the absence of proteases, while MOLT-4 cells were less affected by L-asparaginase treatment. These findings on protease effects are in line with our biochemical data which implied that encapsulation of ScASNaseI significantly elevated its proteolytic resistance as compared to the free enzyme. Light microscopic images of the untreated cells and cells treated with ScASNaseI-loaded capsules are shown in the Supporting Information (Figure S10). Similar results were obtained when both leukemic cell lines were incubated with free or encapsulated EcASNaseII (Figure 8). We observed that SD1 cells were similarly affected by free ScASNaseI and EcASNaseII. In contrast, the proliferation of MOLT-4 cells was influenced more by EcASNaseII than by ScASNaseI. Encapsulation of the bacterial 
enzyme was also proven to be beneficial for its resistance against proteolysis, as evidenced by the lower relative survival resulting from the treatment with capsules in the presence of proteases as compared to the free enzyme in the presence of proteases. Images of untreated cells and of cells treated with EcASNaseII-loaded capsules, are shown in the Supporting Information (Figure S11).

Taken together, our data suggest that encapsulation of LASNases, using biocompatible polyelectrolytes, improves the biochemical stability of the enzymes and protects them against physiologically occurring proteases. We show that this protection holds for both ScASNaseI and EcASNaseII which have distinct catalytic properties $\left(k_{\text {cat }} / K_{\mathrm{M}} 5 \times 10^{4} \mathrm{M}^{-1} \mathrm{~s}^{-1}\right.$ for ScASNaseI, and $1.5 \times 10^{6} \mathrm{M}^{-1} \mathrm{~s}^{-1}$ for EcASNaseII). These findings may form the basis for an improved L-asparaginasedependent antileukemic therapy, though in vitro analyses will have to be complemented by pharmacodynamic and pharmacokinetic studies.

\section{CONCLUSION}

Recent advances in cellular drug delivery have relied on the use of polyelectrolyte microcapsules as carriers for various types of drugs, including proteins. ${ }^{57,58}$ Of great importance is the maintenance of the functionality of the loaded drug during the preparation of the capsules; this is particularly true for enzymes whose activity can be strongly affected by different materials, or by reaction conditions required for capsule fabrication. In the present work, we have highlighted the importance of removing the $\mathrm{CaCO}_{3}$ template under mild conditions of dialysis to preserve catalytic activity of the therapeutic enzyme Lasparaginase (L-ASNase) which is an approved drug for the treatment of leukemia. We focused on the up to now poorly characterized yeast asparaginase, ScASNaseI, which is a glutaminase-free enzyme exhibiting catalytic properties on asparagine that are similar to those of the currently approved E. coli protein. Therefore, this enzyme of eukaryotic origin could form the basis for the development of an improved anticancer drug showing less side effects than the bacterial enzyme

To develop a potential strategy for the amelioration of LASNase's half-life in the blood, we packaged the enzyme in microcapsules fabricated by a variable number of layers of biocompatible polymers. In this way, full catalytic activity was maintained, biochemical stability of the enzyme was enhanced, and it can be expected that the encapsulated enzyme will be protected from inactivation by proteases not only in vitro, as demonstrated in this work, but also in an in vivo situation, thus, improving the enzyme's half-life in the blood. Therefore, encapsulation appears as a promising avenue to bypass numerous problems associated with protease degradation of therapeutic peptides and proteins. Moreover, when residing inside capsules, the enzyme's epitopes would be shielded, preventing direct recognition by the immune system. ${ }^{40,41}$ However, the question of whether the capsules themselves could be immunogenic remains to be addressed. ${ }^{59}$ We are presently probing the general applicability of our experimental strategy to more sensitive biomacromolecules such as various enzymes of human origin. Notably, we embarked on encapsulation of a human L-asparaginase that we recently characterized structurally and functionally ${ }^{60}$ and that could replace enzymes of nonhuman origin.

\section{ASSOCIATED CONTENT}

\section{Supporting Information}

Enzyme preparations are described in detail, and cell culture images and complementary results are given. This material is available free of charge via the Internet at http://pubs.acs.org.

\section{AUTHOR INFORMATION}

\section{Corresponding Author}

*Fax: +49 551 2011074. E-mail: mkonrad@gwdg.de.

\section{Notes}

The authors declare no competing financial interest.

\section{ACKNOWLEDGMENTS}

This work was supported by the Max Planck Society, and by an Alexander von Humboldt fellowship to A.M.Y. We thank members of the Laboratory of Cellular Dynamics (Head: T. Jovin) for offering us the use of their Zetasizer Nano Series analyzer, as well as the Department of Cellular Biochemistry (Head: R. Lührmann) for giving us free access to their Confocal Laser-Scanning Microscope.

\section{REFERENCES}

(1) Asselin, B. L.; Ryan, D.; Frantz, C. N.; Bernal, S. D.; Leavitt, P.; Sallan, S. E.; Cohen, H. Cancer Res. 1989, 49, 4363-4368.

(2) Silverman, L. B.; Gelber, R. D.; Dalton, V. K. Blood 2001, 97, 1211-1218.

(3) Pui, C. H.; Sandlund, J. T.; Pei, D. Blood 2004, 104, 2690-2696.

(4) Jaffe, N.; Traggis, D.; Das, L.; Kim, B. S.; Hann, H. W.; Moloney, W. C.; Dohlwitz, A. Pediatrics 1972, 49, 590-595.

(5) Sallan, S. E.; Hitchcock-Bryan, S.; Gelber, R.; Cassady, J. R.; Frei, E., III; Nathan, D. G. Cancer Res. 1983, 43, 5601-5607.

(6) Tallal, L.; Tan, C.; Oettgen, H.; Wollner, N.; McCarthy, M.; Helson, L.; Burchenal, J.; Karnofsky, D.; Murphy, M. L. Cancer 1970, $25,306-320$.

(7) Ertel, I. J.; Nesbit, M. E.; Hammond, D.; Weiner, J.; Sather, H. Cancer Res. 1979, 39, 3893-3896.

(8) Capizzi, R. L.; Bertino, J. R.; Skeel, R. T.; Creasey, W. A.; Zanes, R.; Olayon, C.; Peterson, R. G.; Handschumacher, R. E. Ann. Intern. Med. 1971, 74, 893-901.

(9) Haskell, C. M.; Canellos, G. P. Biochem. Pharmacol. 1969, 18 $2578-2580$.

(10) Capizzi, R. L.; Bertino, J. R.; Skeel, R. T.; Creasey, W. A.; Zanes, R.; Olayon, C. Ann. Intern. Med. 1971, 74, 893-901.

(11) Holleman, A.; den Boer, M. L.; Kazemier, K. M.; Janka-Schaub, G. E.; Pieters, R. Blood 2003, 102, 4541-4546.

(12) Cantor, J. R.; Yoo, T. H.; Dixit, A.; Iverson, B. L.; Forsthuber, T. G.; George, Georgiou. Proc. Natl. Acad. Sci. U.S.A. 2011, 108, 12721277.

(13) Larson, R. A.; Fretzin, M. H.; Dodge, R. K.; Schiffer, C. A. Leukemia 1998, 12, 660-665.

(14) Whitecar, J. P., Jr; Bodey, G. P.; Harris, J. E.; Freireich, E. J. N. Engl. J. Med. 1972, 282, 732-734.

(15) Zubrod, C. G. Pediatrics 1970, 45, 555-559.

(16) Derst, C.; Henseling, J.; Röhm, K. H. Protein Sci. 2000, 9, 2009_ 2017.

(17) Szewczyk, B. L.; Andrzejewski, W.; Mlynarski, W.; Danska, K. R.; Witas, H.; Bodalski, J. Leuk. Lymphoma 2007, 48, 931-936.

(18) Mueller, H. J.; Boos, J. Crit. Rev. Oncol. Hematol. 1998, 28, 97113.

(19) Asselin, B. L.; Whitin, J. C.; Coppola, D. J.; Rupp, I. P.; Sallan, S. E.; Cohen, H. J. J. Clin. Oncol. 1993, 11, 1780-1786.

(20) Patel, N.; Krishnan, S.; Offman, M. N.; Krol, M.; Moss, C. X.; Leighton, C.; van Delft, F. W.; Holland, M.; Liu, J. Z.; Alexander, S.; Dempsey, C.; Ariffin, H.; Essink, M.; Eden, T. O. B.; Watts, C.; Bates, P. A.; Saha, V. J. Clin. Invest. 2009, 119, 1964-1973. 
(21) Baran, E. T.; Ozer, N.; Hasirci, V. J. Mater. Sci.: Mater. Med. 2002, 13, 1113-1121.

(22) Wolf, M.; Wirth, M.; Pittner, F.; Gabor, F. Int. J. Pharm. 2003, 256, 141-152.

(23) Rizzari, C.; Citterio, M.; Zucchetti, M.; Conter, V.; Chiesa, R.; Colombini, A.; Malguzzi, S.; Silvestri, D.; D’Incalci, M. Haematologica 2006, 91, 24-31.

(24) Cao, S. G.; Zhao, Q. Y.; Ding, Z. T.; MaL, Y. T.; Wang, J. H. Ann. N.Y. Acad. Sci. 1990, 613, 460-467.

(25) Kawashima, K.; Takeshima, H.; Higashi, Y.; Hamaguchi, M.;

Sugie, H.; Imamura, I. Leuk. Res. 1991, 15, 525-30.

(26) Fernandes, A. I.; Gregoriadis, G. Int. J. Pharm. 2001, 217, 215224.

(27) Kwon, Y. M.; Chung, H. S.; Moon, C.; Yockman, J.; Park, Y. J.; Gitlin, S. D.; David, A. E.; Yang, V. C. J. Controlled Release 2009, 139, 182-189.

(28) Volodkin, D. V.; Petrov, A. I.; Prevot, M.; Sukhorukov, G. B. Langmuir 2004, 20, 3398-3406.

(29) Sukhorukov, G. B.; Volodkin, D. V.; Gunther, A. M.; Petrov, A. I.; Shenoya, D. B.; Mohwald, H. J. Mater. Chem. 2004, 14, 2073-2081.

(30) Volodkin, D. V.; Larionova, N. I.; Sukhorukov, G. B. Biomacromolecules 2004, 5, 1962-1972.

(31) De Cock, L. J.; De Koker, S.; De Geest, B. G.; Grooten, J.; Vervaet, C.; Remon, J. P.; Sukhorukov, G. B.; Antipina, M. N. Angew. Chem., Int. Ed. 2010, 49, 6954-6973.

(32) De Temmerman, M. L.; Demeester, J.; De Vos, F.; De Smedt, S. C. Biomacromolecules 2011, 12, 1283-1289.

(33) Caruso, F.; Trau, D.; Mohwald, H.; Renneberg, R. Langmuir 2000, 16, 1485-1488.

(34) Gao, C. Y.; Liu, X. Y.; Shen, J. C.; Möhwald, H. Chem. Commun. (Cambridge, U.K.) 2002, 17, 1928-1929.

(35) Yashchenok, A. M.; Delcea, M.; Videnova, K.; Jares-Erijman, E. A.; Jovin, T. M.; Konrad, M.; Möhwald, H.; Skirtach, A. G. Angew. Chem., Int. Ed. 2010, 49, 8116-8120.

(36) Radtchenko, I. L.; Sukhorukov, G. B.; Möhwald, H. Colloids Surf., A 2002, 202, 127-133.

(37) Zelikin, A. N.; Becker, A. L.; Johnston, A. P. R.; Wark, K. L.; Turatti, F.; Caruso, F. ACS Nano 2007, 1, 63-69.

(38) Tanner, P.; Onaca, O.; Balasubramanian, V.; Meier, W.; Palivan,

C. G. Chem.-Eur. J. 2011, 17, 4552-4560.

(39) Ariga, K.; Ji, Q.; Mori, T.; Naito, M.; Yamauchi, Y.; Abe, H.; Hill, J. P. Chem. Soc. Rev. 2013, 42, 6322-6345.

(40) Sakr, O. S.; Borchard, G. Biomacromolecules 2013, 14, 21172135.

(41) Najer, A.; Wu, D.; Vasquez, D.; Palivan, C. G.; Meier, W. Nanomedicine (London, U.K.) 2013, 8, 425-447.

(42) Bradford, M. M. Anal. Biochem. 1976, 72, 248-254.

(43) Ho, P. P.; Milikin, E. B.; Bobbitt, J. L. J. Biol. Chem. 1970, 245, $3708-3715$.

(44) Artigas, J. M.; Garcia, M. E.; Faure, M. R.; Gimeno, A. M. Postgrad. Med. J. 1981, 57, 219-222.

(45) Putnam, F. W. The Plasma Proteins; Academic Press: IN, 1984.

(46) Petrov, A. I.; Volodkin, D. V.; Sukhorukov, G. B. Biotechnol. Prog. 2005, 21, 918-925.

(47) She, Z.; Antipina, M. N.; Li, J.; Sukhorukov, G. B. Biomacromolecules 2010, 11, 1241-1247.

(48) Derwich, K.; Stencel, D.; Warzywoda, M.; Leda, M. Pract. Oncol. Radiother. 1999, 4, 15-22.

(49) Tanaka, K. A.; Key, N. S.; Levy, J. H. Anesth. Analg. 2009, 108, $1433-1446$.

(50) Vogt, G.; Woell, S.; Argos, P. J. Mol. Biol. 1997, 269, 631-643.

(51) Tiourina, O. P.; Antipov, A. A.; Sukhorukov, G. B.; Larionova,

N. I.; Lvov, Y.; Möhwald, H. Macromol. Biosci. 2001, 1, 209-214.

(52) Fachin, D.; Smout, C.; Verlent, I.; Nguyen, B. L.; Van Loey, A. M.; Hendrickx, M. E. J. Agric. Food Chem. 2004, 52, 2697-2703.

(53) Querol, E.; Perez-Pons, J. A.; Mozo-Villarias, A. Protein Eng., Des. Sel. 1996, 9, 265-271.

(54) Vogt, G.; Argos, P. Fold. Des. 1997, 2, 40-46.

(55) Schmid, R. D. Adv. Biochem. Eng. 1979, 12, 42-118.
(56) Gianfreda, L.; Modafferi, M.; Greco, G., Jr. Enzyme Microb. Technol. 1985, 7, 78-82.

(57) Vergaro, V.; Scarlino, F.; Bellomo, C.; Rinaldi, R.; Vergara, D.; Maffia, M.; Baldassarre, F.; Giannelli, G.; Zhang, X.; Lvov, Y. M.; Leporatti, S. Adv. Drug Delivery Rev. 2011, 63, 847-864.

(58) Anandhakumar, S.; Nagaraja, V.; Raichur, A. M. Colloids Surf., B 2010, 78, 266-274.

(59) De Temmerman, M. L.; Rejman, J.; Vandenbroucke, R. E.; De Koker, S.; Libert, C.; Grooten, J.; Demeester, J.; Gander, B.; De Smedt, S. C. J. Controlled Release 2012, 158, 233-239.

(60) Su, Y.; Karamitros, C. S.; Nomme, J.; McSorley, T.; Konrad, M.; Lavie, A. Chem. Biol. 2013, 20, 533-540. 
Preserving catalytic activity and enhancing biochemical stability of the therapeutic enzyme asparaginase by biocompatible multilayered polyelectrolyte microcapsules.

Christos S. Karamitros ${ }^{1}$, Alexey M. Yashchenok ${ }^{2}$, Helmuth Möhwald², Andre G. Skirtach ${ }^{2,3}$, Manfred Konrad ${ }^{*}$

\section{Supplementary Information}

The open reading frame (ORF) coding for yeast cytoplasmic L-asparaginase ScASNaseI (UniProt P38986, gene ASP1) consisting of 1143 bp, was amplified with PCR using as template genomic DNA from the Saccharomyces cerevisiae S288C wildtype strain. NdeI and BamHI sites were incorporated at the 5'- and 3'-oligonucleotides' ends, respectively: 5'-oligo GG A At T C C ATAt G A A A A GCGATt C A G T t G A A ATC; 3' - oligo CGCGGATCCTCACCCACCATAGACGCCAGTG. The PCR reaction mixture consisted of oligonucleotide mix, KAPA high fidelity buffer, dNTPs and KAPA HiFi DNA polymerase. The reaction was initiated at $95{ }^{\circ} \mathrm{C}$ for $3 \mathrm{~min}$, followed by 25 cycles of denaturation at $98{ }^{\circ} \mathrm{C}$ for $20 \mathrm{~s}$, primer annealing at $60{ }^{\circ} \mathrm{C}$ for $30 \mathrm{~s}$, and extension at $72{ }^{\circ} \mathrm{C}$ for $30 \mathrm{~s}$. The amplification reaction was terminated after a 5 min polishing step at $72{ }^{\circ} \mathrm{C}$. The PCR product (Figure S1) was gelpurified, digested with NdeI and BamHI H.F., purified with PCR clean-up kit and then ligated overnight at $16{ }^{\circ} \mathrm{C}$ into pET14b-SUMO vector (Figure S2) using T4 DNA ligase. The ligation mixture was used to transform DH5a E.coli cells. Positive clones were determined following colony PCR screening using one primer for the vector (Forward) and one primer for the insert (Reverse), restriction digestion with NdeI and BamHI H.F., and finally sequencing of the cloned 
DNA insert. The expressed protein construct includes an N-terminal 6-histidine tag, followed by

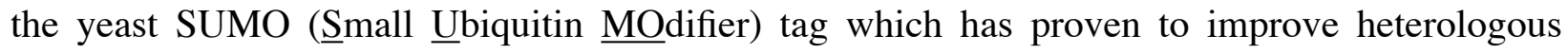
protein solubility and stability.

E.coli BL21(DE3) C41 cells containing the S.c.ASNaseI plasmid were cultured overnight at 37 ${ }^{\circ} \mathrm{C}$ in TB medium supplemented with $200 \mu \mathrm{g} / \mathrm{ml}$ ampicillin. A fraction of this culture was used to inoculate fresh TB culture (dilution 1:100) supplemented with $200 \mu \mathrm{g} / \mathrm{ml}$ ampicillin. When O.D. 600 reached $\sim 0.5-0.7$, the expression was induced by adding IPTG to a final concentration of 1 $\mathrm{mM}$. After incubation at $37^{\circ} \mathrm{C}$ for $8 \mathrm{~h}$, the culture was centrifuged at $4,000 \mathrm{~g}$ for $30 \mathrm{~min}$, the cells were harvested, resuspended in affinity matrix binding buffer $\left(50 \mathrm{mM} \mathrm{Na}_{2} \mathrm{HPO}_{4}, 0.5 \mathrm{M} \mathrm{NaCl}, 10\right.$ $\mathrm{mM}$ imidazole $\mathrm{pH} 8.0$ ), and ultimately lysed by sonication. The cell lysate was centrifuged at $17,200 \mathrm{~g}$ for $45 \mathrm{~min}$, the resulting supernatant mixed with pre-equilibrated nickel agarose beads, and incubated at $4{ }^{\circ} \mathrm{C}$ for $3 \mathrm{~h}$ under rotation. Subsequently, the mixture was filled in a $5 \mathrm{~mL}$ polypropylene column and dried by gravity. The nickel resin was washed with 25 bed volumes of washing buffer ( $50 \mathrm{mM} \mathrm{Na} 2 \mathrm{HPO}_{4}, 1 \mathrm{M} \mathrm{NaCl}, 20 \mathrm{mM}$ imidazole, $\mathrm{pH}$ 8.0). Finally, the bound protein was eluted from the column by applying $300 \mathrm{mM}$ imidazole, and dropwise collection of fractions. All purification steps were performed at $4{ }^{\circ} \mathrm{C}$. The collected fractions were mixed, and buffer was exchanged against $50 \mathrm{mM} \mathrm{Na}_{2} \mathrm{HPO}_{4}, 0.5 \mathrm{NaCl}$, pH 7.5 using a PD-10 column (GE). The eluted protein fraction was incubated with yeast SUMO protease (molar ratio protease:protein $\sim 1: 100$ ) at $30{ }^{\circ} \mathrm{C}$ for $2 \mathrm{~h}$ in order to cleave the $\mathrm{N}$-terminal 6-His-SUMO tag. In a last purification step, the protein was subjected to size exclusion chromatography by passing it through a Supradex 200 column (Pharmacia/GE) to remove the cleaved tag. Protein purity was evaluated by SDS-PAGE and was estimated to exceed $95 \%$. The protein sample was aliquoted, mixed with $25 \%$ glycerol, and stored at $-20{ }^{\circ} \mathrm{C}$ until use. The E.coli L-ASNase II (EcASNaseII) 
was expressed and purified analogously.

The fusion protein eGFP-ScASNaseI, was constructed using a modified pET14b vector which was generated for the needs of the present study (Figure S3). The N-terminus included the His $6^{-}$ eGFP part followed by a short hydrophilic flexible linker consisting of (Gly-Ser) $)_{2}$. Downstream of the 6-His-eGFP plus linker moiety, the coding region for ScASNaseI was inserted via NdeI and BamHI sites. The final construct was verified by control digestion and sequencing. E.coli BL21(DE3) C41 cells harboring the pET14b eGFP-ScASNaseI plasmid were used for expression of the fusion protein at $22{ }^{\circ} \mathrm{C}$ in auto-induction culture medium containing lactose as inducer. The culture was inoculated with a single colony and was incubated at $22{ }^{\circ} \mathrm{C}$ for $24 \mathrm{~h}$. The fusion enzyme was purified and stored as described above for the 6-His-SUMO-ScASNaseI fusion.

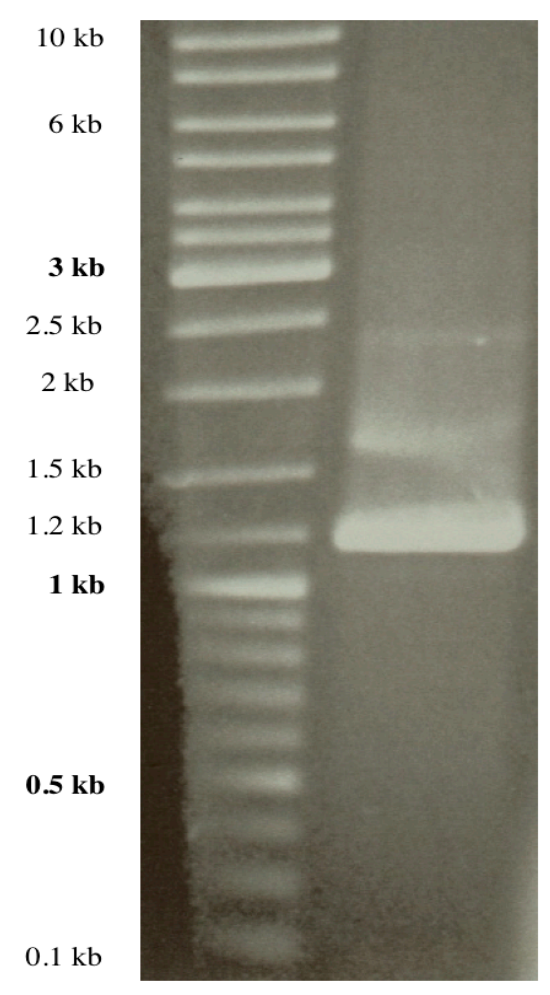

Figure S1. PCR amplification of $\boldsymbol{S c A S N a s e I ~ A S P 1 ~ g e n e ~ u s i n g ~ a s ~ t e m p l a t e ~ g e n o m i c ~ D N A ~ f r o m ~}$ Saccharomyces cerevisiae. The main amplified fragment shows the expected size of $1143 \mathrm{bp}$. 


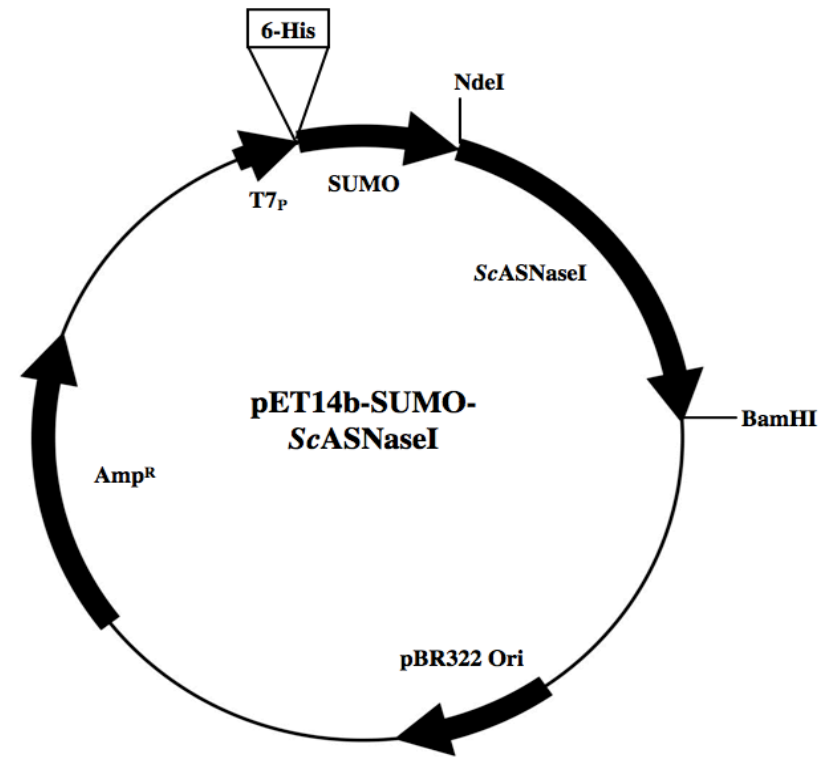

Figure S2. The final construct of the 6-His-SUMO-ScASNaseI cloned into pET14b plasmid. The purified intact $S c$ ASNaseI protein (after removal of 6-His-SUMO tag) is shown below in Figure S4.

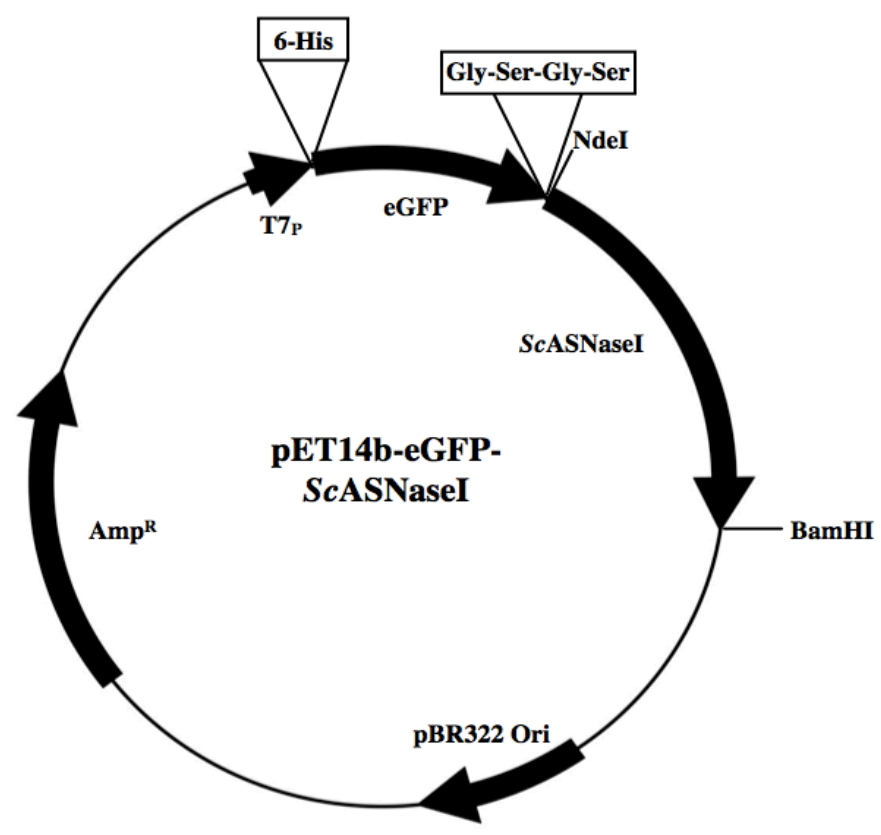

Figure S3. Construct for the expression of 6-His-eGFP-ScASNaseI fusion protein. Also indicated is the position of the hydrophilic linker (Gly-Ser) 2 . The purified fusion protein is in Figure 4 


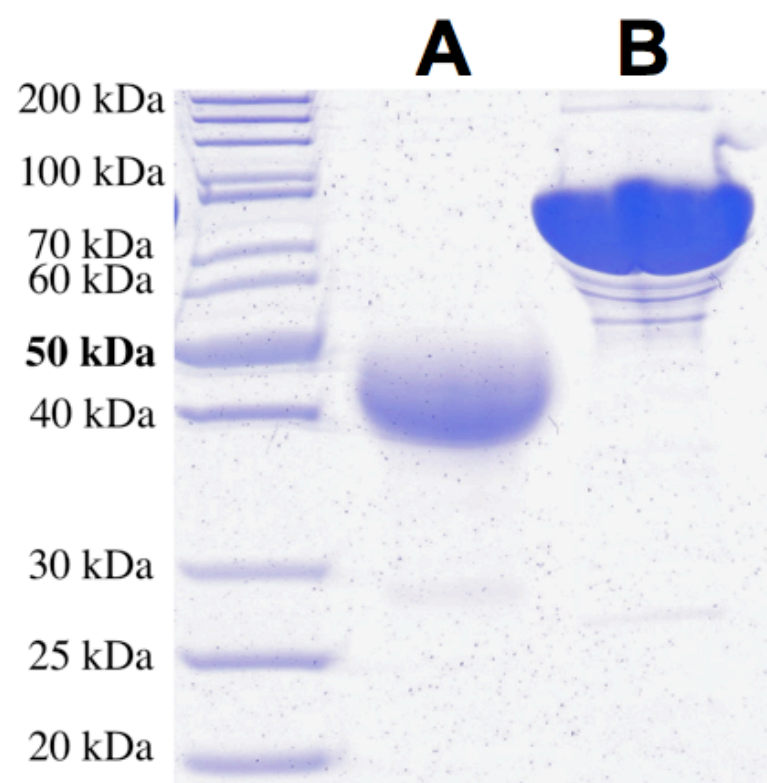

$15 \mathrm{kDa}$

\section{0 kDa}

Figure S4. SDS-PAGE analysis of ScASNaseI after removal of 6-His-SUMO-tag (A), which corresponds to the expected size of $\sim 40 \mathrm{kDa}$ and of the 6-His-eGFP-ScASNaseI fusion (B) showing an apparent $\mathrm{Mr} \sim 70 \mathrm{kDa}$ (theoretical Mr: $69 \mathrm{kDa}$ ). 


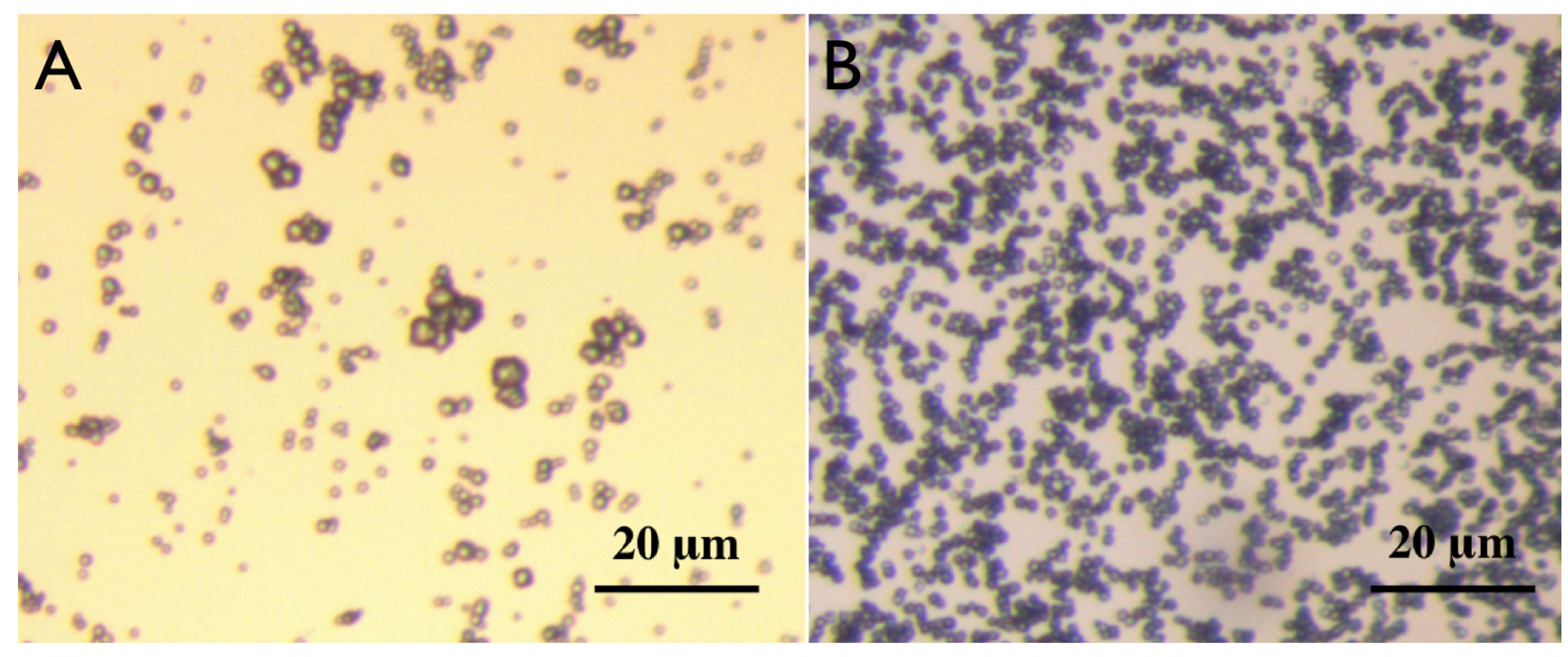

Figure S5. Protein-loaded calcium carbonate particles formed by following the co-precipitation method and imaged by transmission light microscopy. Figures A and B show the L-asparaginasefilled cores, obtained when using $1 \mathrm{M}(\mathrm{A})$ or $0.15 \mathrm{M}$ (B) $\mathrm{Na}_{2} \mathrm{CO}_{3} / \mathrm{CaCl}_{2}$.

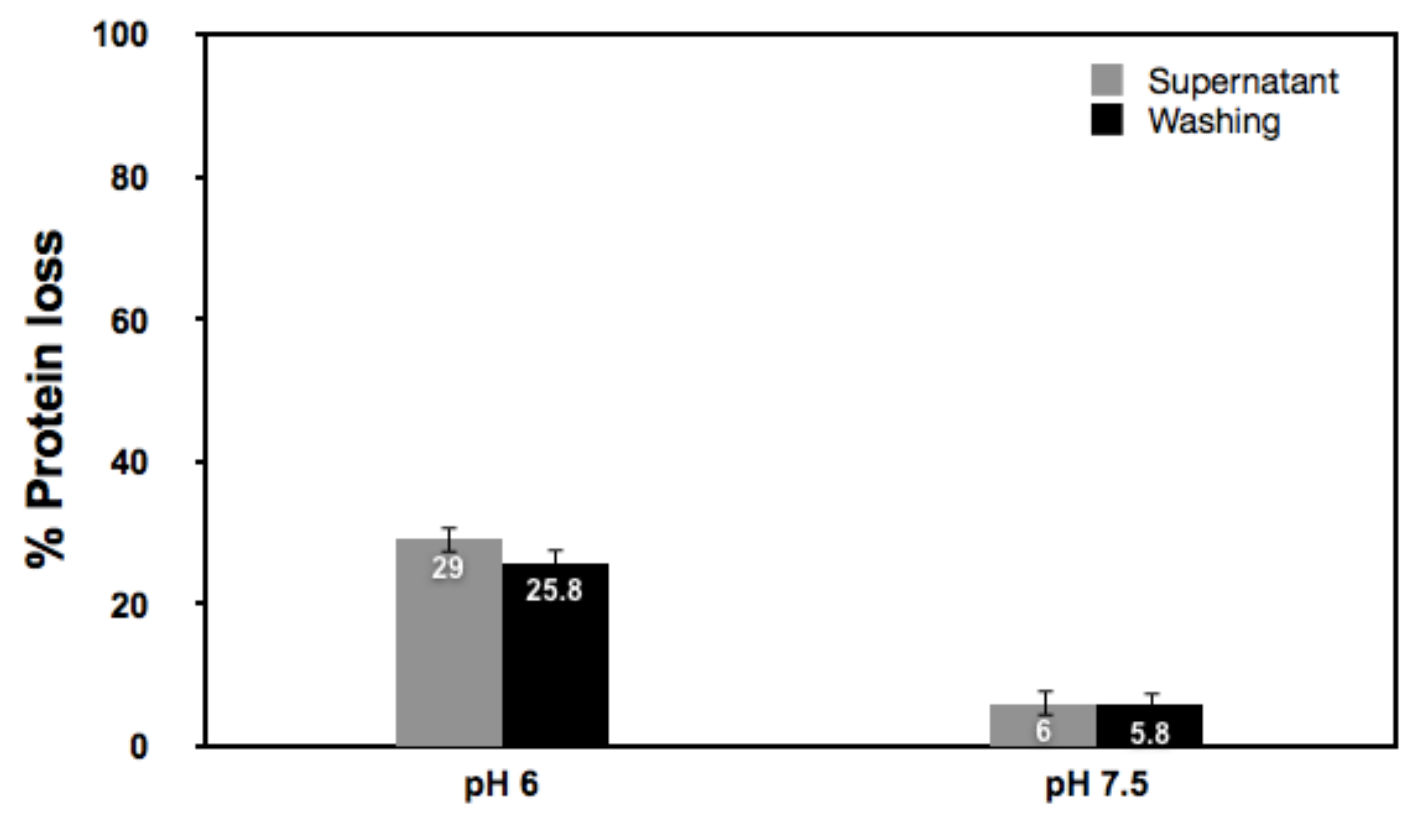

Figure S6. Comparison of the calcium carbonate/ScASNaseI loading efficiency under 2 different $\mathrm{pH}$ conditions following the co-precipitation method. For quantification of protein loss, the supernatant and the washing fractions were collected after centrifugation and the total amount of 
protein was determined by the Bradford method. The initial amount of enzyme used was $3 \mathrm{mg}$. Results are means \pm S.D. of 2 independent experiments.

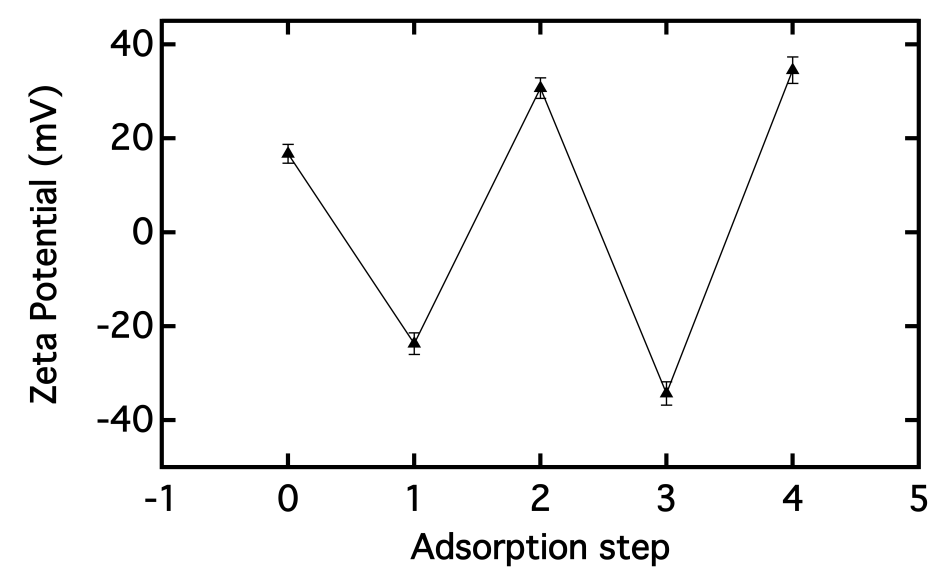

Figure S7. Zeta potential of particles determined after individual polymer adsorption steps. Step 0 defines the $\mathrm{Z}$ potential of the initial uncoated $\mathrm{CaCO}_{3}$ particles in the presence of enzyme. Steps 1 and 3 correspond to dextran sulfate adsorption, while 2 and 4 indicate poly-L-arginine deposition. The $\mathrm{Z}$ potential values alternate between negative and positive upon coating with either dextran sulfate or poly-L-arginine, indicating the adsorption of poly-anions or polycations, respectively. Shown are the average values and the S.D. of 3 independent measurements. 


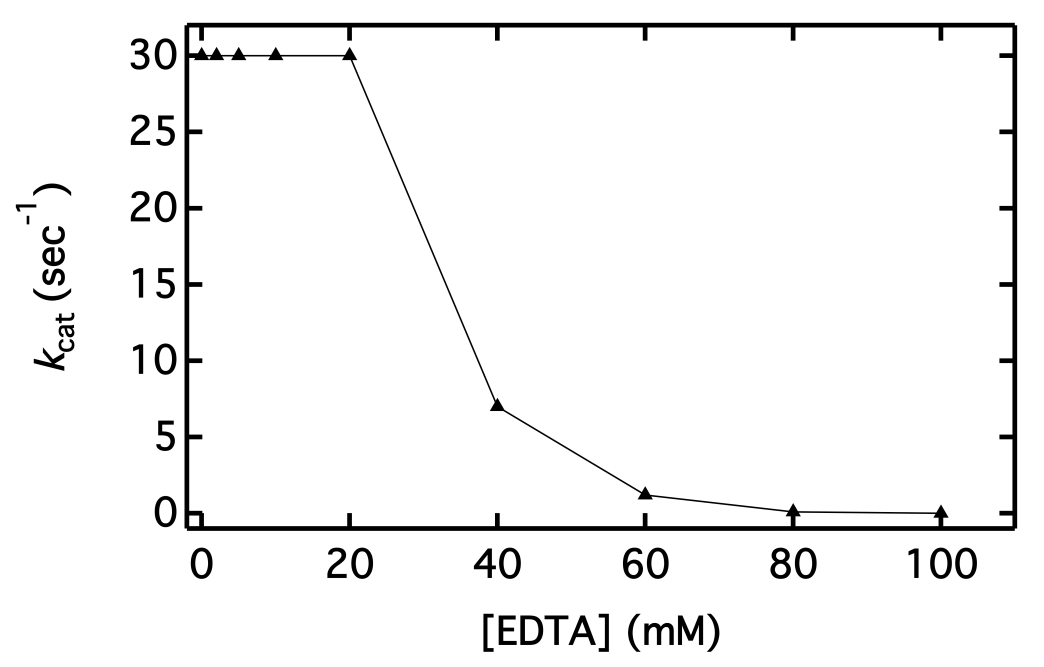

Figure S8. Inhibitory effect of EDTA on ScASNaseI activity. Measurements were performed between 0 and $100 \mathrm{mM}$ EDTA using $10 \mu \mathrm{g}$ of purified enzyme. The plot demonstrates the decrease of the steady-state turn-over rate as a function of different EDTA concentrations tested.

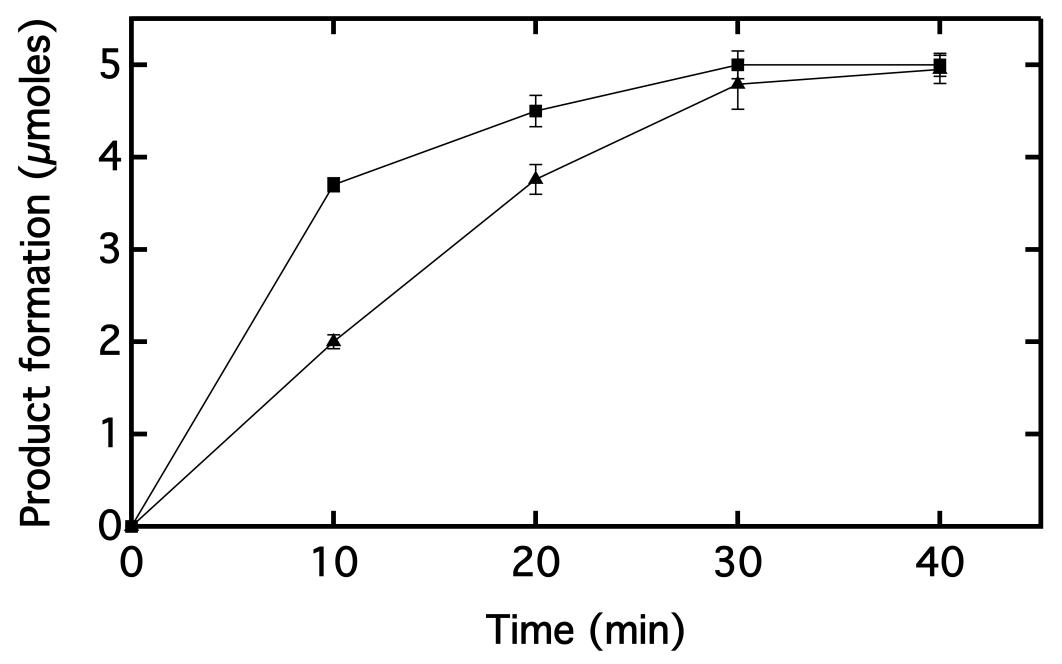

Figure S9. Time course of the enzymatic reaction when using ScASNaseI-filled capsules with either two ( $\mathbf{\square})$ or three ( $\mathbf{\Lambda})$ bilayers. The enzymatic assay was performed as described in the Methods section using L-Asn at $5 \mathrm{mM}$ final concentration. For direct comparison of data, the number of capsules per volume which were used for measuring the enzymatic activity was kept 
equal in both cases. Assuming that the amount of enzyme per capsule is the same in both cases and that the loaded enzyme is equally active, the observed slower time course of the reaction may be attributed to restricted substrate, or product diffusion in case of the three-bilayer capsules. Shown are the average values and the S.D. of 2 independent experiments. 


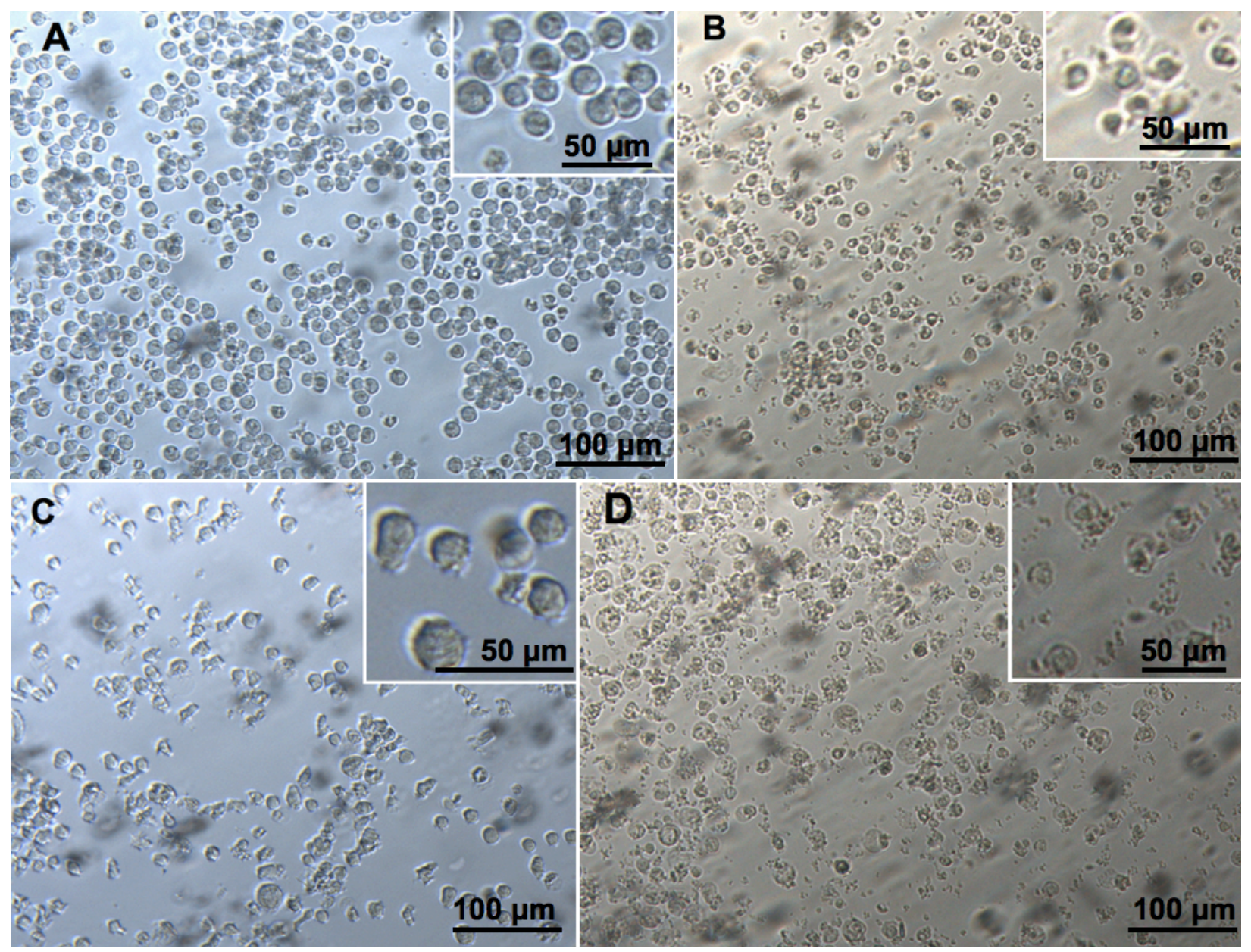

Figure S10. Transmission light microscopy images of untreated MOLT-4 (A) and SD1 (C) and treated MOLT-4 (B) and SD1 (D) with capsules containing ScASNaseI. Images for the untreated cells were taken at the point where the drugs were added $(\mathrm{t}=0)$ and for the treated $72 \mathrm{~h}$ after incubation; point at which the proliferation state was evaluated. 

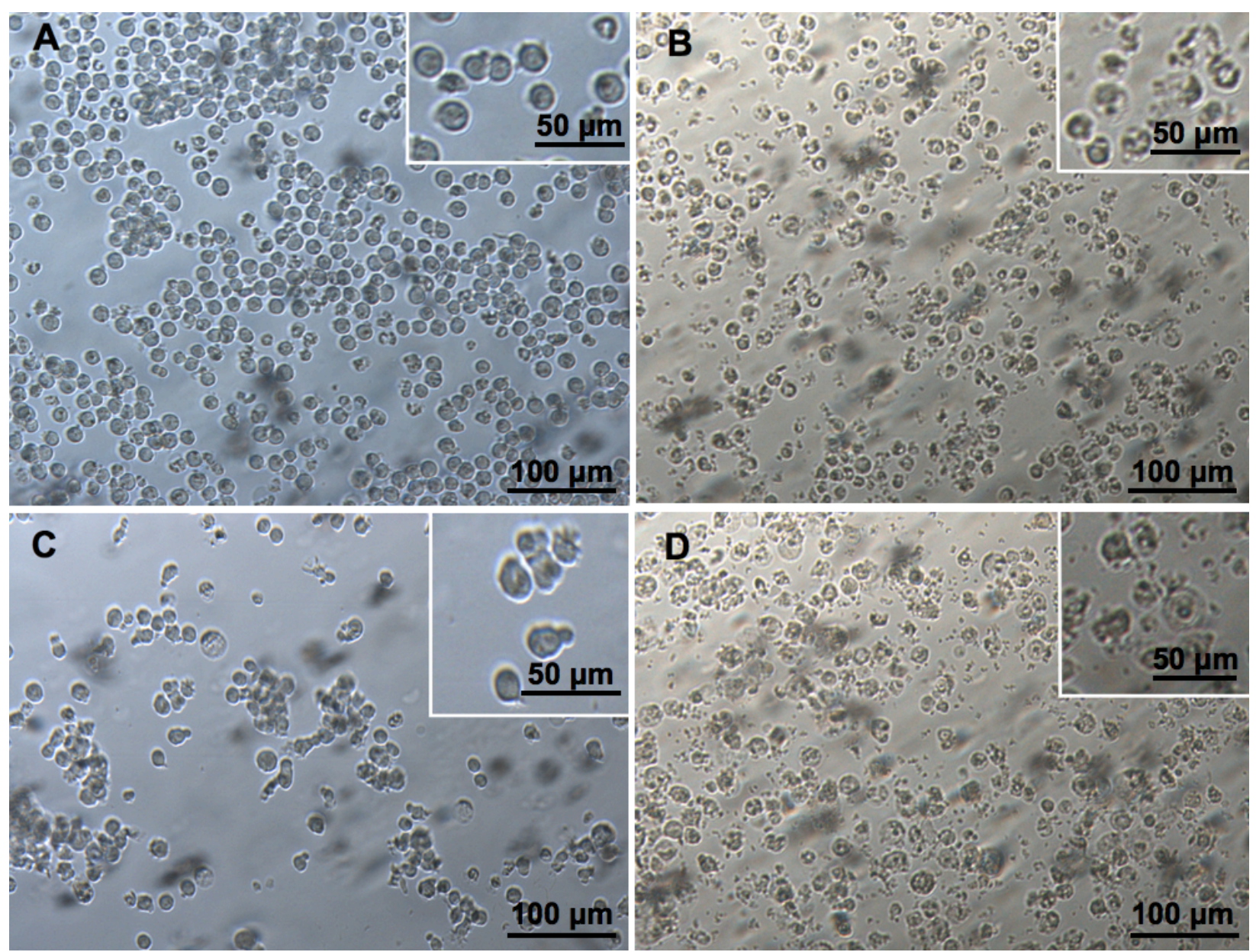

Figure S11. Transmission light microscopy images of untreated MOLT-4 (A) and SD1 (C) and

treated MOLT-4 (B) and SD1 (D) with EcASNaseII-filled capsules. Similarly to Figure S10, images for the untreated cells were taken at the point where the drugs were added $(t=0)$ and for the treated $72 \mathrm{~h}$ after incubation. 


\section{Discussion}

\subsection{General introduction}

The present work centered on the development of improved protein therapeutics for the treatment of acute lymphoblastic leukemia (ALL) following complementary protein engineering and drug delivery approaches $[134,212]$. We focused mainly on the biochemical, biophysical and structural characterization of two human L-ASNases, designated hASNase 1 [57] and hASNase3 [36,38]. Both enzymes hydrolyze the amino acid L-Asn but with poor catalytic properties $[32,38]$. Bacterial L-ASNases have been extensively used as antileukemic protein therapeutics for the last 50 years. However, this treatment is associated with a plethora of side effects mainly attributed to the bacterial origins of the used enzymes [100-106]. Inspired by the necessity of development of a protein drug of human origin, our ultimate goal has been the generation of catalytically improved human variants which could be used as alternative protein therapeutics to the current bacterial L-ASNases for the treatment of ALL. To this end, we centered on the biochemical and structural characterization of hASNase3, an enzyme which belongs to the N-terminal nucleophile superfamily [18]. We reasoned that the structural elucidation could provide a solid basis for the rational design of point mutations which could improve the enzyme's catalytic features.

\subsection{Characterization of hASNase 3}

\subsubsection{Structural characterization and glycine-induced intramolecular activation of hASNase3}

The determination of the hASNase3 structure revealed a dimeric state with the, highly conserved among the Ntn-hydrolases, $\alpha \beta \beta \alpha$ motif for each monomer (Introduction, Figure 4) [36-38]. The tertiary structure of the human enzyme significantly resembles the respective structure of its bacterial homolog, EcASNase3, which was solved several years ago [5]. A major characteristic of these Ntn-hydrolases is their property of being translated as inactive precursors, followed by an intramolecular cleavage at a scissile peptide bond, thereby generating two subunits ( $\alpha$ and $\beta$ ) which remain non-covalently associated and comprise the 
active form the enzyme [22]. However, particularly in case of hASNase3, recombinant expression in E.coli results in only partial cleavage of the produced enzyme population with fully activated and non-activated enzyme species; and importantly, this autoactivation process never reaches completion even after incubation for several days at $37{ }^{\circ} \mathrm{C}$, though rather fast intramolecular processing happens with other Ntn-hydrolases (thus, e.g.the E.coli homolog) $[5,38]$. We discovered that the amino acid glycine dramatically accelerates the autoproteolytic step of hASNase3 in a glycine concentration-dependent manner, thus allowing completion of the enzyme's self-activation [38]. Strikingly, other amino acids which are structurally related to glycine or other small molecule metabolites, cannot induce similar activation effects, thereby establishing glycine as a very specific molecule for hASNase3 intramolecular cleavage acceleration. This discovery helped to determine the structure of the fully cleaved hASNase3, as well as the uncleaved enzyme in complex with glycine [38]. The latter structure allowed us to propose the mechanism of this glycine-induced activation, explaining the ability of this amino acid to promote the enzyme's cleavage. Two bound glycine molecules were observed in the uncleaved form of hASNase3. The first occupies the same position as the substrate L-asparagine, while the second is oriented towards the residue Thr168 which serves as the primary nucleophile of the active enzyme. More specifically, the second glycine molecule is positioned such that its carboxylic moiety interacts directly with the hydroxyl group of Thr168. This interaction would allow glycine to act as a base which subtracts the proton from the hydroxyl group, thereby activating it for the subsequent attack on the preceding Gly167 carbonyl group, ultimately initiating the cleavage reaction. Interestingly, when unprocessed hASNase3 was incubated simultaneously with glycine and aspartate at 1:1 molar ratios (tested concentrations: 10 and $50 \mathrm{mM}$ each), no inhibition against the glycine-induced activation effect was observed [38]. Those results suggest that glycine has a dominant role on the activation of hASNase3, and its effect is not influenced by the presence of the enzymatic reaction's product (L-Asp). The question of whether the first glycine molecule, which occupies the same position as the substrate, is still present or is displaced in the presence of aspartate remains open, and to answer it, crystallization of hASNase 3 in the presence of both glycine and aspartate may have to be attempted. This proposed mechanism is further supported by the $\mathrm{pH}$-dependent cleavage rate we determined by incubating uncleaved hASNase 3 with glycine spanning a range of different $\mathrm{pH}$ values (7.5, 8.5 and 9.5) [38]. The cleavage occurs slightly faster at $\mathrm{pH} 9.5$ as compared to $\mathrm{pH} 7.5$ 
and 8.5, possibly because at higher $\mathrm{pH}$ the nucleophilicity of glycine is further increased resulting in a faster activation rate. Aiming at the investigation of the generality of this glycine effect possibly occurring in other members of Ntn-hydrolase superfamily as well, we recombinantly produced and purified not only E.coli ASNase3, but also another member of this family, the enzyme Taspase1. Multiple attempts to purify the E.coli homolog of hASNase 3 in its uncleaved form totally failed. Even when the purification steps were done at $4{ }^{\circ} \mathrm{C}$, the final state of the enzyme was the fully-cleaved form as was evidenced by SDSPAGE analysis. This prompted us to test another human Ntn-hydrolase. Taspase1 also is a threonine aspartase which plays a crucial role in regulation of the mixed lineage leukemia (MLL) nuclear protein [21]. Production of this enzyme in E.coli results in a mixture of cleaved and uncleaved species similar to hASNase3. However, incubation of Taspase1 with high concentrations of glycine (even up to $1 \mathrm{M}$ ) did not show any effect on the activation of this enzyme (Appendix, Part 1). These findings further support the specificity of the glycine-hASNase3 interaction, though it is absolutely necessary to test more Ntn-hydrolases in order to draw a more general conclusion.

The next question which plausibly comes up and we tried to address was, what would be the state of the enzyme if it were overexpressed in human cells, and, more importantly, what is the state of the endogenous hASNase 3 in human cells. Transiently transfected HEK293 cells with a plasmid carrying the ORF of hASNase 3 expressed the enzyme in its partially cleaved form in standard medium and in its fully-cleaved form in the presence of glycine in the growth medium, in a glycine concentration-dependent manner as evidenced by immunoblotting analysis using polyclonal antibodies raised in rabbits specifically against hASNase3 [38]. Interestingly, this happened when using glycine concentrations in the range of $5-10 \mathrm{mM}$ though it must be underlined the necessity of determining the intracellular concentration of free glycine upon treatment of the cells with glycine in the growth medium. In principle, the exogenous supply of glycine in the medium does not provide any information about the amount of glycine which is uptaken by the cells and is available in its free form to interact with the inactive translated hASNase3. On the other hand, it must be highlighted the fact that, the quantitative determination of intracellular concentrations of metabolites is experimentally very challenging and it can be done either by developing very sensitive assays which can detect the molecule of interest or by using radio-labeled compounds, which require additional expertise and the availability of special facilities. 
Significantly, immunoblotting analysis of cell extracts of two breast cancer cell lines, namely SKBR3 and MCF7, without including high glycine concentration in the growth medium, revealed that endogenous hASNase3 exists in both cleaved and uncleaved states in approximately 1:1 stoichiometric ratio (Appendix, Part 2). This suggests that hASNase3 is present in both states under steady-state in vivo conditions. Perhaps, an experiment using translation inhibitors could terminate the production of the inactive precursor and allow the capture of the enzyme's state shortly after its expression.

A reasonable argument which could be brought up is whether the glycine concentrations which are required in order to observe a considerable activation effect on hASNase3, are physiologically relevant. Concentrations in the range of 5-10 $\mathrm{mM}$ might not appear physiological at first glance. However, a recent metabolite-profiling report on cancer cell lines correlated increased glycine levels with proliferation rates, thereby highlighting the role of this amino acid in rapidly proliferating cells [286]. Strikingly, another study [287] which coincided with our discovery about glycine and focused on the role of pyruvate kinase isoform M2 in glycolytic flux in cancer cells, uncovered an intracellular accumulation of glycine in the range of $10 \mathrm{mM}$, a concentration which would fully activate hASNase 3 processing [38]. Those observations are in direct relevance with the fact that hASNase3 is predominantly expressed in a number of different types of cancers as evidenced by a systematic study a few years ago [34]. The discovered effect of glycine on hASNase3 was proven to be a very efficient in-vitro tool for the activation of the enzyme by accelerating and completing its intramolecular cleavage, though its in vivo relevance remains to be further investigated. However, based on the findings of other independent studies focusing on the metabolite profiling of cancer cells, there are good reasons to believe that the glycine-induced phenomenon on hASNase3 has physiological impact in-vivo as well.

\subsubsection{Circular permutant-like version of hASNase3}

Our studies have shown that full activation of hASNase3 was really essential for its biochemical characterization. Working with a mixture of fully-cleaved and uncleaved enzyme molecules does not allow the accurate and precise kinetic, biochemical, and biophysical characterization of a particular enzyme. Fast processing of hASNase3 can be achieved by including up to $200 \mathrm{mM}$ glycine in the growth medium of E.coli during the recombinant 
expression. However, this did not always result in full activation, and a short incubation period after the purification of the enzyme was required. In addition, glycine inhibits the enzymatic reaction exhibiting a $\mathrm{K}_{\mathrm{i}} \sim 10 \mathrm{mM}$ (data not shown) and, therefore, it must be removed by dialysis once the activation is complete. Those steps add to the already several experimental steps from the expression to the purification of the enzyme. For that reason, we developed a co-expression system to circumvent the requirement of intracellular activation [288]. Several co-expression combinations of the $\alpha$ and $\beta$ subunits were investigated (coexpression from the same plasmid, from different plasmids, expression from different plasmids, followed by purification and final mixing together). However, all trials failed for a number of different reasons which are discussed in detail in our recently published work [288]. The only co-expression system which resulted in the production of soluble and active hASNase 3 relied on the co-expression of both $\alpha$ and $\beta$ subunits in an operon-like way. The design of this construct depended on the concept of circular permutation [289]. Circular permutation in protein engineering is the process during which certain parts of the protein which form a concise folding unit (e.g. several $\alpha$-helices or $\beta$-sheets) are produced in a rearranged format, not following the natural order of the wildtype gene. The final folded molecule is identical to the wildtype, though quite often with improved biochemical properties as has been reported in several cases [290]. Normally, a circular permutant version of a protein has new $\mathrm{N}$ - and $\mathrm{C}$ - termini, while the two old ones are usually linked by artificially introduced peptide linkers with a variable number of amino acids which span the distance between the old $\mathrm{N}$ - and C- termini [291].

The first naturally existing circular permutant protein was discovered in 1979 by Bruce Cunningham and his colleagues, who noticed that the peptide sequence of the lectin protein favin was similar to an already known protein, namely concanavalin A, with the only difference being that the ends of the lectin protein favin were circularly permuted [292]. Later, it was further shown that concanavalin A undergoes posttranslational cleavage, coupled with a ligation reaction finally resulting in its circular permutant version [293]. However, it was not before 1995 when scientists found for the first time a circularly permuted pair of genes, encoding a class of proteins which are called saposins and are involved in sphingolipid catabolism and lipin antigen presentation in humans. The last years, there has been a rising interest towards the generation of circular permutant proteins and enzymes as a protein engineering tool. Perhaps, the most major challenge is to design an appropriate peptide linker 
which will connect the old termini in a way that will not cause any misfolding impediments. Many permutants have been successfully produced in the lab and their similar, to the wildtype, biochemical and biophysical properties have been experimentally demonstrated [294]. On the other hand, there have been reported several cases where circular permutants exhibited improved catalytic activity and thermostability, reduced proteolytic susceptibility, as well as altered substrate or ligand binding affinities [295]. Therefore, the researchers' motivations for the generation of circular permutants may vary depending on the final goal. In case of hASNase3, we tried to uncouple the intramolecular autoactivation step by generating a circular permutant version, without linking the old termini. We reasoned that, since the two final $\alpha$ and $\beta$ subunits remain non-covalently strongly associated after the intramolecular cleavage, they could be produced by co-expression and interact forming the active final form of the enzyme, despite the absence of a connecting linker. After performing a systematic study of different co-expression constructs, we found that the only one which results in the soluble production of both $\alpha$ and $\beta$ subunits and, most importantly, in active hASNase 3 was the circular permutant-like construct expressed from the pJC20-hASNase3[ $\beta$ $+\alpha]$ plasmid. The rearrangement of the two subunits, by expressing at the $\mathrm{N}$-terminus the $\beta$ and at the C-terminus the $\alpha$ subunit, facilitated the removal of the N-terminal methionine by the bacterial endogenous methionine amino peptidase (MAP), thereby exposing the catalytically essential Thr168 at the N-terminus of the $\beta$ subunit rendering the enzyme catalytically active. The kinetic properties of the permutant were found to be similar to the wildtype enzyme, though a more extensive study would shed more light on their biochemical and biophysical distinctions. In summary, in this study we established an attractive alternative method for the production of fully active hASNase3 through its expression by a circular permutant-like construct, thus totally precluding the necessity for intramolecular activation by glycine. 


\subsubsection{Engineering of hASNase3}

\subsubsection{FACS-based high-throughput screening of hASNase3 mutant libraries}

The ultimate goal of the present study was the engineering of hASNase 3 aiming at the identification of catalytically improved variants, envisioning future replacement of the current bacterial L-ASNases as protein therapeutics against ALL. The determination of the hASNase 3 structure did not actually help us to rationally design a set of mutations which could lead to improved variants. The reason is that, hASNase 3 belongs to a family of enzymes which, strictly speaking, are not primarily L-ASNases. All hASNase3 homologs from E.coli, plants and other mammals display similar, poor catalytic properties against LAsn. Therefore, the existence of certain amino acid "fingerprints" attributable to better catalytic rates against L-Asn, of other homologs was not available. In other words, there is no reference wildtype enzyme structurally similar to hASNase3, which exhibits favorable kinetic properties against the L-Asn hydrolysis and could form the basis for a rational engineering enterprise. Taken together all the aforementioned facts, it becomes plausible that the main avenue for engineering of hASNase3 was directed evolution. However, a major challenging task in directed evolution experiments is the development of a sensitive high-throughput screening system, which is capable of discriminating catalytically improved variants among others. In the present study we dealt with the application of a FACS-based high-throughput screening system for the analysis of a number of hASNase 3 mutant libraries. In addition, we focused on the development of a novel screening system for directed evolution applications by capitalizing on droplet-based microfluidic setups, whose great potentials as compartmentalization systems have been demonstrated in several studies [198-207].

Our FACS-based screening system relied on the use of a 5-gene-deletion E.coli strain (deletion of all genes which contribute to the biosynthesis of L-Asp) whose survival is solely dependent on the availability of L-Asp from the growth medium [179]. Genetic complementation of hASNase 3 mutants can rescue those E.coli cells through the supply of LAsp, product of the enzymatic reaction, thereby the growth of the cells is proportional to the activity of the hASNase 3 mutants. The co-expression of eGFP provided an additional quantification level of the availability of L-Asp, allowing the correlation of intracellular 
eGFP fluorescence with the mutants' L-ASNase activity, followed by FACS-sorting of the most highly fluorescent cells, as higher availability of L-Asp results in higher expression levels of eGFP which, in turn, leads to higher fluorescent levels. This system has been successfully used in the past for the deimmunization of EcASNase2 [179], though in that study the ultimate goal was not the isolation of catalytically improved mutants, but of variants which were exhibiting kinetic properties similar to the wildtype enzyme. For our purpose, we tested this system by comparing the mean fluorescence signal obtained from cells expressing the EcASNase2 and hASNase3, and we found that those which were expressing the E.coli enzyme showed $\sim 20$-fold higher mean fluorescent signal as compared to those expressing the human enzyme (Results section 5.1.3.1, Figure 1). Interestingly, despite the $\sim 2,000$-fold difference between the $k_{\mathrm{cat}} / \mathrm{K}_{\mathrm{M}}$ values of those enzymes as determined in-vitro using purified enzymes (with the E.coli being more efficient), the intracellular mean fluorescence signal resulted from the complementation assay differs only 20 -fold. This indicates that this assay provides only a semi-quantitative means of evaluating the enzyme's activity in the cells through its correlation with eGFP fluorescence. In addition, it must be underscored the complex intracellular environment consisting of multiple metabolic processes which might interfere with the availability of L-Asp, thus not allowing a strict quantitative correlation between the L-Asp concentration (result of L-ASNase activity), with the eGFP production and ultimately the fluorescent signal. Yet, considerably improved hASNase 3 variants in comparison to the wildtype enzyme, should possibly be discriminated through the intracellular eGFP fluorescence, followed by sorting and further biochemical characterization of the purified enzyme variants.

Subsequently, we analyzed five hASNase 3 mutant libraries and more specifically, one epPCR and four site-saturation mutagenesis libraries. The average number of mutations in case of epPCR library was $\sim 5$ per kb (ORF of hASNase3 has $930 \mathrm{bp}$ ) as described in detail in the Methods section. However, after four sorting rounds, no fluorescence enrichment was observed, possibly due to the fact that, the generated mutations did not have a positive impact on the catalytic properties of the enzyme. On the other hand, it could well be that the mutations totally impaired the ability of the enzyme's intramolecular activation, thereby resulting in inactive precursors despite the presence of glycine in the medium for the activation induction. This is another particular factor which must be considered and plays a crucial role in the engineering of hASNase 3: mutations must not influence the ability of the 
enzyme to undergo the intramolecular activation step. Taking into account the fact that, the enzyme's amino acid regions which can affect autoproteolysis are unknown and possibly unpredictable (unless a systematic site-directed mutagenesis study is performed highlighting such pivotal regions), it becomes understandable that additional, uncontrollable factors play notable roles in the successful hASNase3 engineering task. The generation of more epPCR libraries with higher error rates could provide a better basis for searching improved variants. It has been shown experimentally in certain cases that, proteins with considerably improved properties or novel functions can be isolated from high error-rate epPCR libraries $(\sim 15$ mutations per $\mathrm{kb}$ ); possibly due to the fact that, high mutation rates "unlock" unique sequences of the proteins' sequence space, which are enriched in beneficially coupled mutations [296]. In other words, the more the mutations, the higher is the probability that those mutations interact in a positive manner (positive epistasis), thereby improving the proteins' functions [297]. However, on the other hand, high mutation rates can lead to protein populations within which few sequences retain their functions. Therefore, an optimal error rate must be adjusted that provides a balance between functionality and novel sequences, and this is protein-dependent and experimentally challenging and time-consuming.

Given the known structure of hASNase3, we additionally employed site-saturation mutagenesis (SSM) experiments at regions which are located at the first and second notional shell from the enzyme's active site (see Methods section). Non-highly conserved amino acids were randomized since they most likely tolerate mutations without considerable influence on the enzyme's stability [298]. Four libraries were constructed by randomizing either four or two amino acids (two libraries with 4-codon and two libraries with 2-codon randomization, respectively) using degenerate primers with NNS (S: G, C) codons. NNS codons were chosen because only one stop codon is encoded (instead of three), and additionally the overrepresentation of the commonest variants is minimized (Arg, Leu and Ser are encoded each by six codons and are overrepresented when NNN codons are used) [299]. Simultaneously, all twenty amino acids are still encoded, thus the diversity of the library is not restricted. The first SSM libraries were generated by randomizing four codons. The construction of big libraries consisting of the mutation of many amino acids (in the range of 4-5) samples more extensively the protein's sequence space, thus the likelihood of the discovery of variants with improved properties is higher as compared to smaller libraries (1-3 amino acid randomization). Unfortunately, in case of hASNase3 the FACS-based screening 
of both 4-codon randomization libraries did not lead to the identification of an improved variant. The mean fluorescence of the cells expressing the mutants was lower than the respective value of the wildtype enzyme, suggesting that, both libraries predominantly consisted of variants with worse catalytic properties than the starting molecule. Those results suggest that, the chosen enzyme's regions for randomization cannot tolerate mutations despite the lack of high conservativity. In addition, the impact of each amino acid mutation on the enzyme's activity and/or stability remains unknown, unless deconvolution experiments are performed. For example, in case of SSM-LIB2 which did not show any fluorescence enrichment, it is unclear whether the first set (Met193-Val194) or the second set of mutations (Cys202-Leu203) had the most negative affect on the enzyme's activity. In order to address this question, the original 4-codon library must be dissected into two independent 2-codon randomization libraries (one for Met193-Val194 and one for Cys202-Leu203), followed by further screening and characterization. This analysis would provide more information about the tolerance of specific regions towards mutations and would also indicate whether two regions interact in an additive or non-additive manner. Therefore, in future experiments, more combinations of amino acid randomization schemes must be attempted aiming at the sampling of more pathways within the fitness landscape.

In contrast to the SSM-LIB1 and SSM-LIB2 (4-codons randomization), the next two smaller libraries SSM-LIB3 and SSM-LIB4, which were based on the mutation of two residues following the NNS scheme showed fluorescence enrichment during the screening process. After two rounds of sorting, we identified finally three variants, with the best one exhibiting up to $\sim 6$-fold better $k_{\text {cat }} / K_{M}$ in comparison to the wildtype enzyme (see Results section). At this point, it is important to note that despite the 6-fold improvement of the enzyme's catalytic properties, the respective arithmetic fluorescence mean of the cell population was not increased by 6-fold. In contrast, it showed a less than 3 -fold increase in comparison to the wildtype. This confirms what was mentioned above regarding the only $\sim 20$-fold difference between the EcASNase2 and hASNAse3, despite their 2,000-fold difference of their catalytic properties, further supporting the conclusion that it is a semi-quantitative approach for the enzyme activity determination.

The aforementioned best variant (DM1) was isolated from SSM-LIB3 which was constructed by randomizing two amino acid residues very close to the binding pocket ( $5 \AA$ ), the Ile189 and Val190. The sequencing of the mutated variant revealed the substitution of Ile189 by a 
Thr and Val190 by an Ile residue. Strikingly, the second improved variant (DM2) which was isolated from this library ( $\sim 2$-fold $k_{\mathrm{cat}} / \mathrm{K}_{\mathrm{M}}$ improvement as compared to the wildtype) had the original residues mutated in a reversed manner i.e. Ile189 was substituted by Val and Val190 by Ile. In the wildtype hASNase3, the side chain of Ile189 points towards the active site, thus providing a space-filling hydrophobic group to the substrate's binding pocket. The replacement of this Ile by Thr most likely offers more space for substrate binding, though space alone does not necessarily lead to higher activity. An additional hint to the possible rationalization of the effect of those mutations can be obtained by the DM2, which has a Val residue at this position. The two variants (DM1 and DM2) carry the same mutation at position 190 (Ile), but DM1 has a Thr, and DM2 has a Val at position 189, respectively. The two mutants share almost the same $\mathrm{K}_{\mathrm{M}}(1.3 \mathrm{mM}$ for DM1 and $1.58 \mathrm{mM}$ for DM2), but they are characterized by considerably different $k_{\text {cat }}$ values with the one of DM1 being $\sim 4$-fold higher. Those observations strongly suggest that the presence of a polar residue at position 189 , favors particularly the substrate turnover rather than the substrate binding. Though, it must be emphasized that both sets of mutations lowered equally $(\sim 50 \%)$ the $\mathrm{K}_{\mathrm{M}}$ value. The third improved variant (DM3) was isolated from SSM-LIB4, which was generated by randomizing two amino acids (Arg143 and Arg147) located at the surface of hASNase3, quite distant from the binding pocket ( $17 \AA$ for Arg143, and $14 \AA$ for Arg147). Interestingly, the variant has replaced the positively charged Arg143 by a negatively charged Glu residue, and the Arg147 by Lys, which is also positively charged. This variant exhibited the lowest $\mathrm{K}_{M}$ value in comparison to the other isolated mutants, which is $\sim 4$-fold lower than that of the

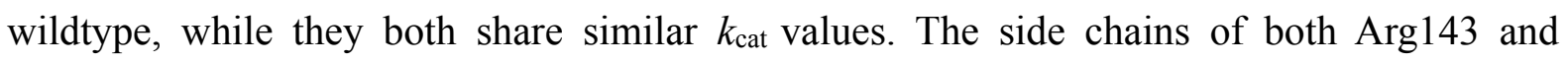
Arg147 point outwardly with respect to the enzyme's active site, while the distance between them $(\sim 5 \AA)$ is somewhat longer than the upper allowable limit for van der Waals interactions (normally, side chains interact via van der Waals interactions when they have less than $4 \AA$ distance [300]). Yet, this does not exclude the possibility that the oppositely charged side chains of Glu143 and Lys147 may interact, thereby stabilizing the $\alpha$-helix which accommodates them. In protein engineering it is generally believed that mutations at specific surface locations can considerably increase stability, possibly because the propensity of these regions to initiate unfolding is decreased [301]. Indeed, the stability of DM3 increased in comparison to the wildtype as well as to the other isolated variants, as evidenced by the increase of the enzyme's $T_{\mathrm{m}}$ value, determined by differential scanning fluorimetry (DSF). 
Furthermore, this set of mutations on the surface of hASNase 3 is another counterexample where amino acid substitutions far away from the active site have a notable impact on the catalytic properties of enzymes [302].

Considering the fact that we isolated three variants from two independent sets of randomized mutations, we reasoned that combination of those amino acid substitutions, resulting in two quadruplet variants (QDM), could have a positive cooperative effect, thus further improving the catalytic properties of hASNase 3 . Therefore, we combined the mutations of DM1 with DM3, and DM2 with DM3, resulting in the generation of QDM1 and QDM2 variants. However, despite the improved thermal stability of both QDM (both showed a $T_{\mathrm{m}}$ value of $\sim$ $68{ }^{\circ} \mathrm{C}$ ) in comparison to the initial combined variants, their catalytic efficiencies were not ameliorated. In case of QDM1, which combines the Thr189-Ile190 and Glu143-Lys147 mutations, both $k_{\text {cat }}$ and $\mathrm{K}_{\mathrm{M}}$ deteriorated. In contrast, QDM2 displayed an improved $k_{\mathrm{cat}}$, (very close to this from DM1) which is the highest among all mutants characterized in this work, though this improvement was more than neutralized by its $\mathrm{K}_{M}$ value, which was reconverted at the wildtype's levels. The overall catalytic efficiency $k_{\text {cat }} / K_{M}$ for both QDMs, were found to be lower than those of the initial combined variants and therefore, it appears reasonable to suggest that the two combined set of mutations interact in a negative cooperative manner regarding the catalytic enzyme's properties.

The effect of more than one mutation on proteins' properties such as stability, substrate binding and specificity and catalytic efficiency has been at the center of protein engineering studies for many years and brings up the question about additivity and non-additivity. Are the effects of two distinct point mutations, or more general of two sets of mutations, added up mathematically when they are combined in a double or multiple mutant? Or do they interact in a non-additive (epistatic) manner, leading to either positive or antagonistic effects? Those questions are very difficult to answer, and the literature is full of examples covering both cases (additivity and non-additivity) [303, 304]. It is generally believed that epistasis may occur when the side chains of two residues are in close proximity, or when one or both cause a change in the reaction mechanism of the rate-limiting step [305]. On the other hand, in cases where mutations which are located far away from each other, epistasis is more challenging to interpret and explain. In case of hASNase3, the combination of the three sets of mutations did not lead to further catalytically improved mutants, though their thermal stability was increased. More in-depth analyses need to be carried out involving urea- 
denaturation experiments or differential scanning calorimetry, in order to determine the $\Delta \Delta \mathrm{G}$ (difference between the free energy of the wildtype and the mutant) of all mutants studied in this work. Those informations will provide more definite answers to the question of additivity and non-additivity for hASNase 3 . If the $\Delta \Delta \mathrm{G}$ could be guessed based on the thermal stability results, then indeed the final stability of the QDMs increased, pointing to positive epistasis concerning the stability, in contrast to their catalytic efficiencies.

The fitness landscape of proteins is vast [306]. For example, for a relatively small protein of 300 residues (hASNase3 has 309) there are 5700 single amino acid substitutions and more than $3 \times 10^{6}$ ways to make simply two amino acid replacements. According to experimental results obtained from a different number of proteins, most of the mutational pathways lead to dead-ends and finally to nonfunctional and worse variants [307]. Therefore, the identification of efficient pathways to initiate a directed evolution experiment is very challenging, since only few pathways in Darwinian evolution lead to fitter proteins [308]. The generation of large libraries by randomizing many amino acids (equal or more than four) is very essential for a higher coverage of the sequence space. On the other hand, the likelihood of getting trapped in local minima (pathways of the fitness landscape which are dead-ends) is higher because accumulated mutations become nonviable [309]. Analysis of the directed evolution literature suggests that, in several cases successful engineering involved simple uphill walks in the fitness landscape, one mutational step at a time [310]. Quite often, single amino acid mutations are responsible for the functional change, despite the fact that many mutations may have been introduced [311]. In case of hASNase3, the 4-codon randomization libraries did not result in the isolation of catalytically improved variants. By contrast, the $\sim 10^{3}$-fold smaller, 2-codon randomization libraries allowed us to unlock enzyme's regions which can have a positive influence on the catalytic properties upon mutagenesis. Yet, it must be emphasized that in the case of the large libraries, the lack of improved variants might be due to the mutational intolerance of the specific regions, rather than the number of randomized amino acids. In order to evaluate this assumption, additional libraries must be generated by mutating a smaller number of residues around those regions. The engineering of hASNase 3 appears to be a very challenging task for different reasons. The necessity for the intramolecular activation step adds an additional difficulty to the system, and it must always be taken into consideration before the design of mutant libraries. Perhaps, it would be beneficial and contributive before the construction of a library for subsequent screening 
rounds, if a preliminary short mutational analysis around the region of interest were performed, thereby investigating the tolerance (regarding the ability of the enzyme to get autocleaved) of this region against mutations. A possible way to circumvent this activation step, is the use of the most recent circular permutant-like construct development, though this would entail further standardizations of the screening system, using different plasmids (this construct did not produce protein when it was plugged into pET14b vector). In addition, it would be desirable a codon-optimized for E.coli sequence to be used for the circular permutant-like version, because the expression levels were found to be dramatically lower than the wildtype enzyme [288]. Otherwise, it appears to be a promising alternative to the wildtype hASNase3, as template for mutagenesis.

The catalytic properties of this enzyme are considerably poor and far away of any potential anti-leukemic application. When the wildtype enzyme was incubated with two leukemic cell lines (MOLT-4 and SD1) no effect was observed (data not shown), while EcASNase2 killed the cells within one doubling event [257]. This lack of effect is due to the very high $\mathrm{K}_{\mathrm{M}}$ of the enzyme $(3 \mathrm{mM})$ and the very low L-Asn concentration in the culture medium $(\sim 100 \mu \mathrm{M})$ which is similar to the steady-state serum concentration [312]. Furthermore, the turnover of hASNase3 is significantly lower $\left(\sim 1 \mathrm{~s}^{-1}\right.$ at $25{ }^{\circ} \mathrm{C}$ and $\sim 3.5 \mathrm{~s}^{-1}$ at $\left.37{ }^{\circ} \mathrm{C}\right)$ than that of EcASNase2. Given the fact that, in case of ALL a very fast depletion of the L-Asn serum levels must be achieved in order to induce remission, the $k_{\text {cat }}$ must also be substantially improved (EcASNase2 has a $k_{\mathrm{cat}} \sim 22 \mathrm{~s}^{-1}$ at $37^{\circ} \mathrm{C}$ and the other FDA approved enzyme from ALL treatment from Erwinia chrysanthemi exhibits a $k_{\text {cat }}>300 \mathrm{~s}^{-1}$ at $37{ }^{\circ} \mathrm{C}$ ). The lack of a Ntn-hydrolase homolog which would display very promising catalytic properties and could form an interesting model for the rational engineering of hASNase3, further complicates the situation. In addition, systematic analysis of the protein engineering literature shows that, there are very few examples where both kinetic parameters $\left(k_{\text {cat }}\right.$ and $\left.\mathrm{K}_{\mathrm{M}}\right)$ for the wildtype substrate were improved. In certain cases, indeed, dramatic improvements regarding the catalytic efficiency of enzymes have been reported, but they are predominantly related to substrate analogs or new substrates, but not against the enzyme's natural substrate [313]. The isolation of the improved mutants which were characterized in this work forms a good starting point for further mutagenesis and screening experiments. Notably the DM1 and DM3 which showed the most improved properties can be used as templates for epPCR or SSM. Perhaps, a cell-killing effect of the three DMs against leukemic cells is worth testing, though 
based on their catalytic properties it appears reasonable to assume that such an effect is difficult to observe. Finally, it is progressively becoming clearer through the different directed evolution studies that, the best indicator for the evolvability of an enzyme is its natural history [314]. Enzymes from large families displaying diverse substrate activities are easier to evolve given the fact that the same natural evolutionary mechanisms govern and dictate the obtainment of new functions at the laboratory level as well. Connecting this belief with hASNase3, we could be more optimistic about its evolvability since it satisfies the two aforementioned characteristics: it is a member of a very large family of enzymes (Ntnhydrolases) and accepts a different number of substrates (primarily several derivatives of LAsp dipeptides, and secondarily L-Asn). What is needed, is to uncover the correct pathway among the vast number of pathways of the fitness landscape, which lead to local maxima.

\subsubsection{Development of a droplet-based microfluidic high-throughput screening system}

In the present work we developed a high-throughput enzyme-activity screening platform by capitalizing on the droplet-based microfluidic technology. The great potential of this technology has been recently demonstrated in a wide range of applications, including highthroughput screening setups in directed evolution approaches [200-205]. The major challenge for enzyme evolution experiments relying on the droplet-based microfluidic setups is the availability of a highly sensitive fluorescence assay. Given the very small droplets volume (range of $\mathrm{pL}$ or $\mathrm{fL}$ ) within which the reactions take place assays utilizing absorbance as output, fail to be used due to severe lack of sensitivity (the light path length is extremely small). On the other hand, prior to this work, there was no available fluorescent assay utilizing the natural substrate of L-ASNase (L-Asn) to monitor its activity. This was our motivation to develop a novel L-ASNase assay, which would enable the monitoring of the enzyme's activity in the fluorescence mode solely relying on L-Asn as the natural substrate. To this end, we coupled the L-ASNase activity step to a second one in which the generated LAsp is oxidized to iminoaspartate and hydrogen peroxide. Hydrogen peroxide can be converted by horseradish peroxidase in the presence of Amplex Red to Resorufin, which shows excellent fluorescent and absorption properties. The assay can be used equally well either to the fluorescent or to the absorbance modes as demonstrated in our published work 
[265]. In addition, it can be used to detect very low levels of L-Asn from samples (down to $0.1 \mu \mathrm{M}$ ) performing end-point measurements or, to detect L-ASNase activities using aliquots of serum spiked with L-ASNase. The latter possibility might be very useful in clinics where the determination of residual L-ASNase activities from serum samples obtained from patients treated with L-ASNase is very essential [315].

Once the assay was standardized using macroscopic volumes, we tested its applicability in microscopic volumes (droplets). As evidenced by our results described in the respective section (Results section 5.2.3.1, Figure 5), the assay behaves almost identically under both conditions enabling the accurate determination of L-ASNase activity using purified enzyme. The next steps included trials to determine L-ASNase activity at the single-cell level, attempting to form the basis for the development of a high-throughput system for directed evolution experiments. The use of droplet-based microfluidic setups as screening platforms for the screening of enzyme mutant libraries has been demonstrated in many cases, and there is an exponentially rising interest towards this technology. In many of the reported cases of directed evolution experiments, yeast was used as a host organism for the expression of the mutated sequences, by displaying the molecule of interest on the outer surface [199]. Alternatively, in-vitro expression systems have been also successfully employed [203]. The use of E.coli cells for the expression of enzymes subjected to mutagenesis cycles, followed by compartmentalization in emulsions has been reported a few years ago [198]. In this case, the enzyme of interest (arylsulfatase from Pseudomonas aeruginosa) was produced in the cytoplasm, and, consequently, the cells were lysed in the droplets using commercially available detergents, in order to liberate the enzyme and make it accessible to the substrate. However, such approaches were not proven beneficial in our case, because the use of detergents inactivated the helper enzymes (L-AspOx and HRP), thereby not allowing us to monitor the L-ASNase activity (see Results section 5.2.3.2)

Therefore, in the present study, we made use of the anchored periplasmic expression (APEx1) in order to display the EcASNase2 onto the periplasmic side of the inner membrane of E.coli cells via fusion to the signal peptide and the first six N-terminal amino acids of the inner membrane lipoprotein NlpA [155,275]. This technology has been shown to have great potential in the directed evolution of single chain antibody fragments, which were anchored in the inner membrane, and upon disruption of the outer membrane by chemical and enzymatic means, they interacted with the respective antigens for subsequent FACS analysis. 
The use of the APEx1 system helped us to circumvent a great number of issues related to cell lysis in droplets. Multiple attempts to lyse the cells using lysing agents like lysozyme and polymyxin B failed. Different droplets exhibited varying degrees of enzymatic activities, most likely attributed to the different degrees of lysis of the compartmentalized cells. In addition, the lysis of the cells would entail additional transformation steps in order to recover the plasmid DNA of the sorted droplets for the next round of selection. The use of the APEx1 system allowed us to display EcASNase2 in the inner membrane of the cells and monitor enzymatic activity upon incubation with the assay compounds. The amino acid L-Asn is a small molecule, thus its diffusion in the membrane via crossing only the outer membrane is not limited. On the other hand, the produced L-Asp can be exported from the periplasm in a similar manner, thereby being accessible to the L-AspOx for the subsequent Amplex Redcoupled reaction steps. Initial attempts to disrupt the outer membrane by lysozyme and EDTA making the substrates directly accessible to the displayed enzyme, resulted in significant loss of L-ASNase activity $(\sim 50 \%)$. This was to be expected since it has been shown that EDTA inhibits the L-ASNase reaction [257]. Yet, this was not proven to be a significant impediment of the system, since as mentioned above, the non-disruption of the outer membrane did not limit the reaction, mainly due to the small size of L-Asn and L-Asp enabling them to diffuse easily into and out of the periplasm.

At this point it is important to note that, there are several other display technologies which can be applied using E.coli cells, though with certain disadvantages. The periplasmic display of proteins anchored in the outer membrane is also possible, by creating fusions between the protein of interest and endogenous outer membrane proteins. A noteworthy example which has been utilized several times is the Lpp-OmpA system [316]. This hybrid system is based on the properties of the E.coli major lipoprotein Lpp and the outer membrane protein A (OmpA). Lpp is an outer membrane lipoprotein which faces the periplasmic side and is absolutely essential for the correct localization of the heterologous protein to the outer membrane [317]. OmpA consists of eight anti-parallel $\beta$-stands forming a $\beta$-barrel which span the whole outer membrane and is responsible for the display of the protein of interest on the outer surface of the cell [318]. Neither Lpp nor OmpA system can independently display proteins and their combination is required for this goal [319]. What is combined is the peptide signal sequence and the first nine amino acids of the Lpp protein and a truncated form of OmpA. The major disadvantage of this outer surface display system is the need to 
generate very big constructs considering the large parts of the Lpp-OmpA fusion which must be combined. The situation becomes even more complex if the protein of interest is a large protein. In addition, the mechanism of display is quite complicated involving many steps from the expression of the fusion construct in the cytoplasm, transport to the periplasm, and from there crossing the protein of interest through the outer membrane, followed by anchoring of the OmpA protein's loops in the membrane. Furthermore, inserting large proteins into the external loops, frequently interferes with localization of the fusion protein, thereby resulting in unsuccessful display [317]. Other systems involve the simple periplasmic expression of proteins, similar to the APEx1 approach without anchoring the proteins on the inner membrane. However, it has been found that periplasmic expression may lead to spontaneous disruption of the outer membrane due to accumulation of high amounts of proteins in the periplasm [320]. Assuming that this may occur in an uncontrollable manner, it would result in loss of the expressed mutants during expression in the growth medium, and what would be compartmentalized in emulsions would be simple spheroplasts without the protein of interest.

In contrast, the APEx 1 system comprises a number of certain advantages as compared to the aforementioned systems. It involves only a short $\mathrm{N}$-terminal fusion consisting of the peptide leader and the first six amino acid of the mature lipoprotein NlpA while the translocation to the periplasm is mediated by the Sec pathway, only by crossing the inner membrane. The lipoprotein NlpA display may provide a more uniform display as compared to periplasmic expression, thus resulting in a more homogeneous signal during the analysis by any means (droplets, FACS e.t.c.). If the protein of interest is to interact with a big substrate molecule which cannot cross the outer membrane (e.g. antigen in case of antibody display, or big organic compounds), the outer membrane can be permeabilized chemically and enzymatically without loss of the anchored molecule, thereby offering the possibility for direct interaction [155].

The displayed EcASNase2 in the inner membrane of E.coli cells was assayed successfully applying the 3-step Amplex Red fluorescent assay. Based on the activity rates using known concentrations of purified enzyme, we calculated the amount of the anchored enzyme, which was approximated very well by quantitative immunoblotting analysis of the membrane fraction obtained after cell fractionation using anti-His 6 antibodies (see Results section 5.2.3.2). The cells after the activity measurements maintained their viability, and this was 
tested by plating them onto agar plates supplemented with the respective antibiotic. The cells grew forming almost equal number of colonies as before their compartmentalization in emulsions. This is another great advantage of this display approach which enables cell recovery excluding additional transformation or amplification via PCR steps for the recovery of the encoding DNA sequences of interest. The next steps for the development and the standardization of the droplet-based microfluidic screening system include the compartmentalization of cells displaying the hASNase3 enzyme and monitoring its enzymatic activity. For the first preliminary experiments the E.coli enzyme was used because it is 2,000-fold more efficient, its preparation is relatively easy since no activation step is required, thus facilitating overall the activity measurements. In addition, the mixing of two different droplet populations, one carrying EcASNase2 and the other hASNase3, followed by subsequent sorting of the most highly fluorescent emulsions based on the distinct enzymatic activities is very essential. Provided that this droplet discrimination is possible and the isolation of the desired events can take place, then the system would be ready for the screening of real hASNase3 mutant libraries.

In summary, it must be underscored that the availability of two or more different highthroughput screening systems for the analysis of enzyme mutant libraries is a privilege. In our case, by applying the FACS-based screening approach and analyzing overall five libraries, we managed to identify eventually three hASNase 3 variants with several fold improved catalytic properties. Perhaps, the analysis of more libraries sampling more the sequence space might yield more efficient mutants. However, the FACS approach provides an indirect means of measuring the enzymatic activity and this is largely evidenced by the fact that, EcASNase2 and hASNase 3 display catalytic properties differing by a factor of $\sim 2,000$-fold, while the respective genetic complementation control experiment using FACS, showed a difference around 20 -fold. In addition, the variant which exhibited a 6 -fold higher $k_{\mathrm{cat}} / \mathrm{K}_{\mathrm{M}}$ value as compared to the wildtype, was isolated from a library which showed a less than 3-fold fluorescence enrichment ( $\mu \sim 50$ for wildtype, and $\mu \sim 130$ for the library enriched with 6fold improved mutant). On the other hand, the microfluidic approach provides a more direct means of enzymatic activity determination than FACS, given that what is fluorescently detected, is the enzyme's catalytic activity measured in-vitro. Both technologies can be proven to be extremely powerful for the characterization of mutant libraries and must be 
considered complementary towards a common ultimate goal, which is the isolation of catalytically improved hASNase 3 variants.

\subsection{Biochemical characterization of hASNase1}

Inspired by the necessity to develop a human L-ASNase for an alternative treatment of leukemia, we searched for other enzymes of human origins which could possess L-ASNase activity. Performing BLASTP analysis in the database using as template the cytoplasmic and periplasmic E.coli enzymes, we identified a 573-residue enzyme, designated 60-kDa human lysophospholipase, whose N-terminal domain showed high amino acid sequence identity (47\%) with the EcASNase1 [57]. This enzyme comprises two domains; the N-terminal domain ( the $40 \mathrm{kDa}$ part of the enzyme) resembles significantly the EcASNase 1 enzyme, while the C-terminal domain (the rest $20 \mathrm{kDa}$ ) is homologous to ankyrin repeat-like domains $[55,56]$. Strikingly, this is a very poorly characterized protein, and this is evident of a single study which is available in the literature [56]. In this work, it was demonstrated that the fulllength $60-\mathrm{kDa}$ human lysophospholipase interacts with a serine/threonine protein kinase, named Sgk1, an enzyme which is involved in various cell proliferation pathways. More than a decade ago, a Japanese group had reported on the rat homolog of the 60-kDa human lysophospholipase, isolated from rat liver [55]. In this study, the authors had shown that the full-length rat enzyme possesses three distinct activities acting as lysophospholipase against lysophosphatidylcholine, platelet-activating factor (PAF) acetylhydrolase as well as LASNase; though these enzymatic activities were determined only qualitatively using lysates from HEK293 cells overexpressing the respective gene rather than purified enzyme preparations. Several years later, the lipase activity was confirmed for the human $60-\mathrm{kDa}$ lysophospholipase, but the ability of this enzyme (full-length) to hydrolyze L-Asn was not demonstrated [56].

We generated a series of N-terminus truncations of the full-length $60-\mathrm{kDa}$ lysophospholipase, corresponding to the L-ASNase domain [57]; the expression of the full-length enzyme was not successful using E.coli as host organism, though different vectors, tags and expression conditions must be further tested. Interestingly, one of the tested constructs produced a soluble truncated L-ASNase domain, which we designated hASNasel because of the high homology with EcASNase1. Most importantly, the purified recombinant enzyme showed L- 
ASNase activity. However, when we tested it for lysophospholipase activity using a mixture of lysophospholipids as substrates, we did not detect any activity [Appendix, Part 3]. Those data raise several questions concerning the catalytic properties of this enzyme: Does this enzyme eventually possess lysophospholipase activity? If yes, then does the active site for the lipase activities reside in the L-ASNase active site? What is the role of the C-terminus ankyrin repeats? Could the absence of the C-terminus ankyrin repeat of the truncated LASNase domain cause the lack of the lipase activity we could not measure? Ankyrin repeats mediate exclusively protein-protein interactions, and no ankyrin repeat domain has been found to hold catalytic properties [321]. All the upper questions are more difficult to answer given the unknown cellular functional role of the full-length enzyme. Mammalian cells encode a great number of enzymes which act as lysophospholipases, forming two large families: the small and the big mammalian lysophospholipases [322]. Those enzymes catalyze the conversion of the substrates which are supposed to be hydrolyzed by the $60-\mathrm{kDa}$ rat and human lysophospholipases. Those facts plausibly bring up the question about the physiological role of this enzyme in cells, considering in addition the existence of the Nterminus L-ASNase domain.

When we kinetically characterized hASNase1, we found that the enzyme does not follow Michaelis-Menten kinetics but instead, it exhibited sigmoidal kinetic behavior similar to its homolog EcASNase1 [57]. The structural determination of the E.coli enzyme revealed a tetrameric oligomeric state, as well as additional, distinct from the active site, binding sites for L-Asn. This fact, in combination with the enzyme's sigmoidal kinetics showing a positive response against increasing concentrations of L-Asn, defined the E.coli homolog as an allosteric enzyme which shows positive cooperativity with a Hill coefficient $\left(\mathrm{n}_{\mathrm{H}}\right) \sim 2.6$ and $\mathrm{S}_{0.5} \sim 1.2 \mathrm{mM}$ [51]. Those values do not considerably differ from what we determined for the E.coli enzyme $\left(\mathrm{n}_{\mathrm{H}} \sim 3.5\right.$ and $\left.\mathrm{S}_{0.5} \sim 0.4 \mathrm{mM}\right)$ [57]. hASNase1 displayed also sigmoidal steadystate kinetics with a $\mathrm{n}_{\mathrm{H}} \sim 3.9$ and $\mathrm{S}_{0.5} \sim 11.5 \mathrm{mM}$. However, when we analyzed the native molecular weight of this enzyme in a size exclusion chromatography column, astonishingly we found that the enzyme was running as a monomer. In addition, when we repeated the same experiment in the presence of the substrate L-Asn, we observed a shift of the main chromatographic peak to higher molecular weights, indicating that the presence of substrate induces the formation of hASNase 1 oligomers. Yet, the main state of the enzyme was monomeric, and notably under assay conditions where we used lower enzyme concentrations 
than in the size-exclusion chromatography experiment, the predominant form of the enzyme is monomeric.

This pronounced allosteric behavior of hASNase1 with very high Hill coefficient, given its monomeric state is difficult to explain. However, despite the steady-state kinetic analysis which clearly supports the enzyme's allosteric behavior, we further probed the putative allosteric site by fluorescence labeling of a Cys residue which is located in the binding cavity of this site according to a homology structural model we built based on the EcASNase1 structure [57]. Titration of the labeled enzyme by L-Asn caused fluorescence quenching, indicating interactions between the substrate and this enzyme's domain, thereby further supporting the existence of an allosteric site. Furthermore, mutational analysis of hASNase1 revealed that the enzyme does not tolerate any mutation of crucial residues located in either the active site or the allosteric site. In addition, despite the high Hill coefficient, hASNase1 exhibited a $\mathrm{S}_{0.5}$ value of $\sim 11.5 \mathrm{mM}$ raising more questions about its physiological role in cells. However, this relatively high $\mathrm{S}_{0.5}$ value falls well within the range of free concentrations of intracellular amino acids $(>10 \mathrm{mM})$, including L- Asn, reported for mammalian cells [323], thus making the enzyme operate efficiently particularly at elevated substrate concentrations.

In fact, a number of monomeric enzymes displaying allosteric behavior have been reported, with human glucokinase enzyme being the most thoroughly characterized [324]. Glucokinase (also called hexokinase IV), unlike L-asparaginase, is a two-substrate (ATP plus glucose) enzyme and displays a moderate degree of allostery [325]. Nevertheless, monomeric enzymes with single binding sites like hASNase1, can also show allostery [326]. Two basic models have been proposed aiming at the mechanistic explanation of monomeric allostery: The mnemonic model [327], and the Ligand-Induced Slow Transition (LIST) model [328]. Both models assume the existence of two different enzyme conformations which are characterized by distinct affinities for the substrate (low and high affinity states). Depending on the substrate concentration, certain conformational changes may occur, thus perturbing the equilibrium of the two states in favor of the high-affinity state and an increased catalytic activity. Based on the observed sigmoidal kinetic behavior and the monomeric state of hASNase1, it appears reasonable to assume that this is another example of a monomeric enzyme exhibiting positive allosteric regulation. Additionally, given the fact that L-Asn plays a dual role of being both substrate and regulator of hASNase1, L-Asn can be considered as a 
homotropic allosteric effector of this enzyme which adds to the constantly growing number of allosterically regulated proteins [52].

Summarizing, it is very important to note that despite the classification of hASNase1 as a bacterial-type L-ASNase, its kinetic properties are not better than those of hASNase3. Considering especially its marked allosteric properties with very high both Hill coefficient and $\mathrm{S}_{0.5}$ values, it becomes apparent that the engineering of this enzyme is more challenging than the engineering of hASNase3. On the other hand, hASNase1 displays a higher turnover number than hASNase3 $\left(\sim 7 \mathrm{~s}^{-1}\right.$ as opposed to $3 \mathrm{~s}^{-1}$ at $\left.37^{\circ} \mathrm{C}\right)$, though hASNasel operates at its maximal velocity under L-Asn concentrations which are remarkably higher than the steadystate serum concentration $(\sim 100 \mu \mathrm{M})$. Furthermore, the thermal stability of hASNase 3 is considerably higher than that of hASNase 1 as evidenced by the $\sim 10{ }^{\circ} \mathrm{C}$ difference performing DSF analysis. Overall, based on our accumulated biochemical and biophysical data for both human L-ASNases, hASNase3 has been studied more extensively, and the recent isolation of the three catalytically improved mutants has formed a solid basis for further improvement. Regarding hASNase1, its structural determination would be beneficial as an immediate next step, in order to shed more light on structural characteristics responsible for its pronounced allosteric behavior; offering the possibility of a more rational engineering design aiming at its catalytic amelioration.

\subsection{Characterization of polyelectrolyte microcapsules filled with catalytically active $S c$ ASNase1 and EcASNase2}

In the present work, despite our efforts to characterize and engineer L-ASNases of human origin as alternatives for treatment of ALL, we additionally focused on complementary technologies for the improvement of the currently available drugs, or for the future enhancement of improved hASNases. Those technologies have defined the field of drug delivery systems and have found a vast number of biomedical applications in recent years [215-219]. There are excellent reviews which highlight the most recent advances in this field $[122,123,128]$. We exclusively capitalized on the technology of Layer-by-Layer (LbL) polyelectrolyte microcapsules (PECs). This technology has been introduced in the field during the last fifteen years and offers the possibility of packaging different biomolecules of interest in spherical structures consisting of biocompatible and biodegradable polyelectrolytes 
[125-128]. The advantages of this approach are severalfold and are discussed in detail in the Introduction. In case of L-ASNases, given their biomedical applications as anti-leukemic regimens, their encapsulation could potentially increase their in-vivo half-life by protecting them against the proteases' attacks and by preventing their interaction with antibodies which cause their so-called silent inactivation [105]. By packaging the Saccharomyces cerevisiae LASNase1 (ScASNase1) and EcASNase2 (the currently FDA-approved anti-leukemic drug) in PECs, we investigated the encapsulation effect on their biochemical stability, resistance against proteases degradation, as well as their efficacy against leukemic cells [257].

The fabrication of PECs involves the use of an initial sacrificial template, typically $\mathrm{CaCO}_{3}$, where the biomolecules of interest (e.g. enzymes, proteins, antibodies, DNA etc) are adsorbed $[222,224]$. The loading of the molecules is followed by the successive deposition of negatively and positively charged polyelectrolytes onto the spherical surface of the initial template. Once, the desirable number of polyelectrolyte bilayers is deposited, the sample is subjected to a final treatment for the dissolution of the inner template, resulting in the eventual entrapment of the molecules in the polyelectrolyte shell forming the so-called hollow microcapsules. This final treatment for the template dissolution usually involves the use of quite harsh conditions such as lowering the $\mathrm{pH}$ to 3 or 2 , raising the temperature up to 50-60 ${ }^{\circ} \mathrm{C}$, or using EDTA concentrations in the range of 0.2-0.3 $\mathrm{M}$ aiming at the complexation of the $\mathrm{Ca}^{2+}$ by EDTA, thereby dissolving the inner $\mathrm{CaCO}_{3}$ cores [124]. However, while certain molecules like small organic compounds or even DNA can tolerate such extreme treatments, for proteins and notably enzymes this sort of manipulations can result in loss of their function. Indeed, when we used 0.2 M EDTA to dissolve $\mathrm{ScASNase1-filled} \mathrm{CaCO}_{3}$ cores, the enzyme totally lost its activity [257]. EDTA titrations against ScASNase 1, revealed that the enzyme can only tolerate up to $20 \mathrm{mM}$ EDTA concentrations without being affected. Based on these observations, we developed a protocol for PEC fabrication using very low EDTA concentrations in comparison to the standard protocols [223,224]. After the polyelectrolyte layers deposition, the sample was subjected to exhaustive dialysis $(1: 1000$ volume ratio) against the working buffer containing $20 \mathrm{mM}$ EDTA. Following this approach, we managed to minimize the exposure of the enzyme to high concentrations of EDTA which has inhibitory effects on the enzymatic activity and simultaneously, the dialysis against a large buffer:sample volume ratio mildly dissolved the inner $\mathrm{CaCO}_{3}$ cores, thus forming PECs with fully active ScASNase1 [257]. 
The final capsules were characterized by confocal and scanning electron microscopy, which confirmed the formation of intact, hollow PECs with an average diameter of $2 \mu \mathrm{m}$. This fabrication protocol might be useful for other cases in which labile molecules are to be used for the preparation of PECs. Subsequently, we investigated the stability of the encapsulated ScASNase1 by treating the capsules with proteases such as thrombin and trypsin, two enzymes which have been shown to degrade the therapeutic EcASNase2 during the treatment of ALL. In addition, the ScASNase1-filled PECs were incubated at the physiologically relevant temperature of $37{ }^{\circ} \mathrm{C}$, and at $4{ }^{\circ} \mathrm{C}$ and their residual activity was assessed at different time points by assaying an aliquot from the initial mixture, in order to determine the isothermal inactivation rate and the storage stability of the encapsulated enzyme. Our results convincingly showed that the encapsulation of ScASNase1 dramatically improved the storage stability of the enzyme as well as its stability at $37{ }^{\circ} \mathrm{C}$ in comparison to the free enzyme. Moreover, the resistance against trypsin and thrombin proteolysis was considerably improved. For example, trypsin totally inactivated the free enzyme after one hour of incubation at $37^{\circ} \mathrm{C}$, while the encapsulated enzyme retained its activity even after seven hours of incubation. To extend our in-vitro findings to the cell culture level, we incubated two different leukemic cell lines (MOLT-4; T lymphoblastoid type of cells, and SD; T leukemic cells) with free and encapsulated ScASNase1 including as control the EcASNase2 (FDAapproved drug) in independent treatments, aiming at the simulation of conditions in the patients' serum. In parallel, similar cell-PECs samples were prepared by including in the mixtures the combination of thrombin and trypsin at concentrations which are present under physiological and pancreatitis conditions, respectively [257]. We found that incubation of the free enzyme with the proteases resulted in limited cell killing, while the encapsulated enzymes caused cell death to similar levels with the samples which were lacking proteases. Those data clearly indicate that, the presence of the proteases degraded the enzymes, thereby not allowing them to deplete the L-Asn levels of the growth medium which would cause cell death. On the other hand, the encapsulated enzymes were protected within the shell of the PECs preventing the proteases from inactivating them, allowing them to deprive the L-Asn in the medium causing cell killing.

Our preliminary in-vitro results concerning the benefits of the encapsulation of therapeutic enzymes like L-ASNase are very promising. However, it would be an exaggeration to praise the PECs-L-ASNase system as immediately available for treatment. Certain important issues 
must be addressed in the future for better characterization and improvement of PECs-based systems. One of the greatest challenges in the field is the prevention of aggregation of the polyelectrolyte capsules [242]. Due to their cationic and anionic properties, the capsules have an inherent tendency to form clusters and aggregate. This is a major limitation for their biomedical applications, because the aggregated capsules could cause severe problems in blood stream through the blood vessels. Another challenge in the field is related to the quantification of the loaded drug onto the capsules, and more specifically of proteins. The following of the drug amount which is loaded onto the initial sacrificial template is possible, but it is no longer doable once the deposition of the poly-amino acid electrolytes is initiated. The reason is that, all the available assays for the quantification of proteins interfere with such polyelectrolytes. Additionally, during the successive cycles of the polyelectrolytes deposition and washing, a certain amount of the initially used protein amount is lost. Therefore, the only possible route for estimation of the final loaded protein amount is indirectly by subtracting from the initial amount, the lost protein fractions during deposition and washing. However, this approach includes a very high degree of experimental error, thereby often leading to inaccurate measurements of encapsulated protein. The use of enzymes offers the privilege to evaluate approximately the efficacy of the capsules through enzymatic activity determinations, though this is not possible with other proteins. Last but not least, it is highly debatable whether the PECs themselves are immunogenic or not, and this issue certainly must more carefully and systematically be addressed [329,330]. We are currently probing the general applicability of our experimental strategy to more sensitive biomacromolecules such as hASNase1 and hASNase3. Notably, we embarked on encapsulation of hASNase 3 and the different mutants we recently isolated and could replace enzymes of nonhuman origin. 


\section{Appendix}

Part 1. To test whether free glycine or other, structurally related, amino acids would accelerate autoproteolytic processing of Ntn-hydrolases other than hASNase3, we cloned, recombinantly expressed in E.coli and purified the human Taspase1, which was incubated with various compounds as shown in Figure1.

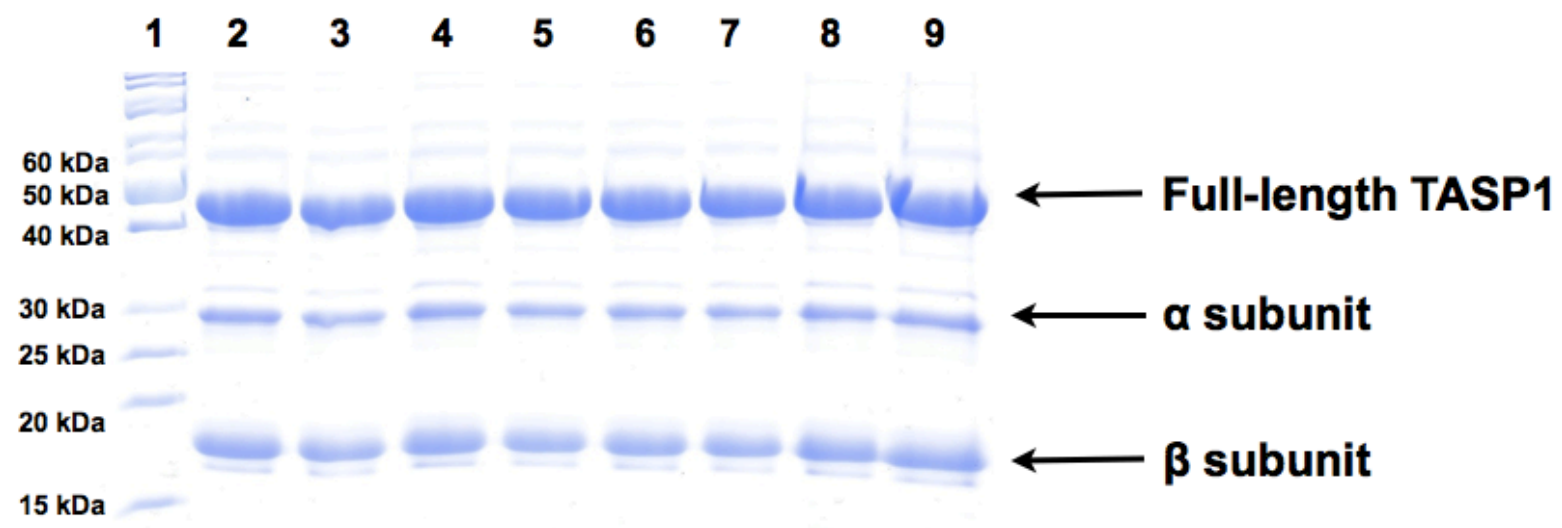

Figure 1. Incubation of Taspase1 with different concentrations of glycine, serine, alanine and threonine. Lane 1: molecular mass markers; Lane 2: control (bacterially produced and purified human Tasp1, without any compound added); Lane 3: $50 \mathrm{mM}$ glycine; Lane 4: 100 $\mathrm{mM}$ glycine; Lane 5: $200 \mathrm{mM}$ glycine; Lane 6: $500 \mathrm{mM}$ glycine; Lane 7: $500 \mathrm{mM}$ serine; Lane 8: $500 \mathrm{mM}$ alanine; Lane 9: $500 \mathrm{mM}$ threonine. The cloning of Tasp1 ORF (cDNA obtained from Source Bioscience, UK) was done similar to the cloning of hASNase3 as described in the Methods section (5.1.2.1). The expression was performed using the C41 (DE3) E.coli strain overnight at $16{ }^{\circ} \mathrm{C}$ with $0.5 \mathrm{mM}$ IPTG final concentration. The expression yield was $\sim 2 \mathrm{mg}$ of purified enzyme per $1 \mathrm{~L} 2 \mathrm{xYT}$ medium. The cells were lysed, the mixture was centrifuged, and the soluble fraction of the cellular extract was subjected to NiNTA affinity chromatography followed by size-exclusion chromatography. The final enzyme preparation was concentrated at a final concentration of $5 \mathrm{mg} / \mathrm{mL}$, mixed with $30 \%$ glycerol (final concentration), and stored at $-20{ }^{\circ} \mathrm{C}$. Aliquots of this stock were mixed with different concentrations of glycine, serine, alanine and threonine, and were incubated at $37{ }^{\circ} \mathrm{C}$ for $24 \mathrm{~h}$. Subsequently, the samples were analyzed by SDS-PAGE in order to evaluate the state of the intramolecular processing of the enzyme. None of the amino acids had an effect on the enzyme's autoactivation as evidenced by the fact that all treatments showed the same proteolytic cleavage profile as the control sample. The three black arrows show the fulllength Tasp1 precursor,and the generated $\alpha$ and $\beta$ subunits, respectively. In addition, the partially activated Tasp1 was tested for L-Asn hydrolysis, though with no detectable LASNase activity. 
Part 2. Since the physiological role of hASNase 3 is largely unknown, we analyzed its endogenous status of proteolytic processing in the natural cellular environment using polyclonal antibodies and cell extracts of two breast cancer cell lines, namely MCF-7 and SKBR-3, as shown in Figure 2.

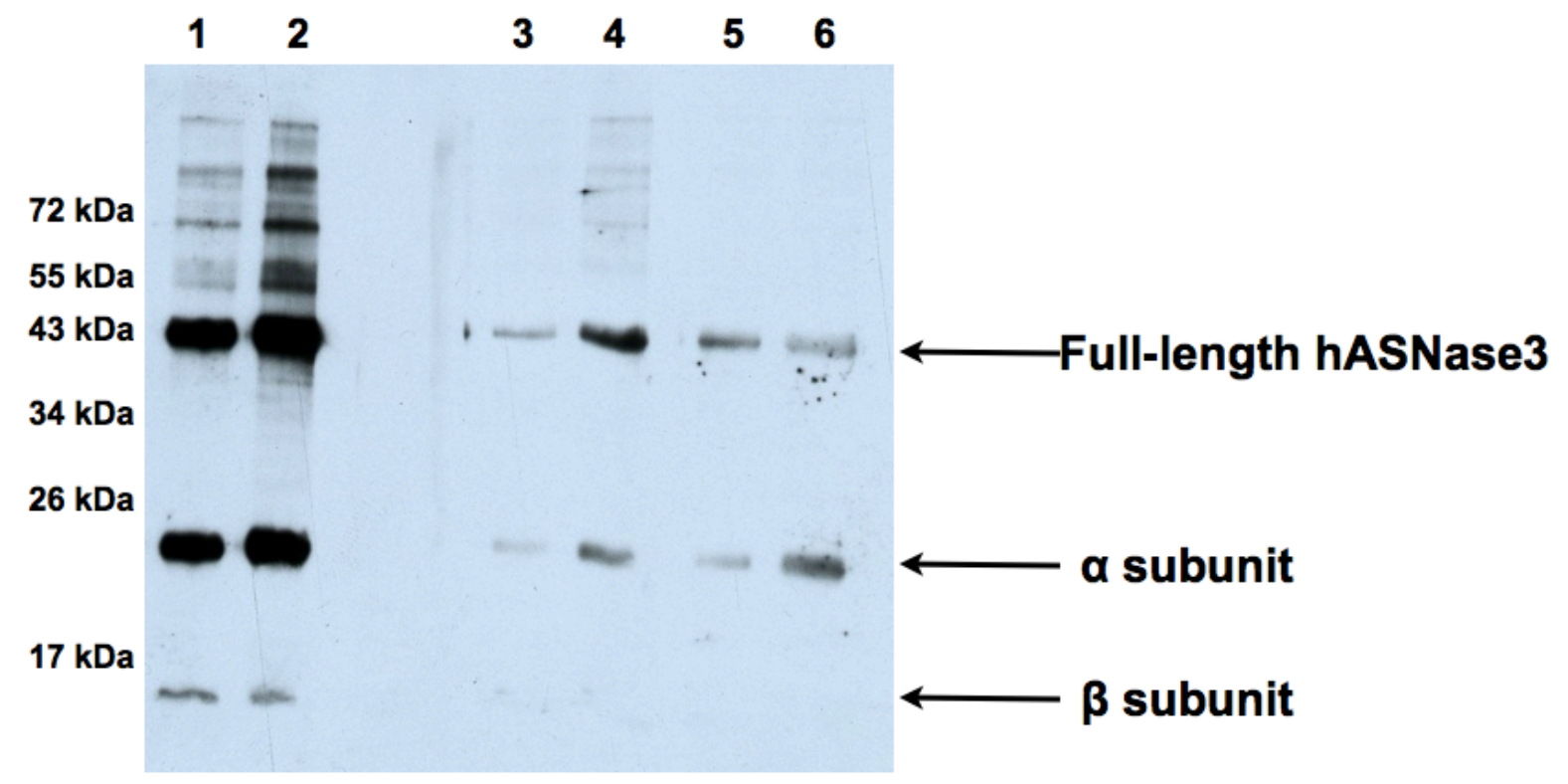

Figure 2. Immunoblot analysis of endogenous hASNase3 using cell extracts from MCF-7 and SKBR-3 breast cancer cell lines. Lane 1: control (50 ng purified recombinant, partially cleaved hASNase3); Lane 2: control (100 ng purified recombinant hASNase3); Lane 3: $1 \mu \mathrm{g}$ MCF-7 cell extract; Lane 4: $5 \mu \mathrm{g}$ MCF-7 cell extract; Lane 5: $1 \mu \mathrm{g}$ SKBR-3 cell extract and lane 6: $5 \mu \mathrm{g}$ SKBR-3 cell extract. Immunoblot analysis was performed as described in Chapter 3. The dilution of the primary polyclonal anti-hASNase 3 antibodies (generated using the full-length protein for immunization) was 1:5,000, and that of the secondary HRPconjugated anti-rabbit antibody was 1:10,000. The extracts of the cell lines were a kind gift from Dr. Theresa McSorley. The three black arrows show the full-length hASNase3 precursor, and the $\alpha$ and $\beta$ subunits, respectively. The $\beta$ subunit is not recognized as efficiently as the $\alpha$ subunit by the polyclonal antibodies, though Ponceau staining revealed an estimated 1:1 molar ratio for both subunits (see Chapter 3). 
Part 3. Human L-ASNase1 that was studied in this work (Chapter 6) resides in the Nterminal domain of the $60 \mathrm{kDa}$ Lysophospholipase protein to which the following lipase activities have been assigned: lysophospholipase and platelet-activating factor acetylhydrolase. We wanted to test whether the N-terminal truncated domain we worked with holds such activities. Therefore, we applied a sensitive fluorescent assay to detect potential lysophospholipase activity associated with the hASNase1 domain. The following four Figures show the assay standardization using as positive control the enzyme A2 Phospholipase, a titration binding curve generated by one of the expected products of the lipase hydrolytic activity (palmitic acid), and the plots obtained after incubating hASNase1 with lysophospholipids provided as mixtures of fatty acids.

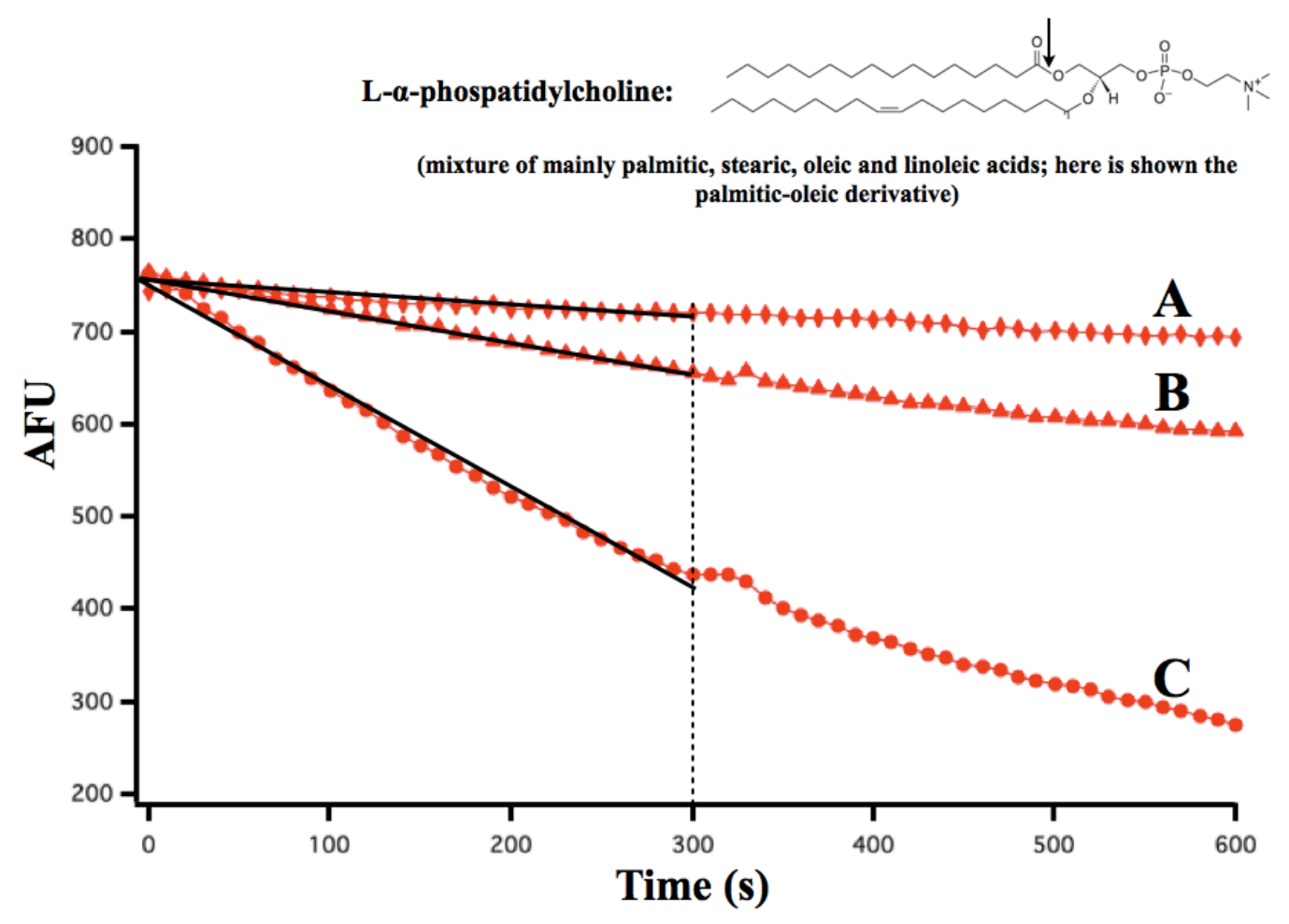

Figure 3. Standardization of the ADIFAB fluorescent assay using phospholipase A2 as positive control and L- $\alpha$-phosphatidylcholine as substrate (provided as a mixture of different fatty acid derivatives). This assay detects free fatty acid molecules which are released upon hydrolysis of lysophospholipids by lysophospholipases, or hydrolysis of phospholipids by phospholipases. The free fatty acid then is complexed with the intestinal fatty acid-binding protein conjugated with the fluorescent probe acrylodan, commercially known as a free fatty acid indicator and abbreviated as ADIFAB. Free fatty acid binding to ADIFAB induces a fluorescence signal decrease due to changes of the position of the acrylodan fluorophore, which is located close to the free fatty acid binding pocket of intestinal fatty acid-binding protein (FABP) [57]. (A): background reaction (assay buffer, 1 $\mu \mathrm{M}$ ADIFAB and $7 \mu \mathrm{g}$ phospholipase $\mathrm{A} 2$ without substrate), fitting parameters of the $0-300 \mathrm{~s}$ curve: $y=745-0.088 x, R^{2}=0.95$; (B): assay buffer, $1 \mu \mathrm{M}$ ADIFAB, $7 \mu \mathrm{g}$ phospholipase $\mathrm{A} 2$ and $100 \mu \mathrm{M}$ L- $\alpha$-phosphatidylcholine, $\mathrm{y}=750-0.3 \mathrm{x}, \mathrm{R}^{2}=0.97$ and $(\mathrm{C})$ : assay buffer, $1 \mu \mathrm{M}$ $\mathrm{ADIFAB}, 15 \mu \mathrm{g}$ phospholipase $\mathrm{A} 2$ and $100 \mu \mathrm{M}$ L- $\alpha$-phosphatidylcholine, $\mathrm{y}=752-0.92 \mathrm{x}$, $\mathrm{R}^{2}=0.93$. The assay buffer was $50 \mathrm{mM}$ HEPES, $140 \mathrm{mM} \mathrm{NaCl}, 5 \mathrm{mM} \mathrm{KCl}, 1 \mathrm{mM} \mathrm{Na} 2 \mathrm{HPO}_{4}$, 
$\mathrm{pH}$ 7.5. The final volume of the assay mixture was $200 \mu \mathrm{L}$, and the fluorescence intensity was recorded continuously for $10 \mathrm{~min}$ at $25{ }^{\circ} \mathrm{C}$ using a Jasco FP 8300 spectrofluorometer in the high sensitivity mode (excitation at $386 \mathrm{~nm}$ and emission at $432 \mathrm{~nm}$ with $2.5 \mathrm{~nm}$ bandwidths). The figure also shows the general structure of L- $\alpha$-phosphatidylcholine which is commercially available as a mixture of different fatty acids. The black arrow in the chemical structure indicates the point where the enzymatic hydrolysis of the ester bond takes place resulting in the liberation of the fatty acid. Plot was generated by Igor Pro (Wavemetrics).

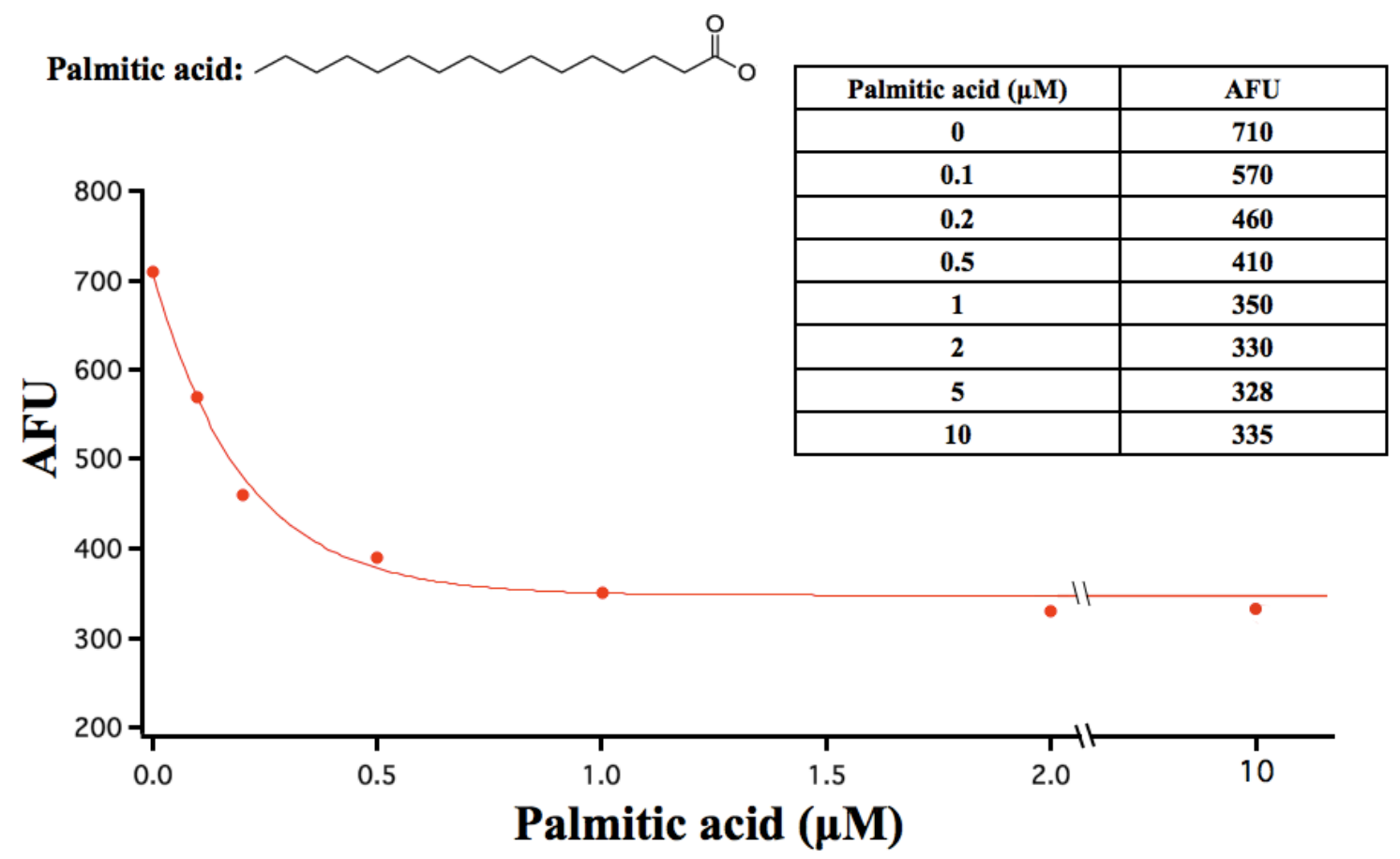

Figure 4. Standardization of the ADIFAB fluorescent assay using free palmitic acid. Different concentrations of palmitic acid (dissolved initially in ethanol) as shown in the table in the figure were titrated against $1 \mu \mathrm{M}$ ADIFAB (final concentration) using the buffer conditions described in Figure 3 above. The samples were placed at $25{ }^{\circ} \mathrm{C}$ for $10 \mathrm{~min}$ to equilibrate, and subsequently the fluorescence intensities were recorded using the same settings as those described in Figure 3. From the resulting curve, a $\mathrm{K}_{\mathrm{d}} \sim 0.16 \mu \mathrm{M}$ was calculated $\left(\mathrm{R}^{2}=0.956\right)$. 


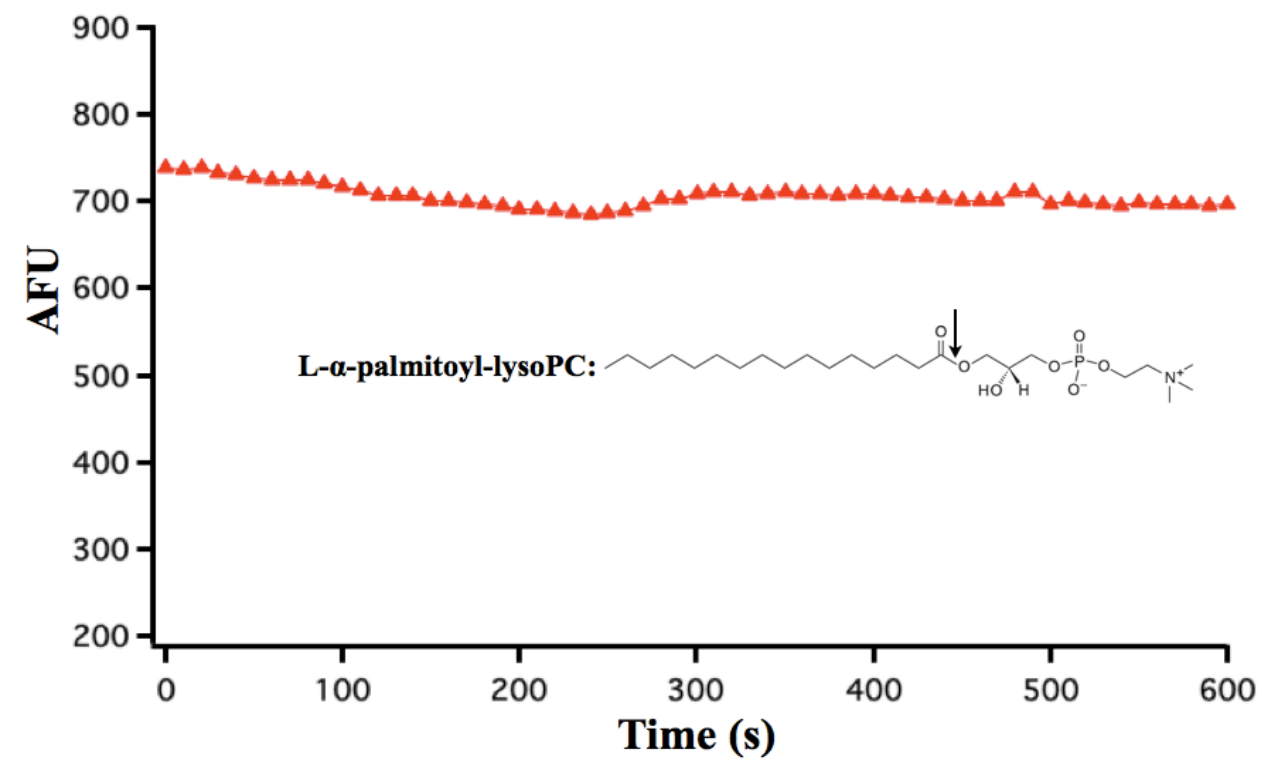

Figure 5. Test of L-a-palmitoyl-lysophosphatidylcholine hydrolysis by hASNase1 applying the ADIFAB assay. The assay was performed as described in the previous figures using $1 \mu \mathrm{M}$ ADIFAB, $100 \mu \mathrm{M}$ L- $\alpha$-palmitoyl-lysoPC, and $1.8 \mu \mathrm{M}$ hASNase1 (15 $\mu \mathrm{g}$ in 200 $\mu \mathrm{L}$ assay solution) as final concentrations.

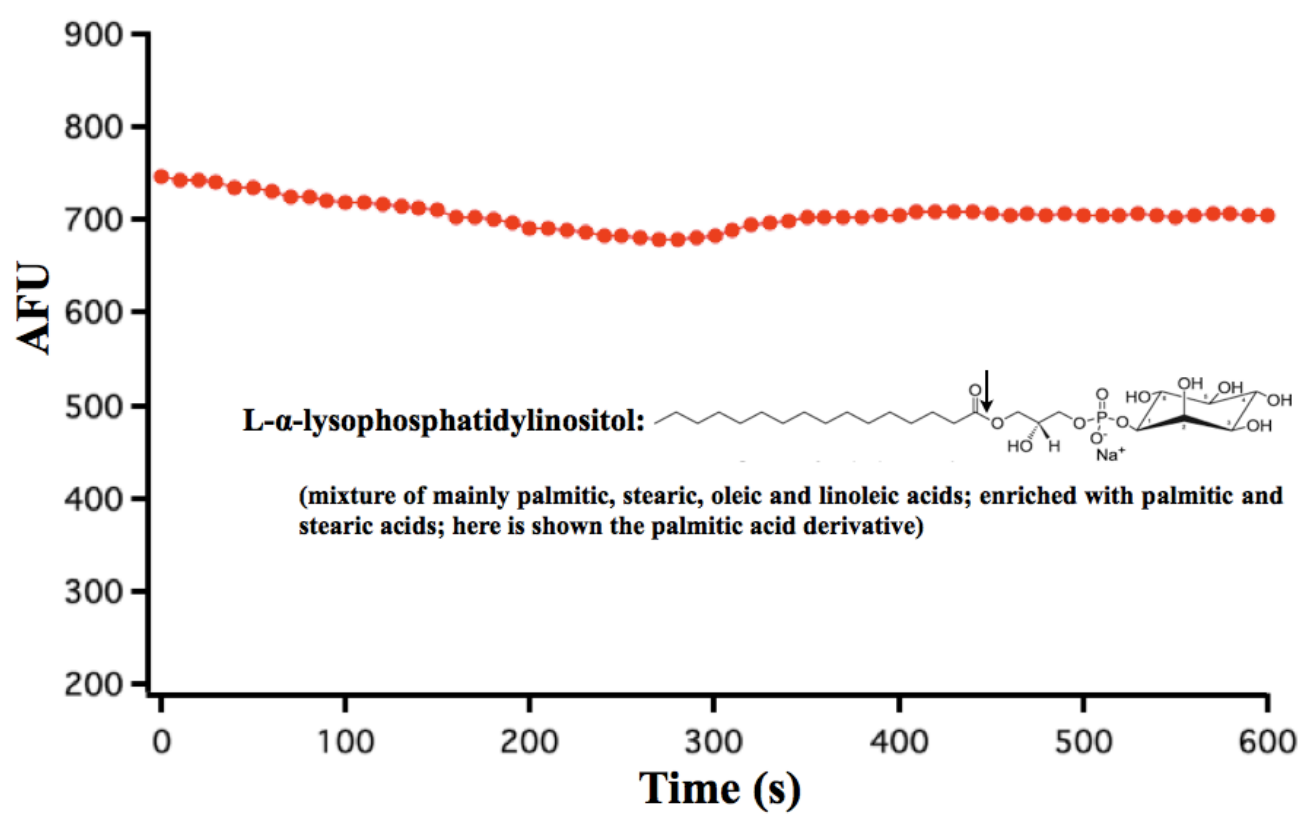

Figure 6. Test of L- $\alpha$-lysophosphatidylinositol hydrolysis by hASNase1 applying the ADIFAB assay. The assay was performed as described above using $1 \mu \mathrm{M}$ ADIFAB, $100 \mu \mathrm{M}$ L- $\alpha$-lysophosphatidylinositol, and $1.8 \mu \mathrm{M}$ hASNase 1 as final concentrations. 


\section{Bibliography}

1. Brown, H. D., Chattopadhyay, S. K., and Matthews, W.S. (1970) L-Asparaginase activity in several mammalian species. Int. J. Biochem. 1, 357-360

2. Willis, R. C., and Woolfolk, C. A. (1975) L-Asparagine uptake in Escherichia coli. J. Bacteriol. 123, 937-945

3. Credali, A., García-Calderon, M., Dam, S., Perry, J., Díaz-Quintana, A., Parniske, M., Wang, T. L., Stougaard, J., Vega, J. M., and Marquez, A. J. (2013) The $\mathrm{K}^{+}$-dependent asparaginase, NSE1, is crucial for plant growth and seed production in Lotus japonicus. Plant Cell Physiol. 54, 107-118

4. Michalska, K., Bujacz, G., and Jaskolski, M. (2006) Crystal structure of plant asparaginase. J. Mol. Biol. 360, 105-116

5. Borek, D., Michalska, K., Brzezinski, K., Kisiel, A., Podkowinski, J., Bonthron, D. T., Krowarsch, D., Otlewski, J., and Jaskolski M. (2004) Expression, purification and catalytic activity of Lupinus luteus asparagine $\beta$-amidohydrolase and its Escherichia coli homolog. Eur. J. Biochem. 271, 3215-3226

6. Cedar, H. and Schwartz, J. H. (1967) Localization of the two L-asparaginases in anaerobically grown Escherichia coli. J. Biol. Chem. 242, 3753-3755

7. Schwartz, J. H., Reeves, J. Y. and Broome, J. D. (1966) Two L-asparaginases from E.coli and their action against tumors. Proc. Natl Acad. Sci. USA, 56, 1516-1519

8. Pui, C. H. and Evans, W. E. (1998) Acute lymphoblastic leukemia. New Engl. J. Med. 339, 605-615

9. Pui, C. H., Campana, D. and Evans, W. E. (2001) Childhood acute lymphoblastic leukaemia- current status and future perspectives. The Lancet Oncology, 2, 597-607

10. Asselin, B. L., Ryan, D., Frantz, C. N., Bernal, S. D., Leavitt, P., Sallan, S. E., and Cohen, H. J. (1989) In vitro and in vivo killing of acute lymphoblastic leukemia cells by Lasparaginase. Cancer Res. 49, 4363-4368

11. Palm, G. J., Lubkowski, J., Derst, C., Schleper, S., Rohm, K. H., Wlodawer, A. (1996) A covalently bound catalytic intermediate in Escherichia coli asparaginase: crystal structure of a Thr-89-Val mutant. FEBS Lett. 390, 211-216

12. Jerlstrom, P. G., Bezjak, D. A., Jennings, M. P. and Beacham, I. R. (1989) Structure and expression in Escherichia coli $\mathrm{K}-12$ of the L-asparaginase I-encoding ansA gene and its flanking regions. Gene 78, 37-46

13. Swain, A. L., Jaskolski, M., Housset, D., Rao, J. K., and Wlodawer, A (1993) Crystal structure of Escherichia coli L-asparaginase, an enzyme used in cancer therapy. Proc. Natl Acad. Sci. USA 90, 1474-1478

14. Sodek, L., Lea, P. J., and Miflin, B. J. (1980) Distribution and properties of a potassiumdependent asparaginase isolated from developing seeds of Pisum sativum and other plants. Plant Physiol. 65, 22-26.

15. Michalska, K., and Jaskolski, M. (2006) Structural aspects of L-asparaginases, their friends and relations. Acta Biochim. Pol. 53, 627-640

16. Smith, J. L., Zaluzec, E. J., Wery, J. P., Niu, L., Switzer, R. L., Zalkin, H., and Satow, Y. (1994) Structure of the allosteric regulatory enzyme of purine biosynthesis. Science 264, 1427-1433

17. Oinonen, C. and Rouvinen, J. (2000) Structural comparison of Ntn-hydrolases. Protein Sci. 9, 2329-2337 
18. Brannigan, J. A., Dodson, G., Duggleby, H. J., Moody, P. C., Smith, J. L., Tomchick, D. R., and Murzin, A. G. (1995) A protein catalytic framework with an N-terminal nucleophile is capable of self-activation. Nature 378, 416-419

19. Oinonen, C., Tikkanen, R., Rouvinen, J., and Peltonen, L. (1995) Three-dimensional structure of human lysosomal aspartylglucosaminidase. Nat. Struct. Biol. 2, 1102-1108

20. Duggleby, H. J., Tolley, S. P., Hill, C. P., Dodson, E. J., Dodson, G., and Moody, P. C. E. (1995) Penicillin acylase has a single-amino-acid catalytic center. Nature 373, 264-268

21. Khan, J. A., Dunn, B. M., and Tong, L. (2005) Crystal structure of human Taspase1, a crucial protease regulating the function of MLL. Structure 13, 1443-1452

22. Borek, D., Jaskolski, M. (2001) Sequence analysis of enzymes with asparaginase activity. Acta Biochim Polon 48, 893-902

23. Dodson, G., Wlodawer, A. (1998) Catalytic triads and their relatives. Trends Biochem. Sci. 23, 347-352

24. Galperin, M. Y., and Koonin, E. V. (2012) Divergence and convergence in enzyme evolution. J. Biol. Chem. 287, 21-28

25. Guo, H. C., Xu, Q., Buckley, D., and Guan, C. (1998) Crystal structures of Flavobacterium glycosylasparaginase. An N-terminal nucleophile hydrolase activated by intramolecular proteolysis. J. Biol. Chem. 273, 20205-20212

26. Michalska, K., Brzezinski, K., and Jaskolski, M. (2005) Crystal structure of isoaspartyl aminopeptidase in complex with L-aspartate. J. Biol. Chem. 280, 28484-28491

27. Almonte, A. G., and Sweatt, J. D. (2011) Serine proteases, serine protease inhibitors, and protease-activated receptors: roles in synaptic function and behavior. Brain Res. 1407, 107-122

28. Michalska, K., Hernandez-Santoyo, A., and Jaskolski, M. (2008) The mechanism of autocatalytic activation of plant-type L-asparaginases. J. Biol. Chem. 283, 13388-13397

29. McGovern, M. M., Aula, P., and Desnick, J. R. (1983) Purification and properties of human hepatic aspartylglucosaminidase. J. Biol. Chem. 258, 10743-10747

30. Jackson, M., Clayton, P., Grunewald, S., Keir, G., Mills, K., Mills, P., Winchester, B., Worthington, V., and Young, E. (2005) Elevation of plasm aspartylglucosaminidase is a useful marker for the congenital disorders of glycosylation type I (CDG I). J. Inherit Metab. Dis. 28, 1197-1198

31. Saito, S., Ohno, K., Sugawara, K., Suzuki, T., Togawa, T., and Sakuraba, H. (2008) Structural basis of aspartylglucosaminuria. Biochem. Biophys. Res. Commun. 377, 11681172 .

32. Cantor, J. R., Stone, E. M., Chantranupong, L., and Georgiou, G. (2009) The human asparaginase-like protein $1 \mathrm{hASRGL1}$ is an Ntn hydrolase with $\beta$-aspartyl peptidase activity. Biochemistry 48, $11026-11031$

33. Dieterich, D. C., Landwehr, M., Reissner, C., Smalla, K. H., Richter, K., Wolf, G., Bockers, T. M., Gundelfinger, E. D., and Kreutz, M. R. (2003) Gliap: a novel untypical Lasparaginase localized to rat brain astrocytes. J. Neurochem. 85, 1117-1125

34. Weidle, U. H., Evtimova, V., Alberti, S., Guerra, E., Fersis, N., and Kaul, S. (2009) Cell growth stimulation by CRASH, an asparaginase-like protein overexpressed in human tumors and metastatic breast cancers. Anticancer Res. 29, 951-963

35. Borek, D., and Jaskolski, M. (2000) Crystallization and preliminary crystallographic studies of a new L-asparaginase encoded by Escherichia coli genome. Acta Cryst. D. 56, 1505-1507 
36. Nomme, J., Su, Y., Konrad, M., and Lavie,A. (2012) Structures of apo and product-bound human L-asparaginase: insights into the mechanism of auto-proteolysis and substrate hydrolysis. Biochemistry 51, 6816-6826

37. Li, W., Cantor, J. R., Yogesha, S. D., Yang, S., Chantranupong, L., Liu, J. Q., Agnello, G., Georgiou, G., Stone, E. M., and Zhang, Y. (2012) Uncoupling intramolecular processing and substrate hydrolysis in the N-terminal nucleophile hydrolase hASRGL1 by circular permutation. ACS Chem. Biol. 7, $1840-1847$

38. Su, Y., Karamitros, C. S., Nomme, J., McSorley, T., Konrad, M., and Lavie, A. (2013) Free glycine accelerates the autoproteolytic activation of human asparaginase. Chem. Biol. 20, 533-540

39. Aswad, D. W., Paranandi, M. V., and Schurter, B. T. (2000) Iso-aspartate in peptides and proteins: Formation, significance, and analysis. J. Pharm. Biomed. Anal. 21, 1129-1136.

40. Marti-Arbona, R., Fresquet, V., Thoden, J. B., Davis, M. L., Holden, H. M., and Raushel, F. M. (2005) Mechanism of the reaction catalyzed by isoaspartyl dipeptidase from Escherichia coli. Biochemistry 44, 7115-7124

41. Larkin, M. A., Blackshields, G., Brown, N. P., Chenna, R., McGettigan, P. A., McWilliam, H., Valentin, F., Wallace, I. M., Wilm, A., Lopez, R., Thompson, J. D., Gibson, T. J., and Higgins, D. G. (2007) Clustal W and Clustal X version 2.0. Bioinformatics 23, 2947-2948

42. Waterhouse, A. M., Procter, J. B., Martin, D. M., Clamp, M., and Barton, G. J. (2009) Jalview Version 2: a multiple sequence alignment editor and analysis workbench. Bioinformatics 25, $1189-1191$

43. DeLano, D. W. (2002) The PyMOL Molecular Graphics System, Schrödinger, LLC New York

44. Jaskolski, M., Kozak, M., Lubkowski, J., Palm, G., and Wlodawer, A. (2001) Structures of two highly homologous bacterial L-asparaginases : a case of enantiomorphic space groups. Acta Crystallogr. D. Biol. Crystallogr. 57, 369-377

45. Lubkowski, J., Palm, G. J., Gilliland, G. L., Derst, C., Rohm, K. H. and Wlodawer, A. (1996) Crystal structure and amino acid sequence of Wolinella succinogenes Lasparaginase. Eur. J. Biochem. 241, 201-207

46. Miller, M., Rao, J. K., Wlodawer, A. and Gribskov, M. R. (1993) A left-handed crossover involved in amidohydrolase catalysis. Crystal structure of Erwinia chrysanthemi Lasparaginase with bound L-aspartate. FEBS Letters 328, 275-279

47. Lubkowski, J., Wlodawer, A., Ammon, H. L., Copeland, T. D. and Swain, A. L. (1994) Structural characterization of Pseudomonas $7 \mathrm{~A}$ glutaminase-asparaginase. Biochemistry 33, 10257-10265

48. Harms, E., Wehner, A., Aung, H. P. and Rohm, K. H. (1991) A catalytic role for threonine-12 of E.coli asparaginase II as established by site-directed mutagenesis. FEBS Letters 285, 55-58

49. Wehner, A., Harms, E., Jennings, M. P., Beacham, I. R., Derst, C., Bast, P. and Rohm, K. H. (1992) Site-specific mutagenesis of Escherichia coli asparaginase II. None of the three histidine residues is required for catalysis. Eur. J. Biochem. 208, 475-480

50. Ehrman, M., Cedar, H. and Schwartz, J. H. (1971) L-Asparaginase II of Escherichia coli. Studies on the enzymatic mechanism of action. J. Biol. Chem. 246, 88-94

51. Yun, M. K., Nourse, A., White, S. W., Rock, C. O., and Heath R. J. (2007) Crystal structure and allosteric regulation of the cytoplasmic Escherichia coli L-asparaginase I. J. Mol. Biol. 369, $794-811$ 
52. Huang, Z., Mou, L., Shen, Q., Lu, S., Li, C., Liu, X., Wang, G., Li, S., Geng, L., Liu, Y., Wu, J., Chen, G., and Zhang, J. (2014) ASD v2.0: updated content and novel features focusing on allosteric regulation. Nucleic Acids Res. 42, D510-D516

53. Yao, M., Yasutake, Y., Morita, H. and Tanaka, I. (2005). Structure of the type I Lasparaginase from the hyperthermophilic archaeon Pyrococcus horikoshii at $2.16 \AA$ resolution. Acta Crystallog. sect. D 61, 294-301

54. Sinclair, K., Warner, J. P., and Bonthron, D. T. (1994) The ASP1 gene of Saccharomyces cerevisiae, encoding the intracellular isozyme of L-asparaginase. Gene 144, 37-43

55. Sugimoto, H., Odani, S., and Yamashita, S. (1998) Cloning and expression of cDNA encoding rat liver $60-\mathrm{kDa}$ lysophospholipase containing an asparaginase-like region and ankyrin repeat. J. Biol. Chem. 273, $12536-12542$

56. Menniti, M., Iuliano, R., Foller, M., Sopjani, M., Alesutan, I., Mariggio, S., Nofziger, C., Perri, A. M., Amato, R., Blazer-Yost, B., Corda, D., Lang, F., and Perrotti, N. (2010) 60 $\mathrm{kDa}$ lysophospholipase, a new Sgk1 molecular partner involved in the regulation of ENaC. Cell Physiol. Biochem. 26, 587-596

57. Karamitros, C. S., and Konrad, M. (2014) Human 60-kDa lysophospholipase contains an $\mathrm{N}$-terminal L-asparaginase domain that is allosterically regulated by L-asparagine. J. Biol. Chem. 289, 12962-12975

58. du Plessis, D. J., Nouwen, N., and Driessen, A. J. (2011) The Sec translocase. Biochim. Biophys. Acta 1808, 851-865

59. Willis, R. C. and Woolfolk, C. A. (1974) Asparagine utilization in Escherichia coli. J. Bacteriol. 118, 231-241.

60. Derst, C., Henseling, J. and Rohm, K. H. (2000) Engineering the substrate specificity of Escherichia coli asparaginase. II. Selective reduction of glutaminase activity by amino acid replacements at position 248. Protein Sci. 9, 2009-2017.

61. Kim, K. W., Kamerud, J. Q., Livingston, D. M., and Room, R. J. (1988) Asparaginase II of Saccharomyces cerevisiae. Characterization of the ASP3 gene. J. Biol. Chem. 263, 11948-11953

62. League, G. P., Slot, J. C., and Rokas, A. (2012) The ASP3 locus in Saccharomyces cerevisiae originated by horizontal gene transfer from Wickerhamomyces. FEMS Yeast Res. 12, 859-863

63. Asselin, B. L., Ryan, D., Frantz, C. N., Bernal, S. D., Leavitt, P., Sallan, S. E., and Cohen, H. J. (1989) In vitro and in vivo killing of acute lymphoblastic leukemia cells by Lasparaginase. Cancer Res. 49, 4363- 4368

64. Pui, C. H. \& Evans, W. E. (1998) Acute lymphoblastic leukemia. New Engl. J. Med. 339, 605-615

65. Stecher, A. L., de Deus, P. M., Polikarpov, I., and Abrahão-Neto, J. (1999) Stability of Lasparaginase: an enzyme used in leukemia treatment. Pharm. Acta Helv. 74, 1-9

66. Duval, M., Suciu, S., Ferster, A., Rialland, X., Nelken, B., Lutz, P., Benoit, Y., Robert, A., Manel, A. M., Vilmer, E., Otten, J., and Philippe, N. (2002) Comparison of Escherichia coli-asparaginase with Erwinia-asparaginase in the treatment of childhood lymphoid malignancies: results of a randomized European Organization for Research and Treatment of Cancer- Children's Leukemia Group phase 3 trial. Blood 99, 2734 -2739

67. Keating, G. M. (2013) Asparaginase Erwinia chrysanthemi (Erwinaze $\left.{ }^{R}\right)$ : A guide to its use in Acute Lymphoblastic Leukemia in the USA. BioDrugs 27, 413-418

68. Kotzia, G. A. and Labrou, N. E. (2005) L-asparaginase from Erwinia chrysanthemi 3937: Cloning, expression and characterization. J. Biotechnol. 127, 657-669 
69. Aghaiypour, K., Wlodawer, A., and Lubkowski, J. (2001) Structural basis for the activity and substrate specificity of Erwinia chrysanthemi L-asparaginase. Biochemistry 40, 5655-5664

70. Avramis, V. I., and Panosyan, E. H. (2005) Pharmacokinetic/pharmacodynamic relationships of asparaginase formulations: the past, the present and recommendations for the future. Clin. Pharmacokinet. 44, 367-393

71.Vitale, A., Guarini, A., Chiaretti, S., Foa, R. (2006) The changing scene of adult acute lymphoblastic leukemia. Curr. Opin. Oncol. 18, 652-59

72. Pui, C. H., Robison, L. L., and Look, T. A. (2008) Acute lymphoblastic leukemia. Lancet 371, 1030-1043

73. Pui, C. H., Campana, D., Evans, W. E. (2001) Childhood acute lymphoblastic leukaemia: current status and future perspectives. Lancet Oncol. 2, 597-607

74. Corbin, A. S., Agarwal, A., Loriaux, M., Cortes, J., Deininger, M. W., and Druker, B. J. (2011) Human chronic myeloid leukemia stem cells are insensitive to imatinib despite inhibition of BCR-ABL activity. J. Clin. Invest. 121, 396-409

75. Döhner, H., Estey, E. H., Amadori, S., Appelbaum, F. R., Büchner, T., Burnett, A. K., Dombret, H., Fenaux, P., Grimwade, D., Larson, R. A., Lo-Coco, F., Naoe, T., Niederwieser, D., Ossenkoppele, G. J., Sanz, M. A., Sierra, J., Tallman, M. S., Löwenberg, B., and Bloomfield, C. D. (2010) Diagnosis and management of acute myeloid leukemia in adults: recommendations from an international expert panel, on behalf of the European LeukemiaNet. Blood 115, 453-474

76. Juliusson G, Antunovic P, Derolf $\AA$, et al. (2009) Age and acute myeloid leukemia: real world data on decision to treat and outcomes from the Swedish Acute Leukemia Registry. Blood 113, 4179-4187

77. Avramis, V. I. (2012) Asparaginases: Biochemical pharmacology and modes of drug resistance. Anticancer Res. 32, 2423-2438

78. Weir, E. G., and Borowitz, M. J. (2001) Flow cytometry in the diagnosis of acute leukemia. Semin. Hematol. 38, 124-138

79. Teitel, M. A., and Pandolfi, P. P. (2009) Molecular genetics of acute lymphoblastic leukemia. Annu. Rev. Pathol. Mech. Dis. 4, 175-198

80. Mounira, A. G. (2006) Bloom syndrome, genomic instability and cancer: the SOS-like hypothesis. Cancer Lett. 236, 1-12

81. Pui, C. H., and Campana, D. (2000) New definition of remission in childhood acute lymphoblastic leukemia. Leukemia 14, 783-785

82. El-Chennawi, F. A., Al-Tonbary, Y. A., Mossad, Y. M., and Ahmed, M. A. (2008) Immune reconstitution during maintenance therapy in children with acute lymphoblastic leukemia, relation to co-existing infection. Hematology 13, 203-209

83. Kang, M. H., Kang, Y. H., Szymanska, B., Wilczynska-Kalak, U., Sheard, M. A., Harned, T. M., Lock, R. B., and Reynolds, C. P. (2007) Activity of vincristine, L-ASP, dexamethsone against acute lymphoblastic leukemia is enhanced by the BH3-mimetic ABT-737 in vitro and in vivo. Blood 110, 2057-2066

84. Garcia-Manero, G. and Kantarjian, H. M. (2000) The hyper-CVAD regimen in adult acute lymphocytic leukemia. Hematol. Oncol. Clin. North Am. 14, 1381-1396

85. Kobrinsky, N. L., Sposto, R., Shah, N. R., Anderson, J. R., DeLaat, C., Morse, M., Warkentin, P., Gilchrist, G. S., Cohen, M. D., Shina, D., and Meadows, A. T. (2001) Outcomes of treatment of children and adolescents with recurrent non-Hodgkin's lymphoma and Hodgkin's disease with dexamethasone, etoposide, cisplatin, cytarabine, 
and 1-asparaginase, maintenance chemotherapy, and transplantation: Children's Cancer Group Study CCG-5912. J. Clin. Oncol. 19, 2390-2396

86. Kidd, J. G. (1953) Regression of transplanted lymphomas induced in vivo by means of normal guinea pig serum. I. Course of transplanted cancers of various kinds in mice and rats given guinea pig serum, horse serum, or rabbit serum. J. Exp. Med. 98, 565-582

87. Broome, J. D. (1961) Evidence that the L-asparaginase activity of guinea pig serum is responsible for its antilymphoma effects. Nature 191, 1114-1115

88. Yellin, T.O., Wriston, J. C. Jr (1966) Antagonism of purified asparaginase from guinea pig serum toward lymphoma. Science 151, 998-999

89. Rytting, M. E. (2012) Role of L-asparaginase in acute lymphoblastic leukemia: focus on adult patients. Blood and Lymphatic Cancer: Targets and Therapy 2, 117-124

90. Holleman, A., den Boer, M.L., Kazemier, K.M., Janka-Schaub, G.E., and Pieters, R. (2003) Resistance to different classes of drugs is associated with impaired apoptosis in childhood acute lymphoblastic leukemia. Blood 102, 4541-4546

91. Appel, I.M., Kazemier, K. M., Boos, J., Lanvers, C., Veerman, A. J., van Wering, E., den Boer, M. L., and Pieters, R. (2008) Pharmacokinetic, pharmacodynamic and intracellular effects of PEG-asparaginase in newly diagnosed childhood acute lymphoblastic leukemia: results from a single agent window study. Leukemia 22, 1665-1679

92. Sen, S., and D'Incalci, M. (1992) Apoptosis: biochemical events and relevance to cancer chemotherapy. FEBS Lett. 307, 122-127

93. Emadi, A., Zokaee, H., and Sausville, E. A. (2014) Asparaginase in the treatment of nonALL hematologic malignacies. Cancer Chemother. Pharmacol. 73, 875-883

94. Reinert, R. B., Oberle, L. M., Wek, S. A., Bunpo, P., Wang, X. P., Mileva, I., Goodwin, L. O., Aldrich, C. J., Durden, D. L., McNurlan, M. A., Wek, R. C., and Anthony, T. G. (2006) Role of glutamine depletion in directing tissue-specific nutrient stress responses to Lasparaginase. J. Biol. Chem. 281, 31222-31233

95. Cantor, J. R., and Sabatini, D. M. (2012) Cancer cell metabolism: one hallmark, many faces. Cancer Discovery 2, 881-898

96. Kalhammer, R. and Sethuraman, N. (2008) New Directions in Tumor Therapy-Amino Acid Depletion with GlutaDON ${ }^{\circledR}$ as Treatment for Cancer, in Modern Biopharmaceuticals: Design, Development and Optimization (ed. J. Knäblein), Wiley-VCH Verlag GmbH, Weinheim, Germany. doi: 10.1002/9783527620982.ch21

97. Glazer, E. S., Stone, E. M., Zhu, C., Massey, K. L., Hamir, A. N., and Curley, S. A. (2011) Bioengineered human arginase I with enhanced activity and stability controls hepatocellular and pancreatic carcinoma xenografts. Transl. Oncol. 4, 138-146

98. Agrawal, V., Woo, J. H., Mauldin, J. P., Jo, C., Stone, E. M., Georgiou, G., and Frankel, A. E. (2012) Cytotoxicity of human recombinant arginase I (Co)-PEG5000 in the presence of supplemental L-citrulline is dependent on decreased argininosuccinate synthetase expression in human cells. Anticancer Drugs 23, 51-64

99. Covini, D., Tardito, S., Bussolati, O., Chiarelli, L. R., Pasquetto, M. V., Digilio, R., Valentini, G., and Scotti, C. (2012) Expanding targets for a metabolic therapy of cancer: 1asparaginase. Recent Pat. Anticancer Drug Discov. 7, 4-13

100. Müller, H. J., Beier, R., Löning, L., Blütters-Sawatzki, R., Dörffel, W., Maass, E., Müller-Weihrich, S., Scheel-Walter, H. G., Scherer, F., Stahnke, K., Schrappe, M., Horn, A., Lümkemann, K., and Boos, J (2001) Pharmacokinetics of native Escherichia coli asparaginase (Asparaginase medac) and hypersensitivity reactions in ALL-BFM 95 reinduction treatment. Br. J. Haematol. 114, 794-799 
101. Kearney, S. L., Dahlberg, S. E., Levy, D. E., Voss, S. D., Sallan, S. E., Silverman, L. B. (2009) Clinical course and outcome in children with acute lymphoblastic leukemia and asparaginase-associated pancreatitis. Pediatr Blood Cancer. 53, 162-167

102. Fu, C. H., Sakamoto, K. M. (2007) PEG-asparaginase. Expert Opin Pharmacother. 8, 1977-1984

103. Parsons, S. K., Skapek. S. X., Neufeld. E. J., Kuhlman, C., Young, M. L., Donnelly, M., Brunzell, J. D., Otvos, J.D., Sallan, S. E., and Rifai, N. (1997) Asparaginase-associated lipid abnormalities in children with acute lym- phoblastic leukemia. Blood 89, 1886-1895

104. Villa, P., Corada, M., and Bartosek, I. (1986) L-asparaginase effects on inhibition of protein synthesis and lowering of the glutamine content in cultured rat hepatocytes. Toxicol. Lett. 32, 235-241

105. Zalewska-Szewczyk, B., Andrzejewski, W., Mlynarski, W., Jedrychowska-Danska, K., Witas, H., and Bodalski, J. (2007) The anti-asparagines antibodies correlate with Lasparagines activity and may affect clinical outcome of childhood acute lymphoblastic leukemia. Leuk. Lymphoma 48, 931-936

106. Patel, N., Krishnan, S., Offman, M. N., Krol M., Moss, C. X., Leighton, C., van Delft, F. W., Holland, M., Liu, J. Z., Alexander, S., Dempsey, C., Ariffin, H., Essink, M., Eden, T. O. B., Watts, C., Bates, P. A., and Saha V. (2009) A dyad of lymphoblastic lysosomal cysteine proteases degrades the antileukemic drug 1-asparaginase. J. Clin. Invest. 119, 1964-1973

107. Asselin, B.L. (1999) The three asparaginases. Comparative pharmacology and optimal use in childhood leukemia. Adv. Exp. Med. Biol. 457, 621-629

108. Rizzari, C., Zucchetti, M., Conter. V., Diomede, L., Bruno, A., Gavazzi, L., Paganini, M., Sparano, P., Nigro, L. L., Arico, M., Milani, M., and D’Incalci, M. (2000) L-asparagine depletion and L-asparaginase activity in children with acute lymphoblastic leukemia receiving i.m. or i.v. Erwinia C. or E.coli L-asparaginase as first exposure. Ann Oncol. 11, 189-193

109. Jones, B., Holland, J. F., Glidewell, O., Jacquillat, C., Weil, M., Pochedly, C., Sinks, L., Chevalier, L., Maurer, H. M., Koch, K., Falkson, G., Patterson, R., Seligman, B., Sartorius, J., Kung, F., Haurani, F., Stuart, M., Burgert, E. O., Ruymann, F., Sawitsky, A., Forman, E., Pluess, H., Truman, J. and Hakami, N. (1977) Optimal use of 1-asparaginase (NSC-109229) in acute lymphocytic leukemia. Med. Pediatr. Oncol. 3, 387-400

110. Graham, M. L. (2003) Pegaspargase: a review of clinical studies. Adv. Drug Deliv. Rev. 55, 1293-1302

111. Panetta, J. C., Gajjar, A., Hijiya, N., Hak, L.J., Cheng, C., Liu, W., Pui, C. H., and Relling, M. V. (2009) Comparison of native E.coli and PEG asparaginase pharmacokinetics and pharmacodynamics in pediatric acute lymphoblastic leukemia. Clin. Pharmacol. Ther. 86, 651-658

112. Hawkins, D.S., Park, J. R., Thomson, B. G., Felgenhauer, J. L., Holcenberg, J. S., Panosyan, E. H., and Avramis, V. I. (2004) Asparaginase pharmacokinetics after intensive polyethylene glycol-conjugated 1-asparaginase therapy for children with relapsed acute lymphoblastic leukemia. Clin. Cancer Res. 10, 5335-5341

113. Jevsevar. S., Kunstelj, M., Porekar, V.G. (2010) PEGylation of therapeutic proteins. Biotechnol. J. 5, 113-128

114. Harding, F.A., Liu, A. D., Stickler, M., Razo, O. J., Chin, R., Faravashi, N., Viola, W., Graycar, T., Yeyng, V. P., Aehle, W., Meijer, D., Wong, S., Rashid, M. H., Valdes, A. M., Schellenberger, V. (2005) A beta-lactamase with reduced immunogenicity for the targeted 
delivery of chemotherapeutics using antibody-directed enzyme prodrug therapy. Mol Cancer Ther 4, 1791-1800

115. Yeung, V. P., Chang, J., Miller, J., Barnett, C., Stickler, M., and Harding F. A. (2004) Elimination of an immunodominant CD4+ $\mathrm{T}$ cell epitope in human IFN-beta does not result in an in vivo response directed at the subdominant epitope. $J$ Immunol. 172, 66586665

116. Van Regenmortel, M. H. (2009) What is a B-cell epitope? Methods in Mol. Biol. 524, $3-20$

117. Holgate, R. G., and Baker, M.P. (2009) Circumventing immunogenicity in the development of therapeutic antibodies. IDrugs 12, 233-237

118. Wang. P., Sidney, J., Courtney, D., Mothe, B., Sette, A., and Peters, B., (2008) A systematic assessment of MHC class II peptide binding predictions and evaluation of a consensus approach. PLoS Comput Biol 4, e1000048

119. Jones, E. Y., Fugger, L., Strominger, J. L., and Siebold, C. (2006) MHC class II proteins and disease: A structural perspective. Nat. Rev. Immunol. 6, 271-282

120. Benkovic, S.J., and Hammes-Schiffer, S. (2003) A perspective on enzyme catalysis. Science 301, 1196-1202

121. Warmerdam P. A., Plaisance, S., Vanderlick, K., Vandervoort, P., Brepoels, K., Collen, D., and De Maeyer, M. (2002) Elimination of a human T-cell region in staphylokinase by T-cell screening and computer modeling. Thromb. Haemostasis 87, 666-673

122. Tiwari, G., Tiwari, R., Sriwastawa, B., Bhati, L., Pandey, S., Pandey, P., and Bannerjee

S. K. (2012) Drug delivery systems: An updated review. Int. J. Pharm. Investig. 2, 2-11

123. Paolino, D., Sinha, P., Fresta, M. and Ferrari, M. (2006) Drug Delivery Systems. Encyclopedia of Medical Devices and Instrumentation

124. Tong, W., Song, X., and Gao, C. (2012) Layer-by-Layer assembly of microcapsules and their biomedical applications. Chem. Soc. Rev. 41, 6103-6124

125. Delcea, M., Yashchenok, A., Videnova, K., Kreft, O., Möhwald, H., and Skirtach, A. G. (2010) Multicompartmental micro- and nanocapsules: hierarchy and applications in biosciences. Macromol. Biosci. 10, 465-474

126. Skirtach, A. G, Karageorgiev, P., Bedard, M. F., Sukhorukov, G. B., and Möhwald, H. (2008) Reversibly permeable nanomembranes of polymeric microcapsules. J. Am. Chem. Soc. 130, 11572-11573

127. Vergaro, V., Scarlino, F., Bellomo, C., Rinaldi, R., Vergara, D., Maffia M., Baldassarre F., Giannelli, G., Zhangd, X., Lvovd, Y. M., and Leporatti S. (2011) Drug-loaded polyelectrolyte microcapsules for sustained targeting of cancer cells. Adv. Drug Deliv. Rev. 63, 847-864

128. De Jong, W. H., and Borm, P. J. A. (2008) Drug delivery and nanoparticles: applications and hazards. Int. J. Nanomed. 3, 133-149

129. Decher, G. (1997) Fuzzy nanoassemblies: toward layered polymeric multicomposites, Science 277, 1232-1237

130. Moghimi, S. M., Hunter, A. C., and Murray, J. C. (2005) Nanomedicine: current status and future prospects. FASEB J. 19, 311-330

131. Galbiati, A., Tabolacci, C., Morozzo Della Rocca, B., Mattioli, P., Beninati, S., Paradossi, G., Desideri, A. (2011) Targeting tumor cells through chitosan-folate modified microcapsules loaded with camptothecin. Bioconjugate Chem. 22, 1066-1072

132. Buckel, P. (1996) Recombinant proteins for therapy. Trends Pharmacol. Sci. 17, 450-456 
133. Katsuhiko, A., Qingmin, J., and Hill, J. P. (2010) Enzyme-encapsulated Layer-by-Layer assemblies: Current status and challenges toward ultimate nanodevices. Adv. Polym. Sci. 229, 51-87

134. Chen, R. (2001) Enzyme engineering: rational redesign versus directed evolution. Trends Biotechnol. 19, 13-14

135. Chen, R. (1999) A general strategy for enzyme engineering. Trends Biotechnol. 17, 344 345

136. Hurley, J. H. Chen, R., and Dean, A. M. (1996) Determinants of cofactor specificity in isocitrate dehydrogenase: structure of an engineered $\mathrm{NADP}^{+} \rightarrow \mathrm{NAD}^{+}$specificity-reversal mutant. Biochemistry 35, 5670-5678

137. Lutz. S. (2010) Beyond directed evolution--semi-rational protein engineering and design. Curr. Opin. Biotechnol. 21, 734-743

138. Kuchner, O. and Arnold, F. H. (1997) Directed evolution of enzyme catalysis. Trends Biotechnol. 15, 744-749

139. Romero, P. A. and Arnold, F. H. (2009) Exploring protein fitness landscapes by directed evolution. Nat. Rev. Mol. Cell Biol. 10, 866-876

140. Pritchard, L., Corne, D., Kell, D., Rowland, J., and Winson M. (2005) A general model of error-prone PCR. J. Theor. Biol. 234, 497-509

141. Bloom, J. D., Meyer, M. M., Meinhold, P., Otey, C. R., MacMillan, D., and Arnold F. H. (2005) Evolving strategies for enzyme engineering. Curr. Opin. Struct. Biol. 15, 447-452

142. Stemmer, W. P. (1994) Rapid evolution of a protein in vitro by DNA shuffling. Nature 370, 389-391

143. Griswold, K. E., Kawarasaki, Y., Ghoneim, N., Benkovic, S., Iverson, B. L. and Georgiou G. (2005) Evolution of highly active enzymes by homology-independent recombination. Proc. Natl. Acad. Sci. USA 102, 10082-10087

144. Bornscheuer, U. T. and Pohl, M. (2001) Improved biocatalysts by directed evolution and rational protein design. Curr. Opin. Chem. Biol. 5, 137-143

145. Farinas, E. T., Schwaneberg, U., Glieder, A., and Arnold, F. H. (2001) Directed evolution of a cytochrome P450 monooxygenase for alkane oxidation. Adv. Synth. Catal. 343, 601-606

146. Zaccolo, M., and Gherardi, E. (1999) The effect of high-frequency random mutagenesis on in vitro protein evolution: A study on TEM-1 $\beta$-Lactamase. J. Mol. Biol. 285, 775-783

147.Smith, J. M. (1970) Natural selection and the concept of a protein space. Nature 225, 563-564

148. Reetz, M. T., Kahakeaw, D., and Lohmer, R. (2008) Addressing the numbers problem in directed evolution. ChemBioChem 9, 1797-1804

149. Guo, H. H., Choe, J. and Loeb, L. A. (2004) Protein tolerance to random amino acid change. Proc. Natl Acad. Sci. USA 101, 9205-9210

150. Rennell, D., Bouvier, S. E., Hardy, L. W. and Poteete, A. R (1991) Systematic mutation of bacteriophage-T4 lysozyme. J. Mol. Biol. 222, 67-87

151. You, L. and Arnold, F. (1996) Directed evolution of subtilisin E in Bacillus subtilis to enhance total activity in aqueous dimethylformamide. Protein Eng. 9, 77-83

152. Varadarajan, N., Cantor, J. R., Georgiou, G., and Iverson, B. L. (2009) Construction and flow cytometric screening of targeted enzyme libraries. Nat. Protoc. 4, 893-901

153. Levin, I., and Aharoni, A. (2012) Evolution in microfluidic droplet. Chem. Biol. 19, 929-931 
154. Alvarez-Barrientos, A., Arroyo, J., Canton, R., Nombela, C., and Sanchez-Perez, M. (2000) Applications of flow cytometry to clinical microbiology. Clin. Microbiol. Rev. 13, 167-195

155. Harvey, B. R., Georgiou, G., Hayhurst, A., Jeong, K. J., Iverson, B. L., and Rogers, G. K. (2004) Anchored periplasmic expression, a versatile technology for the isolation of high-affinity antibodies from Escherichia coli-expressed libraries. Proc. Natl Acad. Sci. USA 101, 9193-9198

156. Chen, G., Hayhurst, A., Thomas, J. G., Harvey, B. R., Iverson, B. L. and Georgiou G. (2001) Isolation of high-affinity ligand-binding proteins by periplasmic expression with cytometric screening (PECS). Nat. Biotechnol. 19, 537-542

157. Ruff, A. J., Dennig, A., Wirtz, G., Blanusa, M., and Schwaneberg, U. (2012) Flow cytometer-based high-throughput screening system for accelerated directed evolution of P450 monooxygenases. ACS Catal. 2, 2724-2728

158. Dröge, M.J., Boersma, Y. L., van Pouderoyen, G., Vrenken, T. E., Rüggeberg C. J., Reetz, M. T., Dijkstra, B. W., and Quax, W. J. (2006) Directed evolution of Bacillus subtilis lipase A by use of enantiomeric phosphonate inhibitors: crystal structures and phage display selection. ChemBioChem. 7, 149-157

159. Jackson, W. C., Bennett, T. A., Edwards, B. S. Prossnitz, E., Lopez, G. P., and Sklar, L. A. (2002) Performance of in-line microfluidic mixers in laminar flow for high-throughput flow cytometry. Biotechniques 33, 220-226

160. He. L., Olson, D. P., Wu, X., Karpova, T. S., McNally, J. G., and Lipsky, P. E. (2003) A flow cytometric method to detect protein-protein interactions in living cells by directly visualizing donor fluorophore quenching during CFP-->YFP fluorescence resonance energy transfer (FRET). Cytometry A 55, 71-85

161. Lazebnik, Y. A., Poletaev, A. I., and Zenin V. V. (1992) Drop-delay measurement using enzyme-coated particles. Cytometry 13, 649-652

162. Ibrahim, S. F. and van den Engh, G. (2007) Flow cytometry and cell sorting. Adv. Biochem. Eng. Biotechnol. 106, 19-39

163. Plovins, A., Alvarez, A. M., Ibanez, M., Molina, M. \& Nombela, C. (1994) Use of fluorescein-di-B-d-galactopyranoside (Fdg) and C-12-Fdg as substrates for $\beta$-galactosidase detection by flow-cytometry in animal, bacterial, and yeast-cells. Appl. Environ. Microbiol. 60, 4638-4641

164. Johnsson, N. and Johnsson, K. (2007) Chemical tools for biomolecular imaging. ACS Chem. Biol. 2, 31-38

165. Shaner, N. C., Steinbach, P. A. and Tsien, R. Y. (2005) A guide to choosing fluorescent proteins. Nature Methods 2, 905-909

166. Georgiou, G. (2000) Analysis of large libraries of protein mutants using flow cytometry. Adv. Protein Chem. 55, 293-316

167. Zaslaver, A., Bren, A., Ronen, M., Itzkovitz, S., Kikoin, I., Shavit S., Liebermeister, W., Surette M. G., and Alon, U. (2006) A comprehensive library of fluorescent transcriptional reporters for Escherichia coli. Nature Methods 3, 623-628

168. Valdivia, R. H. and Falkow, S. (1997) Fluorescence-based isolation of bacterial genes expressed within host cells. Science 277, 2007-2011

169. Nguyen, A. W. and Daugherty, P. S. (2005) Evolutionary optimization of fluorescent proteins for intracellular FRET. Nature Biotechnol. 23, 355-360

170. Link, A. J., Jeong, K. J., and Georgiou, G. (2007) Beyond toothpicks: new methods for isolating mutant bacteria. Nat. Rev. Microbiol. 5, 680-688 
171. Eklund, B. I., Edalat, M., Stenberg, G., and Mannervik, B. (2002) Screening for recombinant glutathione transferases active with monochlorobimane Anal. Biochem. 309, 102-108

172. Schuster, S., Enzelberger, M., Trauthwein, H., Schmid, R. D. and Urlacher, V. B. (2005) pHluorin-based in vivo assay for hydrolase screening. Anal. Chem. 77, 2727-2732

173. Olsen, M. J., Stephens, D., Griffiths, D., Daugherty, P., Georgiou, G., and Iverson, B. L. (2000) Function-based isolation of novel enzymes from a large library. Nature Biotechnol. 18, 1071-1074

174. Santoro, S. W., Wang, L., Herberich, B., King, D. S. and Schultz, P. G. (2002) An efficient system for the evolution of amino-acyl-tRNA synthetase specificity. Nature Biotechnol. 20, 1044-1048

175. Wang, J. D., Herman, C., Tipton, K. A., Gross, C. A., and Weissman, J. S. (2002) Directed evolution of substrate-optimized GroEL/S chaperonins. Cell, 111 1027-1139

176. Santoro, S. W., and Schultz P. G. (2002) Directed evolution of the site specificity of Cre recombinase. Proc. Natl. Acad. Sci. USA 99, 4185- 4190

177. Link, A. J., Vink, M. K. S., Agard, N. J., Prescher, J. A., Bertozzi, C. R., and Tirrell, D. A. (2006) Discovery of aminoacyl-tRNA synthetase activity through cell-surface display of noncanonical amino acids. Proc. Natl Acad. Sci. USA 103, 10180-10185

178. Schuster, S., Enzelberger, M., Trauthwein, H., Schmid, R. D., Urlacher, V. B. (2005) pHluorin-based in vivo assay for hydrolase screening. Anal. Chem. 77, 2727-2732

179. Cantor, J. R., Yoo, T. H., Iverson, B. L., Forsthuber, T. G., and Georgiou G. (2011) Therapeutic enzyme deimmunization by combinatorial T-cell epitope removal using neutral drift. Proc. Natl Acad. Sci. USA 108, 1272-1277

180. Sackmann, E. K., Fulton, A. L., and Beebe D. J. (2014) The present and the future role of microfluidics in biomedical research. Nat. Review 507, 181-189

181. Theberge, A. B., Courtois, F., Chaerli, Y., Fischlechner, M., Abell, C., Hollfelder, F., and Huck, W. T. S. (2010) MIcrodroplets in Microfluidics: An evolving platform for discoveries in chemistry and biology. Angew. Chem Int. Ed. 49, 5846-5868

182. Teh, S. Y., Lin, R., Hung, L. H., Lee, A. P. (2008) Droplet microfluidics. Lab Chip 8, $198-220$

183. Cygan, Z. T., Cabral, J. T., Beers K. L. and Amis, E. J. (2005) Microfluidic platform for the generation of organic-phase microreactors. Langmuir 21, 3629-3634

184. Baroud, C. N., Gallaire, F., and Dangla, R. (2010) Dynamics of microfluidic droplets. Lab Chip 10, 2032-2045

185. Friend, J. and Yeo, L. (2010) Fabrication of microfluidic devices using polydimethylsiloxane. Biomicrofluidics 4, 026502

186. Chen, C. C., Hirdes, D., and Folch, A. (2003) Gray-scale photolithography using microfluidic photomasks. Proc. Natl Acad. Sci. USA 100, 1499-1504

187. Madou, M. J. (1997) Fundamentals of Microfabrication (CRC, Boca Raton, FL).

188. Choi, S., and Park, J. K. (2010) Two-step photolithography to fabricate multilevel microchannels. Biomicrofluidics 4, 046503

189. Thorsen, T., Roberts, R. W., Arnold, F. H., and Quake, S. R. (2001) Dynamic pattern formation in a vesicle-generating microfluidic device. Phys. Rev. Lett. 86, 4163-4166

190. Carbonell, C., Stylianou, K. C., Hernando, J., Evangelo, E., Barnett, S. A., Nettikadan, S., Imaz, I., and Maspoch, D. (2013) Femtolitre chemistry assisted by microfluidic pen lithography. Nat. Commun. 4, 2173

191. Baret, J. C. (2012) Surfactants in droplet-based microfluidics. Lab Chip 12, 422-433 
192. DeMello A. J. (2006) Control and detection of chemical reactions in microfluidic systems. Nature 442, 394-402

193. Mazutis, L., Gilbert, J., Ung, W. L., Weitz, D. A., Griffiths, A. D., and Heyman, J. A. (2013) Single-cell analysis and sorting using droplet-based microfluidics. Nat. Protoc. 8, 870-891

194. Seemann, R., Brinkmann, M., Pfohl, T., and Herminghaus, S. (2012) Droplet based microfluidics. Rep. Prog. Phys. 75, 016601

195. El Debs, B., Utharala, R., Balyasnikova, I. V., Griffiths, A. D., and Merten, C. A. (2012) Functional single-cell hybridoma screening using droplet-based microfluidics. Proc. Natl Acad. Sci. USA 109, 11570-11575

196. Craighead, H. (2006) Future lab-on-a-chip technologies for interrogating individual molecules. Nature 442, 387-393

197. Brouzes, E., Medkova, M., Savenelli, N., Marran, D., Twardowski, M., Hutchison, J. B., Rothberg, J. M., Link, D. R., Perrimon, N., and Samuels, M. L. (2009) Droplet microfluidic technology for single-cell high-throughput screening. Proc. Natl Acad. Sci. USA 106, 14195-14200

198. Kintses, B., Hein, C., Mohamed, M. F., Fischlechner, M., Courtois, F., Laine, C., and Hollfelder, F. (2012) Picoliter cell lysate assays in microfluidic droplet compartments for directed evolution. Chem. Biol. 19, 1001-1009

199. Wang, B. L., Ghaderi, A., Zhou, H., Agresti, J., Weitz, D. A., Fink, G. R., and Stephanopoulos G. (2014) Microfluidic high-throughput culturing of single cells for selection based on extracellular metabolite production or consumption. Nat. Biotechnol. doi:10.1038/ nbt. 2857

200. Clausell-Tormos, J., Lieber, D., Baret, J. C., El-Harrak, A., Miller, O. J., Frenz, L., Blouwolff, J., Humphry, K. J., Köster, S., Duan, H., Holtze, C., Weitz, D. A., Griffiths, A. D., and Merten, C. A. (2008) Droplet-based microfluidic platforms for the encapsulation and screening of mammalian cells and multicellular organisms. Chem. Biol. 15, 427-437

201. Baret, J. C., Miller, O. J., Taly, V., Ryckelynck, M., El-Harrak, A., Frenz, L., Rick, C., Samuels, M. L., Hutchison, J. B., Agresti, J. J., Link, D. R., Weitz, D. A., and Griffiths, A. D. (2009) Fluorescence-activated droplet sorting (FADS): efficient microfluidic cell sorting based on enzymatic activity. Lab Chip 9, 1850-1858

202. Agresti, J. J., Antipov, E., Abate, A. R., Ahn, K., Rowat, A. C., Baret, J. C., Marquez, M., Klibanov, A. M., Griffiths, A. D., and Weitz, D. A. (2010) Ultrahigh-throughput screening in drop-based microfluidics for directed evolution. Proc. Natl Acad. Sci. USA 107, 4004-4009

203. Fallah-Araghi, A., Baret, J. C., Ryckelynck, M., and Griffiths, A. D. (2012) A completely in vitro ultrahigh-throughput droplet-based microfluidic screening system for protein engineering and directed evolution. Lab Chip 12, 882-891

204. Dittrich, P. S., Jahnz, M., and Schwille, P. (2005) A new embedded process for compartmentalized cell-free protein expression and on-line detection in microfluidic devices. Chembiochem 6, 811-814

205. Courtois, F., Olguin, L. F., Whyte, G., Bratton, D., Huck, W. T., Abell, C., and Hollfelder, F. (2008) An integrated device for monitoring time-dependent in vitro expression from single genes in picolitre droplets. Chembiochem 9, 439-446

206. Pekin, D., Skhiri, Y., Baret, J. C., Le Corre, D., Mazutis, L., Salem, C. B., Millot, F., El Harrak, A., Hutchison, J. B., Larson, J. W., Link, D. R., Laurent-Puig, P. Griffiths, A. D., 
and Taly, V. (2011) Quantitative and sensitive detection of rare mutations using dropletbased microfluidics. Lab Chip 11, 2156-2166

207. Shim, J., Ranasinghe, R. T., Smith, C. A., Ibrahim, S. M., Hollfelder, F., Huck, W. T. S., Klenerman, D., and Abell, C. (2013) Ultrarapid generation of femtoliter microfluidic droplets for single-molecule-counting immunoassays. ACS Nano 7, 5955-5964

208. Ehrl, B. N., Liebherr, R. B., and Gorris, H. H. (2013) Single molecule kinetics of horseradish peroxidase exposed in large arrays of femtoliter-sized fused silica chambers. Analyst. 138, 4260

209. Cristobal, G., Arbouet, L., Sarrazin, F., Talaga, D., Bruneel, J. L., Joanicot, M., and Servant, L. (2006) On-line laser Raman spectroscopic probing of droplets engineered in microfluidic devices. Lab Chip 6, 1140-1146

210. Han, Z., Li, W., Huang, Y. and Zheng B. (2009) Measuring rapid enzymatic kinetics by electrochemical method in droplet-based microfluidic devices with pneumatic valves. Anal. Chem. 81, 5840-5845

211. Ahn, K., Kerbage, C., Westervelt, R. M., Link, D. R., and Weitz D. A. (2006) Dielectrophoretic manipulation of drops for high-speed microfluidic sorting devices. Appl. Phys. Lett. 88, 024104

212. Beyer, S., Mak, W. C., and Trau, D. (2007) Reverse-phase LbL-encapsulation of highly water soluble materials by layer-by-layer polyelectrolyte self-assembly. Langmuir 23, 8827-8832

213. Immordino, M. L., Dosio, F., and Cattel, L. (2006) Stealth liposomes: review of the basic science, rationale, andclinical applications, existing and potential. Int. $J$. Nanomedicine 1, 297-315

214. Sahay, G., Querbes, W., Alabi, C., Eltoukhy, A., Sarkar, S., Zurenko, C., Karagiannis, E., Love, K., Chen, D., Zoncu, R., Buganim, Y., Schroeder A., Langer R., and Anderson, D. G. (2013) Efficiency of siRNA delivery by lipid nanoparticles is limited by endocytic recycling. Nat. Biotechnol. 31, 653-658

215. Svenson, S., and Tomalia, D. A. (2005) Dendrimers in biomedical applications-reflections on the field. Adv. Drug Deliv. Rev. 57, 2106-2129

216. McBain, S. C., Yiu, H. P., and Dobson, J. (2008) Magnetic nanoparticles for gene and drug delivery. Int. J. Nanomedicine 3, 169-180

217. Vardharajula, S., Ali, S. Z., Tiwari, P. M., Eroglu, E., Vig, K., Dennis, V. A., and Singh, S. R. (2012) Functionalized carbon nanotubes: biomedical applications. Int. J. Nanomedicine 7, 5361-5374

218. Peyratout, C. S., and Daehne, L. (2004) Tailor-made polyelectrolyte microcapsules: from multilayers to smart containers. Angew. Chem. Int. Ed. 43, 3762-3783

219. De Geest, B. G., De Koker, S., Sukhorukov, G.B., Kreft, O., Parak, W. J., Skirtach, A. G., Demeester, J., De Smedt, S. C., Hennink, W. E. (2009) Polyelectrolyte microcapsules for biomedical applications. Soft Matter 5 282-291

220. Bédard, M. F., De Geest, B. G., Skirtach, A. G., Möhwald, H., Sukhorukov G.B. (2010) Polymeric microcapsules with light responsive properties for encapsulation and release. Adv. Colloid Interface Sci. 158 2-14

221. Caruso, F., Caruso, R. A., and Möhwald, H. (1998) Nanoengineering of inorganic and hybrid hollow spheres by colloidal templating. Science 282, 1111-1114

222. Gil, R. P., del Mercato, L.L., del-Pino, P., Muñoz-Javier, A., Parak, W.J. (2008) Nanoparticle-modified polyelectrolyte capsules. Nanotoday 3, 12-21 
223. Sukhorukov, G. B., Volodkin, D. V., Günther A. M., Petrov, A. I., Shenoy, D. B., and Möhwald, H. (2004) Porous calcium carbonate microparticles as templates for encapsulation of bioactive compounds. J. Mater. Chem. 14, 2073-2081

224. Petrov, A. I., Volodkin, D. V., Sukhorukov, G. B. (2005) Protein-calcium carbonate coprecipitation: a tool for protein encapsulation. Biotechnol. Prog. 21, 918-925

225. Volodkin, D. V., von Klitzing, R., and Möhwald, H. (2010) Pure protein microspheres by calcium carbonate templating. Angew. Chem. 122, 9444-9447

226. Kreft, O., Georgieva, R., Baumler, H., Steup, M., Muller-Rober, B., Sukhorukov, G.B., Möhwald, H. (2006) Red blood cell templated polyelectrolyte capsules: a novel vehicle for the stable encapsulation of DNA and proteins. Macromol. Rapid Commun. 27, 435-440

227. Delcea, M., Yashchenok, A., Videnova, K., Kreft, O., Möhwald, H., Skirtach, A.G. (2010) Multicompartmental micro- and nanocapsules: hierarchy and applications in biosciences. Macromol. Biosci. 10, 465-474

228. Doll, T. A. P. F., Raman, S., Dey, R., and Burkhard, P. (2013) Nanoscale assemblies and their biomedical applications. J. R. Soc. Interface 10, 20120740

229. Petrov, A. I., Volodkin, D. V., and Sukhorukov, G. B. (2005) Protein-calcium carbonate coprecipitation: a tool for protein encapsulation. Biotechnol. Prog. 21, 918-925

230. Dejugnat, C., and Sukhorukov, G.B. (2004) pH-Responsive properties of hollow polyelectrolyte microcapsules templated on various cores. Langmuir 20, 7265-7269

231. Volodkin, D. V., Larionova, N. I., and Sukhorukov, G. B. (2004) Protein encapsulation via porous $\mathrm{CaCO}_{3}$ microparticles templating. Biomacromolecules 5, 1962-1972

232. Yeo, Y., and Park, K. (2004) Characterization of reservoir-type microcapsules made by the solvent exchange method. AAPS PharmSciTech. 5, 10-17

233. Mauser, T., Dejugnat, C., and Sukhorukov, G. B. (2004) Reversible pH-dependent properties of multilayer microcapsules made of weak polyelectrolytes, Macromol. Rapid Commun. 25 1781-1785

234. Xu, W., Choi, I., Plamper, F. A., Synatschke, C. V., Müller A. H. E., and Tsukruk, V. V. (2013) Nondestructive Light-Initiated Tuning of Layer-by-Layer Microcapsule Permeability. ACS Nano 7, 598-613

235. Antipov, A. A., Sukhorukov, G. B. and Mohwald, H. (2003) Infuence of the ionic strength on the polyelectrolyte multilayers' permeability. Langmuir 19, 2444-2448

236. Tong, W., and Gao, C. (2008) Multilayer microcapsules with tailored structures for biorelated applications. J. Mater. Chem. 18, 3799-3812

237. Cortez, C., Tomaskovic-Crook, E., Johnston, A. P. R., Scott, A. M., Nice, E. C., Heath, J. K., Caruso, F. (2007) Influence of size, surface, cell line, and kinetic properties on the speci_c binding of A33 antigen-targeted multilayered particles and capsules to colorectal cancer cells, ACS Nano 1, 93-102

238. Gullotti, E., and Yeo, Y. (2009) Extracellularly activated nanocarriers, a new paradigm of tumor targeted drug delivery. Mol. Pharm. 6, 1041-1051

239. Santos, J. L., Nouri, A., Fernandes, T., Rodrigues, J., and Tomas, H., (2012) Gene delivery using biodegradable polyelectrolyte microcapsules prepared through the layer-bylayer technique. Biotechnol. Progr. 28, 1088-1094

240. Maedaet, H. (2001) The enhanced permeability and retention (EPR) effect in tumor vasculature: the key role of tumor selective macromolecular drug targeting. Adv. Enzyme Regul. 41, 189-207 
241. Heuberger, R., Sukhorukov, G., Voros, J., Textor, M., and Mohwald, H. (2005) Biofunctional polyelectrolyte multilayers and microcapsules: control of non-specific and bio- specific protein adsorption. Adv. Funct. Mater. 15, 357-366

242. Skirtach, A. G., Yashchenok, A. M. and Möhwald, H. (2011) Encapsulation, release and applications of LbL polyelectrolyte multilayer capsules. Chem. Commun. 47, 12736-12746

243. Bedard, M. F., De Geest, B. G., Skirtach, A. G., Möhwald, H., and Sukhorukov, G. B. (2010) Polymeric microcapsules with light responsive properties for encapsulation and release. Adv. Colloid Interface Sci. 158, 2-14

244. Gaponik, N., Radtchenko, I. L., Sukhorukov, and Rogach, A. L. (2004) Luminescent polymer microcapsules addressable by a magnetic field. Langmuir 20, 1449-1452

245. Pavlov, A. M., Saez, V., Cobley, A., Graves, J., Sukhorukov, G. B., and Mason, T. J. (2011) Controlled protein release from microcapsules with composite shells using high frequency ultrasound-potential for in vivo medical use. Soft Matter 7, 4341-4347

246. Tian, Y. Z., Li, Y. L., Wang, Z. F., and Jiang, Y. (2014) Nuclease-responsive DNA-PEI hollow microcapsules for bio-stimuli controlled release. J. Mater. Chem. B 2, 1667-1672

247. Abbaspourrad, A., Datta, S. S., and Weitz, D. A. (2013) Controlling release from pHresponsive microcapsules. Langmuir 29, 12697-12702

248. Skirtach, A. G., Antipov, A. A., Shchukin, D. G., and Sukhorukov, G. B. (2004) Remote activation of capsules containing Ag nanoparticles and IR dye by laser light. Langmuir 20, 6988-6992

249. Shchukin, D. G., Gorin, D. A., and Mohwald H. (2006) Ultrasonically induced opening of polyelectrolyte microcontainers. Langmuir 22, 7400-7404

250. Itoh, Y., Matsusaki, M., Kida, T., and Akashi, M. (2006) Enzyme-responsive release of encapsulated proteins from biodegradable hollow capsules. Biomacromolecules 7, 27152718

251. Kazakova, L. I., Shabarchina, L. I., Anastasova, S., Pavlov, A. M., Vadgama, P., Skirtach, A. G., and Sukhorukov, G. B. (2013) Chemosensors and biosensors based on polyelectrolyte microcapsules containing fluorescent dyes and enzymes. Anal. Bioanal. Chem. 405, 1559-1568

252. Floretti, F., Mendoza-Palomares, C., Helms, M., Al Alam, D., Richert, L., Arntz, Y., Rickenbach, S., Garnier, F., Haikel, Y., Gangloff, S. C., and Benkirane-Jessel, N. (2010) Nanostructured assemblies for dental application. ACS Nano 4, 3277-3287

253. De Cock, L. J., Lenoir, J., De Koker, S., Vermeersch, V., Skirtach, A. G., Dubruel, P., Adriaens, E., Vervaet, C., Remon, J. P., and De Geest, B. G. (2011) Mucosal irritation potential of polyelectrolyte multilayer capsules. Biomaterials 32, 1967-1977

254. Shimomura, H., Gemici, Z., Cohen, R. E., and Rubner, M. F. (2010) Layer-by-layerassembled high-performance broadband antireflection coatings. ACS Appl. Mater. Interfaces 2, 813-820

255. Jain, R. K. (1987) Transport of molecules in the tumor interstitium :a review. CancerRes. 47, 3039-3051

256. Serpe, L. (2006) Conventional chemotherapeutic drug nanoparticles for cancer treatment, in: C.S.S.R. Kumar (Ed.). Nanomaterials for Cancer Therapy 6, Wiley-VCH

257. Karamitros, C. S., Yashchenok, A. M., Möhwald, H., Skirtach, A. G., and Konrad, M. (2013) Preserving catalytic activity and enhancing biochemical stability of the therapeutic enzyme asparaginase by biocompatible multilayered polyelectrolyte microcapsules. Biomacromolecules 14, 4398-4406 
258. Jabr-Milane, L.S., van Vlerken, L.E., Mansoor, S.Y., and Amiji, M. (2008) Multifunctional nanocarriers to overcome tumor drug resistance. Cancer Treat. Rev. 34, 592-602

259. Palamà, I.E., Coluccia, M.A.L., della Torre, A., Vergaro, V., Perrone, E., Cingolani, R., Rinaldi, R., and Leporatti S. (2010) Multilayered polyelectrolyte capsules and coated colloids: cytotoxicity and uptake by cancer cells. Sci. Adv. Mater. 2, 138-150

260. De Koker, S., De Geest, B.G., Cuvelier, C., Ferdinande, L., Deckers, W., Hennink, W.E, De Smedt, S., and Mertens, N. (2007) In vivo cellular uptake, degradation, and biocompatibility of polyelectrolyte microcapsules. Adv. Funct. Mater. 17, 3754-3763

261. Peroutka, R. J., 3rd, Orcutt, S. T., Strickler, J. E., and Butt, T. R. (2011) SUMO fusion technology for enhanced protein expression and purifica- tion in prokaryotes and eukaryotes. Methods Mol. Biol. 705, 15-30

262. Bradford, M. M. (1976) A rapid and sensitive method for the quantitation of microgram quantities of protein utilizing the principle of protein-dye binding. Anal. Biochem. 72, 248 $-254$

263. Derst, C., Henseling, J., and Röhm, K. H., (1992) Probing the role of thre- onine and serine residues of E.coli asparaginase II by site-specific mu- tagenesis. Protein Eng. 5, 785-789

264. Balcao, V. M., Mateo, C., Fernández-Lafuente, R., Malcata, F. X., and Guisa 'n, J. M. (2001) Structural and functional stabilization of L-asparagi- nase via multisubunit immobilization onto highly activated supports. Biotechnol. Prog. 17, 537-542

265. Karamitros, C. S., Lim, J., and Konrad, M. (2014) An Amplex Red-based fluorometric and spectrophotometric assay for L-asparaginase using its natural substrate. Anal. Biochem. 445, 20-23

266. Cadwell, R. C., and Joyce, G. F. (1992) Randomization of genes by PCR mutagenesis. Genome Res. 2, 28-33

267. Cadwell, R. C., and Joyce, G. F. (1942) Mutagenic PCR. Genome Res. 3, 136-140

268. Ho, S. N., Hunt, H. D., Horton, R. M., Pullen, J. K., and Pease, L. R. (1989) Sitedirected mutagenesis by overlap extension using the polymerase chain reaction. Gene $\mathbf{7 7}$, 51-59

269. Matulis, D., Kranz, J. K., Salemme, F. R., and Todd, M. J. (2005) Thermodynamic stability of carbonic anhydrase: measurements of binding affinity and stoichiometry using ThermoFluor. Biochemistry 44, 5258 -5266

270. Boivin, S., Kozak, S., and Meijers, R. (2013) Optimization of protein purification and characterization using Thermofluor screens. Protein Expr. Purif. 91, 192-206

271. Drummond, D. A., Iverson, B. L., Georgiou, G., and Arnold, F. H. (2005) Why higherror-rate random mutagenesis libraries are enriched in functional and improved proteins. J. Mol. Biol. 350, 806-816

272. Reetz, M. T. (2013) The importance of additive and non-additive mutational effects in protein engineering. Angew. Chem. Int. Ed. 52, 2-11

273. McCullum, E. O., Williams, B. A. R., Zhang, J., and Chaput, J. C. (2010) Random mutagenesis by error-prone PCR. Methods in Molecular Biology 634, 103-109

274. Lim, J., Vrignon, J., Gruner, P., Karamitros, C. S., Konrad, M., and Baret, J. C. (2013) Ultra-high throughput detection of single cell $\beta$-galactosidase activity in droplets using micro-optical lens array. App. Phys. Lett. 103, 203704 
275. Jeong, K. J., Seo, M. J., Iverson, B. L., and Georgiou, G. (2007) APEx 2-hybrid, a quantitative protein-protein interaction assay for antibody discovery and engineering. Proc. Natl Acad. Sci. USA 104, 8247-8252

276. Holtze, C., Rowat, A. C., Agresti, J. J., Hutchison, J. B., Angile, F. E., Schmitz, C. H., Köster, S., Duan, H., Humphry, K. J., Scanga, R. A., Johnson, J. S., Pisignano, D., and Weitz, D. A. (2008) Biocompatible surfactants for water-in-fluorocarbon emulsions. Lab Chip 8, 1632-1639

277. Hueber, A., Srisa-Art, M., Holt, D., Abell, C., Hollfelder, F., deMello, A. J., and Edel, J. B. (2007) Quantitative detection of protein expression in single cells using droplet microfluidics. Chem. Commun. 1218-1220

278. Skhiri, Y., Gruner, P., Semin, B., Brosseau, Q., Pekin, D., Mazutis, L., Goust, V., Kleinschmidt, F., El Harrak, A., Hutchison, J. B., Mayot, E., Bartolo, J. F., Griffiths, A. D., Taly, V., and Baret, J. C. (2012) Dynamics of molecular transport by surfactants in emulsions. Soft Matter 8, 10618-10727

279. Myers, J. A., Curtis, B. S., and Curtis, W. R. (2012) Improving accuracy of cell and chromophore concentration measurements using optical density. BMC Biophysics 6:4

280. Daugelavicius, R., Bakiene, E., Bamford, D. H. (2000) Stages of Polymyxin B interaction with the Escherichia coli cell envelope. Antimicrob. Agents Chemother. 44, 2969-2978

281. Hayhurst, A., and Harrie, W. J. (1999) Escherichia coli Skp chaperone coexpression improves solubility and phage display of single-chain antibody fragments. Protein Expr. Purif. 15, 336-343

282. Liang, W., Mason, A. J., and Lam, J. K. W. (2013) Western blot evaluation of siRNA delivery by $\mathrm{pH}$ responsive peptides. Methods Mol. Bio. 986, 73-87

283. Arayanarakool, R., Shui, L., Kengen, S. W. M., van den Berg, A., and Eijkel, J. C. T. (2013) Single-enzyme analysis in a droplet-based micro- and nanofluidic system. Lab Chip 13, 1955-1962

284. Gorris, H. H. and Walt, D. R. (2010) Analytical chemistry on the femtoliter scale. Angew. Chem., Int. Ed. 49, 3880-3895

285. Yakushi, T., Masuda, K., Narita, S., Matsuyama, S., and Tokuda, H. (2000) A new ABC transporter mediating the detachment of lipid-modified proteins from membranes. Nat. Cell Biol. 2, 212-218

286. Jain, M., Nilsson, R., Sharma, S., Madhusudhan, N., Kitami, T., Souza, A. L., Kafri, R., Kirschner, M. W., Clish, C. B., and Mootha, V. K. (2012). Metabolite profiling identifies a key role for glycine in rapid cancer cell proliferation. Science 336, 1040-1044

287. Chaneton, B., Hillmann, P., Zheng, L., Martin, A. C., Maddocks, O. D., Chokkathukalam, A., Coyle, J. E., Jankevics, A., Holding, F. P., Vousden, K. H., et al. (2012). Serine is a natural ligand and allosteric activator of pyruvate kinase M2. Nature 491, 458-462

288. Karamitros, C. S., and Konrad, M. (2014) Bacterial co-expression of the $\alpha$ and $\beta$ protomers of human 1-asparaginase-3: Achieving essential N-terminal exposure of a catalytically critical threonine located in the $\beta$-subunit. Protein Expr. Purif. 93, 1-10

289. Plainkum, P., Fuchs, S. M., Wiyakrutta, S. and Raines R. T. (2002) Creation of a zymogen. Nat. Struct. Biol. 10, 115-119

290. Bliven, S., and Prlic, A. (2012) Circular permutation in proteins. PLoS Comp. Biol. 8, e1002445 
291. Boanca, G., Sand, A., and Barycki, J. J. (2006) Uncoupling the enzymatic and autoprocessing activities of Helicobacter pylori-glutamyltranspeptidase. J. Biol. Chem. 281, 19029-19037

292. Cunningham, B. A., Hemperly, J. J., Hopp, T. P., Edelman, G. M. (1979) Favin versus concanavalin A: Circularly permuted amino acid sequences. Proc Natl Acad Sci U S A 76, 3218-3222

293. Carrington, D. M., Auffret, A., Hanke, D. E. (1985) Polypeptide ligation occurs during post-translational modification of concanavalin A. Nature 313, 64-67

294. Yu, Y., and Lutz, S. (2011) Circuler permutation: a different way to engineer enzyme structure and function. Trends Biotechnol. 29, 18-25

295. Daugherty, A. B., Govindarajan, S., and Lutz, S. (2013) Improved biocatalysts from a synthetic circular permutation library of the flavin-dependent oxidoreductase old yellow enzyme. J. Am. Chem. Soc. 135, 14425-14432

296. Romero, P. A., and Arnold, F. H. (2009) Exploring protein fitness landscapes by directed evolution. Nat. Rev. Mol. Cell Biol. 10, 866-876

297. Skinner, M. M., and Terwilliger, T. C. (1996) Potential use of additivity of mutational effects in simplifying protein engineering. Proc Natl Acad Sci U S A 93, 10753-10757

298. Kim, S. H., Pokhrel, S., and Yoo, Y. J. (2008) Mutation of non-conserved amino acids surrounding catalytic site to shift $\mathrm{pH}$ optimum of Bacillus circulans xylanase. J. Mol. Catal., B Enzym. 55, 130-136

299. Patrick, W. M., and Firth, A. E. (2005) Strategies and computational tools for improving randomized protein libraries. Biomol. Engineering 22, 105-112

300. Luscombe, N. M., Laskowski, R. A., and Thornton, J. M. (2001) Amino acid-base interactions: a three-dimensional analysis of protein-DNA interactions at an atomic level. Nucleic Acids Res. 29, 2860-2874

301. Yuen, C. M., and Liu, D. R. (2007) Dissecting protein structure and function using directed evolution. Nat. Methods 12, 995-997

302. Guo, H. H., Choe, J., and Loeb, L. A. (2004) Protein tolerance to random amino acid change. Proc Natl Acad Sci U S A 101, 9205-9210

303. Aita, T., Uchiyama, H., Inaoka, T., Nakajima, M., Kokubo, T. and Husimi, Y. (2000) Analysis of a local fitness landscape with a model of the rough Mt. Fuji-type landscape: application to prolyl endopeptidase and thermolysin. Biopolymers 54, 64-79

304. Huang, Z., Wagner, C. R., and Benkovic, S. J. (1994) Nonadditivity of mutational effects at the folate binding site of Escherichia coli dihydrofolate reductase. Biochemistry 33, 11576-11585

305. Wells, J. A., Powers, D. B., Bott, R. R., Graycar, T. P., and Estell, D. A. (1987)Designing substrate specificity by protein engineering of electrostatic interactions. Proc Natl Acad Sci U S A 84, 1219-1223

306. Wong, T. S., Roccatano, D., and Schwaneberg, U. (2007) Steering directed protein evolution: strategies to manage combinatorial complexity of mutant libraries. Environ. Microbiol 9, 2645-2659

307. Bloom, J. D., Arnold, F. H., and Wilke, C. O. (2007) Breaking proteins with mutations: threads and thresholds in evolution. Molecular System Biology 3:76

308. Weinreich, D. M., Delaney, N. F., Depristo, M. A., Hartl DL (2006) Darwinian evolution can follow only very few mutational paths to fitter proteins. Science 312, 111-114 
309. Gumulya, Y., Sanchis, J., Reetz, M. T. (2012) Many pathways in laboratory evolution can lead to improved enzymes: how to escape from local minima. ChemBioChem 13, 1060-1066

310. Tracewell, C. A. and Arnold, F. H. (2009) Directed enzyme evolution: climbing fitness peaks one amino acid at a time. Curr. Opin. Chem. Biol. 13, 3-9

311. Carballeira, J. D., Krumlinde, P., Bocola, M., Vogel, A., Reetz, M. T., Backvall, J. E. (2007) Directed evolution and axial chirality: optimization of the enantioselectivity of Pseudomonas aeruginosa lipase towards the kinetic resolution of a racemic allene. Chem Commun (Camb)1913-1915

312. Cooney, D. A., Capizzi, R., and Handschumacher, R. (1970) Evaluation of l-asparagine metabolism in animals and man. Cancer Res 30, 929-935

313. Reetz, M. T., Wang, L. and Bocola, M. (2006) Directed evolution of enantioselective enzymes: iterative cycles of CASTing for probing protein-sequence space. Angew. Chem. Int. Ed. 45, 1236-1241

314. O'Loughlin, T. L., Patrick, W. M., Matsumura, I. (2006) Natural history as a predictor of protein evolvability. Protein Eng. Des. Sel. 19, 439-442

315. Lanvers, C, Pinheiro, J. P. V., Hempel, G., Wuerthwein, G. and Boos, J. (2002) Analytical validation of a microplate reader-based method for the therapeutic drug monitoring of L- asparaginase in human serum. Anal. Biochem. 309, 117-126

316. Francisco, J. A., Earhart, C. F., and Georgiou G. (1992) Transport and anchoring of betalactamase to the external surface of Escherichia coli. Proc. Natl. Acad. Sci. U S A 89, 2713-2717

317. Georgiou, G., Stathopoulos, C., Daugherty, P. S., Nayak, A. R., Iverson, B. L. and Curtiss, R. 3rd. (1997) Display of heterologous proteins on the surface of microorganisms: from the screening of combinatorial libraries to live recombinant vaccines. Nat. Biotechnol. 15, 29-34

318. Freudl, R., MacIntyre, S., Degen, M. and Henning, U. (1986) Cell surface exposure of the outer membrane protein OmpA of Escherichia coli K-12. J. Mol. Biol. 188, 491-494

319. Earhart, C. F. (2000) Use of an Lpp-OmpA fusion vehicle for bacterial surface display. Methods Enzymol. 326, 506-516

320. Brundiek, H. B., Evitt, A. S., Kourist, R., and Bornscheuer, U. T. (2012) Creation of a lipase highly selective for trans fatty acids by protein engineering. Angew. Chem. Int. Ed. 51, 412-414

321. Li, J., Mahajan, A., and Tsai, M. D. (2006) Ankyrin repeat: a unique motif mediating protein-protein interactions. Biochemistry 45, 15168 -15178

322. Wang, A., and Dennis, E. A. (1999) Mammalian lysophospholipases. Biochim. Biophys. Acta 1439, 1-16

323. Hansen, H. A., and Emborg, C. (1994) Extra- and intracellular amino acid concentrations in continuous Chinese hamster ovary cell culture. Appl. Microbiol. Biotechnol. 41, $560-564$

324. Kamata, K., Mitsuya, M., Nishimura, T., Eiki, J., and Nagata, Y. (2004) Structural basis for allosteric regulation of the monomeric allosteric en- zyme human glucokinase. Structure 12, $429-438$

325. Zhang, J., Li, C., Chen, K., Zhu, W., Shen, X., and Jiang, H. (2006) Conformational transition pathway in the allosteric process of human glucoki- nase. Proc. Natl. Acad. Sci. U.S.A. 103, $13368-13373$ 
326. Porter, C. M., and Miller, B. G. (2012) Cooperativity in monomeric enzymes with single ligand-binding sites. Bioorg. Chem. 43, 44-50

327. Ricard, J., Meunier, J. C., and Buc, J. (1974) Regulatory behavior of monomeric enzymes. 1. The mnemonical enzyme concept. Eur. J. Biochem. 49, 195-208

328. Cardenas, M. L., Rabajille, E., and Niemeyer, H. (1984) Suppression of kinetic cooperativity of hexokinase D (glucokinase) by competitive inhibitors. A slow transition model. Eur. J. Biochem. 145, 163-171

329. De Temmerman, M. L., Rejman, J., Vandenbroucke, R. E., De Koker, S., Libert, C., Grooten, J., Demeester, J., Gander, B., and De Smedt, S. C. (2012) Polyelectrolyte LbL microcapsules versus PLGA microparticles for immunization with a protein antigen. $J$ Control. Release 158, 233-239

330. Dierendonck, M., De Koker, S., Vervaet, C., Remon, J. P., and De Geest, B. (2012) Interaction between polymeric multilayer capsules and immune cells. J. Control. Release 161, 592-599 


\section{Publication List}

1. Su, Y., Karamitros, C. S., Nomme, J., McSorley, T., Konrad, M., and Lavie, A. (2013) Free glycine accelerates the autoproteolytic activation of human asparaginase. Chem. Biol. 20, 533- 540

2. Karamitros, C. S., Lim, J., and Konrad, M. (2014) An Amplex Red-based fluorometric and spectrophotometric assay for L-asparaginase using its natural substrate. Anal. Biochem. 445, 20-23

3. Karamitros, C. S., and Konrad, M. (2014) Bacterial co-expression of the $\alpha$ and $\beta$ protomers of human 1-asparaginase-3: Achieving essential N-terminal exposure of a catalytically critical threonine located in the $\beta$-subunit. Protein Expr. Purif. 93, 1-10

4. Karamitros, C. S., Yashchenok, A. M., Möhwald, H., Skirtach, A. G., and Konrad, M. (2013) Preserving catalytic activity and enhancing biochemical stability of the therapeutic enzyme asparaginase by biocompatible multilayered polyelectrolyte microcapsules. Biomacromolecules 14, 4398-4406

5. Karamitros, C. S., and Konrad, M. (2014) Human 60-kDa lysophospholipase contains an $\mathrm{N}$ - terminal L-asparaginase domain that is allosterically regulated by L-asparagine. J. Biol. Chem. 289, 12962-12975

6. Lim, J., Vrignon, J., Gruner, P., Karamitros, C. S., Konrad, M., and Baret, J. C. (2013) Ultra-high throughput detection of single cell $\beta$-galactosidase activity in droplets using micro-optical lens array. Appl. Physic. Lett. 103, 203704-1

\section{Book Chapters}

1. Platis, D., Kotzia, G. A., Axarli, I. A., Chronopoulou, E., Karamitros, C.S., Labrou, N.E (2012). Biocatalysis, Enzyme Engineering and Enzyme Biotechnology, In B. Simpson, et al., (Ed), Food Biochemistry and Food Processing (2nd Edition), June 2012, WileyBlackwell Publishing Company USA 


\section{Curriculum Vitae}

Full Name: Christos S. Karamitros

Date of Birth: 26.11.1984

Place of Birth: Athens, Hellas

Citizenship: Hellenic

\section{Education}

June 2002: High school graduation.

September 2002-September 2008: B.Sc., Agricultural University of Athens, Department of Biotechnology.

November 2008-June 2010: M.Sc. in Biotechnology, Agricultural University of Athens, Department of Biotechnology, Laboratory of Enzyme Technology.

October 2010-May 2014: Ph.D. studies, Goettingen University and Max Planck Institute of Biophysical Chemistry

\section{$\underline{\text { Research Experience }}$}

May 2007 - September 2007: Four months practical exercise at the Agricultural University of Athens, Department of Biotechnology, Laboratory of Enzyme Technology. This practical course aimed at the improvement of my knowledge on modern techniques in molecular biology and enzyme biotechnology.

September 2007-September 2008: Last year undergraduate dissertation project. The research project focused on the immobilization and study of the structural stability of the enzyme glutathione S-transferase (GST) from maize.

November 2008-March 2010: Master thesis experimental project. The research work focused on the: cloning, expression and purification of the anticancer enzyme L-asparaginase from Erwinia chrysanthemi, extracellularly produced in E.coli.

October 2010-May 2014: Ph.D. thesis work at Max Planck Institute for Biophysical Chemistry.

June 2012-September 2012: Visitor at the Institute of Cellular and Molecular Biology (ICMB) at the University of Texas at Austin under the supervision of Dr. George Georgiou. Research work: FACS-based (Fluorescence Activated Cell Sorting) high-throughput screening analysis of enzyme mutant libraries aiming at the isolation of catalytically improved variants. 


\section{Honors and Awards}

- Max Planck fellowship for doctoral studies.

- Selected for participation to the EMBO practical course "Modern biophysical methods for protein-ligand interactions", 21-25th October 2013, Oulu, Finland (limited number of participants, who were selected according to their CV and academic achievements).

\section{Conference and Scientific Meetings Participations}

- International conference of Molecular Biology, December 2007, Athens, Hellas.

- International conference of Biotechnology, February 2008, Athens, Hellas.

- First Hellenic conference of Agricultural Biotechnology, October 2009, Agricultural University of Athens, "The interaction of hGSTP1-1 with herbicides and insecticides: development of enzyme biosensor for environmental use".

- First Workshop on Pharmacokinetic/Pharmacodynamic Modeling, 7-8th December 2009, Patras, Hellas, 'Basic PK/PD modeling concepts and the role and utility of PK/PD in drug development" (limited number of participants, who were selected according to their CV and academic achievements).

- GGNB Science Day, 29th November 2011, Goettingen, Germany, “Asparaginase as anticancer drug. New Challenges and Perspectives".

- EMBO Chemical Biology conference, 26-29th September 2012, Heidelberg, Germany, "Enzymes for Leukemia Therapy: Asparaginase as anti-cancer Drug. Autoproteolytic activation of human asparaginase induced by free glycine".

- Protein Society Meeting, 20-23nd July 2013, Boston, MA, USA, Poster presentation "Free glycine accelerates the activation of human Asparaginase 3".

- Horizons in Molecular Biology, 9-12th September 2013, Goettingen, Germany, "Enzymes for Leukemia Therapy: Asparaginase as anti-cancer Drug. Autoproteolytic activation of human asparaginase induced by free glycine".

- International Symposium of the German Society for Biochemistry and Molecular Biology, 3-6th October 2013, Frankfurt, Germany, "Free glycine accelerates the activation of human Asparaginase 3".

- GGNB Science Day, 9th December 2013, Goettingen, Germany, "Therapeutic enzymes for the treatment of leukemia: Autoproteolytic activation of human asparaginase by free glycine and in vitro killing of cancer cells by encapsulated E.coli asparaginase".

\section{Oral Presentations}

- Max Planck Campus seminar, 23nd May 2012, "Engineering of enzymes for amino acid depletion therapy in leukemia"

- EC COST Thematic Workshop: Nano-scaled arrangements of proteins, aptamers, and other nucleic acid structures- and their potential applications, 8-9th October 2013, Leipzig, Germany, "Biocompatible nanoparticles and polyelectrolyte microcapsules as carriers of protein drugs: Asparaginases as approved enzymes in therapy of leukemia" 